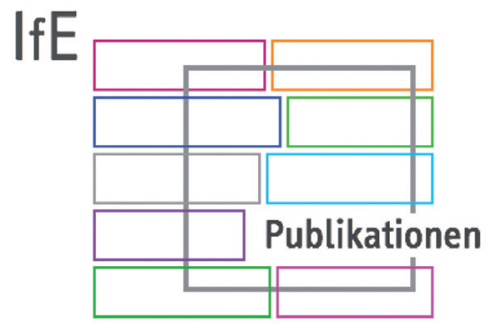

Erziehungswissenschaftliche

Studien

Band 1

Christina Radicke

Familiale Tradierungsprozesse in einer Drei-Generationen-Perspektive

Kontinuierliche Veränderungen veränderliche Kontinuitäten 

Christina Radicke

Familiale Tradierungsprozesse in einer Drei-Generationen-Perspektive

Dieses Werk ist lizenziert unter einer

Creative Commons

Namensnennung - Weitergabe unter gleichen Bedingungen

4.0 International Lizenz.

(c) (†) (?) 
erschienen als Band 1 der Reihe „Erziehungswissenschaftliche Studien“ im Universitätsverlag Göttingen 2014 
Christina Radicke

Familiale

Tradierungsprozesse in einer Drei-Generationen-

Perspektive

Kontinuierliche Veränderungen veränderliche Kontinuitäten

Erziehungswissenschaftliche Studien

Band 1

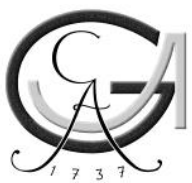

Universitätsverlag Göttingen

2014 


\section{Bibliographische Information der Deutschen Nationalbibliothek}

Die Deutsche Nationalbibliothek verzeichnet diese Publikation in der Deutschen Nationalbibliographie; detaillierte bibliographische Daten sind im Internet über $<$ http://dnb.ddb.de $>$ abrufbar.

Herausgeberinnen der Reihe „Erziehungswissenschaftliche Studien“:

Prof. Dr. Klaus-Peter Horn (Schriftleitung), Prof. Dr. Kerstin Rabenstein, Prof. Dr. Tobias C. Stubbe, Prof. Dr. Hermann Veith

Georg-August-Universität Göttingen

Institut für Erziehungswissenschaft

Waldweg 26

37073 Göttingen

(http://www.uni-goettingen.de/ife)

Anschrift der Autorin

Christina Radicke

Email: cr@radicke.org

Dieses Buch ist auch als freie Onlineversion über die Homepage des Verlags sowie über den Göttinger Universitätskatalog (GUK) bei der Niedersächsischen Staats- und Universitätsbibliothek Göttingen (http://www.sub.uni-goettingen.de) erreichbar. Es gelten die Lizenzbestimmungen der Onlineversion.

Satz und Layout: LaTeX, LyX; Lars Müller

Umschlaggestaltung: Margo Bargheer

(C) 2014 Universitätsverlag Göttingen

http:/ / univerlag.uni-goettingen.de

ISBN: 978-3-86395-172-6

ISSN: 2199-5133 


\section{Inhaltsverzeichnis}

Einleitung $\quad 9$

1 Familiale Tradierungsprozesse: Einblicke in den Forschungsstand 13

1.1 Die Familie im Blickpunkt der sozialwissenschaftlichen Forschung . . . . 14

1.1 .1 Die Familie . . . . . . . . . . . . . . . . . . . . . 14

1.1.2 Die Familie im gesellschaftlichen Wandel . . . . . . . . . . 17

1.2 Familiale Erziehung . . . . . . . . . . . . . . . . . . . 21

1.2.1 Der Erziehungsbegriff . . . . . . . . . . . . . . . . 21

1.2.2 Familiale Beziehungssystematiken . . . . . . . . . . . 22

1.2.3 Die Wertorientierungen im gesellschaftlichen Wandel . . . . . . 25

1.2.4 Familienerziehung im gesellschaftlichen Wandel . . . . . . . . . . 28

1.2.5 Familiale Rollen im gesellschaftlichen Wandel . . . . . . . . . . 36

1.3 Familiale Tradierungsprozesse . . . . . . . . . . . . . . 42

1.3.1 Die innerfamiliale Verhandlung von Geschichte . . . . . . . . . 42

1.3.2 Tradierung von Werteeinstellungen _... . . . . . . . 43

1.3.3 Tradierungsprozesse im Zeichen gesellschaftlichen Wandels . . . . 45

2 Die theoretische Rahmung familialer Tradierungsprozesse: Gedächtnisund Generationenperspektive

2.1 Die soziale Prägung des Gedächtnisses . . . . . . . . . . . . . . . . . 50

2.1.1 Halbwachs' kollektives Gedächtnis . . . . . . . . . . . . . . 50

2.1.2 Kommunikatives und kulturelles Gedächtnis . . . . . . . . . . . 52

2.1.3 Implizites und explizites Gedächtnis . . . . . . . . . . . . 53

2.1.4 Konstruktivität und Gegenwartsbezug . . . . . . . . . . . . . . 54

2.2 Das Familiengedächtnis als intergenerationelles Gedächtnis . . . . . . 57

2.3 Dynamisierung des Erinnerungsparadigmas durch die Einbeziehung der Generationenperspektive . . . . . . . . . . . . . . . . . 59

2.3.1 Die historisch-soziologische Generationenperspektive . . . . . . . 60

2.3.2 Die familial-pädagogische Generationenperspektive . . . . . . . 63 
3 Methode und Forschungsdesign 69

3.1 Methodologische und methodische Grundannahmen . . . . . . . . . . . 69

3.2 Interviewkonzeption . . . . . . . . . . . . . . 75

3.3 Durchführung und Sample . . . . . . . . . . . . . . . . . 76

4 Wege und Brücken durch die Zeit: Familiale Tradierungsprozesse in $\begin{array}{ll}\text { einer Drei-Generationen-Perspektive } & \mathbf{8 1}\end{array}$

4.1 Flexibel erscheinende Tradierungslogiken . . . . . . . . . . . . 82

4.1.1 Familie Dienel/Ebel: Religiöse Alltagsprägung . . . . . . . . . 82

4.1.2 Familie Cronert/Jäger: Intergenerationelle Solidarität und Hilfe . 109

4.1.3 Einordnung der Tradierungslogik unter Berücksichtigung der gesellschaftlichen Bezüge . . . . . . . . . . . . . . . . 134

4.2 Geschlossene Tradierungslogiken . . . . . . . . . . . . . . . . . 143

4.2.1 Familie Mahler/Ahrens: Vertraute Horizonte vs. Horizonterweiterung oder Milieuorientierung . . . . . . . . . . . 143

4.2.2 Familie Nolte/Gümper: Repräsentative Höchstleistungen . . . 168

4.2.3 Einordnung der Tradierungslogik unter Berücksichtigung der gesellschaftlichen Bezüge . . . . . . . . . . . . . . . 193

4.3 Diffuse Tradierungslogiken . . . . . . . . . . . . . . . . . . 202

4.3.1 Familie Beyer/Schmidt: Familiale Bindungen und Verantwortlichkeiten . . . . . . . . . . . . . . . . 202

4.3.2 Einordnung der Tradierungslogik unter Berücksichtigung der gesellschaftlichen Bezüge . . . . . . . . . . . . . . . . 229

4.4 Familiale Tradierungslogiken im Überblick . . . . . . . . . . . . . 233

5 Bildungsstatus, Generation und Region: Familiale Tradierungslogiken $\begin{array}{ll}\text { und ihre gesellschaftliche Einbettung } & 237\end{array}$

5.1 Bildungsmilieu . . . . . . . . . . . . . . . . . . . 237

5.1 .1 Religiosität . . . . . . . . . . . . . 238

5.1 .2 Bildung . . . . . . . . . . . . . . . . 239

5.1.3 Familiale Gemeinschaft . . . . . . . . . . . . . . . . . 240

5.2 Intragenerationelle Gemeinsamkeiten und intergenerationelle Unterschiede . . . . . . . . . . . . . . . . . . . . . . . . . 240

5.2.1 Generationendifferenz und generationelle Topoi . . . . . . . . . 241

5.2.2 Erziehungsarrangements: Von der selbstverständlichen Ordnungszur Angebotsstruktur . . . . . . . . . . . . . . . . . . 249

5.2.3 Familienleitbilder: Von der Dethematisierung zur Thematisierung von Konflikten . . . . . . . . . . . . . . . . . . . 257

5.3 Regionale Besonderheiten und Bezüge . . . . . . . . . . . . . . . 260

5.3.1 Familienleitbilder . . . . . . . . . . . . . . . . 261

5.3.2 Familie und Gesellschaftssystem . . . . . . . . . . . . . 263

5.3.3 Optimale Kindesförderung und elterliche Ratsuche . . . . . . . 265 
Inhaltsverzeichnis

5.3.4 Großelternschaft . . . . . . . . . . . . . . . . . . . . 269

6 Familiale Tradierungsprozesse im gesellschaftlichen Wandel: Fazit und Ausblick

7 Danksagung 283

8 Literatur 285

9 Anhang 307

9.1 Sampleübersicht . . . . . . . . . . . . . . . . . . . . . . . . 307

9.2 Transkripitionsregeln . . . . . . . . . . . . . . . . . 308 



\section{Einleitung}

Im Zentrum dieser Studie stehen familiale Tradierungsprozesse im Verlauf von drei Generationen. Konkret stellt sich die Frage, welche Inhalte im Zuge der Erziehung in Familien weitergegeben, angepasst, verändert oder aufgegeben werden und wie die Prozesse der Vermittlung und Aneignung gestaltet sind.

Die Aktualität der Fragestellung lässt sich aus den zahlreichen populärwissenschaftlichen Publikationen zum Thema ableiten (z.B. Bueb 2006, Gaschke 2001). Der Grundtenor dieser Veröffentlichungen lautet: Es finde ein Werteverlust in der Gesellschaft statt, der u.a. in einem Zusammenhang mit der veränderten bzw. nicht mehr erfolgenden elterlichen Erziehung stehe. Die Kritik mündet in die Forderung, zu ,alten Werten' in der Erziehung zurückzukehren. Dieses skizzierte Verlustszenario findet Widerhall im öffentlichen Raum und führt zu einer verstärkten, öffentlichen - auch kontroversen Diskussion der Thematik, sei es durch eine erneute Bestimmung der elementaren Bestandteile von Erziehung (Hentig 2009) oder durch eine kritische Auseinandersetzung mit den beschworenen Verfallsszenarien und vorgeschlagenen Kurskorrekturen sowie durch das Einbringen eigener Vorschläge zur Erziehung (Brumlik 2007, Andresen/ Brumlik/Koch 2010). Statt sich in diese normative Debatte um Erziehung einzureihen, wird in dieser Studie ein anderer Weg eingeschlagen. Die angedeutete Verfallsperspektive wird zum Anlass genommen, Formen und Inhalte der Erziehung im Generationenverlauf genauer zu betrachten und neben Wandlungsprozessen - die sich übrigens weder automatisch als negativ noch als positiv konnotieren lassen - auch Kontinuitäten zu beachten, also die vielfach beschworene Verfallsdiagnose kritisch zu hinterfragen.

Zur Bearbeitung des Themas wurde ein qualitatives Forschungsdesign gewählt. Es wurden in zehn Familien einer ländlichen, katholisch geprägten Region in Niedersachsen und Thüringen, die in der Vergangenheit zum innerdeutschen Grenzgebiet gehörte, offene Leitfadeninterviews mit narrativen Passagen geführt. Jeweils drei Familienangehörige aufeinanderfolgender Generationen wurden befragt. Anhand dieser Daten sollen Muster der Tradierung herausgearbeitet werden, Aussagen über ihre repräsentative Verteilung hingegen werden nicht angestrebt.

Die Einbeziehung von drei familialen Generationen erlaubt es, Tradierungsprozesse in ihrer Komplexität zu erfassen, da sowohl die Sichtweise der Erziehenden als auch der Erzogenen eingebunden sowie die Entwicklung von den Großeltern bis zu ihren Enkeln verfolgt werden kann. Die Leitfragen in diesem Zusammenhang lauten: Welche Inhalte der Erziehung finden sich in den einzelnen Familien und wie entwickeln sie sich im Generationenverlauf? Erhalten sie sich relativ unverändert, werden sie modifiziert oder setzen sich nachfolgende Generationen von ihnen ab? Wie versucht die jeweils erziehende Generation die Inhalte zu vermitteln und wie verhält sich die nachfolgende 


\section{Einleitung}

Generation hierzu? Gibt es unterschiedliche inhaltliche Schwerpunkte und Arten der Tradierung in den beiden untersuchten Teilgebieten? Inwiefern korrespondieren diese Entwicklungslinien mit den jeweiligen historischen Kontexten? Wie wirken sich die jeweiligen gesellschaftlichen Rahmenbedingungen und ihre Veränderungen auf die konkreten Formen der Erziehung in den einzelnen Familien aus?

Mit dieser Fragestellung lassen sich in vertikaler Perspektive familiale Tradierungsprozesse untersuchen, die erst durch die Einbeziehung der gesellschaftlichen Rahmenbedingungen in ihrer Vielschichtigkeit erfasst werden. Es können Aussagen darüber getroffen werden, welche Inhalte der Erziehung bedeutsam bleiben bzw. an Relevanz verlieren. Ebenso lässt sich bestimmen, inwiefern sich die Erziehung der mittleren Generation von der vorhergehenden unterscheidet bzw. welche Anknüpfungspunkte vorhanden sind. Hierbei spielt die Bewertung der eigenen Erziehungserfahrungen eine zentrale Rolle, die durch das gewählte Sample für alle drei Generationen beschreibbar ist. Insgesamt können auf diese Weise bezogen auf die Inhalte und Formen der Tradierung fundierte Erkenntnisse über Kontinuität und Wandel bzw. deren Zusammenwirken gewonnen werden.

Ein Wechsel der Analyseebene erlaubt es darüber hinaus, regionale und generationelle Spezifika sowie den Bildungsstand einzubeziehen. Die besondere Lage der Untersuchungsregion eröffnet interessante Vergleichsperspektiven. Es stellt sich die Frage, ob die unterschiedlichen gesellschaftlichen Systeme in der BRD und der DDR mit unterschiedlichen Inhalten und Formen der Erziehung und Tradierung in den einzelnen Familien der beiden Teilgebiete einhergingen, die sich bis heute auswirken. Durch diesen Vergleichshorizont sind die Einflüsse der gesellschaftlichen Verhältnisse auf die einzelnen Familien schärfer konturierbar. Ein horizontaler Quervergleich ermöglicht es zudem, nach intragenerationellen Gemeinsamkeiten und ihren potenziellen Einflüssen auf Inhalte und Formen der Tradierung zu suchen. Schließlich sind die möglichen Auswirkungen des Bildungsmilieus in die Reflexionen einzubeziehen.

Grundlegend für diese Studie ist die Annahme, dass die jeweils Erziehenden Inhalte im Zuge der Erziehung an die nächste Generation weitergeben möchten. Mit diesen müssen sich die Erzogenen auseinandersetzen, indem sie sie übernehmen, modifizieren oder sich von ihnen abgrenzen. D.h. die nachfolgende Generation stellt eine zentrale Einflussgröße in Bezug auf Tradierungsprozesse dar. Dabei sind nicht zwangsläufig Brüche anzunehmen, vielmehr ist von einem komplexen Ineinandergreifen von Kontinuität und Wandel auszugehen, das im Zuge der Analyse nachgezeichnet werden soll. Es ist zu vermuten, dass die Elemente weitergegeben bzw. angenommen werden, die sich für die jeweiligen Generationen als anschlussfähig erweisen. Das wiederum ist abhängig vom jeweiligen historischen Kontext, was nochmals die Bedeutung dieser Ebene für die Untersuchung familialer Tradierungsprozesse unterstreicht. Zusätzlich sind in den einzelnen Familien je spezifische Konstellationen zu erwarten, die im Zusammenspiel mit der gesellschaftlichen Ebene auf Formen und Inhalte der Tradierung einwirken, sodass verschiedene Arten der Tradierung im Sample zu erwarten sind. Insbesondere sind Unterschiede zwischen den Familien des westlichen und östlichen Teilgebietes möglich. 
Das erste Kapitel widmet sich den für den Kontext dieser Studie zentralen Begriffen Familie, Erziehung und Tradierung. Neben der Erörterung des Familienbegriffs erfolgt ein Überblick über die Entwicklung der Familienstrukturen. Im Anschluss werden der dieser Studie zugrunde liegende Erziehungsbegriff sowie Modelle zur Erfassung von Erziehungsprozessen thematisiert. Leitend ist dabei die Frage, inwiefern sie tradierungsrelevante Aspekte enthalten. Danach wird der Wertewandel als ein gesellschaftliches Phänomen skizziert, das sich auf Erziehungsprozesse auswirkt. Zugleich werden wesentliche Entwicklungslinien von der Nachkriegszeit bis in die Gegenwart dargestellt, die die Erziehung sowie die familialen Rollen betreffen. Auf der Grundlage dieses Wissens werden Fragen aufgeworfen, die auf die möglichen Konsequenzen für Tradierungsprozesse zielen.

Das zweite Kapitel fokussiert auf die theoretische Rückbindung familialer Tradierungsprozesse. Hierzu dienen Halbwachs' Gedächtnistheorie $(1985,1991)$ sowie die Generationenperspektive. Nachdem die Überschneidungslinien zwischen Tradierung und Erinnerung aufgezeigt worden sind, werden Halbwachs' Gedächtnistheorie sowie sein Modell des Familiengedächtnisses unter Einbeziehung der neueren kulturwissenschaftlichen wie psychologischen Erkenntnisse in Hinblick auf ihre Eignung zur Analyse familialer Tradierungsprozesse diskutiert. Die Hinzuziehung der Generationenperspektive stellt eine geeignete Ergänzung dar, um Kontinuität und Wandel im Rahmen familialer Tradierung erfassen zu können. Hierzu werden die Möglichkeiten erläutert, die die einzelnen Generationenkonzepte in diesem Zusammenhang bieten. Koselleck (1989) und Olson u.a. (1989) liefern weitere Anknüpfungspunkte, mit denen die vorgestellten Überlegungen ergänzt und ausdifferenziert werden können.

Nachdem auf diese Weise familiale Tradierungsprozesse theoretisch rückgebunden worden sind sowie ein Instrumentarium für ihre Analyse entwickelt worden ist, werden im dritten Kapitel die für diese Studie zentralen methodologischen und methodischen Annahmen qualitativer Sozialforschung sowie der dokumentarischen Methode zusammengefasst. Nach einer Skizzierung der Erhebungsmethode werden die Besonderheiten der Erhebungssituation sowie der -region beschrieben und das Sample kurz vorgestellt.

Die nächsten beiden Kapitel widmen sich den empirischen Ergebnissen der Studie. Im vierten Kapitel werden die im Sample identifzierbaren Tradierungslogiken in vertikaler Perspektive rekonstruiert. Sie werden exemplarisch an Fallbeispielen in eigenen Unterkapiteln vorgestellt. Sofern eine Logik in beiden Teilregionen existiert, werden Familien aus beiden Gebieten in die Darstellung einbezogen. Gleiches gilt für Unterschiede im Bildungsstand. Das Interesse konzentriert sich auf die Leitfrage, wie sich Formen und Inhalte der Tradierung in den Familien im Generationenverlauf entwickeln. Hierzu wird untersucht, welche Inhalte Eltern auf welche Weise an ihre Kinder weitergeben möchten und wie sich die Kinder hierzu verhalten. Die Betrachtung der jeweiligen Erziehungsarrangements schließt u.a. die Gestaltung der intergenerationellen Beziehungen, der innerfamilialen Regeln, ihre Handhabung, die existierenden Spielräume sowie Anforderungen ein. Auf diese Weise lassen sich Kontinuitäten, Modifikatio- 


\section{Einleitung}

nen und Umarbeitungen im Generationenverlauf herausarbeiten. Am Ende jedes Unterkapitels erfolgt eine zusammenfassende Beschreibung der wesentlichen Kennzeichen der jeweiligen Tradierungslogik. Zudem wird die Entwicklung der familialen Tradierungsprozesse an die jeweiligen historischen Kontexte rückgebunden. Im letzten Unterkapitel erfolgt ein zusammenfassender Überblick über die herausgearbeiteten Tradierungslogiken sowie eine Auseinandersetzung mit existierenden Forschungsergebnissen zum Thema.

Im fünften Kapitel wird die Analyseebene gewechselt. Anfangs steht die Frage im Zentrum, ob sich Verbindungslinien zwischen dem jeweiligen Bildungsstand und der verfolgten Tradierungslogik in den jeweiligen Familien erkennen lassen. Ausführlicher wird anschließend die Frage nach generationellen Verortungen in horizontaler Perspektive behandelt. Die Aufmerksamkeit richtet sich auf folgende Fragen: Werden im- oder explizit Unterschiede zwischen den Generationen thematisiert? Sind generationenspezifische Topoi erkennbar? Können unterschiedliche Positionierungen zur selbst erfahrenen sowie praktizierten Erziehung generationell rückgebunden werden? Verändern sich die Familienleitbilder im Generationenverlauf? Wie lassen sich die Ergebnisse historisch kontextualisieren? Abschließend erfolgt eine systematische Betrachtung der regionalen Ebene. Von Interesse ist, ob sich Inhalte und Formen der Tradierung in bestimmten Bereichen regional unterscheiden: Sind bis heute fortwirkende regionale Spezifika zu erkennen, die an die jeweils unterschiedlichen gesellschaftlichen Kontexte der Vergangenheit in der BRD und DDR rückgebunden werden können?

Im Fazit werden die wichtigsten Ergebnisse noch einmal zusammengefasst und im Kontext der existierenden Studien zur Tradierung betrachtet. Darüber hinaus wird im Rückblick auf die empirische Auswertung noch einmal das Potenzial herausgestellt, das Halbwachs' Gedächtnistheorie gekoppelt mit einer Generationenperspektive bietet, um Tradierungsprozesse in ihrer Komplexität zu erfassen. Schließlich werden weitere Facetten familialer Tradierung aufgezeigt, die sich mit dem entwickelten Analyseinstrumentarium bearbeiten lassen. 


\section{Familiale Tradierungsprozesse: Einblicke in den Forschungsstand}

Für die Vermittlung und Aneignung von Inhalten, worunter Werte, Einstellungen und Orientierungen zu verstehen sind, werden in der Literatur verschiedene Begriffe wie Tradierung (Kraul 2003b, Ecarius 2001), Transmission (Berteaux/Berteaux-Wiame 1991), Generationenlernen (Liegle/Lüscher 2004), generative Sozialisation (Lüscher 2008) sowie kulturelle Transferbeziehungen (Stecher/Zinnecker 2007) verwendet. Das verbindende Element zwischen diesen Bezeichnungen ist in der Tatsache zu sehen, dass alle aufgeführten Begriffe in irgendeiner Form auf einen intergenerationellen Austausch zwischen älteren und jüngeren Mitgliedern einer Gesellschaft bzw. auf die Herstellung eines Kontinuitätszusammenhangs zwischen Vergangenheit, Gegenwart und Zukunft rekurrieren. ${ }^{1}$ Am geläufigsten erscheinen die Bezeichnungen Tradierung und Transmission, weswegen die folgenden Ausführungen sich auf diese Termini konzentrieren. Tradierung leitet sich vom lateinischen Verb tradere ab und bedeutet, weitergeben' (Pfeifer 1993, 1444). Bei dem Wort Transmission handelt es sich um eine Übernahme aus der englischen Literatur (Zinnecker 2009, 141); es lässt sich als ,Übermittlung, Übersendung'verstehen. Die beiden Begriffe unterscheiden sich folglich nicht fundamental in ihren wörtlichen Bedeutungen und werden in der vorliegenden Untersuchung synonym verwendet. Ein wesentliches Charakteristikum des Tradierungs- bzw. Transmissionsbegriffs besteht in seiner Doppelnatur. Er bezieht sich zum einen auf die Inhalte, die weitergegeben werden, und zum anderen auf den Prozess der Weitergabe selbst (Kellerhals 2002, 213/214).

In den gängigen pädagogischen und erziehungswissenschaftlichen Wörterbüchern finden sich keine eigenen Einträge zu den Stichworten Tradierung oder Transmission, sodass ein Umweg über den Traditionsbegriff notwendig ist. Tradition umfasst alles, was aus der Vergangenheit in die Gegenwart im Generationenverlauf weitergegeben wird (Shils 1981, 12). ${ }^{2}$ Hierbei kann es sich um materielle Dinge handeln (Shils 1981, 12), sowie um immaterielle Elemente wie z.B. Werte, Regeln, Lebensformen, Deutungsmuster, Sitten, etc. (Böhm 2005, 635, Brüggen 1996, 1528, Schaub/Zenke 1995, 343, Tenorth/ Tippelt 2007, 719). Schönpflug zählt als weitere Elemente noch soziale Orientierungen und Fähigkeiten, wie z.B. das Lesen, Wissen und Verhaltensweisen auf (Schönpflug

\footnotetext{
${ }^{1}$ Neben der intergenerationellen Ebene beziehen einige Autoren, wie z.B. Liegle/Lüscher (2004), auch die intragenerationelle Ebene mit ein.

${ }^{2}$ Es finden sich zwar keine eigenen Artikel von Shils in pädagogischen und erziehungswissenschaftlichen Publikationen, aber seine Ausführungen werden von einigen Autoren wie z.B. Brüggen (1996) berücksichtigt.
} 


\section{Familiale Tradierungsprozesse: Einblicke in den Forschungstand}

2009, 9). Ein konstitutives Merkmal einer Tradition bildet der wiederholte interpersonale Austausch (Shils 1981, 15). Mit der Wiederholung ist ein wesentliches Charakteristikum der Tradition und Tradierung benannt, das es ermöglicht, diese Begriffe von anderen abzugrenzen.

Tradierungsprozesse vollziehen sich einmal auf der Ebene direkter interpersonaler Kontakte, die im Zentrum dieser Studie stehen. Daneben ist aber auch eine indirektere Form denkbar, z.B. mittels Büchern, die keine konkreten interpersonalen Kontakte erfordern. ${ }^{3}$ Letzteres bietet zudem die Möglichkeit, in Vergessenheit geratene Tradierungsinhalte wieder aufzunehmen oder an sie anzuknüpfen.

Das führt zu der Frage nach den Mechanismen der Weitergabe. Für Shils sind Traditionen und Tradierung grundlegende Bestandteile jeder Gesellschaft. Vertreter ${ }^{4}$ jeder Generation beziehen sich in irgendeiner Weise zumindest auf einige Ideen vorangegangener Generationen, und die von den jeweiligen Generationen verwendeten Orientierungen und Denkmuster werden nur zum Teil von ihnen selbst erschaffen (Shils 1981, 38). Das ist ein unmittelbar überzeugender Gedanke, existieren die einzelnen Generationen doch nicht getrennt voneinander, sondern stehen im wechselseitigen Austausch, sodass zumindest die Übertragung einiger Ideen und Verhaltensmuster wahrscheinlich ist. Das bedeutet zugleich, dass eine einseitige Konzentration auf Wandel, Generationenkonflikte und Tradierungsbrüche der Komplexität der Thematik nicht gerecht werden würde. Die Bezugnahme muss nicht unbedingt bewusst erfolgen (Brüggen 1996, 1528). Viele tradierte Elemente gehen als tacit knowledge in die Verhaltens- und Denkmuster der nachfolgenden Generation ein, ohne dass die Tradierung bewusst wäre (Shils 1981, 22). Tradierungsprozesse sind demnach keine Ausnahmeerscheinungen, sondern elementare Bestandteile des gesellschaftlichen Lebens. Sie müssen nicht unbedingt reflektiert oder hinterfragt werden, sondern können sich durchaus in habitualisierter Form vollziehen.

\subsection{Die Familie im Blickpunkt der sozialwissenschaftlichen Forschung}

\subsubsection{Die Familie}

Die familiale Sphäre wird als ein wichtiger Ort für Tradierungsprozesse betrachtet. Ihre besondere Relevanz ergibt sich daraus, dass sie i.d.R. als primäre Sozialisationsinstanz eingestuft wird (Ecarius 1998, 42); die meisten Menschen in dieser Gesellschaft verbrin-

\footnotetext{
${ }^{3}$ Benner benennt in Bezug auf Erziehungsprozesse nochmals drei verschiedene Formen der Tradierung, die sich als konservierend, bewahrend und verändernd sowie unterbrochen oder abgerissen bezeichnen lassen (Benner 2004, 163/164).

${ }^{4}$ Wenn an dieser Stelle von dem Vertreter die Rede ist, ist selbstverständlich auch die Vertreterin gemeint. Die überwiegende Verwendung der männlichen Form in diesem Text erfolgt lediglich aus Gründen der besseren Lesbarkeit.
} 


\subsection{Die Familie im Blickpunkt der sozialwissenschaftlichen Forschung}

gen zumindest die Anfangszeit ihres Lebens in einer Familie oder familienähnlichen Struktur und sammeln dort Erfahrungen, die i.d.R. als stark prägend aufgefasst werden, was sich „in lebenslangen Beziehungen zu den nächsten Angehörigen (zumeist Eltern und Geschwister)" niederschlägt (Lettke/Lange 2007, 9). Mit anderen Worten: Die Familie wird als eine relativ dauerhafte und die Mitglieder stark beeinflussende Gruppe betrachtet. Ihr wird eine besondere Bedeutung zugesprochen, da in der Familie die junge Generation mit der älteren interagiert und in der Familie „Tradierung und Wandel aufeinander bezogen“ sind (Ecarius 2003, 539). Ähnlich argumentiert bereits Claessens, indem er die Familie als Ort beschreibt, an dem Werte „erhalten, aber auch verändert“ werden und „durch die Familie auf die nächste Generation“ wirken (Claessens 1972, 55). Das geschieht nicht nur durch absichtsvolles Handeln, sondern bereits durch die Teilhabe an familialen Praxen erfährt die heranwachsende Generation eine „kulturelle Prägung“ (Müller/Borg/Falkenreck 2010, 56), die folglich einen Weg der Tradierung darstellt.

Die Frage, was unter Familie zu verstehen ist, entzieht sich einer einfachen Bestimmung. Die allseits bekannte Vorstellung von der modernen Kleinfamilie, womit „die rechtlich gesicherte Lebensgemeinschaft eines Ehepaars mit seinen eigenen (unmündigen) Kindern im eigenen privaten Haushalt“" (Gukenbiehl 1995, 69) skizziert ist, kann der Vielzahl der existierenden Familienformen, wie sie die Familienforschung beschreibt und wofür diese das Schlagwort von der Pluralisierung der familialen Lebensformen geprägt hat (Nave-Herz 5 2000, 20-22, Peuckert 2008, 21-28, Lange/Lettke 2007, 17), nicht gerecht werden. ${ }^{6}$

Die Redewendung von der Pluralisierung familialer Lebensformen ist zugleich ein gutes Beispiel, um auf zwei unterschiedliche Phänomene hinzuweisen, die zu unterscheiden sind: Familienleitbilder und tatsächlich existierende Familienformen (Fuhs 2007, 20). Von einer Pluralisierung der Familienformen zu sprechen, birgt die Gefahr, eine Familienform, die der modernen Kleinfamilie, als die bis in die jüngste Vergangenheit einzige Familienform zu imaginieren, was leicht zu dem Fehlschluss führen kann, die Vielfalt familialer Lebensformen als ein Zeichen für den Verfall der Familie zu deuten (Fuhs 2007, 22). Wie die historische Familienforschung jedoch zeigt, existierten auch in der Vergangenheit verschiedene familiale Lebensformen parallel nebeneinander (Rosenbaum 1978, 23-25, Rosenbaum 1982b, Fuhs 2007, 27, Lenz/Böhnisch 1997, 11-22), ${ }^{7}$ und die moderne Kleinfamilie dominierte lediglich während der 1950er und 1960er Jah-

\footnotetext{
${ }^{5}$ Nave-Herz setzt sich ausführlich und kritisch mit der These zur Pluralisierung familialer Lebensformen auseinander (Nave-Herz 2000, 20-22).

${ }^{6}$ Eine Übersicht über die verschiedenen Familienformen findet sich bei Nave-Herz (Nave-Herz 2007, 17). Neben der modernen Kleinfamilie, bestehend aus einem lebenslang verheirateten Ehepaar mit Kindern, ist die Zahl Alleinerziehender, Patchwork- oder Stieffamilien sowie lediger Paare mit Kindern in den vergangenen Jahren gestiegen (Peuckert 2008, 23-28).

${ }^{7}$ Die historische Familienforschung hat sich eingehend mit den unterschiedlichen Lebenslagen der verschiedenen Familienformen der Vergangenheit beschäftigt und die jeweiligen Spezifika der einzelnen
} 


\section{Familiale Tradierungsprozesse: Einblicke in den Forschungsstand}

re (Nave-Herz 2007, 23 und Peuckert und 2007, 36). Die Leitbilder wiederum verweisen auf die sozialen Orientierungen der Individuen (Wahl 1997, 103/104), ohne mit den vorfindbaren Familienform deckungsgleich sein zu müssen (Fuhs 2007, 20).

Die jeweiligen gesellschaftlichen Rahmenbedingungen wirken sich auf die konkreten Familienstrukturen aus, was bedeutet, dass Familienkonstellationen sich im Zuge der gesellschaftlichen Entwicklung verändern können (Rosenbaum 1978, 9 und 12). ${ }^{8}$ Die Familie stellt folglich ein gesellschaftliches Phänomen dar und es gibt keine allgemeingültige Definition von Familie, die für alle historischen Epochen Gültigkeit beanspruchen könnte (Rosenbaum 1978, 12 und 20). Hierbei unterliegt nicht nur „die Art und Weise des familialen Zusammenlebens" Veränderungen, sondern, mindestens genauso wichtig, „das Verständnis von Familie ${ }^{9}$ und Generationenbeziehungen“ (Lettke/Lange 2007, 10). Somit werden auch die Familienleitbilder vom gesellschaftlichen Entwicklungen beeinflusst.

Ein Zugang, der in den letzten Jahren verstärkt für die Definition der Familie herangezogen wurde, besteht darin, die Generationenbeziehungen bzw. -differenzierung in den Mittelpunkt der Betrachtung zu stellen (Bertram 2000, 106, Baader/Sager 2008, 293, Böhnisch 2005, 283, Ecarius 2003, 539). Dieser Ansatz eignet sich insbesondere im Zusammenhang mit familialen Tradierungsprozessen, in deren Mittelpunkt die intergenerationellen Austauschprozesse stehen. Für Lenz/Böhnisch ist das zentrale Merkmal der Familie „die Zusammengehörigkeit von zwei (oder mehreren) aufeinander bezogenen Generationen, die zueinander in einer Elter[n]-Kind-Beziehung stehen" (Lenz/Böhnisch 1997, 28). ${ }^{10}$ Damit ist ein Abgrenzungsmerkmal zu anderen Formen der Generationenbezie-

Familien herausgearbeitet. Differenzierte Betrachtungsweisen familialer Lebensformen seit dem Ende des 18. Jahrhunderts finden sich z.B. bei Rosenbaum (1982a) und bei Sieder (1987).

${ }^{8}$ Das soll nicht darüber hinwegtäuschen, dass das Individuum als handelnder Akteur diesen Strukturen nicht völlig ausgeliefert ist, sondern sie gestalten kann. Die Strukturen determinieren letztlich nicht vollständig das individuelle Handeln (Lettke/Lange 2007, 10/11).

${ }^{9}$ Das lässt sich exemplarisch durch die Betrachtung verschiedener Familiendefinitionen der letzten Jahrzehnte illustrieren. Während Claessens/Menne die Familie 1970 noch als Gruppe betrachten, „in der wenigstens zwei gegengeschlechtliche psycho-sozial erwachsene Menschen eine weitere Generation produzieren und mindestens so erziehen, daß diese nächste Generation dazu motiviert wird, ihrerseits die folgende Generation zu produzieren und ebenso zu motivieren“ (Claessens/Menne 1970, 170), fehlen in jüngeren Begriffsbestimmungen wie der von Lenz/Böhnisch (1997, 28), Ecarius (2001, 559) oder Hofer $(2002,6)$ die Verweise auf zwei Personen sowie Gegengeschlechtlichkeit. Bereits Mollenhauer, der diese Definition aufgreift, verweist darauf, dass die Konzentration auf zwei erwachsene Personen aufgrund der gesellschaftlichen Entwicklung nicht haltbar ist (Mollenhauer 1996, 605). Aus heutiger Perspektive ist auch die Gegengeschlechtlichkeit als Merkmal obsolet geworden. Angesichts von Adoptionen oder auch der modernen Reproduktionsmedizin ist die biologische Reproduktionsfunktion nicht ausschließlich der familialen Sphäre zuzuordnen. Hierauf weist z.B. Liebau im Zusammenhang mit dem familialen Generationenbegriff hin (Liebau 1997, 25/26). Bereits diese Beispiele verweisen auf die historische Gebundenheit der Auffassungen von Familie.

${ }^{10}$ Die Generationenbeziehungen mit einem besonderem Fokus auf die Eltern-Kind-Beziehung in den Mittelpunkt der Betrachtung zu stellen, verweist zugleich auf die historische Kontextabhängigkeit 


\subsection{Die Familie im Blickpunkt der sozialwissenschaftlichen Forschung}

hungen gegeben, da die Eltern-Kind-Beziehung mit spezifischen Rollen und Funktionen verknüpft ist, die sie von anderen Arten der Generationenbeziehungen unterscheidet. ${ }^{11}$ Auch hierbei handelt es sich um gesellschaftlich beeinflusste Phänomene, die sich im Zeitverlauf verändern können.

In Anlehnung an und in Weiterführung von Lenz/Böhnisch definiert Ecarius die Familie als intergenerationelle und interaktive Gemeinschaft, deren Mitglieder „in unterschiedlichen sozialen und biographischen Zeitstrukturen den Erziehungs- und Sozialisationsprozess durchlaufen und in der Vergangenheit als auch in der Gegenwart miteinander verbunden sind" (Ecarius 2001, 559). Durch die Einbeziehung der unterschiedlichen Zeitstrukturen wird der historische Kontext explizit erfasst, wodurch die gesamtgesellschaftlichen Bezüge bei der Definition von Familie berücksichtigt werden. Die Ausführungen von Lenz/Böhnisch und Ecarius ermöglichen es, Angehörige einer dritten Generation in die Familiendefinition aufzunehmen und über ein Kernfamilienmodell hinauszugehen, was für die in dieser Studie verfolgte Mehrgenerationenperspektive besonders relevant ist.

Ein weiteres Charakteristikum der Familie ist mit dem Modell des Familienzyklus bzw. der Familienkarriere umschrieben. Es wird angenommen, dass Familien während ihres Bestehens verschiedene Phasen durchlaufen. Mit dem Übergängen zwischen den einzelnen Phasen sind Familienentwicklungsaufgaben verbunden (Hofer 2002, 19-24); Veränderungen innerhalb einer Familie werden als systematisches Element von Familie betrachtet (Hofer 2002, 4). Die Familie ist folglich weder in historischer Perspektive noch bezogen auf die Entwicklung jeweils einzelner Familien als statisch zu betrachten. Insgesamt ist die Familie sowohl als eine relativ dauerhafte als auch dynamische Einheit konzeptualisiert, die sowohl Kontinuitäten als auch Veränderungen ermöglicht.

\subsubsection{Die Familie im gesellschaftlichen Wandel}

In historischer Perspektive lassen sich verschiedene familienstrukturelle Entwicklungen erkennen: Die Geburtenzahlen sind seit dem Beginn des 20. Jahrhunderts und die Heiratsquoten seit den 1960er Jahren nahezu kontinuierlich gesunken, die Scheidungshäufigkeit ist ebenso wie die Anzahl der Familienformen jenseits des klassischen Familienmodells gestiegen und der Anteil der familialen Haushaltsformen ist in der Gesamtschau zurückgegangen. ${ }^{12}$ Diese Auflistung erweckt zunächst den Eindruck, die Familie,

des Begriffs. Für andere historische Kontexte kann z.B. „die Teilnahme an der Gemeinschaft der Arbeit“ (Rosenbaum 1978, 31) oder die Zugehörigkeit zur Haushaltsgemeinschaft (Mitterauer 1997, 16) der entscheidende Definitionsfaktor sein.

${ }^{11}$ Die spezifische familiale Rollenstruktur sowie -definitionen sind ein wichtiges Kriterium, um die Familie von anderen sozialen Gruppen abzugrenzen (Nave-Herz 2007, 15).

${ }^{12}$ Eine wesentliche Veränderung der familialen Strukturen stellt die abnehmende Kinderzahl pro Familie dar. Diese ist von durchschnittlich 4,1 Kinder um 1900 auf 1,29 Kinder (West) bzw. 1,22 Kinder (Ost) im Jahr 2003 gesunken (Schäfers 2004, 126). In den 1960er Jahren lag die zusammengefasste Geburtenziffer in beiden deutschen Teilstaaten bei ca. 2,5 Kindern pro Frau. Mit Ausnahme einiger 


\section{Familiale Tradierungsprozesse: Einblicke in den Forschungstand}

verstanden als moderne Kleinfamilie, befinde sich in einer ernsthaften Krise und eine Beschäftigung mit dieser Lebensform werde zunehmend obsolet. Diese Diskussion um den Verfall der Familie stellt in der Öffentlichkeit auch ein regelmäßig wiederkehrendes Thema dar. ${ }^{13}$

Dieser Sichtweise folgend konstatieren einige Autoren, dass die Verkopplung von Ehe und Familie als Leitnorm an Verbindlichkeit verliert (Tyrell 1988, 148, Schneewind 1992, 20). ${ }^{14}$ In eine ähnliche Richtung zielen die Ausführungen von Beck und BeckGernsheim. Sie beschreiben als eine Folge der Individualisierung die Freisetzung des Individuums aus traditionalen Bezügen und Bindungen (Beck/Beck-Gernsheim 1990, 12/13 und Beck-Gernsheim 1990b, 67). Für sie büßt die Lebensform Familie an Verbindlichkeit ein (Beck/Beck-Gernsheim 1994, 11), und sie wird zu einer nicht mehr selbstverständlichen Wahloption. Die inhaltliche Ausgestaltung des Familienlebens ist ebenso offen (Beck/Beck-Gernsheim 1990, 13). Angesichts der sinkenden Verbindlichkeit bedeutet das zugleich einen Anstieg von Unsicherheiten (Beck-Gernsheim 1990b, 67). ${ }^{15}$ Büchner hingegen verbindet die strukturellen Veränderungen der Familie mit einer positiveren Konnotation, indem für ihn der Rückgang an Konformitätsansprü-

Schwankungen ist diese Ziffer seitdem gesunken und erreicht 2009 einen Wert von 1,36 Kindern pro Frau. Hierbei wies die zusammengefasste Geburtenziffer in Westdeutschland bis 1990 einen geringeren Wert auf als in der DDR (Statistisches Bundesamt 2011b).

Nimmt man beide deutschen Staaten zusammen, so sinkt die Zahl der Eheschließungen pro 1000 Einwohner von 9,4 im Jahr 1960 auf 4,6 im Jahr 2009 (Statistisches Bundesamt 2011a). Im selben Zeitraum steigen die Ehescheidungen in beiden Teilgebieten von 1,0 je 1000 Einwohner im Jahr 1960 auf 2,3 im Jahr 2009 (Statistisches Bundesamt 2011a).

Lebten 1972 im früheren Bundesgebiet 93,4\% der Kinder bei verheirateten Erwachsenen, so beträgt dieser Anteil im Jahr 2000 83,9\%. In den neuen Bundesländern liegt der Anteil deutlich niedriger bei 69,0\% (Engstler/Menning 2003, 25). 2009 stellt sich die Verteilung der Familienformen mit minderjährigen Kindern folgendermaßen dar: Bei $72 \%$ der Familien handelt es sich um Ehepaare mit ihren Kindern, 19\% sind alleinerziehend und bei $9 \%$ handelt es sich um Lebensgemeinschaften (Statistisches Bundesamt 2010, 7).

Der Anteil der Ein-Personen-Haushalte betrug 1972 im früheren Bundesgebiet 26,2\%. Die Haushaltsform von ,Eltern mit ledigen Kindern', worunter hier Ehepaare mit ledigen Kindern und Alleinerziehende gefasst werden, lag bei 44,4\%. Für das Jahr 2000 werden für Deutschland insgesamt $36,1 \%$ Ein-Personen-Haushalte sowie 32,1\% Haushalte von ,Eltern mit ledigen Kindern' ausgewiesen (Engstler/Menning 2003, 34). Für das Jahr 2005 wird der Anteil der Familien mit Kind mit ca. 36\% beziffert (Nave-Herz 2007, 27).

${ }^{13}$ Wie Tyrell und Kaufmann herausstellen, finden sich diese Klagen bereits bei Riehl (Tyrell 1988, 148 und Kaufmann 1988, 393).

${ }^{14}$ Für Tyrell betrifft dies auch die Elternschaft und er verweist auf die rückläufige „Inklusion der Erwachsenenbevölkerung in die Elternrolle“ (Tyrell 1988, 150/151).

${ }^{15} \mathrm{Wie}$ Beck/Beck-Gernsheim aufzeigen, ist dies jedoch nicht einseitig mit Freiheitszuwächsen gleichzusetzen, da im Gegenzug institutionelle Vorgaben steigen würden (Beck/Beck-Gernsheim 1994, 12). Die Kehrseite der wachsenden Wahloptionen bilden die ebenso zunehmenden Zwänge. 


\subsection{Die Familie im Blickpunkt der sozialwissenschaftlichen Forschung}

chen die Realisierung vielfältiger, gesellschaftlich anerkannter Familienentwürfe eröffnet (Büchner 2010, 531).

Kaufmann beschränkt im Gegensatz zu Tyrell den Attraktivitäts- und Stabilitätsverlust auf den Normbereich der Ehe und nimmt Elternschaft hiervon aus (Kaufmann 1988, 410). Die aufgezeigten Pluralisierungstendenzen betrachtet Kaufmann weniger als einen Bedeutungsverlust der Leitbilder von Partnerschaft und Elternschaft als vielmehr durch die gesellschaftliche Entwicklung bedingte Schwierigkeiten, diese Leitbilder umzusetzen (Kaufmann 1988, 411). Das Leitbild der Ehe als „Form des Zusammenlebens von Partnern, Eltern und Kindern" gilt auch für die Familie in der DDR, in der die Mehrheit der Bevölkerung mindestens einmal im Leben geheiratet und die Ehe unter Jugendlichen einen hohen Stellenwert eingenommen hat (Gysi 1990, 37).

Für andere Autoren stellt die Kernfamilie nach wie vor das dominierende Leitbild dar, das den institutionalisierten Rahmen für das kindliche Heranwachsen bildet (Wilk 2000, 29). Die Statistiken unterstützen diese Annahme. Auch wenn der Anteil der ZweiEltern-Familien insgesamt von 90,5\% (1981) auf 72\% (2009) gesunken ist, wächst die Mehrzahl der Kinder nach wie vor in Zwei-Eltern-Familien auf (Kaufmann 1988, 397 und Statistisches Bundesamt 2010, 7). ${ }^{16}$ Das zeigt, dass diese Familienform nach wie vor eine gesellschaftlich relevante Gruppe darstellt, die für die erziehungswissenschaftliche Forschung allgemein und die Frage nach familialen Tradierungsprozessen im Besonderen von Interesse ist. Die Familie genießt in der Bevölkerung eine hohe Wertschätzung (Nave-Herz 2000, 25) und die Wissenschaft betrachtet die Familie weiterhin als zentrale Sozialisationsinstanz (Büchner 2010, 519, Ecarius 2001, 559, Büchner/Fuhs 1996, 159; Lukesch 1996, 165). Langness/Leven/Hurrelmann bezeichnen die Familie ausdrücklich als „den wichtigsten sozialen ,Heimathafen', von dem aus sie [die Kinder und Jugendlichen, C.R.] die anderen Lebenswelten erschließen" (Langness/Leven/ Hurrelmann 2006, 49). Erst mit zunehmendem Alter werden andere Bezugsgruppen bedeutsam, ohne aber den Einfluss der Familie völlig aufzuheben (Lukesch 1996, 165). Folglich lässt sich aus dieser Perspektive keineswegs von einem Niedergang der Familie sprechen.

Unter Einbeziehung des demografischen Wandels werden die zweifelsohne existierenden strukturellen Veränderungen der Familie von anderen Autoren differenziert betrachtet. Neben der abnehmenden Kinderzahl pro Familie stellt das Ansteigen der durchschnittlichen Lebenserwartung eine zentrale Veränderung mit weitreichenden Folgen für das familiale Zusammenleben dar. ${ }^{17}$ Die Dominanz nichtfamilialer Haushalts-

\footnotetext{
${ }^{16}$ Differenziert nach Ost- und Westdeutschland zeigt sich allerdings ein deutliches Gefälle. Peuckert, der mit Datenmaterial aus dem Jahr 2005 arbeitet, beziffert den Anteil der Kinder, die bei verheirateten Paaren leben, für Ostdeutschland mit 62\% und für Westdeutschland mit 81\% (Peuckert 2007, 48).

${ }^{17}$ Im letzten Viertel des 19. Jahrhunderts betrug die durchschnittliche Lebenserwartung von Männern 46,5 und von Frauen 48,1 Jahre. 1999/2000 ist das durchschnittliche Lebensalter von Männern auf 75,1 und von Frauen auf 81,1 Jahre angestiegen (Schäfers 2004, 91/92).
} 


\section{Familiale Tradierungsprozesse: Einblicke in den Forschungstand}

formen erklärt sich z.B. aus der gestiegenen Lebenserwartung, in deren Folge die Familienphase nur noch einen (tendenziell abnehmenden) Teil der Lebensspanne ausfüllt (Nave-Herz 2007, 27/28). Gleichzeitig gründet ein Teil der Bevölkerung im Vergleich zur rezenten Vergangenheit erst zu einem späteren biografischen Zeitpunkt eine Familie; bei einem Teil der Individuen, die alleine oder ohne Kinder leben, handelt es sich demnach um Personen in der nach- bzw. vorelterlichen Phase (Nave-Herz 2007, 25/26), sodass von der Verteilung der Haushaltsformen nicht ohne Weiteres auf dauerhafte Kinderlosigkeit und einen Bedeutungsverlust der Familie geschlossen werden kann. Ein Wechsel zu einer Längsschnittperspektive zeigt, dass die meisten Personen in Deutschland zweimal in ihrem Leben Bestandteil einer Familie sind, einmal als Kind und einmal als Elternteil (Bien 1994, 6, Nave-Herz 2007, 28). Auch aus den steigenden Scheidungszahlen lässt sich kein Bedeutungsrückgang der Familie ableiten. Denn auch wenn inzwischen fast jede dritte Ehe geschieden wird, handelt es sich meist um Ehepaare ohne Kinder bzw. um Paare in der nachelterlichen Phase (Nave-Herz 2007, 24). Das relativiert ebenfalls die Annahme familialer Instabilität, zumindest was Familien mit minderjährigen Kindern betrifft.

Auch Bertram sieht Veränderungen innerhalb der familialen Beziehungen, die für ihn kein Ausdruck von Individualisierung oder Singularisierung sind, sondern vielmehr den „Übergang von der neolokalen Gattenfamilie mit kleinen Kindern zu einer multilokalen Mehrgenerationenfamilie mit lebenslangen Beziehungen zwischen Generationen, ohne daß diese Generationen noch unter einem Dach leben müssen" (Bertram 1996, 78/79) kennzeichnet. Lange/Lettke sehen sogar eine konzeptuelle Erweiterung von Familie: Großeltern- und Urgroßelternschaft (soziale Erweiterung) sind heute keine Ausnahmen mehr, und die gemeinsame Lebensspanne von Eltern und Kindern ist im Vergleich zur Vergangenheit größer geworden (zeitliche Erweiterung) (Lange/Lettke 2007, 20-31). ${ }^{18}$ Durch diese veränderten Konstellationen ergebe sich ein erweitertes Potenzial für ein Generationenlernen (Lange/Lettke 2007, 21-25).

Tyrell und Beck/Beck-Gernsheim ist sicherlich insofern zuzustimmen, als die Optionen zur Gestaltung des Familienlebens vielfältiger werden und die rigide Kopplung von Ehe und Elternschaft an Verbindlichkeit abnimmt. Hieraus allerdings einen Niedergang der Familie generell abzuleiten, wäre übereilt. Angesichts der hohen Wertschätzung der Familie in der Bevölkerung erscheinen die Erklärungsmodelle überzeugender, die die Veränderung der Familienstrukturen unter Einbeziehung des demografischen Wandels betrachten und in erster Linie eine Umgestaltung des familialen Zusammenlebens herausarbeiten, ohne ein Verfallsszenario der Familie zu zeichnen. Die Zwei-Eltern-Familie stellt nach wie vor gelebte Realität dar und ist als Leitbild präsent, ohne das ausschließlich dominierende zu sein. Die strukturellen Veränderungen der Familie haben tatsächlich weitreichende Folgen, jedoch nicht im Sinne einer zunehmenden Irrelevanz der Familie als Lebensform. Vielmehr führen insbesondere die steigende Lebenserwartung ge-

\footnotetext{
${ }^{18}$ Darüber hinaus wird noch eine sachliche Erweiterung angeführt, in deren Folge die Trennung von Privatheit und Öffentlichkeit abgeschwächt wird (Lange/Lettke 2007, 27).
} 
koppelt mit der sinkenden Geburtenrate dazu, dass die vertikalen Familienbeziehungen im Vergleich zu den horizontalen zahlreicher werden. Das rückt die Austauschprozesse zwischen den Generationen ins Zentrum der Aufmerksamkeit und unterstreicht ihre zunehmende Relevanz. Die Auseinandersetzung mit der Entwicklung familialer Strukturen zeigt, wie zentral es ist, familiale Tradierungsprozesse insbesondere in vertikaler Perspektive zu untersuchen und das Ineinandergreifen von Kontinuität und Wandel näher zu betrachten, was zur familialen Erziehung überleitet.

\subsection{Familiale Erziehung}

\subsubsection{Der Erziehungsbegriff}

In Anbetracht des steten Zugangs neuer sowie des Abgangs früherer „Kulturträger“ durch Geburt und Tod ist für Mannheim die Tradierung der bereits akkumulierten Kulturgüter unumgänglich, um diese den neuen Kulturträgern zur Verfügung zu stellen (Mannheim 1964/1928, 530-538). Erziehung stellt ein Mittel dar, um die nicht genetisch vererbbaren Güter der nächsten Generation zur Verfügung zu stellen (Winkler 2000, 58/59), und wird von verschiedenen Autoren als ein zentrales Mittel für Tradierungsprozesse angeführt (Kraul 2003b, Benner 2004, Brüggen 1996). Erziehung ist somit nicht nur eine elementare familiale Aufgabe (Schäfers 2004, 116), sondern lässt sich als ein Schlüsselelement für Tradierungsprozesse auffassen.

Für die Untersuchung familialer Tradierungsprozesse sind Erziehungsauffassungen am geeignetsten, die die interaktive Komponente sowie die Generationenbeziehungen in den Mittelpunkt für die theoretische Fundierung von Erziehung stellen. Letzteres wurde bereits von Schleiermacher in seine erziehungstheoretischen Reflexionen eingebaut und wird verstärkt wieder aufgegriffen (Kraul 2003b und 2003a, Ecarius 2008, Brüggen 1996, Sünkel 1996 und 1997, Winkler 1998). Ecarius betrachtet Erziehung allgemein als „Anleitung und Heranführung einer jüngeren Generation zur Selbsttätigkeit in Auseinandersetzung mit einer älteren Generation" (Ecarius 2008, 9) und die familiale Erziehung mit Bezug auf Mollenhauer und Mead als „eine Form familialer Interaktion zwischen älteren und einer jüngeren Generation" (Ecarius 2002, 45). Noch pointierter hebt Sünkel die aktive Beteiligung von Erziehenden und Zu-Erziehenden am Erziehungsprozess hervor: „Um Erziehung handelt es sich immer dann, wenn, und immer dort, wo nicht-genetische Tätigkeitsdispositionen vermittelt und angeeignet werden" (Sünkel 1997, 198). ${ }^{19}$ Das verweist nachdrücklich darauf, dass auch die Absichten und Wünsche des Educandus bedeutsam für den Erziehungsprozess sind (Kron 2001, 55). Erziehung umfasst zudem habitualisierte Komponenten. Eine Vielzahl von ,Transmissionseffekten' sind beiläufige Produkte „der elterlichen Lebensform, ihres ,Vor'-Bildes, ihrer Art und Weise, das Leben zu gestalten und zu bewältigen“ (Fend 2009, 84). Um

\footnotetext{
${ }^{19}$ Das zeigt zugleich, dass die mit ,Sozialmachung' und ,Sozialwerdung' umschriebenen Prozesse (Fend 1969, 37-39) wechselseitig aufeinander bezogen sind und eng ineinandergreifen.
} 


\section{Familiale Tradierungsprozesse: Einblicke in den Forschungstand}

diese Elemente zu integrieren, verweist Liebenwein auf Schneewinds erweiterten Erziehungsbegriff (Liebenwein 2008, 29). Mit dieser Terminologie bezeichnet Schneewind „alle kindbezogenen Erlebnis- und Handlungsweisen [...], die Elternpersonen mit oder ohne Beeinflussungsabsicht äußern" (Schneewind 1980, 21). Diese Definition erlaubt es, explizite und implizite Absichten der Akteure unter dem Erziehungsbegriff zu subsumieren. Zudem ist die familiale Erziehung in gesellschaftliche Kontexte eingebunden und wird durch diese beeinflusst (Ecarius 2007, 145). ${ }^{20}$

Hieraus ergibt sich ein Erziehungsverständnis für diese Studie, das den intergenerationellen Austausch in den Mittelpunkt stellt, Erziehung als einen wechselseitigen Prozess auffasst, der sowohl explizite Ziele als auch implizite Facetten und habitualisierte Formen einschließt und davon ausgeht, dass die jeweiligen gesellschaftlichen Kontexte sich auf das konkrete Erziehungsgeschehen auswirken.

Erziehung stellt zwar ein zentrales Instrument für die Tradierung von Inhalten dar, ist aber nicht identisch mit dieser. Während sich Erziehung auf ein interaktives, interpersonales Geschehen bezieht, ist das für Tradierung nicht unmittelbar konstitutiv. Tradierung bezieht sich ausschließlich auf die Weitergabe und Aneignung bereits vorhandener Inhalte. Erziehung hingegen ist nicht auf dieses Merkmal beschränkt, sondern kann auch Innovationen (Kraul 2003b) umfassen, die sich unabhängig oder in Abgrenzung von tradierten Inhalten entwickeln können.

\subsubsection{Familiale Beziehungssystematiken}

In diesem Abschnitt werden zentrale Modelle zur Erfassung von Eltern-Kind-Beziehungen vorgestellt und in Hinblick auf ihre Eignung für die Erfassung von Tradierungsprozessen betrachtet. Die Erziehungsstilforschung hat sich eingehend mit den verschiedenen Variationen elterlichen Erziehungsverhaltens beschäftigt. Einen hohen Bekanntheitsgrad hat das Modell von Baumrind (1991) erlangt. ${ }^{21}$ Eine prominente Einteilung unterscheidet zwischen autoritativem, autoritärem, permissivem und vernachlässigendem Erziehungsstil (Baumrind 1991, 750). ${ }^{22}$ Autoritativ erziehende Eltern sind u.a. sowohl fordernd als auch responsiv, sie stellen klare Regeln auf, ihr Verhalten ist eher untersützend als strafend, sie fördern die kindliche Eigenständigkeit, das Verantwor-

\footnotetext{
${ }^{20}$ Angesichts dieser Ausführungen ist die auf den ersten Blick eingängige Erziehungsdefinition Brezinkas (Brezinka 1990, 95) für den Kontext dieser Studie ungeeignet; insbesondere da Brezinka die gesellschaftlichen Kontexte zu wenig berücksichtigt, sich zu einseitig auf die erziehende Person konzentriert und den Erzogenen demgegenüber als „Objekt von Handlungsinteressen“ konzeptionalisiert (Mollenhauer 1976, 27). Weitere Auseinandersetzungen mit Brezinkas Definition der Erziehung finden sich u.a. bei Koller (2008), Kron (2001) Liebenwein (2008) und Schwenk (1996).

${ }^{21}$ Bereits zuvor haben Lewin/Lippitt/White (1939) ein Modell entwickelt, das die Stile in autoritär, demokratisch sowie laissez-faire unterteilt.

${ }^{22}$ In ihrem Aufsatz von 1991 erweitert Baumrind ihre Typologie in Bezug auf Heranwachsende und unterscheidet Authoritative, Democratic, Good-Enough, Directive, Nondirective sowie Unengaged homes bzw. families (Baumrind 1991, 752/753).
} 


\subsection{Familiale Erziehung}

tungsbewusstsein und die Kooperationsfähigkeit (Baumrind 1991, 750 und Papastefanou/Hofer 2002, 185). Autoritär agierende Eltern sind fordernd und gehen wenig auf die kindlichen Bedürfnisse ein. Sie greifen auf Machtmittel zurück, um ihre Vorstellungen durchzusetzen, erwarten Gehorsam sowie die Akzeptanz von Anordnungen, überwachen die kindlichen Aktivitäten sorgfältig und stellen klare Regeln auf (Baumrind 1989, 356 und Baumrind 1991, 750, Papastefanou/Hofer 2002, 185). Permissiv erziehende Eltern sind gegenüber ihren Kindern wenig fordernd, gehen stark auf die Bedürfnisse der Kinder ein und sind bemüht, Disziplinierungen zu vermeiden (Baumrind 1989, 356 und Baumrind 1991, 750). Eltern, die einen vernachlässigenden Erziehungsstil praktizieren, stellen wenig Forderungen an die Kindern, gehen kaum auf sie ein, schaffen keine intellektuell anregende Umwelt und kontrollieren die kindlichen Aktivitäten nicht (Baumrind 1989, 357 und Baumrind 1991, 750).

Schneewind kritisiert an der traditionellen Erziehungsstilforschung, dass sie von einer deterministischen Wirkung der elterlichen Erziehung ausgeht (Schneewind 2000, 197). Er schlägt vor, eine systemisch-kontextualistische Perspektive einzunehmen und betont die interaktionistische Komponente der Erziehung. Hiernach beeinflussen sich Erziehende und Erzogene wechselseitig im Erziehungsprozess (Schneewind 2000, 199). Mit Schneewinds Familienklima-Modell lassen sich die verschiedenen Facetten der familialen Beziehungsstrukturen erfassen, indem die emotionale Seite (positiv-emotionales Klima), das Ausmaß der Offenheit nach innen und außen (anregendes Klima) sowie der Normorientierung (normativ-autoritäres Klima) innerhalb von Familien als verschiedene Dimensionen der Beziehungsebene betrachtet werden (Schneewind 2010, 114/115).

Für Ecarius sind die Beziehungen zwischen den Generationen, also das jeweilige familiale Generationengefüge, die ambivalente Beziehungsstruktur von Nähe und Distanz, die jeweilige Position in der Generationenbeziehung sowie die konkret umgesetzte Machtbalance wesentliche Komponenten des Erziehungsprozesses (Ecarius 2002, 49). Das Ausmaß der erfahrenen Unterstützung und emotionalen Zuwendung durch die Erziehenden ist ein wesentlicher Faktor, der beeinflusst, wie die Erzogenen sich zu dem erfahrenen Erziehungsarrangement positionieren (Ecarius 2002, 239). Das unterstreicht die Bedeutung der aneignenden Seite in Bezug auf Erziehungsprozesse.

Lüscher/Liegle befassen sich mit familialen Generationenbeziehungen unter dem Aspekt der Generationenambivalenzen. Grundlegend ist für sie die Annahme, dass die Beziehungen zwischen den Generationen mit Ambivalenzerfahrungen verbunden sind (Lüscher/ Liegle 2003, 286). ${ }^{23} \mathrm{Je}$ nach Familienkonstellation werden Ambivalenzen in den einzelnen Familien zugunsten der Solidarität zurückgedrängt, offen angesprochen, massiv erfahren oder aber kaum reflektiert und besprochen (Lüscher 2005, 75/ 76). Überzeugend ist die Betonung, dass die jeweils konkreten familialen Generationenbeziehungen nicht einseitig geprägt sind, sondern komplexe Strukturen aufweisen und

\footnotetext{
${ }^{23}$ Unter Ambivalenzen verstehen sie die Existenz gleichzeitiger „Gegensätze des Fühlens, Denkens, Wollens, Handelns und der Beziehungsgestaltung, die [...] zeitweise oder dauernd als unlösbar interpretiert werden“ (Lüscher/Liegle 2003, 288).
} 


\section{Familiale Tradierungsprozesse: Einblicke in den Forschungsstand}

sowohl Konflikte als auch Aspekte der Solidarität umfassen können. Mit den sozialen Dimensionen Kontrolle und Wärme, den verschiedenen Facetten des Familienklimas, der Wechselseitigkeit von Erziehungsprozessen, dem Ausmaß der elterlichen Unterstützung sowie den intergenerationellen Ambivalenzen sind Aspekte angesprochen, die auch für die Untersuchung von Tradierungsprozessen relevant sind. Aus diesem Grund werden sie in heuristischer Perspektive im empirischen Teil berücksichtigt, ohne jedoch den Anspruch zu erheben, Tradierungsprozesse unter eines der vorgestellten Modelle zu subsumieren. In den bisherigen Ausführungen fehlt jedoch eine historische Kontextualisierung. Inwiefern z.B. das Maß an Kontrolle oder emotionaler Zuwendung förderlich oder hinderlich für den Erziehungsprozess ist, hängt nicht zuletzt auch mit den gesellschaftlichen Rahmenbedingungen und den jeweils dominanten Leitbildern zusammen, denen Erziehende und Erzogene folgen. Tradierungsprozesse sind folglich nicht unabhängig von den jeweiligen Zeitbezügen zu betrachten.

Bois-Reymond bindet in ihren Ansatz zur Einordnung der elterlichen Erziehung modernisierungstheoretische Überlegungen ein. ${ }^{24}$ In ihrer Typologie der Eltern-KindBeziehungen bilden der Befehlshaushalt einerseits und der Verhandlungshaushalt andererseits die zentralen Eckpfeiler. Im Befehlshaushalt existieren u.a. viele Regeln, die nicht an die Bedürfnisse des Kindes angepasst werden, das Kind hat kaum Mitspracherechte, abweichendes Verhalten wird mit zumeist zuvor bekannten Strafen sanktioniert und das Kind wird nicht mit „abweichenden Meinungen, Normen und Werten“ vertraut gemacht. Die Machtressourcen des Kindes sind insgesamt gering. Das Familienklima ist warm oder kalt (Bois-Reymond 1994, 152). Der Verhandlungshaushalt ${ }^{25}$ zeichnet sich dadurch aus, dass Regeln meist im Eltern-Kind-Gespräch festgelegt werden, dem Kind viel Raum zur Selbstentfaltung eingeräumt wird, die Eltern pädagogisch interessiert sind, abweichendes Verhalten angesprochen wird, es keine Tabuthemen gibt und ein warmes Familienklima herrscht (Bois-Reymond 1994, 155/156). Gesamtgesellschaftlich sieht Bois-Reymond einen Übergang vom Befehls- zum Verhandlungshaushalt, den sie auf den sozialen Wandel zurückführt, in dessen Zuge sich die Machtbalance zwischen den Geschlechtern und Generationen verschiebt (Bois-Reymond 1994, 143-148). Hiermit sind explizit die Machtverhältnisse zwischen den Generationen angesprochen, die zweifellos auch für Tradierungsprozesse bedeutsam sind. Mit einer asymmetrischen Machtbalance zwischen den Generationen sind andere Optionen in Bezug auf die Vermittlung und Aneignung von Inhalten verbunden als mit einer symmetrischer gestalteten. Es lässt sich einerseits vermuten, dass im Befehlshaushalt eher Tradierungsinhalte

\footnotetext{
${ }^{24}$ Als wichtige Schlagworte nennt Bois-Reymond Intimisierung, Informalisierung und Pluralisierung von Familienverbänden, die steigende Relevanz kommunikativer Fähigkeiten sowie der Erziehung zur Selbstständigkeit, ein steigendes Erziehungsrisiko sowie die ungleichzeitige Entwicklung, in deren Zuge „traditionale und moderne Konstellationen koexistieren“ (Bois-Reymond 1994, 144-148).

${ }^{25}$ Bois-Reymond unterteilt den Verhandlungshaushalt selbst nochmals in regelgeleitet und offen. Der offene Verhandlungshaushalt unterscheidet sich vom regelgeleiteten dadurch, dass „die Machtbalance zwischen Eltern und Kindern [...] noch ausgewogener“ ist (Bois-Reymond 1994, 156).
} 


\subsection{Familiale Erziehung}

unverändert weitergegeben werden. Andererseits erscheint es auch plausibel, dass sie eher zurückgewiesen werden.

Bois-Reymonds Modell fokussiert ebenso wie das von Baumrind auf die Beziehungen zwischen zwei Generationen, und das konkrete Erziehungsverhalten wird nicht mit der individuellen Erfahrungsaufschichtung und -bearbeitung, verknüpft. ${ }^{26}$ Die intergenerationellen Aushandlungsprozesse lassen sich zwar untersuchen, sie stehen aber nicht im Zentrum der Betrachtung. Für die Analyse von Tradierungsprozessen sind die angeführten Punkte jedoch zentral. Durch die Einbeziehung einer dritten Generation sowie die Berücksichtigung der individuellen Lebensgeschichten lässt sich erfassen, wie die jeweiligen Erziehungssettings von den Eltern gestaltet, von der nächsten Generation verarbeitet und bei der Erziehung der nächsten Generation angewendet werden, die sich wiederum hierzu positioniert. Das leitet über zu den für Tradierungsprozesse relevanten Entwicklungen im Bereich familialer Erziehung. Neben den gesellschaftlichen Wertetrends werden im folgenden familiale Erziehungsvorstellungen sowie die hiermit verbundenen Rollenzuschreibungen in historischer Perspektive betrachtet.

\subsubsection{Die Wertorientierungen im gesellschaftlichen Wandel}

Die Werteforschung skizziert einige zentrale Veränderungen im Bereich der Wertorientierungen. ${ }^{27}$ Einen hohen Bekanntheitsgrad genießen in diesem Zusammenhang Ingleharts Studien zum Wertewandel, in denen er eine Verlagerung von materialistischen zu postmaterialistischen Werten für die westlichen Industriegesellschaften nachweist (Inglehart 1977, 1979, 1989, 1998). ${ }^{28}$ Diese betrachtet er als Bestandteil eines größeren kulturellen Wandels, der Veränderungen religiöser Einstellungen, sozialer, kultureller und sexueller Normen sowie der Geschlechterrollen umfasst (Inglehart 1989, 90, Inglehart 1998, 380-405). Auch wenn Ingleharts Studien kritisch diskutiert werden, scheinen zu-

\footnotetext{
${ }^{26}$ Mit den beiden Modellen können zwar die allgemeinen Erziehungstendenzen in einer Familie beschrieben werden, es ist aber fraglich, ob dieser Stil für alle Inhalte und Situationen gilt. Mit den Modellen lassen sich die expliziten Seiten der Erziehung gut erfassen; problematischer gestaltet sich das bei der Einbeziehung der implizten Seiten von Erziehung.

${ }^{27}$ Der Wertebegriff wird in vielfältiger Weise gebraucht (Kmieciak 1976, 147 und Thome 2005, 389). Häufig wird die Definition Kluckhohns bzw. die Kmieciaks deutsche Übersetzung verwendet (z.B. Meulemann 1996b, Müller-Schneider 2001, 91, Klages, 2001b, 727, Thome 2005, 389, Kmieciak 1976, 148): „A value is a conception, explicit or implicit, distinctive of an individual or characteristic of a group, of the desirable which influences the selection from available modes, means, and ends of action" (Kluckhohn 1952, 395).

${ }^{28}$ Der materialistische Typus präferiert Sicherheitswerte, die sich in der Zustimmung zu Zielen wie „Kampf gegen höhere Preise“ oder „Aufrechterhaltung der Ordnung in der Nation“ niederschlagen (Inglehart 1979, 285 und 1989, 101). Der postmaterialistische Typus hingegen favorisiert Ziele wie „verstärktes Mitspracherecht bei wichtigen Regierungsentscheidungen“ und „Schutz der freien Meinungsäußerung" (Inglehart 1979, 285 und 1989, 101). In einigen seiner Untersuchungen verwendet Inglehart einen erweiterten Fragenkatalog (Inglehart 1989, 101).
} 


\section{Familiale Tradierungsprozesse: Einblicke in den Forschungsstand}

mindest die Verschiebungen der Wertorientierungen im Kern nicht bestritten zu werden. ${ }^{29}$

Für Klages vollzieht sich ein Wertewandel, der Mitte der 1960er Jahre einsetzt ${ }^{30}$ und zu einer Abnahme von Pflicht- und Akzeptanzwerten innerhalb der Bevölkerung führt sowie zu einer Zunahme von Selbstentfaltungswerten ${ }^{31}$ (Klages 2001b, 730/731 und 2002, 3). ${ }^{32}$ Er entwirft eine im Vergleich zu Inglehart komplexere Typologie, die fünf Typen ${ }^{33}$ umfasst. Bemerkenswert an Klages Typenkonzeption ist, dass Pflicht- und Akzeptanzwerte sowie Selbstentfaltungswerte sich nicht gegenseitig ausschließen müssen, sondern wie im Fall des aktiven Realisten miteinander kombiniert werden, was Klages als Wertesynthese bezeichnet (Klages 1984, 23, 164/165, 2001a, 10 und Gensicke 2002a, 33). In diese Richtung tendiert offensichtlich ein nicht unerheblicher Teil der Bevölkerung. $^{34}$

Meulemann beschreibt für die alte Bundesrepublik ebenfalls einen Wertewandel, der sich zwischen Mitte der 1960er und Mitte der 1970er Jahre ereignet (Meulemann 1996b). Die Werte Leistung und Akzeptanz verlieren an Bedeutung, die Mitbestimmung im politischen und privaten Bereich wird wichtiger und der Wert der Gleichheit bleibt konstant (Meulemann 1996b, 130 und 87). Die Veränderungen setzt Meulemann in Be-

${ }^{29}$ Die Kritik bezieht sich vielmehr auf die Anlage der Studie, die theoretischen Prämissen und Interpretationen (Lehner 1979, Meulemann 1996b, Oesterdiekhoff 2001). Klages fordert eine mehrdimensionale Konzeption des untersuchten Werteraumes sowie eine konsequente Auseinandersetzung mit der bei Inglehart als „Mischtypus“ bezeichneten Teilgruppe, die sich weder eindeutig dem materialistischen noch dem postmaterialistischen Typus zuordnen lässt (Klages 1984, 23ff und Klages 1992, 21 und 23). Für Gensicke ist Ingleharts Typologie wegen der weitestgehenden Nichtbeachtung von Mischtypen ungeeignet für die Untersuchung eines Wertewandels in Ostdeutschland, da gerade diese dort verbreitet seien (Gensicke 1992b, 692).

${ }^{30}$ Die Veränderungen führt Klages allgemein auf den gesellschaftlichen Modernisierungs- und Individualisierungsprozess zurück und benennt als Faktoren u.a. „Vollmotorisierung der Bevölkerung“, „Medienrevolution“ sowie die Bildungsexpansion (Klages 2001b, 729/730).

${ }^{31}$ Die Selbstentfaltungswerte unterteilt Klages nochmals in „hedonistisch-materialistische“ sowie „idealistische Selbstentfaltung“" (Klages 2001b, 734).

${ }^{32}$ Die Daten beziehen sich bis zur Wende nur auf die westlichen Bundesländer.

${ }^{33}$ Die Typologie besteht aus Konventionalisten, die in hohem Maße zu Pflicht- und Akzeptanzwerten und nur in geringem Maße zu den Dimensionen der hedonistisch-materialistischen sowie idealistischen Selbstentfaltung neigen. Bei den perspektivenlos Resignierten sind alle drei Wertebereiche niedrig ausgeprägt. Die aktiven Realisten zeigen hohe Ausprägungsgrade auf allen drei Wertedimensionen. Die hedonistischen Materialisten haben vor allem eine Affinität zu den hedonistischmaterialistischen Selbstentfaltungswerten und die nonkonformen Idealisten stimmen vor allem der idealistischen Selbstentfaltungsdimension zu (Klages 2001b, 734/735).

${ }^{34}$ In der Speyerer Wertestudie bilden die aktiven Realisten die größte Gruppe mit 30\% (Klages 2001b, 735 ) bzw. 36\% (West) sowie 35\% (Ost) (Gensicke 2002a, 34, 36). Ähnliches gilt für Jugendliche. Wie die Jugendshellstudien von 2002 und 2006 zeigen, folgen sie einem Trend zum Pragmatismus, indem sie trotz einiger Verschiebungen zwischen den Erhebungsjahren Leistung, Kreativität und Sicherheitsorientierung miteinander kombinieren (Gensicke 2002b, 140/141 und 2006, 176). 


\subsection{Familiale Erziehung}

ziehung zueinander und interpretiert sie zusammenfassend als zweite Säkularisierung: Institutionelle Vorgaben sowie die Arbeit dienen immer weniger als Vorlagen, aus denen ein Lebenssinn bezogen werden könnte. In der Folge verlagert sich die Sinnsuche auf die Mitbestimmung, im familialen wie im politischen Bereich (Meulemann 1996b, 131-133).

Inwiefern in der DDR ein Wertewandel stattgefunden hat, ist hingegen strittig. Meulemann konstatiert zwar auch für die DDR Veränderungen, wie den Bedeutungsverlust des Wertes der Akzeptanz und die zunehmende Relevanz von Autonomiewerten bei Lehrlingen zwischen 1975 und 1990, die er teils als Folge einer erzwungenen Säkularisierung einstuft, nicht aber als Ausdruck eines Wertewandels (Meulemann 1996b, 198/ 199, 202-207, 238/239).

Gensicke hingegen stuft die Verschiebungen als Wertewandel ein, der für ihn mit dem in der Bundesrepublik der 1960er Jahre vergleichbar ist (Gensicke 1992b, 688 und Gensicke 1995, 73). Gleichzeitig findet er als spezielle Variante in Ostdeutschland neben den Wandlungen in Richtung Individualismus, Materialismus und Hedonismus ein „Bedürfnis nach sozialer Sicherheit", Hochschätzung von Hilfsbereitschaft, Gleichberechtigung sowie informeller Umgangsformen (Gensicke 1992a, 61). Insgesamt wird ein ähnlicher, wenn auch später einsetzender Verlauf des Wertewandels postuliert (Gensicke 1992b, 692 und Klages 1993, 222), der als Ausdruck eines systemübergreifenden Modernisierungsprozesses interpretiert wird (Gensicke 1995, 73).

Nach der Wiedervereinigung finden sich ähnliche Wertepräferenzen und eine ähnliche Verteilung der fünf Klages'schen Wertetypen in Ost- und Westdeutschland (Gensicke 2002a, 31-33). Jedoch werden in den westlichen Bundesländern Religiosität und Gleichheit stärker betont, in den östlichen Bundesländern der Wert der Leistung (Gensicke 2002a, 31 und Meulemann 1996b, 289, 303 und 347). Für Meulemann sind das, gekoppelt mit der stärker ausgeprägten Religiosität in Westdeutschland, Indizien für fortbestehende Unterschiede in den Wertepräferenzen, die er als „ein Fortwirken der DDRSozialisation oder ihre Verstärkung durch die Situation der Transformation“ (Meulemann 2002, 22) betrachtet.

Zusammenfassend lässt sich als Grundtendenz festhalten, dass es im Verlauf der letzten Jahrzehnte in Deutschland - entgegen manch anderslautender öffentlicher Einschätzungen - keinen Werteverfall ${ }^{35}$ gegeben hat. ${ }^{36}$ Stattdessen wird zumindest für den west-

\footnotetext{
${ }^{35}$ Einen Werteverfall suggerieren z.B. die Erziehungsratgeber von Gaschke (2001) und Bueb (2006). Nach Klages Einschätzung steigt in der Bevölkerung die Ansicht, Werte wie „,Moral', ,Pflichtbewusstsein', ,Recht und Ordnung' sowie ,Fleiß““ würden zu wenig geachtet. Das betrachtet er als Beleg, wie die Bevölkerung durch die Medien verbreitete negative Einschätzungen wie die einer „Egoistengesellschaft“ internalisiert habe (Klages 2001a, 7/8).

${ }^{36}$ Lediglich Hillmann fürchtet, dass mit einem zunehmend reflexiven Wertebewusstsein die Gefahr einer Werterschütterung, eines Werterelativismus' und -verfalls verbunden ist (Hillmann 2001, 35).
} 


\section{Familiale Tradierungsprozesse: Einblicke in den Forschungstand}

lichen Teil Deutschlands ein Wertewandel ${ }^{37}$ beschrieben. Oesterdiekhoff bezeichnet als allgemeinen Konsens für die Stoßrichtung des Wandels: „Von der Akzeptanz- und Pflichtkultur zur Kultur der Selbstverwirklichung" (Oesterdiekhoff/Jegelka 2001, 8). Die Frage, ob die beschriebenen Veränderungen in der DDR als Wertewandel einzustufen sind, ist für den Zusammenhang dieser Studie weniger bedeutsam als die Tatsache, dass es Veränderungen gibt, die später einsetzen als in den alten Bundesländern, nämlich in den 1980er Jahren.

Insgesamt liefert die Werteforschung den allgemeinen Hintergrund, vor dem sich familiale Tradierungsprozesse abspielen. Zwar widmet sich Gensicke dem Zusammenhang von elterlicher Erziehung und der Herausbildung der verschiedenen Wertetypen (Gensicke 2000, 143-147), wie die einzelnen Familien allerdings mit bestimmten Werten im Generationenverlauf umgehen, wird weder bei Gensicke noch bei den anderen vorgestellten Autoren ausführlicher behandelt. ${ }^{38}$ Die Frage, wie Familien die beschriebenen Verschiebungen intergenerationell bearbeiten, stellt folglich ein zu untersuchendes Forschungsdesiderat dar. Für die vorliegende Studie ist von Interesse, ob der beschriebene Wertewandel von den Familien aufgegriffen wird und zu Tradierungsbrüchen führt, die familialen Kontinuitäten den Wandel überlagern oder Veränderungen und Konstanten miteinander kombiniert werden, wie insbesondere Meulemanns Ausführungen und Klages' Konzept der Wertesynthese nahelegen. Lassen sich hierbei generationelle Schwerpunktverlagerungen erkennen, um Tradierungsinhalte auch unter veränderten gesellschaftlichen Rahmenbedingungen anschlussfähig zu halten? Finden sich unterschiedliche Entwicklungslinien in den beiden untersuchten Teilgebieten oder sind die von der Werteforschung beschriebenen Unterschiede für Tradierungsprozesse eher irrelevant? Die beschriebenen Entwicklungen der Wertorientierungen lenken zunächst allerdings das Augenmerk auf die familiale Erziehung im Zeitverlauf.

\subsubsection{Familienerziehung im gesellschaftlichen Wandel}

Die Veränderungen beziehen sich auf die Erziehungsmuster, den Stellenwert des Kindes sowie die Bedeutung der Beziehungsqualität.

„Vom Befehlen und Gehorchen zum Verhandeln“39 Ein Blick auf ausgewählte Erziehungsziele für Westdeutschland zeigt, dass der Stellenwert von „Selbständigkeit und freier Wille“ zwischen 1951 und 1995 von $28 \%$ auf 60\% angestiegen ist. Im gleichen Zeitraum sank die Bedeutung von „Gehorsam und Unterordnung“ von 25\% auf 7\%. „Ord-

\footnotetext{
${ }^{37}$ Wie Duncker folgerichtig festhält, sind es weniger die Werte, die sich ändern, als vielmehr die Bedeutung, die ihnen von den Mitgliedern einer Gesellschaft zugesprochen werden (Duncker 1998, 22).

${ }^{38} \mathrm{Z}$ war untersucht Fritzsche, welches elterliche Erziehungsverhalten die Herausbildung verschiedener Wertorientierungen unterstützt (Fritzsche 2000, 120-126), es lassen sich aber genauso wenig wie bei Gensicke Aussagen über Inhalte und Modi der Tradierung im Generationenverlauf treffen.

${ }^{39}$ Mit dieser prägnanten Formel beschreibt Büchner den Wandel der Erziehung (Büchner 2010, 533).
} 


\subsection{Familiale Erziehung}

nungsliebe und Fleiß“ wiederum bleibt im Vergleichszeitraum relativ konstant bei $40 \%$ (Reuband 1997, 134). ${ }^{40}$ Auch wenn sich der Schwerpunkt der Erziehungsziele auf Autonomiewerte verlagert, schließen sich Konventions- und Autonomiewerte in der Erziehung nicht aus (Meulemann 1996b, 123) ${ }^{41}$ - ein Indiz, dass in der Erziehung Kontinuität und Wandel zeitgleich existieren und einander ergänzen. Schneewind/Ruppert stellen in ihrer Längsschnittuntersuchung Liberalisierungstendenzen in der Erziehung sowie eine Abnahme normativer Verbindlichkeiten fest (Schneewind/Ruppert 1995, 147 und 151). Schneewind spricht in diesem Zusammenhang von einer stillen Revolution der Erziehung (Schneewind 2010, 76/77). Im Zuge dieser Veränderungen werden körperliche Strafpraktiken praktisch bedeutungslos (Schütze 2002, 85) und die Generationenbeziehungen egalitärer (Bois-Reymond 1994, 143/144, Peuckert 2007, 51 und Peuckert 2008, 160). Laut Reuband sind Jugendliche seit Beginn des 20. Jahrhunderts in steigendem $\mathrm{Maße}$ bereit, elterlichen Entscheidungen zu widersprechen, ihre familialen Mitspracherechte nehmen kontinuierlich zu und der Konformitätsdruck verringert sich (Reuband 1988, 87 und 1997, 145). Das Prinzip des Gehorsams wird vom Prinzip des Diskutierens und Aushandelns abgelöst (Reuband 1997, 145, Schütze 2002, 86).

Auch in der DDR ändert sich die Erziehung und die Generationenbeziehungen werden egalitärer (Meulemann 1996b, 227). Die Familien versuchen, ihre Privatsphäre zu schützen und schaffen sich ihre eigenen Nischen und Netzwerke (Ahnert/Lamb 2001, 1858). In der Folge wird die Familie zum wichtigen Rückzugsort, zum Refugium, was die Entfaltung ausgeglichenerer Machtbalancen in der Familie ebenso gefördert haben könnte wie die stärkere gegenseitige Abhängigkeit (Meulemann 1996b, 224, 228 und 229). Reuband und Pollmer/Hurrelmann stellen eine ähnliche Entwicklung der Erziehungsmuster in Ost- und Westdeutschland fest (Reuband 1997, 149, Pollmer/Hurrelmann 1992, 7). ${ }^{42}$ Demnach können die politischen Beeinflussungsversuche innerhalb der DDR die fortbestehenden Übereinstimmungen in den Erziehungskulturen nicht aufheben (Ahnert/Lamb 2001, 1852).

Neben diesem allgemeinen Trend lassen sich bis heute jedoch auch milieuspezifische Unterschiede in der Erziehung ausmachen (Liebenwein 2008, 245). ${ }^{43}$ Einige Milieus orientieren sich am Erziehungsstil der Eltern, andere setzen sich davon ab (Liebenwein

\footnotetext{
${ }^{40}$ Die größten Verschiebungen fallen in den Zeitraum von ca. Mitte der 1960er bis Mitte der 1970er Jahre (Reuband 1997, 134 und Meulemann 1996b, 121/122). Jüngere und Personen mit höherem Schulabschluss sind dabei überproportional am Wandel beteiligt (Reuband 1988, 81).

${ }^{41} \mathrm{Zu}$ den Autonomiewerten zählen z.B. Toleranz, Menschenkenntnis und Wissensdurst. Die Konventionswerte beinhalten u.a. Orientierungen wie Sparsamkeit und Ordnung (Meulemann 1996b, 122/ 123).

${ }^{42}$ In Ostdeutschland wird das Erziehungsverhalten bis in die 1960er Jahre sogar als liberaler eingeschätzt, wofür Reuband den bis in die 1930er Jahre höheren Modernisierungsgrad in Ostdeutschland anführt (Reuband 1995, 228/229).

${ }^{43}$ Liebenwein orientiert sich an den sozialen Milieus nach SINUS 2007 (Liebenwein 2008, 44). Eine autoritäre Erziehung findet sich nur noch in den traditionsverwurzelten Milieus. Im hedonistischen Milieu wird permissiv-verwöhnend und im Konsum-materialistischen Milieu permissiv-
} 


\section{Familiale Tradierungsprozesse: Einblicke in den Forschungstand}

2008, 246-261). Auch finden sich Indizien für unterschiedlich ausgeprägte Nuancierungen der Erziehungsmuster in Ost- und Westdeutschland nach der Wende, die mit den unterschiedlichen gesellschaftlichen Rahmenbedingungen vor der Wiedervereinigung assoziiert werden. Das Erziehungsziel Autonomie steht in beiden Landesteilen hoch im Kurs, allerdings findet sich im Osten eine stärkere Akzentsetzung auf Konventionen (Meulemann 1996b, 327 und Gensicke 2002a, 33, 38). ${ }^{44}$ Während Meulemann letzteres als eine Folge des Transformationsprozesses betrachtet, die um die Jahrtausendwende fast verschwunden ist (Meulemann 1996a, 68 und 2002, 19 und 21), führt Gensicke dieses Erziehungsmuster als Reaktion der Familien auf die spezifischen DDR-Bedingungen zurück (Gensicke 2002a, 40), das sich offenbar über die Wende hinweg halten konnte. Für ihn sind das „Achten auf Ordnung, die Erziehung zur Selbständigkeit und zur Verantwortungsübernahme“ in Westdeutschland nicht so eng miteinander verknüpft wie in Ostdeutschland (Gensicke 2002a, 40). Auch Schmidtchen beschreibt Unterschiede im Erziehungsverhalten. Er konstatiert, dass in Ostdeutschland der reife Erziehungsstil stärker vertreten ist, worunter er die Kopplung von emotionaler Zuwendung mit Anforderungen versteht. In Westdeutschland ist demgegenüber in den 1990er Jahren der naive Erziehungsstil verbreiteter, womit ein elterliches Erziehungsverhalten charakterisiert ist, das den Kindern emotionalen Rückhalt gewährt ohne Anforderungen zu stellen (Schmidtchen 1997, 114/15). ${ }^{45}$ Für Uhlendorff waren in der ehemaligen Bundesrepublik Toleranz, Verhandlungsbereitschaft und das Einräumen von individuellen Freiheiten zur Förderung der kindlichen Entwicklung Bestandteile des erwarteten Erziehungskanons (Uhlendorff 2004, 81). Die Erziehung in Ostdeutschland beurteilt er demgegenüber als etwas traditionaler, kontrollierender und beschützender. Zudem existiert in Ostdeutschland eine starke Familienorientierung, die er als eine Reaktion auf politische Steuerungsversuche interpretiert (Uhlendorff 2004, 80). ${ }^{46}$

Im Anschluss an diese Ausführungen ist für die vorliegende Untersuchung von besonderem Interesse, ob und wie überlieferte und neue Erziehungswerte, -grundsätze und -praxen in den einzelnen Familien kombiniert werden und sich in einer Mehrgene-

vernachlässigend erzogen. In den übrigen Milieus dominieren autoritative bzw. demokratische Erziehungsstile (Liebenwein 2008, 245).

${ }^{44}$ Gensicke spricht von Pflicht- und Akzeptanzwerten der Konvention, womit „Fleiß und Ehrgeiz, Ordnung und Sicherheit, Disziplin und Pflichterfüllung" gemeint sind (Gensicke 2002a, 33). Die Differenz zwischen Ost- und Westdeutschland ist interessanterweise unmittelbar nach der Wende geringer gewesen und hat sich erst in der Folgezeit etwas verstärkt. Meulemann zieht hieraus den Schluss, dass nicht das System der DDR die Ostdeutschen autoritärer gemacht habe, sondern die Transformation nach der Wende (Meulemann 1996a, 68).

${ }^{45}$ Daneben gibt es noch den gleichgültigen und den paradoxen Erziehungsstil. Der erste zeichnet sich dadurch aus, dass weder Forderungen existieren noch emotionaler Rückhalt geboten wird. Beim paradoxen Erziehungsstil stellen die Eltern Forderungen, ohne einen emotionalen Rückhalt zu gewähren (Schmidtchen 1997, 113/114). Insgesamt ähnelt die Typologie Baumrinds (1989 und 1991) Einteilung.

${ }^{46}$ Die zu Grunde liegende Studie wurde Anfang der 1990er Jahre durchgeführt (Uhlendorff 2004, 73). 


\subsection{Familiale Erziehung}

rationenperspektive entwickeln. Daneben stellt sich die Frage, ob die skizzierten unterschiedlichen Nuancen der Erziehung in Ost- und Westdeutschland bis heute existieren oder sich, wie Meulemann argumentiert, inzwischen aufgelöst haben.

Von der Eltern- zur Kindzentrierung Eine wesentliche Veränderung stellt die Verschiebung von der Eltern- zur Kindzentrierung dar (Büchner 2010, 533). Für diese Entwicklung finden sich bereits Indizien in Studien der 1950er Jahre, wie z.B. bei Wurzbacher (1958). Er arbeitet verschiedene Muster der intergenerationellen Beziehungsgestaltung $^{47}$ heraus und identifiziert ein zunehmend bedeutsamer werdendes Leitbild, in dem der Eigenwert des Kindes stark zur Geltung kommt und „die Eigenständigkeit des kindlichen Wesens" beachtet wird (Wurzbacher 1958, 214), was auf eine Kindorientierung verweist.

Trotz der bei Wurzbacher zu erkennenden Tendenz ${ }^{48}$ scheint dieses Leitbild Anfang der 1950er bei Weitem noch nicht flächendeckend umgesetzt. Schütze/Geulen können kaum Anzeichen entdecken, dass Eltern sich Anfang der 1950er Jahre kindzentriert verhalten würden: Sie haben wenig Zeit für die Kinder, das Mitbringen von Spielkameraden ist unüblich oder unerwünscht, körperliche Strafen sind Bestandteil der Erziehung, die schulischen Leistungen werden registriert und kommentiert, sie stehen aber offenbar nicht im Zentrum der Aufmerksamkeit (Schütze/Geulen 1983, 36). ${ }^{49}$ Die Angehörigen der Jahrgänge um 1940 erinnern sich, viel Zeit im Freien verbracht zu haben, wobei sie teilweise der Kontrolle der Erwachsenen entzogen gewesen seien (Schütze/Geulen 1983, 33). Im Verlauf der 1950er Jahre werden die bestehenden Freiräume der Kinder eingegrenzt und die elterlichen Kontrollen verstärkt (Schütze/Geulen 1983, 38).

\footnotetext{
${ }^{47}$ Wurzbachers Studie basiert auf Daten aus den Jahren 1949 bis 1951 und widmet sich der Gestaltung der intergenerationellen Beziehungen in den Familien. Er identifiziert vier Haupttypen: 1. Ausübung starker elterlicher Verfügungsgewalt über das Kind; 2. Zentrale Stellung des Kindes; 3. Eigenständigkeit des Kindes; 4. Kinderlose Familien (Wurzbacher 1958, 198, 161/162). Unabhängig von Königs Kritik der mangelnden Repräsentativität $(1974,222 / 223)$ und der erkennbaren Bezüge auf eine traditionelle Frauen- und Mutterrolle (Wurzbacher 1958, 168 und 209) ist interessant, dass Wurzbacher verschiedene Umgangsarten mit Kindern in den Familien herausarbeiten kann.

${ }^{48}$ Inwiefern es sich bei der von Forschern der 1940er und 1950er Jahre postulierten einsetzenden Kindorientierung um Anzeichen für eine liberalere Erziehung gehandelt hat, ist Gegenstand der Diskussion. Schütze weist darauf hin, dass Kindorientierung zu verschiedenen Zeiten mit unterschiedlichen Bedeutungen versehen worden ist. So sei z.B. Ende der 1940er eine frühe Reinlichkeitserziehung, die ein Bestrafen von Einnässen umfasste, als Ausdruck der Erziehung zur Selbstständigkeit gedeutet worden. In den 1970er Jahren galt das als Maßnahme, die die Entwicklung der kindlichen Selbstständigkeit behinderte (Schütze 2002, 74). Allerdings berücksichtigen Forscher der 1940er und frühen 1950er Jahre auch die kindlichen Mitbestimmungsmöglichkeiten und die Machtstrukturen in den Familien (Baumert 1954 und Wurzbacher 1958). Diese beiden Indikatoren sind durchaus geeignet, autoritäres oder liberales Erziehungsverhalten im heutigen Verständnis zu bestimmen.

${ }^{49}$ Einige dieser Erziehungselemente sind sicherlich auf die wirtschaftlich prekäre Situation vieler Familien in der Nachkriegszeit zurückzuführen.
} 


\section{Familiale Tradierungsprozesse: Einblicke in den Forschungstand}

Für die Kindheit der um 1960 Geborenen ergibt sich kein einheitliches Erziehungsbild. Die Erziehungsarrangements reichen von sehr streng bis betont nachgiebig (Schütze/Geulen 1983, 45/46). Als Relikte einer autoritären Erziehung finden sich körperliche Strafen. Im Gesamtbild sind aber Liberalisierungstendenzen ${ }^{50}$ sowie eine Tendenz der mütterlichen Überbehütung erkennbar (Schütze/Geulen 1983, 44-46), die eine zunehmende Kindzentrierung vermuten lassen. ${ }^{51}$

In den 1990er Jahren hat sich eine an das Kindeswohl geknüpfte Kindorientierung als dominantes Leitbild für die Eltern-Kind-Beziehungen durchgesetzt (Büchner/Fuhs 1996, 198 und Büchner 2010, 533). ${ }^{52}$ Hierbei unterscheiden sich Ost- und West-Familien kaum. Einzig auf das kindliche Wohlverhalten wird in den östlichen Bundesländern stärker geachtet und das Strafverhalten ist tendenziell etwas ausgeprägter (Büchner/Fuhs 1996, 196). Interessanterweise existieren auch eher traditional ausgerichtete Eltern-KindBeziehungen, die zugleich ein hohes Maß an Kindorientierung aufweisen, sowie eher moderne Eltern-Kind-Beziehungen, in denen Reste einer Elternzentriertheit und traditionalen Strafverhaltens überdauert haben (Büchner/Fuhs 1996, 195). Diese Kombinationen demonstrieren exemplarisch, wie gesellschaftliche Entwicklungen in die familialen Erziehungssettings integriert werden, was u.U. auf den ersten Blick zu paradoxen Verbindungen führt.

Im Zuge der Entwicklung von der Eltern- zur Kindzentrierung verändern sich die Anforderungen an Kinder und Eltern nachhaltig. Leistungsansprüche gegenüber den Kindern gehen zurück (Schneewind/Ruppert 1995, 160), gleichzeitig nehmen die elterlichen Aufgaben und Pflichten enorm zu (Beck-Gernsheim 1990a, 167, Engelbert, u.a. 2000, 7). ${ }^{53}$ Die Kindorientierung ist mit dem Anspruch der optimalen Kindesförderung verbunden (Beck-Gernsheim 1990a, 168). Die Kindesentwicklung ist von Anfang an unter Berücksichtigung der kindlichen Bedürfnisse zu fördern, und der Druck auf Eltern ist gestiegen, sich diesen Anforderungen anzupassen (Peuckert 2007, 52 und Engelbert u.a. 2000, 7). Hiermit verbunden ist die Notwendigkeit, sich beständig zu informieren, um dem Anspruch der optimalen kindlichen Förderung gerecht zu werden (BeckGernsheim 1990a, 170 und Peuckert 2007, 52). ${ }^{54}$ Gleichzeitig werden die Eltern mit

\footnotetext{
${ }^{50}$ Liberalere Vorstellungen sind auch in westdeutschen Erziehungsratgebern der späten 1960er Jahre erkennbar (Höffer-Mehlmer 2007, 79). Erziehungsratgeber aus der DDR enthalten sogar recht früh liberalere Auffassungen in Bezug auf Geschlechtererziehung und elterliche Erziehungsstrafen (Höffer-Mehlmer 2007, 83).

${ }^{51}$ Eine zunehmende Kindorientierung konstatiert Meulemann mit Rekurs auf Reubands (1995) Untersuchungen auch für die DDR (Meulemann 1996b, 224/225).

${ }^{52}$ Das Eltern-Kind-Verhältnis ist in Büchner/Fuhs' Studie mehrheitlich (ca. zwei Drittel) durch eine Kindorientierung gekennzeichnet (Büchner/Fuhs 1996, 192). Dieses Muster findet sich insbesondere in höheren sozialen Statusgruppen, die somit als diejenigen gelten können, die zur Verbreitung der Kindorientierung beitragen.

${ }^{53}$ Nach der Wiedervereinigung gilt das für beide Landesteile (Peuckert 2008, 156, 164).

${ }^{54}$ Peuckert spricht in diesem Zusammenhang von professionalisierter Elternschaft und Büchner von einer Protoprofessionalisierung (Peuckert 2007, 52 und Büchner 2010, 532).
} 
der Botschaft konfrontiert, dass ein Leistungsversagen der Kinder letztlich ein Resultat ihres erzieherischen Fehlverhaltens sei (Peuckert 2007, 52 und Beck-Gernsheim 1990a, 171).

Die optimale kindliche Förderung ist ein Leitbild, das sich in den Erziehungsarrangements der Eltern niederschlägt (Beck-Gernsheim 1990a, 169). In Fragen der wissenschaftlichen und populärwissenschaftlichen Erziehungsratschläge sind Eltern gut informiert, ${ }^{55}$ und Mütter investieren ein hohes Maß an Zeit, Energie und finanziellen Mitteln in die Erziehung ihrer Kinder (Beck-Gernsheim 1990a, 172 und Nave-Herz 2007, 34). Gefördert wird diese Entwicklung zweifelsohne durch die abnehmende Kinderzahl pro Familie. Für ostdeutsche Eltern ergeben sich nach der Wende neue Verantwortlichkeiten in Bezug auf die existierenden Fördermöglichkeiten, Fragen der Schulwahl oder der Freizeitorganisation. Aufgaben, die zuvor teils von staatlicher Seite wahrgenommen wurden, sind nun entscheidungsoffen und müssen unter dem Blickwinkel der optimalen Förderung beurteilt werden (Uhlendorff 2004, 72).

Aus Kindersicht ist gegenwärtig die elterliche Aufmerksamkeit für das kindliche Wohlverhalten hoch (Schütze 2002, 85). Da frühere Elterngenerationen vermutlich ebenso hierauf geachtet haben, ist eine Kontinuitätslinie beschrieben. Eine zweite bildet die Aufmerksamkeit für das schulische Fortkommen der Kinder (Schütze 2002, 85). Die hiermit einhergehenden Anforderungen an die Eltern haben sich allerdings gravierend erhöht und es wird eine maximale Unterstützung erwartet (Schütze 2002, 85). ${ }^{56}$

Die beschriebene Entwicklung ist nicht mit dem Schluss zu verwechseln, vorangehenden Elterngenerationen sei es nicht um eine optimale Förderung ihrer Kinder gegangen. Allerdings erscheinen die Anforderungen an die elterlichen Leistungen wenn auch nicht unbedingt anspruchsvoller, so doch voraussetzungsvoller geworden zu sein, was Art und Umfang des Engagements betrifft. Hierbei treffen sich zwei gegensätzliche Tendenzen: Einerseits besteht die Forderung, die Individualität des Kindes zu schätzen (Gebhardt 2009a, 181), andererseits geht von dem Primat der optimalen Förderung ein erheblicher Normierungsdruck aus. ${ }^{57}$ Insbesondere scheint ein (unreflektierter) Rückgriff auf eigene Erziehungserfahrungen keine selbstverständliche Option mehr darzustellen. Hieraus ergibt sich die Frage, inwiefern die beschriebenen Entwicklungen von den Familien dieser Studie im Generationenverlauf aufgegriffen werden und wie sich das auf Tradierungsinhalte und -formen auswirkt. Schließen die einzelnen Generationen an

\footnotetext{
${ }^{55}$ Das betrifft alle sozialen Schichten (Beck-Gernsheim 1990a, 169).

${ }^{56}$ Die Motivation der Eltern, die Schulkarriere der Kinder zu fördern, ist weniger dem Wunsch nach sozialem Aufstieg geschuldet, als vielmehr Ausdruck der Furcht, die Kinder könnten durch fehlende Unterstützung Wettbewerbsnachteile erleiden (Schütze 2002, 86).

${ }^{57}$ Der steigende Normierungsdruck an sich stellt keine neue Erscheinung dar, sondern lässt sich bereits in Elternratgebern um die Wende zum 20. Jahrhundert erkennen und nimmt nach $1945 \mathrm{zu}$ (Gebhardt 2009a, 45 und 172). Allerdings sprachen die Ratgeber in früheren Zeiten nur einen begrenzten Kreis an (Gebhardt 2009a, 182). Demgegenüber haben sich der heutige Adressatenkreis sowie die zu beobachtenden und zu fördernden Bereiche des Aufwachsens erweitert.
} 


\section{Familiale Tradierungsprozesse: Einblicke in den Forschungsstand}

die eigenen Erziehungserfahrungen an, orientieren sie sich neu? Finden sich aktuell elterliche Verunsicherungen in Bezug auf Erziehungsfragen?

Steigende Aufmerksamkeit für die Beziehungsqualität Eine weitere Entwicklung stellt die verstärkte Aufmerksamkeit für die emotionale Qualität der Beziehungen in der Erziehung dar. ${ }^{58}$ Bereits gegen Ende des 18. Jahrhunderts finden sich in einigen Milieus Vertrauen und Zuneigung als Leitbilder für die Gestaltung der intergenerationellen Beziehungen (Reuband 1992, 103). Allerdings dauert es bis Ende der 1960er Jahre, bis diese bei der Erziehung in den Mittelpunkt der Betrachtung rücken. Gebhardt arbeitet in ihrer Studie das Konzept der „Lebensbemeisterung“ als ein zentrales Leitbild der 1930er und 1940er Jahre aus, das bis in die 1970er Jahre in der familialen Erziehung zu finden ist (Gebhardt 2009b, 52-54). ${ }^{59}$ Das emotionale Wohlbefinden des Kindes oder eine innige Eltern-Kind-Beziehung stehen bei diesem Leitbild nicht im Mittelpunkt. ${ }^{60}$

In der westdeutschen Sozialisationsforschung rückt gegen Ende der 1960er, Anfang der 1970er Jahre die emotionale Qualität der Mutter-Kind-Beziehung in den Fokus der Aufmerksamkeit und geht mit einer verstärkten emotionalen Zuwendung zum Kind einher (Schütze 2002, 75 und Gebhardt 2009a, 180). ${ }^{61}$ Schülein verwendet für die Entwicklung der 1960er Jahre das Schlagwort der „Beziehungsrevolution“ (Schülein 1990, 79-88). Hiermit verbunden werden die Primärkontakte zunehmend emotional differenzierter und komplexer, was tendenziell sowohl für die Partnerbeziehungen als auch für die Eltern-Kind-Beziehungen in allen sozialen Schichten gilt (Schülein 1990, 86). Für Eltern wird es zunehmend bedeutsam, die emotionale Zuneigung und Verbundenheit gegenüber ihren Kindern zum Ausdruck zu bringen (Schneewind/Ruppert 1995, 151). Im Zuge dieser Entwicklung erfahren Leitbilder der Erziehung, wie z.B. die emotionale Unterstützung der Kinder, die Anpassung elterlichen Verhaltens an die kindlichen Bedürfnisse und die Empathie für das Kind eine enorme Bedeutungssteigerung (Schü-

\footnotetext{
${ }^{58}$ Das heißt nicht, dass frühere Generationen nicht liebevoll mit ihren Kindern umgegangen seien.

${ }^{59}$ Hiermit ist ein Sozialisationsmuster beschrieben, das dem Kind bereits im Säuglingsalter durch entsprechendes Verhalten vermittelt, „dass es in dieser Welt nicht um Bedürfnisbefriedigung gehen würde, sondern um die Beherrschung der eigenen körperlichen und emotionalen Bedürfnisse zum Zwecke einer Immunisierung gegen die Härten des Lebens“ (Gebhardt 2009b, 36).

${ }^{60}$ Die Fokussierung auf das frühkindliche Sozialisationsziel der „Lebensbemeisterung“ (Gebhardt 2009a, 92) impliziert zugleich ein generelles Ziel, das - sofern internalisiert - auch nach der frühkindlichen Phase in der elterlichen Erziehung eine Rolle gespielt haben dürfte.

In der DDR wurde früher als in Westdeutschland ein anderes Verständnis in der Frühsozialisation propagiert. Es ging weniger um eine Machtprobe, sich gegen das Kind durchzusetzen, sondern vielmehr um Anleitung in der Früherziehung (Gebhardt 2009a, 147). Zugleich hielt sich länger als in Westdeutschland eine technisch-rationale Sichtweise der Erziehung (Gebhardt 2009, 155).

${ }^{61}$ Die Hinwendung zur emotionalen Qualität der Beziehungen, insbesondere der Mutter-KindBeziehung, ist eng mit Bowlbys Bindungstheorie verknüpft (Schütze 2002, 75 und Gebhardt 2009a, 167).
} 


\subsection{Familiale Erziehung}

lein 1990, 134/135). ${ }^{62}$ Hierbei werden keine gravierenden Ost-West-Unterschiede in der intergenerationellen emotionalen Bindungsqualität angenommen (Schütze 2002, 82). Heutige Eltern stehen vor der schwierigen Aufgabe, eine enge Bindung zum Kind bei gleichzeitiger Förderung der kindlichen Eigenständigkeit zu realisieren (Schütze 2002, 78).

Der zunehmende Fokus auf die emotionale Qualität der Beziehungen geht mit steigenden Erwartungen an diese einher. In der Konsequenz werden die Familienbeziehungen sowohl auf der Partner- als auch der Eltern-Kind-Ebene konfliktanfälliger (Engelbert u.a. 2000, 9) ${ }^{63}$ Hieraus allerdings zunehmend als dramatisch empfundene, grundsätzliche intergenerationelle Konflikte abzuleiten, wäre voreilig.

Zwar erinnern Erwachsene (Jahrgänge 1930 bis 1939) Mitte der 1980er Jahre seltener Konflikte und Meinungsverschiedenheiten mit den Eltern in ihrer Jugendzeit als Jugendliche der 1980er Jahre (Zinnecker 1985, 115 und 186), diese Entwicklung sieht Zinnecker jedoch weniger als Beleg für zunehmend belastete Eltern-Kind-Beziehungen, sondern vielmehr als Ausdruck einer veränderten Streitkultur, in der Konflikte thematisierbar werden. Die Machtbalancen verschieben sich zugunsten der Jugendlichen und die Ansprüche gegenüber den Eltern steigen (Zinnecker 1985, 165/166, 170). Aktuelle Befragungen zeigen, dass Jugendliche mehrheitlich „trotz gelegentlicher Meinungsverschiedenheiten mit ihren Eltern klarkommen“ (Leven/Quenzel/Hurrelmann 2010, 65). Das untermauert die Annahme einer veränderten Streitkultur, die Konflikte als Bestandteile intergenerationeller Beziehungen und nicht als Bedrohung der Beziehungen betrachtet.

Zinneckers Ergebnisse lassen sich als Ausdruck einer Umbruchphase interpretieren. Kindheit und Jugend der älteren der 1985 befragten Jugendlichen fällt in eine Zeit, in der liberalere Erziehungsvorstellungen in der Öffentlichkeit zwar diskutiert, aber noch nicht zwangsläufig in den Familien umgesetzt werden. Das Ansteigen der Meinungsverschiedenheiten zwischen Jugendlichen und Eltern ist als Ausdruck jugendlicher Forderungen an ihre Eltern zu verstehen, die öffentlich verhandelten liberaleren Erziehungsansätze in der eigenen Familie umzusetzen. Die zwischen Mitte der 1950er und Mitte der 1980er Jahre steigende Anzahl Jugendlicher, die ihre eigenen Kinder anders erziehen möchte (Zinnecker 1985, 160), stützt diese Interpretation.

\footnotetext{
${ }^{62}$ Diese Leitvorstellungen moderner Eltern-Kind-Beziehungen arbeitet Schülein anhand von Zeitschriften wie ,Eltern“ heraus (Schülein 1990, 134). Als historischen Vorläufer stellt Schülein den traditionalistischen Typus vor. Wesentliche Kennzeichen dieses Leitbildes waren z.B. in bürgerlichen Schichten die geringe emotionale Differenzierung sowie die Forderung nach Distanz in den Beziehungen (Schülein 1990, 132/133). Neben dem modernen Typus beschreibt Schülein noch den avantgardistischen, bei dem die Merkmale des modernen Typus gesteigert hervortreten. Ein wesentliches Kennzeichen ist die „bedingungslose Hinwendung zum Kind“ (Schülein 1990, 138/139).

${ }^{63}$ Beck-Gernsheim thematisiert als Kehrseiten der zunehmenden Emotionalisierung und Kindzentrierung der Eltern-Kind-Beziehungen die Gefahr von überzogenen Erwartungen, Überforderungen und ,Überemotionalisierung' (Beck-Gernsheim 1990a, 180 und 182).
} 


\section{Familiale Tradierungsprozesse: Einblicke in den Forschungstand}

Der Anteil der Jugendlichen, der mit dem elterlichen Erziehungsstil zufrieden ist und die eigenen Kinder ähnlich erziehen würde, wächst gegenüber 1985 zwischen 2000 und 2010 deutlich an (Leven/Quenzel/Hurrelmann 2010, 64/65). Das lässt sich als Indiz für einen zwischen 1985 und den 2000er Jahren erzielten, intergenerationellen Konsens bezüglich der Erziehungsmethoden deuten. Die These einer „frühzeitigen, konflikthaften Ablösung vom Elternhaus“" (Leven/Quenzel/Hurrelmann 2010, 63) steht heute nicht mehr im Mittelpunkt. ${ }^{64}$ Vielmehr interessiert die Frage, wie „lebenslange Beziehungen mit Eltern und Großeltern bei gleichzeitiger Selbständigkeit von frühester Jugend an" in den Familien realisiert werden (Bertram 1996, 78). Ebenso scheint eine Übereinstimmung zwischen den Generationen erreicht zu sein, was die Wertorientierungen betrifft. Nach Noelle-Neumann/Petersen hat sich die Anfang der 1970er Jahre entstandene Generationenkluft ${ }^{65}$ bezüglich der Wertorientierungen fast gänzlich aufgelöst. Traditionelle Werte erleben eine Renaissance und werden mit Erziehungszielen wie Durchsetzungsfähigkeit und einem Trend zum Lebensgenuss kombiniert (Noelle-Neumann/Petersen 2001, 16-21). Für diese Studie stellt sich die Frage, ob sich die Erziehungsarrangements zwischen den Generationen in Bezug auf die emotionale Beziehungsgestaltung und die familiale Streitkultur unterscheiden und welche Bedeutung diesen Aspekten jeweils zukommt.

\subsubsection{Familiale Rollen im gesellschaftlichen Wandel}

Kindheit Im Zuge des gesellschaftlichen Wandels hat sich die Sicht auf das Kind verändert. Fungierten Kinder in der Nachkriegszeit als „Hoffnungsträger“ und in den 1970er und 1980er Jahren als „Sinnstifter“, so gelten sie in den 1990er Jahren als „Akteur[e] in eigener Sache" (Schütze 2002, 83). Der Status der Kinder wechselt von Zögling zum aktiven Mitgestalter (Engelbert u.a. 2000, 8/9) ${ }^{66}$ Sie werden zunehmend als aktiv am Erziehungsprozess Beteiligte betrachtet. Die Verschiebung vom Befehls- zum Verhandlungshaushalt ist ebenso ein Ausdruck dieser Veränderungen wie die steigende Kindorientierung und die zunehmend als partnerschaftlich (Baader/Sager 2008, 305) bezeichneten Eltern-Kind-Beziehungen.

Auch in anderen Bereichen verändern sich die Anforderungen an Kinder. Kinder verfügen zwar einerseits heute über vergleichsweise viele Freiheiten und sie können ihre Freizeit in steigendem Maße an ihren Interessen ausgerichtet verbringen (Peuckert 2008, 147, 154), andererseits scheint der kindliche Alltag zunehmend verregelt. Im Zuge der

\footnotetext{
${ }^{64}$ Laut Oswald sind die innerfamilialen Generationenbeziehungen im Verlauf der letzten Jahrzehnte nicht durch generationenbedingte Konflikte geprägt gewesen und die zwar populäre, aber empirisch nicht belegte Annahme eines generellen Generationenkonflikts stellt eine Überschätzung der ElternKind-Konflikte dar (Oswald 1989, 368, 377, Zimbardo 1992, 91).

${ }^{65}$ Die Autoren beziehen sich auf die Verhältnisse in Westdeutschland.

${ }^{66}$ Die Forderung, die aktive Rolle der Kinder bei „der Konstruktion und Bestimmung ihres eigenen Lebens“ (Qvortrup 1993, 121) zu beachten, spiegelt eine wichtige Veränderung der Perspektive auf Kinder.
} 


\subsection{Familiale Erziehung}

Institutionalisierung von Kindheit werden immer mehr speziell an Kinder adressierte Freizeitaktivitäten angeboten, die mit relativ festen Terminen und Verbindlichkeiten einhergehen sowie „von Erwachsenen geplant, betreut und kontrolliert werden“, wodurch die Eigenorganisation des Kinderlebens durch Kinder eingeschränkt wird (Peuckert 2008, 152). Die zunehmende Verhäuslichung ${ }^{67}$ von Kindheit, in deren Rahmen immer mehr kindliche Aktivitäten in Privaträume verlagert werden (Peuckert 2008, 152), erhöht die Kontroll- und Steuerungsmöglichkeiten der Erwachsenen. ${ }^{68}$ Die zunehmende Mediennutzung (Peuckert 2008, 149) dürfte die Tendenz der Verhäuslichung zusätzlich befördern. Diese Entwicklung geht übrigens nicht mit einer Abnahme sozialer Kontakte einher (Peuckert 2008, 151). Insgesamt ist Kindheit in Westdeutschland in stärkerem Maße als in Ostdeutschland durch das „Muster einer Termin- und Vereinskindheit“ geprägt (Peuckert 2008, 153). Dieses Muster könnte durch den Umstand gefördert worden sein, dass Kinder als Folge des demografischen Wandels in den ersten Lebensjahren in erster Linie in enger Beziehung zu Erwachsenen aufwachsen und Spielgruppen in Anbetracht der sinkenden Kinderzahl zunehmend organisiert werden müssen (Nave-Herz 2007 35/36). Kinder verfügen heute über viel Freizeit (Peuckert 2008, 148/149).$^{69}$ Es ist aber auch eine zunehmende Verschulung der Freizeit erkennbar (Peuckert 2008, 154), was angesichts des Postulats der optimalen kindlichen Förderung nicht verwunderlich ist.

Insgesamt scheint Kindheit heute durch zunehmende Freiheiten und Entfaltungsmöglichkeiten bei gleichzeitiger Einschränkung dieser Freiheiten durch steigende Kontrollmöglichkeiten der Erwachsenen sowie steigender Anforderungen an die kindlichen Kompetenzen gekennzeichnet zu sein. Neben diesen erheblichen Veränderungen von Kindheit, die mit veränderten Rollenanforderungen einhergehen, existieren aber auch Kontinuitäten, wie z.B. die Tatsache, dass trotz allen Wandels große Teile der kindlichen Sozialisation und Erziehung in der Familie erfolgen (Büchner 2010, 519).

Mutterschaft Eine wesentliche Veränderung betrifft die mütterliche Erwerbstätigkeit, die zwischen 1961 und 2004 in Westdeutschland stark angestiegen ist. 1961 arbeiteten 34,7\% der Mütter mit Kindern unter 18 Jahren bzw. 29,7\% der Mütter mit Kindern unter sechs Jahren. In der DDR gab es eine höhere Quote. 1989 betrug der Anteil erwerbstätiger Mütter innerhalb der Gesamtgruppe erwerbstätiger Frauen 92\% (Sommerkorn/Liebsch 2002, 113). Nach der Wende waren Frauen verstärkt von Ar-

\footnotetext{
${ }^{67}$ Zinnecker beschreibt mit der Verhäuslichung eine Entwicklung, in der die kindlichen Handlungsräume immer stärker in „geschützte Räume hineinverlagert“ werden (Zinnecker 1990, 142). Im Zuge der Verhäuslichung können soziale Handlungen voneinander separiert und „stabile und berechenbare Handlungsräume“ geschaffen werden (Zinnecker 1990, 143).

${ }^{68}$ Die Verlagerung kindlicher Aktivitäten in geschlossene Räume trifft in höherem Maße für Mädchen $\mathrm{zu}$ und ist insgesamt in Westdeutschland stärker ausgeprägt als in Ostdeutschland (Peuckert 2008, 147, 150).

${ }^{69}$ Jungen verfügen insgesamt über mehr freie Zeit als Mädchen (Peuckert 2008, 148/149).
} 


\section{Familiale Tradierungsprozesse: Einblicke in den Forschungstand}

beitsplatzverlusten betroffen (Sommerkorn/Liebsch 2002, 119), wodurch sich der Anteil erwerbstätiger Frauen und Mütter in Ostdeutschland verringert hat. Für 2004 weisen die Statistiken 63,3\% erwerbstätiger Mütter mit Kindern unter 18 Jahren bzw. 52\% erwerbstätiger Mütter mit Kindern unter sechs Jahren aus (Nave-Herz 2007, 39). ${ }^{70}$

Die unterschiedliche Erwerbsbeteiligung in den beiden Landesteilen vor der Wende korrespondiert mit unterschiedlichen Mutterbildern. Während in der DDR die berufstätige Mutter das dominante und staatlich propagierte Leitbild darstellte (Sommerkorn/ Liebsch 2002, 113), das angesichts der nach wie vor bestehenden höheren Erwerbsbeteiligung in Ostdeutschland offensichtlich bis heute wirksam ist, dominierte in Westdeutschland lange Zeit die „soziale Festschreibung der Frauenrolle auf den Lebensbereich Familie“ (Sommerkorn/Liebsch 2002, 103). Erst Ende der 1980er Jahre ist das Leitbild der Doppelorientierung an Familie und Beruf keine Ausnahmeerscheinung mehr (Sommerkorn/Liebsch 2002, 112). Bowlbys Bindungstheorie mit der Forderung nach einer „warme[n], intensive[n] und beständige[n] Beziehung“ (Bowlby 1972, 69) des Kindes zur Mutter übt laut Schütze in der zweiten Hälfte des 20. Jahrhunderts einen starken Einfluss auf das Bild von der Mutter aus; kindliche Fehlentwicklungen werden in der Folge dem bewussten oder unbewussten Verhalten und Gefühlen der Mutter angelastet (Schütze 2000, 95/96). Die sich hieraus ergebenden Rollenzwänge wirken sich bis heute auf westdeutsche Mütter ${ }^{71}$ aus (Schütze 2000, 96). Allerdings erfuhr die Mutterrolle in den letzten Jahrzehnten insofern eine Entlastung, als die Bedeutung des Vaters für die Erziehung des Kindes entdeckt wurde (Schütze 2000, 97). Es stellt sich jedoch die Frage, ob das Postulat der optimalen Kindesförderung, das die Rolle einer allseitigen Entwicklungshelferin (Beck-Gernsheim 1990a, 142, 171 und 172) umfasst, die Entlastung nicht wieder aufhebt. ${ }^{72}$

Das Thema der mütterlichen Erwerbsarbeit scheint bis heute zu polarisieren. Die Favorisierung einer mütterzentrierten Erziehung, die zumindest eine mehrjährige Erwerbsunterbrechung impliziert, hat in Westdeutschland nicht ausgedient ${ }^{73}$ und findet dort in weit höherem Maße Zustimmung als in Ostdeutschland (Gerhards/Hölscher 2003, 213/214).

Hieraus ergeben sich eine Reihe von Fragen für diese Studie: Wie gingen und gehen die Mütter des hier untersuchten Samples mit den an sie herangetragenen Anforderun-

\footnotetext{
${ }^{70} 2004$ wird nicht mehr zwischen Ost- und Westdeutschland unterschieden. Die letzten Daten für Westdeutschland stammen aus dem Jahr 2000 und liegen auf einem ähnlichen Niveau wie 2004 (Nave-Herz 2007, 39).

${ }^{71}$ Ein Mutterbild, das die Erziehung allein als mütterliche Aufgabe sah und ihr zugleich die alleinige Verantwortlichkeit für die Entwicklung des Kindes zuschrieb, konnte in Ostdeutschland schon allein wegen der staatlich forcierten außerfamilialen Sozialisation nicht im gleichen Maße greifen wie in Westdeutschland.

${ }^{72}$ Diese Aufgabe stellt sich spätestens seit der Wende auch für Eltern in Ostdeutschland verstärkt.

${ }^{73}$ Auch wissenschaftliche Erkenntnisse, die zeigen, dass mütterliche Erwerbsarbeit und außerhäusliche Betreuung der Kinder sich nicht automatisch negativ auf die kindliche Entwicklung auswirken (Nave-Herz 2007, 44), können an dieser Einstellung offensichtlich nichts ändern.
} 


\subsection{Familiale Erziehung}

gen um? Orientieren sie sich an den praktizierten Familienmodellen ihrer Mütter oder setzen sie sich von diesen ab? Inwiefern unterschieden bzw. unterscheiden sich die Lebensentwürfe der Mütter im östlichen und westlichen Teilgebiet in der vorliegenden Studie?

Vaterschaft Hämmerle verdeutlicht, dass das Deutungsmuster des abwesenden Vaters zwar populär, aber zu pauschal ist, um den vielfältigen gelebten Vaterschaftskonzepten in historischer Perspektive gerecht zu werden (Hämmerle 2010, 141). Die Forschung zum Thema zeigt die Heterogenität der existierenden Familien- und Erziehungsarrangements in der Geschichte (Friebertshäuser/Matzner/Rothmüller 2007, 182 und Hämmerle 2010, 128/129), und Väter sind auch in der Vergangenheit in die Erziehung der Kinder involviert gewesen (Nave-Herz 2007, 55).

Eine weit verbreitete Vorstellung vom Leitbild der Vaterrolle der 1950er Jahre in der Bundesrepublik ist die des Vaters als Familienernährer ${ }^{74}$ und als oberste familiale Autorität. Es wurde von vielen gesellschaftlichen Gruppierungen vertreten und durch sozialpolitische Maßnahmen gestützt (Plötz 2006, 65 und 67/68). ${ }^{75}$ Aus empirischer Sicht hingegen ist die Umsetzung dieses Leitbildes auch für die 1950er Jahre längst nicht so eindeutig. Vielmehr lassen sich bereits für diese Zeitperiode auf der Mikroebene Konflikte um die Position des Vaters ausmachen (Plötz 2006, 71) und zeitgenössische Studien sehen in Westdeutschland die Vorrangstellung der väterlichen Autorität in Abbau begriffen (Baumert 1952, 84 und König 1974, 230). Trotz dieser Veränderungstendenzen finden sich auch noch einige Jahre später bei Heranwachsenden in das Elternbild eingelagerte geschlechtsrollenstereotype Zuschreibungen. ${ }^{76}$

Angesichts des in der DDR propagierten Leitbildes der berufstätigen Frau und Mutter (Schlegel 1999, 382) ließe sich annehmen, dass in der DDR ein anderes Vaterbild existiert hätte. Die gesetzlichen Grundlagen, die eine Gleichberechtigung der Ehegatten, geteilte Haushalts- und Erziehungspflichten sowie eine Förderung der weiblichen Erwerbsarbeit vorsahen ( $\$ 2,9$ und 10 Familiengesetzbuch der Deutschen Demokratischen Republik vom 20.12.1965 und GBl. Nr. 111, 1037 vom 27.09.1950 zit.n. Hille 1985, 32/33), schlossen eine ähnliche Diskussion um Familie und die Vorrangstellung des Vaters in der Familie aus. Aber auch wenn Gysi 1989 postuliert, in der Betreuung und Erziehung der Kinder schiene „die traditionelle Geschlechtstypik am weitesten ab-

\footnotetext{
${ }^{74}$ Anfang der 1960er Jahre waren in einer westdeutschen Studie für zwei Fünftel der befragten 23jährigen Frauen die Erwerbsarbeit des Mannes Voraussetzung für eine Eheschließung, wohingegen die befragten Männer dies umgekehrt nicht von ihren Partnerinnen erwarteten (Pfeil 1968, 76).

${ }^{75}$ Bis 1959 fand sich die Vormachtstellung des Vaters in der Familie zudem juristisch fixiert im BGB (Plötz 2006, 64/65).

${ }^{76}$ Mütter werden vermehrt Attribute wie zärtlich und milde zugeschrieben, Vätern hingegen vermehrt Eigenschaften wie gleichbleibend, stark, festbleibend; sowohl das Vater- als auch das Mutterbild der Befragten fallen überwiegend positiv aus; inwiefern das mit dem tatsächlichen elterlichen Verhalten deckungsgleich ist, lässt sich nicht feststellen (Friedrichs 1968, 135 und 129).
} 


\section{Familiale Tradierungsprozesse: Einblicke in den Forschungstand}

gebaut“, muss sie gleichzeitig eingestehen, dass in der Familie trotzdem eine stereotype Rollenverteilung herrschte und Frauen sich im größeren Umfang um die Kinder kümmerten (Gysi 1989, 192). ${ }^{77}$ Ende der 1980er Jahre ist eine Mehrheit der Männer der Ansicht, Männer sollten die Rolle des Familienernährers einnehmen sowie Oberhaupt der Familie sein (Schlegel 1999, 384). Trotz der Veränderungen, die sich zweifelsohne in der Frauen- und Mutterrolle in der DDR vollzogen haben, hat sich offenbar ein recht patriarchalisches Leitbild vom Vater erhalten.

Aktuell werden die Bedeutung des Vaters für die kindliche Entwicklung sowie die Frage der Vereinbarkeit von Familie und Beruf für Väter thematisiert (Baader 2006, 121, 129, Kortendiek 2004, 387). Studien nehmen eine wachsende väterliche Involviertheit in die Erziehung wahr, die mit einem gewandelten Selbstverständnis der Väter einhergeht: Väter betrachten sich zunehmend als Erzieher ihrer Kinder (Friebertshäuser/ Matzner/Rothmüller 2007, 185).$^{78}$ Es werden verschiedene Vaterschaftskonzepte identifiziert. Neben dem traditionellen und dem modernen Ernährer gibt es z.B. den ganzheitlichen sowie familienzentrierten Vater (Matzner 2004, 339-435).

Diese Forschungsergebnisse zeigen, dass Väter am Erziehungsprozess beteiligt waren und sind, wenn auch in unterschiedlicher Weise. Das führt zu der Frage, wie die Väter des in der vorliegenden Studie untersuchten Samples ihre Elternschaft gestalten und ob zwischen der ältesten und mittleren Generation Unterschiede zu finden sind.

Großelternschaft Elemente der spätestens seit der ersten Hälfte des 20. Jahrhunderts etablierten großelterlichen Rollennormen sind eine feststellbare Intensivierung der Großeltern-Enkel-Beziehungen bei gleichzeitiger Anerkennung der elterlichen Vorrangstellung, die Gewährung größerer Freiräume für die Enkelkinder sowie die Attribuierung der Großeltern als altmodisch (Chvojka 2003, 128, 129, 143, 151, 161, 198, 201 und 349/350), die bis heute aktuell sind (Höpflinger/Hummel/Hugentobler 2006, 18, 20). ${ }^{79}$ Mit der Zuschreibung „altmodisch“ verbindet sich zugleich die Vorstellung konstitutiver Unterschiede in Werthaltungen und Verhalten zwischen Großeltern und Enkeln, was dazu beiträgt, das Konfliktpotenzial von vornherein gering zu halten (Höpflinger/Hummel/Hugentobler 2006, 18). Insgesamt werden mit der Großelternrolle heute überwiegend positiv konnotierte Stereotype verbunden und die Großeltern als wichtige Bezugspersonen für die Enkel betrachtet (Höpflinger/Hummel/Hugentobler 2006, 21, Uhlendorff 2009, 2). Zugleich werden Großeltern relativ große Freiräume bei der Ausgestaltung der Großeltern-Enkel-Beziehungen eingeräumt (Höpflinger/Hummel/

\footnotetext{
${ }^{77}$ Auf letzteres verweist auch Hille $(1985,113)$.

${ }^{78}$ Allerdings besteht eine Diskrepanz zwischen der wachsenden Anzahl an Vätern, die sich in die Erziehung der Kinder aktiv einbringen möchten, und der tatsächlichen Praxis (Baader 2006, 128).

${ }^{79}$ Daneben entwickelte sich gegen Ende des 19. Jahrhunderts auch eine negative Sichtweise auf Großeltern, die ihnen veraltete Ansichten in Bezug auf die Kindeserziehung sowie letztendlich negative Einflüsse auf das Kind unterstellte (Chvojka 2003, 291). Diese Negativstereotype finden sich in der Literatur bis in die Nachkriegsjahre und in einem Einzelfall sogar noch 1980 (Gebhardt 2009a, 54).
} 


\subsection{Familiale Erziehung}

Hugentobler 2006, 21). Großeltern engagieren sich z.B. in der Enkelbetreuung, beraten in Krisensituationen, haben eine ratgebende Funktion, vermitteln kulturelle Werte, bewahren Familientraditionen und leisten finanzielle Unterstützung (Brake/Büchner 2007, 209-214)..$^{80}$

Herlyn/Kistner arbeiten drei Gestaltungsmuster von Großmutterschaft heraus. Für die „familienorientierten Großmütter“ hat die Großmutterschaft eine „zentrale Bedeutung“, für „doppelorientierte Großmütter“ ist eine „Gleichgewichtung von Großmutterschaft und anderen außerfamilialen Interessen“ wichtig und bei „außerfamilial orientierten Großmüttern“ dominieren außerfamiliale Interessen (Herlyn/Kistner 1997, 10). Trotz des weitaus höheren Maßes an Vollzeiterwerbstätigkeit sind familienorientierte Großmütter verstärkt in Ostdeutschland zu finden (Herlyn/Kistner 1997, 10). ${ }^{81}$ In der Gesamtschau existiert eine relativ hohe Kontakthäufigkeit zwischen Großmüttern und Enkeln (Herlyn/Kistner 1997, 12). ${ }^{82}$ Viele Großmütter möchten ihre Enkel verwöhnen und sich nicht in deren Erziehung einmischen (Herlyn/Kistner 1997, 9 und 12.)

Wie sich zeigt, orientieren sich Großmütter einerseits an den gängigen Rollenklischees, andererseits gestalten sie ihre Rolle jenseits dieser Stereotype unterschiedlich. Verschiedene Muster der Rollengestaltung sind hierbei nicht nur für die Großmutterschaft anzunehmen, sondern für Großelternschaft generell (Roseann/Silverstein 1996, Haumann 2006). ${ }^{83}$ Lüscher entfaltet auf theoretischer Ebene eine Typologie von Großelternschaft: Großeltern können die Rolle von Autoritätspersonen, Gefährten, Patriarchen oder Unbekannten annehmen (Lüscher 2008, 51/52).

Höpflinger/Hummel/Hugentobler identifizieren drei Sichtweisen von Enkeln auf Großeltern. Dominierend ist „ein gefühlsorientiertes positives Grosselternbild“, das mit Eigenschaften wie „grosszügig, liebevoll und gesellig" sowie "humorvoll und tolerant" assoziiert ist (Höpflinger/Hummel/Hugentobler 2006, 55, 57). Daneben gibt es ein eher negativ konnotiertes „Bild von strengen und ungeduldigen Grosseltern, die für nachkommende Generationen wenig Verständnis aufweisen“ (Höpflinger/Hummel/

\footnotetext{
${ }^{80}$ Beratende oder kulturelle Werte vermittelnde Großeltern widersprechen der Attribuierung als ,altmodisch', sofern es sich um anschlussfähige Inhalte für die nachfolgenden Generationen handelt. Es ist anzunehmen, dass diese Zuschreibung eher ein gängiges Klischee ist, dem auf empirischer Ebene keineswegs durchgängig entsprochen wird.

${ }^{81}$ Das korrespondiert mit den Ergebnissen der Werteforschung, die in Ostdeutschland eine höhere Familienorientierung konstatiert.

${ }^{82}$ Die Kontakthäufigkeit ist von der Wohnortentfernung sowie vom Alter der Enkel und Großmütter abhängig (Herlyn/Kistner 1997, 12). Hierbei sind kaum schichtspezifische Unterschiede festzustellen; Großmütter aus höheren Schichten sind lediglich kulturell interessierter, was sich auf die gemeinsamen Aktivitäten mit den Enkeln auswirkt (Herlyn/Kistner 1997, 14).

${ }^{83}$ Neugarten/Weinstein (1964) untersuchen in den USA der 1960er Jahre die verschiedenen Aspekte von Großelternschaft wie die Zufriedenheit mit der Rollengestaltung, Bedeutung der Großelternrolle sowie Großelternstil. Neben Unterschieden in Zufriedenheit und Bedeutung der Großelternrolle können sie fünf verschiedene Großelternstile identifizieren: Formal, Fun-Seeking, Parent-Surrogate, Reservoir of Family Wisdom sowie Distant Figure (Neugarten/Weinstein 1964, 201-204).
} 


\section{Familiale Tradierungsprozesse: Einblicke in den Forschungsstand}

Hugentobler 2006, 57). Die dritte Perspektive zeichnet ein „Bild von altmodischen und dazu gleichzeitig geizigen Grosseltern, die nicht mehr à jour sind“ (Höpflinger/ Hummel/Hugentobler 2006, 57).

Uhlendorff beklagt, in Deutschland gebe es kaum Forschung, die direkte oder indirekte Einflüsse von Großeltern auf Enkel untersucht (Uhlendorff 2003, 120/121). Da die vorliegende Studie ausdrücklich Tradierungsprozesse in einer Drei-GenerationenPerspektive zum Gegenstand hat, sind zumindest die indirekten Einflüsse ausdrücklich thematisiert. Von besonderem Interesse für diese Studie ist, wie die Großelternschaft in den einzelnen Familien gestaltet wird und welche Vorstellungen von Großelternschaft vorhanden sind. Lassen sich starke Kontinuitäten finden, wie die erstaunliche Dauerhaftigkeit einiger Rollenstereotype nahelegt, oder führt der gesellschaftliche Wandel zu spürbaren Veränderungen? Unterscheiden sich die praktizierten Großelternkonzepte in den beiden Teilregionen, wie angesichts der aufgelisteten Ost-West-Unterschiede zu vermuten ist?

\subsection{Familiale Tradierungsprozesse}

\subsubsection{Die innerfamiliale Verhandlung von Geschichte}

Familiale Tradierungsprozesse sind in Bezug auf unterschiedliche Aspekte untersucht und belegt. Es existieren diverse Beiträge und Studien, die sich damit befassen, wie bestimmte historische Ereignisse bzw. die Handlungen von Familienmitgliedern während des Nationalsozialismus innerfamilial thematisiert und verhandelt werden, wie von einzelnen Familienmitgliedern erlebte historische Ereignisse auf nachfolgende Generationen wirken und welche Deutungsmuster zu einzelnen Zeitepochen wie dem Nationalsozialismus oder auch der DDR innerhalb der einzelnen Familiengenerationen existieren (Rosenthal 2000, Welzer/Moller/Tschugnall 2002, Wachsmuth 2008, Radebold/ Bohleber/Zinnecker 2009, Lehmann 2009). Im Mittelpunkt steht die Verarbeitung individueller Lebensgeschichten und deren intergenerationelle Weitergabe im Zusammenspiel mit herausgehobenen historischen Ereignissen oder Epochen. Diese Studien liefern wertvolle Einblicke, wie bestimmte Tradierungsinhalte bewusst und unbewusst weitergegeben, umgearbeitet oder abgelehnt werden. Es wird deutlich hervorgehoben, dass nachfolgende Generationen keine passiven Rezipienten, sondern aktiv an der Bearbeitung des familialen Erbes beteiligt sind (Rosenthal 1997, 59/60, Rosenthal 2000, 166 und Welzer/Moller/Tschugnall 2002).

Auch wenn Tradition üblicherweise mit Bewahren bzw. Dauerhaftigkeit gleichgesetzt wird, zeigen die Ausführungen, dass Traditionen und Tradierungsinhalte sich verändern können und dies i.d.R. auch laufend geschieht, wobei sich die Umformungen auf der Grundlage des Bestehenden vollziehen und sich auf diese Weise Varianten bilden können (Shils 1981, 13/14). Dieser Gedanke findet sich auch bei Giddens, der Traditionen - und wie sich ergänzen lässt: Tradierung - folgerichtig als „etwas aktives und interpre- 


\subsection{Familiale Tradierungsprozesse}

tatives" betrachtet (Giddens 1993, 451). Um ein vollständiges Bild von Tradierungsprozessen zu erhalten, sind bei der Analyse diese Bearbeitungen zu berücksichtigen.

\subsubsection{Tradierung von Werteeinstellungen}

In seiner Drei-Generationen-Studie stellt Bengston Ähnlichkeiten in den Werteeinstellungen zwischen den Generationen sowie bedeutende intragenerationelle Varianzen fest und schlussfolgert, dass es keinen substantiellen generation gap gebe (Bengston 1975, 368). Vielmehr nehmen Troll/Bengston beträchtliche innerfamiliale Kontinuitäten für Bereiche wie den politischen oder religiösen Sektor an (Troll/Bengston 1979, 136 und 139). Auch neuere Studien können Zusammenhänge zwischen den Werteeinstellungen der einzelnen familialen Generationen feststellen bzw. begründet vermuten (Bertram 1994, 132, Boehnke/Welzel 2006, 357, Grob/Weisheit/Gomez 2009, 363). Es gibt aber auch Facetten, wie z.B. geschlechtsrollenstereotype Vorstellungen und Fragen des Lebensstils, die stärker von Kohorten- und Periodeneffekten beeinflusst zu werden scheinen (Troll/Benston 1979, 142). Dem Freundeskreis schreiben sie eine ergänzende Funktion zu (Troll/Bengston 1979, 146). Hiermit sind unterschiedliche Akteursgruppen thematisiert, die den Vermittlungs- und Aneignungsprozess beeinflussen. Schönpflug nennt in diesem Zusammenhang drei Gruppen: Eltern (vertikal), Gleichaltrige (horizontal) sowie weitere erwachsene Kontaktpersonen, wie z.B. Lehrer (Schönpflug 2001, 133). Auch wenn viele Inhalte sowohl von der einen als auch der anderen Gruppe tradiert werden (Schönpflug 2001, 133), zeigt sich hier die Komplexität familialer Tradierungsprozesse. Groß/Wößner lenken das Augenmerk in diesem Zusammenhang unter Rückgriff auf Bronfenbrenner auf das Zusammenspiel von Mikro-, Meso- und Makroebene (Groß/Wößner 2008, 137).

Die verschiedenen Studien arbeiten vielfältige Kriterien heraus, die die innerfamiliale Weitergabe von Werten beeinflussen. Zunächst sind hier Filter zu nennen, wie die elterliche Motivation, bestimmte Inhalte weiterzugeben sowie die Bereitschaft der Kinder, die elterlichen Vorgaben zu akzeptieren (Schönpflug/Bilz 2009, 229/230). Letzteres hängt nicht zuletzt vom Inhalt ab (Knafo/Schwartz 2009, 257). Mit den altersspezifischen Entwicklungsabschnitten werden intragenerationelle Gemeinsamkeiten in Bezug auf Lebensziele erklärt (Grob/Weisheit/Gomez 2009, 358 und 360) und - zumindest indirekt - intergenerationelle Varianzen plausibel. Die Bereitschaft der Kinder, elterliche Tradierungsinhalte zu akzeptieren, ist daneben von ihrem Lebensalter abhängig. Schönpflug/Bilz gehen davon aus, dass im Kindesalter größere Kongruenzen zwischen Kindern und ihren Eltern zu finden sind als während der Jugend und der Phase der Ablösung vom Elternhaus (Schönpflug/Bilz 2009, 216). Bengston weist darüber hinaus auf die jeweilige Biografie und die individuelle Verarbeitung sozio-historischer Ereignisse hin, die sich auf die Ausbildung globaler Wertorientierungen stärker auswirken würden als die familialen oder gesellschaftlichen Bedingungen (Bengston 1975, 368).

Schönpflug/Bilz (2009) identifizieren in ihrer quantitativen Studie verschiedene transmission belts (Transmissionsriemen), worunter sie Faktoren verstehen, die die Weiterga- 


\section{Familiale Tradierungsprozesse: Einblicke in den Forschungsstand}

be von Werten ${ }^{84}$ unterstützen. Ein empathischer Erziehungsstil unterstützt in ihrem Sample z.B. die Weitergabe kollektivistischer Werte (Schönpflug/Bilz 2009, 219). Weitere transmission belts stellen ein höheres und hohes Ausbildungsniveau der Eltern sowie eine harmonische Ehebeziehung dar (Schönpflug/Bilz 2009, 220, 235). Für die Tradierung bestimmter Werte werden zudem Gender- und Umwelteinflüsse konstatiert (Knafo/Schwartz 2009, 260 und 261). Troll/Bengston hingegen finden keine herausgehobenen Hinweise für substantiell differierende Einflüsse von Vater und Mutter für die Tradierung von bestimmten Orientierungen, wie z.B. die religiöse Einstellung (Troll/ Bengston 1979, 144/145). Bertram stellt deutliche schichtspezifische Varianten fest. Werte wie Pflicht, Leistung, Gehorsam und Unterordnung beispielsweise sind häufiger bei Personen mit niedrigeren Schulabschlüssen zu finden (Bertram 1994, 133) ${ }^{85}$

Wie die Studien nachdrücklich zeigen, existieren familiale Wertetradierungsprozesse. Sicherlich ist Bengstons Argumentation insofern zuzustimmen, als nicht von deterministischen Auswirkungen familialer oder generationeller Faktoren auszugehen ist. Gleichwohl ist die Annahme berechtigt, dass individuelle Entscheidungen und Orientierungen von diesen Sphären beeinflusst werden. Die Varianz der Wertepräferenzen in Abhängigkeit vom jeweiligen Altersstadium zu betrachten, ist ein überzeugender Gedanke. Trotzdem können familiale Leitthemen existieren, die zwar je nach Altersstufe mal mehr mal weniger bedeutend für die einzelnen Familienmitglieder sind, die aber trotzdem als Orientierungsmuster in den einzelnen Generationen vorhanden sind. In den vorgestellten Studien werden der gesellschaftliche Wandel und die innerfamiliale Bearbeitung von Tradierungsinhalten und -formen als potenzielle Einflussfaktoren eher randständig behandelt - Aspekte, die in dieser Studie im Mittelpunkt stehen. Es ist von Interesse, ob die familiale Tradierung durch gesellschaftlichen Wandel beeinflusst wird und ob sich generationell rückbindbare Verarbeitungen von Tradierungsinhalten und -formen entdecken lassen. Um ihre innerfamiliale Bearbeitung in vertikaler Perspektive nachzeichnen zu können, wird nicht wie in den meisten der vorgestellten Wertestudien auf zuvor festgelegte Items zurückgegriffen. Vielmehr werden die zentralen Orientierungen der Familienmitglieder aus dem Interviewmaterial heraus rekonstruiert. Auf diese Weise lässt sich nachzeichnen, ob und auf welche Weise Inhalte und Formen der Tradierung im Generationenverlauf bearbeitet werden.

\footnotetext{
${ }^{84}$ Die Konzeptionalisierung der Werte und Wertedimensionen folgt der Schwartz'schen Wertetypologie (Schönpflug/Bilz 2009, 217). Schwartz fasst die Wertetypen Self-Direction, Stimulation, Security, Conformity, Tradition, Power, Achievement, Hedonism, Benevolence und Universalism in einem Kreisdiagramm zu den bipolar angeordneten Wertedimensionen Openness to change vs. Conservation sowie Self-Enhancement vs. Self-Transcendence zusammen (Schwartz 1992, 43 und 1996, 5).

${ }^{85}$ Die Wertevermittlung findet nicht allein im Elternhaus statt, sondern auch in der Schule (Bertram 1994, 134).
} 


\subsubsection{Tradierungsprozesse im Zeichen gesellschaftlichen Wandels}

Zinnecker geht davon aus, dass Tradierung von der älteren zur jüngeren Generation nach wie vor bedeutsam ist und den Eltern z.B. bei der Vermittlung von Religion an die Kinder eine wichtige Rolle zukommt (Zinnecker 1998, 346 und 348 und Zinnecker/ Hasenberg 1999, 456). Von besonderer Bedeutung für die religiöse Erziehung in der Familie sind das elterliche Engagement „für Religion und Erziehung“ sowie die Frage, inwiefern die Eltern selbst religiös erzogen worden sind (Zinnecker/Hasenberg 1999, 456 und Zinnecker/Georg 1996, 348). Der Bildungsstand der Eltern, das praktizierte Familienmodell, das Ausmaß der elterlichen Kontrolle, die Erziehung zur Selbstständigkeit oder der Umfang der elterlichen Hilfeleistungen sind demgegenüber nachrangig (Zinnecker/Hasenberg 1999, 455). Auch die 15. Shell Jugendstudie arbeitet einen Zusammenhang zwischen einem religiösen Elternhaus und den religiösen Vor- und Einstellungen der Kinder heraus (Gensicke 2006, 224) ${ }^{86}$ Der allgemeine Bedeutungsrückgang kirchlicher Institutionen verstärkt hierbei den Stellenwert der Familie zusätzlich. ${ }^{87}$

Alheit/Bast-Haider/Drauschke untersuchen die Mentalitätslage der Menschen in einer ostdeutschen Grenzregion (Alheit/Bast-Haider/Drauschke 2004, 11). ${ }^{88}$ Sie stellen als dominantes Muster eine intergenerationale Modernisierungsresistenz fest (Alheit/ Bast-Haider/Drauschke 2004, 324). Ihre wesentlichen Kennzeichen sind „veränderungsresistente Grundstimmungen“, die sich auch auf intergenerationale Botschaften beziehen (Alheit 2005, 35). Teile der Bevölkerung weigern sich, „ihre soziale Situation zu verändern" und geben „diese ,Botschaft" auch an die Folgegenerationen weiter" (Alheit/Bast-Haider/Drauschke 2004, 324) ${ }^{89}$ Die aufgeführten Studien können insgesamt die Tradierung von Inhalten aufzeigen. Die sich hieran anschließende spannende Frage lautet, wie die Tradierungsprozesse konkret gestaltet sind.

Berteaux/Berteaux-Wiame untersuchen die soziale Mobilität von Familien im Generationenverlauf (Berteaux/Berteaux-Wiame 1991). Den Gedanken der Veränderung berücksichtigend unterscheiden sie als Tradierungsvarianten Transmissionsprozesse zum

\footnotetext{
${ }^{86}$ Hierbei existieren deutliche Ost-West-Unterschiede. Der Prozess der Entkirchlichung sowie ein Bedeutungsrückgang der Religiosität sind z.B. unter Kindern und Jugendlichen in Ostdeutschland sehr viel ausgeprägter als in Westdeutschland (Zinnecker/Strozda/Georg 1996, 335 und Gensicke 2006, 223).

${ }^{87}$ Indizien für den Bedeutungsrückgang kirchlicher Institutionen sind die abnehmende Nachfrage nach kirchlichen Riten, wie Taufen und Trauungen, sowie die sinkende Zahl an Gottesdienstteilnahmen in den letzten Dekaden (Wolf 2003, 55). Letzteres gilt auch für Jugendliche (Schwab 2007, 505). Wolfs Feststellung, dass zwar die Gruppe derjenigen, „die eine stärkere Religiosität tradieren könnten, kleiner geworden ist“, diese aber offensichtlich in diesem Bereich relativ effektiv ist (Wolf 1995, 352), unterstützt wiederum Zinneckers Ausführungen.

${ }^{88}$ Die Untersuchung basiert vor allem auf qualitativen Interviews von Großeltern und Enkeln (Alheit/ Bast-Haider/Drauscke 2004, 134).

${ }^{89}$ Alheit/Bast-Haider/Drauschke vermuten, dass dieses Muster auf andere Bereiche der DDRGesellschaft übertragbar ist (Alheit/Best-Haider/Drauschke 2004, 340).
} 


\section{Familiale Tradierungsprozesse: Einblicke in den Forschungstand}

Identischen und Transmissionsprozesse zum Äquivalenten. Im ersten Fall werden Inhalte unverändert von einer Generation an die nächste weitergegeben (Berteaux/BerteauxWiame 1991, 23). Im zweiten Fall werden die Inhalte im Zuge der Vermittlung und Aneignung transformiert. Sie gehen zudem von einem „Nebeneinander von Bewahrung und Transformation“ aus (Berteaux/Berteaux-Wiame 1991, 38); die gleichzeitige Existenz von Bestehendem und Veränderungen sind als Regelfall anzunehmen und nicht als Ausnahme. Es wäre auch ungewöhnlich und unwahrscheinlich, wenn es in der Familie bzw. der Gesellschaft ausschließlich zu Veränderungen käme oder das Bestehende bewahrt werden würde. Viel plausibler ist die Annahme, dass Neues und Bestehendes häufig wechselseitig aufeinander bezogen sind, Veränderungen sich also auf Basis des Bestehenden vollziehen. In Anbetracht dieser Prämisse stellt die ausschließliche Konzentration auf eine Generation, selbst wenn soziale Wandlungsprozesse und Brüche im Zentrum der Betrachtung stehen, eine unzulässige Verkürzung der Perspektive dar. Eine Einbeziehung der intergenrationellen Austauschprozesse ist unabdingbar, um sowohl Brüche und Veränderungen erkennen zu können als auch Kontinuitäten, die trotz Wandels vorhanden sind, nicht aus dem Blick zu verlieren. Hierzu ist eine Mehrgenerationenperspektive erforderlich. Innerhalb dieser Mehrgenerationenperspektive erscheint es lohnend, Berteaux/Berteaux-Wiames Idee auf die Frage anzuwenden, wie Tradierungsinhalte und -formen im Zuge der familialen Erziehung bearbeitet und ggf. transformiert werden.

Für Ziegler hat die familiale Tradierung nach wie vor einen hohen Stellenwert, die sich in der Regel als Transformation vollzieht (Ziegler 2000, 25 und 231). In seiner Studie untersucht er familiale Tradierungsprozesse anhand einer österreichischen Familie. Das soziale Erbe ${ }^{90}$ wird zwar im familialen Prozess von der vermittelnden Generation übertragen, inwiefern die nachfolgende Generation das soziale Erbe jedoch übernimmt, umformt oder die Annahme verweigert, wird für Ziegler entscheidend beeinflusst von den kulturellen Erfahrungen, die die aneignende Generation außerhalb der Familie sammelt (Ziegler 2000, 23/24). ${ }^{91}$ Ziegler konstatiert in seiner Studie mit Rekurs auf Koselleck generationell unterschiedliche Arten des Umgangs mit Erfahrungsräumen und Erwartungshorizonten (Ziegler 2000, 248-250). Hiermit ist eine weitere interessante Perspektive aufgezeigt, die für die Analyse familialer Tradierungsprozesse weiter auszudifferenzieren ist. So lässt sich z.B. fragen, ob sich die Familien des Samples in Bezug auf die Umgangsweise mit den je eigenen Erfahrungsräumen und Erwartungshorizonten unterscheiden.

Wohlrab-Sahr/Karstein/Schmidt-Lux identifizieren in einem ostdeutschen Sample unterschiedliche familiale Strategien für den Umgang mit Generationendifferenzen. Neben

\footnotetext{
${ }^{90}$ Das soziale Erbe bezeichnet für Ziegler „soziale Güter wie Werte, Einstellungen, Grundorientierungen“, die im Generationenverlauf tradiert werden (Ziegler 2000, 57).

${ }^{91}$ Interessant ist Zieglers Feststellung, dass es in der mittleren Generation, die Ende der 1940er Jahre geboren ist, weniger zu einer Herauslösung aus familialen Bindungen und Traditionen kommt, als vielmehr zu einer steigenden Reflexivität im Umgang mit den Traditionen (Ziegler 2000, 239).
} 


\subsection{Familiale Tradierungsprozesse}

der Möglichkeit, potenzielle Differenzen zu vermeiden, indem die Gemeinsamkeiten wie etwa tradierte berufliche Orientierungen oder die Tradition eines Familienbetriebes in den Vordergrund gestellt werden (Wohlrab-Sahr/Karstein/Schmidt-Lux 2009, 77), identifizieren sie drei Strategien: Sie können überblendet, externalisiert oder problematisiert werden (Wohlrab-Sahr/Karstein/Schmidt-Lux 2009, 84-115). ${ }^{92}$ Das führt zu der Annahme, dass sich Familien auch in Bezug auf Tradierungsmuster unterscheiden; ein Aspekt, der im empirischen Teil dieser Studie zu untersuchen ist.

Schneewind/Ruppert können anhand einer Längsschnittstudie zu Eltern-Kind-Beziehungen das Zusammenspiel von Familien- und Zeitgeschichte aufzeigen. Zum einen existieren deutliche Unterschiede im Erziehungsverhalten von Eltern und ihren erwachsenen Kindern, was zumindest teilweise als ein Generationeneffekt gedeutet wird (Schneewind/Ruppert 1995, 146). Zum anderen wirken sich aber die jeweils eigenen Erziehungserfahrungen auf die nachfolgende eigene Erziehungspraxis aus (Schneewind/ Ruppert 1995, 153). Demnach beeinflusst der elterliche Erziehungsstil das Erziehungsverhalten der nachfolgenden Generation ebenso wie gesamtgesellschaftliche Einflüsse in Form eines Generationeneffektes (Schneewind 1996, 126). ${ }^{93}$ Bei der Analyse familialer Tradierungsprozesse ist folglich das interdependente Geflecht aus individuellen Erfahrungen und gesellschaftlichen Rahmenbedingungen zu berücksichtigen.

Büchner/Brake (2006) stellen in ihrem Sammelband die Bildungsbedeutsamkeit von Familien sowie die unterschiedlichen Strategien der Weitergabe und Aneignung von Bildung und Kultur (Büchner 2006, 13/14) in den Mittelpunkt. In dem Band werden Beispiele für die Vielfalt familialer Bildungsstrategien aufgezeigt, wie z.B. die Wirksamkeit vorreflexiver Strategien des Habitus (Brake 2006, 108), die Bildungswirkungen religiös geprägter kultureller Alltagspraxen (Krah/Büchner 2006, 136) oder die Bedeutung der sozialen Beziehungsmuster in der Familie (Gohlke/Büchner 2006, 172), wobei die Transformationen nicht aus dem Auge gelassen werden. Zugleich wird an einem Beispiel aufgezeigt, wie bestimmte Bildungsstrategien im Zuge des gesellschaftlichen Wandels für nachfolgende Generationen immer prekärer werden und die Anschlussfähigkeit im Sinne eines erfolgreichen Bildungsverlaufs massiv behindern (Wahl 2006). Die Ergebnisse dieser Untersuchungen lassen eine systematische Suche nach unterschiedlichen Mustern der Tradierung aussichtsreich erscheinen.

Vierzigmann/Kreher können anhand eines Fallbeispiels über drei Generationen die Existenz und je generationenspezifische Bearbeitung von familialen Leitmotiven bzw. -themen aufzeigen (Vierzigmann/Kreher 1998, 23 und 32/33, Kreher 2002, 193, 202). Das Leitmotiv stellt eine Art Motto dar, das die erinnerte Biografie der Befragten strukturiert (Kreher 2002, 202). Thon untersucht weibliche Bildungsverläufe in Familien

\footnotetext{
${ }^{92}$ Mit Ausnahme der Problematisierung, in der die Familieneinheit brüchig wird, handelt es sich um familiale Kommunikationsmuster, die dazu dienen, die familiale Einheit aufrechtzuerhalten.

${ }^{93}$ Konkret heißt dies, dass z.B. ein Vater, der in den 1970ern selbst vergleichsweise streng erzogen wurde, in den 1990ern sein Kind zwar liberaler erzieht als er selbst erzogen wurde, er aber im Vergleich zu anderen Vätern zu den weniger liberalen zählt (Schneewind 1996, 126).
} 


\section{Familiale Tradierungsprozesse: Einblicke in den Forschungstand}

über drei Generationen hinweg und kann generationenübergreifende Strategien herausarbeiten im Umgang mit der „Realisierung erweiterter Bildungschancen innerhalb von veränderten Gelegenheitsstrukturen" (Thon 2010, 164). Die Suche nach generationenübergreifenden Wertorientierungen und Strategien, die zentral für die individuelle Lebensgestaltung der Familienmitglieder sind, erscheint auch aufgrund dieser Ergebnisse vielversprechend.

Ecarius untersucht in ihrer Drei-Generationen-Studie die Erziehungsmuster der zwischen 1908-1929, zwischen 1939-1952 sowie zwischen 1968-1975 Geborenen in einer ostdeutschen Region (Ecarius 2002, 80). Sie kann sowohl die Tradierung von Erziehungsmustern wie „Verweilen im Befehlen“ und „Verweilen im Verhandeln“ als auch die Transformation „,vom Befehlen zum Verhandeln“ in einzelnen Familien herausarbeiten (Ecarius 2002, 83-100). Insgesamt vollzieht sich ein Wandel vom Befehls- zum Verhandlungshaushalt (Ecarius 2002, 183, 221). Die Verlagerung deutet Ecarius als Ausdruck eines durch gesellschaftliche Veränderungsprozesse ausgelösten generationellen Wandels (Ecarius 2002, 255-265). Die Beurteilung der erfahrenen Erziehung hängt dabei u.a. von der Einschätzung der emotionalen Qualität der Generationenbeziehungen sowie der wahrgenommenen Unterstützung ab (Ecarius 2002, 238/239). Zusätzlich sind die jeweils gesellschaftlich dominierenden Vorstellungen zur Erziehung relevant (Ecarius 2002, 240/241). Damit sind die gesellschaftlichen Rahmenbedingungen explizit als Faktoren eingeführt, die die familialen Tradierungsprozesse beeinflussen und folglich in die Analyse einzubeziehen sind. Es stellt sich die Frage, welche gesellschaftlichen Entwicklungen auf welche Weise die Vermittlung und Aneignung beeinflussen; ein Aspekt, der im empirischen Teil der Untersuchung zu bearbeiten ist. 


\section{Die theoretische Rahmung familialer Tradierungsprozesse: Gedächtnis- und Generationenperspektive}

In diesem Kapitel erfolgt die theoretische Einbettung familialer Tradierungsprozesse. Hierzu werden Halbwachs' Überlegungen zum kollektiven Gedächtnis mit einer Generationenperspektive verbunden. Es bietet sich an, Forschungen zu den Bedingungen individuellen Erinnerns einzubeziehen, da zwischen Tradierung und Erinnerung Überschneidungspunkte bestehen. In beiden Fällen steht die Übertragung von Inhalten aus der Vergangenheit in die Gegenwart bzw. Zukunft im Zentrum. ${ }^{94}$ Erinnerungen und ihre Rahmungen stellen das Bindeglied zwischen Vergangenheit, Gegenwart und Zukunft dar und lassen sich allgemein als eine wichtige Voraussetzung für Tradierungsprozesse bezeichnen, da nur das weitergegeben werden kann, was explizit oder implizit erinnert wird. Erinnerungen beziehen sich zwar auf vergangene Ereignisse, eine ihrer wichtigen Funktionen ist es aber, Orientierungen in der Gegenwart und „für zukünftiges Handeln zu ermöglichen“" (Welzer 2010, 8). Das ist auch für den Tradierungskanal der Erziehung relevant. Die jeweils praktizierte Erziehung ist mit Vorstellungen davon verbunden, wie der Erzogene werden soll (Ecarius 2002, 47) und somit auf die Zukunft ausgerichtet. Zugleich speisen sich die Ziele der Erziehenden aus ihren eigenen Erziehungserfahrungen, also aus ihrer Vergangenheit - unabhängig davon, ob sie sie übernehmen, modifizieren oder ablehnen. Die Auseinandersetzung mit den eigenen Erinnerungen wird von den jeweiligen historischen Kontext beeinflusst und dient der Orientierung für die Ausgestaltung der gegenwärtigen und zukünftigen Erziehung.

Zur Bearbeitung dieses komplexen Themenfelds werden eingangs die für diese Studie wesentlichen sozialwissenschaftlichen, kulturwissenschaftlichen und psychologischen Gedächtniskonzepte skizziert und in Hinblick auf den Gegenstand dieser Arbeit diskutiert. Dabei werden die Leerstellen oder ,blinden Flecken` herausgearbeitet, die durch die Einbeziehung einer generationellen Perspektive gefüllt werden können. Die Kopplung von Halbwachs' Gedächtnistheorie mit der Generationenperspektive ermöglicht es, die zu vermutenden Dynamiken familialer Tradierungsprozesse in diachroner wie synchroner Perspektive in den Blick zu nehmen und zu untersuchen, wie sich Tradierungsprozesse im Zeitverlauf entwickeln und welchen Einfluss die jeweilige Lagerung im sozial-historischen Raum hierbei ausübt.

\footnotetext{
${ }^{94}$ Zum Aspekt der Zeitlichkeit und der Verbindung von Halbwachs' Überlegungen zum kollektiven Gedächtnis mit einer generationellen Perspektive siehe auch Radicke (2013).
} 


\subsection{Die soziale Prägung des Gedächtnisses}

Die Erinnerungsforschung bildet ein äußerst facettenreiches und interdisziplinäres Forschungsfeld. Zur Begriffsklärung lässt sich allgemein sagen, dass Erinnern ein Prozess ist und die Erinnerungen das Ergebnis dieses Vorganges sind (Erll 2005, 7). Das Gedächtnis bezeichnet „die Art und Weise, in der Ereignisse das Gehirn und seine zukünftigen Aktivitäten beeinflussen können“" (Siegel 2006, 23).

\subsubsection{Halbwachs' kollektives Gedächtnis}

Eine für die Sozialwissenschaften zentrale Theorie zur Erinnerung hat Halbwachs mit dem kollektiven Gedächtnis vorgelegt. Diese wurde vielfach aufgegriffen, diskutiert und ausdifferenziert. Das Konzept bezieht sich zum einen auf das individuelle Gedächtnis und seine Geprägtheit durch die soziale Umwelt und zum anderen auf den Bereich der kulturellen Überlieferung, auf in irgendeiner Form objektivierte Bezüge auf Vergangenes (Erll 2005, 97). Diese doppelte Verwendung findet sich bereits bei Halbwachs (Erll 2005, 14/15 und Ollick 1999, 336). Für diese Arbeit ist vor allem die erste Lesart relevant. Die zentrale Annahme Halbwachs' in diesem Zusammenhang lautet, dass die individuelle Erinnerung von der jeweiligen Bezugsgruppe geprägt wird. Zwar ist es immer das Individuum, das sich erinnert, dies geschieht aber in Abhängigkeit von der jeweiligen Bezugsgruppe, die sein Denken und Fühlen prägt (Halbwachs 1991, 2 und 15). Individuelle Erinnerungen lassen folglich in gewissem Maße auch Rückschlüsse auf die vorherrschenden Orientierungen der Gruppe zu. Die Gedanken und Sichtweisen der Gruppe sind eine notwendige Voraussetzung, damit der Einzelne dauerhafte Erinnerungen produzieren kann. Die Gruppe stellt den Individuen Bezugsrahmen für die jeweilige Erinnerung zur Verfügung (Halbwachs 1985, 201). Sie ermöglichen es dem Einzelnen, Erinnerung zu ordnen und zu kategorisieren. Von der Bezugsgruppe gehen zugleich starke Tendenzen aus, ihre ,sozialen Rahmen' an ihre Mitglieder weiterzugeben, womit die Verbindung zur Tradierung hergestellt ist. Diese Rahmen können nicht nur Jahreszahlen, Namen und Formeln sein, sondern auch Denk- und Erfahrungsströmungen (Halbwachs 1991, 50). Mit den Denk- und Erfahrungsströmungen ist ein zentraler Punkt eingeführt. Um zu verdeutlichen, dass sich diese Arbeit vor allem auf diese - oder genauer gesagt auf leitende Vorstellungen und Orientierungen, die in den einzelnen Familien existieren - bezieht, die die Erinnerung des Einzelnen prägen, werden diese im Folgenden als familiale Bezugsrahmen ${ }^{95}$ bezeichnet. Das bedeutet, das Interesse richtet sich weniger auf die Erinnerung an sich oder den intergenerationell geteilten Fundus gemeinsamer Erinnerungen, sondern auf die sich in den Erinnerungen manifestierenden Bezugsrahmen.

\footnotetext{
${ }^{95}$ Halbwachs selbst benutzt diesen Terminus und spricht davon, dass Gedächtnis ohne Bezugsrahmen nicht denkbar ist (Halbwachs 1985, 121).
} 


\subsection{Die soziale Prägung des Gedächtnisses}

Jedes Individuum hat Anteil an mehreren kollektiven Gedächtnissen im Sinne eines sozial geprägten Gedächtnisses (Halbwachs 1991, 64/65). Hierdurch ergibt sich die Möglichkeit, jedes Individuum mehrdimensional verorten zu können, z.B. als Mitglied seiner Familie oder seiner Generation. Die Individualität des einzelnen Gedächtnisses resultiert aus den jeweils spezifischen Kombinationen der Gruppenzugehörigkeiten der Individuen (Erll 2005, 16 und Jureit 2006, 116). Somit können sich beim Einzelnen auch ganz unterschiedliche Zusammenstellungen sozialer Bezugsrahmen ergeben. Hierbei können sich Konkurrenzen, Ergänzungen, etc. der jeweiligen Bezugsrahmen ergeben, von denen denkbar ist, dass sie in die verschiedenen Gruppen zurückwirken. Anders ausgedrückt: Die Teilhabe an verschiedenen Gruppen kann dazu führen, dass die Bezugsrahmen in den einzelnen Gruppen verändert, erweitert oder ggf. aufgegeben werden. Diese potenziellen Wechselwirkungen zwischen den einzelnen Gruppen sind von Halbwachs nicht systematisch aufgegriffen und ausgearbeitet worden; sie sind jedoch relevant, ergibt sich doch so ein komplexeres Bild des kollektiven Gedächtnisses. Durch Beachtung des möglichen Wirkungsgeflechts zwischen verschiedenen Gruppen wird das etwas statisch wirkende Konzept dynamisiert und zeigt zwei gegenläufige Tendenzen auf: zum einen das Streben nach Kontinuität und Einheit innerhalb der Gruppe und zum anderen die Potenziale eines möglichen Wandels, bedingt durch die gleichzeitige Teilhabe der Gruppenmitglieder an verschiedenen kollektiven Gedächtnissen.

Bei Verlassen der Gruppe können die gruppengebundenen Erinnerungen mit ihren Bezugsrahmen verblassen (Halbwachs 1991, 7). Das unterstreicht nochmals, dass Erinnerungen und ihre Bezugsrahmen keine statischen, sondern relationale und wandelbare Größen sind, und verweist auf das Gegenstück zur Erinnerung: das Vergessen. Die bestehenden Erinnerungen des Einzelnen mit ihren Bezugsrahmen wiederum geben Aufschluss über sein Relevanzsystem, seine Wertvorstellungen und Orientierungen.

Der mit Halbwachs' Theorie verbundene Begriff der Bezugsrahmen verdeutlicht die Gruppengebundenheit individueller Erinnerung und hat gegenüber anderen Begriffen wie Orientierungsrahmen (Bohnsack 1997), Habitus (Bourdieu 1987), oder Familienthemen (Ecarius 2003) für die hier verfolgte Fragestellung Vorteile. Dem Habitusbegriff liegt als ein Schwerpunkt die Annahme von Kontinuitäten zugrunde. In dieser Studie stehen daneben aber auch Wandlungsprozesse im Mittelpunkt, die über die Bezugsrahmen erfassbar sind, besonders unter Berücksichtigung der Generationenperspektive. Halbwachs' Konzeption des Familiengedächtnisses, dessen zentraler Bestandteil die familialen Bezugsrahmen sind, impliziert bereits intergenerationelle Vermittlungsprozesse und bezieht sich konkret auf die Gruppe der Familie. Hier liegt ein wichtiger Unterschied zu Bohnsacks Orientierungsrahmen, der keinen speziellen Fokus auf die Familie richtet und nicht so stark von der Annahme intergenerationeller Vermittlungsprozesse bestimmt ist. Ecarius' Familienthemen sind ausschließlich auf die Familie fokussiert. Halbwachs' kollektives Gedächtnis hingegen erlaubt eine mehrfache Verortung des Individuums, da jeder Einzelne Anteil an verschiedenen kollektiven Gedächtnissen mit den jeweiligen Rahmungen hat, z.B. als Teil der Familiengruppe, einer regionalen und generationellen Gruppe. 


\section{Die theoretische Rabmung familialer Tradierungsprozesse}

\subsubsection{Kommunikatives und kulturelles Gedächtnis}

Assmann hat für die eben skizzierte Verwendungsweise des kollektiven Gedächtnisses den Begriff kommunikatives Gedächtnis eingeführt. Das kommunikative Gedächtnis als individuelles Gedächtnis, das sich in Auseinandersetzung mit der jeweiligen sozialen Umwelt bildet, bezieht sich auf die Alltagskommunikation, umfasst einen Zeitraum von 80 bis 100 Jahren, wandert mit der Gegenwart mit, entsteht durch Interaktion im Alltag, hat Geschichtserfahrungen der Individuen zum Inhalt, ist informell und seine Träger sind „unspezifisch, [die, C.R.] Zeitzeugen einer Erinnerungsgemeinschaft" (Assmann 1988, 10 und 2002, 56). Ollick bezeichnet diese Form des kollektiven Gedächtnisses als collected memory (Ollick 1999). Das individuelle Gedächtnis mit seinen Bezügen zur sozialen Umwelt steht im Mittelpunkt. Es wird betont, dass es immer der Einzelne ist, der sich erinnert. In der Gesellschaft vorhandene Symbole oder auch generell ,Rahmen“ können ihre Wirkung nur in dem Maße entfalten, wie der Einzelne ihnen Bedeutung zumisst (Ollick 1999, 338).

Unter kulturellem Gedächtnis fasst Assmann „den jeder Gesellschaft und jeder Epoche eigentümlichen Bestand an Wiedergebrauchs-Texten, -Bildern und Riten zusammen, in deren ,Pflege' sie ihr Selbstbild stabilisiert und vermittelt, ein kollektiv geteiltes Wissen vorzugsweise (aber nicht ausschließlich) über die Vergangenheit, auf das eine Gruppe ihr Bewusstsein von Einheit und Eigenart stützt“ (Assmann 1988, 15). Vom kulturellen Gedächtnis ist die Rede, wenn die lebendige Kommunikation übergegangen ist in die verschiedensten Formen der objektivierten Kultur, seien es nun Texte, Bilder, Riten, Denkmäler, etc. (Assmann 1988, 11). Das kulturelle Gedächtnis bedarf der Einweisung, befasst sich mit dem Außeralltäglichen und hat spezielle Träger (Assmann 2002, 54/ 55). ${ }^{96}$ Diese beiden Formen des kollektiven Gedächtnisses stehen in Wechselwirkung zueinander (Assmann 2002, 51/52). D.h. Texte, Bilder, Riten, etc. können die individuelle Erinnerung und vielleicht auch die ,sozialen Rahmen' einer Gruppe beeinflussen. Hierin liegt aber auch ein Problem, da sich diese beiden Formen anscheinend nur theoretisch exakt trennen lassen. Zudem gerät Halbwachs' Feststellung, jeder Einzelne habe Anteil an mehreren kollektiven Gedächtnissen, etwas aus dem Blickfeld. Damit treten folglich auch die Wechselwirkungen zwischen den gleichzeitig existierenden verschiedenen Gruppengedächtnissen in den Hintergrund.

In den Modellen des kommunikativen Gedächtnisses bzw. collected memory wird das Individuum demgegenüber stärker als handelnder Akteur betrachtet. Es wird deutlich, dass der Einzelne aktiv am Erinnerungsprozess sowie der Internalisierung der Bezugsrahmen beteiligt ist, was u.a. eine partielle Aneignung sowie Veränderung dieser Rahmen im Zuge von Interaktion und Kommunikation einschließt. In den Konzepten ist die Möglichkeit der Veränderung angelegt, was für diese Studie von besonderem Interesse ist, da es nicht nur um Fragen der Kontinuität geht, sondern auch um die des Wandels

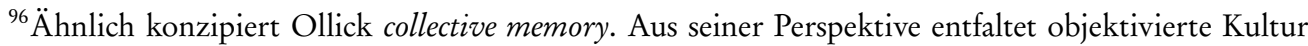
unabhängig von subjektiv zugesprochenem Sinn Bedeutung und Wirkung (Ollick 1999, 336 und 341).
} 


\subsection{Die soziale Prägung des Gedächtnisses}

der Bezugsrahmen. Assmanns und Ollicks Ausführungen ergänzen somit Halbwachs' Überlegungen zum sozial geprägten individuellen Gedächtnis, das am geeignetsten für diese Studie ist: Durch die gleichzeitige Teilhabe an mehreren kollektiven Gedächtnissen können familiale und generationelle Einflüsse in die Betrachtung einbezogen werden.

\subsubsection{Implizites und explizites Gedächtnis}

Die jeweiligen Bezugsrahmen müssen den Individuen nicht unbedingt bewusst sein, sondern können sich auch als implizite Erinnerungen darstellen (Moller 2010, 89). Das leitet über zur Aufteilung des individuellen Langzeitgedächtnisses ${ }^{97}$ in implizites und explizites Gedächtnis, wie sie die Psychologie vornimmt. Das explizite Gedächtnis beinhaltet die bewusst zugänglichen Erinnerungen (Siegel 2006, 28-30). Es enthält das semantische und das episodische Gedächtnis. Grob skizziert, besteht das episodische Gedächtnis aus persönlichen Erfahrungen in Form von Ereignissen, Orten und Dingen (Hirst/Manier 2002, 41). Episodische Erinnerungen sind mit dem Gefühl eines autonoetischen Bewusstseins verbunden, also dem Eindruck des eigenen Erlebens (Tulving 2006, 51 und Piefke/Markowitsch 2010, 16). Das semantische Gedächtnis enthält Faktenwissen, wobei die Informationen über den Wissenserwerb verloren gegangen sind (Hirst/Manier 2002, 41). Ein Großteil des episodischen Gedächtnisses bildet zusammen mit einem Teil des semantischen Gedächtnisses das autobiografische Gedächtnis (Pohl 2010, 75). ${ }^{98}$ Interessant sind die Wechselwirkungen zwischen episodischem und semantischem Gedächtnis. Unabhängig von der unmittelbaren Teilnahme an bestimmten Ereignissen können Inhalte des semantischen Gedächtnisses die episodischen Erinnerungen des Einzelnen beeinflussen. Das Faktenwissen, z.B. über tagesaktuelle Themen, kann sich in lebensgeschichtlichen Erinnerungen niederschlagen und u.U. die Bezugsrahmen der Erinnerung verändern (Hirst/Manier 2002, 43/44). ${ }^{99}$ Das verweist zugleich

\footnotetext{
${ }^{97}$ Neben dem Langzeitgedächtnis gibt es noch Kurzzeit- und Arbeitsgedächtnis (Markowitsch 2009, 37-40), die aber im Zusammenhang mit dieser Studie nicht relevant sind.

${ }^{98}$ Kurze Überblicksdarstellungen zu den verschiedenen, individuellen Gedächtnisformen finden sich bei Siegel, Baddeley und Markowitsch (Siegel 2006, 24-25, Baddeley 2009, 10 und Markowitsch 2009, 72-75).

${ }^{99}$ Hirst/Manier unterteilen das semantische Gedächtnis nochmals in das distant semantic und das lived semantic memory. Ersteres bezieht sich auf das Wissen über eine Zeit, die die Menschen nicht selbst miterlebt haben und die für ihre Selbstdefinition relativ unwichtig ist (Hirst/Manier 2002, 43/44). Das lived semantic memory bezieht sich auf Wissen über das tagesaktuelle Geschehen der Gruppenmitglieder. Hirst/Manier erörtern das am Beispiel des Vietnamkrieges, der auch für den Alltag derjenigen prägend war, die zwar nicht an den Kampfhandlungen teilgenommen haben, sich aber in ihrem Umfeld mit dem Thema auseinandersetzten (Hirst/Manier 2002, 43). Die Relevanz für das persönliche Leben und die persönlichen Erinnerungen hängt also nicht von der unmittelbaren Anwesenheit bei den entsprechenden Ereignissen ab. Vielmehr ist ausschlaggebend, ob die Personen sich mit den Ereignissen in ihrem Alltag auseinandersetzen.
} 


\section{Die theoretische Rabmung familialer Tradierungsprozesse}

auf die Interdependenz von historischer Zeit, dominanten gesellschaftlichen Denk- und Verarbeitungsströmungen sowie ihrer individuellen Rahmungen.

Inhalte des impliziten Gedächtnisses hingegen werden i.d.R. nicht bewusst erinnert (Baddeley 2009, 10). Gleichwohl können sie das Denken, Handeln und die Vorstellungen des Einzelnen beeinflussen (Siegel 2006, 26 und Pohl 2007, 21). Zentral in diesem Zusammenhang ist, dass lebensgeschichtliche Erinnerungen sowohl vom impliziten als auch vom expliziten Gedächtnis beeinflusst werden (Siegel 2006, 33). Beide schlagen sich in den Denk- und Handlungsmustern des Einzelnen sowie in den Formen der Vermittlung und Aneignung der Bezugsrahmen nieder und sind der Analyse zugänglich.

\subsubsection{Konstruktivität und Gegenwartsbezug}

Zwei weitere wichtige Merkmale der Erinnerung sind ihr konstruktiver Charakter und ihr Gegenwartsbezug. Bereits Halbwachs hat nachdrücklich darauf verwiesen, dass Erinnerung ein konstruktiver Prozess ist. Die Vergegenwärtigung der Vergangenheit kann immer nur ansatzweise geschehen (Halbwachs 1985, 133 und 161). Erinnerungen geben die Vergangenheit nicht detailgetreu und nur ausschnitthaft wieder. Es handelt sich bei ihnen um keine objektiven Spiegelungen vergangener Ereignisse. Vielmehr enthalten sie das, was die erinnernde Person für ihre Vergangenheit hält. Zudem handelt es sich bei Erinnerungen um keine endgültig feststehenden Abbilder der Vergangenheit, sondern sie sind wandelbar (Halbwachs 1991, 56, 72). Hiermit ist der Gegenwartsbezug der Erinnerung angesprochen. Für Halbwachs ist die Erinnerung in weiten Teilen „eine Rekonstruktion der Vergangenheit mit Hilfe von der Gegenwart entliehenen Gegebenheiten und wird im übrigen durch andere, zu früheren Zeiten unternommene Rekonstruktionen vorbereitet, aus denen das Bild von ehemals schon recht verändert hervorgegangen ist" (Halbwachs 1991, 56, Halbwachs 1985, 132, 136). Der Konstruktionsprozess geht von der Gegenwart aus, wobei vergangene Erinnerungen an ein Ereignis die folgenden Erinnerungen beeinflussen können. Auch wenn die Veränderung von Erinnerungen bereits in Halbwachs' Überlegungen eine wichtige Rolle spielt, hat er nicht genauer ausgeführt, wie genau sich der mögliche Wandel von Erinnerungen, gerade auch in Auseinandersetzung mit der jeweiligen Bezugsgruppe, vollzieht.

Auch die psychologischen und neurowissenschaftlichen Disziplinen betonen den konstruktiven Charakter, den Gegenwartsbezug und die Veränderbarkeit von Erinnerungen (Schacter 1999, 22, 27 und Piefke/Markowitsch 2010 17/18). Aus diesen Disziplinen stammen differenzierte Einblicke in den Erinnerungsvorgang, die für diese Studie bedeutsam sind. Um eine Information im Gedächtnis zu verankern, muss sie codiert ${ }^{100}$ werden. Die Art der Codierung beeinflusst die Dauerhaftigkeit einer Erinnerung. Eine

\footnotetext{
${ }^{100}$ Biologisch betrachtet führt der Codierungsprozess zur Stärkung der neuronalen Verbindungen zwischen den am Codierungsprozess beteiligten Neuronengruppen und es entstehen flüchtige oder dauerhafte Veränderungen im Gehirn, was als Engramm bezeichnet wird (Schacter 1999, 100 und Korte 2001, 142).
} 


\subsection{Die soziale Prägung des Gedächtnisses}

elaborierte Codierung fördert eine höhere Gedächtnisleistung. Die neuen Informationen oder Wahrnehmungen werden in den Pool der bereits vorhandenen integriert und es findet eine Verknüpfung mit bereits vorhandenem Wissen statt; je vielfältiger das ausfällt, desto einfacher ist eine Erinnerung wieder zugänglich (Schacter 1999, 79 und Krüger 2001, 137).

Ferner hängt die Art der Codierung von der emotionalen Befindlichkeit, dem Grad der Aufmerksamkeit sowie der Bedeutung ab, die der jeweiligen Information zugemessen wird (Schacter 1999, 77 und Markowitsch 2009, 10). Das bereits vorhandene Wissen bestimmt, welche Wahrnehmungen ausgewählt und codiert werden (Schacter 1999, 80). Schacter beschreibt quasi eine Pfadabhängigkeit des Gedächtnisses und verweist dadurch auf seine soziale Geprägtheit. Mit Verweis auf Halbwachs bedeutet das, dass die jeweiligen Bezugsgruppen bzw. ihre Rahmungen beeinflussen, was die Aufmerksamkeit auf sich zieht und was elaboriert codiert wird. Die erinnerten Episoden bieten zugleich Hinweise auf die relevanten Bezugsrahmen. ${ }^{101}$ Ähnliches gilt für den Abruf von Erinnerungen ${ }^{102}$, der durch Hinweisreize ausgelöst und beeinflusst ${ }^{103}$ wird. Eine Erinnerung wird um so eher dauerhaft im Gehirn verankert, je häufiger sie abgerufen wird (Schacter 1999, 146). Die Umwelt, von der Art und Umfang der Hinweisreize ausgehen, beeinflusst in starkem Maße die Erinnerung. Das ist für die Auswertung der Interviews von Bedeutung, da davon auszugehen ist, dass eine flüssig vorgetragene Erinnerung bereits häufiger abgerufen worden ist und somit eine besondere Relevanz aufweist.

Trotz des konstruktiven Charakters der Erinnerung finden sich in der psychologischen Disziplin Stimmen, die Erinnerungen im Allgemeinen eine hohe Aussagekraft zusprechen. Schacter bilanziert, dass die persönlichen Erinnerungen der Individuen an die „allgemeinen Umrisse“ des eigenen Lebens grundsätzlich richtig seien, und sie den „allgemeinen Charakter längerer Zeitperioden ihrer Vergangenheit aus der Rückschau" betrachtet in der Regel zutreffend einschätzen würden (Schacter 199, 157/158). Das belegt er mit Untersuchungen zu Kindheitserinnerungen von Geschwistern, die die grundlegenden Merkmale des Familienlebens übereinstimmend erinnerten. ${ }^{104}$ Spe-

${ }^{101}$ Interessant ist in diesem Zusammenhang auch, welche Ereignisse vergessen werden, was ebenfalls Hinweise auf die Bezugsrahmen liefert. Ortlepp führt aus, dass das Vergessen genau wie das Erinnern ein soziales Phänomen ist, das in Rahmungen eingebunden ist. Änderungen der Bezugsrahmen können Vergessen begünstigen oder verhindern (Ortlepp 2002, 315).

${ }^{102}$ Es werden nur Bruchstücke der Erfahrung im Gedächtnis eingespeichert. Hieraus werden beim Abruf Erinnerungen konstruiert (Schacter 1999, 72). Hierbei notwendigerweise entstehende Lücken werden aufgefüllt (Pohl 2010, 76).

${ }^{103}$ So bleiben häufig wiederholte Geschichten in der Regel gleich, egal durch welchen Hinweisreiz die Erinnerung ausgelöst wird (Schacter 1999, 134). Anders verhält es sich mit selten abgerufenen Erinnerungen. Hier könnte die Art des Abrufreizes wesentlich mehr Einfluss auf das Erinnerungserlebnis haben (Schacter 1999, 134).

${ }^{104}$ Schacter zitiert zur Untermauerung eine Studie Barclays, in der Versuchspersonen „die allgemeine Bedeutung ihrer Erlebnisse, obwohl sie sich in vielen Einzelheiten täuschten“, richtig erinnerten (Schacter 1999, 159). 


\section{Die theoretische Rabmung familialer Tradierungsprozesse}

zifische Ereignisse hingegen können von einigen Familienmitgliedern vergessen werden oder es können sich Diskrepanzen in Bezug auf konkrete Gegebenheiten ergeben, was Schacter auf die jeweilige Bedeutung zurückführt, die die einzelnen Familienmitglieder dem Ereignis beimessen (Schacter 1999, 157 und 158). Wenn sich in dieser Studie Familienmitglieder in unterschiedlicher Weise auf dieselben Ereignisse beziehen, könnte das bedeuten, dass unterschiedliche Relevanzen und Bezugsrahmen, die die Erinnerung beeinflussen, zum Tragen kommen.

Erlebnisse werden retrospektiv oft positiver erinnert als sie waren, was als Positivitätsbias bezeichnet wird (Pohl 2010, 82). ${ }^{105}$ Dieser steige mit zunehmenden Alter, sodass z.B. der Anteil der Personen, die ihre Kindheit als glücklich bezeichnen, mit dem Alter wachse. Zudem können Erinnerungen den jeweils gültigen Konventionen angepasst werden, um z.B. Erlebnisse für die Zuhörer plausibler zu gestalten (Pohl 2010, 82). Diese Erkenntnisse - insbesondere der Positivitätsbias - sind bei der Auswertung zu berücksichtigen.

Die angeführten Positionen belegen deutlich, dass es sich bei individuellen Erinnerungen weder um exakte Wiedergaben der Vergangenheit handeln muss, noch dass sie völlig abgelöst von vergangenen Ereignissen sind und eine Annäherung an diese möglich ist. Unabhängig von der Frage, ob erzählte Erinnerungen Aussagen über die Vergangenheit ermöglichen, kommt Erinnerungen eine handlungsleitende Relevanz für Gegenwart und Zukunft zu. Gleichgültig, wie ein Ereignis einstmals erlebt wurde, die aktuelle Reinterpretation bildet die Basis für die gegenwärtigen Beurteilungen, Entscheidungen und Handlungen des Einzelnen. Erinnerungen wirken folglich auf die Gestaltung von Gegenwart und Zukunft ein. Sie bieten insgesamt einen guten Zugang zur subjektiven Wirklichkeit der erinnernden Personen. Sie geben Hinweise darauf, wie „die jeweilige Person ihr Leben und die Welt deutet“ (Pohl 2010, 78). Diese Sichtweisen, Konstruktionen und Deutungen lassen sich rekonstruieren, ebenso wie die dahinter stehenden Logiken, die kollektiven Orientierungsmuster (Meuser 2006, 142), womit ein zentrales Anliegen qualitativer Sozialforschung benannt ist.

In Anbetracht des konstruktiven und selektiven Charakters von Erinnerungen sind die präsentierten Erinnerungen besonders aussagefähig, was die zentralen Bezugsrahmen des Einzelnen betrifft. Die erinnerten Lebensereignisse und die in sie eingelagerten und zu rekonstruierenden Bezugsrahmen sind für gegenwärtige und zukünftige Denk- und Handlungsmuster relevant. Bezogen auf den Gegenstand dieser Untersuchung geben sie wertvolle Einblicke in die persönlichen Erziehungserfahrungen und -bewertungen, sei es als Erzogene oder als Erzieher, die wiederum die Folie für die aktuellen und zukünftigen Denkweisen, Einschätzungen und Handlungen bilden.

\footnotetext{
${ }^{105}$ Abgesehen von extrem negativen Ereignissen werden positive Erlebnisse generell besser erinnert (Pohl 2010, 82).
} 


\subsection{Das Familiengedächtnis als intergenerationelles Gedächtnis}

\subsection{Das Familiengedächtnis als intergenerationelles Gedächtnis}

Halbwachs selbst thematisiert das Zusammenspiel von Erinnerung und Generation, indem er ein intergenerationelles Gedächtnis, das Familiengedächtnis, näher betrachtet (Halbwachs 1985, 203-242). Für Halbwachs sind Familien Erinnerungsgemeinschaften mit je eigenen kollektiven Gedächtnissen. ${ }^{106}$ Familien „reproduzieren nicht nur ihre Vergangenheit, sondern sie definieren ihre Wesensart, ihre Eigenschaften und Schwächen" (Halbwachs 1985, 209/210). Bestimmte Elemente der Vergangenheit, die mit der Familie oder einzelnen Familienmitgliedern in Zusammenhang gebracht werden, werden erinnert und bilden eine Art Rahmen für die einzelnen Familienmitglieder. Hierbei kann es sich z.B. um Vorstellungen handeln, wie: „In unserer Familie wird man alt“ oder „....ist man stolz“ (Halbwachs 1985, 210). Solche Vorstellungen oder auch Leitlinien und Wertorientierungen stellen die familialen Bezugsrahmen dar, über die für Halbwachs jede Familie verfügt und die für diese Studie relevant sind. Das Interesse gilt weniger den Erinnerungen selbst als vielmehr den gemeinsamen bzw. unterschiedlichen Bezugsrahmen der Familienmitglieder, die sich aus den Erinnerungen rekonstruieren lassen.

Allerdings erscheint die Konzeption des Familiengedächtnisses bei Halbwachs relativ statisch, da er besonders die Gemeinsamkeiten in den Blick nimmt, wodurch der Eindruck eines homogenen Gebildes entsteht. Anpassungen und Veränderungen der familialen Bezugsrahmen laufen in dieser Perspektive Gefahr, zu stark in den Hintergrund zu treten. Das Individuum wird zu wenig in seiner Funktion als handelnder Akteur beachtet, das sich seine Umwelt aktiv aneignet. Zum einen bleibt unberücksichtigt, inwiefern sich die Teilhabe der Familienmitglieder an jeweils unterschiedlichen kollektiven Gedächtnissen auf die familialen Bezugsrahmen der Familie auswirkt. Zum anderen ist die Familie der Ort, an dem unterschiedliche Generationen aufeinandertreffen. Die einzelnen Generationen verfügen über je unterschiedliche Erfahrungsaufschichtungen und Sozialisationserlebnisse, von denen anzunehmen ist, dass sie auf die Vermittlung und Aneignung der familialen Bezugsrahmen rückwirken. Wie die Aushandlungen, die Mechanismen der Weitergabe, Anpassung und Veränderung in den Familien gestaltet sind, bleibt in den Ausführungen Halbwachs' vage. Hier sind Dynamiken zu vermuten, die durch die konsequente Einbeziehung der Generationenperspektive herausgearbeitet werden können.

Keppler und Welzer haben sich jeweils eingehender mit dem Familiengedächtnis beschäftigt. Für Keppler besteht die Einheit der Familiengeschichte in der kontinuierlichen Praxis des Erinnerns im Familienkreis (Keppler, 1992, 207). Welzer stellt fest, dass das Familiengedächtnis weniger aus einem einheitlichen Repertoire an Geschichten be-

\footnotetext{
${ }^{106}$ Auch die psychologische Forschung betont den Einfluss der Familie. Nelson verweist auf die Bedeutung sozialer Beziehungen für die Entwicklung des autobiografischen Gedächtnisses. Der Familie als primärer Sozialisationsinstanz wird eine besondere Rolle zugeschrieben. Die Art, wie Mütter über Erinnerungen reden (memory talk), beeinflusst die kindlichen Erinnerungen und die Fähigkeiten des Kindes, Erinnerungen zu kommunizieren (Nelson 2006, 82-86).
} 


\section{Die theoretische Rabmung familialer Tradierungsprozesse}

steht, als vielmehr in der kommunikativen Vergegenwärtigung von Episoden (Welzer 2002, 150). Es scheint im Kern darum zu gehen, sich über die jeweiligen familialen Bezugsrahmen zu verständigen. Diese stellen den zentralen Bestandteil des Familiengedächtnisses dar.

Welzer/Moller/Tschugnall (2002) zeigen in ihrer Studie anhand der Weitergabe von biografischen Erinnerungen der ältesten Familiengeneration an den Zweiten Weltkrieg, dass die nachfolgenden Generationen recht unterschiedliche Versionen aus diesen weitergegebenen Episoden entwickeln können. Einerseits ist einschränkend anzumerken, dass im Zuge der Weitergabe die autobiografischen Erinnerungen der ältesten Generation zu semantischen Erinnerungen der jüngeren Generationen konvertiert werden, die jüngeren Generationen also keine eigenen Erinnerungen an die betreffenden Ereignisse haben. Es handelt sich um überlieferte Berichte aus einer anderen Zeit, die für sie Sinn- und Verständnislücken aufweisen können, die durch eigene Schlussfolgerungen aufgefüllt werden, wodurch sich die ursprüngliche Geschichte erheblich verändern kann (Welzer 2002, 150 und Welzer 2001, 162/163). Zusätzlich handelt es sich in diesem Falle um einen sensiblen Themenbereich, geht es doch auch um die Frage des elterlichen bzw. großelterlichen Verhaltens während des Nationalsozialismus und damit letztlich auch um Fragen der Schuld bzw. Unschuld. Es stellt sich die Frage, ob diese Umarbeitungen bei gemeinsamen autobiografischen Erinnerungen oder emotional weniger stark besetzten Themen ebenso intensiv ausfallen würden. Andererseits ist unabhängig hiervon das Ausmaß der Umarbeitungen und -deutungen erstaunlich. Es wird sehr deutlich, dass die erheblich voneinander abweichenden Versionen der Erinnerungen an bestimmte Ereignisse die jeweils unterschiedlichen Reinterpretationen - die individuellen Perspektiven auf den betreffenden Gegenstand - spiegeln, die sich personen- und kontextabhängig ändern können.

Die voneinander abweichenden Erinnerungen eignen sich jedoch besonders, um Rückschlüsse auf die familialen Bezugsrahmen zu ziehen. Für Welzer dient das jeweilige Familiengedächtnis dazu, die soziale Identität der Wir-Gruppe zu bestätigen (Welzer 2002, 151). In den Einzelerinnerungen manifestiert sich der generationenspezifische Blickwinkel auf das Verhältnis „von Persönlichkeit, Lebensgeschichte und historischer Zeit“ (Welzer 2002, 155). Die Familien bemühen sich, die hierbei entstehenden Unterschiede zu glätten. Das Familiengedächtnis hat die Funktion „den transgenerationellen und überhistorischen Zusammenhang der Wir-Gruppe“ herzustellen und die „Fiktion einer gemeinsamen Erinnerung und Geschichte" sicherzustellen (Welzer 2002, 155/156). Hiermit ist quasi ein grundlegender familialer Bezugsrahmen beschrieben: das Zusammengehörigkeitsgefühl. Welche familialen Bezugsrahmen sich unterhalb dieser übergeordneten Ebene finden, wurde bei Welzer nicht weiter ausgearbeitet und steht im Zentrum dieser Studie.

Ähnlich wie bei Halbwachs richtet sich das Augenmerk bei Welzer auf die Übereinstimmungen des Familiengedächtnisses bzw. auf die homogenisierenden Tendenzen, nicht in Bezug auf die übereinstimmenden Erinnerungen, wohl aber was den gemeinsa- 
men familialen Bezugsrahmen betrifft. Das von Welzer beobachtete Streben nach Übereinstimmung zwischen den Familienmitgliedern impliziert die Annahme, diese seien bemüht, sich an gemeinsamen Bezugsrahmen zu orientieren. Auch wenn davon auszugehen ist, dass diese Rahmen sehr wirkmächtig sind, dürfen hierüber die Möglichkeiten der Anpassungen und Veränderungen nicht vergessen werden. Zugleich verdeutlichen Welzers Ausführungen: Homogenität ist nicht automatisch gegeben, sondern muss aktiv hergestellt werden.

Wie die Erläuterungen belegen, sind die Individuen als aktiv handelnde Akteure relevant. Die einzelnen Familienmitglieder tragen unterschiedliche Impulse in die Familie hinein, was die Wahrscheinlichkeit von Veränderungen und Anpassungen erhöht. Deshalb ist es sinnvoll, neben den Kontinuitäten den Blick für Anpassungen und Veränderungen der familialen Bezugsrahmen zu schärfen, sowie die Frage zu betrachten, wie die familialen Bezugsrahmen weitergegeben, angepasst oder verändert werden. Eine vielversprechende Möglichkeit stellt die Einbeziehung der soziologisch-historischen und familial-pädagogischen Generationenperspektive dar.

\subsection{Dynamisierung des Erinnerungsparadigmas durch die Einbeziehung der Generationenperspektive}

Der Gedanke, Erinnerungs- und Generationenperspektive miteinander zu verknüpfen, findet sich bereits bei Ortlepp. Er schlägt vor, Halbwachs' Theorie des kollektiven Gedächtnisses mit Mannheims Generationenkonzept zu verbinden (Ortlepp 2002). Mit Bezug auf Mannheim geht Ortlepp davon aus, dass nur Angehörige einer Generationseinheit in der Lage seien, ähnliche Erinnerungen zu konstruieren und damit quasi ein kollektives Gedächtnis zu bilden. Unterschiedliche Generationen verfügen für ihn über unterschiedliche kollektive Gedächtnisse, die u. U. untereinander ausgetauscht werden, wodurch möglicherweise ein kollektives Gedächtnis mit Fremdeinflüssen entstehen könne (Ortlepp 2002, 318/319). Zugleich sind für Ortlepp Generationenbeziehungen, wie z.B. in der Familie, durch Erinnerungen geprägt (Ortlepp 2002, 321). Die Erinnerungen an die eigene Erziehungserfahrung bilden den Hintergrund, vor dem angesichts der aktuellen sozialen Rahmenbedingungen individuell entschieden wird, was an die nachfolgenden Generationen weitergegeben werden soll und was nicht (Ortlepp 2002, 322). Ortlepps Konzeption ist allerdings weiter zu differenzieren. Zum einen wird die Annahme, nur Angehörige einer Generationeneinheit könnten ein kollektives Gedächtnis bilden, mit Verweis auf Halbwachs' Familiengedächtnis obsolet. Zum anderen spricht Ortlepp zwar einen familialen und pädagogischen Generationenbegriff an, bindet ihn aber nicht konsequent ein. Wenn es um Erziehung und Tradierung in der Familie geht, greift die alleinige Konzentration auf Mannheims Generationenkonzept zu kurz und ist um die familial-pädagogische Generationenperspektive zu ergänzen. Die Verknüpfung soll dazu beitragen, die in der Generationenforschung geäußerte Kritik zu überwinden, es werde zu einseitig entweder auf familiale 


\section{Die theoretische Rabmung familialer Tradierungsprozesse}

oder auf historisch-soziologische Generationsphänomene fokussiert (Karstein 2009, 53, Wohlrab-Sahr/Karstein/Schmidt-Lux 2009, 21).

\subsubsection{Die historisch-soziologische Generationenperspektive}

Mannheim hat mit seinem Aufsatz „Das Problem der Generationen“ ein Konzept zur Bestimmung historisch-gesellschaftlicher Generationen vorgelegt (Mannheim 1964/ 1928). Sein Verdienst besteht darin, herausgearbeitet zu haben, dass der gemeinsame Geburtsjahrgang, also eine ähnliche Lagerung im sozial-historischen Raum (Generationenlagerung), allein nicht ausreichend ist, um von einer Generation sprechen zu können. Vielmehr müssen eine Orientierung an „derselben historischen Problematik“ (Generationszusammenhang) sowie ähnliche Verarbeitungsweisen dieser Erlebnisse (Generationseinheit) hinzukommen (Mannheim 1964/1928, 542-544). Mit anderen Worten: Erst das Zusammenspiel von objektiver Zugehörigkeit zu einem Jahrgang mit der subjektiven Zugehörigkeit (Liebau 1997, 21/22), also der Entwicklung ähnlicher Einstellungen und Verarbeitungsmuster, konstituieren eine Generation. Die Aufmerksamkeit konzentriert sich in horizontaler Perspektive auf die Gemeinsamkeiten innerhalb einer als Generation zu bestimmenden Gruppe. Zugleich erfolgt hierdurch eine Abgrenzung zu anderen Generationengruppen (Weigel 2002, 163/164). Diese Form der Auseinandersetzung mit dem Generationenbegriff zielt darauf ab, gesellschaftlichen Wandel durch Einbeziehung der Generationenperspektive zu erklären, wobei der Jugend eine besondere Rolle als Initiator dieser Prozesse zugesprochen wird (Liebau 1997, 22).

Mannheims Konzept ist vielfach kritisch diskutiert worden. ${ }^{107} \mathrm{Z}$ weifel beziehen sich u.a. darauf, ob sich in makro-soziologischer Perspektive noch Generationseinheiten und generationelle Großgruppen identifizieren lassen (Wimmer 1998, 86 und Liebau 1997, 24). ${ }^{108}$ Angesichts der Ausdifferenzierung verschiedener Lebensformen und gestiegener Wahlmöglichkeiten ist dieser Einwand plausibel. Trotzdem ist es sinnvoll, die historisch-soziologische Perspektive nicht gänzlich auszublenden. Die Akteure der einzelnen Generationen bilden ihre Einstellungen und Handlungsweisen nicht losgelöst von gesamtgesellschaftlichen Entwicklungslinien aus, sondern werden durch diese beeinflusst. Auch wenn in dieser Studie keine generationellen Großgruppen auf der Makroebene identifiziert werden sollen und können, lässt sich fragen, ob sich innerhalb der einzelnen familialen Generationen in horizontaler Perspektive gemeinsame Rahmenbedingungen des Aufwachsens identifizieren lassen, die explizit oder implizit die Ausbildung ähnlicher Orientierungen unterstützen und auf die Tradierung und Erziehung einwirken.

\footnotetext{
${ }^{107}$ Exemplarisch seien hier Zinnecker (2003), Weisbrod (2005) und Matthes (1985) genannt.

${ }^{108}$ Liebau bezieht seine Kritik in erster Linie auf die mögliche Identifizierung von Generationseinheiten. Auch der Bestimmung von Generationszusammenhängen steht er skeptisch gegenüber. Die Generationslagerung hingegen hält er für elementar, um die Rahmenbedingungen des Aufwachsens einer Generation beschreiben zu können (Liebau 1997, 24).
} 
Mannheims starker Fokus auf die Jugendzeit als prägende Phase (Mannheim 1964/ 1928, 536/537) ist mittlerweile vielfach kritisiert worden (Zinnecker 2003, 51, Jureit 2006, 27, Rosenthal 2000, 165). Zum einen lässt sich die Kindheit nicht ausklammern (Zinnecker 2003, 51), zum anderen wird Sozialisation inzwischen als ein lebenslanger Prozess aufgefasst (Lüscher 1993, 27). Die Engführung auf die Jugendphase ist auch mit den Erkenntnissen der Erinnerungsforschung nicht zu vereinbaren. Die Gegenwart mit ihren relevanten Bezugsrahmen wirkt auf die Erinnerung ein, was eine im Lebensverlauf veränderte Perspektive bedingen kann. Das schließt die Möglichkeit ein, dass relevante Ereignisse erst retrospektiv als (generationen-)prägend betrachtet werden, dass also aktuelle Erfahrungen den Blick auf Vergangenes prägen. Ereignissen kann dabei im Nachhinein eine Relevanz zugeschrieben werden, die sie zum Zeitpunkt des Erlebens weder individuell noch gesellschaftlich hatten. ${ }^{109}$ Folglich ist prinzipiell allen Lebensphasen ein prägendes Potenzial inhärent.

Durch eine ausschließliche Fokussierung auf intragenerationelle Gemeinsamkeiten ergibt sich die Gefahr, zu einseitig auf Wandlungsprozesse und generationelle Konflikte Bezug zu nehmen und möglicherweise vorhandene intergenerationelle Gemeinsamkeiten aus dem Blick zu verlieren, deren Einbezug aber einen differenzierten Blick auf gesellschaftliche Entwicklungstendenzen ermöglichen würde. Wie Jureit hervorhebt, hat Mannheim bei seiner Generationenkonzeption tiefgreifende konflikthafte Umbrüche im Fokus (Jureit 2006, 30). Eine Konzentration auf historische Großereignisse birgt das Risiko, gesellschaftliche Entwicklungen jenseits dieser herausgehobenen Wechsel zu vernachlässigen. Hiermit wird die Chance vertan, gesellschaftliche Rahmenbedingungen zu entdecken, die weniger hervorstechend sind, aber trotzdem innerhalb einer Generationenlagerung zur Herausbildung ähnlicher Denk- und Handlungsmuster führen und somit generationenbildend wirken können. Folglich ist unabhängig von der gesamtgesellschaftlichen Relevanz innerhalb der einzelnen Generationen nach ähnlichen Erinnerungen, Themen und darin enthaltenen Bezugsrahmen zu suchen, die sich generationell verankern lassen. Ein weiteres Manko besteht darin, dass die Aushandlungsprozesse zwischen den Generationen - wenn überhaupt - nur implizit in den Blick geraten. Das erschwert es, zu einer differenzierteren Erklärung historischen Wandels zu gelangen, die fortbestehende Kontinuitäten nicht vernachlässigt.

Generation kann auch als Selbstbeschreibungskategorie dienen (Jureit 2009, 126). Gleichaltrige thematisieren sich selbst als „gefühlte Gemeinschaften“. Sie betrachten ihr Denken, Fühlen und Handeln als altersspezifisch geprägt (Jureit 2009, 126 und Jureit 2006, 41), was sie als Deutungsangebot offerieren und sich für andere Mitglieder derselben Jahrgänge als anschlussfähig erweisen kann (Jureit 2006, 40/41 und Jureit 2009, 131). Werden diese Angebote aufgegriffen, ließe sich das aus gedächtnistheoretischer

\footnotetext{
${ }^{109}$ Den konstruktiven Charakter von Erinnerungen einbeziehend, impliziert das - konsequent weitergedacht -, dass das Gefühl der Generationenzugehörigkeit eine temporäre Erscheinung von Individuen sein kann.
} 


\section{Die theoretische Rabmung familialer Tradierungsprozesse}

Perspektive als ein generationelles kollektives Gedächtnis ${ }^{110}$ bezeichnen. Nun ist nicht selbstverständlich davon auszugehen, dass sich die einzelnen Familienmitglieder selbst als Teile einer wie auch immer gearteten Generationengruppe beschreiben. $\mathrm{Da}$ in der Familie Angehörige verschiedener Generationen im Austausch miteinander stehen, ist es naheliegender, nach einer relationellen Verortung der einzelnen Generationenangehörigen zueinander zu suchen. Von Interesse ist, ob die Interviewpartner selbst Generation als Argument (Bohnenkamp/Manning/Silies 2009, 10/11) einführen, um sich abzugrenzen, indem sie z.B. auf unterschiedliche historische Kontexte verweisen oder andere Verarbeitungsweisen aufgrund der unterschiedlichen Erfahrungsaufschichtung annehmen. Diese Verortung kann explizit oder implizit erfolgen. Wichtig ist, dass Unterschiede oder Gemeinsamkeiten auf die gesellschaftlichen und historischen Rahmenbedingungen zurückgeführt werden können und nicht auf individuelle Unterschiede. Dieser Ansatz leistet einen Beitrag, um die gesellschaftlichen und historischen Rahmenbedingungen in die Analyse einzubeziehen, ohne sogleich davon auszugehen, dass diese generationenstiftend im Sinne einer sich öffentlich artikulierenden Generation sind. ${ }^{111}$

Generation kann dabei als analytische Kategorie verwendet, aber auch von den Akteuren selbst aufgegriffen werden, um sich und andere zu verorten (Jureit 2006, 8/9). Das verweist auf den Begriff der Generationalität, womit „die subjektive Selbst- und Fremdverortung von Menschen in ihrer Zeit und deren damit verbundenen Sinnstiftungen“ sowie dem Einzelnen aufgrund seiner generationellen Verortung zugesprochenen Eigenarten gemeint sind (Reulecke 2003, VIII). Die von den Akteuren vorgenommenen, generationell aufgeladenen Selbst- und Fremdverortungen liefern Hinweise, wie sich die Akteure selbst in Raum und Zeit verorten, was die Tradierungsprozesse beeinflussen kann.

Einen Ausgangspunkt für die generationelle Selbst- und Fremdverortung bietet die Generationendifferenz, die aus Altersdifferenzen resultiert und sowohl für den historisch-soziologischen als auch für den genealogisch-pädagogischen Generationenbegriff relevant ist. Wimmer betrachtet Altersdifferenz als ein grundlegendes Merkmal jeder Generationsdefinition (Wimmer 1998, 83). Die Altersdifferenz bezeichnet Unterschiede zwischen den Generationen, die unmittelbar an Zeitlichkeit gekoppelt sind: „Was die Menschen verschiedener Lebensalter existentiell voneinander trennt, das ist die Zeit selbst, und erst sekundär der Status, das Wissen, der Besitz oder die Macht" (Wimmer 1998, 92). Die vom Einzelnen entwickelten Relevanzstrukturen hängen nicht nur von den jeweiligen Inhalten ab, sondern in ebenso starkem Maße davon, zu welchem Zeitpunkt des Lebens diese Inhalte erfahren werden (Wimmer 1998, 100). Hinzu kommt, dass die unterschiedlichen Lebensalter mit Bedeutungen aufgeladen werden, indem den

\footnotetext{
${ }^{110}$ Schuman/Scott (1989) stellen fest, dass unterschiedliche Kohorten, befragt nach den wichtigsten nationalen oder internationalen Ereignissen der letzten Jahrzehnte, unterschiedliche Relevanzsetzungen vornahmen, was auf unterschiedliche kollektive Gedächtnisse hindeutet.

${ }^{111}$ Massenmediale Vorlagen können den Blick der einzelnen Generationen mitprägen - sowohl auf ihre Vergangenheit als auch auf die Gegenwart (Jureit 2006, 90/91).
} 
einzelnen Generationen je spezifische Rollen, Funktionen und Bilder zugeschrieben werden (Wimmer 1998, 89 und Winterhager-Schmid 2000, 18/19, 24). Generationelle Aspekte sind damit keine rein sozialen Konstruktionen, zugleich erhalten sie ihre besondere Bedeutung erst durch gesellschaftliche Zuschreibungsmechanismen, die wiederum historischem Wandel unterliegen. Ein wesentliches Kennzeichen des intergenerationellen Verhältnisses ist also die Generationendifferenz, die sich aus der Altersdifferenz und der ihr zugeschriebenen Bedeutung ergibt. Die Generationendifferenzen aus Akteursund Fremdperspektive zu untersuchen, erlaubt es, generationelle Unterschiede sowie die Ausgestaltung der Generationenbeziehungen zu erfassen. Zugleich handelt es sich bei dem Begriff der Generation um eine „relationale Kategorie, die auf ein Beziehungsverhältnis verweist“" (Baader/Sager 2008, 292). D.h. die Untersuchung generationeller Aspekte erfordert in der Regel eine Vergleichsperspektive, um das je Spezifische einer Generation herausarbeiten zu können, wie es die familial-pädagogische Generationenperspektive ermöglicht.

\subsubsection{Die familial-pädagogische Generationenperspektive}

Der genealogisch-familiensoziologische Generationenbegriff stellt die genealogische Abstammungslinie sowie die mikrosoziale Ebene ins Zentrum der Aufmerksamkeit (Liebau 1997, 25). ${ }^{112}$ In Anbetracht des Untersuchungsgegenstands und der Ausführungen $\mathrm{zu}$ Tradierung, Familie und Erziehung ist es naheliegend, dieser Perspektive eine besondere Beachtung zu schenken. Eine Gliederung anhand der Eltern-Kind-Abfolge bietet zudem den Vorteil, sich an vorfindbaren Strukturen orientieren zu können und den Gegenstand „nicht erst voluntaristisch“ (Kohli 2007, 49) definieren zu müssen.

In der Familie treffen unterschiedliche familiale Generationen aufeinander, also Großeltern, Eltern und Kinder, die sich durch eine unterschiedliche Erfahrungsaufschichtung auszeichnen. In der Familie existiert eine Ansammlung verschiedener Erfahrungen aus verschiedenen historischen Zeiten mit ihren jeweiligen strukturellen Bedingungen (Ecarius, 2007, 144). Zugleich ist mit Großeltern, Eltern und Kindern eine Gruppe versammelt, deren Mitglieder sich in unterschiedlichen Lebensphasen befinden, die mit unterschiedlichen Aufgaben, Rollen und Bedeutungen versehen sind. Hieraus können unterschiedliche Relevanzsetzungen und Gewichtungen einzelner familialer Bezugsrahmen resultieren. Das bildet die Ausgangslage, vor deren Hintergrund das Familienleben und die Erziehung gestaltet werden, und stellt die Familienmitglieder und -generationen vor die Aufgabe, mit der damit verbundenen Generationendifferenz umzugehen.

Wie die familiensoziologische Forschung zeigt, dominieren in Familien reziprok geprägte Beziehungen und Generationensolidarität zwischen den Familiengenerationen (Lüscher/Liegle 2003, 164-150, 264 und Kohli/Szydlik 2000, 11). Das widerspricht zu-

\footnotetext{
${ }^{112}$ Liebau resümiert, dass trotz sozialer Wandlungsprozesse der familiale Generationenbegriff seine Überzeugungskraft beibehalten habe, auch wenn er empirisch neu zu bestimmen sei (Liebau 1997, $25 / 26$ und 30$)$.
} 


\section{Die theoretische Rabmung familialer Tradierungsprozesse}

mindest auf der Mikroebene der konkreten Familienbeziehungen der Annahme existentieller Generationenkonflikte, und die Familienforschung hat sich inzwischen von dieser Prämisse gelöst (Ecarius 2002, 25 und 28). Zwar soll hier die Existenz von konflikthaften Auseinandersetzungen und Brüchen in der familialen Generationenfolge nicht geleugnet werden, sie sind aber nicht als Regelfall anzunehmen. Mit Verweis auf die Gedächtnisforschung ist nicht davon auszugehen, dass die nachfolgende Generation zwangsläufig mit den familialen Bezugsrahmen sowie der Erziehungspraxis der Vorgängergeneration bricht. Die Tradierung vollzieht sich oftmals in Form von Anpassungen, die durch die jeweiligen gesellschaftlichen und historischen Rahmenbedingungen beeinflusst werden, aber in Auseinandersetzung mit dem Gegebenen entstehen und sich nicht als absolute Brüche bezeichnen lassen. Demzufolge sind vielmehr fließende Übergänge und ein langsamer Wandel zu erwarten.

Innerhalb der Generationenforschung werden familiale Generationen des Öfteren mit dem Aspekt der Kontinuität und historisch-soziologische Generationen stärker mit Wandel assoziiert (Karstein 2009, 55, Wohlrab-Sahr/Karstein/Schmidt-Lux 2009, 65, Ehmer 2003, 59). Diese Perspektive vernachlässigt die Dynamiken familialer Austauschprozesse, die bei der Betrachtung des pädagogischen Generationenbegriffs deutlich hervortreten. Dieser geht auf Schleiermacher zurück, dessen Ausführungen zum Generationenverhältnis Helsper u.a. als ersten systematischen Generationsentwurf betrachten (Helsper u.a. 2009, 15). Schleiermacher befasst sich mit der Frage: „Was will denn eigentlich die ältere Generation mit der jüngeren?" (Schleiermacher 2000, 9). ${ }^{113}$ Die gleichzeitige Existenz verschiedener Lebensalter rückt ins Zentrum, wobei im Unterschied zu Mannheim die Aufmerksamkeit nicht den Gemeinsamkeiten innerhalb einer Generation gilt, sondern den Unterschieden zwischen den Generationen (Weigel 2002, 184). In Schleiermachers Argumentation gibt es immer nur zwei Generationen: eine ältere und eine jüngere (Schleiermacher 2000, 9). Er zeichnet eine klare Rollenaufteilung, wonach die ältere Generation kulturelle Wissensbestände an die jüngere weiterreicht. Allerdings wird letztere nicht zu einer unveränderten Übernahme verpflichtet. Ihr werden Gestaltungsspielräume zugestanden, sofern sie das Erhaltene verbessern kann (Schleiermacher 2000, 34). ${ }^{114}$ Hierin manifestiert sich die „Offenheit der Schleiermacherschen Pädagogik“, die die „Antizipation einer möglichen Weiterentwicklung“ (Kraul 2003b, 286) berücksichtigt.

Sünkel greift Schleiermachers Überlegungen auf und reformuliert den pädagogischen Generationenbegriff (Sünkel 1997). Der bei Schleiermacher hervortretende Fortschrittsoptimismus entfällt. Ausgehend von erziehungstheoretischen Überlegungen erfolgt ei-

\footnotetext{
${ }^{113}$ Unübersehbar stehen die Generationenbeziehungen im Zentrum der Betrachtung, die für Winkler das Fundament von Schleiermachers Erziehungstheorie bilden $(1998,122)$.

${ }^{114}$ Ein kurzer Überblick über Schleiermachers Verwendung des Generationenbegriffs sowie zu der Frage, wie Wissen zwischen den Generationen tradiert und zugleich Raum für neue Entwicklungen geschaffen wird, findet sich bei Ecarius (2008, 21-29).
} 
ne Einteilung in vermittelnde und aneignende Generation (Sünkel 1997, 199). ${ }^{115} \mathrm{Im}$ Unterschied zu Schleiermacher gehört die ältere Generation nicht automatisch zur vermittelnden. Je nach Kontext kann der Einzelne mal der einen, mal der anderen Generation angehören (Sünkel 1997, 199/200). ${ }^{116}$

Im Unterschied zur historisch-soziologischen Perspektive stehen weniger die inhaltliche Bestimmung historischer Generationen im Zentrum als vielmehr Fragen der Vermittlung, Übernahme und Veränderung immaterieller kultureller Güter und Einstellungen (Helsper u.a. 2009, 22). Bei der Weitergabe von Wissensbeständen, zu denen u.a. familiale Bezugsrahmen zählen, handelt es sich um einen interaktiven Prozess und alle beteiligten Generationen tragen aktiv dazu bei, was auf welche Weise weitergegeben wird. Im intergenerationellen Dialog wird die Sicht auf die Vergangenheit wechselseitig ausgehandelt und reinterpretiert (Rosenthal 2000, 177), was einen Effekt auf die Bezugsrahmen haben kann. Bezogen auf die Aneignungsebene formuliert Schmidt explizit: „Dem tradere kommt ein accipere entgegen, welches die Tradita immer irgendwie verändert" (Schmidt 1970, 1202), womit die Adressatenseite explizit als Gegengewicht zum Übermittler in die Überlegung eingebracht wird. ${ }^{117}$ Die Adressaten beeinflussen, inwiefern Tradierungsinhalte angenommen, modifiziert, nicht übernommen oder abgelehnt werden, wobei diese Prozesse sowohl bewusst als auch unbewusst ablaufen können.

Allerdings unterliegen Weitergabe, Anpassung bzw. Veränderung keiner völligen Beliebigkeit. Sowohl vermittelnde als auch aneignende Generation treffen auf existierende familiale Bezugsrahmen, zu denen sie sich verhalten müssen, indem sie an diese anknüpfen oder sich von ihnen absetzen. Durch die Fokussierung auf Vermittlung und Aneignung tritt der dynamische Aspekt von Tradierungsprozessen hervor, und es wird deutlich, dass die Weitergabe familialer Bezugsrahmen kein Automatismus mit bereits feststehendem Ergebnis ist. Wie diese Prozesse gestaltet werden, beeinflusst, welche Bezugsrahmen in welcher Weise tradiert werden. In der familialen Erziehung verknüpfen sich genealogischer und pädagogischer Generationenbegriff, indem Eltern in der Regel (aber nicht immer) eine Vermittlungs- und den Kindern eine Aneignungsfunktion zugeschrieben wird (Sünkel 1997, 200). Die familiale Erziehung ist „im Spannungsfeld von individuellen Interessen und historischen, gesellschaftlichen Strukturen" angesiedelt (Ecarius 2007, 144), die sich auf die konkrete Erziehung auswirken. Die Tradierung familialer Bezugsrahmen ist hierbei zwischen den Polen von Kontinuität und Veränderung angesiedelt. Zum einen geht es um die Weitergabe kultureller Wissensbestände. Zum anderen

\footnotetext{
${ }^{115}$ Vermittlung und Aneignung sind auch für das Konzept der generativen Sozialisation bedeutsam (Liegle/Lüscher 2008, 146/147).

${ }^{116}$ Mit der Möglichkeit einer wechselnden Generationenzuordnung ist ein wesentlicher Unterschied zur historischen Generationenzuordnung angesprochen.

${ }^{117}$ Leider wird gerade der Aspekt des accipere oder vielleicht treffender der Aneignung in den einzelnen Artikeln zur Tradierung nicht immer deutlich formuliert bzw. scheint nicht immer die ihm zustehende Aufmerksamkeit zu erhalten, sodass es leicht zum Missverständnis kommen kann, Tradierung sei ein einseitiger Vermittlungsprozess.
} 


\section{Die theoretische Rabmung familialer Tradierungsprozesse}

bedarf es angesichts gesellschaftlicher Veränderungsprozesse einer gewissen Flexibilität, damit die nachfolgende Generation sich auch unter veränderten Rahmenbedingungen zurechtfinden kann. Der Zusammenschluss der pädagogischen mit der familialen Perspektive kann dabei ein Manko des pädagogischen Generationenbegriffs ausgleichen: die Beschränkung auf zwei Generationen. Eine Kombination von familialem und pädagogischem Generationenbegriff lässt eine differenziertere Betrachtungsweise zu, indem sich z.B. großelterliche von elterlichen Vermittlungsprozessen unterscheiden lassen.

Die Verwendung eines familial-pädagogischen Generationenbegriffs unter Berücksichtigung der gesellschaftlichen Rahmenbedingungen kann dazu beitragen, das Verhältnis von familialem und historisch-soziologischem Generationenbegriff sowie den ihnen zugeschriebenen dominanten Konnotationen von Kontinuität und Wandel zu klären, indem der vermeintliche Gegensatz aufgelöst wird. Historisch-soziologischer und familialer Generationenbegriff sind aufeinander bezogen, da gesellschaftliche Veränderungsprozesse auf der Mikroebene, u.a. in der Familie, verarbeitet werden. ${ }^{118}$ Gesellschaftliche und familiale Sozialisationsprozesse vollziehen sich nicht unabhängig voneinander, wie Jureit hervorhebt $(2006,64)$. Die Einbeziehung einer familial-pädagogischen sowie soziologisch-historischen Generationenperspektive ermöglicht es, das Familiengedächtnis und die familialen Bezugsrahmen dynamischer und flexibler zu konzipieren, indem Kontinuitäten und Wandel erfasst werden können.

In diesem Zusammenhang ist ein Blick auf die individuelle Auseinandersetzung mit der eigenen Lebensgeschichte notwendig. Ecarius betrachtet es in ihrer Drei-Generationen-Studie als charakteristisch für die älteste Generation, dass sie die eigenen Erziehungerfahrungen bei der Erziehung der Kinder übernimmt; die mittlere Generation favorisiert aufgrund ihrer eigenen Erziehungserfahrungen andere Erziehungsinhalte für die jüngste Generation (Ecarius 2002, 256 und 259). An dieser Stelle bietet es sich an, Kosellecks Begriffspaar von Erfahrungsraum und Erwartungshorizont (1989, 349-359) in die Überlegungen zur Charakterisierung der Erziehung zu integrieren. ${ }^{119}$ Die Terminologie wird von Koselleck ursprünglich benutzt, um eine gesellschaftliche Entwicklung zu skizzieren, in der der Erfahrungsraum der älteren Generation nicht mehr umstandslos auf den Erwartungshorizont für die Zukunft übertragbar ist $(1989,359-369) .{ }^{120} \mathrm{Die}$ Begrifflichkeiten lassen sich auf die Mikroebene und das konkrete Erziehungsgeschehen übertragen. Der Erfahrungsraum der Zu-Erziehenden besteht aus den Bezugsrahmen, die an sie herangetragen werden sowie aus der Art und Weise, wie das geschieht. Zum Erwartungshorizont zählen die Vorstellungen der Erziehenden, wie die Erzogenen werden und welche Bezugsrahmen sie internalisieren sollen. Der Erfahrungsraum der eigenen Erziehung während der Kindheit bildet für die Erziehenden den Hintergrund für

\footnotetext{
${ }^{118}$ Möglicherweise strahlen umgekehrt Veränderungen auf der Mikroebene in den gesellschaftlichen Raum aus.

${ }^{119}$ Zur Eignung von Kosellecks Begriffspaar von Erfahrungsraum und Erwartungshorizont für die Analyse familialer Tradierungsprozesse siehe auch Radicke (2013).

${ }^{120}$ Inwiefern diese Beschreibung zutrifft, ist für den Kontext dieser Arbeit nicht ausschlaggebend.
} 


\subsection{Dynamisierung des Erinnerungsparadigmas}

die Entwicklung von Erwartungshorizonten, die über Erziehung an die nächste Generation herangetragen werden. Je nach Positionierung zum eigenen Erfahrungsraum und den darin eingelagerten Bezugsrahmen werden bei der Erziehung der eigenen Kinder ähnliche oder abweichende Erwartungshorizonte entworfen, zu denen sich die Kinder wiederum positionieren. Die Ausgestaltung der jeweiligen Erwartungshorizonte wird vom jeweiligen historischen Kontext beeinflusst, sodass zudem die zeitliche Perspektive eingebunden werden kann.

In der vorliegenden Studie werden die Erwartungshorizonte als zentrale Bezugsgrößen für die nähere Charakterisierung von Tradierungsprozessen verwendet. Durch die Konzentration auf die Frage, wie die Familien die Erwartungshaltungen gegenüber der nächsten Generation gestalten, lassen sich familiale Tradierungslogiken bestimmen und unterscheiden. Für die nähere Ausdifferenzierung der Tradierungslogiken ist zudem Olsons u.a. (1989) Circumplex-Modell instruktiv. Sie untersuchen anhand der Dimensionen Kohäsion und Adaptabilität, wie Familien mit Veränderungen umgehen, die sich im Verlauf des Familienzyklus ${ }^{121}$ ergeben. Kohäsion misst den Grad der emotionalen Verbundenheit der Familienmitglieder untereinander, und Adaptabilität fokussiert auf die Fähigkeiten der Familie, in Abhängigkeit vom situativen Kontext die Macht- und Rollenbeziehungen sowie die Beziehungsregeln zu ändern (Olson u.a. 1989, 48), indem sie diese entweder flexibel anpassen oder strikt an ihnen festhalten. ${ }^{122}$ Wie Familien mit Veränderungen umgehen, ist nicht nur in Bezug auf die verschiedenen Stadien des Familienzyklus von Bedeutung, sondern auch in abgewandelter Form für Tradierungsprozesse. Die Dimension der Adaptabilität rekurriert im Kern auf das Ausmaß an familialer Flexibilität. Übertragen auf familiale Tradierungsprozesse stellt sich die Frage, wie flexibel Familien auf gesellschaftliche Entwicklungsprozesse reagieren, und ob sie Tradierungsinhalte und -modi anpassen oder nicht.

Angesichts des sozialen Wandels ist zu vermuten, dass ein gewisses Maß an Anpassungsbereitschaft seitens der Erziehenden - sowohl was die Inhalte als auch was die Modi der Tradierung betrifft - dazu beiträgt, familiale Bezugsrahmen an die nächste Generation weiterzugeben. Gerade in Zeiten gesellschaftlichen Wandels dürfte es der nächsten Generation die Anschlussfähigkeit erleichtern. In Anbetracht dieser Erörterungen ist die Annahme, dass die Inhalte weitergegeben werden, die von der älteren Generation als nützlich betrachtet werden (Kraul 2003b, 295), um die Aneignungsperspektive zu ergänzen: die Inhalte werden übernommen, die der aneignenden Generation anschlussfähig und nützlich erscheinen. Damit lautet die zentrale Frage nicht, ob Elemente tradiert werden, sondern was aus der Fülle der möglichen Inhalte wie tradiert wird. Konkret auf diese Studie bezogen bedeutet das zu untersuchen, ob sich innerhalb der einzelnen Familien gemeinsame Orientierungen herausarbeiten lassen, wie diese tra-

\footnotetext{
${ }^{121}$ Eine Übersicht über die Stadien des Familienzyklus findet sich bei Hofer $(2002,19)$.

${ }^{122}$ Die Kommunikation stellt eine dritte Dimension dar, die die Verortung auf den beiden anderen Dimensionen entscheidend beeinflusst (Olson u.a. 1989, 49).
} 
2 Die theoretische Rabmung familialer Tradierungsprozesse

diert werden, welche Tradierungslogiken sich erkennen lassen und wie gesellschaftliche Entwicklungen innerfamilial verarbeitet werden. 


\section{Methode und Forschungsdesign}

\subsection{Methodologische und methodische Grundannahmen}

Die zentrale Fragestellung dieser Studie nach Inhalten und Formen familialer Tradierung rückt die beteiligten Akteure ins Zentrum der Betrachtung. Deswegen wird ein qualitatives Forschungsdesign verwendet, in dessen Zentrum qualitativ erhobene Interviews stehen. Grundlegend für diese Forschungsrichtung ist die Annahme, dass „, $[\mathrm{u}] \mathrm{nser}$ gesamtes Wissen von der Welt, sei es im wissenschaftlichen oder im alltäglichen Denken“ Konstruktionen beinhaltet und folglich alle Tatsachen bereits ,immer interpretierte Tatsachen" darstellen (Schütz 2004/1953, 158). Die Konstruktionen der lebensweltlichen Akteure stellen die Grundlage für die Ableitung sozialwissenschaftlicher Konstruktionen dar, die Schütz „Konstruktionen zweiten Grades“ nennt (Schütz 2004/ 1953, 159). Im Mittelpunkt des Interesses steht also nicht die Suche nach irgendwie gearteten objektiven Tatsachen, sondern nach den Perspektiven, mit deren Hilfe die Akteure ihre Wirklichkeit konstruieren. Vergangene Erfahrungen beeinflussen hierbei die gegenwärtigen und zukünftigen Konstruktionen der Individuen, ohne sie jedoch deterministisch festzulegen. Die Relevanz, die den individuellen Perspektiven auf die Wirklichkeit zukommt, liegt somit auf der Hand. Sie beeinflussen das individuelle Handeln und sind dadurch unmittelbar wirklichkeitskonstituierend. Die auf den individuellen Erfahrungen beruhenden Konstruktionen sind darüber hinaus nicht so beliebig, wie anzunehmen wäre, sondern folgen bestimmten Strukturen (Alheit/Dausien 2000, 275 und Alheit/Hoerning 1989, 13). In die Biografie des Einzelnen eingelagert finden sich allgemeine Strukturen und Regeln, die das Handeln des Einzelnen leiten, ohne ihm unbedingt bewusst zu sein (Bude 2006, 109). Sie bietet einen Zugang zur Erforschung der individuellen Verarbeitung gesellschaftlicher und milieuspezifischer Erfahrung sowie allgemeiner Strukturen (Marotzki 2000, 176). Im Zentrum des Interesses stehen die Deutungs- und Handlungsmuster der Individuen, von denen angenommen wird, dass sie sozial verregelt sind. Allerdings soll dies nicht heißen, individuelles Handeln ließe sich vollständig aus den vorgegebenen Strukturen ableiten, wie die Frage „nach den Handlungsspielräumen der Subjekte innerhalb der gesellschaftlichen Strukturen“ (Rosenbaum 2001, 746) verdeutlicht. Durch die Einnahme einer akteurszentrierten Perspektive soll hervorgehoben werden, dass die Subjekte Akteure in ihrer sozialen Umwelt sind und sich zu den Regeln und Strukturen verhalten.

Das Anliegen der rekonstruktiven Sozialforschung besteht darin, die impliziten Wissensbestände und Regeln, denen das Handeln der Individuen folgt, herauszuarbeiten und nicht nur den subjektiv gemeinten Sinn der Akteure zu erfassen, sondern die dahinter stehenden Logiken, die kollektiven Orientierungsmuster aufzudecken, denen der 


\section{Methode und Forschungsdesign}

Einzelne folgt (Meuser 2006, 140 und 142). Hierfür stellt die dokumentarische Methode ein ausdifferenziertes Instrumentarium bereit. Sprachliche Äußerungen in Interviews werden unter Verweis auf die Ethnomethodologie als indexikalisch aufgefasst, d.h. als „ein Hinweis auf das mit diesen Äußerungen verbundene, durch diese Äußerungen selbst aber nicht explizierte, Orientierungsmuster" (Bohnsack 1997, 53). Das Ziel der dokumentarischen Methode besteht darin, nicht nur das explizite Wissen der Akteure $\mathrm{zu}$ erfassen, sondern das der Handlungspraxis des Einzelnen zugrunde liegende Orientierungswissen herauszuarbeiten (Bohnsack/Nentwig-Gesemann/Nohl 2001, 9). Die in den individuellen Äußerungen zum Ausdruck kommenden Relevanzen der Akteure werden bei der Analyse berücksichtigt, wobei die dokumentarische Methode darauf zielt, diese zu transzendieren und über die subjektiven Intentionen und Common-SenseTheorien der Akteure hinauszugelangen (Bohnsack/Nentwig-Gesemann/ Nohl 2001, 12). Hierzu werden Beobachtungen erster Ordnung von Beobachtungen zweiter Ordnung unterschieden. Erstere erfassen den „kommunikativ-generalisierende[n], wörtlichen oder ,immanenten“ Sinngehalt“, letztere den „konjunktiven, metaphorischen oder eben dokumentarischen Sinngehalt“ (Bohnsack/Nentwig-Gesemann/ Nohl 2001, 14). Neben den Sinnzuschreibungen der Akteure geht es folglich darum, das die Handlungspraxis leitende Wissen zu explizieren. In der Praxis alltäglicher Verständigung sind zumeist beide Dimensionen präsent, also der kommunikative wie der konjunktive Aspekt, die somit nur analytisch trennbar sind (Bohnsack 1997, 54).

Die Absicht der dokumentarischen Methode besteht darin, nicht nur die Konstituierung einer Handlung zu erklären, indem der dahinter stehende Entwurf im Sinne eines „Um-Zu-Motivs“ herausgearbeitet wird - was Bohnsack mit dem Begriff des Orientierungsschemas belegt -, sondern es geht im Kern darum, die „Konstituierung des Entwurfs“ unter Berücksichtigung vorausgegangener Erfahrungen im Sinne eines „WeilMotivs“ zu erfassen, also den Orientierungsrahmen herauszuarbeiten (Bohnsack 1997, 51/52). Neben der Erstellung einer Typik geht es darum, anhand der jeweiligen Orientierungsrahmen zu erfassen, für welche konjunktiven Erfahrungsräume sie gelten; also zu klären, in welchen sozialweltlichen Bereichen sie verankert sind (Nentwig-Gesemann 2001, 295 und Bohnsack 2001, 245).

Diese Annahmen berücksichtigend, besteht das Analyseverfahren der dokumentarischen Methode aus einem mehrstufigen Prozess. Zu Beginn erfolgt die formulierende Interpretation, die sich nach dem Relevanzsystem der Akteure richtet (Bohnsack 2007, 34). Im Zuge dieses Arbeitsschrittes, der danach fragt, „was die gesellschaftliche Realität in der Perspektive der Akteure ist", werden die immanenten Sinngehalte erschlossen (Bohnsack/Nentwig-Gesemann/Nohl 2001, 12-15). Es soll ein inhaltlicher Überblick über das Material erstellt werden, wozu zunächst ein thematischer Verlauf der Interviews erstellt wird, der nach Ober- und Unterthemen gegliedert wird. Anschließend werden die Themen ausgewählt, die für die forschungsleitende Fragestellung als relevant betrachtet werden, sowie die Stellen, zu denen sich die befragten Personen besonders ausführlich äußern (Bohnsack 2007, 134/135 und Nohl 2006, 46). Die sich an- 


\subsection{Methodologische und methodische Grundannahmen}

schließende reflektierende Interpretation orientiert sich an der Frage, wie diese Realität hergestellt wird, und zielt darauf, den konjunktiven Sinngehalt zu erfassen (Bohnsack/ Nentwig-Gesemann/Nohl 2001, 12-15 und Bohnsack 2007, 34, Nohl 2006, 51). Der Rahmen, innerhalb dessen ein Thema behandelt wird, soll in diesem Analyseschritt herausgearbeitet werden (Bohnsack 2007, 135). Es erfolgt zunächst eine formale Auswertung, die die Unterscheidung zwischen Erzählung, Beschreibung und Argumentation ${ }^{123}$ umfasst, wobei davon ausgegangen wird, dass Argumentationen vor allem den Bereich des kommunikativen Wissens umfassen und konjunktives Wissen in Beschreibungen und Erzählungen eingelagert ist (Nohl 2006, 48/49). Letztere sind für die Analyse und die Rekonstruktion der Rahmungen von besonderem Interesse, wobei anzumerken ist, dass die Art und Weise, „wie jemand seine Handlungsweisen rechtfertigt“, Rückschlüsse auf den jeweils relevanten Orientierungsrahmen erlaubt (Nohl 2006, 50). Diese werden im Zuge der komparativen Sequenzanalyse ermittelt. Leitend ist hierbei der Gedanke, dass ein Thema von einem Individuum in einer bestimmten Art und Weise erfahren wird. Auf einen ersten Erzählabschnitt kann nur ein bestimmter, dem jeweiligen Rahmen entsprechender zweiter Abschnitt folgen, der sich im nächsten Schritt ratifizieren lässt (Nohl 2006, 11 und 52/53).

Bei der Auswertung ergibt sich das Problem, dass der Forscher selbst aufgrund seiner Standortgebundenheit, d.h. seiner jeweiligen Sozialisation und Zugehörigkeit zu einem bestimmten Milieu (Fuchs 1984, 136), über bestimmte Normalitätsannahmen verfügt, die in die Interpretation einfließen (Nohl 2006, 54). Um dieses Problem zu relativieren und zu kontrollieren, wird die Interpretation so früh wie möglich an einen Vergleich mit anderen empirischen Fällen gebunden (Nohl 2006, 54/55), worin ein wichtiger Mehrwert der dokumentarischen Methode liegt.

Die Auswertung des Datenmaterials in dieser Studie orientiert sich an diesem Vorgehen. $\mathrm{Zu}$ Beginn der Analyse stand die Frage, welche zentralen Themen sich jeweils in den einzelnen Interviews identifizieren lassen. Durch eine vergleichende Betrachtung der drei befragten Generationen einer Familie lassen sich auf diese Weise die für die einzelnen Familien relevanten Inhalte herausarbeiten, die in den einzelnen Interviews meist schon in der Eingangserzählung thematisch relevant werden. Über die Kontrastierung mit den anderen Familien des Samples können die Orientierungen der einzelnen Familien geschärft und als spezifisch für die jeweils untersuchte Familie abgesichert werden. Die so herausgearbeiteten Orientierungen werden innerhalb dieser Studie als familiale Bezugsrahmen bezeichnet. Es wird also nicht auf die Terminologie der dokumentarischen Methode mit ihrer Unterscheidung von Orientierungsschema und Orientierungsrahmen zurückgegriffen. Hiermit soll zum einen die Verbindung zu Halbwachs' Gedächtnistheorie betont werden, und zum anderen wird berücksichtigt, dass die Bezugsrahmen eine Doppelnatur aufweisen. So lassen sie sich in einigen Familien in bestimmten Situationen zum Bereich des kommunikativen Wissens zuordnen; bei der

\footnotetext{
${ }^{123}$ In diesem Schritt wird die Textsortentrennung vorgenommen, die Schütze zur Auswertung narrativer Interviews verwendet (Schütze 1987).
} 


\section{Methode und Forschungsdesign}

Schilderung anderer Situationen wiederum weisen sie eher die Qualität eines konjunktiven Wissens auf. Mit anderen Worten: Die Bezugsrahmen können situationsabhängig sowohl einen expliziten als auch impliziten Charakter annehmen. In einigen Familien sind die Bezugsrahmen zudem insgesamt eher der impliziten Sphäre zuzuordnen und in anderen Familien stärker der expliziten.

Nachdem die familialen Bezugsrahmen bestimmt worden sind, richtet sich die Fragestellung auf die Tradierung dieser Bezugsrahmen. Die Analyse konzentriert sich dabei auf alle Stellen des Interviews, die die inter- und intragenerationelle Ebene thematisieren. Hierzu zählen beispielsweise die familialen Beziehungen und die jeweiligen Erziehungsarrangements mit ihren intergenerationellen Machtbalancen, Regeln und Strukturen. Das Augenmerk gilt der Entwicklung der Bezugsrahmen und Erziehungssettings im Generationenverlauf, wobei das Ziel darin besteht, die dem Tradierungsprozess zugrundeliegenden Logiken zu explizieren. Da diese als Bestandteil des Erziehungsprozesses sowohl explizite wie implizite Züge aufweisen können, wird auch hier auf die Terminologie von Orientierungsschema, -rahmen bzw. -muster verzichtet und stattdessen von Tradierungslogik gesprochen.

Bezogen auf das Erhebungsverfahren des Interviews wird darauf verwiesen, dass Interviewte sich in einer bestimmten Weise selbst präsentieren und ein Bild vermitteln möchten, wie sie „gesehen werden möchten oder sich selbst sehen“ und Interviews auf einer „reflektierten ,vorzeigbaren“ Version der eigenen Person“ basieren, die „ein kommunikativ auf den Zuhörer und die Situation zugeschnittenes Bild erkennen" lassen (Schmidt-Lauber 2001, 168 und 180). Sicherlich wirkt sich die jeweilige Einschätzung der Interviewsituation darauf aus, welche Inhalte der Interviewte anspricht und welche nicht (Rosenthal 2009, 51). Hierbei geben „[g]esellschaftliche, institutionelle und familiale Regeln bzw. die Regeln unterschiedlicher Diskurse [...] vor, was, wie, wann und in welchen Kontexten thematisiert werden darf und was nicht" (Rosenthal 2009, 51). Dieser Verweis auf die den Äußerungen zugrundeliegenden Regeln bedeutet zugleich, dass die Selbstpräsentationen der Individuen nicht so beliebig ausfallen, wie sich denken ließe. Sie folgen vielmehr bestimmten Regeln und Orientierungen und lassen Rückschlüsse auf die jeweils relevanten Bezugsrahmen der Befragten zu. Wie der interfamiliale Vergleich zeigt, unterscheiden sich die einzelnen Familien hinsichtlich ihrer Bezugsrahmen durchaus und in ihren Selbstpräsentationen sind unterschiedliche Prioritäten und Relevanzsetzungen zu erkennen. Dies unterstreicht die strukturierende Kraft der familialen Bezugsrahmen, unabhängig davon, inwiefern diese von den Individuen tatsächlich umgesetzt werden oder nicht.

In diesem Zusammenhang sei kurz auf die immer wieder aufgeworfene Frage nach der Aussagekraft der in narrativ orientierten Interviews erhobenen Inhalte eingegangen, bei denen es sich um die Präsentation von lebensgeschichtlichen Erinnerungen handelt. Die Zweifel beziehen sich darauf, ob die erinnerten Inhalte aufgrund ihres retrospektiven Charakters Rückschlüsse auf das Leben der Individuen in der Vergangenheit bzw. ihr Erleben in der Vergangenheit zulassen. Einige Autoren gehen davon 


\subsection{Methodologische und methodische Grundannahmen}

aus, dass Erinnerung, wie sie in lebensgeschichtlichen Interviews erhoben wird, keine „vergangene Wirklichkeit abbildet, sondern allenfalls eine heutige Sicht auf etwas, das jemand erlebt zu haben glaubt“ (Welzer/Markowitsch 2006, 15). Entgegen dieser radikalen Einschätzung erläutert Rosenthal mit Rückgriff auf die Gestalttheorie, dass lediglich die Welt, wie sie sich dem Individuum darbietet, erfassbar ist. Letzteres ist von Husserl mit dem Terminus des Noemas belegt (Rosenthal 1995, 27). Bei der kommunikativen Präsentation von vergangenen Erinnerungen unterscheidet sich das Erinnerungsnoema in der Regel vom Erlebnisnoema in der Vergangenheit, gleichwohl bezieht es sich auf das damalige Erlebnis und ist nicht losgelöst von diesem: „Das Erlebnis wird ja nicht verändert, sondern heute nur anders gesehen und verstanden. Dabei kann diese heutige Sicht nun durchaus eine sein, die damals die Erfahrung mitbestimmte, bisher jedoch nicht als solche realisiert wurde" (Rosenthal 1995, 95). Folglich ermöglichen mündliche Darstellungen vergangener Erlebnisse, wie etwa in Form von narrativ orientierten Interviews, Rückschlüsse auf die Vergangenheit der erzählenden Personen zu ziehen (Rosenthal 2005, 168 und Bohnsack 2007, 93). Für Rosenthal eröffnen insbesondere die als Erzählung präsentierten Erinnerungen eine Annäherung an den vergangenen Handlungsablauf (Rosenthal 2005, 141). Bohnsack misst den durch eine Stegreiferzählung evozierten mündlichen Präsentationen einen besonderen Stellenwert zu. Durch diese wird ein Erinnerungs- und Erzählfluss angeregt, der im abnehmenden Maße einer bewussten Steuerung unterliegt und im Zuge dessen „theoretisch-reflexiv weniger überformte Ebenen der Selbsterfahrung" (Bohnsack 2007, 93) reproduzierend dargestellt werden, die eine Annäherung an das Erleben in der Vergangenheit darstellen (Bohnsack 2007, 93 und Rosenthal 2005, 143). Dies bedeutet natürlich nicht, dass erzählte Erinnerungen immer dem tatsächlichen Handlungsablauf, wie er von den Erzählenden erlebt wurde, entsprechen. Bei der Analyse ist deswegen besonders auf Brüche, Widersprüche und Inkonsistenzen $\mathrm{zu}$ achten, die allerdings weniger dazu dienen, $\mathrm{zu}$ in irgendeiner Weise objektiven Tatsachen zu gelangen, sondern vielmehr als Ausgangsbasis dienen, um hierüber die Regeln und Orientierungsstrukturen der Befragten zu explizieren.

Durch das Design dieser Studie, in der mehrere Generationen einer Familie befragt werden, ergibt sich zudem die Möglichkeit eine mehrfache Perspektive auf das Zusammenleben der jeweiligen Familien zu erhalten. Der Vergleich der Sichtweisen der einzelnen Akteure auf ihr Familienleben verweist auf erstaunliche Kongruenzen. Die Familienmitglieder beschreiben die allgemeinen Charakteristika ihres Familienlebens in ähnlicher Weise. Anders formuliert: Es findet sich in diesem Sample keine Familie, in der beispielsweise die älteste Generation die Erziehung der eigenen Kinder als antiautoritär beschreiben und die entsprechende Kindergeneration die Erziehung als autoritär bezeichnen würde oder umgekehrt. In den Familien dieses Samples gibt es eine gemeinsame Sicht auf das Familienleben inklusive seiner Vergangenheit; die Erinnerungen der einzelnen Familienmitglieder verfügen über eine gemeinsame Basis. ${ }^{124} \mathrm{Je}$ nach

\footnotetext{
${ }^{124}$ Die Erkenntnis der psychologischen Forschung, derzufolge Individuen den allgemeinen Rahmen ihrer Vergangenheit i.d.R. zutreffend erinnerten (Schacter 1999, 157/158), wird damit unterstützt.
} 


\section{Methode und Forschungsdesign}

familialem Setting existieren jedoch gravierende Unterschiede, was die Beurteilung des Familienlebens und insbesondere der Erziehungssettings betrifft. Auch werden teilweise unterschiedliche Aspekte von den einzelnen Familienmitgliedern besonders betont.

Als Schwachstelle narrativ orientierter Interviews wird gelegentlich angeführt, dass Interviewpartner Inhalte verschweigen, auslassen oder umgehen und hierdurch Leerstellen entstehen, die sich der Analyse entziehen. Auch wenn diese Auslassungen bereits im Einzelinterview auffallen, bietet die Mehrfachperspektive, wie sie in dieser Studie angewendet wird, den Vorteil, dass diese Leerstellen durch den innerfamilialen Vergleich teils deutlicher hervortreten und teils durch andere Familienmitglieder - zumindest partiell aus einer anderen Perspektive - aufgefüllt werden können. Hierüber lässt sich für die jeweiligen Teilbereiche analysieren, inwiefern innerfamilial teils unterschiedliche Leitbilder und Relevanzstrukturen vorhanden sind, die diesen unterschiedlichen Thematisierungen bzw. Dethematisierungen zugrunde liegen. Erst durch die dreifache Perspektive auf das Familienleben geraten die Dynamiken familialer Tradierungsprozesse deutlich in den Blick, worin ein relevanter Mehrwert zu sehen ist.

Im Rahmen der dokumentarischen Methode wird eine mehrdimensionale Typenbildung angestrebt. Zunächst erfolgt die sinngenetische Typenbildung, die auf dem herausgearbeiteten generativen (Sinn-)Muster basiert (Bohnsack 2001, 231). Die Annahme der Mehrdimensionalität des Einzelfalles bildet die Voraussetzung für den nächsten Schritt, die soziogenetische Interpretation. Der Umstand, dass der Einzelfall verschiedene Dimensionen oder „Räume“ aufweist, führt zu der Frage, welchen dieser Sphären der im Zuge der Interpretation entwickelte Orientierungsrahmen zugeordnet werden kann (Bohnsack/Nentwig-Gesemann 2006, 164/165). Es gilt, bestimmte Erfahrungsräume zu lokalisieren, in denen die Rahmungen verankert sind, je nachdem ob es sich z.B. um eine „migrations- oder aber auch alters-, generations-, geschlechts- und milieutypischen Orientierung" (Bohnsack/Nentwig-Gesemann 2006, 165) handelt. Zugleich bedeutet dies, die Grenzen der entwickelten Typik aufzuzeigen, indem „fallspezifische Beobachtungen aufgewiesen werden, die anderen Typen zuzuordnen sind“ (Bohnsack/ Nentwig-Gesemann 2006, 164/165).

Ausgehend von einer Basistypik, die durch die Forschungsfrage vorgegeben ist, werden verschiedene konjunktive Erfahrungsräume, die sich auf Alter, Generation, Geschlecht, etc. beziehen können, in den Blick genommen und die erfasste Orientierung einem konjunktivem Erfahrungsraum zugeordnet (Bohnsack 2001, 237). Während im Zuge der sinngenetischen Typenbildung der modus operandi herausgearbeitet wird, ist im Zuge der soziogenetischen Interpretation die Genese des modus operandi zu rekonstruieren (Bohnsack 2001, 250). Das Ziel besteht nicht darin, Aussagen über die numerische Verteilung der identifizierten Typen zu treffen, sondern es geht um Verallgemeinerungen auf theoretischer Ebene (Rosenthal 2005, 13 und 75).

Die in der dokumentarischen Methode dargestellte mehrdimensionale Typenbildung harmoniert in besonderer Weise mit den theoretischen Grundlagen, wie sie sich aus der Gedächtnistheorie von Halbwachs ergeben. Sie bietet ein geeignetes Instrumentarium, um die bei Halbwachs beschriebene Gruppengebundenheit des Einzelnen sowie die $\mathrm{Zu}$ - 
gehörigkeit jedes Einzelnen zu verschiedenen Bezugsgruppen analytisch zu fassen. Den Ausgangspunkt der Typenbildung stellt in dieser Studie der konjunktive Erfahrungsraum der Familie dar. Die entworfene Typologie bezieht sich auf die familialen Tradierungslogiken, deren Genese und jeweilige Gestaltung im konjunktiven Erfahrungsraum der Familie verankert werden kann. Durch die Einbeziehung einer bildungsmilieu-, generations- sowie teilregionalspezifischen Perspektive lassen sich neue Facetten erkennen, die sich auf die Tradierungsinhalte und -modi auswirken, sich aber auf andere konjunktive Erfahrungsräume als die Familie beziehen. Die Berücksichtigung dieser außerfamilialen Aspekte zeigt, dass auch andere konjunktive Erfahrungsräume bzw. Zugehörigkeiten zu anderen Gruppen sich auf den Tradierungsprozess auswirken und bestimmte Phänomene der Tradierungsprozesse erklären können. Die Einbindung eines bildungsmilieu-, generations- und teilregionalspezifischen Blickwinkels ist somit für die Analyse familialer Tradierungsprozesse ertragreich und vermittelt ein detailliertes Bild der Tradierungsvorgänge unter Einbeziehung des gesellschaftlichen Wandels, das über die familiale Sphäre hinaus reicht.

Eine vielversprechende Ergänzung ist die Einbindung von Topoi in die Analyse, insbesondere in Bezug auf die generationellen Erfahrungsräume. Unter Topoi sind „bestimmte wiederkehrende, meist kürzere“ ähnlich lautende Sätze zu verstehen, die auf den ersten Blick unscheinbar sind und erst durch ein wiederholtes Auftreten Aufmerksamkeit erregen, die kontextuell einzubetten sind, und die als Mittel dienen, über die Aussagen transportiert werden (Schröder 2005, 19-20). Sie „stehen im Schnittpunkt sozialer und individueller Wertmaßstäbe. Sie sind subjektive Äußerungen und zugleich Indikatoren für kulturelle Normen“ (Lehmann 1991, 205). Eine Beachtung dieser Sätze erscheint lohnenswert, um sie als Ausgangspunkt für eine tiefergehende Analyse zu nutzen. So können sie z.B. Hinweise für generationelle Gemeinsamkeiten darstellen, indem sie Vertretern einer Generation als Einleitung bzw. Bilanzierung für bestimmte Inhalte dienen. Neben der Schärfung des Blicks für solche als Topoi bezeichneten Sätze ist es sinnvoll, auf Metaphern und kommunizierte Sachverhalte zu achten, die sich generationell rückbinden lassen und über die zumindest latent generationelle Gemeinschaftszugehörigkeiten bzw. umgekehrt Generationendifferenzen markiert werden.

\subsection{Interviewkonzeption}

Es wurden offene Leitfadeninterviews mit narrativen Passagen erhoben. Den Ausgangspunkt für die Gestaltung der Interviews bilden folgende Überlegungen. Im Unterschied zur quantitativ angelegten Werteforschung beispielsweise sollten die möglichen Tradierungsinhalte nicht vorab festgelegt, sondern im Zuge der Auswertung der Interviews rekonstruiert werden. Es ist davon auszugehen, dass sowohl Tradierungsinhalte wie auch die Mechanismen der Tradierung den Befragten nur teilweise als explizites Wissen zur Verfügung stehen. Im Laufe der Tradierung werden darüber hinaus implizit Inhalte weitergegeben, und auch die Gestaltung der Tradierungsprozesse vollzieht sich 


\section{Methode und Forschungsdesign}

teils in habitualisierter Form. Aus diesen Gründen liegt es nahe, eine möglichst offene Herangehensweise zu wählen, die sich zugleich allerdings auf den Themenbereich der Fragestellung konzentriert und die Gestaltung des Familienlebens, der Generationenbeziehungen und der Erziehung ins Zentrum rückt. Um dies zu erreichen, wurde eine Mischform aus narrativen und Leitfadeninterview gewählt. Auf einen erzählgenerierenden Eingangsimpuls, der sich auf die Erziehung und das Familienleben bezog, folgte ein immanenter Nachfrageteil. Auf diese Weise sollte den Interviewpartnern die Möglichkeit gegeben werden, sich ausführlich zum Thema zu äußern, ohne sie zu stark durch Kategorievorgaben zu lenken (Rosenthal 2005, 53 und 2002, 177). Zusätzlich wurde ein Interviewleitfaden entwickelt, dessen Fragen - sofern nicht bereits in den vorherigen Phasen des Interviews angeschnitten - im Anschluss gestellt wurden. Die Fragen bezogen sich auf verschiedene Aspekte des Familienlebens, der intergenerationellen Beziehungen sowie die Erziehung und wurden offen formuliert, um weitere Erzählungen anzuregen. Auch hier bestand die Absicht darin, den Interviewpartnern Raum zur Entfaltung ihres Relevanzsystems zu geben und auf diese Weise die Gefahr zu minimieren, lediglich sozial erwünschte Antworten zu erhalten. Den Abschluss bildete ein Bilanzierungsteil. Diese Vorgehensweise weist eine große Nähe zum episodischen Interview auf (Flick 2002, 160 und Lamnek 2010, 331). Um jedoch zu unterstreichen, dass die Eingangserzählung die Basis für weitere erzählgenerierende Nachfragen darstellte, wird hier der Terminus des offenen Leitfadeninterviews mit narrativen Passagen zur Kennzeichnung des Interviewtyps gewählt.

\subsection{Durchführung und Sample}

Als Untersuchungsregion wurde eine ländliche Region gewählt, die während der Zeit der deutschen Teilung zum innerdeutschen Grenzgebiet zählte. Das westliche Teilgebiet liegt in Niedersachsen und Hessen, das östliche in Thüringen. Hierbei handelt es sich um eine ländliche und kleinstädtisch geprägte Region, die ehemals zum Kurfürstentum Mainz gehörte und eine katholische Enklave in einem protestantisch geprägten Gebiet darstellt (Behrens 2009, 177). In der Geschichte der Region gibt es einige erwähnenswerte Besonderheiten. Im 19. Jahrhundert galt die Region als ökonomisches Krisengebiet, und viele Bewohner waren zu einer saisonalen Wanderarbeit gezwungen (Behrens 2009, 178). Viele der Wanderarbeiter organisierten sich in Vereinen, deren zentrale Bezugspunkte die Aufrechterhaltung eines Heimatbewusstseins sowie des katholischen Glaubens darstellten. Um die Wende zum 20. Jahrhundert hatte sich ein regionales Bewusstsein etabliert, zu dessen Merkmalen u.a. die konfessionelle Zugehörigkeit zählte (Behrens 2009, 179/180). Dieses Bewusstsein wird von denjenigen Vertretern der ältesten Generation, die in der Region geboren wurden, als charakteristische Rahmenbedingung des eigenen Aufwachsens bezeichnet, was sich beispielsweise in einer Äußerung von Herrn Friese zeigt. Er betrachtet Kirche und Familie als feste Bestandteile der regionalen „Denkweise“ (Herr Friese, 1:00:18). Die religiöse Zugehörigkeit bildet insgesamt 


\subsection{Durchführung und Sample}

bis heute ein wesentliches Merkmal für die Selbstbeschreibungen, wie die Interviews dieses Samples zeigen. ${ }^{125}$ Mit der innerdeutschen Teilung wurde die Bevölkerung mit unterschiedlichen politischen und gesellschaftlichen Rahmenbedingungen konfrontiert, die sich auf das Alltagsleben auswirkten. Im Westen vollzog sich eine gesellschaftliche Modernisierung, in deren Zuge eine allgemeine Autoritätskritik in Erscheinung trat, die sich auch auf die Hierarchien der katholischen Kirche bezog (Behrens 2009, 195 und Klenke 2003, 42/43). Hinzu gesellte sich eine veränderte Konsum- und Freizeitkultur, die sich mit einer gewissen zeitlichen Verzögerung auch in dem westlichen Untersuchungsraum dieser Studie bemerkbar machte und zu einem Aufweichen „der traditionellen Milieuzusammenhänge führte" (Behrens 2009, 195). Eine steigende Mobilität tat ein Übriges, um räumliche wie soziale Grenzen aufzuweichen (Klenke 2003, 43).

Anders verlief die Entwicklung im östlichen Teilgebiet. Dort befand sich die Bevölkerung in einer doppelten Diaspora-Situation: einer konfessionellen wie ideologischen (Behrens 2009, 195). Die im Sperrgebiet ${ }^{126}$ vorgenommenen Umsiedlungsaktionen von 1952 und $1961^{127}$ trugen dazu bei, dass das Milieu näher zusammenrückte (Behrens 2009, 187). Im Zuge der staatlichen Umsiedlungen wurden die Bewohner darüber im Unklaren gelassen, welche Kriterien für die Umsiedlung der Betroffenen ausschlaggebend waren. Berdahl sieht in dem sich hier manifestierenden nicht nachvollziehbaren Gebrauch von Macht ein besonders effektives Steuerungsmittel (Berdahl 1999, 65). Es dient dazu, die Bevölkerung in einer Art vorauseilendem Gehorsam zu Anpassungen $\mathrm{zu}$ animieren und auf diese Weise Opposition zu unterdrücken. Wie Palmowskis Ausführungen zeigen, fanden die Steuerungsbemühungen des Staates allerdings durchaus ihre Grenzen; die Bevölkerung verschaffte sich Gestaltungsräume und verteidigte ihren Glauben gegenüber staatlichen Bemühungen, die Bedeutung der Religion zurückzudrängen. Der DDR-Staat war in diesem Zusammenhang zwar darum bemüht, offenen Widerstand seitens der Bevölkerung der Region zu vermeiden, verzichtete aber nicht darauf seine Macht zu demonstrieren (Palmowski 2009, 228-258). Angesichts drohender staatlicher Willkür konnte die Kirche in der Region ,ihre Stellung als inoffizielle Interessenvertretung der Bevölkerung“ ausbauen (Klenke 2003, 43) und auf diese Weise ihren Stellenwert in der Bevölkerung festigen. Als positiv für den Erhalt des katholischen Milieus erwies sich in diesem Zusammenhang auch, dass die Bevölkerung die katholische Kirche mit dem freien Westen assoziierte (Klenke 2003, 45). Die innerhalb der Sperr-

\footnotetext{
${ }^{125}$ Berdahl beschreibt ebenfalls einen starken Zusammenhang zwischen Heimat und Glauben für die Untersuchungsregion (Berdahl 1999, 84).

${ }^{126}$ Hierbei handelt es sich um eine innerhalb der DDR angelegte $5 \mathrm{~km}$ breite Zone entlang der innerdeutschen Grenze, in der besondere Bestimmungen galten; u.a. waren die Einreise von Besuchern sowie der Zuzug von Personen reglementiert (Lebegern 2004, 24).

${ }^{127} 1952$ waren in zwei Landkreisen der untersuchten Region des Sperrgebiets 661 Personen von den Umsiedlungen betroffen, 1961 war die Zahl geringer (Bennewitz/Potratz 1994, 56 und 152). Eine ausführliche Übersicht zu den Planungen und Durchführungen der beiden Umsiedlungsmaßnahmen geben Bennewitz und Potratz (Bennewitz/Potratz 1994).
} 


\section{Methode und Forschungsdesign}

gebiete besonders eingeschränkte Mobilität der Bevölkerung dürfte ebenfalls eine konservierende Wirkung entfaltet und dazu beigetragen haben, das katholische Milieu zu erhalten. Die katholische Kirche war somit in den beiden Teilgebieten mit unterschiedlichen Zuschreibungen konfrontiert. Während sie im westlichen Teilgebiet Gefahr lief, als altmodisch und überholt betrachtet zu werden, galt sie im östlichen Teilgebiet als modern. Vor der Wende wurde der Staat in der östlichen Region als Gegner betrachtet, was dazu beitrug, die Bevölkerung an die Kirche zu binden; nach dem Systemumbruch fiel dieser Aspekt weg (Klenke 2003, 45).

Mit der Auswahl dieser Region verbindet sich nicht der Anspruch, zu repräsentativen Aussagen zu Inhalten und Modi der Tradierung zu gelangen, die für die gesamte BRD, DDR bzw. das wiedervereinigte Deutschland gelten. So ist zweifelsohne die für das Sample beschriebene Bedeutung der religiösen Zugehörigkeit weder für die BRD, die DDR noch für das wiedervereinigte Deutschland repräsentativ. Es lassen sich aber grundlegende Mechanismen der Tradierung aufzeigen sowie die Frage danach stellen, wie Familien die gesellschaftlichen Rahmenbedingungen verarbeiten. Die Auswahl der Region ermöglicht es, die Auswirkungen zweier unterschiedlicher politischer Systeme vergleichend in die Untersuchung einzubeziehen und der Frage nachzugehen, ob sich Unterschiede in den beiden Teilregionen finden, die sich auch heute noch - mehr als zwanzig Jahre nach der Wiedervereinigung - bemerkbar machen.

Das Sample dieser Studie besteht aus 30 Interviews, die in zehn Familien mit je einem Vertreter der Großeltern-, Eltern- und Kindergeneration geführt wurden. ${ }^{128}$ In neun Fällen handelt es sich um direkte genealogische Linien, und in einem Fall wurde die Schwiegermutter als Vertreterin der ältesten Familiengeneration in die Untersuchung einbezogen, da die Eltern der interviewten Angehörigen der mittleren Generation bereits verstorben waren. In dieser Familie ist folglich keine Mehrfachperspektive für die Erziehung der mittleren Generation gegeben. Fünf der Familien stammen aus dem niedersächsischen Teilgebiet der beschriebenen Region und fünf Familien aus der östlichen Region. Vier dieser fünf Familien haben darüber hinaus während der Zeit der deutschen Teilung innerhalb des Sperrgebiets gelebt. Alle Vertreter der mittleren Generation sind in der beschriebenen Region aufgewachsen. Zwei Angehörige der mittleren Generation sind inzwischen mit ihren Familien aus der Region weggezogen. Innerhalb des Samples ist lediglich eine Vertreterin der mittleren Generation geschieden. Die übrigen Vertreter dieser Generation sind verheiratet und leben mit ihren leiblichen Kindern zusammen. Die Namen, Geburtsjahre und angegebenen Berufe der Interviewten wurden verändert, allgemein gehalten oder auf den Ausbildungsgrad reduziert. Sofern es geboten erschien, wurden zudem Freizeitaktivitäten und Hobbys verfremdet.

Die interviewten Familien unterscheiden sich in einigen Fällen in Bezug auf die Altersstruktur. In den meisten Familien sind die Mitglieder der jetzigen Großelterngeneration in den 1920er und 1930er Jahren geboren. Die Elterngeneration ist zwischen Mitte der 1950er bis Anfang der 1970er Jahre geboren und die Geburtsjahrgänge der jüngsten

${ }^{128}$ Eine tabellarische Übersicht der interviewten Familienangehörigen findet sich im Anhang. 


\subsection{Durchführung und Sample}

Generation liegen zwischen Ende der 1980er und Anfang der 1990er Jahre. In zwei thüringischen Familien sind die Angehörigen der Kindergenerationen jedoch um ca. zehn Jahre älter. Da diese unter anderen Rahmenbedingungen als die jüngeren Mitglieder der Kindergeneration aufgewachsen sind, werden sie aus dem generationellen Quervergleich ausgeklammert. Drei Interviewteilnehmerinnen aus Thüringen sind Ende der 1940er und Anfang der 1950er Jahre geboren und zählen in einem Fall zur Großeltern- und in den beiden anderen Fällen zur Elterngeneration. Die Streuung der Geburtsjahrgänge lässt sich für die beiden älteren Generationen produktiv nutzen, indem sie Rückschlüsse erlaubt, ob sich zwischen den Generationen ein eher langsamer Wandel vollzieht oder ob Brüche dominieren. Im vorliegenden Sample sind sowohl Frauen als auch Männer vertreten, wobei der Frauenanteil dominiert. Die Genderperspektive steht nicht im Zentrum der Fragestellung, wird aber an entsprechenden Stellen berücksichtigt. Die Familien sind allesamt eher im mittleren und höheren Bildungsmilieu zu verorten.

Die Kontaktaufnahme zu den einzelnen Familien erfolgte überwiegend im Schneeballsystem. ${ }^{129}$ Einige der interviewten Familienmitglieder sprachen Weiterempfehlungen aus, über die jedoch nicht immer weitere Familien für die Untersuchung gewonnen werden konnten; entweder gab es in den Familien keine drei Generationen, die interviewt werden konnten, oder ein Familienmitglied hatte kein Interesse, an der Studie teilzunehmen. Die meisten Angesprochenen waren aber bereit, weitere Empfehlungen auszusprechen, auch wenn sie selbst nicht in die Untersuchung aufgenommen werden konnten oder wollten. Auf diese Weise entstand ein breiter gefächertes Netzwerk, das über das unmittelbare Nahfeld der beteiligten Familien hinausreicht. Allerdings gibt es in dem Sample keine Familien, in denen niedrige bzw. keine Bildungsabschlüsse existieren, sodass hier von einem Creaming-Off-Effekt gesprochen werden kann. Angesichts des ohnehin sehr hohen Komplexitätsgrades des Forschungsgegenstandes ist diese Einschränkung aber nicht unbedingt von Nachteil. Es erleichtert zunächst einmal das Erkennen von grundsätzlichen Mechanismen der Tradierung, die durch die Einbeziehung einer weiteren Dimension das Erkennen der grundlegenden Muster zusätzlich erschwert hätte. Die Sampleauswahl erlaubt somit Aussagen zu grundlegenden Mustern von Inhalten und Modi der Tradierung, ohne einen Anspruch auf Vollständigkeit zu erheben. Aussagen über die Häufigkeitsverteilung der verschiedenen Muster werden ohnehin nicht angestrebt.

Die Daten wurden in Form von Einzelinterviews erhoben. In einigen Fällen waren auf Wunsch der Interviewten - in den meisten Fällen handelte es sich um Vertreter der ältesten Generation - zeitweise die Ehepartner und in einem Fall die Tochter anwesend. Letztere wurde zu einem späteren Zeitpunkt als Angehörige der mittleren Generation ebenfalls interviewt. Bei der Interpretation ist die Anwesenheit von Familienmitglie-

\footnotetext{
${ }^{129}$ Ein alternativer Zugang wären Zeitungsaufrufe gewesen, worüber sicherlich eine breitere Streuung des Personenkreises hätte erreicht werden können. Jedoch hält auch dieses Vorgehen seine besonderen Probleme bereit, da auf diese Weise in erster Linie Personen angesprochen werden, die sich gerne nach außen präsentieren.
} 


\section{Methode und Forschungsdesign}

dern zu berücksichtigen, da dies zweifelsohne den Gesprächsverlauf beeinflusst. Anstatt dies jedoch als Manko zu betrachten, lässt sich das als eine weitere Information für die Interpretation heranziehen (Rosenthal 2005, 54). So waren beispielsweise bei den Interviews von Frau Dienel und Frau Mahler auf ihren Wunsch hin der Ehemann bzw. die Tochter zumindest teilweise anwesend. In beiden Fällen lässt sich zunächst vermuten, dass die unbekannte Situation des Interviews im Vorfeld als verunsichernd empfunden wurde und deswegen der Beistand von Familienmitgliedern gewünscht wurde. Die Betrachtung des Interviewverlaufs legt im Falle von Frau Dienel jedoch eine weitere Lesart nahe, die Aussagen zu der Außendarstellung der Paarbeziehung zulässt. Herr Dienel wird von seiner Frau gelegentlich in das Interview einbezogen, um von ihr eingebrachte Thematiken zu ergänzen und zu bestätigen. Aspekte, die Herr Dienel anspricht, werden hingegen nicht unbedingt aufgegriffen und von Frau Dienel weiter vertieft. Dies verweist darauf, dass Frau Dienel nicht so unsicher ist, wie eingangs vermutet wurde. Vielmehr scheint es ihr wichtig zu sein, die Einigkeit der Paarbeziehung durch die Einbeziehung ihres Ehemannes in das Interview zu demonstrieren. Für Frau Mahler wiederum scheint die Intention, die Tochter bei dem Interview dabei sein zu lassen, darin zu liegen, eine Rückhalt gebende Unterstützung zu haben. Hierbei versucht Frau Mahler an einigen Stellen des Interviews, die Zustimmung der Tochter zu ihrer Darstellung der Familiengeschichte einzuwerben, die von der Tochter aber umgehend abgelehnt wird. Das verdeutlicht, dass es in dieser Familie unterschiedliche Perspektiven auf das gemeinsame Familienleben gibt, was offensichtlich auch Frau Mahler bewusst ist, was sie aber nicht in der Außendarstellung der Familie gespiegelt sehen möchte. 


\section{Wege und Brücken durch die Zeit: Familiale Tradierungsprozesse in einer Drei-Generationen-Perspektive}

In diesem Kapitel werden die unterschiedlichen Arten familialer Tradierung in einer Drei-Generationen-Perspektive an Fallbeispielen vorgestellt. Hierzu werden vorrangig an den in den jeweiligen Familien als dominant herausgearbeiteten Einstellungen, den familialen Bezugsrahmen, die Wege der Vermittlung und Aneignung nachgezeichnet. Selbstverständlich verfügt jede Person über diverse Bezugsrahmen, allerdings in unterschiedlicher Gewichtung. Eine Konzentration auf die zentralen Bezugsrahmen und deren Entwicklung im Generationenverlauf dient dazu, die grundlegenden Mechanismen der Tradierung pointiert herauszuarbeiten.

Insgesamt lassen sich drei Tradierungslogiken rekonstruieren. Um die Variationsbreite innerhalb der einzelnen Logiken aufzuzeigen, wurde für die erste Logik jeweils eine Familie aus dem östlichen und westlichen Teilgebiet ausgewählt. Innerhalb der zweiten Logik fanden sich nur Familien aus einer Teilregion, sodass sich die vorgestellten Familien lediglich im Bildungsstand unterscheiden. Die der dritten Logik zuzurechnenden Familien sind in Bezug auf die Auswahlkriterien Region und Bildungsstand so ähnlich, dass lediglich eine dargestellt wird.

Für die Analyse halten die Typologien Baumrinds (1991) sowie Bois-Reymonds (1994) wichtige Impulse bereit, die die Relevanz des Ausmaßes an emotionaler Zugewandtheit, dem Umgang mit gesetzten Regeln sowie die Machtbalancen herausarbeiten. Wie aufzuzeigen sein wird, sind diese Elemente zwar wichtige Bestandteile für die Vermittlung und Aneignung, aber nicht ausreichend, um Tradierungsprozesse adäquat zu erfassen. In der folgenden Darstellung werden weitere für Tradierungsprozesse wichtige Aspekte einbezogen. Von besonderem Interesse ist in Anlehnung an Kosellecks Termini von Erfahrungsraum und Erwartungshorizont (Koselleck 1989, 349-359) und Olsons u.a. Ausführungen zum Cicumplex-Modell (1989), wie im Zuge der Tradierung Erwartungen an die nächste Generation herangetragen und von dieser verarbeitet werden. Im Anschluss werden familiale und gesellschaftliche Ebene aufeinander bezogen, und die herausgearbeiteten familialen Entwicklungen mit den gesellschaftlichen Rahmenbedingungen zusammengeführt. 


\section{Familiale Tradierungsprozesse in einer Drei-Generationen-Perspektive}

\subsection{Flexibel erscheinende Tradierungslogiken}

\subsubsection{Familie Dienel/Ebel: Religiöse Alltagsprägung ${ }^{130}$}

Biografische Eckdaten Frau Dienel ist 1939 geboren, hat eine Ausbildung in der Verwaltung absolviert und 1964 geheiratet. Ihr Mann (Jahrgang 1939) ist Akademiker. Das Paar hat zwei Kinder, die 1965 und 1969 geboren sind. Heike Ebel (Jahrgang 1965) hat ebenso wie ihr Mann, der fünf Jahre älter ist, eine akademische Ausbildung. Sie heiratet 1991. Ihre Kinder werden 1991, 1993 und 1999 geboren. Beide Frauen scheiden mit der Geburt ihrer Kinder aus dem Berufsleben aus. Janina Ebel ist die älteste Tochter von Heike Ebel und besucht das Gymnasium. Die Familien sind katholisch und leben in unterschiedlichen Orten in Niedersachsen. Frau Dienel ist in einem katholischen Milieu innerhalb eines evangelischen Umfeldes aufgewachsen. Nach der Heirat zieht sie mit ihrem Mann in eine katholisch geprägte Region, wo ihre Kinder aufwachsen. Heike Ebel kehrt nach ihrem Studium zunächst in ihren Heimatort zurück und gründet eine Familie. Vor einigen Jahren ist sie mit ihrer Familie in eine Region gezogen, in der die evangelische Konfession dominiert.

Aneignungsebene Frau Dienel ${ }^{131}(* 1939)$ : Aufwachsen in behüteten Verhältnissen Die religiöse Alltagsprägung bildet ein zentrales Element in den Kindheitserinnerungen Frau Dienels. Religiöse Alltagspraxen wie der regelmäßige Kirchgang, das Morgen-, Mittags- und Abendgebet sind ein fester Bestandteil des Alltags. Das Abendgebet umfasst zudem eine Tagesbilanz: „Und wenn wir abends in Bett gehen=gingen, wurde ein kurzes Fazit gezogen vor Gottes Angesicht //hmh// in Anführungsstrichen“ (Frau Dienel III 01:56-02:05). ${ }^{132}$

Wie Frau Dienel an mehreren Stellen des Interviews erwähnt, sind alle Familienmitglieder aktiv in das Gemeindeleben eingebunden (III 00:23-01:01, 01:35-01:40, 03:1103:26, 06:48-07:14). ${ }^{133}$ Der Freundeskreis von Eltern und Kindern habe mehrheitlich aus religiös orientierten Menschen bestanden (IV 36:12-36:50), sodass die Familie insgesamt in einem religiös geprägten Umfeld lebt. Vertreter des Klerus seien regelmäßige Gäste des Vaters gewesen, die für die Familie zugleich eine wichtige Informationsquel-

${ }^{130}$ Zum Fallbeispiel Dienel/Ebel siehe auch Kraul/Radicke (2012) und Radicke (2013).

${ }^{131}$ Die Interviews I bis IV wurden mit Frau Dienel am 12.12.2008 und 19.12.2008 geführt; zeitweise war ihr Ehemann anwesend. Um die Zuordnung zur familialen Generationenfolge zu erleichtern, wird die älteste Generation jeweils mit der Anrede Frau bzw. Herr und Nachname genannt, die mittlere Generation wird mit Vor- und Nachnamen aufgeführt und die jüngste Generation nur mit dem Vornamen.

${ }^{132}$ Die Transkripitionsregeln orientieren sich an den Vorschlägen der dokumentarischen Methode (Bohnsack 2007, 235) und sind im Anhang aufgelistet.

${ }^{133}$ Die Zeitangaben, die sich auf die einzelnen Interviews beziehen, dienen dazu, paraphrasierte Interviewpassagen deutlich von Interpretationen abzusetzen. 


\subsection{Flexibel erscheinende Tradierungslogiken}

le zum Weltgeschehen dargestellt hätten (IV 02:36-03:00, 19:34-19:37) und somit die Meinungsbildung und Wertorientierungen der Familie beeinflusst haben dürften.

Die religiösen Praxen sind so präsent, dass z.B. selbst der Gottesdienst in die häuslichen Kinderspiele Eingang gefunden hat (III 03:28-04:04). Mit den offiziellen religiösen Praxen wie der einheitlichen Gottesdienstgestaltung verbindet sie bis heute positive Assoziationen, die ihr an jedem Ort zu jeder Zeit das Gefühl von Vertrautheit und damit Zugehörigkeit, Teilhabe und Gemeinschaft vermittelten (III 04:20-04:46). Diese positiven Konnotationen in Verbindung mit der religiösen Alltagsprägung lassen sich als hilfreiche Katalysatoren für die Aneignung des familialen Bezugsrahmens interpretieren, die als unterschwellig positive Gefühle mit dem Bezugsrahmen assoziiert werden. Zudem sind es Indizien, dass der Glaube ihr Orientierung und Verhaltenssicherheit bietet.

Eng mit der religiösen Alltagsprägung verknüpft findet sich eine Betonung der kulturellen, insbesondere der musischen Bildung. Der Vater erteilt seinen Kindern selbst Musikunterricht, wobei das regelmäßige Üben den Kindern manchmal missfallen habe (IV 06:50:06:54, 09:00-09:20, 30:27-30:54, 1:08:05-1:08:13). Zusätzlich habe er darauf geachtet, die Kinder in den Kanon der klassischen Musik einzuführen. Sobald es die finanziellen Verhältnisse erlaubt hätten, hätten sie als Kinder Opernaufführungen besuchen dürfen, die zuvor am heimischen Klavier durchgearbeitet worden seien (IV 21:12-22:04). Der musische Bereich dient einem doppelten Zweck: Einerseits erfolgt durch die Musik - durch die Beteiligung an kirchlichen Musikgruppen - eine Rückbindung an die Kirchengemeinde. Andererseits werden über die vertraute Rahmung der Musik neue Bereiche erschlossen.

Dem Lesen räumt Frau Dienel einen besonderen Stellenwert ein: „Also Lesen war bei uns ganz groß geschrieben" (IV 10:10:43-11:10). Auch die schulische Ausbildung der Kinder wird aufmerksam begleitet. So hätten sie als Kinder ihre Hausaufgaben zwar relativ eigenständig erledigt, die Ergebnisse seien aber von der Mutter kontrolliert worden. Etwaige Fehler hätten gewissenhaft korrigiert werden müssen (IV 39:24-40:09), sodass ein wesentliches Ziel der Kontrolle in dem Einüben von Sekundärtugenden wie Ordnung, Disziplin und Fleiß besteht. In der Familie wird auf die schulische Bildung Wert gelegt. Die Brüder besuchen das Gymnasium. Bei Frau Dienel selbst hingegen scheint die elterliche Bildungsorientierung durch geschlechtsrollenstereotype Vorstellungen kanalisiert zu werden. Sie besucht die Realschule und erklärt, dies habe ihrem Wunsch entsprochen (I 02:12-02:34). Ihre Schullaufbahn ist für Frau Dienel rechtfertigungsbedürftig und sie bedauert retrospektiv, nicht das Gymnasium besucht zu haben (I 18:03-18:25), was auf den hohen Stellenwert der schulischen Ausbildung für sie hindeutet.

Die Beziehungen zu ihren Eltern beschreibt Frau Dienel als innig und gut. Die Mutter sei sehr fürsorglich gewesen und habe aufgrund ihrer Nichtberufstätigkeit viel Zeit für ihre Kinder gehabt (I 01:41-01:53). Auch der Vater habe sich um die Kinder gekümmert und beide hätten den Kindern jederzeit als Ansprechpartner zur Verfügung gestanden 


\section{Familiale Tradierungsprozesse in einer Drei-Generationen-Perspektive}

(IV 06:07-07:00). Die Eltern hätten z.B. den Kindern Ablauf und Inhalte der Gottesdienste erklärt (III 04:06-04:15). Hier zeigt sich der elterliche Wunsch, ihre Religiosität zu tradieren. Zugleich wird ersichtlich, dass die Eltern nicht von einer selbstverständlichen, unhinterfragten und bedingungslosen Übernahme des Bezugsrahmens durch die Kinder ausgehen, sondern dies der elterlichen Unterstützung bedarf. Anstatt aber dogmatisch den Rahmen festzulegen, wählen die Eltern das Mittel des Erklärens, was es den Kindern erleichtert haben dürfte, eine positive Haltung zum familialen Bezugsrahmen $\mathrm{zu}$ entwickeln. Hierin sind zugleich Anzeichen für ein gewisses Maß an Flexibilität zu sehen. Am Verhalten der Eltern ist abzulesen, dass sie die eigenen Einstellungen nicht als absolute, selbstverständliche Größen annehmen, von denen sie annehmen, dass die nachfolgende Generation sie übernehmen muss, und auf denen sie beharren. Stattdessen versuchen sie, eine Zustimmung durch unterstützendes Handeln zu fördern.

Sie habe eine „behütete“ (I 01:24-01:26) Kindheit gehabt, was für Frau Dienel positiv konnotiert ist und nicht etwa als einengend empfunden wird. Sie hätten viel gemeinsam als Familie unternommen (IV 05:06-05:50). Die Eltern hätten ihr immer als Halt gedient, seien zuverlässig und unterstützend gewesen: „Und die waren eigentlich immer da, wenn wir Probleme hatten: und das hat also so 'ne Sicherheit gegeben, dieses Urvertrauen, $/ / \mathrm{hmh} / /$ was ja bei vielen fehlt, das hatten wir also hundertprozentig" (IV 06:58-07:06). Diese Wahrnehmung der Generationenbeziehungen dürfte die Akzeptanz von Inhalten und Arten der elterlichen Vermittlung positiv verstärkt haben. Die intergenerationelle Gemeinschaft hat jedoch auch ihre Grenzen: Sich den Eltern nackt zu zeigen, sei z.B. ein Tabu gewesen. (IV 1:16:48-1:17:06).

In der Regel seien die Eltern auf die Bedürfnisse der Kinder eingegangen und hätten ihnen in einem gewissen Rahmen Mitspracherechte eingeräumt (IV 44:01-44:10). In manchen Fällen sei der Vater allerdings strikt gewesen:
„Aber mein Vater war dann doch irgendwann so weit, wenn die Diskus- sionen nichts brachten, dann kam ein Machtwort //hmh//: 'Und es nützt jetzt nichts, wenn ihr es nicht einsehen wollt. Vielleicht kapiert ihr es spä- ter mal. Jetzt macht ihr das und das.' //hmh// Und wir wehrten, wehrten uns Natürlich als Kinder gegen Anordnungen [...]. A:ber da wurde dann, wenn wir unsere Einwände [...] vorgebracht hatten und sie nicht als einsich- tig waren bei meinen Eltern, dann mussten wir das machen und machten das auch" (IV 44:11-44:41).

Deutlich wird hier ein Machtgefälle in den Generationenbeziehungen zugunsten der Eltern beschrieben. Ein Austragen von Konflikten mit den Eltern ist nur sehr eingeschränkt möglich. Es gibt aber auch inhaltliche Spielräume, die gemeinsam mit den Kindern bestimmt werden. Es existiert somit eine gewisse Flexibilität, zumindest innerhalb enger Grenzen. Zudem erhalten die Kinder das Gefühl, selbst mitentscheiden zu dürfen. Dies dürfte die Akzeptanz der getroffenen Entscheidungen durch die Kinder erhöht haben. Frau Dienels retrospektives Verständnis sowie die positive Beurteilung dieses Vorgehens untermauern diese Annahme. 


\subsection{Flexibel erscheinende Tradierungslogiken}

Das Gewähren von Gestaltungsspielräumen in engen Begrenzungen durch die Eltern ist jedoch nicht mit Beliebigkeit gleichzusetzen. Fehltritte der Kinder haben Konsequenzen:

„Wir wurden wohl, wenn wir wirklich Mist gebaut hatten, böse ausgeschimpft. //hmh// Das war klar. //hmh// Aber äh sie haben immer durchblicken lassen, dass sie uns geliebt haben dabei, $/ / \mathrm{hmh} / /$ egal was wir machten" (IV 49:53-50:05).

Frau Dienel ist durchaus bewusst, dass bestimmte Regeln einzuhalten sind und Verletzungen dieser Regeln nicht ungeahndet bleiben: „[I]ch bin zwar mit verdammt schlechtem Gewissen nach Hause gegangen, //hmh// aber ich wusste immer, ich komm da lebend wieder raus, //hmh// ohne Schaden //hmh// zu nehmen oder so" (IV 50:3050:36). Auch wenn Regelverstöße mitunter unangenehme Konsequenzen haben können, kann sich Frau Dienel der emotionalen Zugewandtheit der Eltern sicher sein.

Die Eltern sind aber nicht omnipräsent, und Frau Dienel und ihren Geschwistern verbleiben Gestaltungsspielräume in Form der eigenständigen Freizeitgestaltung mit Freunden (IV 25:43-26:28). Die Einbettung des Freundeskreises in ein religiöses Milieu (IV 36:19-36:50) dürfte es sicherlich den Eltern erleichtert haben, ihren Kindern hier Spielräume zu gewähren. Das situative Gewähren von Freiräumen kann auf kindlicher Seite dazu beitragen, die Generationenbeziehungen sowie die religiöse Alltagsprägung als nicht einengend und anschlussfähig zu erleben.

Aus einigen Kindheitserinnerungen Frau Dienels lässt sich ableiten, dass die Eltern bereit gewesen sind, ihre Regeln in Abhängigkeit von den jeweiligen Gegebenheiten anzupassen. Das betrifft z.B. die Frage, ob Namens- oder Geburtstage gefeiert werden. Die Eltern orientieren sich nach ihrem Umzug an den Gepflogenheiten des neuen Wohnortes und feiern Geburts- statt Namenstage. Denn obwohl ein Großteil der freundschaftlichen Kontakte über die Kirchengemeinde zustande gekommen sei, habe es auch evangelische Freunde, etwa durch die Schule, gegeben, deren Gewohnheiten die Familie übernommen habe (III 02:27-03:02). Als die Mutter herausgefunden habe, dass sich Frau Dienel für die Auftritte des Kirchenchors heimlich Perlonstrümpfe von einer Freundin geborgt habe, habe es zunächst eine Auseinandersetzung mit der Mutter gegeben. Sobald sie jedoch erfahren habe, dass alle anderen Mitglieder des Frauenchors solche Strümpfe für die Auftritte gehabt hätten, habe die Mutter ihre Vorwürfe zurückgenommen und der Tochter ebenfalls Perlonstrümpfe für die Chorauftritte gekauft (IV 12:10-13:02). Hier zeigt sich deutlich, wie die Mutter auf die Einflüsse der Nachkriegsgesellschaft reagiert und ihre Grundsätze in Abhängigkeit von diesen zu revidieren in der Lage ist. Eine ähnliche Milieuorientierung findet sich auch in anderen Fällen, und die Eltern sind bereit, Verbote zurückzunehmen, wenn als vertrauensvoll eingestufte Erwachsene hierum bitten (IV 03:42-05:04). Bei einer anderen Gelegenheit gelingt es Frau Dienel, ihren Vater von der Ungerechtigkeit seines Handelns ihr gegenüber zu überzeugen (IV 40:10-41:40). 


\title{
4 Familiale Tradierungsprozesse in einer Drei-Generationen-Perspektive
}

Insgesamt übt Frau Dienel wenig Kritik, weder an der Art der Vermittlung noch an den Inhalten, und stellt ihr Familienleben während ihrer Kindheit als relativ harmonisch dar. Auch wenn es einige Konflikte mit den Eltern gegeben hat, sind sie für Frau Dienel keine dominierenden Kennzeichen des Familienlebens. Zudem werden sie von Frau Dienel tendenziell eher positiv als Diskussionen dargestellt. Zwar betrachtet sie Konflikte als notwendig, allerdings beziehen sie sich auf ihren Bruder und nicht die Eltern:

\begin{abstract}
„Weil, wenn alles so harmonisch ist in der Familie, //hmh// man muss ja irgendwo auch mal Konflikte austragen. //hmh// Und mit meinen Eltern war das nich möglich, außer als wir älter wurden, diese Beschränkungen auf die Uhrzeiten, wann wir zu Hause zu sein hatten. //hmh// Da, da waren also schon heiße Diskussionen. Und ähm diese ganzen anderen (Kon-) äh Konflikte habe ich eigentlich mit meinem jüngeren Bruder $/ / \mathrm{hmh} / /$ ausgetragen“ (IV 33:26-33:53).
\end{abstract}

Hierin manifestiert sich ein weiteres Mal die Asymmetrie der Machtbalancen in der Familie. Die Eltern beanspruchen offenbar die Deutungshoheit darüber, welche Konflikte diskutierbar sind und welche nicht. Das partielle Gewähren von Mitspracherechten verdeckt diese Asymmetrien teilweise und mag dazu beitragen, dass Frau Dienel ihre Beteiligungsmöglichkeiten als ausreichend betrachtet und die elterliche Steuerung anerkennt.

Die Eltern zeigen insgesamt eine gewisse situationsabhängige Flexibilitätsbereitschaft, die es Frau Dienel ermöglicht, die familiale Lebenswelt innerhalb eines bestimmten Rahmens als offen wahrzunehmen und die religiöse Prägung als festen Bestandteil des Alltags zu akzeptieren. Zwar ist der Rahmen und in weiten Teilen auch die Art der Vermittlung festgelegt; wie dieser Rahmen ausgefüllt wird, besitzt jedoch zumindest in Grenzen Gestaltungsräume für die Kinder. Der familiale Bezugsrahmen wird nicht autoritär durchgesetzt, sondern vorzugsweise erklärt und einige Richtlinien werden von den Eltern in Auseinandersetzung mit ihren Kindern verändert.

Vermittlungsebene Frau Dienel $\left({ }^{*} 1939\right)$ : Engmaschige Begleitung und gestaltete (Frei-)räume Frau Dienel orientiert sich an der eigenen Erziehungserfahrung, ohne sie exakt zu wiederholen. Der familiale Bezugsrahmen bleibt trotz eigener Schwerpunktsetzungen erhalten und wird im Zuge der Erziehung als deutlich konturierte Erwartung an die Kinder herangetragen:

„[W]eil auch wir versucht haben, unseren Kindern vorzuleben, dass man die Schöpfung als wertvoll ansehen muss, und dass es irgendwo ein höheres Wesen geben muss, dem man auch verpflichtet ist. //hmh// Nich einfach in der Weltgeschichte äh rumzutoben und zu machen, was man will, ohne Verantwortung für andere zu übernehmen“ (I 16:35-16:54). 


\subsection{Flexibel erscheinende Tradierungslogiken}

Die über die Gebetspraxis der eigenen Kindheit eingeübte Maxime, für das eigene Handeln verantwortlich zu sein, findet sich als Erwartung gegenüber den Kindern in der eigenen Erziehungspraxis wieder. Zugleich ist eine Auseinandersetzung mit der Relation von fremden und eigenen Bedürfnissen oder - in der Terminologie der Wertewandelsforschung - dem Verhältnis von Pflicht- und Akzeptanz- sowie Selbstentfaltungswerten (Klages 2002, 1) erkennbar - ein Thema, das in den Kindheitserinnerungen Frau Dienels keine derart prominente Rolle einnimmt. Sie favorisiert eindeutig die Pflichtund Akzeptanzwerte, die sich in dem Anspruch ausdrücken, für andere verantwortlich zu sein. Mit Verweis auf ihren Glauben möchte sie den Kindern Fürsorglichkeit und Selbstlosigkeit vermitteln: „Aber die Grundtendenz der Lehre Jesu [...] dass die Menschen sich gegenseitig lieben und Gott ein liebender Gott ist, //hmh// das ist unsere Lehre, die wir weitergeben“ (I 21:01-21:15).

Diese Prioritätensetzung schwächt sich im Lebensverlauf Frau Dienels ab. Während die eigenen Kinder im Z weifelsfall die eigenen Bedürfnisse zurückstellen sollten, verschiebt sich der Fokus bei den Enkeln. Ihnen wird mit der Einschränkung, nicht egoistisch zu werden, Selbstbewusstsein zugestanden (IV 1:51:56-1:52:27). Hier lässt sich eine Auseinandersetzung mit der Relation von fremden und eigenen Bedürfnissen erkennen, deren Schwerpunkt sich im Zeitverlauf zugunsten der eigenen Anliegen verschiebt. Auch der eigene Selbstanspruch ist hiervon betroffen. Die Eltern und Schwiegereltern im Alter zu unterstützen, ist für Frau Dienel unter Verweis auf ihren Glauben eine selbstverständliche Pflicht, auch wenn es ihr Leistungsvermögen überschritten habe. Erst die Intervention des Ehemannes sowie die externe Bestätigung durch eine außenstehende Respektsperson, ermöglichen es ihr, die partielle Delegation der Pflege zu akzeptieren (I 33:08-34:26; 39:03-39:10). Das zeigt, wie stark sie die mit der religiösen Alltagsprägung verknüpfte Maxime verinnerlicht hat, die sie nicht ohne Hilfe relativieren kann. Zugleich wird deutlich, dass zumindest in gewissen Grenzen die an den familialen Bezugsrahmen geknüpften Erwartungen auch intraindivduell veränderbar sind.

Ähnlich wie in ihrer eigenen Kindheit versucht Frau Dienel, ihre Kinder in ein Milieu einzubinden, von dem sie annehmen kann, dass dort mit ihren Vorstellungen konforme Einstellungen vor allem in Bezug auf die Religiosität herrschen. Auf diese Weise besteht die Möglichkeit religiöse Einstellungen wie generell Orientierungen des Milieus en passant zu vermitteln, ohne dass sie als explizite Forderungen an die Kinder herangetragen werden müssten. Im Unterschied zu ihrer eigenen Kindheit erfolgt diese Einbindung weniger über die Kirchengemeinde als vielmehr über das Netzwerk der Verwandtschaft (I 28:23-29:29).

Wie bei den eigenen Eltern ist das Vorleben der religiösen Praxen und Einstellungen ein zentrales Element, um die Kinder an den Glauben heranzuführen. Die eigenen religiösen Praxen umfassen Gebete, Gottesdienstbesuche, das Lesen religiöser Texte in der Vorweihnachtszeit sowie Besuche theologischer Expertenrunden (I 05:42-06:23, 07:00-07:49, 09:03-13:47, 15:21-15:26; II 07:57-08:08, IV 05:06-06:12). Ihre selbstlose Einstellung manifestiert sich außerfamilial in ihrem ehrenamtlichen Engagement in 


\section{Familiale Tradierungsprozesse in einer Drei-Generationen-Perspektive}

kirchlichen Organisationen. Zugleich dient es aber auch zweifelsohne einem Eigeninteresse: „Und ich muss sagen, als was mir rel- ziemlich schnell fehlte, war außerhalb der Kinder irgendein Betätigungsfeld“ (I 05:09-05:17). Innerfamilial konzentriert sich die Aufmerksamkeit auf die Kinder: „Die Kinder sind vorrangig und was für die gut ist, mache ich“ (I 24:16-24:20). Die hier bereits anklingende Kindzentrierung Frau Dienels schlägt sich im (gesamten) Erziehungssetting nieder.

Im Unterschied zu den eigenen Eltern, die den familialen Bezugsrahmen hauptsächlich durch ihr praktisches Vorleben vermittelt hätten (I 14:29-14:51), ist es Frau Dienel wichtig, Glaubensinhalte und -praxen kritisch zu reflektieren. Sie möchte wissen, „was im Glauben im Moment überhaupt modern ist“ (I 18:51-18:54), wie die Bibel „nach heutigem Verständnis zu lesen ist“ (I 10:54-10:58) und welche Inhalte und Praxen des Glaubens zeitgemäß sind (I 23:05-23:11). Die Impulse, die vom zweiten Vatikanum ${ }^{134}$ ausgehen, stellen für sie ein Aufbruchsignal dar, das sie begeistert aufnimmt (I 22:4623:10). Inhalte von religiösen Texten betrachtet sie nicht als festgeschriebene Dogmen, die im Generationenverlauf unverändert weiterzugeben sind. Vielmehr sind sie - abhängig von den jeweiligen gesellschaftlichen Kontexten - modifizierbar und damit in gewisser Weise flexibel. Der Glaube werde sich ,immer weiterentwickeln. Was wir jetzt glauben, wird in 50 Jahren schon wieder anders aussehen. $/ / \mathrm{hmh} / /$ Und dadurch bleibt aber auch der Glaube lebendig" (III 06:33-06:40).

„Diskussion über alles“ (I 14:52-15:09) ist folgerichtig für Frau Dienel eine zentrale Maxime, die u.a. für die Erziehung der eigenen Kinder - im Unterschied zur eigenen Kindheit - leitend ist. D.h. zugleich, den Kindern Mitspracherechte zu gewähren, die so den Eindruck gewinnen können, ihre Anliegen und Ansichten werden berücksichtigt. Auf diese Weise können sie das Erziehungsarrangement zumindest in gewissen Umfang als flexibel erleben.

Insbesondere möchte Frau Dienel mit ihren Kindern über Glaubensinhalte diskutieren. Hierin ist gleichzeitig ein Beitrag zur kulturellen Bildung zu sehen. Abgesehen davon ist das Angebot, den Glauben gemeinsam (kritisch) zu reflektieren, jedoch nicht mit einer Freiheit der Kinder zu verwechseln, sich nach Belieben zum familialen Bezugsrahmen zu positionieren. Es gibt Forderungen, wie z.B. sich selbstlos zu verhalten, die nicht zur Disposition stehen. Bei Bedarf interveniert Frau Dienel, ohne jedoch auf autoritäre Praktiken zurückzugreifen. Stattdessen bedient sie sich einer vordergründigen Liberalität und versteht es, ihre Vorstellungen über Argumente bzw. den sichtbaren Ausdruck ihrer Enttäuschung durchzusetzen, wie folgende Episode illustriert: Als die Tochter nicht am sonntäglichen Gottesdienst habe teilnehmen wollen, akzeptiert Frau Dienel das scheinbar. Allerdings lässt Frau Dienel durchblicken, dass sie traurig darüber sei. Das wiederum veranlasst die Tochter dazu, aus Rücksicht auf die Gefühle der

\footnotetext{
${ }^{134}$ Das zweite vatikanische Konzil begann im Oktober 1962. Es wurden verschiedene Standpunkte der katholischen Kirche thematisiert und es erfolgten Veränderungen; z.B. wurden Laien Beteiligungsmöglichkeiten im kirchlichen Rahmen eingeräumt und die Idee des Dialogs zwischen den christlichen Gemeinschaften gestärkt (Schäfer 2013 und Pottmeyer 1996, 144/145).
} 


\subsection{Flexibel erscheinende Tradierungslogiken}

Mutter am Gottesdienst teilzunehmen (I 15:12-16:05). ${ }^{135}$ Auch in Hinblick auf Moralvorstellungen zur Sexualität bedient sich Frau Dienel argumentiver Strategien, um ihre Tochter von ihrem Standpunkt zu überzeugen:

„Ich hab versucht, ihr klarzumachen: ,Du bist ganz kostbar. //hmh// Und das müssen die Jungen erkennen. //hmh// Du bist nicht einfach so jemand, der das ausprobiert am laufenden Band [...]. Sondern, wenn mal wirklich einer kommt, der es ernst meint, $/ / \mathrm{hmh} / /$ der mag das auch nicht““ (IV 1:27:51-1:28:09).

Unverkennbar ist an diesem Beispiel eine Orientierung an einer konservativen Sexualmoral, die Frau Dienel durch ihr argumentatives Vorgehen und eine vordergründig unvoreingenommene und offene Thematisierung kaschiert. Auch bei der Vermittlung von Einstellungen wie Dankbarkeit und Bescheidenheit, auf die Frau Dienel großen Wert legt, kommen argumentative Strategien zum Einsatz (IV 29:32-29:53, 1:48:10-1:48:19).

Indem die Kinder an die Interessensgebiete der Eltern herangeführt werden, werden Gemeinsamkeiten zwischen den Generationen gefördert. Das betrifft z.B. die Musik. Alle Kinder werden an einem Musikinstrument ausgebildet (I 30:09-30:22). Dienten die musischen Praxen in der Herkunftsfamilie Frau Dienels u.a. der Verankerung der Familienmitglieder in der Kirchengemeinde, leistet das gemeinsame Musizieren während der eigenen Familienphase vor allem einen Beitrag zur Ausgestaltung der privaten Praxen, wie z.B. dem Weihnachtsfest: „Und ähm das Weihnachtsfest [...] wurde dann mit Geschwistern, Eltern und Kindern $/ / \mathrm{hm} / /$ begangen. Und äh da wurde musiziert also bald stundenlang [...]. Das hat allen total Freude gemacht" (I 29:49-30:09). Über das gemeinsame Musizieren wird nicht nur der Aspekt der kulturellen Bildung performativ umgesetzt, sondern auch zugleich die Familiengemeinschaft gestärkt.

Zugleich werden die Kinder in den Kanon der klassischen Musik eingeführt. Dieses Projekt wird genauso ernsthaft verfolgt wie im Elternhaus Frau Dienels (IV 22:0622:27). Hierdurch werden offenbar erfolgreich weitere Gemeinsamkeiten geschaffen, da die Tochter sich kaum für jugendkulturelle Musikstile interessiert habe: „Also sie hat sich auch nicht so sehr für die supermoderne Musik interessiert" (IV 1:36:46-1:36:49).

Weitere Schnittpunkte werden über gemeinsame Sportaktivitäten hergestellt. Hier engagiert sich vor allem Herr Dienel. Er habe die Kinder dazu ermuntert, ihre Leistungsgrenzen auszutesten und ggf. zu überwinden (IV Herr und Frau Dienel, 56:2757:40, IV 1:12:41-1:13:32). Alle Familienmitglieder betreiben darüber hinaus gemeinsam eine Individualsportart (IV 57:46-58:12). Für Herrn Dienel dient der Sport nicht nur gesundheitlichen Zwecken, sondern auch als Ausgleich „gegen Fernsehen und andere Dinge“ (IV Herr Dienel, 58:39-58:47). Die pädagogischen Intentionen sind unverkennbar. Über sportliche Aktivitäten sollen Ausdauer, Fleiß und ein als sinnvoll

\footnotetext{
${ }^{135}$ Zugleich signalisiert Frau Dienel hiermit, dass die Tochter eine selbstlose Orientierung verinnerlicht hat.
} 


\section{Familiale Tradierungsprozesse in einer Drei-Generationen-Perspektive}

betrachteter Umgang mit der Freizeit eingeübt sowie die intergenerationelle Gemeinschaft gestärkt werden. Allerdings insistieren die Eltern nicht darauf, gemeinsam Sport zu betreiben. Es ist eher als Angebot konzipiert, das die Kinder annehmen oder ablehnen können. So hätten sie es toleriert, als die Tochter nicht mehr an den gemeinsamen sportlichen Aktivitäten habe teilnehmen wollen (58:15-58:39).

Wie aus der Maxime Herrn Dienels ersichtlich, entspricht Fernsehen nicht den familialen Vorstellungen von einer angemessenen Freizeitbeschäftigung. Stattdessen folgen sie eher einem hochkulturellen Schema. Entsprechend seien Kindersendungen während der Kindheit stark reglementiert gewesen: „Kriegten sie zu wenig zu sehen“ (IV 1:37:07-1:37:10). Später habe die Familie gemeinsam Familiensendungen gesehen (IV 1:37:12-1:37:23). Damit wird auch das Fernsehen zu einer Familienangelegenheit, die die intergenerationelle Gemeinschaft unterstützen soll. Das Herstellen intergenerationeller Nähe durch eine Vielzahl gemeinsamer Aktivitäten und Interessen ist eine wesentliche Komponente des Erziehungsarrangements. Frau Dienel sieht sich hier in der Fortführung des elterlichen Erziehungssettings: „[W]ir haben so viel zusammen gemacht. //hmh// Im Grunde wiederholt sich das wieder" (IV 1:06:22-01:06:25). Die vielen gemeinsamen Aktivitäten unterstützen zum einen die optimale Förderung der Kinder, erlauben jedoch zugleich eine engmaschige Kontrolle.

Die Gymnasiallaufbahn der eigenen Kinder scheint eine Selbstverständlichkeit zu sein; die Frage nach der zu besuchenden Schulform wird im Interview gar nicht thematisiert. Es entsteht der Eindruck, als sei ein Studium der Kinder für Frau Dienel selbstverständlich. Das zeigt sich u.a. daran, dass die Berufsausbildung des Sohnes ausführlicher erläutert wird (II 19:38-20:30) und sie am Studium der Tochter mit großem Interesse partizipiert (I 18:26-18:57). Letzteres lässt sich u.a. als Weg betrachten, die fehlenden eigenen Bildungstitel zumindest symbolisch zu kompensieren.

Weitere grundlegende Komponenten des Erziehungsarrangements sind: Zeit für die Kinder sowie kommunikativer Austausch mit den Kindern. Während der gemeinsamen Mahlzeiten, denen ein zentraler Stellenwert zugeschrieben wird, hätten die Kinder Gelegenheit gehabt, „alles, was in der Schule war, hier an Dampf“ abzulassen „oder an Freudigem“ (IV 1:26:18-1:26:50) zu erzählen. Frau Dienel versucht eine Atmosphäre der Nähe zu schaffen, die das emotionale Wohlbefinden der Kinder fördert. Sie nimmt sich ausgiebig Zeit, um sich die Erlebnisse und Sorgen anzuhören und interessiert sich für den gesamten Alltag der Kinder. Die gemeinsamen Essenszeiten dienen somit einem doppelten Zweck: Die Kinder haben eine verlässliche Ansprechpartnerin und Frau Dienel wird im Gegenzug nebenbei über den Alltag ihrer Kinder informiert. Damit erhält sie zugleich die Möglichkeit, ungeplante Entwicklungen ihrer Kinder frühzeitig bemerken und ggf. intervenieren zu können. Es ergeben sich quasi beiläufig Möglichkeiten der Kontrolle und Steuerung, die in das Gewand der Aufmerksamkeit und Kind- 


\subsection{Flexibel erscheinende Tradierungslogiken}

orientierung gekleidet sind und den Kindern nicht als Zwang, sondern als Zuwendung erscheinen können. ${ }^{136}$

Über die starke Einbindung der Kinder in die Familiengemeinschaft versucht Frau Dienel, ein engmaschiges Netz der Sicherung um ihre Kinder auszubreiten. Sie merkt selbstkritisch an, dass sie manchmal zu ängstlich und überfürsorglich im Umgang mit ihren Kindern gewesen sei. Manchmal habe sie sich z.B. zu stark in die Angelegenheiten der Tochter eingemischt (IV 1:26:59-1:27:50). Insgesamt habe die eigene ängstliche Haltung dazu geführt, diese teilweise auf ihre Kinder zu übertragen (1:13:40-1:14:22). ${ }^{137}$ Die Ausführungen verweisen auf einige überbehütende Tendenzen, die eine Erziehung zur Selbstständigkeit ${ }^{138}$ erschweren.

Auch wenn im Erziehungsarrangement eine deutliche Kindorientierung zu erkennen ist, sind die Kinder nicht pflichtenlos. Frau Dienel habe beiden Kindern Aufgaben im Haushalt zugeteilt, die sie in eigener Regie untereinander hätten aufteilen können (1:09:17-1:09:49). Sie folgt dabei keinen geschlechtsrollenstereotypen Vorstellungen, da sowohl Tochter als auch Sohn zu den Arbeiten im Haushalt herangezogen werden.

Ein nahezu klassischer Konfliktfall betrifft das regelmäßige Üben des Musikinstruments. In diesem Punkt verhält sich Frau Dienel sehr strikt (I 30:38-31:17) und versucht ihre diesbezügliche Rigidität durch kommunikative Strategien abzumildern. Sie hätten ihrer Tochter gut zugeredet: „Heike, irgendwann siehst du es ein. Tu uns den Gefallen und mach es" (IV 1:08:22-1:08:27). Auch auf die töchterliche Begabung wird verwiesen:
„Aber ich habe ihr auch gesagt: Man muss die Begabung haben. //hmh// Also wenn wir gewusst hätten, es ist nichts zu machen, [...] dann wären wir die letzten gewesen, die gesagt hätten: 'Du übst aber weiter'. [...] Aber sie war begabt. //ja, hmh// Und dann war es für uns einfach schade, ich meine, dass man sich drückt" (IV 1:08:56-1:09:16).

Frau Dienel argumentiert mit einer für die Tochter nicht einsehbaren Zukunft, appelliert an ihr Mitgefühl, den Eltern eine Freude zu bereiten, und bedient sich einer scheinbaren Objektivierung, dem Talent der Tochter, um ihre Position durchzusetzen. Neben dem Erlernen eines Musikinstruments werden en passant Leistungserbringung sowie das Zurückstellen aktueller Bedürfnisse eingeübt. Allerdings lässt Frau Dienel auch eine situationsabhängige Flexibilität erkennen, indem sie z.B. nicht auf das Einhalten der Übungsstunden besteht, wenn ihre Tochter andere Termine hat. Auch in anderen Bereichen ist Frau Dienel bereit, ihre Vorstellungen flexibel anzupassen und auf die Wünsche der Kinder einzugehen. Obwohl sie eigentlich gegen Haustiere gewesen sei, habe ihre

\footnotetext{
${ }^{136}$ Zur Funktion und Relevanz familialer Tischgespräche und Tischrituale siehe Keppler (1994) und Audehm (2007).

${ }^{137}$ Ihre Kinder würden retrospektiv diese Facette kritisieren und Frau Dienel würde einsehen, diesbezüglich einen „bösen Fehler“ (IV 1:49:19) begangen zu haben.

${ }^{138}$ Ecarius definiert Erziehung als „Anleitung und Heranführung einer jüngeren Generation zur Selbsttätigkeit in Auseinandersetzung mit einer älteren Generation“ (Ecarius 2008, 9).
} 


\section{Familiale Tradierungsprozesse in einer Drei-Generationen-Perspektive}

Tochter welche bekommen, als sie gemerkt habe, dass es ihr „Herzenswunsch“ gewesen sei (IV 1:41:36-1:44:12).

Generell ist Frau Dienel bemüht, Konflikte nicht zu stark eskalieren zu lassen. Wenn sie sich z.B. im Streit von ihrer Tochter getrennt habe, hätten sie Zettel als Kommunikationsmittel benutzt, um wieder ins Gespräch zu kommen und den Streit beizulegen:

„Oder ähm ich hab mal 'nen Zettel hingelegt, wenn sie dann aus dem Haus ging im Krach, [...] Und diese Zettel waren auch immer die Brücke wieder. [...] Und dann [...] waren wir beide also unheimlich selig, wenn wir wieder miteinander reden konnten, weil wir es nicht aushalten konnten, //hmh// Krach zu haben. //hmh// Also, das: ging einfach nicht" (IV 1:30:03-1:30:43).

Deutlich erkennbar ist ein ausgeprägtes Harmoniebestreben Frau Dienels, von dem sie annimmt, dass es auch für die Tochter gelte, und das für ihr Familienleitbild zentral ist. Sie ist bestrebt, potenzielle Bedrohungen des als äußerst eng beschriebenen intergenerationellen Verhältnisses durch Konflikte sofort abzuwehren und durch Versöhnungen wieder Einigkeit herzustellen. Konflikte werden eher als Ausnahmen thematisiert und dienen letztlich der Bestärkung des harmonischen Zusammenlebens.

Die religiöse Alltagsprägung ist als Bezugsrahmen im Interview präsent, ohne jederzeit in reflektierter Form explizit das Handeln zu leiten. Analog zu den eigenen Kindheitserfahrungen ist er für die Erziehung der Kinder leitend, ohne eine exakte Wiederholung darzustellen. Frau Dienel setzt jeweils neue Akzente, was den Bezugsrahmen und die Erziehung betrifft. Sie möchte die religiöse Alltagsprägung an die Kinder vermitteln, wobei die konkrete Umsetzung durch das Erziehungsarrangement unterstützt wird, indem den Kindern innerhalb gewisser Grenzen Spielräume zugestanden werden. Hierin manifestiert sich ebenso wie in den von ihr vorgenommenen Schwerpunktverlagerungen ihre Flexibilität, die sie mit dem Hinweis begründet, dass „jede Generation ihre Kinder“ zumindest teilweise „anders“ erziehe (IV 47:01-47:10). Hiermit entbindet sie sich zugleich auf reflexiver Ebene von dem Anspruch, tradierte Positionen unverändert zu übernehmen, und eröffnet Veränderungsoptionen.

Aneignungsebene Heike Ebel ${ }^{139}\left({ }^{*} 1965\right)$ : Eine geborgene Kindheit Heike Ebel beschreibt, ebenso wie ihre Mutter, eine Kindheit, in der religiös konnotierte Orientierungen und Praxen äußerst präsent sind. Ein wesentliches Charakteristikum des elterlichen Verhaltens sind für sie die als ehrlich empfundenen Haltungen (III 33:46-34:05; IV 21:22-22:15). Angewendet auf den familialen Bezugsrahmen bedeutet das, dass Gebete und Kirchgang zum Alltag gehören (III 34:42-35:21, III 36:51-37:02) und die Eltern religiöse Orientierungen und Einstellungen im Alltag umsetzen. Das Vorleben des familialen Bezugsrahmens ist somit für Heike Ebel unmittelbar erfahrbar, was die Aneignung des familialen Bezugsrahmens unterstützt.

${ }^{139}$ Das Interview (I-IV) mit Heike Ebel wurde am 02.02.2009 geführt. 


\subsection{Flexibel erscheinende Tradierungslogiken}

Auch wenn Heike Ebel den Glauben „nich so am Kirchgang festmachen“ (III 34:4234:45) würde, sei der Gottesdienstbesuch ein fester Bestandteil des Familienlebens gewesen. Die Teilnahme erlebt sie jedoch nicht als verpflichtend, sondern als freiwillig:

„Ja genau, und Glauben war natürlich irgendwas, was dazugehörte. [...] Wir sind jede Woche zur Kirche gegangen. [...] [M]eine Eltern haben mich nie gezwungen, zur Kirche zu gehen. //hmh// Also es gab auch Zeiten, wo ich gesagt habe: ,Ich will das heute nich. Ich find das doof, ich seh es nich ein.' Und dann bin ich auch nich gegangen. Und das war auch okay $/ / \mathrm{hmh} / /$ beziehungsweise ich wusste, dass sie es nich gut fanden, aber sie haben mich dann gelassen. //hmh// Also ich bin da nie erpresst worden oder so. Und ich glaube, das haben sie genau richtig gemacht." (III $34: 34-35: 21)$.

Interessanterweise erinnert Heike Ebel die Frage der Gottesdienstteilnahme etwas anders als ihre Mutter, laut der die Tochter zur Kirche gegangen sei und dem Wunsch der Mutter entsprochen habe. Sie schreibt sich stattdessen selbst eine aktive und eigeninitiative Rolle zu und sieht sich keiner elterlichen Willkür ausgeliefert. Folglich kann das elterliche Verhalten von ihr als sehr tolerant und flexibel betrachtet werden.

Heike Ebel nimmt die Eltern als unterstützend wahr. Während der Pubertät seien ihr Glaubenszweifel gekommen und sie habe sich mit philosophischen Glaubensfragen beschäftigt. Hier hätten die Eltern ihr zwar nicht selbst helfen können, sie vermitteln ihr aber einen Kontakt zu einem Priester, mit dem sie sich hierüber habe austauschen können (IV 11:55-12:31). Die Eltern orientieren sich in dieser Darstellung an den Bedürfnissen der Tochter und bemühen sich um ihre Unterstützung. Die Auseinandersetzung mit Glaubensfragen in ihrer Jugend betrachtet Heike Ebel als interessant und eigenmotiviert (III 37:42-38:27). Entsprechend erlebt sie die Beschäftigung mit diesem Thema als bereichernd, ohne dass dies für ihr weiteres Leben einen so hohen Stellenwert einnimmt wie bei ihrer Mutter.

Auch wenn Heike Ebel die elterliche Unterstützung und Hilfe in Glaubensfragen schätzt, hätten sich die Eltern aus ihrer Perspektive nicht so viele Gedanken um die Vermittlung des Glaubens machen müssen (IV 20:49-20:59). Die Aneignung des familialen Bezugsrahmens empfindet Heike Ebel vielmehr als eine selbstverständliche Entwicklung. Die pubertären Glaubenszweifel und das kritische Hinterfragen betrachtet sie als Bestandteil des Glaubens, der nichts an der grundlegenden Bedeutung der Religion für ihren Alltag ändert (III 37:42-38:50, IV 11:55-12:31). Zugleich erfüllt sie die mütterliche Forderung, den Glauben kritisch zu diskutieren. Die anklingende, wenn auch moderate Kritik ist ein Indiz, dass Heike Ebel die steuernden Komponenten der Eltern zur Vermittlung des familialen Bezugsrahmens zumindest unterschwellig registriert. Gleichzeitig möchte sie aber die Eltern eher als tolerant und flexibel wahrnehmen bzw. sind das die Elemente der Erziehungshaltung, die für sie überwiegen. Das drückt sich z.B. in ihrer Feststellung aus: „Ja, meine Eltern haben uns viel machen lassen“ (I 03:14-03:18)). Diese Aussage konkretisiert sie im Nachfrageteil: 


\title{
4 Familiale Tradierungsprozesse in einer Drei-Generationen-Perspektive
}

\begin{abstract}
„Also wir durften uns im Viertel komplett frei bewegen, $/ / \mathrm{hmh} / /$ wir durften einfach irgendwo zu irgendwelchen Freunden gehen, ohne zu sagen, wo wir sind, weil das so ein Pool von Kindern war, den sie kannten $/ / \mathrm{hmh} / /$ und die alle als vertrauenswürdig galten. $/ / \mathrm{hmh} / / \mathrm{Hm}$, ich denke, gewisse Entfernungen hätten wir wahrscheinlich nich überschreiten dürfen. Das stand aber auch nicht an. Das Viertel war klar begrenzt. Das waren zwei Straßen, die das begrenzten und innerhalb dieses Radius konnten wir uns frei bewegen. //hmh// Und ich glaube, wenn wir darüber hinaus uns hätten bewegen dürfen, hätten wir sowieso gefragt“ (IV 00:49-01:16).
\end{abstract}

Heike Ebel beschreibt hier praktisch ein nicht explizit formuliertes, wechselseitiges Abkommen, das den Kindern innerhalb bestimmter Grenzen Freiräume zugesteht. Über die Kinder des Viertels erfolgt quasi beiläufig eine Milieueinbindung, da davon auszugehen ist, dass innerhalb des Viertels überwiegend Familien mit ähnlichen Wertpräferenzen wohnen. Eine offene Steuerung der Freundeswahl ist deswegen nicht notwendig. Hier zeigt sich eine deutliche Parallele zum Erziehungsarrangement, das die Mutter Heike Ebels während ihrer eigenen Kindheit erfahren hat. Weitere wichtige Kontaktpersonen sind die Verwandten (I 06:50-06:59). Auch hier erfolgt en passant die Einbindung in ein Milieu, in dem vermutlich ähnliche Orientierungen wie in der eigenen Familie existieren, sodass Heike Ebel die verdeckte Lenkung übersehen und sich als eigenaktiv beschreiben kann.

Über die Milieueinbindung die Entwicklung der Kinder beiläufig zu beeinflussen, scheint als Strategie aufzugehen; zumindest berichtet Heike Ebel von keinen grundsätzlichen Konflikten mit den Eltern, die sich auf gegensätzliche Orientierungen beziehen würden. Vielmehr verweisen diverse Beispiele auf ein konsensuales Miteiander. Regelungen in Bezug auf die Schule seien nicht notwendig gewesen, weil sie „fleißig und ehrgeizig" (IV 18:28-18:44) gewesen sei. Auch die Kleidung habe keinen Anlass zu Auseinandersetzungen gegeben. Zwar habe Heike Ebel sich während der „Ökophase mit diesen Schlabberkleidern“ (IV 19:47-1949) an dieser Mode orientiert, die Mutter habe sie das aber „auch machen lassen“ (IV 20:04-20:05). Der Tochter werden offensichtlich Freiheiten gelassen, solange sie gewisse Grenzen einhält. An Verbote kann Heike Ebel sich nicht erinnern:

„Nee, Verbote. Ich hätte auch abends weggehen dürfen, //hmh// aber ich w:ar sehr häuslich [...] Ich bin in meinem Leben vielleicht zweimal in der Disco gewesen. $/ / \mathrm{hmh} / /$ Hat mich nich gereizt. [...] Und mein Freundeskreis war auch nicht entsprechend. Wir haben uns dann lieber $/ / \mathrm{hmh} / /$ nachmittags irgendwo getroffen" (IV 19:17-19:35).

Offenbar schätzt Heike Ebel die familiale Gemeinschaft und zeigt wenig Neigung, den familialen Kreis zu verlassen. Sie präsentiert sich nicht als Jugendliche, die Grenzen austestet und gegen elterliche Vorgaben rebelliert. Ähnliches scheint für die Peergroup zu 


\subsection{Flexibel erscheinende Tradierungslogiken}

gelten. Heike Ebel beschreibt sich als sehr angepasst, die elterlichen Regeln akzeptierend und realisiert selbst, dass ihr das in bestimmten Bereichen Freiräume verschafft. Die Eltern stehen gar nicht erst vor der Aufgabe, Verbote aussprechen zu müssen. Entsprechend großzügig können die Eltern in der Erziehung erscheinen, indem sie z.B. der Tochter freigiebig Mitspracherechte zugestehen oder die töchterlichen Verweigerungen akzeptieren, sofern das nicht grundlegend dem familialen Bezugsrahmen widerspricht:

„Klar, meine Kleidung, das war ja nun (so) ganz selbst gemacht. //hmh// Was und wie ich mich anziehe. (.) Wann ich mich mit wem treffe, das konnte ich auch selbst entscheiden. $/ / \mathrm{hmh} / /$ Dadurch, dass es in der Schule auch so unkompliziert lief, hatte $/ / \mathrm{hmh} / /$ ich da auch viele Freiheiten. Ähm (.) meine Freizeitaktivitäten, klar mit dem Jazzdance das wollte ich gerne. $/ / \mathrm{hmh} / /$ Dann haben sie noch versucht, mich in Richtung Squash zu drängen, weil der Rest der Familie Squash spielte, //hmh// das haben sie aber schnell gemerkt, dass das nicht funktioniert. Dann haben sie mich mein Jazzdance machen lassen. $/ / \mathrm{hmh} / / \mathrm{Hm}{ }^{\circ}$ es gab wenig Einschränkungen, eigentlich. ${ }^{\circ}$ Waren auch nich nötig. Also (.) ich war ja jetzt nicht irgendwie so 'n Straßenfeger, der so viel Unsinn oder so gemacht hätte. //hmh// Was hätten sie da viel einschränken sollen. $/ / \mathrm{hmh} / /$ Es lief ja alles so ganz rund eigentlich" (IV 23:14-23:54).

Die situationsspezifische Flexibilitätsbereitschaft der Eltern zeigt sich auch an weiteren Beispielen. Während ihrer Kindheit sei zunächst Fernsehen generell verboten gewesen, Heike Ebel und ihr Bruder hätten das aber missachtet und regelmäßig heimlich bei den Nachbarn die im Elternhaus verpönte Sesamstraße angesehen. Sie sei bereits als Kind überzeugt gewesen, dass die Eltern das geduldet hätten (I 03:14-03:36): „Und sie haben es auch gewusst, aber sie haben uns machen lassen. Das war irgendwie okay" (I 03:32-03:36). Für Heike Ebel stellt das Umgehen des Fernsehverbots keine Regelverletzung dar, die grundlegende Maßstäbe der Eltern erschüttern würde. Das ermöglicht es ihr, sich über diese elterlichen Vorgaben hinwegzusetzen. Die Eltern wiederum beharren nicht kategorisch auf ihren Regeln, zumal es weder den familialen Bezugsrahmen noch ihre grundlegenden Einstellungen betrifft.

Die bisher geschilderten Facetten des Erziehungssettings tragen zweifelsohne dazu bei, dass Heike Ebel die Generationenbeziehungen, als Bestandteil des Erziehungsarrangements, zwar differenziert, aber in der Gesamtschau dennoch gut einschätzt und diese Botschaft deutlich nach außen kommunizieren möchte:

„Nee, jetzt muss ich nochmal was Grundsätzliches sagen. //hmh// Also wenn ICH an meine Kindheit denke, dann denke ich an Geborgenheit, an Wärme [...]. Wenn wir gefragt haben, haben wir immer 'ne Antwort gekriegt zu allen Themen. [...] Es war 'ne sehr ehrliche Atmosphäre. Wir haben viel geredet (.) über alles Mögliche. [...] Also [...] es war wirklich 'ne gute Kindheit, doch, sie haben es gut gemacht. Bis auf dieses zu ängstliche $[. .$.$] erziehen, haben sie es gut gemacht“" (III 33:37-34:30).$ 


\section{Familiale Tradierungsprozesse in einer Drei-Generationen-Perspektive}

Heike Ebel charakterisiert ihre Eltern als verlässlich (IV 04:23-05:27, 21:02-21:14). Insbesondere die emotionale Komponente des Erziehungssettings wird von ihr mit dem positiv konnotierten Attribut der Wärme lobend hervorgehoben und findet sich wiederholt im Interview (IV 25:15-25:23, 26:01-26:14). Diese Sichtweise auf die Eltern-KindBeziehungen, die Geborgenheit, emotionale Zugewandtheit sowie insbesondere auch den Eindruck der elterlichen Zuverlässigkeit beinhaltet, sind wichtige Elemente, die die Annahme von Vermittlungsinhalten unterstützen. Die als offen empfundene Kommunikation über sämtliche Aspekte des Alltags trägt dazu bei, dass Heike Ebel sich als gleichberechtigte Kommunikationspartnerin der Eltern sehen kann. Sie fühlt sich weder unter Druck gesetzt noch von den Eltern gegen ihren Willen in eine bestimmte Richtung gedrängt. Das sind weitere Voraussetzungen, die die Aneignung des familialen Bezugsrahmens unterstützen. Der kommunikative Austausch über sämtliche Themen verweist auf eine detaillierte, wechselseitige Informiertheit über das Leben der einzelnen Familienmitglieder und somit auf eine ausgesprochene Nähe zwischen den Generationen. Auch wenn Heike Ebel dies sehr schätzt, hat es für sie auch eine Kehrseite, sodass sie das Beziehungsarrangement der Nähe sowie die ausgesprochene mütterliche Fürsorglichkeit durchaus ambivalent beurteilt. ${ }^{140}$ Die Beziehung zur Mutter sei in der Retrospektive teilweise zu eng gewesen und das Verhalten der Mutter sei der Ausbildung der Eigenständigkeit abträglich gewesen:

„Hmm, das einzige was nich gut, das wa:r, da:ss äh:m meine Mutter uns so viel abgenommen hat $/ / \mathrm{hmh} / /$ (.) an Auseinandersetzungen. Also wir mussten viele Auseinandersetzungen nicht führen oder nicht ausfechten, weil die das dann für uns geregelt hat. [...] Also, ich bin dadurch lange Zeit sehr unselbstständig gewesen und auch, ja, sehr ängstlich gewesen. $/ / \mathrm{hmh} / /$ Also wenn ich meinen Eltern was vorzuwerfen hätte, dann wär es das. //hmh// Dass sie uns zu viel abgenommen haben in Konfliktsituationen" (I 05:38-06:38).

Als Heike Ebel z.B. Probleme mit ihrer Jazzdancelehrerin gehabt habe, habe die Mutter interveniert und an Stelle ihrer Tochter diesen Konflikt mit der Trainerin ausgetragen (I 06:07-06:20). Während der Pubertät habe sich die Mutter in die Freundeswahl der Tochter eingemischt. Damals sei ihr das nicht negativ aufgefallen, im Gegenteil, sie habe den Rat der Mutter gesucht. Erst retrospektiv empfinde sie das mütterliche Verhalten als unangemessen (I 09:16-10:54). Was sich im Interview mit der Mutter, Frau Dienel, bereits andeutet, wird durch die Aneignungsperspektive der Tochter manifest: Das Erziehungssetting beinhaltet Elemente der over-protection, die für die Tochter insofern problematisch sind, als sie die Ausbildung von Selbstständigkeit behindern. Die ten-

\footnotetext{
${ }^{140}$ Hier bestätigt sich exemplarisch die Annahme von Lüscher/Liegle, dass Generationenbeziehungen durch „Gegensätze des Fühlens, Denkens, Wollens, Handelns und der Beziehungsgestaltung, die [...] zeitweise oder dauernd als unlösbar interpretiert werden“, gekennzeichnet sind (Lüscher/Liegle 2003, 288).
} 


\subsection{Flexibel erscheinende Tradierungslogiken}

denzielle Vermeidung von Konfrontationen stört zudem die Entwicklung einer selbstbewussten, angstfreien Haltung. Indirekt kritisiert sie zugleich, manchmal zu stark im Mittelpunkt der mütterlichen Aufmerksamkeit gestanden zu haben.

Insbesondere in der Pubertät hätte es jedoch Konflikte gegeben, die sich auf das Verhältnis von eigenen und fremden Bedürfnissen bezogen, die Heike Ebel vor allem mit ihrem Vater geführt habe (IV 12:41-13:12, 13:18-14:22). Ihm habe es missfallen, wenn sie bei Diskussionen zur Erläuterung ihres Standpunktes ihre Empfindungen angeführt habe. Seine unwillige Reaktion: „Ich, ich, ich“ (IV 13:20-13:22) seien der Auftakt für anschließende Auseinandersetzungen gewesen. Der Vater habe befürchtet, die Tochter könne sich zur „Egoistin“ entwickeln (IV 7:10-07:20). Während die Mutter Verweise auf die eigenen Gefühle durchaus als Argumentationshilfen bei der Erziehung nutzt, akzeptiert der Vater dieses Muster bei der Tochter offenbar nicht. Das könnte ein Versuch sein, die familiale Interaktion nicht zu sehr von Emotionen bestimmen zu lassen. Heike Ebels Verweis auf die Pubertät dient zugleich als Entschuldigung und Rechtfertigung des damaligen Verhaltens. Davon abgesehen scheint Heike Ebel aber durchaus der Meinung, eine stärkere Beachtung der eigenen Bedürfnisse sei legitim.

Das zeigt sich in ihrer Einschätzung, die Mutter habe ihre eigenen Bedürfnisse manchmal zu stark zurückgestellt: „Da muss meine Mutter auch sehr aufpassen, denke ich, weil sie sehr oft auch ins andere Extrem gleitet. Sie ist zu altruistisch. Sie hat zu wenig gelernt, sich um sich selber und ihre Bedürfnisse zu kümmern" (IV 07:36-07:45). Dies gelte auch für den Vater (IV 13:32-13:40). Diese Erklärungen lassen sich gleichsam als ein Versuch lesen, das Verhalten der Eltern zu entschuldigen und etwaigen negativen Lesarten durch den Hörer vorzubeugen. Heike Ebel bemüht sich den geschilderten Konflikt um die eigenen und fremden Bedürfnisse zu relativieren und eine positive Wendung zu geben:

„Und sonst, ${ }^{\circ} \mathrm{kla}: \mathrm{r}^{\circ}$, ich liebte schon die Auseinandersetzung. [...] Meine Eltern mussten sich auch viel mit mir auseinandersetzen $/ / \mathrm{hmh} / /$ und viel streiten, aber das war nie so, dass ich den Eindruck hatte, ich krieg einen auf den Deckel $/ / \mathrm{hmh} / /$ oder ich darf was nich sagen oder mhmh. $/ / \mathrm{hmh}$, also// Ich durfte alles sagen, was ich denke." (IV 13:59-14:19).

Heike Ebel zeichnet das Bild einer toleranten und freiheitlichen Erziehung ohne Redeverbote. Die Eltern beharren nicht auf ihrer Machtposition und zwingen den Kindern nicht ihren Willen auf. Der intergenerationelle kommunikative Austausch sowie die Reflektiertheit Heike Ebels mögen dazu beitragen, dass die Inhalte, die die Eltern ihren Kindern vermitteln möchten, für sie relativ explizit zu Tage liegen.

Heike Ebel betrachtet retrospektiv ihre Kindheit relativ positiv, was in der von ihr verwendeten Metapher „es war immer Sommer“ (I 00:58-01:03, IV 06:27-06:34) zum Ausdruck kommt. Auch wenn aus ihren Erzählungen schnell ersichtlich wird, dass für sie während ihrer Kindheit nicht „immer Sommer“ gewesen ist, ist die affirmative Sichtweise sicherlich förderlich für die Aneignung der elterlichen Vermittlungsinhalte. Trotz der vorgenommenen Relativierungen schätzt sie insgesamt die elterliche Erziehung. Die 


\section{Familiale Tradierungsprozesse in einer Drei-Generationen-Perspektive}

erkennbare Kritik bezieht sich weniger auf die Inhalte an sich als vielmehr auf die überbehütenden Elemente der Erziehung.

Vermittlungsebene Heike Ebel $\left({ }^{*} 1965\right)$ : Agieren im Hintergrund Die religiöse Alltagsprägung ist auch in der eigenen Familienphase präsent und spielt für die Kindeserziehung eine Rolle, Heike Ebel setzt aber eigene Schwerpunkte. Die Diskussionen um Glaubensinhalte nehmen keinen so prominenten Platz ein wie bei ihrer Mutter, Frau Dienel. Für Heike Ebel sind vor allem die Aspekte „Heimat“, „Geborgenheit“ und eine vertraute „Atmosphäre“ (I 07:50-08:02, 19:33-19:35, III 14:28-14:36, 35:22-36:12, 38:34-38:47, 39:10-39:23) relevant. Besonders betont sie den emotionalen Rückhalt, den ihr der Glaube bietet. Da für sie der Glaube elementarer Bestandteil des Alltags ist, muss sie ihn nicht besonders durch Diskussionen gegenüber ihren Kindern hervorheben (III 38:43-38:59). Stattdessen wünscht sie, dass die Kinder den Glauben aus eigenem Antrieb verinnerlichen (IV 43:45-44:12), was in ihrer Feststellung, ihre Tochter Janina sei mit Überzeugung zur Firmung gegangen, zum Ausdruck kommt (III 42:19-42:23). Sie möchte den Kindern die Entscheidung für den Glauben weder aufzwingen noch abnehmen. Wie sie feststellt, gehen ihre Kinder anders mit Glaubensfragen um:
„Wobei äh::m meine Kinder nich so kritisch hinterfragend sind in der Pu- bertät, wie ich das war. Also das is mehr so 'ne Selbstverständlichkeit. Die müssen auch nich so viel drüber reden. //hmh// Ich hatte immer 'n un- heimliches Redebedürfnich = bedürfnis, das haben die nich so. $/ / \mathrm{hmh} / /$ Vielleicht kommt's noch. Mal sehen, was da noch kommt“ (III 42:24-42:42).

Heike Ebel zeigt sich flexibel, indem sie die eigenen Erfahrungen nicht umstandslos auf den an die Kinder gerichteten Erwartungshorizont überträgt. Sie akzeptiert, dass ihre Kinder sich anders verhalten als sie selbst in ihrer Jugend. Sie versucht nicht, die eigenen Kindheitserfahrungen zu wiederholen und Diskussionen zu forcieren. Im Gegenteil: Heike Ebel gibt sich in dieser Passage aus mehreren Gründen relativ gelassen. In Auseinandersetzung mit der Kritik an ihren eigenen Eltern, sich zu viele Gedanken um die Vermittlung des Glaubens gemacht zu haben, ist es verständlich, die Erziehung nicht zu explizit auf diesen Aspekt auszurichten. Die von Heike Ebel in der eigenen Kindheit vermisste Erziehung zur Eigenständigkeit dürfte ihre Zurückhaltung zusätzlich fördern.

Doch sind auch bei Heike Ebel steuernde Elemente zu finden, um den Bezugsrahmen der religiösen Alltagsprägung zu tradieren. Sie sorgt für die Präsenz religiöser Netzwerke im Alltag ihrer Kinder, wodurch sie in eine bestimmte Richtung gelenkt werden (III 41:22-42:17). Die Einbindung in religiöse Milieus bleibt somit im Generationenverlauf konstant. Ähnliches gilt für das Vorleben religiöser Praktiken und Einstellungen. Die Kinder werden von klein auf mit zum Gottesdienst genommen (III 40:01-40:45), sodass dieser zum kindlichen Alltag gehört. Inzwischen hätten die älteren Kinder aber die Wahl, ob sie am Gottesdienst teilnehmen möchten: 


\subsection{Flexibel erscheinende Tradierungslogiken}

„[U]nd auch heute bei den Großen, wir stellen es ihnen frei, (.) wenn sie mitgehen wollen, gehen sie mit. Meistens wollen sie aber mitgehen. $/ / \mathrm{hmh} / /().(\mathrm{Na})$ ja gut, Timo [...] zieht sich aus allen Dingen des Lebens gerne mal raus. Schiebt dann wichtige Lateinvokabeln vor, die er natürlich dann gerade sonntags abends um sechs lernen muss, @wenn wir in die Kirche wollen@. Aber das is dann auch okay. Also ähm Timo is Messdiener und er is Pfadfinder. Also es, alles freiwillig, //ja// @also es is alles@, nich so, dass wir gesagt haben, ,Mensch mach doch mal. Die brauchen dringend.' (Nee), er hat einfach 'n Freundeskreis, der entsprechend is" (III 40:46-41:21).

Im Vergleich zu den Eltern hat sich der Stellenwert des Gottesdienstbesuches verschoben. Während die Eltern Heike Ebels noch deutlich ihre Meinung geäußert haben, wenn die Tochter nicht am Gottesdienst teilnehmen wollte, reagiert diese gelassener und nimmt die Entscheidung des Sohnes hin. Ein Grund dafür ist sicherlich, dass der Sohn sich nicht grundsätzlich weigert. Zudem betrachtet sie die Gottesdienstteilnahme im Gegensatz zu ihren Eltern nicht mehr als elementaren Bestandteil der eigenen Religiosität und der religiösen Alltagsprägung. ${ }^{141}$ Diese Einstellung manifestiert sich in ihrer Praxis: Der Gottesdienstbesuch ist für die Kinder nicht verpflichtend. Indem sie die Mitgliedschaft des Sohnes in verschiedenen kirchlichen Gruppierungen anführt, verdeutlicht sie, dass der Glaube dennoch eine Bedeutung für den Alltag des Sohnes hat; diese Mitgliedschaften fungieren quasi als Substitut für den Gottesdienstbesuch. Für sie kann der Glaube durch verschiedenste Praktiken ausgedrückt werden. Die hier zum Ausdruck kommende situationsspezifische Flexibilität und Offenheit manifestiert sich auch bei der mittäglichen Gebetspraxis, die anders als in ihrer Herkunftsfamilie gestaltet wird:

„Das mache ich heute anders (.) mit meinen Kindern. [...] Ähm (.) heute singen wir zum Essen, //hmh// weil ich das mit dem Beten ((Telefonklingeln)) immer so 'n bisschen peinlich finde. //hmh// Also nee, [...] ich denke für die Kinder is es 'n bisschen peinlich. Die genieren sich da immer, hab ich so den Eindruck. Wir singen ja alle sehr gerne, //hmh// und wir singen zum Essen“ (III 37:09-37:33).

Sie gestaltet das Ritual ausgehend von der Annahme, was den Kindern Freude bereiten könnte, um den Kindern die Akzeptanz zu erleichtern. In der Abänderung der Gebetspraxis ist zugleich ihre starke Kindorientierung zu erkennen.

Das leitet über zu der von Heike Ebel betonten Erziehung zur Selbstständigkeit, die aus der Verarbeitung ihrer eigenen Erziehungserfahrungen resultiert. Anders als die eigene Mutter bemüht sich Heike Ebel, nicht so stark im Alltag der Kinder präsent zu

\footnotetext{
${ }^{141}$ Die abnehmenden Besuchszahlen des Gottesdienstes (Wolf 2003, 55) legen nahe, dass die Familie hier einem allgemeinen Trend folgt. Allerdings verliert damit die Religiosität nicht automatisch an Bedeutung.
} 


\section{Familiale Tradierungsprozesse in einer Drei-Generationen-Perspektive}

sein. Sie möchte stärker im „Hintergrund“ als Rückhalt agieren (III 42:44-43:01) und den Alltag der Kinder weniger stark steuern. Zwar geht es auch ihr um eine emotionale Unterstützung ihrer Kinder und sie steht ihnen jederzeit als Ansprechpartnerin zur Verfügung (IV 43:23-43:36), sie achtet aber darauf, sofern möglich, die Handlungsinitiativen bei ihren Kindern zu belassen. Das führt Heike Ebel an Vergleichen aus (III 46:58-51:35). Während ihre Mutter z.B. Konflikte für sie geregelt habe, habe Heike Ebel in einer aus ihrer Sicht ähnlich gelagerten Situation, die sich auf den Wunsch ihrer Tochter bezogen habe, die Musiklehrerin zu wechseln, bewusst entschieden: „[I]ch mach das nicht wie meine Mutter. Ich guck einfach mal, was passiert" (III 50:10-50:14). Sie habe ihre Tochter zwar beraten, die Ausführung habe sie aber ihrer Tochter überlassen: "Joa, und dann is sie hingegangen und hat das ihrer alten Lehrerin gesagt. //hmh// Da bin nich ich hingegangen, ich hab die nich angerufen, ich musste nich mitkommen. Das macht sie dann alleine" (III 51:02-51:11). Ausgehend von ihren eigenen Kindheitserfahrungen bemüht sich Heike Ebel um ein anderes Verhältnis von Fürsorge und Aufforderung zu eigenständigem Handeln. Sie versucht, zu stark behütende Tendenzen zu vermeiden und den Kindern mehr Raum für eigene Erfahrungen zu gewähren, worauf sie auch explizit hinweist: „Ich lasse meine Kinder auch mehr Sachen machen, die dann auch mal schief gehen" (III 51:37-51:41). Das gilt sowohl im positiven Sinne, wie am Beispiel ihrer älteren Tochter zu ersehen ist, als auch im negativen. So habe ihre jüngste Tochter trotz Warnung mit dem Rad der älteren Geschwister fahren wollen. Sie habe die Tochter gewähren lassen und diese sei gestürzt (III 51:42-52:04). Das kommentiert sie mit den Worten: „haben wir was draus gelernt“ (III 52:04-52:07). Im Unterschied zu ihrer eigenen Mutter ist sie weniger darauf fokussiert, ihre Kinder von möglichen Enttäuschungen und negativen bzw. ggf. auch schmerzhaften Erfahrungen fernzuhalten. Vielmehr begreift sie auch Fehler und Fehlschläge als wichtige Lernquellen. Scheint ihre Mutter noch stärker daran orientiert, eine idyllische Welt für ihre Kinder aufrechtzuerhalten und sie zu behüten, betrachtet Heike Ebel negative Erfahrungen als solche erst einmal nicht als unmittelbare Bedrohung für das Heranwachsen ihrer Kinder.

Für den familialen Bezugsrahmen der religiösen Alltagsprägung spielt die außerfamiliale Sphäre eine wichtige Rolle. Heike Ebel berichtet von verschiedenen Gruppen der Kirchengemeinde, in denen die Kinder aktiv sind (III 42:07-42:16). Ihr ist daran gelegen, dass die Kinder selbst aktiv werden, außerhalb der familialen Sphäre Erfahrungen sammeln und hierdurch Eigenständigkeit einüben. Die religiöse Alltagsprägung wird in diesem Setting weniger über familiale Diskussionen wie in ihrer Herkunftsfamilie vermittelt, sondern stärker durch eine von den Kindern erfahrbare außerfamiliale Praxis.

Eine im Vergleich zu ihrer Mutter neue und wichtige Erziehungsmaxime ist der ,lange Atem', den sie bereits in der frühen Kindheit ihrer ältesten Tochter Janina anwendet. Heike Ebel habe sich mit dem Problem eines die abendlichen Schlafenszeiten verweigernden Kleinkindes konfrontiert gesehen, das nach dem Zu-Bett-Bringen immer wieder aufgestanden sei. Inspiriert durch Erziehungsratgeber, die den Eltern vorschlagen, den ,längeren Atem` zu haben, habe sie entsprechend reagiert (III 00:34-02:19): 


\title{
4.1 Flexibel erscheinende Tradierungslogiken
}

\begin{abstract}
„Und habe an diesem Abend dieses Kind ungelogen fünfzigmal ins Bett zurückgebracht. Ich habe das gezählt, weil ich //hmh// äh dachte, so würde es sein. Und sie ist fünfzigmal wieder rausgekommen, und ich habe sie fünfzigmal kommentarlos auf den Arm genommen und wieder ins Bett zurückgelegt. Und nach fünfzigmal ist sie dann eingeschlafen“(III 01:41-01:57).
\end{abstract}

Der Ratschlag steht im Einklang mit ihrem aus dem familialen Bezugsrahmen resultierenden Anspruch der Selbstlosigkeit und dem Zurückstellen der eigenen Bedürfnisse. Sie versucht geduldig, das Problem zu lösen, ohne dem Kind autoritär mittels Drohungen oder Strafen den eigenen Willen aufzuzwingen. Die ersten Jahre mit ihren Kindern seien einerseits schön, im Rückblick aber auch ,anstrengend“ gewesen (III 24:21-24:30). Seitdem sie aber mit ihrer ältesten Tochter diskutieren könne, seien alle Probleme lösbar: "Ja und seitdem macht es eigentlich richtig, macht es mir richtig Spaß, //hmh// Mutter zu sein. Muss ich 'nen langen Atem haben, wenn ich so im Rückblick denke" (III 26:14-26:20). Wie sich zeigt, ist die kommunikative Auseinandersetzung mit den Kindern das zentrale Erziehungsinstrument. Das stellt eine deutliche Parallele zu den eigenen Erziehungserfahrungen dar, wenn es auch nicht explizit auf den familialen Bezugsrahmen angewendet wird. Es erklärt, warum Ereignisse wie die oben beschriebenen, die nicht so leicht durch rationale Argumentationen aufzulösen sind, besondere Herausforderungen darstellen. Das geduldige Aushalten, der lange Atem, lässt sich in diesem Zusammenhang als eine Strategie lesen, mit diesen Situationen umzugehen und sie auszuhalten. Im Zusammenspiel mit der Anleitung zur Eigenständigkeit könnte eine weitere Absicht darin bestehen, durch möglichst minimale Interventionen die Kinder so wenig wie möglich einzuschränken und zu dirigieren. Das markiert einen wichtigen Unterschied zum Erziehungssetting der eigenen Mutter.

Zugleich betrachtet Heike Ebel das Zurückstellen der eigenen Bedürfnisse zwar kritisch, übernimmt aber eben diese selbstlose Haltung mehrfach. ${ }^{142}$ Im Unterschied zu ihren Eltern ist es ihr jedoch eher möglich, die eigenen Interessen zu artikulieren. Insgesamt scheint sie eine ausgeglichenere Balance zwischen fremden und eigenen Bedürfnissen anzustreben, was wiederum das konkrete Erziehungssetting beeinflusst.

Der kommunikative Austausch sowie die Maxime ihrer Eltern „Diskussion über alles“ erfahren im eigenen Erziehungsarrangement eine spezifische Ausprägung. Heike Ebel wendet dies zwar nicht auf die Diskussion von Glaubensfragen an, aber in anderer Hinsicht geht sie sogar über das Setting ihrer eigenen Eltern hinaus, indem sie, zweifelsohne von Gordons Bestsellern zur Thematik inspiriert, Kommunikation und „Diskussion über alles" in Form von Familienkonferenzen ${ }^{143}$ institutionalisiert (IV 39:30-40:18).

\footnotetext{
${ }^{142}$ Ihre fürsorgliche Einstellung zeigt sich auch in ihrer karitativen Tätigkeit in verschiedenen kirchlichen Gruppen (I 13:16-13:38, III 27:39-29:30). Ihr selbstloses Engagement scheint eng mit den Lebensbedingungen des eigenen Milieus verknüpft und stößt an Grenzen, sobald sie mit sozialen Problemen konfrontiert wird, die außerhalb ihrer Vorstellungswelt liegen (I 20:01-21:54).

${ }^{143}$ Hierbei handelt es sich um einen Ratgeber, der 1972 erstmals auf deutsch publiziert wurde und sich der konstruktiven Bearbeitung familialer Konflikte widmet. Hierzu werden bestimmte kommuni-
} 


\section{Familiale Tradierungsprozesse in einer Drei-Generationen-Perspektive}

Während der Familienkonferenzen darf jedes Familienmitglied nach einem bestimmten Regelsystem seine Anliegen vorbringen. Auf diese Weise versucht Heike Ebel, die Interessen aller Beteiligten zu berücksichtigen. Die Einberufung von Familienkonferenzen zeugt von dem Bestreben, einvernehmliche Lösungen für intergenerationelle Konflikte $\mathrm{zu}$ finden. Das Einbeziehen der Sichtweisen, Meinungen und Bedürfnisse der Kinder ist ein Versuch, die Asymmetrien im intergenerationellen Machtverhältnis zu minimieren, indem die Kinder in den Entscheidungsprozess aktiv mit einbezogen werden. Am Beispiel der Familienkonferenzen manifestiert sich zudem Heike Ebels Offenheit, ihr Vorgehen anzupassen und nicht dogmatisch auf ihrem Prozedere zu bestehen. So gebe es aktuell keine Familienkonferenzen mehr, weil die älteren Kinder sie mittlerweile „megadoof“ (IV 39:53-39:54) fänden. Stattdessen werden Probleme und Konflikte am Mittagstisch ohne die Regularien der Familienkonferenz besprochen (IV 39:55-39:57). Die elterlichen Vorstellungen stellen keine absoluten, nicht verhandelbaren Größen dar und für ihre situationsabhängige Offenheit gibt es weitere Beispiele. Sie sei zwar irritiert, dass ihre Tochter mit ihren Freundinnen abends chatte, könne aber die Argumente der Tochter für dieses Kommunikationsmittel akzeptieren (III 43:18-43:42).

Im Bedarfsfall werden die Kinder allerdings aufgefordert, die Bedürfnisse anderer zu berücksichtigen:

„Ja, dieses Fürsorgliche oder dieser Aspekt der Nächstenliebe das ist was, was ich versuche jetzt, meiner pubertären Tochter immer mal wieder so ein bisschen nahe zu legen. [...] Es gibt noch ein Leben neben Janina und es gibt auch noch andere Menschen neben Janina“ (IV 33:08-33:30).

Die Tochter wird ermahnt, nicht nur ihren eigenen Interessen nachzugehen, sondern auch auf die Wünsche ihrer Schwester einzugehen (IV 33:45-34:00).

Mitspracherechte, das Recht auf eigene Erfahrungen und Berücksichtigung der kindlichen Bedürfnisse münden nicht in Beliebigkeit und Regellosigkeit. Auch wenn Heike Ebel ihre Kinder zur Selbstständigkeit erziehen möchte mit dem Ziel, „dass die Kinder selber sehen, wie sie klarkommen“ (IV 42:05-42:13), sind die Grenzen dieser Erziehungsmaxime erreicht, wenn es um schulische Belange geht. Bei Leistungsproblemen kommt ein differenziertes Regelsystem zum Einsatz. Als der Sohn z.B. schulische Probleme gehabt habe, seien ihm verschiedene Optionen angeboten worden. Seine Wahl wird mit Bedingungen verknüpft, die schriftlich fixiert und überprüft werden (IV 40:05-41:17). Durch diese Taktik kann der Sohn zunächst den Eindruck gewinnen, selbst entschieden zu haben, was es den Eltern erleichtert, Forderungen zu stellen. Keinerlei Verhandlungsspielraum besteht in Bezug auf die Hausaufgaben, die erledigt werden müssen (IV 38:22-38:26). Als weitere Regel gelte „theoretisch“ regelmäßig mit den Musikinstrumenten zu üben, was „natürlich ganz schlecht“ funktioniere (IV

kative Techniken vorgeschlagen (Gordon 1972). Es ist ein sehr populärer Ratgeber, von dem diverse Auflagen erschienen sind. Die letzte stammt aus dem Jahre 2012. 


\subsection{Flexibel erscheinende Tradierungslogiken}

38:26-38:33). Die Wortwahl lässt auf eine geringere Verbindlichkeit der letzten Regelung schließen, vermutlich weil der musische Sektor stärker als Angebot aufgefasst wird. Zugleich korrespondiert das bereichsabhängig variable Vorgehen mit Heike Ebels Feststellung: „Also es gibt Kämpfe, die wichtig sind, und es gibt Kämpfe, die kann man sich sparen“ (IV 45:10-45:13).

Heike Ebel strebt im Vergleich zu ihrer Mutter ein anderes Verhältnis von Nähe und Distanz zu ihren Kindern an. Während sie zu ihrer eigenen Mutter in der Pubertät „mehr so 'ne Freundinbeziehung hatte“, was sie retrospektiv als problematisch bewertet, sei Janina stärker „für sich, //hmh// in ihrer Welt, mit ihrem Freundeskreis“ (III 43:02-43:17). Das impliziert, dass Heike Ebel ggf. nicht so umfassend über das Leben ihrer Tochter informiert ist, wie ihre Mutter es ihrerzeit gewesen ist. Die Generationenbeziehungen sind nicht ganz so eng wie in der eigenen Kindheit, was Heike Ebel positiv beurteilt, zumal das die Entwicklung der töchterlichen Eigenständigkeit unterstützen kann. ${ }^{144}$ Die Auseinandersetzung mit den eigenen Erziehungserfahrungen führt dazu, den kontrollierenden Aspekt im Erziehungsarrangement zu verringern und den Kindern im Vergleich zur eigenen Kindheit größere Spielräume zuzugestehen.

Während ihre Mutter, Frau Dienel, intergenerationelle Konflikte eher marginalisiert, sind sie für Heike Ebel ein selbstverständlicher Teil des Familienlebens. Auseinandersetzungen mit den Kindern werden von ihr im Interview bereits ohne Aufforderung erörtert und auf die explizite Nachfrage antwortet sie: „Na klar. Das ganze Leben ist @voller Konflikte@. //@(.)@// Na klar“ (IV 35:46-35:49). An anderer Stelle führt sie aus:

„Natürlich gab und gibt es immer noch Probleme und Reibereien. Aber ähm es lässt sich jetzt alles irgendwie regeln. Und wenn sich mal was nich regeln lässt, is es auch nich schlimm. Also ich denk, wenn fünf Leute aufeinander leben, geht es nich ohne Reibereien, $/ / \mathrm{hmh} / /$ und ohne Streit“ (III 25:51-26:07).

Heike Ebel stellt sich an dieser Stelle sehr reflektiert dar und betrachtet Konflikte nicht als Bedrohung für das Familienleben. Diese im Vergleich zur Vorgängergeneration offenere Thematisierung von intergenerationellen Auseinandersetzungen könnte einen Versuch darstellen, das von Heike Ebel trotz einiger Relativierungen relativ harmonisch betrachtete Familienleben der „heilen Welt“ (I 21:16-21:19) während der eigenen Kindheit durch eine leichter umsetzbare Alternative zu ersetzen. Allerdings ist sie auch bemüht, die angesprochenen Konflikte zu relativieren, indem sie z.B. auf die Pubertät der Kinder verweist. Einen wichtigen Beitrag zu dieser Entwicklung, Konflikte als selbstverständlichen Bestandteil des Familienlebens zu akzeptieren sowie den Kindern mehr Mitspracherechte einzuräumen, dürften hierbei auch die Kinder selbst leisten, die anscheinend

\footnotetext{
${ }^{144}$ In diesem Zusammenhang ist sie auch überzeugt, dass sich die Kinder nicht mit allen Angelegenheiten an sie wenden, was sie hinnehmen kann, auch wenn sie bei Bedarf jederzeit ansprechbar sei (IV 43:35-43:42). Heimlichkeiten in Form von Lügen hingegen sind für sie nicht hinnehmbar und werden thematisiert (IV 42:53-43:20).
} 


\section{Familiale Tradierungsprozesse in einer Drei-Generationen-Perspektive}

ihre eigenen Bedürfnisse sehr viel energischer thematisieren und einfordern als Heike Ebel in ihrer Kindheit.

\section{Aneignungsebene Janina Ebel ${ }^{145}\left({ }^{*} 1991\right)$ : Eigene Entscheidungen und die Fa- milie als Rückhalt Im Großen und Ganzen scheint Janina mit der elterlichen Er-} ziehung und dem Bezugsrahmen der religiösen Alltagsprägung einverstanden zu sein. Letzteres führt sie auf die Einbindung in ein religiöses Umfeld zurück. Die hiermit von den Eltern vorgenommene Steuerung betrachtet sie offenbar als selbstverständlich und unproblematisch (03:05-03:12, 17:23-17:42, 18:02-19:02). Religiöse Praxen wie der gemeinsame Gottesdienstbesuch gehören zum Familienleben (19:05-19:25), sodass das Vorleben als Bestandteil zur Vermittlung des familialen Bezugsrahmens betrachtet werden kann, wenn dieses Element auch nicht so prominent in den Schilderungen Janinas zum Tragen kommt wie bei ihrer Mutter und Großmutter.

In Janinas Erzählung spielen die Aspekte der Eigenständigkeit und Eigeninitiative eine wichtige Rolle, die sich u.a. auf den familialen Bezugsrahmen der religiösen Alltagsprägung beziehen. Sie möchte z.B. im Zusammenhang mit ihrer Firmung öffentlich zeigen, „dass mir Kirche nich total egal is u:nd dass ich da halt auch wirklich freiwillig hingehe“ (06:01-06:06). Sie betont den Angebotscharakter, den sie dem Bezugsrahmen zuschreibt. Sie fühlt sich nicht von den Eltern gedrängt, den familialen Bezugsrahmen zu übernehmen. Vielmehr erlebt sie die Eltern als offen, flexibel und an den Interessen ihrer Tochter orientiert. Sie betrachtet die eigenen Wünsche und Bedürfnisse als ausschlaggebend für ihre Entscheidung und die Orientierung an der religiösen Alltagsprägung bildet für sie eine Option. Ähnlich wie ihre Mutter betrachtet sie die eigenen Eltern nicht als steuernd. Hiermit sind für die Aneignungsebene zentrale Aspekte eingeführt.

Janina engagiert sich in kirchlichen Jugend- und Musikgruppen (18:02-18:16, 19:2519:36, 20:23-21:10). Neben Eigenständigkeit spielen in diesem Zusammenhang Freiwilligkeit sowie die Freude an den Aktivitäten eine wichtige Rolle. Die Idee, der kirchlichen Musikgruppe beizutreten, stamme zwar initial von ihrer Mutter, die letztliche Entscheidung habe aber Janina getroffen (20:23-21:10). Mitgestaltete Gottesdienste bringen sie zu der Erkenntnis, dass der Gottesdienst „nich nur langweilig is und sich ewig hinzieht“, sondern dass er auch „durchaus interessant sein kann“ (20:15-20:20). Über die Jugendgruppe erfährt Janina zugleich eine Gemeinschaftseinbindung, wobei für sie die Gruppenaktivitäten besonders erwähnenswert sind (22:18-23:30). Somit könnte in Janinas aktueller Lebensphase die intragenerationelle Gemeinschaftsorientierung im Vordergrund stehen und den religiösen Aspekt überlagern.

Janina erlebt in ihrer Familie ein differenziertes System aus Regeln und Freiräumen. Für sie selbst gebe es aktuell keine Regeln in Bezug auf die Schule. In der Vergangenheit sei sie zwar auf ihre zu erledigenden Aufgaben hingewiesen worden, aber inzwischen könne sie sich das „frei einteilen“, da sie selbst einschätzen könne, was sie in welchem

${ }^{145}$ Das Interview mit Janina Ebel wurde am 02.02.2009 geführt. 


\subsection{Flexibel erscheinende Tradierungslogiken}

Umfang lernen müsse und das „auch so hinkriege“ (43:25-43:28 und 27:41-27:53), was die Eltern inzwischen registriert hätten (44:01-44:20). Die Eltern achten allerdings auf Janinas Zeiteinteilung und bäten sie, zu einer bestimmten Uhrzeit zu Hause zu sein, damit sie genügend Zeit für ihre diversen schulischen und musischen Aufgaben habe (42:33-42:53). Ihrem Bruder hingegen würden die Eltern mehr Regeln setzen, da er im Gegensatz zu ihr eine Zeit lang schlechte Schulleistungen erbracht habe (43:29-43:46). Die Eltern sind folglich in der Lage, situationsabhängig die Regeln anzupassen. Die Eltern vollziehen selbst einen Lernprozess, indem sie den Kindern in Abhängigkeit vom jeweiligen Entwicklungsstand Freiräume und Eigenständigkeit zugestehen. Die Fähigkeit zum Selbstmanagement wird von Janina auch als wichtige Voraussetzung erkannt, um von den Eltern Freiräume zu erhalten, und wird aus diesem Grunde von ihr besonders betont. Zudem nimmt sie die elterlichen Vorgaben nicht als Befehle, sondern als Bitten wahr. Das impliziert zugleich, dass sie gewisse Spielräume sieht, sich zu diesen zu positionieren. Das verweist zugleich auf die elterliche Absicht, ihre Kinder zu eigenständigem Verhalten zu erziehen.

Im Bereich der musikalischen Ausbildung ist ein ähnliches Muster erkennbar. Beim Erlernen des ersten Musikinstruments hätten die Eltern auf das Einhalten regelmäßiger Übungszeiten bestanden, worüber Janina in der Retrospektive „echt froh“ sei (51:2752:30). Aktuell würden die Eltern sie lediglich bitten: „Üb doch noch mal“ (27:58-28:00). Auch wenn Janina „theoretisch“ einsieht, dass die Eltern recht hätten, sei sie manchmal zu „k.o.“, um dem elterlichen Wunsch nachzukommen (27:55-28:27). Die Eltern überlassen aber die Entscheidung Janina: „Das mit dem Üben zum Beispiel teile ich mir letzten Endes doch selbst ein“ (48:39-48:46). Die Eltern zeigen hier eine gewisse Offenheit, indem sie zwar an die Tochter appellieren, sie aber nicht zum Üben zwingen.

Auch in anderen Bereichen empfindet Janina die Eltern als tolerant. Einfluss auf den Freundeskreis zu nehmen, sei z.B. die absolute Ausnahme (42:00-42:42:29). Am Kleidungsstil würden sich kaum Konflikte entzünden, da ihr Kleidungsstil ohnehin nicht so ausgefallen sei, dass er den Eltern missfalle (44:37-45:25). Lediglich auf die Konsummenge achte die Mutter und sei der Meinung, nicht unbedingt notwendige Kleidung könne die Tochter selbst bezahlen (45:27-45:37). Insgesamt scheinen die Eltern grob einen gewissen Rahmen abzustecken, innerhalb dessen Janina sich relativ frei und eigenständig bewegen kann, wobei auch Janina zur Veränderung des abgesteckten Rahmens beitragen kann. Die Mutter habe es anfangs problematisch gefunden, dass Janina aus Sicht der Mutter zu viel Zeit am Computer verbringe. Sie solle „lieber was mit der Familie machen" (32:55-33:04) und außerdem sei die Mutter wegen der potenziellen Gefahren, die sie mit der Internetnutzung verbunden habe, besorgt gewesen. Inzwischen habe sich die Mutter aber damit arrangiert, zumal Janina, wie sie betont, durchaus umsichtig mit dem Medium umgehe (32:55-34:47). Janina kann sich durchsetzen und die Mutter ist bereit, ihre Position zugunsten der Tochter zu relativieren.

Wenn in der Vergangenheit die Eltern etwas für die Tochter entschieden hätten, z.B. im schulischen Bereich, hätten sie das gegenüber Janina erklärt und begründet, sodass es 


\section{Familiale Tradierungsprozesse in einer Drei-Generationen-Perspektive}

sich aus Janinas Sicht um gemeinschaftliche Entscheidungen handelt, die sie akzeptieren kann (02:19-02:46; 17:09-17:21). Eine intergenerationelle Diskussion von Glaubensfragen, wie zwischen ihrer Mutter und Großmutter praktiziert, wird von Janina nicht erwähnt und ist für sie kein zentrales Thema. Das könnte dem mütterlichen Ansatz geschuldet sein, den Glauben als Selbstverständlichkeit zu betrachten.

Aus der Aneignungsperspektive ist das elterliche Erziehungsetting auf Janinas Unterstützung und Förderung ausgerichtet. Dieses Arrangement erleichtert es Janina vermutlich, Tradierungsinhalte anzunehmen. Es gibt aber auch intergenerationelle Spannungen. In der Vergangenheit habe es Konflikte mit der Mutter gegeben, die Janina jedoch eher mit der Pubertät verbindet und weniger mit dem Bezugsrahmen der religiösen Alltagsprägung oder dem elterlichen Vermittlungssetting (10:17-11:04). Janina verfährt bei der Darstellung von Konflikten ähnlich wie ihre Mutter. Über den Verweis auf zeitliche Befristung und biologische Aspekte versucht sie, die Auseinandersetzungen $\mathrm{zu}$ entdramatisieren und zu relativieren. Sie werden auf diese Weise zwar als Bestandteil des Familienlebens präsentiert, ohne ihnen jedoch einen dominierenden Charakter einzuräumen. Lediglich gegen die aus dem familialen Bezugsrahmen resultierende mütterliche Forderung, stärker auf die Bedürfnisse der kleinen Schwester zu achten und mehr Zeit mit ihr zu verbringen, lehnt sie sich auf. Mit Verweis auf ihre knappe Freizeit verteidigt sie ihre Interessen und entzieht sich gelegentlich dem mütterlichen Wunsch (39:53-41:40). Sie kann im Unterschied zur Mutter besser auf die eigenen Bedürfnisse achten, was sich auch bei anderen Gelegenheiten manifestiert. Im Rahmen der Familienkonferenzen habe sie manchmal Probleme gehabt, die eigenen Interessen zugunsten denen der anderen Familienmitglieder zurückzustellen (39:20-39:38). Mit ihrem Verhalten realisiert Janina offenbar das mütterliche Anliegen, den eigenen Bedürfnissen einen höheren Stellenwert zuzugestehen. Allerdings ist Janinas Bestreben nicht immer erfolgreich. Gelegentlich ärgere sie sich über sich selbst, weil sie selbst dann gegenüber anderen „höflich“ und „freundlich“ bleibe, obwohl sie deren Benehmen missbillige (47:12-48:28). Im Gegensatz zu den Vorgängergenerationen ist es Janina möglich, das deutlich als Belastung zu artikulieren.

In Janinas Darstellung unterliegen ihre Beziehungen zu den Eltern einem Wandel:

„Also es ist jetzt nicht mehr so, so ähm, (.) ich sage jetzt mal so intensiv, $/ / \mathrm{hm} / /$ wie es früher vielleicht mal war, weil halt viele Sachen, die ich früher vielleicht eher meinen Eltern erzählt hätte oder so, gehen jetzt eher an meine Freundinnen. $/ / \mathrm{hmh} / /$ (.) $\mathrm{Ähm}$, aber an und für sich eigentlich schon noch ganz gut. $/ / \mathrm{hmh} / /$ (.) Also wir kommen eigentlich ganz gut miteinander klar und so, (.) deswegen halt eigentlich ganz gut. Nur halt eben nicht mehr so ganz so eng, wie es früher mal war" (50:34-51:06).

Janina beschreibt eine lebensphasenspezifische Veränderung der Generationenbeziehungen, einen Ablösungsprozess, in deren Zuge sie eine zunehmende Eigenständigkeit anstrebt. Ihre Peergroup ersetzt teilweise die Eltern als Ansprechpartner. Augenfällig ist 


\subsection{Flexibel erscheinende Tradierungslogiken}

der Unterschied zur Jugend ihrer Mutter, bei der fast der Eindruck einer Abschottung gegen die außerfamiliale Sphäre entsteht. Aktuell wirken die Generationenbeziehungen sehr viel offener, auch für außerfamiliale Einflüsse. Das dürfte die von der Mutter betonte Erziehung zur Eigenständigkeit fördern, und scheint von Janina auch in diesem Sinne genutzt zu werden. Die Eltern versuchen allerdings auch, Janinas Bewegungen aus der Familie hinaus nicht zu weit gehen zu lassen. Ein Beispiel hierfür sind die mütterlichen Ermahnungen, nicht so viel Zeit am Computer und lieber mehr Zeit mit der Familie zu verbringen (32:55-33:04).

Die Eltern nehmen für Janina eine etwas andere Rolle ein als in der Generation zuvor. Sie dienen in erster Linie als Rückhalt. Für Janina, die sich in vielen Dingen als selbstbestimmt betrachtet, ist die elterliche Unterstützung erst bei der Umsetzung ihrer Entscheidungen wichtig, z.B. in Bezug auf ihre Firmung (29:35-30:00). Besonders unterstreicht sie die elterliche Hilfe in Form der Förderung. Speziell in Bezug auf ihre musikalischen Interessen ist sie froh, dass die Eltern „möglichst viel halt irgendwie auch möglich machen“ (30:22-30:26). Janina ist dankbar für das elterliche Engagement, das sie nicht als selbstverständlich betrachtet oder gar einfordert.

Lediglich die partiell zu ängstliche Umsorgung durch die Mutter wird von Janina kritisiert (49:48-50:23), untergräbt sie doch die mütterliche Intention, nicht so stark im Leben der Kinder präsent zu sein, und läuft dem von Janina über die Erziehung internalisierten Bestreben nach Eigenständigkeit zuwider. Das hat zur Folge, dass Janina gelegentlich an ihrem Selbstvertrauen arbeiten muss. Sie musiziere z.B. sehr gerne, habe sich aber vor öffentlichen Auftritten gefürchtet. Mittlerweile habe sie ihre Angst aber bewältigt und hierdurch ihr "Selbstbewusstsein auch noch mal irgendwie gesteigert“ (54:06-55:06).

Trotz der sich abzeichnenden Öffnung für außerfamiliale Einflüsse bleibt auch das Netzwerk der Verwandtschaft für Janina wichtig. Sie habe sich gefreut, durch die Firmung einen Anlass gehabt zu haben, um „mal wieder die ganze Familie hier“ (06:1206:20) zu haben. Die Großeltern werden regelmäßig besucht und es finden Familienurlaube mit Onkel und Cousinen statt (06:29-7:00). Im Unterschied zum Aufwachsen der eigenen Mutter können die Kontakte aufgrund der räumlichen Entfernungen nicht mehr auf alltäglicher Basis realisiert werden. Das könnte zu einer stärkeren außerfamilialen Öffnung beigetragen haben und abgesehen von der aktuellen Lebensphase Janinas ein weiterer Grund sein, warum Janina in ihrer Eingangserzählung Freundschaften zu Gleichaltrigen verstärkt thematisiert (01:40-02:16, 03:36-03:59). Die hier zum Ausdruck kommende Gemeinschaftsorientierung bezieht sie dabei stärker auf Gleichaltrige als auf die Familie.

In der Gesamtschau ist bei Janina eine religiöse Alltagsprägung vorfindbar. Im Unterschied zu ihrer Mutter und Großmutter nimmt diese Thematik in ihren Schilderungen aber eine weniger prägnante Rolle ein. Das ist jedoch nicht unbedingt mit einem Bedeutungsverlust des Bezugsrahmens gleichzusetzen, sondern kann mit der aktuellen Lebensphase zusammenhängen, in der für Janina z.B. Themen wie Freundschaften ei- 


\section{Familiale Tradierungsprozesse in einer Drei-Generationen-Perspektive}

nen höheren Stellenwert einnehmen. Die religiöse Alltagsprägung wird bei Janina explizit an die Elemente Eigenständigkeit, Freude und Aktivsein rückgekoppelt. Darüber hinaus sind die Wahrung der eigenen Bedürfnisse sowie die Ausbildung eines Selbstbewusstseins für Janina relevant. Weitere Rahmungen stellen bildungsbezogene Aspekte dar, wie sie in ihrer hohen Lernmotivation ebenso zum Ausdruck kommen wie in der großen Bedeutung, die Janina der Musik in ihrem Leben einräumt.

Zusammenfassende Betrachtung des familialen Bezugsrahmens Die religiöse Alltagsprägung spielt in allen drei Generationen als Bezugsrahmen eine Rolle. Zugleich sind in den drei Generationen aber auch Schwerpunktverlagerungen zu erkennen. Sind für Frau Dienel im Zusammenhang mit ihrer Religiosität die Auseinandersetzung mit dem Glauben unter besonderer Berücksichtigung der Suche nach den modernen Aspekten relevant, dient der Glaube der Tochter, Heike Ebel, vor allem als Rückhalt, der „Geborgenheit“ bietet und ein „Stück Heimat“ darstellt. Für Janina schließlich ist die eigenaktive, freiwillige Gestaltung der religiösen Elemente zentral. Ein mit dem familialen Bezugsrahmen verbundenes Element stellt das Verhältnis von eigenen und fremden Bedürfnissen dar, das im Generationen- und Zeitverlauf Verschiebungen durchläuft. Für die älteste Generation ist das Zurückstellen der eigenen Bedürfnisse noch eine selbstverständliche Forderung. Die mittlere Generation hingegen bemüht sich um eine ausgewogenere Balance von eigenen und fremden Interessen. In der jüngsten Generation schließlich ist die Beachtung der eigenen Wünsche kein Tabu mehr. Die explizite Rückbindung an religiöse Lehren und Verhaltensmaximen tritt im Generationenverlauf in den Hintergrund. Während Frau Dienel, als Vertreterin der ältesten Generation, Fürsorglichkeit und Verpflichtung für das Wohlergehen anderer noch religiös begründet, wird diese Verbindung von der jüngsten Generation, Janina, nicht mehr ausdrücklich angesprochen. Als Tendenz zeichnet sich ab, dass sich die beschriebene Orientierung im Generationenverlauf vom Status eines mit der eigenen Religiosität verbundenen Elements zum Status eines allgemeinen Prinzips wandelt. Einschränkend ist allerdings auf das Alter Janinas zu verweisen, die ihrerseits noch nicht in der Situation ist, als Mutter ihren Kindern Orientierungen wie Selbstlosigkeit vermitteln zu wollen. Auch die mit der religiösen Alltagsprägung verbundenen Praxen unterliegen wesentlichen Transformationen. War den Eltern Frau Dienels daran gelegen, den Kindern die Praxen durch Erklärungen verständlich zu machen, ohne dass das ihre Gestaltung berührt hätte, passt Frau Dienel sie teils den jeweiligen Rahmenbedingungen flexibel an. Ihre Tochter, Heike Ebel, wird bei der Ausgestaltung religiöser Praxen wie dem Mittagsgebet von den von ihr angenommenen Bedürfnissen der Kinder geleitet. Dieses Zusammenspiel aus Anknüpfen an den familialen Bezugsrahmen und inhaltlichen Neuausrichtungen findet auch in den jeweiligen Erziehungsarrangements ihren Niederschlag. 


\subsection{Flexibel erscheinende Tradierungslogiken}

\subsubsection{Familie Cronert/Jäger: Intergenerationelle Solidarität und Hilfe ${ }^{146}$}

Biografische Eckdaten Herr Cronert ist 1936 in einem Dorf in Thüringen geboren, in dem die Familie heute noch lebt. Der Ort lag zu DDR-Zeiten innerhalb des sogenannten Sperrgebietes. Nach der Schule absolviert er eine Malerlehre, die er 1954 abschließt. 1960 besteht er die Meisterprüfung. 1959 lernt er seine Frau kennen und das Paar heiratet 1965. Die gemeinsame Tochter Friederike wird 1969 geboren. Frau Cronert unterbricht für drei Jahre ihre Erwerbstätigkeit und nimmt danach eine Halbtagsstelle im Ort an. Nach zehnjähriger Schulzeit erlernt Friederike den Beruf der Raumausstatterin. Sie heiratet 1988. Die erste Tochter, Anika, wird 1989 geboren, die zweite Tochter, Cora, 1997. Beide Töchter besuchen das Gymnasium. Anika ist während ihrer Schulzeit für ein Jahr im Ausland und hat inzwischen ihr Abitur. Friederike Jäger lebt mit ihrer Familie in einer eigenen Wohnung im Elternhaus.

Aneignungsebene Herr Cronert (*1936): Regeln und Freiräume ${ }^{147}$ Für Herrn Cronert bildet der familiale Zusammenhalt einen elementaren Topos:
„[V]ielleicht die Nachkriegsjahre, da möchte ich noch etwas mehr drauf eingehen, $/ / \mathrm{hmh} / /$ die ja die Familien in der Richtung noch mehr ge- prägt haben und der Zusammenhalt da vielleicht noch mehr gewesen ist, //hmh// wie dann später. Denn die Nachkriegsjahre äh schon mit der Ver- sorgung, mit dem Leben und mit dem Essen, war ja nich so einfach und da war an und für sich äh der Zusammenhalt zwischen meiner Großmutter und auch meiner, meinen Eltern sehr gegeben, weil wir ja dort gemeinsam auch für uns gesorgt haben“ (II 00:13-00:57).

Herr Cronert beschreibt den intergenerationellen Zusammenhalt - insbesondere während der Nachkriegszeit - als für ihn unmittelbar erfahrbar durch das praktische Vorleben von Eltern und Großmutter. Eine besondere Bedeutung nimmt hierbei seine Großmutter ein, die er gleich im zweiten Satz einführt: „,U]nd ich bin bei meiner Großmutter, die mit im Hause war, äh aufgewachsen“ (I 01:08). Hiermit sind die zentralen Bestandteile von Herrn Cronerts grundlegendem Bezugsrahmen näher spezifiziert: der intergenerationelle Zusammenhalt, insbesondere in Form der Zusammenarbeit, sowie die intergenerationelle Arbeitsteilung, die in einer Aufteilung der Kindererziehung und -betreuung zwischen den Generationen besteht.

Die Großmutter ist aktiv in die Erziehung eingebunden. Herr Cronert erinnert bestimmte Charakteristika der Großmutter:

„[S]o dass sie dann auch äh immer wusste, was sie wollte und da auch konsequent und mitunter auch kaum nachgiebig war. [...] Ansonsten haben

\footnotetext{
${ }^{146}$ Zum Fallbeispiel Cronert/Jäger siehe auch Kraul/Radicke (2012).

${ }^{147}$ Das Interview mit Herrn Cronert wurde am 20.02.2008 geführt.
} 


\section{Familiale Tradierungsprozesse in einer Drei-Generationen-Perspektive}

wir mit, mit unserer Großmutter, mein Bruder war ja dann auch mit da, haben wir ein gutes Verhältnis gehabt und äh sicher mussten wir in der Reihe gehen, wie sich das mitunter auch gehörte, aber Probleme hat es damit nich gegeben. Und es war eben nich so, dass sie auch nicht nur, nicht nachgiebig war, die war auch zu gegebener Zeit war sie dann auch dabei und hat denn auch mal mit uns äh Blödsinn gemacht. //hmh// Und das hat sie auch immer ( ). Es war nicht nur, dass sie 'ne strenge Frau war. //hmh// (.) Wir sind gerne bei ihr gewesen" (II 14:04-15:26).

Herr Cronert betrachtet die Großmutter inner- wie außerfamilial als eine Autoritätsperson. Sie ist in seinen Augen nicht nur willensstark, sondern verfügt auch über klare Prinzipien und Regeln, die sie u.a. auf die Erziehung anwendet. Das führt allerdings zu keinen nennenswerten Konflikten, zumindest werden von Herrn Cronert keine thematisiert. Die Wahrnehmung der Großmutter als Autoritäts- oder Respektsperson mag hierzu einen wichtigen Beitrag geleistet haben, und es ist Herrn Cronert vielleicht gar nicht in den Sinn gekommen, die Regeln der Großmutter anzuzweifeln. Er scheint die Vormachtstellung der Erwachsenen als selbstverständlich zu akzeptieren. Das heißt jedoch nicht, dass die gesetzten Regeln immer eingehalten werden. Herr Cronert beschreibt seine Großmutter gleichzeitig als eine Person, die ihre Grundsätze je nach Kontext variieren kann. Sie kann sowohl direktiv als auch nachgiebig gegenüber den Enkeln sein und auf ihre Wünsche eingehen. Sie beharrt nicht starr auf ihren Maximen, sondern verhält sich gegenüber ihren Enkeln auch flexibel und geht auf sie ein. Seine Bilanzierung, gern bei der Großmutter gewesen zu sein, verweist darauf, dass Herr Cronert im Großen und Ganzen mit dem großmütterlichen Erziehungsarrangement zufrieden ist, zumal ihm offensichtlich in Teilbereichen Freiräume zugestanden worden sind. Die Großmutter ist nicht nur diejenige, die ihren Kindern abends Märchen oder Geschichten aus ihrer Herkunftsfamilie erzählt (II 15:28-15:54, II 29:02-32:05) und somit das Bindeglied zur Familienvergangenheit bildet, sondern auch eine zentrale, Unterstützung leistende Bezugsperson:

„Aber bei Oma //hmh// konnte ich eigentlich immer kommen. //hmh// (.) Hat mit mir auch durchgestanden, wenn ich nach Hause kam und war, wir haben unmittelbar hier am Wasser gewohnt, //hmh// und wir haben als Kinder mehr oder weniger auch immer mit 'm Wasser oder nachher im Wasser gespielt. //hmh// Und wer so dicht am Wasser (ist), der fällt auch rein. U:nd $/ / \mathrm{hmh} / /$ wenn es da Probleme gab und wir sind nach Hause gekommen, dann hat Oma das geregelt“ (II 34:46-35:14).

Die Großmutter wird als ausgleichende Instanz zwischen Eltern und Sohn skizziert, die durch ihre Interventionen dafür sorgt, etwaiges Konfliktpotenzial zwischen Eltern und Kindern möglichst gering zu halten, was dazu beigetragen haben dürfte, die Generationenbeziehungen als positiv und gut erleben zu können. Auch ist die Großmutter diejenige, an die sich Herr Cronert in seiner Jugendzeit wendet, wenn er Geld brauchte: 


\subsection{Flexibel erscheinende Tradierungslogiken}

„Und wenn das Geld knapp wurde, war eigentlich meine Oma immer da, wo ich hingehen konnte, wo ich mich dann auch getraut habe und wo ich gewusst habe, da krieg ich es" (II 34:30-34:39). Die Großmutter ist für ihn eine verlässliche und zentrale Ansprechpartnerin, der er vertraut; Indizien für ein nahes Großmutter-Enkel-Verhältnis.

Neben der Großmutter sind die Eltern wichtige Bezugspersonen. Sowohl Großmutter als auch Eltern hätten ihn immer unterstützt (II 32:43-33:37), wodurch Herr Cronert die Beziehung zu den Eltern insgesamt als positiv einordnen kann. Auch wenn sich aus verschiedenen Beispielen eine emotionale Verbundenheit zwischen den Generationen annehmen lässt, etwa wenn Herr Cronert sich erinnert, die Mutter habe ihn als Kind „ganz gerne“ zu Besorgungen mitgenommen oder die Eltern hätten sich über die gemeinsamen Sonntagsausflüge gefreut (II 46:29-46:58 und 49:32-50:08), wird die emotionale Seite der Beziehungen von Herrn Cronert nicht ausdrücklich als solche thematisiert. Das ist ein Hinweis, dass es sich hier für Herrn Cronert eher um ein Tabuthema handeln könnte.

Während der Kindheit habe es einige Regeln und Maximen gegeben. $\mathrm{Zu}$ diesen zählten die obligatorischen Sekundärtugenden Sauberkeit und Ehrlichkeit, der regelmäßige Kirchgang, Erledigung der Schularbeiten und Pünktlichkeit (II 45:08-46:03, 40:56-41:04, 36:07-37:35). Auf die Einhaltung dieser Regeln sei geachtet worden und die Eltern hätten Orientierungen wie Ehrlichkeit auch an sich selbst als Maßstab angelegt (II 50:55-51:31): „Wichtig is, äh, dass ich den Eindruck (.) immer bei meinen Eltern gehabt habe, dass sie ehrlich zu uns waren, //hmh// dass sie das, was sie gesagt haben, dazu gestanden haben", auch gegenüber Dritten (II 52:33-52:48). Sie ermuntern die Kinder, ihnen gegenüber ehrlich zu sein und sich ihnen anzuvertrauen:

„Auch wenn wir irgendwas gemacht hatten, äh das haben die immer gesagt, da könnt ihr jederzeit nach Hause kommen, äh aber wir wollen die Wahrheit wissen, wie es war. //hmh// Und äh (.) dann standen die da auch zu und haben dann auch versucht, äh das zur rechtfertigen gegenüber Dritten oder wenn irgendwelche Streitereien waren, wie das so geht mit Kindern, $/ / \mathrm{hmh} / /$ oder wir irgendwo mal was (wer weiß, was) weiß ich, weil wir Ältere geärgert haben oder so, das is ja wie das bei Kindern is. U:nd äh sich dann beschwert haben bei den Eltern, ja dann haben wir das gesagt und dann wurde das mit ernsten Worten, haben gesagt, ,na ja, so geht's nich ganz““ (II 41:08-41:52).

Solange die Kinder sich an der Leitlinie orientieren, greifen die Eltern auf eine differenzierte Strategie zurück, um ihnen Rückhalt zu bieten. Sie verteidigen ihre Kinder, sind aber auch bereit, ein kindliches Fehlverhalten einzugestehen. Sie versuchen durch Kompromisse einen Ausgleich herzustellen und das Problem auf diese Weise zu lösen, ohne starr auf einem Standpunkt zu beharren. Die angebotene Unterstützung ist eine wichtige Komponente, um sowohl die Rahmung der intergenerationellen Solidarität als auch die der Ehrlichkeit erfolgreich an die Kinder zu vermitteln. 


\section{Familiale Tradierungsprozesse in einer Drei-Generationen-Perspektive}

Insgesamt entsteht der Eindruck, Herr Cronert habe eine Kindheit erlebt, in der der kindliche Aktionsradius und bestimmte Verhaltensstandards grob abgesteckt gewesen seien, ohne dass der kindliche Alltag detailliert verregelt gewesen ist. Ähnliches gilt für die Machtverhältnisse, die vordergründig klar geregelt sind:

„[D]a haben wir eigentlich auch nie äh in der Richtung äh uns drum bemüht, [...] [von, C.R.] den Eltern da was zu erpressen. Denn das äh (.) war eigentlich $[\ldots]$ nich möglich, wenn die einmal gesagt hatten ,nein', dann war's auch so. //hmh// Dann gab es da äh kein Zurück mehr. //hmh// Sicher mitunter mit leichten Kompromissen, aber in der Regel war's //hmh// (dann so)“ (II 47:22-47:54).

Herr Cronert nimmt die Eltern zugleich konsequent und flexibel wahr. Dieser tendenzielle Widerspruch lässt sich dahingehend auflösen, dass die Eltern zwar den generellen Rahmen vorgegeben haben, aber gleichzeitig zu bestimmten Zugeständnissen bereit gewesen sind. Darauf verweist auch Herrn Cronerts Charakterisierung der Eltern: Sie

„[w]aren mitunter auch konsequent, $/ / \mathrm{hmh} / /$ waren aber auch äh bei bestimmten Dingen, wo sie gesagt haben, na ja, da können wir uns nochmal drüber unterhalten, und haben auch versucht über einen Kompromiss oder einen Umweg dann doch dieses oder jenes noch zuzugeben“ (II 51:01-51:20).

Die Eltern stellen wohl das grundsätzliche Regelwerk auf, sind aber teilweise bereit, die Bedürfnisse des Sohnes zu berücksichtigen, sodass er das Gefühl entwickeln kann, bis zu einem gewissen Grad Entscheidungen zu beeinflussen. In einigen Bereichen habe es auch Mitspracherechte gegeben, z.B. in Bezug auf gemeinsame Unternehmungen (II 46:20-46:34).

Auch wenn Herr Cronert zunächst den Eindruck erweckt, die elterlichen Entscheidungen jeweils akzeptiert zu haben, hält er sich nicht immer an die elterlichen Vorgaben. Er habe z.B. trotz Verbotes als Kind mit Fundmunition und ausgedientem Kriegsgerät gespielt (II 18:52-22:43). Dieser Regelverstoß ist ihm sicherlich auch aufgrund der in der Nachkriegszeit durchaus typischen, von Erwachsenen unkontrollierten Freiräume (Schütze/Geulen 1983, 33) möglich, die verhinderten, dass die Regelübertretung bemerkt wurde; zumindest solange, bis Herr Cronert sich verletzt. Das führt zwar zu Konflikten, es habe aber keine gravierenden Folgen für Herrn Cronert gehabt: „[a]ber wenn es passiert war, dann war es da dann" (II 23:01-23:04). Anscheinend sind keine weiteren Sanktionen gegen Herrn Cronert wegen der Verbotsmissachtung verhängt worden. Das sind Indizien, dass die Eltern das Wohlergehen des Kindes höher bewertet haben als die Einhaltung der von ihnen aufgestellten Verbote. Die Eltern reagieren insgesamt kontextabhängig unterschiedlich. Mittags sei Herr Cronert des Öfteren zu spät zum Essen gekommen, weil er so in Spiele vertieft gewesen sei. Die Mutter habe 


\subsection{Flexibel erscheinende Tradierungslogiken}

dann mit ihm geschimpft, es habe auch mal „ganz harte Worte gegeben“ oder sie habe gedroht, ihm kein Essen aufzuheben, habe das aber nie umgesetzt. Hierauf scheint Herr Cronert auch gesetzt zu haben, da er gewusst habe, dass seine Mutter nicht die „Allerhärteste“ gewesen sei (II 43:53-44:57). An diesem Beispiel werden zwei Aspekte deutlich. Zum einen weiß Herr Cronert, in welchem Umfang und in welchen Bereichen ihm Spielräume für abweichendes Verhalten zur Verfügung stehen, die er auch nutzt. Er ist praktisch derjenige, der mit Tolerierung durch die Mutter für eine Flexibilisierung der Vorgaben gesorgt hat. Folglich kann er zumindest in einem gewissen Umfang das elterliche Erziehungssetting als flexibel wahrnehmen. Zum anderen ist erkennbar, dass die Mutter ihre Anweisungen nicht autoritär durchsetzt, sondern zumindest implizit die Wünsche und Regelübertretungen ihres Sohnes akzeptiert. Die kindlichen Bedürfnisse werden berücksichtigt und das Verhalten der Mutter ist kindorientiert. Auch das sind Aspekte, die dazu beitragen, das Familienleben als positiv zu erleben und in der Folge die elterlichen Orientierungen zu übernehmen.

Während die Mutter trotz „harter Worte“ dennoch letztlich als nachgiebig beschrieben wird, wird der Vater als konsequenter erlebt. Einmal habe er auf dem Weg zur Arbeit Herrn Cronert dabei überrascht, wie er heimlich mit Freunden geraucht habe (42:04-43:28). Der Vater habe sich mit den Worten verabschiedet: „Wir sprechen uns dann heute Abend“ (II 42:48-42:51). Abends habe sich der Vater dann mit seinem Sohn auseinandergesetzt: „Dann hat er mich da ins Gebet genommen. Ich habe da keinen Schlag bekommen, [...] aber ernsthaft ins Gebet genommen. Und ich habe das auch eingesehen dann" (II 42:52-43:03). Hier deutet sich ein nahezu klassisches Bild der Erziehungsaufteilung an: Der Vater als durchgreifende Autoritätsperson, die für die Ahndung von Regelverstößen zuständig ist, wohingegen die Mutter nicht ganz so strikt agiert. Herr Cronert betont im Zusammenhang mit seinen kindlichen Regelverstößen, nicht körperlich bestraft worden zu sein: „Aber ansonsten äh muss ich sagen, ich habe eigentlich äh keine Prügelstrafe von zu Hause gekriegt, auch mein Bruder nich. $/ / \mathrm{hmh} / / \mathrm{Da}$ waren meine Eltern eigentlich äh nich für eingestellt und da standen die über der Sache" (II 41:53-42:03 und 43:31-43:45). Wie die Formulierung nahelegt, betrachtet Herr Cronert körperliche Strafen während seiner Kindheit durchaus noch als ein gängiges Erziehungsmittel. Der einschränkende Zusatz „eigentlich“ verweist darauf, dass die Eltern vermutlich generell Konflikte mit den Kindern anders gelöst haben, ohne unbedingt konsequent auf körperliche Strafen verzichtet zu haben. Körperliche Strafen sind aber zumindest für Herrn Cronert, wenn überhaupt, eher Ausnahmen gewesen, sodass er die intergenerationellen Beziehungen nicht als gewalttätig, sondern eher als am kindlichen Wohlbefinden orientiert wahrnehmen kann, was für die Aneignung der elterlichen Vorgaben förderlich gewesen sein dürfte.

Als prägend für seine Kindheit betrachtet Herr Cronert die Religiosität. Sie sei „ein bewusster Ausdruck für unser ganzes Leben auch als Kinder" gewesen (II 39:18-39:23). Die Großmutter und die Eltern seien regelmäßig zur Kirche gegangen und haben hierdurch diese Facette der Religiosität den Kindern praktisch vorgelebt. Für die Kinder sei der Gottesdienstbesuch obligatorisch gewesen, und es hätte außer im Krankheitsfall 


\section{Familiale Tradierungsprozesse in einer Drei-Generationen-Perspektive}

keine Ausnahmen gegeben. Die Gottesdienstteilnahme stellt für ihn eine früh eingeübte, verpflichtende Routine dar (39:02-40:23). Zusätzlich sei er noch als Messdiener tätig gewesen (37:12-37:14). Das religiöse Milieu seiner Kindheit entfaltet offenbar eine stark normierende Kraft. Auch in das weitere familiale Umfeld werden die Kinder durch die regelmäßig stattfindenden Familienfeiern eingebunden, die Herr Cronert auf Wunsch der Eltern zumindest bis zur Volljährigkeit besucht und die er in positiver Erinnerung hat (II 24:25-27:06). Die wechselseitigen familialen Einladungen sind ein Mittel, über das zugleich die Familiengemeinschaft gestärkt wird. Die Eltern achten somit auf eine doppelte Einbindung in das familiale und religiöse Netzwerk. Hierin ist ein geschickt gewähltes Mittel zu sehen, um eine Orientierung der Kinder an den elterlichen Vorgaben zu unterstützen - zumal Herr Cronert diese Einbindung als selbstverständlich ansieht.

Aus den Äußerungen Herrn Cronerts spricht insgesamt eine positive Grundeinstellung, die er auch seinen Eltern zuschreibt. Die Eltern hätten es in Zusammenarbeit mit der Großmutter verstanden, die Versorgung der Familie sicherzustellen; die Kinder seien sowohl mit dem Notwendigen als auch darüber hinaus mit Spielzeug versorgt gewesen (II 00:01-04:14). Wie die vorgestellten Interviewpassagen zeigen, reagieren die Eltern auf Probleme im Erziehungsalltag mit einer gewissen Pragmatik und verhalten sich stärker lösungs- als prinzipienorientiert. Insgesamt werden sie eher als aktiv und eigeninitiativ denn als passiv charakterisiert.

Zusammenfassend ist das Aufwachsen für Herrn Cronert durch das Erleben intergenerationeller Solidarität und die Existenz eines insofern klaren Regelwerks gekennzeichnet, als dieses den Rahmen absteckt, innerhalb dessen Herrn Cronert Freiheiten und Eigenständigkeiten zugebilligt werden. Großmutter und Eltern nimmt er als unterstützend und an der Entwicklung der Kinder interessiert wahr. Hierbei sind die Eltern in einem gewissen Maße bereit, auf die Bedürfnisse und Wünsche der Kinder einzugehen. Sie beharren nicht dogmatisch auf ihren Standpunkten, sondern sind eher pragmatisch und lösungsorientiert. Weitere wichtige Rahmungen sind die Religiosität sowie als wesentliches Merkmal der Eltern deren Aufrichtigkeit. All diese Aspekte werden von Herrn Cronert bei der Erziehung der eigenen Tochter aufgegriffen.

\section{Vermittlungsebene Herr Cronert $\left({ }^{*} 1936\right)$ : Arbeitsteilung und situative Kompro- missbereitschaft Die intergenerationelle Zusammenarbeit in Form der Einbindung der eigenen Eltern in die Erziehung der Tochter betrachtet Herr Cronert explizit als ein besonderes Kennzeichen seiner Familie, das in der Generationenfolge weitergege- ben wird:}

„Unser Kind ist dann, hatte ich schon gesagt, hier aufgewachsen bei der Oma, bei meiner Mutter dann $/ / \mathrm{hmh} / /$ mehr oder weniger. Und äh wir haben im Wesentlichen das, was vorher meine Großmutter gemacht hat, war dann meine Mutter für unsere Tochter. //hmh// Sodass wir im Nachgang praktisch die eine Generation, die dann verstorben war, an der Stelle 


\subsection{Flexibel erscheinende Tradierungslogiken}

dann meine Mutter oder meine Eltern $/ / \mathrm{hmh} / /$ getreten sind, um dann auch für uns als Familie da zu sein. //hmh// Für uns als Sohn und Schwiegertochter wie auch für unsere Tochter“ (II 04:33-05:15).

Die Einbeziehung der großelterlichen Arbeitskraft in das Familienleben der jüngeren Generation wird quasi nicht als freiwillige Leistung, sondern als selbstverständliche Pflicht der älteren Generation skizziert. In der Familie kommt eine „Ketten-generalisierte-Reziprozität“ zum Tragen: Der Empfänger einer Gefälligkeit erbringt im Austausch nicht für die gebende, sondern für eine dritte Person eine Leistung (Hollstein 2005, 199). Diese Form der Reziprozität ist ein Instrument, das zur Entlastung der Beziehungen zwischen gebender und nehmender Generation beitragen kann. Im Falle der Familie Cronert/Jäger hat das den Vorteil, dass der unmittelbare Verpflichtungscharakter für die empfangende Generation aufgehoben und auf zukünftige Leistungen für eine nachfolgende Generation projiziert wird. Zugleich entwirft Herr Cronert ein Bild vom Alter, das nicht durch nachlassende Kräfte oder Passivität gekennzeichnet ist, sondern durch Aktivitäten und Dienste, die für die jüngere Generation unmittelbar wertvoll sind. In Weiterführung seines Arguments übernehmen Herr Cronert und seine Frau nach der Familiengründung der Tochter die Betreuung der Enkelinnen (II 10:42-11:24, 1:11:34-1:12:47). Die Zeit mit diesen erlebt er als Bereicherung. Ein weiteres Charakteristikum, das von der Kindheit bis zur eigenen Großelternzeit Herrn Cronerts Gültigkeit hat, ist die klare Regelung der Machtstrukturen zwischen Großeltern und Eltern, was die Erziehung betrifft:

„Wobei wir nicht die Pflicht der Eltern [...] übernehmen. //hmh// Dann sagen wir schon, wenn irgendwelche Dinge zur Diskussion kommen und Fragen kommen, ob sie was machen können oder nicht. Wo wir dann sagen: Das können wir nich entscheiden. [...] Also die Elternpflicht übernehmen wir nicht. //hmh// Das war nie so. Auch bei uns früher nicht. $/ / \mathrm{hmh} / /$ Also das is schon. $/ / \mathrm{hmh} / /$ Das letzte Wort wird da gesprochen“" (II 1:16:43-1:17:32).

Dieser Grundsatz ist ein nützliches Instrumentarium, um das Konfliktpotenzial zwischen den beiden erziehenden Generationen gering zu halten und zugleich das Prinzip der familialen Solidarität zu stützen. Durch das generelle Setting, in dem die Großeltern einen aktiven Part bei der Erziehung ihrer Enkel einnehmen, wird der Bezugsrahmen der intergenerationellen Solidarität und Zusammenarbeit auch für die Tochter Herrn Cronerts, Friederike, unmittelbar erfahrbar, sodass auch hier das Vorleben als ein wichtiges Instrument zur Vermittlung des familialen Bezugsrahmens betrachtet werden kann.

Seine Mutter habe sich ähnlich verhalten wie ihrerzeit seine Großmutter und habe genauso eine Vermittlerrolle zwischen Eltern und Kind eingenommen, indem sie möglichen Konfliktpunkten im Vorfeld durch Interventionen vorgebeugt habe (II 35:20- 


\section{Familiale Tradierungsprozesse in einer Drei-Generationen-Perspektive}

35:48). Herr Cronert habe manchmal erst mit zeitlichem Abstand von etwaigen Verfehlungen der Tochter erfahren (II 1:00:11-1:00:38). Da es sich um vergangene Situationen handelt, fühlt er sich nicht veranlasst, nachträglich zu intervenieren. Er gibt sich gelassen und belässt seiner Mutter Entscheidungsspielräume.

Auch wenn Herr Cronert nach eigenem Bekunden weniger in die alltäglichen Routinen der Erziehung involviert ist (II 59:26-59:29), für die hauptsächlich Großmutter und Mutter zuständig sind (II 1:03:27-1:04:39), sieht er sich dennoch aktiv bei der Festund Umsetzung der erziehungsrelevanten Eckkoordinaten beteiligt. Hierbei handelt es sich weniger um konkret ausformulierte Regeln als vielmehr um elterliche Grundhaltungen. Zunächst ist das einmal das Verantwortungsgefühl gegenüber seiner Familie (II 55:10-55:55:24). Es schließt sich die allgemeine Einstellung an, der „Tochter, das was möglich war zu geben, //hmh// alles zu geben. Ganz egal in welcher Richtung" (II 55:49-55:58). Ungeachtet des recht unkonkreten Charakters der Aussage spricht hieraus dennoch das Anliegen, das eigene Kind so weit wie möglich zu fördern, zu unterstützen und ihr ihre Wünsche zu erfüllen, materiell wie immateriell.

Im Vergleich zur eigenen Kindheit legt Herr Cronert offensichtlich einen stärkeren Fokus auf die schulische Ausbildung. Er und seine Frau hätten ihre Tochter ermuntert, nach guten schulischen Leistungen zu streben (II 57:13-57:49). Herr Cronert schlägt seiner Tochter vor, im Anschluss an ihre Ausbildung zu studieren, was diese jedoch ausschlägt. Auch wenn Herr Cronert mit diesem Entschluss der Tochter unzufrieden gewesen sei, habe er sie nicht unter Druck gesetzt, sondern die Entscheidung der Tochter akzeptiert (II 57:50-58:06). Er selbst erklärt sich die Entscheidung der Tochter mit geschlechtsrollenstereotypen Vorstellungen, indem er darauf verweist: „wie das dann so is bei jungen Mädchen" (II 58:14-58:16); wegen ihres Freundes habe sie nicht wegziehen wollen (II 58:07-58:21; 1:08:26-1:08:59). Mit dieser Begründung, mit der Herr Cronert das töchterliche Verhalten quasi als ,natürlich' darstellt, entschärft er den potenziellen Konflikt.

Herr Cronert betrachtet den regelmäßigen Gottesdienstbesuch der Tochter als selbstverständlich (II 40:25-40:36). Ähnlich wie in der Kindheit Herrn Cronerts kommt die Tochter so mit einem religiösen Milieu in Berührung, das über den Familienkreis hinausreicht. Die Tochter durch regelmäßige Verwandtenbesuche in das weitere familiale Netzwerk einzubinden, gestaltet sich für Herrn Cronert als etwas schwieriger, da der Wohnort der Familie nach Gründung der DDR zum Sperrgebiet wird und Besuche von nicht Ortsansässigen erschwert werden. Die Cronerts bemühen sich aber darum, die Kontakte zur Verwandtschaft so gut wie möglich aufrechtzuerhalten (II 06:51-07:55, 1:23:12-1:24:19).

Auch wenn die alltäglichen Erziehungsroutinen im Wesentlichen in die Zuständigkeit seiner Frau gefallen seien, habe Herr Cronert in Konfliktsituationen in Absprache mit seiner Frau die Tochter zur Rede gestellt (II 1:06:44-1:08:03). Generell habe die Tochter bei ihm "ein bisschen mehr Kredit“ gehabt als bei seiner Frau, die konsequenter in 


\subsection{Flexibel erscheinende Tradierungslogiken}

der Erziehung gewesen sei (II 1:09:52-1:10:25). Hier zeigt sich ein weiteres Mal die Flexibilitätsbereitschaft Herrn Cronerts, die er selbst an anderer Stelle expliziert:

„Da: waren wir, (1) denke ich mal, ein bisschen flexibel. Haben gesagt, na ja wenn's nich so, dann müsst ihr (es) so angehen. //hmh// Wobei, äh wir das Ende zwar immer gesehen haben, aber nun nich unbedingt äh gesagt haben, wir müssen da gerade durch. //hmh// Das haben wir dann auch schon mal gesagt. $\mathrm{Na}$ ja, da können wir auch schon mal nach rechts oder nach links //hmh// gehen, aber das: Ziel haben wir eigentlich nich aus 'm Auge verloren" (II 1:06:04-1:06:34).

Die Generationenbeziehungen zwischen Eltern und Tochter sind gemäß der Erzählung Herrn Cronerts gut, und der Tochter wurden offenbar viele Freiräume gelassen, zumindest seitens Herrn Cronerts. Die emotionale Seite der Beziehungen wiederum thematisiert Herr Cronert kaum.

Als Großvater ist Herr Cronert - dem familialen Bezugsrahmen entsprechend - aktiv in die Erziehung der Enkelkinder eingebunden und partizipiert stärker am Alltag der Enkel als seinerzeit am Alltag der Tochter:

„[D]as möchte ich vielleicht auch erwähnen, die Frage der Enkelkinder, den Eindruck habe ich jedenfalls, die is mir, liegt mir näher, $/ / \mathrm{hmh} / /$ und ich weiß eigentlich von der Kindphase von den Enkelkindern mehr, wie ich derzeit von meinem eigenen Kind gewusst habe“ (II 1:11:42-1:12:00).

Es gefällt Herrn Cronert, in den Alltag der Enkelkinder eingebunden zu sein. Die Beziehungen zu den Enkeln beschreibt Herr Cronert als gut: „Wir haben (.) eigentlich bei den Enkelkindern 'nen ganz guten STAND“ (II 1:14:25-1:14:30). Die Enkelkinder würden auf die Großeltern hören und ihnen von sich aus von ihrem Alltag erzählen; es gebe keine Geheimnisse (II 1:16:18-1:16:42, 1:21:48-1:22:05). Als Großeltern seien sie gegenüber ihren Enkeln etwas großzügiger als die Eltern, was Herr Cronert als selbstverständlich betrachtet (II 1:14:34-1:14:46). In einigen Dingen hätten die Enkel andere Einstellungen, was Herrn Cronert aber nicht stört:

"Ja, die sind moderner. Das fängt mit der Musik an $/ / \mathrm{hmh} / /$ und hört mit dem ganzen Leben und mit dem Anziehen und jeder Sache (.) $/ / \mathrm{hmh} / /$ hört das auf. $/ / \mathrm{hmh} / /$ Und das sehe ich ein. Die können mit 19 noch nich Opa sein. //hmh// Oder Oma. //Ja// Da müssen sie schon, da bin ich eigentlich auch immer so eingestellt gewesen, dass ich gesagt habe, die Jüngeren müssen eine andere Meinung haben wie die Oma oder der Opa. //hmh// Wenn das nich wäre, dann würde die Welt ja stehen bleiben. Das kann doch nich wahr sein“ (1:20:47-1:21:21).

Herr Cronert nimmt deutlich eine Generationendifferenz wahr, die ihm zugleich als Argument dient, um unterschiedliche Ansichten anzuerkennen und das Konfliktpotenzial 


\section{Familiale Tradierungsprozesse in einer Drei-Generationen-Perspektive}

einzudämmen. Zudem drückt Herr Cronert in dieser Passage seinen Willen aus, flexibel zu sein und nicht dogmatisch auf der Richtigkeit seines Standpunktes zu beharren.

Seinen Enkeln möchte er neben den Tugenden Fleiß und Ehrlichkeit eine lebenslustige und positive Einstellung vermitteln (1:17:43-1:19:37). Hier orientiert sich Herr Cronert offensichtlich an seinen eigenen Erziehungserfahrungen und möchte die an ihn herangetragenen Orientierungen an seine Enkel weitergeben. Interessanterweise erwähnt Herr Cronert die Orientierung der Ehrlichkeit bei der Erziehung seiner Tochter nicht. Möglicherweise stellt sie für ihn in Bezug auf die eigene Familienphase eine Selbstverständlichkeit dar, die nicht weiter erwähnenswert ist.

\section{Aneignungsebene Friederike Jäger $\left({ }^{*} 1969\right)^{148}$ : Im Zentrum der Aufmerksamkeit} In Friederike Jägers Kindheits- und Jugendgeschichte nehmen die Großeltern ebenfalls einen prominenten Platz ein. Sie sei „mehr oder weniger die meiste Zeit“ (I 01:15-01:18) bei ihren Großeltern gewesen, womit sie für Friederike zentrale Bezugspersonen im Alltag sind. Mit dem Großvater sei sie oft im Garten gewesen, der ihr viele Spielmöglichkeiten geboten habe (I 02:01-02:28). Die Großmutter habe mittags für sie gekocht und viel Zeit mit ihrer Enkelin verbracht (I 08:03-08:12). Die Großeltern integrieren sie in ihren Alltag, ohne auf ausgeklügelte pädagogische Lernumwelten zurückzugreifen.

Ähnlich wie ihr Vater schreibt Friederike Jäger insbesondere der Großmutter eine Vermittler- und Beschützerrolle gegenüber Dritten zu. Sie kann sich nicht erinnern,

„[d]ass die [Großmutter, C. R.] //hmh// jemals mit mir geschimpft hätte. Die hat wirklich alle Hände über mich gehalten: ,Lasst das Kind in Ruhe. $/ / \mathrm{hmh} / /$ Wenn ich denn wirklich mal, was gewesen war, was ich verbrochen hatte oder wie auch immer: ,Das hat sie ja nich so gemeint. Also lasst sie. //hmh// Das is ja nun vergessen und vergeben“" (I 41:21-41:38).

Intergenerationelle Solidarität und Hilfe durch die Großeltern sind im Alltag von Friederike Jäger präsent und haben im Vergleich zur vorherigen Generation an Intensität zugenommen. Die den Enkeln entgegengebrachte Aufmerksamkeit verstärkt sich im Generationenverlauf. Die gelebte Praxis stellt dementsprechend ein wesentliches Element dar, um den familialen Bezugsrahmen zu tradieren. Die kompromisslose Unterstützung durch die Großmutter dürfte zudem dazu beitragen, die Erziehung als flexibel und kindorientiert wahrzunehmen.

Die Eltern wiederum hätten unterschiedlich auf Fehlverhalten ihrer Tochter reagiert. Während die Mutter gelegentlich ungehalten gewesen sei (II 35:45-35:47), habe der Vater sie eindringlich ermahnt: „Hatte dann so 'nen eigenartigen@Tonfall, weiß ich nich@, [...] jetzt wenn der was sagte, oh, dann war es wirklich hart, was du jetzt durchgezogen hast“" (II 35:25-36:16). Friederike Jäger erlebt ihren Vater, der nur in Extremfällen einschreitet, als gelassener im Vergleich zur Mutter. Das hängt wahrscheinlich damit

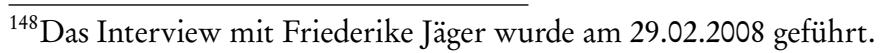




\subsection{Flexibel erscheinende Tradierungslogiken}

zusammen, dass der Vater nicht ganz so stark in den Erziehungsalltag eingebunden ist. Wenn er interveniert, scheint er sich ähnlich zu verhalten wie der eigene Vater und die Tochter ernsthaft zurechtzuweisen.

Die Großmutter nimmt für Friederike Jäger die Rolle einer weisen Ratgeberin ein:

„Ich weiß nicht, wenn ich mich in der Schule über irgendwas geärgert hab, oder (.) in der Lehre nachher. Dann hat sie immer nur gesagt: ,Reg dich nich auf. Wer weiß, wozu es gut is.' $/ / \mathrm{hmh} / /$ Und sie hatte Recht. Sie hatte jedes Mal Recht." (I 46:00-46:14).

Sie erlebt ihre Großmutter als verlässliche und ihr zugewandte Ansprechpartnerin. Mit ihrer gelassenen und abwägenden Haltung demonstriert die Großmutter Flexibilität und wirkt dadurch beruhigend auf ihre Enkelin ein. Kommunikation und argumentative Bearbeitungen sind zentrale Bestandteile der Erziehung, die offenbar im Umgang mit Friederike Jäger in einem stärkerem Maße zum Zuge kommen als noch in der Generation zuvor. Das verweist auf veränderte intergenerationelle Machtverhältnisse, in deren Folge kommunikative Überzeugungstrategien an Bedeutung gewinnen und Gehorsam nicht mehr selbstverständlich erwartet wird.

Dem kommunikativen Austausch widmet Friederike Jäger mehr Aufmerksamkeit als seinerzeit ihr Vater:

"U:nd weiß ich nich. Ich bin auch gerne mit ihr [der Großmutter, C.R.] unterwegs gewesen, oft mit ihr unterwegs gewesen, ja und einfach nur sitzen und reden, das haben wir auch stundenlang gekonnt. //hmh// Auch nachher wo ich schon verheiratet war" (I 42:14-42:30).

Die Großmutter wird als emotional unterstützend, ausgleichend und wenig reglementierend beschrieben, in deren Gegenwart Friederike Jäger sich wohlfühlt. Die Enkelin sieht in ihr eine Autorität, wobei die Großmutter diese nicht ausspielt. Konfliktlinien zwischen Großmutter und Enkelin sind nicht erkennbar. Insgesamt zeichnet Friederike Jäger ein klassisches Großelternbild: verwöhnend und nicht fordernd (Höpflinger/ Hummel/Hugentobler 2006, 18 und 20). Diese Konstellation dürfte es ihr erleichtert haben, den von ihr erlebten familialen Bezugsrahmen der intergenerationellen Solidarität als selbstverständlich anzunehmen. Darüber hinaus ist damit keine unmittelbare Gegenleistung verknüpft. Friederike Jäger profitiert während ihrer Kindheit von diesem Arrangement, ohne selbst direkt mit einer Forderung konfrontiert zu sein. Indirekt erbringt sie dennoch eine Gegenleistung in Gestalt der den Großeltern entgegengebrachten Zuneigung und Wertschätzung, sodass durchaus von einer Reziprozität der Generationenbeziehungen gesprochen werden kann.

Friederike Jäger sieht sich im Zentrum der elterlichen und großelterlichen Aufmerksamkeit, die bestrebt sind, die Konsumwünsche der jüngsten Generation zu realisieren: „Und ich denke meine Eltern und auch meine Großeltern, [...] die haben eigentlich immer versucht, mir alles zu ermöglichen. //hmh// Egal, und wenn das noch so 'n skurriler Wunsch gewesen is, sie haben's halt versucht“"(I 08:36-08:55). Die eingeschränkten 


\section{Familiale Tradierungsprozesse in einer Drei-Generationen-Perspektive}

Konsummöglichkeiten in der DDR münden nicht in Resignation. Vielmehr werden Probleme eigeninitiativ in intergenerationeller Zusammenarbeit bewältigt. Die Großmutter habe z.B. in der BRD Jeansstoff gekauft, den die Mutter in eine Schneiderei gebracht habe, damit Friederike Jäger eine Jeans nach westlichem Vorbild habe anziehen können (II 19:06-20:51). Das Engagement für die jüngere Generation ist eine zentrale Facette im familialen Erziehungsarrangement. Eltern und Großeltern verbinden damit keine Forderungen, was die starke Kindzentrierung unterstreicht und die Aneignung des familialen Bezugsrahmens fördern dürfte.

Allerdings gibt es auch Grenzen der Großzügigkeit. Die Frage nach einem mädchengerechten Verhalten birgt tendenziell Konfliktpotenzial. Für Friederike ist das im Kern eine Auseinandersetzung um geschlechtsrollenstereotype Zuweisungen und Anforderungen. Die Maxime habe gelautet: „[D]as Mädchen muss ein Mädchen bleiben. Und möglichst pink tragen oder rot“ (II 27:58-28:03). Das bezieht sich auch auf die Spiele und das Verhalten:

„Wie gesagt, ich hatte schöne Puppen, //hmh// auch viele Puppen, aber dann doch lieber 'ne @Katze im Puppenwagen@. //hmh// Joa, oft kaputte Knie. ,Mä:DCHEN' haben sie immer gesagt, ,MACH doch langsam. Du musst doch nich mit den Jungens mithalten können', ne. //hmh// ,Spiel doch mal mit dem Puppenwagen. Du hast so schöne Puppen. Spiel doch mal Friseur', oder so, ne. Das war dann so $\mathrm{hm} / / \mathrm{hmh} / /$ richtig typisch Mädchen eben, ne. Aber da konnte ich nich so: viel anfangen. Ich bin lieber auf Bäume geklettert und hab Fußball gespielt. @(.)@//hmh//Fand ich toller. Das is=liegt sicherlich auch daran, weil eben rundrum nur Jungen waren“(II 11:19-11:59).

Die Erwachsenen versuchen Friederike Jäger in eine bestimmte Richtung zu lenken, allerdings nicht besonders energisch. Sie beschränken sich im Wesentlichen auf Vorschläge und sanfte Ermahnungen, verbieten ihr aber nicht, sich über die geschlechtsrollenstereotyp konnotierten Spielanregungen hinwegzusetzen. In der Folge können die von den Erwachsenen gesetzten Maßstäbe von Friederike Jäger in der Praxis als nicht absolut gültig, sondern als formbar und flexibel empfunden werden. Sie orientiert sich in ihren Spielen stärker an ihrer Peergroup als an den Vorstellungen der Eltern. Friederike Jäger betont im Zusammenhang mit ihrer Kindheit besonders die mit körperlicher Aktivität verbundenen Spiele im Freien, was als eine Gegenreaktion auf die an sie herangetragenen Erwartungen interpretiert werden kann. Sie habe hervorragend Fußball spielen können und sich in ihren Spielen an so „typische[n] Jungssachen“ orientiert: „Messer schnitzen, und Pfeile und Bogen, und [...] Zwille und Erbsengewehr, und das gehörte eben alles dazu" (I 48:21-49:39). Friederike Jäger beschreibt sich als Kind, das sich aktiv die Umwelt aneignet und kleinere Risiken nicht scheut. Damit entwirft sie praktisch ein Gegenbild zu den explizit und implizit an sie herangetragenen Geschlechtsrollenklischees. Unterstützung erhält sie dabei von ihrem Großvater: 


\subsection{Flexibel erscheinende Tradierungslogiken}

„Und mein Opa kann ich mich erinnern, der hat mir alles Mögliche gebaut. Stelzen (.) kann ich mich erinnern. Hab ich 'n paar Stelzen bekommen $/ / \mathrm{hmh} / /$ zum Laufen. Oder, ich weiß nich, was hat er noch gemacht? Stelzen, 'ne Zwille (.) so richtig $/ / \mathrm{hmh} / /$ zum Schießen, Erbsengewehre“ (I 42:50-43:10).

Für den Großvater sind vermutlich die eigenen Vorstellungen von begehrenswertem Spielzeug sowie die Wünsche der Enkelin handlungsleitend. Der Enkelin eine Freude zu bereiten, überlagert eine potenzielle Orientierung an Geschlechtsrollenklischees und bringt zugleich seine starke Kindzentrierung zum Ausdruck. Der Großvater unterwandert somit in der Praxis die an seine Enkelin herangetragenen Rollenzuschreibungen.

Die Frage der angemessenen Kleidung sowie des Umgangs mit der Kleidung wird weniger tolerant gehandhabt. Konflikte habe es dabei vor allem mit der Mutter gegeben. Besonders lebhaft erinnert Friederike Jäger ihre Abneigung gegen bestimmte Kleidung:

„@Ein paar knallrote Lackschuhe@ [...] Ich konnt die Schuhe nich leiden, @aber ich musste die ständig anziehen. Ich fand die so schrecklich@ [...]. Aber meine Mutter war immer der Meinung, die passen schön und die werden angezogen mit 'ner schönen weißen Strumpfhose. Bloß ich hatte immer das Problem, die weiße Strumpfhose war bei mir nich@lange weiß@. [...] Und dann hab ich ein Kleid gehabt, [...] da hab ich mich immer gefühlt, wie eingepresst“ (II 28:16-29:14).

Friederike Jäger widersetzt sich vehement den strikten mütterlichen Vorgaben, die ihre Wünsche nach Bequemlichkeit ignorieren, und räumt der Bewegungsfreiheit Vorrang vor dem achtsamen Umgang mit der Kleidung ein. Sie sei z.B. öfter mit Schuhen in den nahe gelegenen Bach gegangen, was für großen Ärger gesorgt habe, da Schuhe teuer und zudem Mangelware gewesen seien (II 29:43-30:42). Allerdings kommt ihr bei diesen Konflikten die Großmutter - entsprechend ihrer Rolle als Beschützerin - zur Hilfe: „Ach', hat meine Oma dann gesagt, ,wir trocknen die [Schuhe, C.R.] wieder und dann cremen wir die schön ein mit Schuhcreme und dann geht das auch wieder"“ (II 30:48-30:55). Die Großmutter als Verbündete ihrer Enkelin unterläuft die mütterlichen Bestrebungen einer geschlechtsrollenstereotypen Erziehung aus emotionaler $\mathrm{Zu}$ gewandtheit zur Enkelin. Die mütterlichen Erwartungen haben zwar einerseits einen relativ starken Aufforderungscharakter und wirken recht starr, andererseits sorgen aber gerade die Großeltern mit ihrem Verhalten im Zusammenspiel mit den Handlungen der Enkelin für eine Durchbrechung und Flexibilisierung dieser statischen Anforderungen.

Obwohl Friederike Jäger sich gegen ein als ,mädchenhaft ${ }^{t}$ empfundenes Verhalten in der familialen Sphäre wehrt, hindert es sie nicht daran, geschlechtsrollenstereotype Vorstellungen als Argumentationshilfe gegen die in der Schule obligatorische Zivilverteidigung heranzuziehen, die für sie offenbar eine Grenzüberschreitung darstellt. Sie habe nie verstanden, dass sie als Mädchen an diesen Veranstaltungen habe teilnehmen und die diesem Anlass entsprechende Kleidung habe tragen müssen (I 27:35-29:26; 30:50-31:15). 


\section{Familiale Tradierungsprozesse in einer Drei-Generationen-Perspektive}

Hier ist eine doppelte Ablehnung erkennbar: Friederike Jäger möchte sich weder an traditionellen Weiblichkeitsentwürfen noch am staatlichen Identifikationsangebot als Gegenpol orientieren. Stattdessen strebt sie eine Zwischenposition an, weder gänzlich den alten noch den neuen Vorstellungen folgend.

Neben den beschriebenen Flexibilisierungstendenzen existieren innerhalb der Familie einige feste Regeln, z.B. bezogen auf den schulischen Bereich. Die Schulsachen hätten in einem ordentlichen Zustand sein müssen: „Super ordentlich. //hmh// Das fing mit der Handschrift an und hörte mit 'm Ranzen Packen auf" (II 26:16-26:23). Zudem wäre sie dazu angehalten worden, „[i]mmer nett und höflich“ (II 26:27-26:29) zu sein. Darüber hinaus sei „nie irgendwas erzwungen worden“ (26:42-26:52). Die hiermit umrissenen Grundregeln belassen allerdings in anderen Bereichen relativ viele Spielräume. Die ältere Generation überträgt offenbar die eigenen Erziehungserfahrungen, in denen Konventionswerte wie Ordnung und Höflichkeit eine wichtige Rolle gespielt haben, auf die nächste Generation, ohne den Kanon zu verändern. Die ältere Generation übt dabei keinerlei Leistungsdruck aus. Das scheint auch nicht notwendig gewesen zu sein. Friederike Jäger berichtet, eine sehr gute Schülerin gewesen zu sein, die ohne größere Anstrengungen einen guten Schulabschluss erlangt habe (II 24:31-24:50). Im sportlichen Bereich habe sie zudem überdurchschnittliche Leistungen erbracht und sei von den Lehrern für die Teilnahme an außerschulischen Wettkämpfen ausgewählt worden (II 31:28-32:12). Großeltern und Eltern achten zwar auf den schulischen Bereich, scheinen Bildungs- und Talentförderung aber stärker als staatliche Aufgabe zu betrachten. Interessanterweise erwähnt Herr Cronert die sportlichen Talente seiner Tochter im Interview überhaupt nicht. Das lässt sich als Indiz betrachten, dass ein ausgeprägtes Leistungsdenken bzw. die Verinnerlichung eines Wettbewerbs- und Konkurrenzprinzips in dieser Familie nicht handlungsleitend ist.

Was den eigenen Berufswunsch betrifft, sieht sich Friederike Jäger nicht von ihrer Familie, wohl aber von staatlicher Seite reglementiert. Da sie weder Parteimitglied gewesen noch zur Jugendweihe gegangen sei, habe der Staat ihr die von ihr favorisierte Ausbildung verwehrt (II 25:03-25:48, I 34:30-35:19), sodass sie eine andere Ausbildung habe wählen müssen. Die Möglichkeit eines anschließenden Studiums schlägt sie aus. Sie begründet diesen Entschluss anders als ihr Vater. Sie habe von ihrem Betrieb nicht die Zusicherung erhalten, nach dem Studium wieder zurückkehren zu können. Sie hätte theoretisch auf eine Stelle in einer anderen Region der DDR versetzt werden können (I 36:40-37:37). Das wiederum hätte die familialen Kontaktmöglichkeiten aufgrund der besonderen Wohnlage der Eltern im Sperrgebiet eingeschränkt und bringt sie letztlich dazu, auf ein Studium zu verzichten. Hieraus spricht eine ausgeprägte Familienorientierung, die als Friederike Jägers Beitrag zur intergenerationellen Solidarität gewertet werden kann. ${ }^{149}$

\footnotetext{
${ }^{149}$ Der Vater wiederum begründet die Entscheidung Friederike Jägers gegen ein Studium anders. Er hat vielleicht das Problem aufgrund seiner optimistischen und pragmatischen Grundeinstellung als lösbar betrachtet oder der Bildung an dieser Stelle eine Priorität eingeräumt.
} 


\subsection{Flexibel erscheinende Tradierungslogiken}

Elementarer Bestandteil des Familienlebens seien die gemeinsamen Mahlzeiten am Wochenende gewesen (I 01:46-01:56). Hierin lässt sich ein Mittel zur Stärkung der familialen Gemeinschaft sehen, in die auch die Großeltern mütterlicherseits einbezogen werden. Sie seien sonntags in ihrem Wohnort außerhalb des Sperrgebiets besucht worden (I 06:15-07:03). Diese institutionalisierte Besuchspraxis lässt sich als ein Versuch der Eltern lesen, trotz der staatlichen Reglementierungen die familialen Kontakte aufrechtzuerhalten und ihre Tochter in das familiale Umfeld einzubinden.

Der regelmäßige Kirchgang ist für Friederike Jäger genauso wie für ihren Vater seinerzeit eine Selbstverständlichkeit (I 08:20-08:33): „,[]der zu meiner Zeit, hatte ich immer das Gefühl, hier is eigentlich jeder katholisch, hier geht jeder in die Kirche, das is normal. $/ / \mathrm{hmh} / / \mathrm{Da}$ hat man auch gar nich drüber nachgedacht, das is eben so und das war so" (II 21:19-21:47). Neben dem familialen erlebt sich Friederike Jäger somit in ein religiöses Umfeld eingebunden. Da ähnlich wie in der Familie Dienel/Ebel starke Kongruenzen zwischen familialer Sphäre und der religiösen Umwelt anzunehmen sind, trägt das zweifelsohne dazu bei, die jeweils relevanten familialen Orientierungen auf die nächste Generation zu übertragen.

Zugleich dient die Religionszugehörigkeit Friederike Jäger als Abgrenzungskriterium, wobei die besonderen Umstände in ihrem Heimatort relevant sind. Neben alteingesessenen Bewohnern katholischen Glaubens sind zu DDR-Zeiten in dem Grenzort Soldaten stationiert worden, die in der Wahrnehmung Friederike Jägers staatstreu sind und in Opposition zum katholischen Glauben stehen (I 24:38-25:30, 37:59-38:31, II 21:55-22:15). Für sie treffen hier unterschiedliche und unvereinbare Überzeugungen aufeinander, die zu einer Separierung der Gruppen und damit letztlich zu einer Teilung des Dorfes führen. Sprachlich wird das deutlich, indem die Gruppe der anderen als Offiziere bzw. Offizierskinder klar vom eigenen „Trupp“, der „eigentlich sUPER zusammengehalten" habe, differenziert wird (I 37:59-38:09), und manifestiert sich zugespitzt in folgender Aussage:

„Es war aber eigentlich nich nur meine Eltern oder Großeltern, ich hab das von meinen Freundinnen auch immer wieder gehört [...]: , Mit denen musst du dich gar nicht abgeben. ' Das waren dann meistens immer so die Offizierskinder. $/ / \mathrm{hmh} / /$ Weil die eher Angst hatten, dass man irgendwo ausgehorcht wurde, //ja// denk ich mal, //hmh// deswegen war das sicherlich so. ,Gib dich nich mit denen ab, das bringt nix." //hmh// So, so eher 'ne Vorsichtsmaßnahme“" (II 31:14-31:39).

Die herrschenden Rahmenbedingungen führen zu einem verstärkten Zusammenschluss innerhalb des religiösen Milieus sowie einer Abschottung nach außen, in deren Folge die Orientierungen des Milieus aufrechterhalten werden sowie der familiale Bezugsrahmen der intergenerationellen Solidarität gefördert wird. Die vorgenommene Kontrolle der Sozialkontakte betrachtet Friederike nicht als Einengung, sondern vielmehr als Selbstschutz. 


\section{Familiale Tradierungsprozesse in einer Drei-Generationen-Perspektive}

Auch wenn Friederike einerseits berichtet, Eltern und Großeltern seien bemüht gewesen, ihr alle Wünsche zu erfüllen, hätten sich ihre Mitspracherechte in Grenzen gehalten: „A:ber im Prinzip //hmh// wurde für mich entschieden“ (II 32:33-32:38). Insgesamt klassifiziert Friederike Jäger die Erziehung als konservativ (II 32:49- 32:50). Dieses Urteil steht in einem scheinbaren Widerspruch zu der betonten Flexibilität des Vermittlungsarrangements. Dieser lässt sich aufösen, da es einige wenige Inhalte sind, die als konservativ zu klassifizieren sind. Sowohl Eltern als auch Großeltern hätten z.B. eine konservative Sexualmoral vertreten, der sich Friederike Jäger zwar gebeugt habe, die sie aber zumindest aus heutiger Sicht als völlig überholt betrachtet (II 33:22-34:44). Ähnliches gilt für die geschlechtsrollenstereotypen Vorstellungen sowie die große Relevanz, die ordentlichen und sauberen Schulsachen beigemessen werden. Abgesehen von diesen wenigen Grundsätzen folgt die Erziehung jedoch weniger konservativen Vorstellungen, sondern ist in vielen Bereichen situationsbedingt flexibel, wozu die Großeltern nicht unerheblich beitragen.

Zwar steht Friederike Jäger im Mittelpunkt der elterlichen und großelterlichen Sorge, es verbleiben ihr aber Spielräume, und sie steht nicht permanent unter der Aufsicht Erwachsener. Sie habe kaum in der Wohnung gespielt, sondern sei meist mit anderen Kindern „durch die Gegend gezogen“ (I 02:32-03:17). Diese Freiräume sorgen dafür, dass die ihr zuteil werdende Aufmerksamkeit und Unterstützung nicht zu einer Überbehütung führen, was es erleichtert, den familialen Bezugsrahmen der gegenseitigen Unterstützung und des Zusammenhalts anzunehmen.

Die intergenerationelle Solidarität stellt für Friederike Jäger eine zentrale Erziehungserfahrung dar, die durch die gesellschaftlichen Rahmenbedingungen zusätzlich gefördert wird. $\mathrm{Zu}$ einigen Inhalten der Erziehung wie der konservativen Sexualmoral und den geschlechtsrollenstereotypen Identifikationsangeboten baut sie hingegen eine kritische Distanz auf.

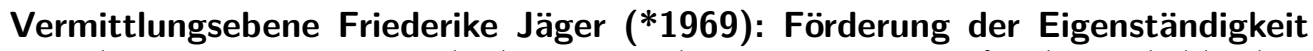
Die Eltern unterstützen Friederike Jäger, indem sie z.B. mittags für ihre Enkel kochen oder ihnen bei den Hausaufgaben helfen (II 51:30-52:20). Die intergenerationelle Solidarität ist auf diese Weise auch für die jüngste Generation im Erziehungssetting als unmittelbar erfahrbar angelegt. Friederike Jäger legt großen Wert auf gemeinsame Mahlzeiten: „[W]ir haben bestimmte Essenszeiten, $/ / \mathrm{hmh} / /$ wo alle zusammen sitzen und essen. Und DIE Zeiten äh möchte ich auch schon, dass alle da sind“ (II 54:53-55:02). Das Austragen von geschwisterlichen Konflikten ist hierbei unerwünscht (II 55:10-55:19, 57:26-58:22). Die somit quasi institutionalisierten Essenszeiten dienen zweifelsohne der Stärkung der familialen Gemeinschaft, werden doch auf diese Weise regelmäßige Gelegenheiten des Austausches geschaffen. Gleichzeitig sollen sie vermutlich als Zeitfenster der Ruhe und Entspannung ein Gegengewicht gegen den von Friederike beklagten gegenwärtigen Alltagsstress (II 37:39-39:12) bilden. Das Unterbinden geschwisterlicher Streitereien unterstreicht dieses Anliegen. Zusätzlich sind ihr gemeinsame Aktivitäten 


\title{
4.1 Flexibel erscheinende Tradierungslogiken
}

mit ihren Töchtern wichtig. Sie gehe z.B. regelmäßig abwechselnd mit der älteren bzw. jüngeren Tochter spazieren (I 10:36-10:47). Hiermit schafft sie weitere Gelegenheiten für gemeinsame Gespräche:

\begin{abstract}
„[W]enn wir jetzt einen Spaziergang machen mit dem Hund, man unterhält sich mehr, //hmh// als wenn man jetzt irgendwo sich ständig aus dem Weg geht, weil der eine Computer spielen muss und der andere Fernsehen gucken muss [...]. Finde ich nich so gut. //hmh// Also man muss sich doch schon mal unterhalten“" (II 55:21-56:02).
\end{abstract}

Friederike Jäger übernimmt ein für sie besonders positiv konnotiertes Element der selbst erfahrenen Erziehung: den kommunikativen Austausch. Hierdurch wird die familiale Gemeinschaft gefördert und zugleich die Möglichkeit geschaffen, sich mit den Kindern auszutauschen, sie zu beraten und bei der Meinungsbildung zu unterstützen. Die von Friederike Jäger eingesetzten Formen erscheinen als beiläufiges, zwangloses Angebot, das den (zumindest implizit ebenfalls vorhandenen) Kontrollaspekt überdeckt. Hierdurch kann dieses Mittel der Erziehung für die Kinder flexibel erscheinen.

Die während der gemeinsamen Spaziergänge geführten Gespräche unterstützen gleichzeitig ein weiteres Erziehungsziel. Friederike Jäger ist es wichtig, dass ihre Kinder eine „eigene Meinung haben und die auch vertreten" und nicht unreflektiert Ansichten von Gleichaltrigen übernehmen (I 13:44-14:17, II 53:09-53:22). Die gemeinsamen Gespräche sind als mütterliche Versuche zu betrachten, den Einfluss der Peergroup zu relativieren. Zugleich lässt sich an diesem Beispiel die Modifikation einer grundlegenden Orientierung im Generationenverlauf aufzeigen. Während für ihren Vater Ehrlichkeit eine wichtige Leitlinie darstellt, wird dieser Aspekt von Friederike Jäger im Zusammenhang mit ihrem Aufwachsen nicht explizit erwähnt. Er taucht allerdings in Gestalt des Unterdrückens der eigenen Meinung auf, wenn sie z.B. berichtet, in Gesellschaft von Personen, die sie als staatstreu betrachtet, lieber geschwiegen zu haben (II 09:26-09:38). Vor diesem Hintergrund einer eingeschränkten Redefreiheit ist Friederike Jägers Insistieren auf der Bildung und Artikulation einer eigenen Meinung zu verstehen. In Auseinandersetzung mit den eigenen Erfahrungen entwickelt Friederike Jäger die Leitlinie der Ehrlichkeit in spezifizierter Form der eigenen Meinung, die sie als Erwartung an ihre Kinder richtet.

Neben der unmittelbaren familialen Gemeinschaft spielt im Erziehungssetting von Friederike Jäger auch die religiöse eine Rolle. Die jüngere Tochter sei Messdienerin und besuche regelmäßig den Gottesdienst. Die ältere Tochter, Anika, könne das hingegen aufgrund anderer Verpflichtungen nicht jede Woche einrichten (II 22:25-23:08). Das wird von Friederike Jäger toleriert:

„Ich will es ihr auch nich aufzwingen, //ja, hmh// muss ich ehrlich sagen, denn das soll sie selber entscheiden. $/ / \mathrm{hmh} / /$ (2) Es macht keinen Sinn, jemanden irgendwas aufzuzwingen, $/ / \mathrm{hmh} / /$ is meine Meinung. $/ / \mathrm{hmh} / /$ 


\section{Familiale Tradierungsprozesse in einer Drei-Generationen-Perspektive}

De:nn dann kann der andere so 'ne starke Antipathie dagegen entwickeln, das macht keinen Sinn“ (II 23:09-23:29).

Auch wenn ebenso wie in den vorherigen Generationen eine Einbindung in ein religiöses Umfeld erfolgt, ist im Vergleich zur Vergangenheit eine deutliche Lockerung festzustellen. Die religiöse Praxis des Kirchgangs entwickelt sich vom Status eines selbstverständlichen, unhinterfragten Bestandteils des Alltags zur (zumindest partiell) eigenverantworteten Option. Friederike Jäger orientiert ihr Erziehungsverhalten stärker am Individuum als an der Gemeinschaft, was nicht nur situative Ausnahmen von der Gottesdienstteilnahme umfasst, sondern auch an die Eigenmotivation der Tochter appelliert. Die Verinnerlichung des Glaubens erfordert für Friederike Jäger eine eigenständige Auseinandersetzung der Tochter mit dieser Thematik. Der Besuch des Gottesdienstes erhält somit eher einen Angebots- als einen Pflichtcharakter, wodurch diese Facette des Erziehungssettings situativ flexibel wirkt.

Friederike Jäger unterstützt ihre Tochter in ihrem Anliegen, über das vertraute Milieu hinaus zu blicken, wie sich am Beispiel des USA-Aufenthalts der Tochter zeigt:

„Die hat damals als sie nach Amerika wollte, hatte sie das irgendwo gelesen, hat sie gesagt: ,So. Ich versuch das jetzt mal, ich bewerbe mich jetzt mal für ein Jahr Amerika.' Habe ich natürlich erstmal geguckt und (habe gesagt): ,Hä? Amerika?‘ Ich sag: ,Wie kommst Du denn darauf?‘ Ja, sie wollte eigentlich immer mal nach Amerika und so 'n Jahr, da würde sie sofort hingehen. Und da hab ich dann gesagt: ,Pass auf, wenn du da hin willst, dann is das DEIN Ding. ICH mache dazu NICHTS. Ich trage dazu auch nichts bei.' Ich sage: ,Das einzigste, was ich mache, ich fahr dich mal nach Hochstadt oder keine Ahnung wohin, aber das war's. //hmh// Mehr nich.' Ich sage: ,Deine BEWERBUNG machst du. Das, was du einreichen musst, machst $D U . ' \mathrm{Na}$ ja, das hat sie dann gemacht. [...] Hat das hingeschickt und is auch prompt eingeladen worden. [...],Gut' hab ich gesagt, ,ich hab's dir gesagt, ich fahr dich hin. Kein Problem.' [...] Und im Februar kriegte sie dann plötzlich den Anruf, sie darf fahren. Da hab ich gesagt: ,Das kann nich sein.‘@(..)@//@(.)@// Also da war ich ein BISSCHEN entsetzt, weil das wirklich so geklappt hat, muss ich ehrlich sagen. //hmh// Joa: und dann kriegte sie irgendwann 'nen Anruf bzw. 'ne email, (.) dass sie eine Gastfamilie $[\ldots]$ hat" (I 15:00-16:42).

Bei der Gastfamilie habe es sich um Native Americans gehandelt. Trotz anfänglicher Bedenken habe sie letztlich dem Vorschlag zugestimmt: „Na ja“ hab ich dann gesagt, ,wenn es unbedingt sein muss'“(I 16:42-17:10).

An dieser Passage lassen sich neben der mütterlichen Bereitschaft, ihrer Tochter neue Erfahrungsräume zu eröffnen, mehrere Facetten des Erziehungssettings aufzeigen. Die Tochter überschreitet mit ihrer Idee den bisherigen familialen Erwartungshorizont - 


\subsection{Flexibel erscheinende Tradierungslogiken}

ein Indiz, dass Friederike Jäger nicht zu den Eltern gehört, die beständig auf der Suche nach neuen Bildungsangeboten sind, um die Förderung ihrer Kinder zu optimieren. Auch wenn eine anfängliche Skepsis deutlich erkennbar ist, verwirft sie nicht sofort den Vorschlag ihrer Tochter, sondern ist bereit, sich mit ihr auszutauschen. Die Generationenbeziehungen sind nicht völlig asymmetrisch konzeptualisiert und die Wünsche der Tochter werden beachtet. Hierbei setzt sie weder auf eine Ent- noch auf eine Ermutigung der Tochter, sondern versucht, eine eher neutrale Haltung einzunehmen. Eine ambivalente Gefühlslage den töchterlichen Wunsch betreffend ist unverkennbar. Während der eigenen Jugendzeit gehörten solche Angebote nicht zum Standardrepertoire, sodass sich Friederike Jäger vor eine völlig neue Situation gestellt sieht. Es lassen sich zumindest indirekt Ängste erkennen, die sich auf die ihr völlig fremde und nicht zu kontrollierende Umgebung beziehen, in der die Tochter leben wird. Es gibt aber auch Anreize, um die Mutter für das töchterliche Vorhaben zu gewinnen. In Friederike Jägers Darstellung des töchterlichen Handelns manifestieren sich Orientierungen, die für Friederike Jäger positiv konnotiert sind und die sie fördern möchte: Offenheit für neue Erfahrungen sowie Eigeninitiative und Durchsetzungsfähigkeit. Das sind Eigenschaften, die von traditionellen Geschlechtsrollenbildern abweichen und für Friederike Jäger aufgrund des eigenen Bestrebens in der Kindheit, diese Klischees abzustreifen, eine große Attraktivität besitzen. ${ }^{150}$ All das sind Gründe, die töchterlichen Ambitionen nicht sofort zu unterbinden. Nachdem sich die implizit anklingende Hoffnung zerschlagen hat, die Angelegenheit werde sich ohne ihre Intervention erledigen, gibt Friederike Jäger ihre Skepsis auf und unterstützt ihre Tochter. Hierin drückt sich Friederike Jägers situationsspezifische Flexibilität als Bestandteil ihres Erziehungsarrangements ebenso aus wie in ihrer Bereitschaft, die für sie überraschende Gastfamilie zu akzeptieren. Die von Friederike Jäger nachdrücklich gestellte Bedingung, die Tochter müsse sich selbst um die Bewerbung kümmern, verweist auf ein weiteres zentrales Erziehungsziel: das Einüben von Selbstständigkeit. Dieses für Friederike Jäger elementare Anliegen formuliert sie selbst an anderer Stelle: „Und meine Kinder habe ich eigentlich immer versucht, so zu erziehen, dass sie irgendwo alleine mal zurechtkommen" (I 11:29-11:38). Dieser Anspruch bezieht sich ebenso auf alltagspraktische Fähigkeiten im Haushalt wie ein selbst organisiertes Handeln (I 11:39-12:19) und die Artikulation einer eigenen Meinung. Um diese Ziele zu erreichen, setzt Friederike Jäger, wie aus der Passage zum USA-Aufenthalt exemplarisch ersichtlich wird, darauf, die Kinder selbst aktiv werden zu lassen und ihnen nicht alles abzunehmen. Den Kindern werden Handlungsspielräume zugesprochen und zugleich wird gefordert, dass sie diese nutzen, es werden quasi Freiheiten mit einem verpflichtenden Charakter gewährt. Der Anspruch, den Kindern nicht alles abzunehmen, werde manchmal von der Großmutter unterlaufen, die insbesondere die jüngere Tochter, Cora, ein „bisschen verhätschelt“, was Cora aber auch einfordere (I 13:25-13:31). Somit scheint die Großmutter eine ähnliche Funktion einzunehmen wie in den Vorgän-

\footnotetext{
${ }^{150}$ Vor diesem Hintergrund ist auch Friederike Jägers Kritik zu verstehen, die sich auf eine zu vorsichtige Haltung bei ihrer jüngeren Tochter bezieht (I 14:15-14:43).
} 


\section{Familiale Tradierungsprozesse in einer Drei-Generationen-Perspektive}

gergenerationen und die mütterlichen Intentionen, wenn auch nicht unbedingt offen zu unterlaufen, so doch zumindest abzuschwächen.

Eine weitere Orientierung, auf die Friederike Jäger bei der Erziehung ihrer Kinder achtet, ist die Hilfsbereitschaft:

„[W]enn man als junges Mädchen, wie sie es is, irgendwohin kommt und sagt: ,So, jetzt lass ich mich mal von allen anderen bedienen.' Ich find das furchtbar, muss ich ehrlich sagen. Irgendwann (.) 's meine Meinung, sollte man dann schon sagen: ,Komm, ich fass mit $\mathrm{zu}, / / \mathrm{hmh} / /$ ich räum mit ab oder ich räum mit hin'“ (I 12:21-12:38).

Findet sich in den Erziehungserfahrungen von Herrn Cronert und Friederike Jäger bereits stark ausgeprägt das Element der familialen Hilfsbereitschaft, so wird hier der Rahmen erweitert zu einer grundsätzlichen Haltung - allerdings auf einem niedrigerem Niveau.

Für den schulischen Sektor finden sich deutliche Parallelen zu den eigenen Erziehungserfahrungen. So achte auch Friederike Jäger auf den sauberen und ordentlichen Zustand der Schulsachen bei der jüngeren Tochter. Die ältere Tochter hingegen werde nicht mehr kontrolliert (II 53:54-54:20). Das mütterliche Verhalten fällt folglich altersabhängig variabel aus, und die Kinder werden stufenweise an ein eigenverantwortliches Handeln herangeführt. Zugleich scheint es für den schulischen Bereich kein detailliert ausgearbeitetes Regelwerk zu geben, lediglich der grobe Rahmen wird vorgegeben, dessen Ausgestaltung weitgehend den Kindern selbst obliegt. Hierbei wird kein Leistungsdruck ausgeübt. Dies zeigt sich exemplarisch an einer mütterlichen Unterhaltung mit der jüngeren Tochter über ihre Berufswünsche:

"Na gut ${ }^{6}$, hat sie gesagt, dann werde ich Tierarzt oder Zookurator; $/ /{ }^{\circ} \mathrm{hmh}$ // Ich sag: ,Oh.' Ich sag: ,Weißt du überhaupt, was ein Zookurator is?‘ Das weiß sie. Dann hat sie mir 'nen Vortrag gehalten, was ein Zookurator alles so macht. [...] Hmhm hab ich gedacht: super. Und dann hab ich gedacht: Ne, eigentlich, Tierarzt, das wird nix mit Cora, die is zu sensibel, [...]. , Oder' hat sie dann gesagt ,ich werde Staatsanwalt.' Ich sag: ,Oh, die Richtung is ja völlig @’ne andere.@' Na ja, mal gucken, was draus wird. Ich weiß es nich" (I 18:45-19:23).

Friederike Jäger zeigt sich interessiert, ohne einen Leistungsdruck aufzubauen. Ihre Bedenken beziehen sich weniger auf die kognitiven Kompetenzen als vielmehr auf die Emotionalität der Tochter. Zugleich findet sich ein Erstaunen über die Informiertheit, Konkretheit und Bandbreite der töchterlichen Vorstellungen. Die Wünsche der Tochter stellen für Friederike Jäger keine konkreten Ziele dar, auf die es hinzuarbeiten gilt, vielmehr sind es Möglichkeiten, deren Entwicklung als offen betrachtet wird.

Das Thema Sexualität wird von Friederike Jäger entschieden anders behandelt als in den vorherigen Generationen: 


\subsection{Flexibel erscheinende Tradierungslogiken}

„Heute sehe ich das völlig anders, völlig locker auch. $/ / \mathrm{hmh} / /$ Ich meine Anika hat auch 'nen Freund. Was soll's denn? //hmh// Wenn ich sage: ,Hier pass auf, du schläfst hier und Armin schläft da', dann sagt die: @Hier, ihr habt wohl 'nen Vogel (.)@'“(II 34:45-34:56).

Friederike Jäger übernimmt nicht die Ansichten ihrer Eltern, sondern orientiert sich an einer aktuellen Einstellung zum Thema. Der Tochter wird die Entscheidungshoheit überlassen, von der die Mutter zudem annimmt, dass Anika sie im Zweifelsfall auch vehement einfordern würde.

Was die Kleidung betrifft, so achte Friederike Jäger darauf, „dass es nich ganz so @verboten@“ aussehe (II 56:10-56:41). Aufgrund ihrer eigenen Erfahrungen hat eine ,mädchenhafte' Kleidung als Vorschrift ausgedient und sie gibt ihren Kindern lediglich eine allgemeine Richtlinie vor. Die Kriterien, die für das äußere Erscheinungsbild gelten, haben sich somit im Generationenverlauf gravierend verändert. Stehen in der Großelterngeneration noch die Aspekte ordentlich und sauber im Vordergrund, erfolgt zur jüngsten Generation eine Transformation, die den Kleidungsstil als solchen in den Mittelpunkt rückt, der allerdings wieder einigen minimalen Ordnungsstandards entsprechen sollte. Die letzten beiden Beispiele verdeutlichen, wie Orientierungen kontextabhängig bearbeitet und auf diese Weise anschlussfähig gehalten werden.

Aneignungsebene Anika Jäger $\left({ }^{*} 1989\right)^{151}$ : Zwischen familialer Unterstützung und Streben nach Freiräumen Für Anika Jäger sind die Großeltern und Eltern zentrale Bezugspersonen, die sie als verlässliche Ansprechpartner wahrnimmt, mit denen sie viel Zeit verbringt, und die sie, wie sie mehrfach anführt, jederzeit unterstützen:

„[I]ch war viel bei meinen Großeltern als ich klein war, und das is auch heute noch so. Wenn ich aus der Schule komme gibt es immer noch Essen bei@Oma@, //hmh// was sehr schön is. Ähm, meine Eltern haben sich viel um mich gekümmert und meine Großeltern auch. Ähm haben mir immer bei den Hausaufgaben geholfen, wenn ich Fragen hatte in der Schule beim Lernen“ (02:07-02:26).

Sie sieht sich im Zentrum der elterlichen und großelterlichen Aufmerksamkeit, sie fühlt sich „umsorgt" (1:10:12-1:10:14) und schätzt die ihr zuteil werdende Fürsorge (02:31-02:50, 14:25-15:27). Intergenerationelle Hilfe und Unterstützung sind für sie ebenso wie in den Vorgängergenerationen unmittelbar erfahrbar. Die Eltern hätten sie nach anfänglicher Skepsis bei der Realisierung ihres Auslandsjahres unterstützt (07:1207:54), worin sich die elterliche Bereitschaft manifestiert, ihre Entscheidungen situationsabhängig zu fällen. Generell seien ihr „viele Freiheiten gelassen“ worden (02:3202:34, 1:34:29-1:34:34, 1:34:58-1:35:02). Zwar habe es insbesondere während ihrer Kindheit einige Regeln gegeben, mit denen sie unzufrieden gewesen sei und über die sie sich

${ }^{151}$ Das Interview mit Anika Jäger wurde am 03.04.2008 geführt. 


\section{Familiale Tradierungsprozesse in einer Drei-Generationen-Perspektive}

gelegentlich hinweggesetzt habe, da sie sich „nichts sagen lassen“ habe (18:50-18:53). In manchen Fällen habe das zu Konflikten mit den Eltern geführt, ohne dass das jedoch gravierende Konsequenzen gehabt habe. In anderen Fällen hätten die Erwachsenen sie gewähren lassen und sie habe aus den Konsequenzen ihres Handelns gelernt, wenn sie sich z.B. nicht dem Wetter entsprechend habe kleiden wollen. Die Regelübertretungen hätten sich aber eher auf „Kleinigkeiten“ bezogen (18:54-21:42). Der Tochter bzw. Enkelin werden die Regeln nicht aufgezwungen, stattdessen setzen die Erwachsenen darauf, dass das Kind anhand der Erfahrungen zur Einsicht kommen werde. Einschränkungen der individuellen Freiheiten werden zumindest retrospektiv als Schutzregeln betrachtet und werden auf diese Weise akzeptabel (1:33:58-1:33:34:08). Die elterlichen Regeln werden nicht als starr empfunden. Die Ausgehzeiten seien z.B. durchaus flexibel gehandhabt worden (21:47-22:20). Die Ahndung von Fehlverhalten habe sich weitestgehend auf mündliche Zurechtweisungen beschränkt und manchmal habe es ein Fernseh- oder Computerverbot gegeben (01:49-01:56). Aktuell gebe es nicht viele Regeln. Am Wochenende helfe sie der Mutter im Haushalt (1:31:45-1:31:56). In Bezug auf den Freundeskreis gebe es keine Vorschriften, die Eltern würden auf ihre „Menschenkenntnis“ vertrauen. Ihre Kleidung suche sie sich selber aus. Bei größeren Entscheidungen, z.B. wenn Anika sich ein Tattoo stechen lassen wollte, würde sie ihre Eltern zuvor fragen und sich nicht über ein eventuelles elterliches Verbot hinwegsetzen (1:30:28-1:32:40, 1:33:19-1:33:34). Sie weiß folglich, dass sie Freiheiten hat, solange ein gewisser Rahmen eingehalten wird.

Eine wichtige Regel bestehe darin, die gemeinsamen Mahlzeiten am Wochenende einzuhalten (1:28:49-1:28:53, 1:30:47-1:30:56). Die bereits von ihrer Mutter als wichtig thematisierte Regel wird auch als solche wahrgenommen. Die hierbei geschaffenen Gelegenheiten des Austausches sowie die Betonung der Familiengemeinschaft stellen zwei Aspekte dar, die für Anika eine zentrale Rolle spielen und eng miteinander verbunden sind. Besonders wichtig ist ihr, dass die Großeltern jederzeit ansprechbar seien (15:14-15:27, 1:28:59-1:29:14). Ihre Eltern hätten ihr nicht nur alle Wissensfragen immer beantwortet, sie seien auch wichtige Bezugspersonen, die sie während ihres Auslandsjahres jederzeit habe anrufen können, wenn sie emotionale Unterstützung gebraucht habe (02:18-02:26, 04:25-04:29, 06:52-07:06, 32:47-33:07, 35:52-37:07, 1:33:51-1:33:56). Hierbei habe sich das Verhältnis zu den Eltern nach dem USA-Aufenthalt intensiviert (22:59-23:58, 1:36:34-1:37:10). Nach der Rückkehr habe sie zwar zunächst einige Eingewöhnungsschwierigkeiten gehabt, was die gemeinsamen Mahlzeiten betreffe, sie seien aber inzwischen von zentraler Bedeutung für sie: „Aber jetzt ähm seit Amerika sitze ich dann bis zum Schluss mit meinen Eltern und wir unterhalten uns noch u:nd //hmh// und das brauch ich auch, das schätz ich auch“ (23:47-23:58). Der kommunikative Austausch wird von Anika in erster Linie als ein Angebot von Eltern und Großeltern betrachtet, das sie emotional und kognitiv unterstützt, und nicht als Steuerungsmittel. Insgesamt beschreibt Anika die Beziehungen zu Eltern und Großeltern als sehr nah und verbunden. Ihre emotionale Zugewandtheit zu Eltern und Großel- 


\subsection{Flexibel erscheinende Tradierungslogiken}

tern lässt sich als Beitrag Anikas zum familialen Bezugsrahmen interpretieren und stellt ihr Äquivalent zur empfangenen Hilfe und Unterstützung dar.

Wie zentral die familialen Beziehungen in Verbindung mit dem kommunikativen Austausch sind, zeigt sich auch an Anikas Schilderungen des Auslandsaufenthalts. Sie fühlt sich in ihrer Gastfamilie herzlich aufgenommen (35:27-35:51, 48:46-50:15). Ihre Gastbrüder hätten sie unterstützt, sie wie eine Schwester behandelt und sich um sie gekümmert (51:35-52:47). Sie sei in das Familienleben integriert worden und habe gerne an den Ritualen der Familie teilgenommen (51:24-51:33). Zur Charakterisierung ihrer Gastfamilie verwendet Anika ähnliche Kategorien, wie sie für ihre Herkunftsfamilie bestimmend sind: Sie stellt die emotionale Verbundenheit sowie die sich für sie als relativ ausgewogen darstellenden intergenerationellen Machtstrukturen in den Vordergrund. Sie fühlt sich keinen Zwängen ausgesetzt und betont die Freiwilligkeit ihrer Handlungen. Es gibt aber einen wichtigen Unterschied im Vergleich zu ihrer Herkunftsfamilie: Ihre Gasteltern verfügen nicht über die notwendigen zeitlichen Ressourcen, um sie in ähnlich intensiver Weise zu umsorgen, wie sie es aus ihrer eigenen Familie gewohnt ist (08:45-9:09). Sie habe zwar größere Freiheiten als zu Hause gehabt, die einzige Direktive habe gelautet: „Ja, ob du nun nach Hause kommst oder nich, Hauptsache du rufst an" (09:06-09:09). Die Kehrseite hiervon besteht jedoch in einer stärkeren Eigenverantwortung sowie der größeren Verwiesenheit auf sich selbst. Diese Leerstelle füllt zumindest teilweise eine andere Gastschülerin aus, die in derselben Familie untergebracht ist. Sie wird für Anika zu einer zentralen Bezugsperson (39:10-39:24), die Rückhalt und kommunikativen Austausch bietet: „Sira war wie eine Schwester für mich. Wir haben fast alles zusammen gemacht. //hmh// und haben uns immer Gesellschaft geleistet. [...] Ja, also mit Sira hab ich auch über alles geredet" (56:37-57:42).

Mit ihrem Auslandsaufenthalt ist für Anika eine deutliche Ambivalenzerfahrung verbunden, die unmittelbar den familialen Bezugsrahmen der intergenerationellen Hilfe und Solidarität betrifft. Zwar schätzt sie die Freiheiten, die die Gasteltern ihr gewähren, vermisst jedoch die Großeltern, das enge Beziehungsnetz, die Unterstützung und das Umsorgen, wie sie es von zu Hause kennt. Nach ihrer Rückkehr sind diese zwar wieder gegeben, jedoch sind die im Vergleich zur Gastfamilie beschränkteren Freiräume zunächst problematisch für Anika (08:45-11:19, 1:08:09-1:10:14, 1:13:06-1:13:26). Der Rahmen ist für Anika in ihrer Herkunftsfamilie etwas enger gesteckt als in der Gastfamilie und die kontrollierende Komponente in den intergenerationellen Beziehungen trotz aller empfundenen Freiheiten etwas stärker ausgeprägt.

Ebenso wie in den Vorgängergenerationen ist die Anbindung an die religiöse Gemeinde zwar gegeben und die Teilnahme an der Kommunion ist für Anika selbstverständlich (1:18:42-1:20:00). Die Bedeutung des Glaubens scheint sich allerdings verändert zu haben. Während für ihren Großvater die regelmäßigen Gottesdienstbesuche noch ein integraler Bestandteil zum Ausdruck seines Glaubens gewesen sind, berichtet Anika hierüber nichts. Entweder sind sie für sie so selbstverständlich, dass sie gar nicht gesondert erwähnt werden müssen, oder in ihrer aktuellen Lebensphase dominieren andere 


\section{Familiale Tradierungsprozesse in einer Drei-Generationen-Perspektive}

Themen. Der Gottesdienstbesuch könnte aber auch an Zentralität für den Ausdruck der eigenen Religiosität verloren haben, die nun quasi privatisiert gelebt wird. ${ }^{152}$

Das persönliche Nahfeld ändert sich im Vergleich zur Vorgängergeneration. Konstant bleibt die Einbindung in den engeren Familienkreis und in das weitere Verwandtschaftssystem durch Familienfeiern (04:45-05:16, 1:01:09-1:03:12). Ebenso ist sie in ein nachbarschaftliches sowie freundschaftliches Netzwerk integriert, wie sich exemplarisch an der für sie organisierten Willkommensfeier nach ihrem Auslandsjahr ablesen lässt (40:54-41:07). Im Unterschied zu ihrer Mutter, deren Sozialkontakte aufgrund der äußeren Rahmenbedingungen der DDR während ihrer Kindheit stärker von ihrem Umfeld beobachtet und reglementiert wurden, ergeben sich für Anika größere Freiheiten. Mit dem Auslandsjahr erweitert sich das Netzwerk der persönlichen Sozialkontakte für Anika, die sie bis heute durch Telefonate und Email-Austausch pflege (55:50-56:34). Dadurch öffnet sich für Anika das sie unmittelbar umgebende Milieu und wird heterogener.

Das ist für Anika ein positiv konnotierter Aspekt. Sie sei „sehr offen geworden für andere Kulturen“ (12:45-12:49). Diese Entwicklung betrachtet sie u.a. als Folge der elterlichen wie großelterlichen Aktivitäten. Durch die vielen Reisen mit ihren Eltern habe sie sich bereits früh für andere Kulturen interessiert (12:45-12:52). ${ }^{153}$ Über ihre Großeltern sei sie während ihrer Kindheit in Kontakt mit einem russischem Geschwisterpaar gekommen, wodurch sich ihr „multikulturelles Denken so 'n bisschen auch schon entwickelt" habe, genau wie durch die Reisen mit den Eltern (06:11-06:35 und 1:34:16-1:34:22). Angeregt durch die eigenen Erfahrungen habe sie die Eltern überzeugen können, ebenfalls eine Gastschülerin aufzunehmen (11:43-12:02). Aus ihrer Sicht tragen Eltern wie Großeltern aktiv zur Erweiterung des Milieus sowie zu ihrer Horizonterweiterung bei, wodurch beide Parteien als offen und tolerant erscheinen können. Ihr Interesse für andere Kulturen und neue Freundschaften stehen für sie im Einklang mit den elterlichen und großelterlichen Intentionen.

In den USA habe Anika eine intensive Hilfsbereitschaft durch andere erlebt. Zurück in Deutschland möchte sie diese positiven Erfahrungen an andere weitergeben. Deswegen sei sie als Ansprechpartnerin für Austauschschüler aktiv, um ihnen bei der Integration in die neuen Kontexte behilflich zu sein (12:55-13:38). Hiermit erweitert Anika den familialen Bezugsrahmen, indem sie das Prinzip der Hilfsbereitschaft auf Personen außerhalb der Familie anwendet. Das kann zum einen mit ihrer aktuellen Lebensphase zusammenhängen oder auch eine Verinnerlichung der bereits im Erziehungsarrangement der Mutter anklingenden Erweiterung darstellen, die ihren Kindern Hilfsbereit-

\footnotetext{
${ }^{152}$ Das ist nicht mit einem Bedeutungsverlust des Glaubens gleichzusetzen, sondern zunächst nur ein Verweis auf eine veränderte Gewichtung der religionsbezogenen Praxen.

${ }^{153}$ Vor diesem Hintergrund sind Anikas Berichte über die diversen Familienurlaube zu verstehen, die nicht nur gemeinsame Familienzeiten sind, sondern auch Gelegenheiten bieten, um neue Erfahrungen zu sammeln und andere Kulturen kennen zu lernen (04:00-04:35, 1:20:28-1:21:58).
} 


\subsection{Flexibel erscheinende Tradierungslogiken}

schaft als generelles Prinzip auch über den familialen Rahmen hinaus vermitteln möchte. ${ }^{154}$

Die Herausbildung einer eigenen Meinung wird von Anika ebenfalls thematisiert und im Interviewverlauf als eine Entwicklung dargestellt. Während der Pubertät sei sie schwierig gewesen, habe sich nichts sagen lassen und habe immer das tun wollen, „was andere Leute auch machen“ (18:52-18:54). Seit ihrem Auslandsaufenthalt interessiere sich Anika für politische Themen und unterhalte sich hierüber mit ihren Eltern (1:12:15-1:12:50). Sie informiere sich und bilde sich zu politischen Themen eine eigene Meinung (1:14:37-1:15:48). Zum einen beschreibt Anika hier u.a. als Folge ihrer Auslandserfahrung eine Erweiterung des sie interessierenden Themenspektrums. Zum anderen vollzieht sie eine Entwicklung, in der sie vom Modus eines eher passiven Rezipierens zu einer aktiven Informationssuche und -bewertung wechselt. Die mütterliche Erwartungshaltung, eine eigene Meinung auszubilden, wird von der Tochter umgesetzt und nicht als Zwang, sondern als eine dem eigenen Interesse entspringende, freiwillige Leistung betrachtet. Diese Überzeugung trägt dazu bei, dass aus der mütterlichen Forderung keine intergenerationellen Konflikte erwachsen.

In anderen Bereichen kommt es zu kleineren Auseinandersetzungen. Ein Streitpunkt betrifft die Einstellung des „easy going“, die sie aus den USA übernommen habe (11:1411:39, 1:10:47-1:11:14). Die Amerikaner hätten „so 'ne Mentalität nach dem Motto: komm ich heut' nich, komm ich morgen" (11:20-11:26), wozu auch Langsamkeit und Unpünktlichkeit zählten (11:27-11:38, 1:11:18-1:11:38). Die Übernahme dieser Maxime habe zu Konflikten mit den Eltern geführt, wenn sie z.B. sonntags nicht zum Mittagessen aufgestanden sei (1:10:57-1:11:11). Die Eltern achten offenbar auf die Einhaltung grundlegender Orientierungen wie Verlässlichkeit und Pünktlichkeit. Hieran kann sich Anika auch schnell wieder gewöhnen, zumal ihr selbst an den gemeinsamen Mahlzeiten gelegen ist, das Einhalten dieser Standards also auch einem Eigeninteresse entspricht.

Bleibt abschließend noch der schulische Sektor. Ähnlich wie in den Generationen zuvor finden sich keine Hinweise, dass Anika sich einem starken Leistungsdruck ausgesetzt sieht. Die Eltern verfolgen zwar aufmerksam die Entwicklung ihrer Tochter in diesem Bereich, ohne ihr allerdings etwas vorzuschreiben. In Hinblick auf die Schulwahl nach der vierten Klasse hätten die Eltern sich mit ihr ausgetauscht, die Entscheidung aber ihrer Tochter überlassen, die sich eindeutig für den Gymnasialbesuch entscheidet: „[I]ch wollte kämpfen. Ich wollte, dass ich auf's Gymnasium gehe. Und das haben meine Eltern akzeptiert und haben mich auf's Gymnasium gehen lassen" (06:37-06:50, 27:15-28:11). Bezogen auf den schulischen Bereich nimmt Anika folglich die Eltern als wenig einengend und flexibel wahr. Als sie Probleme mit einer Lehrerin gehabt habe, hätten die Eltern sich für sie eingesetzt und hätten sich beim Schulleiter beschwert

\footnotetext{
${ }^{154}$ Die intergenerationelle familiale Unterstützung und Hilfe wird dabei nicht außer Kraft gesetzt. Anika bietet im Rahmen ihrer Möglichkeiten innerfamilial ihre Hilfe an, wenn sie z.B. der Mutter am Wochenende im Haushalt hilft oder sich bei Familienfeiern um die Kinder der Verwandtschaft kümmert (1:31:45-1:31:56, 1:03:42-1:03:48).
} 


\section{Familiale Tradierungsprozesse in einer Drei-Generationen-Perspektive}

(25:42-26:21). Leistungsdruck oder -streben geht, wenn überhaupt, von ihr selber aus, wie ihre Äußerung „kämpfen zu wollen“ zeigt. Auch scheint sie stärker als noch in der Generation zuvor ein Wettbewerbs- und Konkurrenzdenken verinnerlicht zu haben. Ihre Entscheidungen, wie z.B. der Auslandsaufenthalt, werden von ihr bereits mit Blick auf die Zukunft getroffen, womit sie wiederum ihre Eltern überzeugen kann: „Ja, sie haben gesehen, [...] dass es auch gut für meine Zukunft ist [...]. Und deswegen, ohne Englisch, bin ich der Meinung, kommt man heutzutage nicht mehr so weit, auch wenn es allein nur wegen Computern ist“ (33:14-34:00).

Zusammenfassende Betrachtung des familialen Bezugsrahmen In der Familie Cronert/Jäger nimmt die intergenerationelle Solidarität und Hilfe in allen drei Generationen eine wichtige Rolle ein und bildet den familialen Bezugsrahmen. Alle drei Generationen erleben eine Kindheit, die durch eine zumindest teilweise Betreuung durch die Großeltern geprägt ist. Auch in dieser Familie wird der familiale Bezugsrahmen in den einzelnen Generationen mit jeweils unterschiedlichen weiteren Orientierungen kombiniert. Für Herrn Cronert sind neben der intergenerationellen Solidarität noch die religiöse Einbettung seiner Kindheit sowie die Orientierungen an Ehrlichkeit, Ordnung und Sauberkeit hervorstechende Merkmale des eigenen Aufwachsens, von denen er für die Erziehung der Tochter zumindest die ersten beiden Elemente übernimmt. Intergenerationelle Solidarität und Hilfe sowie die Einbindung in ein religiöses Umfeld sind auch für die Tochter Friederike Jäger zentrale Komponenten. Zugleich ist eine tendenzielle Erweiterung des Prinzips der Hilfsbereitschaft auf die außerfamiliale Sphäre zu beobachten. Einige an sie herangetragene Orientierungen, wie die von ihr als konservativ empfundene Sexualmoral sowie eine Orientierung an geschlechtsrollenstereotypen Vorstellungen, lehnt sie ab. Das Vertreten einer eigenen Meinung sowie die Erziehung zur Selbstständigkeit werden erst von ihr besonders betont. Auch für Anika als Vertreterin der jüngsten Generation bleiben intergenerationelle Unterstützung und Hilfe wichtige Eckpfeiler, wobei sie das Prinzip der Hilfsbereitschaft von einem familialen zum allgemeinen Verhaltensstandard erweitert. Die religiöse Perspektive tritt im Vergleich zu den vorherigen Generationen stärker in den Hintergrund. Eigenständigkeit unter Einbeziehung einer eigenen Meinung sowie Leistungsorientierung sind für Anika zentrale Aspekte. Dies mag zum Teil ihrer aktuellen Lebensphase geschuldet sein, es kann sich aber auch um die für sie spezifische Ausformung des familialen Bezugsrahmens handeln. Die beschriebenen unterschiedlichen Schwerpunktsetzungen unter Beibehaltung des familialen Bezugsrahmens der intergenerationellen Solidarität und Hilfe beeinflussen wiederum die jeweiligen Erziehungssettings.

\subsubsection{Einordnung der Tradierungslogik unter Berücksichtigung der gesellschaftlichen Bezüge}

Elemente einer autoritativen Erziehung (Baumrind 1991) - die Eltern sind den Kindern jeweils emotional zugewandt, gehen, wenn auch in unterschiedlichem Ausmaß, auf die 


\subsection{Flexibel erscheinende Tradierungslogiken}

Bedürfnisse der Kinder ein, verhalten sich im Allgemeinen stärker unterstützend als strafend, fördern das kindliche Verantwortungsbewusstsein und es existieren den Kindern bewusste Regeln, auf deren Einhaltung geachtet wird (Baumrind 1991, 750 und Papastefanou/Hofer 2002, 185) - sind in den beiden vorgestellten Familien erkennbar. Es finden sich aber auch Aspekte, die sich dieser Zuordnung widersetzen. Die Erziehung zur Eigenständigkeit - als ein weiteres Merkmal autoritativer Erziehung ${ }^{155}$ - ist z.B. im Erziehungsarrangement von Frau Dienel nicht sonderlich ausgeprägt. Das Setting, in dem Herr Cronert aufwächst, weist zudem sowohl autoritäre als auch permissive Züge auf. Autoritäre Elemente existieren in Bezug auf die religiösen Praxen und im Vermittlungssetting der , mitunter kaum nachgiebigen' Großmutter. Die Großmutter agiert aber auch permissiv, etwa wenn sie als ausgleichende Instanz zwischen Kind und Eltern vermittelt. Ähnliches gilt für die Eltern, die Regelübertretungen nicht immer ahnden.

Während des Aufwachsens von Friederike Jäger lassen lediglich die strikte Orientierung an einer konservativen Sexualmoral sowie an geschlechtsrollenstereotypen Vorstellungen noch autoritäre Züge erkennen, die jedoch unter aktiver Beteiligung der Großeltern teilweise außer Kraft gesetzt werden. Zugleich finden sich ausgeprägt permissiv gestaltete Elemente in der Erziehung, die sich in Herrn Cronerts Kompromissbereitschaft ebenso ausdrücken wie in der Bereitschaft der Erwachsenen, Friederike Jäger alle Wünsche zu erfüllen.

Bei der Erziehung der jüngsten Generation dominieren in den Familien Jäger und Ebel die autoritativen Facetten. Anika werden viele Freiheiten gelassen, und das elterliche wie großelterliche Verhalten ist sehr kindbezogen. Die Forderung, Eigenständigkeit bzw. eine eigene Meinung zu entwickeln, wird in beiden Familien als deutlich formulierte Erwartung an die jüngste Generation herangetragen.

In beiden Familien verlagern sich die intergenerationellen Machtbalancen im Zeitverlauf zugunsten der jüngeren Generation. Die Mitspracherechte sind für Herrn Cronert in seiner Kindheit noch eingeschränkt, wohingegen Anikas Meinung meist in den Entscheidungsprozess einbezogen wird. Lässt sich das Erziehungsarrangement, in dem Herr Cronert aufgewachsen ist, unter Rückgriff auf die Typologie Bois-Reymonds tendenziell noch als eine Form des (eingeschränkten) modernisierten Befehlshaushalts (BoisReymond 1994, 195/196) kennzeichnen, so wachsen sowohl Friederike Jäger als auch ihre Tochter in jeweils etwas unterschiedlich ausgeprägten Formen eines Verhandlungshaushalts (Bois-Reymond 1994, 155/156) auf. Ähnlich verläuft die Entwicklung in der Familie Dienel/Ebel. Die Mitsprachrechte Frau Dienels sind in ihrer Kindheit noch begrenzt. Ihr eigenes Verhalten während der eigenen Familienphase beschreibt sie als offener, allerdings lassen sich auch steuernde Komponenten erkennen, wenn sie z.B. die Gefühlsebene einsetzt, sobald inhaltliche Argumente nicht mehr verfangen, oder sich um eine umfassende Informiertheit über den Alltag ihrer Kinder bemüht. Das Erziehungsverhalten ihrer Tochter, Heike Ebel, wirkt demgegenüber gelassener. Sie lässt ihren Kindern größere Freiräume und ist in stärkerem Ausmaß bereit, Argumente der

${ }^{155}$ Zur Charakterisierung der einzelnen Erziehungsstile siehe Kap. 1.2.2. 


\section{Familiale Tradierungsprozesse in einer Drei-Generationen-Perspektive}

Kinder anzuerkennen. Die Machtbalancen sind deutlich ausgewogener als in den vorherigen Generationen, sodass das letzte Erziehungsarrangement als offener Verhandlungshaushalt (Bois-Reymond 1994, 156) bezeichnet werden kann.

Sowohl die Einordnung in Erziehungsstile wie auch die in Erziehungshaushalte erfassen zwar wichtige Charakteristika, lassen aber zentrale Erziehungsmerkmale, die in beiden Familien zu finden sind, außer Acht: die Milieueinbindung sowie den Vorbildcharakter des elterlichen bzw. großelterlichen Handelns, das am familialen Bezugsrahmen ausgerichtet ist. Beide Elemente sind für die Tradierungsprozesse in diesen Familien aber mindestens ebenso relevant. Darüber hinaus sind in Anlehnung an Kosellecks Termini von Erfahrungsraum und Erwartungshorizont (1989, 349-359) das Zusammenspiel der Erziehungserfahrungen und der auf dieser Grundlage entworfenen Erwartungshorizonte der Akteure zu betrachten, um die Komplexität familialer Tradierung adäquat zu erfassen. Angeregt von Olsons u.a. Typologie (1989, 47-51) ist das Ausmaß der Flexibilität, wie sie sich für vermittelnde wie aneignende Generation darstellt, ein geeignetes Merkmal zur Kennzeichnung der jeweils vorliegenden Tradierungslogik in den beiden Familien.

In beiden Familien erscheinen die jeweiligen Erziehungssettings und die für die nachfolgende Generation entworfenen Erwartungshorizonte flexibel. Es existieren Bezugsrahmen, die nicht als unabänderliche Forderungen an die nächste Generation herangetragen werden. Vielmehr werden sie als genereller Rahmen entworfen, der der aneignenden Generation Raum zur Ausgestaltung zugesteht. Das trägt dazu bei, das familiale Konfliktniveau zu begrenzen. Die steuernden Komponenten, die durchaus gegeben sind, werden verdeckt, indem ihnen ein beiläufig erscheinender Charakter gegeben wird. ${ }^{156}$ Kennzeichnend für vermittelnde und aneignende Generation ist der Topos Freiheit. Die jeweils Erziehenden betonen, den Kindern Freiheiten gewährt zu haben, und die Erzogenen nehmen zumindest in den beiden jüngeren Generationen das Thema auf, indem sie hervorheben, Freiheiten gehabt zu haben.

Bei Frau Dienel kommt ihre Anpassungsbereitschaft in ihrer (vermeintlichen) Offenheit, was die Vermittlung des Glaubens betrifft und ihre Maxime: „Diskussion über alles" einschließt, ebenso zum Ausdruck wie in ihrem, sicherlich von den Kindern nicht unwesentlich beeinflussten, flexiblen Umgang mit den familialen Fernsehpraxen, den gemeinsamen Freizeitaktivitäten sowie der zu erkennenden Bereitschaft im Bedarfsfall Regeln anzupassen. Ihre Tochter, Heike Ebel, gibt sich ebenfalls in mehrfacher Hinsicht flexibel: In Bezug auf die religiösen Praxen der Kinder hebt sie die Freiwilligkeit der Teilnahme hervor. Sie bemüht sich darum, den Kindern Freiräume zu gewähren, hält Familienkonferenzen ab, möchte die Eigenständigkeit ihrer Kinder fördern und greift bei Problemen nicht sofort ein, sondern setzt auf den ,langen Atem' als Erziehungsprinzip.

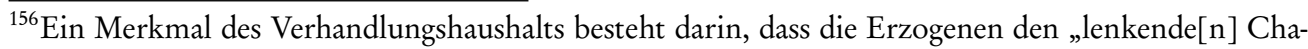
rakter der Erziehung in eine Selbstlenkung " (Ecarius 2002, 231) umdeuten, wodurch der lenkende und disziplinierende Aspekt der Erziehung verdeckt wird.
} 


\subsection{Flexibel erscheinende Tradierungslogiken}

Ein Beispiel für Herrn Cronerts situationsspezifische Flexibilität ist die Akzeptanz der töchterlichen Entscheidung gegen ein Studium. Insgesamt wirkt sein Erziehungsverhalten weniger steuernd als bei Frau Dienel. Charakteristisch für ihn ist eine pragmatische, lösungsorientierte Grundhaltung. Bei seiner Tochter findet sich eine ähnliche Einstellung. Sie besteht z.B. nicht dogmatisch auf ein Befolgen der familial üblichen religiösen Praxen bei ihrer Tochter und kann ihre Skepsis in Bezug auf das Auslandsjahr zugunsten der Wünsche ihrer Tochter überwinden.

Die aneignenden Generationen nehmen in beiden Familien die Vermittlungsarrangements der Eltern als flexibel, offen und Spielräume gewährend wahr. Die Milieueinbindung trägt dazu bei, die elterliche Steuerung zu kaschieren. Das scheint auch erfolgreich zu sein, da die jeweils aneignende Generation diese Steuerung nicht elementar kritisiert. In der Familie Cronert/Jäger ist eine Lockerung der Milieueinbindung bei gleichzeitiger Erweiterung des persönlichen Netzwerkes in der jüngsten Generation festzustellen. Das ist aber der Tradierung des familialen Bezugsrahmens nicht abträglich, da er auf die außerfamiliale Sphäre übertragen zu werden scheint. In der Familie Cronert/Jäger unterstützt das praktizierte Modell des großelterlichen Engagements die Übernahme von Tradierungsinhalten zusätzlich, indem die Großeltern zumindest in den älteren Generationen als ausgleichende Instanzen auftreten und auf diese Weise potenzielle Konflikte bereits im Vorfeld entschärfen.

Das Vorleben stellt ein geeignetes Mittel für eine beiläufige Tradierung dar, da Erwartungen eher indirekt an die Kinder herangetragen werden können. Eine ähnliche Funktion erfüllen die gemeinsamen Familienzeiten, die allerdings im Generationenverlauf unterschiedlich stark ausgeprägt sind. All diese Elemente sind geeignet, um den Eindruck einer natürlichen Selbstverständlichkeit zu erwecken und die Akzeptanz durch die Kinder zu erhöhen. Die starke emotionale Zuwendung und Unterstützung, die alle drei Generationen in ihrer Kindheit erfahren, dürfte die Annahme von Tradierungsinhalten erleichtern und dazu beitragen, das elterliche Erziehungssetting als gestaltbar zu empfinden. Eine ähnliche Wirkung haben die gewährten Mitspracherechte, die in den einzelnen Generationen zwar unterschiedlich stark ausgeprägt sind, den aneignenden Generationen aber das Gefühl vermitteln, aktiv an Entscheidungsprozessen beteiligt zu werden. Alle drei aneignenden Generationen der beiden Familien führen zudem Beispiele an, in denen sie sich gegen die ursprünglichen Vorgaben der Eltern durchsetzen konnten und somit ihre Eltern als flexibel erfahren haben. Die aneignenden Generationen in der Familie Cronert/Jäger sind offensichtlich in stärkerem Ausmaß als in der Familie Dienel/Ebel aktiv an Verschiebungen der elterlichen Vorstellungen beteiligt. Die Entwicklung von Eigenständigkeit schließlich, wenn auch nur in der jüngsten Aneignungsgeneration ein zentraler Aspekt, erfordert geradezu von der vermittelnden Generation ein gewisses Maß an Offenheit, soll das Ziel der Eigenständigkeit erreicht werden.

Flexibel und offen erscheinende Erziehungsarrangements sind das charakteristische Element zur Einordnung der Tradierungsprozesse in diesen Familien, die sich über alle 


\section{Familiale Tradierungsprozesse in einer Drei-Generationen-Perspektive}

drei vorgestellten Generationen nachweisen lassen. Im Zuge der Tradierung werden die jeweiligen Inhalte und Formen leicht abgewandelt und den jeweiligen gesellschaftlichen Gegebenheiten angepasst, ohne dass es zu nennenswerten Konflikten kommen würde. Innerhalb dieser Logik nehmen die nachfolgenden Generationen die elterlichen Erwartungen den familialen Bezugsrahmen betreffend größtenteils an, und die Veränderungen von Inhalten und Formen der Tradierung im Generationenverlauf sind eher als moderate Weiterentwicklungen einzustufen, die den Beteiligten selbst kaum auffallen. Die Auseinandersetzung mit den eigenen Erziehungserfahrungen und der für die nächste Generation entworfenen Erwartungen wird dabei von den jeweiligen gesellschaftlichen Rahmenbedingungen beeinflusst.

Das Zusammenspiel von familialer und gesellschaftlicher Ebene ${ }^{157}$ Die Frage nach der Anschlussfähigkeit der familialen Bezugsrahmen sowie der erfahrenen Erziehungsarrangements erscheint als ein geeigneter Schlüssel, um die erkennbaren Veränderungen im Generationenverlauf zu verstehen. Vermittelnde wie aneignende Generation handeln nicht nur als familiale Akteure, sondern auch als Akteure ihrer Zeit. Sie setzen sich vor dem Hintergrund der gegebenen gesellschaftlichen Bedingungen mit Inhalten und Formen der Tradierung auseinander und bearbeiten sie. Familiale und gesellschaftliche Ebene bilden hierbei ein interdependentes Geflecht, das auf die jeweilige Gestaltung einwirkt. Da sich die gesellschaftlichen Rahmenbedingungen für die Familien Dienel/ Ebel und Cronert/Jäger von der Nachkriegszeit bis 1989 erheblich unterscheiden, werden die beiden Familien im Folgenden getrennt vorgestellt.

Frau Dienel gründet ihre Familie zu einer Zeit, in der überlieferte Orientierungen und Autoritäten in zunehmenden Maße kritisch hinterfragt werden. Das erschwert es, bei der Erziehung der Kinder selbstverständlich auf den eigenen Erfahrungsraum und die hieraus resultierenden Erwartungen zurückzugreifen. Frau Dienels Maxime „Diskussion über alles“, in der sich ihre Auffassung von Modernität manifestiert, lässt sich als ihren Versuch interpretieren, tradierte Elemente der Erziehung mit den sich ändernden gesellschaftlichen Rahmenbedingungen in Einklang zu bringen und so den familialen Bezugsrahmen der religiösen Alltagsprägung im Zeit- und Generationenverlauf anschlussfähig zu halten sowie sich dem wachsenden Trend der Säkularisierung zu entziehen. ${ }^{158}$

Frau Dienel sieht sich gleichzeitig mit dem Mitte der 1960er Jahre einsetzenden Wertewandel konfrontiert, in dessen Zuge Selbstentfaltungswerte und damit die eigenen

\footnotetext{
${ }^{157}$ Zum Ineinandergreifen von familialen und gesellschaftlichen Faktoren in den Familien Dienel/Ebel und Cronert/Jäger siehe auch Kraul/Radicke (2012) und Radicke (2013).

${ }^{158} \mathrm{Als}$ Indizien für die abnehmende Relevanz der Religiosität nennt Meulemann die rückläufige Teilnahme an kirchlichen Praktiken und dem Gemeindeleben zwischen den 1950er und 1980er Jahren (Meulemann 1996b, 126).
} 


\subsection{Flexibel erscheinende Tradierungslogiken}

Interessen und Bedürfnisse an Bedeutung gewinnen. ${ }^{159}$ Entgegen dieser Entwicklung insistiert sie mit Verweis auf den Glauben darauf, dass ihre Kinder die eigenen Bedürfnisse nicht zum obersten Maßstab erheben und ihre Interessen im Zweifelsfall zurückstellen. Auf diese Weise versucht sie, eine für sie religiös verankerte Orientierung zu erhalten. Später modifiziert sie ihre Einstellung, indem sie sich und ihren Enkeln zubilligt, die eigenen Bedürfnisse stärker zu beachten, ohne allerdings die Balance zwischen fremden und eigenen Interessen aus den Augen zu verlieren. $\mathrm{Zu}$ dieser zeitverzögerten Entwicklung tragen einschneidende familiale Ereignisse sowie der intergenerationelle Austausch bei.

Das von Frau Dienel praktizierte Modell der Hausfrauenehe spiegelt das in den 1960er Jahren in der BRD dominante Familienleitbild. ${ }^{160}$ Dieses ermöglicht es ihr, sich ganz der Erziehung ihrer Kinder zu widmen und ist ein Beispiel dafür, wie eigene Kindheitserfahrungen und gesellschaftliche Entwicklung einander ergänzen. Die teilweise unkontrollierten Freiräume, die Kindern aufgrund der prekären Rahmenbedingungen der unmittelbaren Nachkriegszeit zur Verfügung standen, gehören zunehmend der Vergangenheit an und die Erziehung der Kinder rückt in den Mittelpunkt des Interesses (Schütze/ Geulen 1983, 38). Diese Tendenz ermöglicht es Frau Dienel, problemlos an das von ihr wertgeschätzte Erziehungssetting der Eltern anzuschließen, in dem die Mutter den Kindern jederzeit als Ansprechpartnerin zur Verfügung stand. Selbst ihre Tendenz zur Überbehütung stellt hierbei keine Ausnahme dar. ${ }^{161}$

Frau Dienels Modifikationen erlauben es der nachfolgenden Generation, die religiöse Alltagsprägung anzunehmen. Das Zurückstellen der eigenen Bedürfnisse stellt für ihre Tochter, Heike Ebel, jedoch eine widersprüchliche Forderung dar. Denn obwohl sie und ihr Bruder mit ihren Bedürfnissen einerseits die volle Aufmerksamkeit ihrer Mutter genießen, sollen sie andererseits lernen, eben diese Bedürfnisse zurückzustellen. Heike Ebel selbst räumt im Vergleich zur Vorgängergeneration den eigenen Bedürfnissen einen höheren Stellenwert ein, was mit der im Zuge des Wertewandels zunehmenden Bedeutung von Selbstentfaltungswerten korrespondiert. Das beeinflusst ihr Erziehungssetting und die Forderung, die eigenen Bedürfnisse zurückzustellen, verliert an Relevanz.

Heike Ebels Kritik, sie sei zu wenig zur Eigenständigkeit erzogen worden, führt dazu, der Erziehung zur Selbstständigkeit einen hohen Stellenwert einzuräumen. Damit integriert sie einen Erziehungswert, „Selbständigkeit und freier Wille“, der seit den 1950er Jahren eine zunehmende gesamtgesellschaftliche Akzeptanz erfahren hat und mittlerweile mehrheitlich befürwortet wird (Reuband 1997, 134). Gleichzeitig erfordert das Primat der optimalen Kindesförderung (Beck-Gernsheim 1990a, 168) geradezu ein Erziehungssetting, das die Eigenschaften Sicherheit, emotionale Zuwendung und

\footnotetext{
${ }^{159}$ Zum Themenkomplex des Wertewandels siehe Kap. 1.2.3. Zentrale Studien zum Wertewandel stammen von Inglehart (1989), Klages (1984) und Meulemann (1996b).

${ }^{160}$ Die 1950er und 1960er Jahre gelten „als Blütezeit von Ehe und Familie“ (Peuckert 2007, 36).

${ }^{161}$ Die „Neigung zum Überbehüten“ wird in der Literatur mit der Konstellation der bürgerlichen Kleinfamilie in Verbindung gebracht (Schütze/Geulen 1983, 44).
} 


\section{Familiale Tradierungsprozesse in einer Drei-Generationen-Perspektive}

Heranführung zur Eigenständigkeit (Schütze 2002, 78) beinhaltet. Das Erziehungsarrangement von Heike Ebel enthält diese Komponenten ebenso wie die Orientierung am Bezugsrahmen der religiösen Alltagsprägung.

Für Heike Ebel ist insbesondere der emotionale Rückhalt durch den Glauben wichtig, der auch für die Kindeserziehung leitend ist. Diese Schwerpunktsetzung lässt sich als Reaktion auf den Mitte der 1980er Jahre einsetzenden Individualisierungsdiskurs ${ }^{162}$ betrachten, indem sie versucht, durch die Akzentuierung der Aspekte Zugehörigkeit, Heimatgefühl und Rückhalt der über den Individualisierungsdiskurs transportierten Befürchtung einer zunehmenden Vereinzelung etwas entgegenzusetzen und für sich und ihre Kinder Gemeinschaft zu erhalten.

Die Erziehung zur Selbstständigkeit, die den Kindern gleichzeitig größere Freiräume zubilligt, gekoppelt mit dem höheren Stellenwert, der den eigenen Bedürfnissen zugeschrieben wird, erleichtert es der jüngsten Generation, Janina, an den familialen Bezugsrahmen der religiösen Alltagsprägung anzuknüpfen. Die zunehmende Etablierung von Selbstentfaltungswerten ermöglicht es Janina gleichzeitig, die Freude bereitenden Aspekte in den familialen Bezugsrahmen zu integrieren. Der hohe Stellenwert, den sie der Eigenständigkeit zuspricht, korrespondiert mit einer gesamtgesellschaftlichen Entwicklung, die Kinder zunehmend als „Akteur[e] in eigener Sache“ (Schütze 2002, 83) betrachtet, was untrennbar mit dem Attribut der Eigenständigkeit verbunden ist.

Insgesamt scheint die Erziehung im Generationenverlauf einen stärkeren Angebotscharakter zu erhalten, und strikte Forderungen, wie sie in den Kindheitserinnerungen der ältesten Generation gelegentlich zu finden sind, verlieren an Bedeutung. Die intergenerationellen Machtbalancen verschieben sich zugunsten der Kinder. Auch hier findet sich auf gesamtgesellschaftlicher Ebene eine Entsprechung, die mit den Schlagworten vom Befehls- zum Verhandlungshaushalt (Bois-Reymond 1994, 143) benannt ist.

In der Familie Cronert/Jäger wird der familiale Bezugsrahmen der intergenerationellen Hilfe und Solidarität im Generationenverlauf tradiert. Das korrespondiert mit einer in Ostdeutschland stärker ausgeprägteren Familienorientierung, die sich als Verpflichtung gegenüber Eltern und Kindern manifestiert (Meulemann 1996b, 356 und 358). Offenbar überdauert diese Orientierung in der Familie Cronert den Umbruch des politischen Systems bis in die Gegenwart. Insbesondere die Kindererziehung bleibt ein intergenerationelles Projekt. Die Betreuung Friederike Jägers durch die Großeltern mag dazu beigetragen haben, die Einflussmöglichkeiten staatlicher Institutionen auf die Kindeserziehung zu begrenzen. Nach der Wende ermöglicht das großelterliche Engagement Friederike Jäger und ihrem Ehemann, einer Vollzeitbeschäftigung nachzugehen.

Im Generationenverlauf werden zugleich Akzentverschiebungen sichtbar, die Entsprechungen auf gesellschaftlicher Ebene aufweisen und dazu dienen, den Bezugsrahmen im Zeitverlauf anschlussfähig zu halten. Für Herrn Cronert nehmen das gemeinsame Wirtschaften und Arbeiten zur Existenzsicherung der Familie noch einen zentralen Stellenwert ein. Bei Friederike Jäger verlagert sich der Akzent in ihren Kindheitserin-

${ }^{162}$ Zur Individualisierungsthese siehe exemplarisch Beck (1986) und Beck/BeckGernsheim (1990). 


\subsection{Flexibel erscheinende Tradierungslogiken}

nerungen auf die Zusammenarbeit von Eltern und Großeltern, um besondere Konsumwünsche zu erfüllen. Gleichzeitig erhalten die intergenerationelle Kommunikation und die emotionale Zuwendung für Friederike Jäger einen höheren Stellenwert - auch für die Erziehung der eigenen Kinder. Beide Aspekte sind auch für die jüngste Generation zentral. Der skizzierte Verlauf korrespondiert mit der Zunahme des allgemeinen und wirtschaftlichen Wohlstands, auch in der DDR, der es den Familien erlaubt, sich zunehmend auf das emotionale Wohlbefinden der Familienmitglieder zu konzentrieren.

Ein Vergleich der Kindheitserinnerungen lässt im Generationenverlauf eine zunehmende Kindzentrierung erkennen. Bei Friederike Jäger ist dies zum Teil sicherlich ihrem Einzelkindstatus geschuldet. Zum anderen fällt dies aber auch mit einer gesamtgesellschaftlichen Entwicklung zusammen, in deren Zuge das Kind zunehmend ins Zentrum der Aufmerksamkeit rückt. ${ }^{163}$

Die im Vergleich zu Herrn Cronert stärkere Betonung der kommunikativen sowie emotionalen Aspekte der Generationenbeziehungen bei Friederike Jäger und ihrer Tochter sind ein Ausdruck davon, dass gesamtgesellschaftlich in der Erziehung autoritäre Befehlsstrukturen zunehmend ersetzt werden durch intergenerationell ausgehandelte Regeln und Erklärungen bei gleichzeitiger Berücksichtigung der emotionalen Qualität der Beziehungen (Büchner 2010, 533, Schülein 1990, 86).

Bei der von Friederike Jäger thematisierten Selbstständigkeit handelt es sich um ein Erziehungsziel, das im Verlauf der letzten Jahrzehnte gesamtgesellschaftlich an Bedeutung gewonnen hat (Reuband 1997, 134). Die Entwicklung einer eigenen Meinung lässt sich als ein Teilbereich der Erziehung zur Selbstständigkeit subsumieren, reflektiert aber vermutlich zugleich Friederikes Erfahrungen in der DDR-Gesellschaft. Hilfsbereitschaft als allgemeines Sozialverhalten, das nicht mehr auf den Familienkreis begrenzt ist, verweist auf eine Entwicklung, in der den Sozialkompetenzen allgemein eine zunehmende Bedeutung beigemessen wird. Die in der jüngsten Generation im Vergleich $\mathrm{zu}$ den Vorgängergenerationen stärker thematisierte Leistungs- und Wettbewerbsorientierung ist ebenfalls im Zusammenhang mit den seit der Wende veränderten Rahmenbedingungen zu sehen, in deren Zuge diese Orientierungen zunehmend an Bedeutung gewinnen.

Anikas Wertschätzung der elterlichen und großelterlichen Unterstützung und Ratschläge, die sich bei einer gleichzeitig stärkeren Betonung der Eigenständigkeit auch bei Janina findet, korrespondiert mit einem allgemeinen Befund: Jugendliche sehen die Familie als Bereich, der Rückhalt und Schutz bietet (Fritzsche 2000, 104). Sie betrachten ihre Herkunftsfamilien nach wie vor als unterstützend und Eltern bleiben zumindest in bestimmten Bereichen wichtige Ansprechpartner und Ratgeber für ihre Kinder (Brake 2003, 26, o.V. 2006, 29, Hurrelmann, u.a. 2006, 36 und Hofer/Pikowsky 2002, 243).

\footnotetext{
${ }^{163}$ Diese Entwicklung ist für die Bundesrepublik belegt (Büchner 2010, 533, Büchner/Fuhs 1996, 198) und scheint ebenfalls für die Entwicklung in der DDR zu gelten, wie Reubands Ausführungen nahelegen (Reuband 1997, 149 und Reuband 1995, 228/229).
} 


\section{Familiale Tradierungsprozesse in einer Drei-Generationen-Perspektive}

Insbesondere die Schilderungen Herrn Cronerts, der eine familiale Zusammenarbeit von den Nachkriegsjahren bis zum heutigen Tag skizziert, lassen unter Rückgriff auf Reuband zunächst vermuten, dass das jeweilige Gesellschaftssystem nur einen begrenzten Einfluss hat und dem Familienleben praktisch eine familienspezifische Eigenlogik inhärent ist (Reuband 1995, 236). Allerdings ist gleichzeitig festzustellen, dass die prekäre Situation der Nachkriegsjahre sowie die Rahmenbedingungen in der DDR eine Forcierung und Verstärkung des familialen Prinzips der Hilfe und Unterstützung bewirkt haben. Am Bezugsrahmen der Familie Cronert/Jäger manifestiert sich die wechselseitige Durchdringung von familialer und gesellschaftlicher Ebene. Die Familie verarbeitet die gesellschaftlichen Rahmenbedingungen pragmatisch einer familienspezifischen Eigenlogik folgend und integriert sie in ihren familialen Bezugsrahmen.

Der Bezugsrahmen der familialen Unterstützung und Hilfe korrespondiert mit einem in den 1980er Jahren verstärkt einsetzenden Trend in der DDR: dem Rückzug ins Private, in dessen Zuge die Familie zum Refugium avanciert (Gysi 1990, 34). Die spezielle Bevölkerungskonstellation im Wohnort der Familie, eingesessene katholische Einwohner auf der einen Seite und Grenztruppen auf der anderen Seite, mag diese Tendenz zusätzlich verstärkt haben. Die aufgezeigte Lockerung der kindlichen Milieueinbindung in der jüngsten Generation untermauert diese Annahme. Die veränderten Rahmenbedingungen nach der Wende führen zu einer familialen Öffnung. Hierbei wird der familiale Bezugsrahmen keineswegs obsolet, sondern auf einen erweiterten Personenkreis bezogen.

Mit der Familie als dem primären Ort der gegenseitigen Unterstützung und Hilfe, die auf eigenverantwortliche Problemlösungen verweist, ist ein Element der katholischen Soziallehre angesprochen: das Subsidiaritätsprinzip. ${ }^{164}$ Im Falle Herrn Cronerts ist das Prinzip als solches zwar reflexiv zugänglich und tief verinnerlicht, eine mögliche Verankerung in der katholischen Soziallehre hingegen ist, da sie nicht ausdrücklich thematisiert wird, eher als eine implizite aufzufassen. Ungeachtet dessen wird die im Subsidiaritätsprinzip formulierte Einstellung tradiert. Für Friederike Jäger und ihre Tochter ist die familiale Solidarität und Hilfe zwar ebenfalls zentral, aber nicht mehr Gegenstand einer expliziten Reflexion. Diese Entwicklung lässt sich als ein Beispiel auffassen, wie ein Element als tacit knowledge (Shils 1981, 22) im Generationenverlauf weitergegeben wird und den Einstellungen einer Person zu Grunde liegt, ohne explizit zugänglich zu sein.

Durch die Verschränkung von familialer und gesellschaftlicher Ebene lässt sich aufzeigen, dass gesellschaftliche Entwicklungen auf die jeweilige Ausprägung der familialen Bezugsrahmen einwirken, ohne sie für die einzelnen Generationen obsolet werden zu lassen. Die einzelnen Generationen greifen gesellschaftliche Prozesse auf und integrieren sie in den familialen Bezugsrahmen wie in das Erziehungssetting, um hierüber die Anschlussfähigkeit des familialen Bezugsrahmens herzustellen.

\footnotetext{
${ }^{164}$ Eine einführende Übersicht zum Subsidiaritätsprinzip gibt Nothelle-Wildfeuer $(2008,157)$.
} 


\subsection{Geschlossene Tradierungslogiken}

\subsubsection{Familie Mahler/Ahrens: Vertraute Horizonte vs. Horizonterweiterung oder Milieuorientierung}

Biografische Eckdaten Frau Mahler wird 1938 in Pommern geboren. Ihr Vater fällt im Zweiten Weltkrieg. Nach der Flucht lebt sie mit ihrer Mutter und ihrem Bruder zunächst in einem Ort in Niedersachsen. Als ihre Mutter erkrankt, kommt sie in eine Pflegefamilie in einem anderen niedersächsischem Dorf, das katholisch geprägt ist. Dort absolviert sie eine Lehre und heiratet 1959 ihren Mann, der eine Handwerkerausbildung hat. Zunächst leben sie bei den Eltern ihres Mannes. Später ziehen sie mit ihren Kindern in ein eigenes Haus. Das Paar ist katholisch und hat sechs Kinder. Michelle Ahrens, die Interviewpartnerin der mittleren Generation, wird 1961 geboren. Sie ist das zweite Kind und zugleich die älteste Tochter. Nach ihrem Hauptschulabschluss absolviert sie eine Schneiderlehre. Auch nach der Heirat mit Herrn Ahrens, einem Angestellten, führt sie ihren Beruf fort. Inzwischen arbeitet sie in ihrem eigenen Betrieb. 1983 wird ihr Sohn geboren und 1989 ihre Tochter Sonja. Sonja hat inzwischen ihr Abitur und war während der Schulzeit ein Jahr im Ausland. Die Familie von Michelle Ahrens lebt in einem Nachbarort der Eltern in Niedersachsen.

Aneignungsebene Frau Mahler $\left({ }^{*} 1938\right)^{165}$ : Eine prekäre Kindheit Charakteristisch für Frau Mahlers Kindheitserinnerungen ist, dass sich die Kindheit in mehrere Abschnitte unterteilen lässt. Die ersten Lebensjahre verlebt sie mit ihrer Familie in Pommern. Sie lebt zusammen mit Eltern und Bruder im Haus ihrer Großmutter väterlicherseits, das ihr Vater einmal erben soll. Dieser fällt jedoch im Zweiten Weltkrieg. Nach der Flucht der Familie aus Pommern werden die Familienangehörigen sowie die anderen Bewohner des Heimatortes auf verschiedene Dörfer in Niedersachsen verteilt (00:40-01:58, 05:29-05:48). Während Frau Mahler mit ihrer Mutter und ihrem jüngeren Bruder zusammen in einem Ort leben, werden die übrigen Verwandten in anderen Dörfern untergebracht. Ihre Mutter erkrankt in der Folgezeit und ist nicht mehr in der Lage, sich um ihre Kinder zu kümmern. Frau Mahler kommt schließlich zu einer Pflegefamilie in eine andere niedersächsische Region, in der sie heute noch lebt (01:59-02:28).

Für Frau Mahler beginnt mit der Flucht ein prekärer Lebensabschnitt. Die Familie habe in beengten Verhältnissen gelebt, und die Versorgung mit Nahrungsmitteln sei schwierig gewesen (20:53-21:31, 31:12-32:12). Anfangs sei die Mutter zwar noch in der Lage gewesen, sich um ihre Kinder zu kümmern, dies habe sich aber im Laufe der Zeit geändert. Die Mutter sei einerseits „sehr lieb“ gewesen, habe bei Bedarf wohl auch bei den Hausaufgaben geholfen (40:46-40:58, 44:56-45:31), aber die grundsätzliche Organisation des Familienlebens hat sie anscheinend in zunehmenden Maße überfordert. Wie die Interviewpassagen zeigen, fällt die Mutter als verlässliche Bezugsperson aus. Frau

\footnotetext{
${ }^{165}$ Das Interview mit Frau Mahler wurde am 23.02.2009 geführt.
} 


\section{Familiale Tradierungsprozesse in einer Drei-Generationen-Perspektive}

Mahler ist schon früh auf sich allein gestellt und muss Verantwortung übernehmen (35:29-35:42 und 41:00-41:55). Zu dieser Belastung kommt eine Verunsicherung hinzu. Frau Mahler kann sich zwar an keine Konflikte mit der Mutter erinnern, wohl aber daran, dass sie das mütterliche Verhalten teilweise nicht habe verstehen und einordnen können, z.B. wenn die Mutter geschimpft habe „über alles, nicht über uns, sondern über alles" (44:18-44:44).

Die mütterliche Erkrankung sieht Frau Mahler in einem kausalen Zusammenhang stehend mit den radikal veränderten Lebensumständen nach der Flucht:

„Meine Mutter war das erste Mal so auf sich ganz allein gestellt mit uns. //hmh// Weil es waren, ÜBERALL waren Großfamilien. //hmh// So war das früher. //hmh// Und das äh hat sie wohl nicht allein geschafft" (22:2522:35).

Die fehlende Einbindung in einen größeren Gruppenzusammenhang ist für Frau Mahler ausschlaggebend für die familialen Probleme. Bereits hier deutet sich an, dass Frau Mahler als Konsequenz aus dieser Deutung einer solchen Einbindung einen zentralen Stellenwert einräumt. Zugleich zeigt sich in ihrer Argumentation eine starke Orientierung an dem, was von ihr als üblich betrachtet wird. Vor diesem Hintergrund sind ihre Beschreibungen der Kindheit vor der Flucht zu verstehen, die anders und - als Subtext mitschwingend - besser gewesen sei: Die Geburtstage seien z.B. anders gefeiert worden:

„[A]ber früher hatte man doch so, so Freunde und so drum rum. //hmh// Das $=$ das war anders, das war anders. UND Verwandtschaft hatte man auch, ne. //hmh// Ich war hier in, war ich immer alleine, //hmh// ne“ (37:15$37: 27)$.

Auf Nachfrage zu den Geburtstagen führt sie konkretisierend aus:

„[I]ch war natürlich noch sehr klein, aber das war eben, da war die Mama da, da war die Oma da, und=und äh da war die Tante da und so. Hier in $=$ als ich hier war, war ich ja, hatte ich ja GAR keine Verwandtschaft. //hmh// Da hatte ich, war, war, war immer anders“ (38:09-38:22).

Die Geborgenheit in der Verwandtschaft wird zur Projektionsfläche für eine gute Kindheit ohne die für sie nach der Flucht spürbaren Entbehrungen. Sie blickt insofern verklärend in die Vergangenheit, als sie suggeriert, es gebe keine Probleme, sobald nahe Verwandte oder eine Gruppe nahestehender Personen vorhanden seien. Zugleich manifestiert sich deutlich, dass ihr während der späteren Kindheit Rückhalt bietende Bezugspersonen fehlen. In der frühen Kindheit und während der Flucht ist die Mutter offensichtlich noch in der Lage gewesen, diesen Part auszufüllen. Das legt ihre Erinnerung nahe, während der Flucht immer bei der Mutter gewesen zu sein, die sie in schwierigen Situationen, wie bei Kontrollen, „immer festgehalten“ habe (20:10-20:19). Als die Mutter ihre Aufgabe als verlässliche Bezugsperson nicht mehr wahrnehmen kann, 


\subsection{Geschlossene Tradierungslogiken}

übernimmt teilweise die Großmutter diese Funktion, die zu der Zeit in einem anderen Ort gelebt habe: „Aber zu meiner Großmutter, die ich sehr, bis heute noch sehr liebe und verehre, //hmh// war immer der Kontakt da" (02:31-02:41). Die Verlässlichkeit wird von Frau Mahler als wichtiges Charakteristikum der Großmutter hervorgehoben: „We:nn wir Kinder Geburtstag hatten, wir wohnten ja im anderen Ort, //hmh// dann kam die FÜNF Kilometer gelaufen. Die kam. Wir wussten ganz genau, sie kam. [...] Aber sie war immer da“ (06:37-07:14). Die Großmutter habe den Enkeln Kraft gegeben (42:32-42:40) und sei zudem diejenige gewesen, die mit ihr die Verwandten im Umkreis besucht habe (08:19-08:42). Die Großmutter versucht, die familiale Gemeinschaft aufrechtzuerhalten. Auch an den Pfarrer hat sie positive Erinnerungen:

„Wir hatten einen SEHR guten Pfarrer, 'nen guten alten Pfarrer, pommerschen Pfarrer, der hat die Leute zusammengehalten, (.) äh wunderbar. Der hat immer dafür gesorgt, dass auch mal 'ne ZusAmMENKUNFT war. [...] Und er hat sich sehr so immer: ,Was macht ihr denn?‘, hat Hausbesuche gemacht und so, hat sich sehr eingesetzt" (33:30-34:01).

Der Pfarrer sorgt für die von Frau Mahler so sehr vermisste Gemeinschaftseinbindung, zumindest bezogen auf außerfamiliale Strukturen, kümmert sich um seine Gemeindemitglieder und bietet so ein gewisses Maß an Rückhalt und Struktur; beides Elemente, die Frau Mahler aufgrund der familialen Situation fehlen.

Die Religion ist ein fester Bestandteil ihres Aufwachsens. Ursprünglich evangelisch getauft, habe die Mutter die Kinder später, als sie allein gewohnt hätten, mit in die katholische Messe genommen und sie zur Kommunion geschickt (26:21-26:45). Die Großmutter, evangelischen Glaubens, habe dies akzeptiert (32:34-33:06). Die Großmutter habe ihr das „Vater unser“ und die Mutter das „Ave Maria“ beigebracht (42:21-42:24, 43:08-43:10). Die üblicherweise klare Zuordnung zu einer Religionsgemeinschaft ist damit zeitweise aufgehoben - ein Umstand, der möglicherweise zusätzlich verunsichernd gewirkt haben und das Zugehörigkeitsgefühl zu einer Gruppe, diesmal der religiösen, erschwert haben könnte.

Als sie im Alter von ca. 13 Jahren in eine Pflegefamilie kommt, erlebt sie feste Strukturen:

„[I]n der Pflegefamilie, was ich dann so kennen gelernt habe, jeden Sonntag in die Kirche, //ja//, ne. Das war so. (.) Ja, und die Feste wurden so eingehalten. $/ / \mathrm{hmh} / /$ (.) Und eben, wie gesagt, wir mussten alle mit anfassen, $/ / \mathrm{hmh} / /$ alle so ein bisschen $=$ und das, äh das fand ich natürlich auch gut, //hmh// man lernt ja dadurch auch. //hmh// (2) Zum Spielen war immer noch Zeit genug“ (47:08-47:44).

Frau Mahler fügt sich in die ihr vorgegebenen Bahnen ein, sei es, weil ihr die hiermit verbundenen Strukturen Orientierungssicherheit geben oder weil sie den nun gegebenen 


\section{Familiale Tradierungsprozesse in einer Drei-Generationen-Perspektive}

Anschluss an eine Gruppe nicht gefährden möchte. Zudem habe die Großmutter sie gebeten: „Kind sei artig. Verhalte dich richtig“ (49:32-49:39). Frau Mahler habe gehorsam sein und tun sollen, was man ihr sage (50:12-50:17), woran sie sich offensichtlich hält:

„[W]ir mussten auch äh mit zufassen im Haus, //hmh// ne. Und wir mussten mit helfen, äh Wäsche aufhängen oder=oder mal in den den Garten gehen mit, ein bisschen jäten oder ein bisschen hacken oder Gemüse einkochen. Das haben wir alles äh mitgemacht. //hmh// Das war einfach so, ne. //hmh// Und das hat auch Spaß gemacht" (10:10-10:29).

Hier zeichnet sich ein asymmetrisches Machtverhältnis ab. Die Erwachsenen erlegen den Kindern Pflichten auf und bestimmen die Regeln. Den Kindern bleiben kaum Optionen, diese zu verändern. Sofern sich die Kinder an die Vorgaben halten, werden ihnen aber nach Erfüllung der Pflichten Freiräume zugestanden. Die von den Erwachsenen gesetzten Strukturen werden von Frau Mahler nicht in Frage gestellt. Vielmehr wird diese Konstellation durch den Zusatz „das war einfach so “ als üblicher Standard gerechtfertigt, den Frau Mahler akzeptiert. Folglich finden sich auch keine Hinweise für ein Rebellieren gegen die vorgegebenen Strukturen. Im Gegenteil scheint sie sich eher passiv zu verhalten, anpassungsbereit und genügsam zu sein, dankbar für die Ordnungsstrukturen, die ihr Leben erhält, und äußerst gewillt, die positiven Seiten des ihr vorgegebenen Alltags zu betonen. Dies kommt in ihren wiederholten positiven Bilanzierungen, es sei schön gewesen oder es habe Spaß gemacht, zum Ausdruck. Zugleich wirkt die feste Struktur, in der von den Pflegeeltern die Entscheidungen getroffen werden, entlastend, da Frau Mahler der Verantwortung für die Gestaltung ihres Alltags enthoben ist.

Direkte Verbote habe es nicht gegeben, sie scheinen aber auch nicht notwendig gewesen zu sein, da Frau Mahler die zentralen Maximen durchaus bewusst gewesen sind. Wichtige Orientierungen, die man ihr habe vermitteln wollen, seien der Glaube, Gehorsam, Sauberkeit, Ordnung sowie Pünktlichkeit gewesen (47:54-48:14, 52:12-52:27).

Obwohl Frau Mahler sehr bemüht ist, die positiven Seiten des klar geregelten Lebens in der Pflegefamilie hervorzuheben, ist zu erkennen, dass sie nicht völlig zufrieden ist. Vor allem eine emotionale Zuwendung scheint sie vermisst zu haben: „[A]ber (.) äh we:nn, wenn so 'ne Mutter da ist, die ähm (.) nimmt dann auch mal in den Arm, wenn man so Kind ist oder so" (54:28-54:40). Dies habe zwar die Großmutter, die sie weiterhin regelmäßig gesehen habe, zu kompensieren versucht, indem sie „das rübergebracht [hat, C. R.] so, (.) die Liebe“ (07:14-07:55). Allerdings scheint dies für Frau Mahler kein vollwertiger Ersatz für die mütterliche Zuwendung gewesen zu sein.

Die abwesende Mutter, das Vermissen von verlässlichen und emotional zugewandten Bezugspersonen im Alltag, die fehlende Gemeinschaftseinbindung, das Erleben von vorgegebenen Strukturen in der Pflegefamilie, Anpassungsbereitschaft und Genügsamkeit stellen für Frau Mahler wichtige Erfahrungen dar, die die Etablierung ihres zentralen Bezugsrahmens entscheidend beeinflussen. Die familialen Probleme werden von ihr mit dem Eindruck rückgekoppelt, nicht dem von ihr als Standard betrachteten Familienbild $\mathrm{zu}$ entsprechen. In der Folge wird für sie eine Orientierung am sie umgebenden Milieu 


\subsection{Geschlossene Tradierungslogiken}

und dem, was sie als üblichen Standard im Nahfeld betrachtet, elementar bedeutsam. Hierzu zählen die festgeschriebenen Regelungen und Bahnen, die sie in ihrer Pflegefamilie erlebt. Da die geregelten Strukturen und Vorgaben allein jedoch als nicht ausreichend für eine zufriedene und geglückte oder dem Standard entsprechende Kindheit erfahren werden, werden die noch vermissten Elemente, in erster Linie die emotionale Zugewandtheit, auf die Mutterfigur projiziert. Die Verfügbarkeit der Mutter als Bezugs- und Betreuungsperson wird zum Garanten für eine glückliche, dem vermeintlichen Standard folgende Kindheit, die zugleich ein harmonisches Familienleben sicherstellt. Ihr Anspruch, dem angenommenen Standard des Milieus zu entsprechen - unter Berücksichtigung eines traditionellen Mutterideals sowie eines idyllischen und harmonischen Familienlebens -, wird in der Folge für Frau Mahler der zentrale Bezugsrahmen, der die von ihr praktizierte Erziehung lenkt.

Vermittlungsebene Frau Mahler (*1938): Starre Normorientierung Für Frau Mahler bildet die Vorstellung der nicht berufstätigen Mutter, die sich ganz ihren Kindern widmet, die grundlegende Komponente ihres Erziehungssettings:

„[I]ch hatte auch nicht das Verlangen, dass ich hätte müssen arbeiten gehen oder so. $/ / \mathrm{hmh} / /$ Das hat mich, wenn manche immer gesagt haben, ähm, noch so ein bisschen zu verdienen, hab ich immer gedacht, du hast so viel vermisst in deiner Kindheit. //hm// Dann hab ich immer gedacht, was Schöneres gibt es gar nicht für die Kinder, als wenn jemand zu Hause ist, $/ / \mathrm{hmh} / /$ ne. (Also) das fand ich immer" (1:15:00-1:15:21).

Basierend auf ihren eigenen Kindheitserfahrungen entwirft Frau Mahler eine dogmatisch anmutende Einstellung, was das grundlegende Arrangement der Erziehung betrifft. Wie sich ihren Ausführungen entnehmen lässt, ist das von ihr vertretene Mutterideal in ihrem Umfeld zumindest ansatzweise im Wandel. Hierauf reagiert sie mit einer vehementen Ablehnung und dem Festhalten an den von ihr als üblich und richtig angenommenen Strukturen. Ebenso blendet sie die Möglichkeit aus, dass andere Familienmitglieder eine abweichende Perspektive einnehmen könnten. Aus der Aussage spricht eine starke Verinnerlichung des bürgerlichen Familienideals. Das Mutterbild wirkt fast mythologisch aufgeladen als eine Art Heilserwartung: die Anwesenheit der Mutter als Garant für eine glückliche Kindheit. Ansonsten bleibt das hier so stark betonte Mutterbild jedoch recht vage und unkonkret. Wie die Mutter-Kind-Beziehung genau ausgestaltet sein sollte, wird nicht weiter spezifiziert. Hierbei geht Frau Mahler von einer Mutter-Kind-Bindung aus, die quasi natürlich gegeben sei. Dies wird deutlich, wenn sie darüber spricht, dass die Kinder trotz eigener Zimmer immer zu ihr gekommen seien: „Es ist ja so, die haben oben geschlafen. //hmh// Aber wo ziehen die Kinder sich hin? //hmh//Immer unten, wo die Mutter ist, //hmh// in meine Küche“ (1:11:37-1:11:46). Sie selbst orientiert sich an der oben skizzierten Maxime und widmet sich als Hausfrau und Mutter ganz ihren Kindern - zumindest bis das jüngste Kind ein gewisses Alter 


\section{Familiale Tradierungsprozesse in einer Drei-Generationen-Perspektive}

erreicht hat. Sie berichtet von ihren Aufgaben im Haushalt und dem Versorgen der Kinder. Neben der Orientierung an einem Rollenideal dürfte die Versorgung der achtköpfigen Familie Frau Mahlers ganze Aufmerksamkeit beansprucht haben, wie sie selbst feststellt: „Arbeit hatte man genug“ (1:00:00-1:00:05). Unterstützung erhält sie von ihrer Schwiegermutter, die ihr im Haushalt und bei der Betreuung der Kinder geholfen habe (14:11-14:13, 1:00:16-1:00:34). Hierin zeigt sich die praktische Bedeutung der von Frau Mahler in der Kindheit vermissten und nun offensichtlich gegebenen familialen Gemeinschaftsanbindung: Entlastung und Hilfe, die zugleich das Gemeinschaftsgefühl und den Zusammenhalt verstärken können. In der Familie existiert eine klassische Aufgabenteilung zwischen den Geschlechtern: Die Frauen sind für Familie und Haushalt zuständig. Frau Mahlers Rollenideal erfährt somit eine Unterstützung und Verstärkung durch das Umfeld.

Wenn Frau Mahler über ihr Familienleben spricht, so sticht hervor, dass sie wiederholt Umschreibungen wählt, die geeignet sind, Assoziationen eines harmonischen, idyllischen Familienlebens hervorzurufen, ohne dass sie konkret sagen würde, ihr Familienleben sei so gewesen. Frau Mahler charakterisiert ihr Familienleben mit den Worten: „Es war@(.)@//@(.)@// bei uns war immer was los, möcht' mal so sagen“"(13:26-13:30). Diese Umschreibung ist zunächst noch deutungsoffen. Sie kann positiv im Sinne von lebendig und schön interpretiert werden, kann aber auch bedeuten, dass es in der Familie z.B. viele Konflikte gegeben hat, und deswegen „immer was los“ gewesen ist. Gerade der Zusatz „möcht mal so sagen“ verweist darauf. Mit ihrer doppeldeutigen Wortwahl hätte Frau Mahler somit eine euphemistische Umschreibung für ihr Familienleben gefunden, ohne die Aspekte, die das Bild eines harmonischen Familienlebens stören würden, direkt anzusprechen und dem Gesprächspartner vor Augen zu führen. Die weiteren Ausführungen hingegen verweisen auf eine positive Lesart, die Frau Mahler nach außen vermitteln möchte. So habe die Familie ", [s]chön in=in, so in der Natur" (1:03:48-1:03:59) gewohnt, habe viel Platz gehabt und „die Kinder konnten schön draußen spielen“ (13:40-13:51). Die Schwiegermutter sei gern zu ihnen gekommen, weil in ihrer Wahrnehmung in der Familie „wenigstens was los“ gewesen sei (13:33-13:37). Gefragt nach dem Tagesablauf, beschreibt Frau Mahler zuerst ein Bild von ihren „drei Mädchen (.) im Dirndlkleidchen“, das sie bis heute vor Augen habe (15:41-15:56). Hierin ist unschwer eine Versinnbildlichung eines Familienidylls zu erkennen. Wenn sie der Verwandtschaft in der Landwirtschaft geholfen habe, was sie gern getan habe, seien die Kinder nach der Schule zu ihr auf das Feld gekommen und dann seien ,alle über den Frühstückskorb hergefallen" (1:03:23-1:03:34). Am Wochenende, wenn der Ehemann zu Hause gewesen sei, habe sie viel gebacken: „Was hab ich für Kuchen gebacken. O:h, @(.)@ und dann (Grappeln) gebacken. Da war 'ne richtige @Zuckerstraße durch die Küche@“ (1:09:49-1:09:58). Diese Illustrationen des Familienlebens, das Sorgen für das leibliche Wohl der Familienmitglieder sowie die Pausen vom Alltag, rekurrieren auf positiv konnotierte Bilder des Familienlebens. Hierbei entsteht der Eindruck, mit der Sicherstellung des leiblichen Wohlergehens sei auch das psychische Wohlbefinden der Familienmitglieder gewährleistet. 


\subsection{Geschlossene Tradierungslogiken}

Diese Darstellungen überlagern andere Aspekte, die vielleicht zu einer Relativierung dieses idyllischen Bildes beitragen könnten. So wird nicht thematisiert, ob die Kinder in der Landwirtschaft mitarbeiten mussten oder wie die familialen Interaktionen an den Wochenenden konkret gestaltet gewesen sind, ob die gemeinsame Freizeit im Vordergrund gestanden oder ob es Konflikte gegeben hat. Überaus deutlich hingegen wird, dass ein harmonisches Familienleben für Frau Mahler ein wesentlicher Bestandteil ihres Bezugsrahmens ist. Wie zentral diese Vorstellung bis heute für sie ist, wird an ihrer Aussage deutlich, ein Enkel habe sie als „Landliebe-Oma“ (1:21:16-1:21:21) bezeichnet. Hiermit rekurriert sie auf eine Werbung, in der ein idyllisches und harmonisches Familienleben in einer ländlichen, nostalgisch anmutenden Umwelt ohne nennenswerte Alltagsprobleme und -konflikte vorgeführt wird. Die Metapher der "Landliebe-Oma“ versinnbildlicht somit schlagwortartig das Familienideal, dem Frau Mahler bis heute verhaftet ist. Das von ihr favorisierte Familienbild ist sicherlich vor dem Hintergrund ihrer eigenen Kindheitserfahrungen zu verstehen, in dem dieses Ideal unerreichbar gewesen ist. Gleichzeitig orientiert sich Frau Mahler stark daran, was - zumindest ihrer Ansicht nach - in dem sie umgebenden Milieu üblich ist. Dies zeigt sich u.a. an kleinen Randbemerkungen, wie etwa, sie hätten in dem Viertel gewohnt, in dem „die jungen Familien“ gewesen seien, oder an Erziehungsregeln, die als „selbstverständlich“ oder „ganz normal“ klassifiziert werden (13:51-13:57, 16:02-16:16:10). Da Frau Mahler relativ vage und unkonkret bleibt, wenn sie über ihre eigene Familienphase berichtet, ist dies als Indiz zu werten, dass das konkrete Familienleben nicht diesen (überaus hohen) Ansprüchen entsprochen hat, ja vermutlich gar nicht entsprechen konnte. Auch die wiederholten positiven Bilanzierungen von kleineren Begebenheiten sowohl während ihrer Kindheit als auch während ihrer Familienphase deuten hierauf hin.

Angesichts dieses Familienideals, in dem Konflikte nicht vorkommen, ist es nicht verwunderlich, wenn Frau Mahler kaum Auseinandersetzungen und Probleme thematisiert. Dass das Familienleben nicht nur idyllisch, sondern auch mit viel Arbeit für sie und ihren Mann verbunden gewesen ist und die finanzielle Situation manchmal angesichts der Versorgung von sechs Kindern durch einen Alleinverdiener angespannt gewesen sein dürfte, deutet sie lediglich an: „Mein Mann war (.) äh viel in der Fremde. $/ / \mathrm{hmh} / /$ (2) So ein Haus kostet auch viel Geld und wollen alle was essen und=und äh@was anziehen@. //hmh// (8) Manchmal denke ich, wie HAT man das nur alles geschafft" (14:22-14:53). Als die Kinder größer gewesen seien, habe es manchmal Konflikte gegeben, „[w]enn sie auch mal raus wollten und man wollte nicht, und hat gesagt, das gibt es nicht“ (1:05:03-1:05:06). Da die Ausführungen relativ vage bleiben, spricht Frau Mahler vermutlich nicht gerne über dieses Thema. Auch wenn über die Inhalte der Konflikte wenig zu erfahren ist, beinhalten die Ausführungen wichtige Hinweise, was den Umgang mit diesen und die intergenerationelle Machtverteilung betreffen. Weder erklärt Frau Mahler ihre Entscheidung, noch ist sie verhandelbar. Auch wird nicht nach einer Lösung gesucht, die für alle Beteiligten akzeptabel ist. Konflikte werden nicht ausgetragen, sondern mit einem elterlichen Machtwort beendet. Folglich herrscht in 


\section{Familiale Tradierungsprozesse in einer Drei-Generationen-Perspektive}

dieser Familie eine asymmetrische Machtbalance zugunsten der Eltern. Auch hier ist anzunehmen, dass Frau Mahler sich im Einklang mit dem Erziehungsverhalten des sie umgebenden Milieus sieht.

Frau Mahlers starke Fixierung auf eine Aufrechterhaltung der familialen Gemeinschaft ist als Resultat der eigenen Kindheitserfahrungen zu betrachten, die auch während ihrer eigenen Familienphase zum Tragen kommt: „Da hab ich immer, als unsere Kinder klein waren, da hab ich immer gesagt, es is $\triangle С H O ̈ N$, wenn sie alle zusammen sind und $=$ und $=$ und $/ / \mathrm{hmh} / /$ hab das auch immer hochgehalten $/ / \mathrm{hmh} / /$, ne" (36:42-36:49). Zugleich ist hierin ein weiterer Grund zu sehen, warum Konflikte ausgeblendet werden: Sie werden als potenzielle Bedrohung der familialen Gemeinschaft aufgefasst.

Auch in anderen Bereichen der Erziehung folgt Frau Mahler den üblichen, ihr vertrauten Bahnen. Die Kinder hätten abends pünktlich zu Hause sein müssen, Frau Mahler habe auf Ordnung und Sauberkeit geachtet, die Schularbeiten hätten erledigt werden müssen und die Kinder hätten ihre Schulsachen zu Hause ordentlich verstauen müssen (59:29-59:31, 1:06:40-1:07:00). Das gemeinsame Mittagessen sei eine Selbstverständlichkeit gewesen und die Kinder hätten mithelfen müssen (16:02-16:14, 1:10:45-1:10:57, 1:05:59-1:06:11). Ihre Tochter, Michelle Ahrens, habe zudem auf die kleineren Geschwister aufpassen müssen, wenn die Erwachsenen keine Zeit gehabt hätten (17:0717:11). Es fällt auf, dass Frau Mahler im Zusammenhang mit diesen Regeln jeweils das Verb müssen verwendet. Das verweist auf den nicht verhandelbaren Verpflichtungscharakter dieser Regeln. Religiosität und gemeinsame Familienzeiten sind weitere wichtige Aspekte:

„[D]ies Christliche //hmh// haben wir übernommen, in die Kirche mussten sie auch. $/ / \mathrm{hmh} / /$ (1) Das war eigentlich immer so. Sonntags in die Kirche. Die Feste = die, die kirchlichen Feste wurden eingehalten und auch, auch ähm, wir haben immer, sonnabends und sonntags gab es bei uns immer Kaffee und Kuchen. //hmh// Das hab ich, da hab ich auch immer viel gebacken und gemacht und $/ / \mathrm{hmh} / /$ ich fand das auch immer schön. $/ / \mathrm{hmh} / /$ Und die Feste wurden richtig, wie sich das gehört, auch eingehalten" $(1: 09: 00-1: 09: 30)$.

Auf die Nachfrage, ob bei diesen Gelegenheiten alle Familienmitglieder zu Hause gewesen seien, erklärt Frau Mahler: „Ja, das war eigentlich immer so. Das musste auch sein“ (1:09:40-1:09:43). Auch in dieser Familie erfolgt eine Einbindung in das religiöse Milieu durch Partizipation an den religiösen Praxen. Ebenso wie in anderen Familien wird auf gemeinsame Familienzeiten geachtet, über die die Familiengemeinschaft aufrecht erhalten wird und die Gelegenheiten zur beiläufigen Tradierung schaffen. Allerdings hebt Frau Mahler hier ebenso wie bei den zuvor angesprochenen Themen den verpflichtenden Charakter hervor: Die Kinder mussten teilnehmen. Von Freiwilligkeit, die eine Akzeptanz fördern könnte, kann keine Rede sein. Das dürfte zugleich die Möglichkeiten einer beiläufigen Weitergabe von Erziehungsinhalten erschwert haben. Zudem wird 


\subsection{Geschlossene Tradierungslogiken}

in dieser Passage recht deutlich, dass Frau Mahler diese Zusammenkünfte zwar als schön erinnert, sie aber die Perspektive der Kinder nicht berücksichtigt. Ausschlaggebend ist die Sichtweise der Erwachsenen, was abermals die Asymmetrie der intergenerationellen Machtverteilung sowie das Anknüpfen an die eigenen Kindheitserfahrungen unterstreicht.

Dieses hierarchische Machtgefälle scheint Frau Mahler an anderen Stellen auszublenden, indem sie euphemistische Umschreibungen wählt, etwa dass die Kinder hätten ,artig“ (59:14-59:15) sein müssen. Hierdurch wird der Umstand, dass sie aktiv zur Herstellung dieser Machthierarchien beiträgt und Gehorsam verlangt und einfordert, überdeckt. Vielmehr wird die Handlungsebene auf die Kinder verlagert, von denen etwas erwartet wird, nämlich „artig“ zu sein. Diese tendenziell beschönigende Darstellung des Aspekts Gehorsam wird in Verbindung mit dem Mutter- und Familienbild Frau Mahlers verständlich. Der Wunsch nach einem harmonischen, idyllischen Familienleben sowie einer emotional den Kindern zugewandtem Mutter sind nicht leicht mit der Forderung nach Unterordnung der Kinder in Einklang zu bringen.

Die Rigidität, mit der die Regeln umgesetzt worden sind, rechtfertigt Frau Mahler mit der elterlichen Kontrollpflicht und Verantwortung: „Na ja, man musste ja auch immer (.), immer aufpassen, dass ja nichts passiert. Der eine hier, der andere dort und dann immer noch so ein Kleines dabei“ (59:29-59:47). Hier kommt eine generelle Ängstlichkeit zum Ausdruck, die darauf basiert, nicht gern die Verantwortung zu übernehmen, was sich bereits in Frau Mahlers Kindheit zeigt.

Auch wenn offensichtlich die Belange und Wünsche der Kinder in der Regel nicht ausschlaggebend für das elterliche Handeln sind, versucht Frau Mahler an einigen Stellen auf die Bedürfnisse der Kinder einzugehen:

„[W]ir haben auch viel äh gesungen. [...] Wir hatten ja immer einen großen Abwasch. //hmh// Und dann haben wir in der Küche, ich hab abgewaschen und die Kinder haben abgetrocknet, [...] dann hab ich immer, wenn es ging, mit den Kindern gesungen, $/ / \mathrm{hmh} / /$ ne. Um das eben so ein bisschen leichter zu machen" (1:10:24-1:10:41).

Indirekt kommen hier intergenerationelle Alltagskonflikte zum Ausdruck. Die den Kindern zugeteilten Aufgaben werden offenbar nicht immer klaglos ausgeführt. Frau Mahler löst dieses Problem, indem sie beim gemeinsamen Abwasch auf die selbst erfahrene Erziehung zurückgreift: das Singen, das sich in ihren Kindheitserinnerungen als mütterliche Taktik findet, um den Arbeitscharakter von Aufgaben abzumildern (43:16-43:58).

Trotz Frau Mahlers genereller Abneigung, familiale Konflikte zu thematisieren, äußert sie schließlich doch eine Kritik, die nicht die eigene Familienphase betrifft, wohl aber deren zentrale Orientierungen:

„Und wenn ich manchmal heute sehe, ich meine, (hier unsere Kinder), ich war damals zu Hause mit unseren Kindern, was heute aufgestellt wird mit Kindern, $/ / \mathrm{hmh} / /$ von = von klein auf geht das schon los, Babyturnen und 


\section{Familiale Tradierungsprozesse in einer Drei-Generationen-Perspektive}

alles Mögliche, //hmh// mein Gott. //hmh// Es geht auch anders. //ja, $\mathrm{hmh} / /$ Es geht auch mit weniger. (1) Die Hauptsache ist, die Mutter ist da“ (51:30-51:57).

Auch wenn die Kritik als eine allgemeine verpackt ist, zielt sie doch auch deutlich auf die eigene Tochter, Michelle Ahrens, die mit ihren Kindern zum Babyturnen gegangen ist und die nicht dem Modell der Hausfrauenehe folgt. Die Passage liefert darüber hinaus Hinweise auf den Stellenwert der Bildungsförderung in der Vergangenheit. Das Bereitstellen von besonderen Erlebnis- oder Bildungsangeboten sowie die individuelle Förderung der Kinder scheinen demnach nicht im Fokus des elterlichen Handelns gestanden zu haben. Auch dem emotionalen Wohlbefinden der einzelnen Kinder scheint in der Gesamtschau keine besondere Aufmerksamkeit gewidmet worden zu sein. Frau Mahler orientiert sich an der selbst erfahrenen Erziehung und folgt im Wesentlichen den ihr bekannten Bahnen, ohne grundsätzliche Veränderungen an diesem Setting vorzunehmen.

\section{Aneignungsebene Michelle Ahrens $\left({ }^{*} 1961\right)^{166}$ : Die Diskrepanz zwischen müt-} terlichem Ideal und familialer Wirklichkeit Michelle Ahrens' Schilderungen der eigenen Kindheit bilden einen scharfen Kontrast zum mütterlichen Bezugsrahmen des harmonischen und idyllischen Familienlebens. In Michelle Ahrens Erinnerungen an ihre Kindheit nimmt das Thema Strenge einen dominanten Platz ein. Sie sei in einem „diktatorische[n] Elternhaus“ (I 00:39-0:42) aufgewachsen. Retrospektiv empfindet sie die Erziehung als zu streng (I 05:28-05:31). Insbesondere der Vater wird als strafende und teilweise unberechenbare Instanz charakterisiert, der teils zu drakonischen Mitteln, zu denen auch körperliche Strafen gezählt hätten, gegriffen habe, ,[w]enn einer aus der Norm“ gegangen sei (I 02:25-02:27, 09:11-09:18, I 09:30-09:47). Michelle Ahrens macht somit gleich zu Beginn des Interviews deutlich, dass zumindest der Vater sich eines geschlossenen Erziehungssettings bedient und Abweichungen von den von ihm gesetzten Forderungen in keiner Weise toleriert. Bereits kleinere Vergehen, wie ein Zuspätkommen, seien drastisch geahndet worden (I 12:32-14:10). Verfehlungen der Kinder sind für den Vater unentschuldbar, sie können mit keiner Nachsicht rechnen und er ist in keiner Weise bereit, Abweichungen von seinen Vorstellungen zu tolerieren. Die Reaktionen des Vaters erscheinen ungezügelt, sind für die Kinder nicht nachvollziehbar, da sie nicht erklärt werden, was wiederum verunsichernd für die Kinder ist, wie sich an Bemerkungen wie: „Und das habe ich mein Lebtag nich verstanden“ (I 13:35-13:37) oder „[d]as ist also unbegreiflich“ (I 13:42-13:44) zeigt. Verstärkend kommt hinzu, dass die Regeln offensichtlich der väterlichen Willkür unterliegen und für die Kinder nicht berechenbar sind. Ohne Begründung seien z.B. ausgesprochene Strafen, wie ein mehrmonatiger Hausarrest, verändert, aber gleichzeitig mit Auflagen versehen worden (I 13:44-14:10), wodurch der Vater seine Machtposition demonstriert und hervorhebt. Trotz der unnachgiebigen Härte des Vaters sieht Michelle Ahrens zugleich aber auch einen sorgenden

\footnotetext{
${ }^{166}$ Die Interviews mit Michelle Ahrens wurden am 09. und 23.03.2009 geführt.
} 


\subsection{Geschlossene Tradierungslogiken}

Aspekt im väterlichen Verhalten, indem sie z.B. anführt, er hab sie „,irgendwie auch lieb gehabt“ und sie „überall gesucht“, wenn sie z.B. nicht pünktlich zu Hause gewesen sei (I 12:52-13:12). Mit diesen Einschüben versucht sie, die väterliche Rigidität ein wenig zu relativieren, worin sich zugleich eine ambivalente Einstellung zum Vater manifestiert. Einerseits werden die väterlichen Handlungsweisen abgelehnt, andererseits versucht sie den Vater zu verstehen und zumindest ansatzweise zu entschuldigen.

Das mütterliche Verhalten hingegen sei streng, aber „nicht grausam“ gewesen (I 11:2111:32). Zwar sei sie durchaus auch von der Mutter bestraft worden, wenn sie die ihr aufgetragenen Arbeiten nicht richtig erledigt habe, dies sei aber für Michelle Ahrens mehr ein Ausdruck der Überforderung gewesen (I 17:53-18:16). Die mütterlichen Sanktionen sind für die Tochter nicht zügellos oder dem Versuch geschuldet, die eigene Machtposition zu demonstrieren. Die rigorosen Strafpraxen ihres Mannes habe die Mutter jedoch nicht verhindern können (I 09:47-10:04). Aus Sicht von Michelle Ahrens beschützt die Mutter ihre Kinder nicht ausreichend und stützt hierdurch letztlich das väterliche Verhalten. Gegenüber Dritten habe die Mutter die für die Kinder belastenden Aspekte des Familienlebens konsequent verschwiegen. Sie habe sich sehr bemüht, die Fassade eines der Norm entsprechenden Familienlebens aufrecht zu erhalten und potenzielle Hilfsmöglichkeiten abzuwehren (I 11:47-12:24): „Und meine Mutter hat natürlich ni:chts, die hat immer gemauert, ne, //ja// die hat nie was gesagt, ne" (I 12:17-12:21). Der mütterliche Bezugsrahmen des harmonischen und idyllischen Familienlebens ist als imaginiertes Ideal präsent, findet aber keinen Niederschlag in der Lebenswelt der Kinder. Von einem Vorleben des familialen Bezugsrahmens, wie er für die Familien des flexibel erscheinenden Typus charakteristisch ist, kann aus Sicht der Tochter keine Rede sein.

Diese Diskrepanz zwischen Anspruch und Wirklichkeit tritt besonders plastisch am Beispiel der familialen Weihnachtsfeste (I 39:23-40:57) hervor: „Also mir ist das so in Erinnerung gewesen, dadurch dass es immer so streng gewesen ist und dass meine Mutter immer alles so piccobello haben wollte $/ / \mathrm{hmh} / /$ bis auf den letzten Drücker, war immer, immer alles sehr stressig“ (I 39:23-39:33). Im Vorfeld des Weihnachtsfestes habe es regelmäßig Konflikte gegeben, in deren Rahmen eines der Kinder für seine vermeintlichen Verfehlungen bestraft worden sei. Die Gestaltung des Tages sei nach einem ritualisierten und starren Plan ${ }^{167}$ abgelaufen: Nachdem bestimmte Aufgaben für den Vater erledigt worden seien (I 39:33-39:53),

„haben wir in dem einen Zimmer immer gewartet, $/ / \mathrm{hmh} / /$ bis meine Eltern dann geklingelt haben. DANN mussten wir immer erst Kaffee trinken, Stollen und Kekse. Und was ich immer so ganz schlimm fand, das erzählen meine Geschwister aber auch alle so. //hmh// Äh die sagen alle, vorher war die Spannung [...] und anschließend mussten wir uns vor den Baum stellen und singen. Und tun, als wenn alles toll is. [...] Dann mussten wir

\footnotetext{
${ }^{167}$ Michelle Ahrens beschreibt einen ähnlich durchstrukturierten Ablauf für die Gestaltung des Osterfestes (I 31:47-32:22).
} 


\section{Familiale Tradierungsprozesse in einer Drei-Generationen-Perspektive}

meinem Vater immer die Hand geben: ,Frohes Weihnachtsfest' ${ }^{\star}$ //hmh// Das empfanden wir auch immer als schlimm. $/ / \mathrm{hmh} / /$ Meine Mutter hat es ja gut gemeint. Die hatte dann in dem $=$ im Sessel dann immer so diese kleinen Geschenke.“ (I 39:53-40:28).

In der Passage kommen die für die Vermittlung des familialen Bezugsrahmens gültigen Mechanismen und Ansprüche in zugespitzter Weise zum Vorschein. Seitens der Eltern sind extrem hohe und rigorose Ansprüche gegenüber den Kindern vorhanden, die der Tochter auch bewusst sind, wie sich in ihrem Urteil zeigt, es habe „halt zu perfekt" sein sollen (I 41:08-41:11). Das führt zu einer äußerst angespannten Atmosphäre und die mit dem Weihnachstfest verbundenen Ansprüche und Erwartungen werden von den Kindern als Bürde empfunden. Die Kinder selbst erhalten keine Gestaltungsspielräume, sodass sie sich als fremdgesteuert empfinden müssen. Angesichts des Erwartungsdrucks werden Abweichungen von den elterlichen Ansprüchen wahrscheinlicher, was umgehend geahndet wird. Auch in dieser Darstellung ist es gerade der Vater, der nicht bereit ist, seine Erziehung und die hiermit verbundenen Anforderungen an die Kinder situationsspezifisch anzupassen. Zusätzlich demonstriert der Vater über die Rituale seine Macht- und Vorrangstellung, die er generell beansprucht.

Michelle Ahrens beschreibt die häusliche Atmosphäre als angstbesetzt, wozu besonders der Vater beigetragen habe. Er habe den Kindern z.B. „immer so grausame“ Geschichten erzählt, in denen die Kinder bei Ungehorsam mit drastischen Strafen belegt worden seien (I 10:12-10:50). Mit Hilfe solcher „Schauergeschichten“ (III 09:23-10:01) versucht der Vater, die Kinder einzuschüchtern. Ihm geht es weniger darum, seinen Kindern zu erklären, warum sie gehorchen sollen, auch nimmt er keine Rücksicht auf die Gefühle der Kinder, vielmehr besteht die Absicht darin, seine Machtposition autoritär durchzusetzen. Die Angst der Kinder vor dem Vater schlägt sich auch im Alltag nieder. Der Vater sei die Woche über oft berufsbedingt nicht zu Hause gewesen, sodass sich die gemeinsamen Mahlzeiten mit ihm auf die Wochenenden beschränkt hätten. Während dieser Gelegenheiten hätten die Kinder keine Gespräche mit dem Vater geführt: „Wenn wir am Tisch gesessen haben, da hätten Sie eine Nadelpiekse runterfallen hören. Da hat keiner sich getraut, was zu sagen" (I 28:59-29:04). Vielmehr habe der Vater die Unterhaltung bestritten, indem er von seiner Arbeitswoche erzählt habe. Nach dem Alltag der Kinder hingegen habe er sich nicht erkundigt (I 28:56-29:17, 30:32-30:47). Die gemeinsamen Familienessen dienen damit weniger dem kommunikativen Austausch zwischen den Generationen, sondern bieten dem Vater vielmehr eine Plattform zur Demonstration seiner dominanten Stellung. Deutlich wird zudem die Erwachsenenzentrierung des Familienlebens, das nach deren Bedürfnissen gestaltet wird. ${ }^{168}$

Die Angst vor dem nächsten Wutausbruch habe auch die Freizeitgestaltung der Familie ein wenig überschattet: „Es war immer mit etwas Angst dabei. Angst vor Strafe“

\footnotetext{
${ }^{168}$ Dies zeigt sich auch am Ablauf der Kindergeburtstage. Die Kinder hätten sich zwar Freunde einladen dürfen, die Kuchenauswahl sei allerdings danach erfolgt, was die eingeladenen Erwachsenen mochten (III 14:58-15:10).
} 


\subsection{Geschlossene Tradierungslogiken}

(I 27:05-27:25). Hierbei führt Michelle Ahrens relativierend an, dass es manchmal auch unbeschwerter gewesen sei, z.B. während eines Urlaubes (I 32:25-32:45). Indirekt führt sie einen Teil des väterlichen Verhaltens auf den Alltagsstress zurück, zu dem sie auch finanzielle Sorgen aufgrund der Versorgung von sechs Kindern zählt. Das väterliche Handeln ist für sie somit auch Ausdruck einer Überforderung, wie sie explizit feststellt (I 02:31-02:44) und wodurch sie ihn in gewisser Weise entschuldigt. Auch habe der Vater seine Kinder in praktischen Angelegenheiten immer unterstützt (III 20:15-20:21). Diese Relativierungsversuche lassen sich als Ausdruck einer ambivalenten Einstellung zum Vater deuten, die trotz aller negativen Erfahrungen vorhanden ist.

Das Verhältnis zur Mutter charakterisiert Michelle Ahrens retrospektiv mit den Worten: „Meine Mutter mochte ich eigentlich schon immer gern. Wir hatten ja ein gutes Verhältnis, //hmh// ne. Obwohl es war so ein bisschen, na ja, ein bisschen anders, wie es hätte vielleicht sein sollen“"(III 35:47-35:55). Auch wenn sie die Beziehung zur Mutter insgesamt positiv beurteilt, gibt es eine Vielzahl von Beschreibungen, die auf Ambivalenzen in der Beziehung zur Mutter verweisen. Zwischen Mutter und Tochter findet in mancher Hinsicht fast eine Rollenumkehr statt. Michelle Ahrens sei zu vielerlei Haushaltsarbeiten herangezogen worden, habe viel auf die jüngeren Geschwister aufpassen müssen und die Mutter habe sich mit ihren Sorgen, z.B. finanzieller Art, an die Tochter gewandt, die für sie eine Art Vertrauensperson dargestellt habe (I 03:22-03:45, I 17:35-18:29, 18:36-19:22 und I 25:23-25:48). Auch sei Michelle Ahrens zuständig gewesen, wenn die Geschwister Probleme gehabt hätten (I 20:09-20:55, I 21:14-21:36). Die Mutter sei hiervon überfordert gewesen und habe die Verantwortung an ihre Tochter delegiert. Michelle Ahrens sei als älteste Tochter in der Folge zu einer „Ersatzmami“ für ihre Geschwister avanciert (I 21:34-22:11). Die Mutter fordert Hilfe von der Tochter ein, wehrt ihrerseits aber Unterstützungsleistungen an die Tochter ab, was Michelle Ahrens sicherlich auch überlastet hat. Trotzdem beschreibt Michelle Ahrens die Beziehung zur Mutter als wesentlich unbelasteter als das Verhältnis zum Vater. Dies zeigt sich u.a. daran, dass die ganze Familie „so 'n bisschen zu Hause aufgelebt“ sei, als der Vater eine Zeit lang abwesend gewesen sei (04:25-04:50):
„Dann war zwar auch 'ne gewisse (.) äh erzieherische Strenge von Seiten meiner Mutter, dass sie sagte dann und dann (muss ich), musst du zu Hause sein und das muss erledigt sein, aber das galt so mehr der Fürsorge, äh so ich bin jetzt allein verantwortlich und ähm ich muss zusehen, dass nun alles einigermaßen glatt geht. $/ / \mathrm{hmh} / /$ Und das hat ja auch äh wunderbar geklappt“ (I 04:54-05:10).

Zwar sind die mütterlichen Forderungen ebenfalls nicht verhandelbar, sie scheinen aber weniger willkürlich und sind klarer formuliert, sodass den Kindern deutlich ist, was von ihnen erwartet wird. Auch wird sie nicht als derart unkontrolliert wie der Vater beschrieben, was entscheidend dazu beitragen dürfte, die Beziehung zur Mutter als wesentlich entspannter wahrzunehmen. Die Abwesenheit des Vaters habe sich auch auf 


\section{Familiale Tradierungsprozesse in einer Drei-Generationen-Perspektive}

die familialen Machtverhältnisse nach seiner Rückkehr ausgewirkt. Der Vater hätte die Kinder nicht mehr so dominieren können und Michelle Ahrens, zu diesem Zeitpunkt schon Jugendliche, hätte sich „ein bisschen frei gestoßen“ (I 05:11-05:25), was der Vater teilweise akzeptiert habe. Bestimmte Ansprüche seien nicht mehr so rigoros durchgesetzt worden (I 14:55-15:52). Es scheint, als folge der Vater auch hier tradierten Bahnen, indem er den Kindern früh einen Erwachsenenstatus zuspricht. Dies wird zusätzlich dadurch gefördert, dass Michelle Ahrens in etwa zu dieser Zeit mit ihre Ausbildung begonnen und ihr eigenes Geld verdient hat. Ab diesem Zeitpunkt habe sie auch viele Dinge allein entscheiden dürfen (III 33:37-33:46).

Angesichts dieser Schilderungen verwundert es nicht, wenn Michelle Ahrens die Beziehungen zu den Eltern als problematisch beschreibt. Sie habe mit ihren Eltern „nie über etwas reden können“, lediglich über Pflichten und Organisatorisches (I 25:1325:22). Hierin drückt sich ein eher distanziertes Verhältnis zu den Eltern aus, das durch die asymmetrische Machtbalance, in der die Eltern das Machtmonopol beanspruchen, noch verstärkt wird. Sie habe auch kein Vertrauensverhältnis zu ihren Eltern gehabt. Wenn sie Rat gebraucht habe, sei sie auf sich selbst verwiesen gewesen (I 22:18-22:47). Es finden sich kaum Hinweise für eine emotionale Unterstützung durch die Eltern, auch wenn es durch die Mutter eine „gewisse Wärme“ gegeben habe (III 47:44-47:51). Dies scheint die Großmutter väterlicherseits auszugleichen, die von Michelle Ahrens als wichtige Bezugsperson beschrieben wird, die ihr zugehört habe und der sie von Problemen mit dem Vater habe berichten können, ohne von ihr allerdings weitergehende Ratschläge zu erhalten (I 35:15-36:03 und III 19:26-19:42).

Insgesamt nimmt Michelle Ahrens die elterliche Erziehung weniger als regel- denn als verbotsgeprägt wahr (III 25:47-25:50). Das elterliche Erziehungsarrangement ist für sie somit vor allem durch Begrenzungen geprägt, an denen die Eltern unerschütterlich festhalten und die ihr wenig Spielräume lassen. Der kommunikative Austausch als Form der Erziehung spielt eine eher untergeordnete Rolle. Für Michelle Ahrens dominieren stattdessen Erwartungen in Form von Forderungen, die weder begründet werden noch verhandelbar sind. Insbesondere die Mitarbeit in Haushalt und Familie wird erwartet (III 27:57-28:20). Zu den Anforderungen hätten zudem Pünktlichkeit (III 27:45-27:48) sowie die Erwartung, „sauber und adrett“ zu sein (III 32:57-33:02), gezählt. Auch der Kirchgang sei obligatorisch gewesen, und die Missachtung dieser Regel sei geahndet worden: „[W]enn du nicht in die Kirche gewesen bist, kannst du ni:ch rausgehen, Spaß haben" (III 58:03-58:12). Unübersehbar sind hier die Parallelen zur eigenen Erziehungserfahrung der Mutter, die ganz offensichtlich an den ihr vertrauten Bahnen festhält. Wenn sie sich an die Anforderungen gehalten hätte, sei ihr im Anschluss ein „Freiraum“ in Form von Freizeit eingeräumt worden (III 28:21-28:24). Sobald die Kinder sich also einfügen und unterordnen, wird dies von den Eltern honoriert. Während bei der flexibel erscheinenden Tradierungslogik die Regeleinhaltung von der aneignenden Generation als Freiraum empfunden wird, werden in dieser Familie Freiräume durch Gehorsam erkauft. Zudem habe eine Orientierung am umgebenden Milieu stattgefunden: „Es war wichtig, ne, was sollen denn die Leute @sagen (.) @?’ (III 27:25-27:27). Das soziale 


\subsection{Geschlossene Tradierungslogiken}

Umfeld wird den Kindern als Maßstab vorgehalten und dient dazu, die elterlichen Anforderungen $\mathrm{zu}$ unterstreichen und $\mathrm{zu}$ rechtfertigen.

Eine gravierende retrospektive Kritik an der elterlichen Erziehung bezieht sich darauf, dass die Kinder nicht in ihrer Individualität wahrgenommen und gefördert worden seien. Dies habe damit begonnen, die Tochter nicht zum Gymnasialbesuch zu ermuntern, obwohl Michelle Ahrens' Zeugnis dies erlaubt hätte. Vielmehr seien die Eltern von der Annahme geleitet gewesen, die Tochter würde heiraten und bräuchte deswegen keine höhere Schulbildung (III 00:00-00:14). Die Eltern halten auch in diesem Bereich an den aus ihrer Kindheit vertrauten Bahnen fest und sind zu keinen Veränderungen bereit. Zugleich steht dies im Einklang mit einem Element des mütterlichen Bezugsrahmens, wonach zu einem idyllischen und harmonischen Familienleben die nicht berufstätige Mutter zählt. Auch wenn sie in praktischen Dingen von ihren Eltern bis heute unterstützt werde, fände dies seine Grenzen, wenn es „die persönliche Entwicklung“ sowie „die Reife durch Gespräche“ beträfe (I 06:18-06:29 und III 17:26-17:37). Die Talentförderung ihrer Kinder hätte nicht zum Erziehungsrepertoire der Eltern gehört und es sei ihnen nicht in den Sinn gekommen, nach Angeboten Ausschau zu halten, um die Entwicklung ihrer Kinder zu fördern, obwohl es nach Überzeugung von Michelle Ahrens auch damals bereits für finanzschwache Familien Möglichkeiten hierzu gegeben hätte (III 17:48-18:32 und III 37:28-37:40). Aus Sicht der Tochter sind die Eltern nicht bereit, die ihnen vertraute Horizontlinie zu überschreiten, worin sich wiederum die Geschlossenheit des Erziehungsarrangements sowie die Orientierung am Bezugsrahmen manifestieren, die eine solche Förderung der Kinder nicht zwingend erforderlich erscheinen lassen.

In Anbetracht der strengen und rigorosen familialen Atmosphäre während der Kindheit ist es verständlich, dass das Thema „Freiräume“, als Gegenpol zur Strenge, für Michelle Ahrens einen hohen Stellenwert einnimmt. Durch die vielen ihr auferlegten Pflichten sei „viel Freiraum genommen“ und die eigene Entwicklung behindert worden (I 04:13-04:24). Besuche bei Verwandten und bei Gleichaltrigen hätten ihr die Möglichkeit gegeben, den elterlichen Grenzsetzungen zumindest zeitweise zu entgehen (I 02:53-03:19). In der Verwandtschaft erlebt Michelle Ahrens zudem eine andere Gestaltung der intergenerationellen Beziehungen. Ihre Tanten seien sehr kinderlieb gewesen und auf sie eingegangen; Michelle Ahrens sei gerne dort gewesen und habe sich dort frei gefühlt (I 36:53-38:21). Die Tanten bringen ihr Wertschätzung entgegen und zeigen sich kindorientiert; Aspekte, die zu Hause nicht die intergenerationellen Beziehungen prägen. In der Nachbarschaft lernt sie zudem einen anderen Umgang der Erwachsenen mit Kindern kennen. Als ihr im Hause einer Freundin ein Missgeschick passiert sei, habe sie die Reaktion von deren Vater gefürchtet (I 27:25-28:02). Dieser habe jedoch gelassen reagiert und lediglich kommentiert: „Na und, das ist doch nicht so schlimm“ (I 27:50-27:53). Diese Entgegnung habe sie sehr überrascht, weil ihr so ein Missgeschick in ihrer eigenen Familie „nachgehangen“ hätte (I 27:56-28:02). 


\section{Familiale Tradierungsprozesse in einer Drei-Generationen-Perspektive}

Vermittlungsebene Michelle Ahrens (* 1961): Präzise Erwartungen und Angebote Vor dem Hintergrund dieser Wahrnehmung der eigenen Kindheit ist verständlich, dass für Michelle Ahrens ein unmittelbarer Anschluss an den familialen Bezugsrahmen eines harmonischen und idyllischen Familienlebens zumindest problematisch ist. Statt sich wie die Mutter auf ,ihr kleines Nest, ihre kleine Welt“ (III 06:06-06:07) zu konzentrieren, wird für Michelle Ahrens das Streben nach Möglichkeiten zur Horizonterweiterung zentral: „Und dadurch dass zu Hause der Horizont immer so (.) auf den Teller beschränkt gewesen (ist), wollte ich einfach meine Tortenplatte haben" (I 52:34-52:40). Hierin ist ein wichtiger Grund zu sehen, warum sich Michelle Ahrens nicht, wie sie es bei ihrer Mutter erlebt hat, auf das Motto: „Haus, Heim, Herd, Kinder kriegen, glücklich sein, Mann" beschränken möchte und eine Berufstätigkeit für sie elementar wichtig ist (III 05:01-05:33); zumal sie hierdurch das Risiko von Abhängigkeitsverhältnissen und finanziellen Sorgen, wie sie sie in ihrer Kindheit erlebt hat, minimieren kann. In der Rückschau bedauert Michelle Ahrens allerdings, aufgrund ihrer beruflichen Eingebundenheit für ihre Tochter im Jugendalter zu wenig Zeit gehabt zu haben, insbesondere weil sie selbst die mütterliche Aufmerksamkeit in ihrer eigenen Jugendphase vermisst habe und dies nicht habe wiederholen wollen (III 54:59-55:29). Es scheint, als sei ihr Gegenentwurf zur Einstellung ihrer Mutter so stark ausgeprägt, dass es ihr nicht möglich gewesen ist, diesen zu variieren.

Diese Verschiebung des Bezugsrahmens ist ebenso wichtig für das eigene Vermittlungsarrangement wie auch ihr Bestreben „die Minuspunkte“ ihrer eigenen Kindheitserfahrungen zu vermeiden (I 51:22-51:30). Trotz ihrer in vielen Teilen negativen Beurteilung der elterlichen Erziehung lässt sich Michelle Ahrens retrospektive Einschätzung der eigenen Erziehung als ein Wechselspiel aus Anknüpfen an sowie Abgrenzen von der elterlichen Praxis betrachten:

„[I]ch meine ich bin, in der heutigen Zeit würde ich sagen, auch manchmal noch viel zu streng gewesen, ne. //hmh// Aber ich hab zum Beispiel immer versucht, wo äh wo zu Hause praktisch der Horizont, immer gesagt wurde, das geht nicht, das können wir nicht und das machen wir nicht, das habe ich versucht mit meinen Kindern immer zu machen. // hmh// $\mathrm{Ne}$, immer denen versucht [...] viele Möglichkeiten bildungsmäßig oder so (.) (aufgehen) zu lassen. Trotzdem würde ich aber im Nachhinein sagen, ich bin erziehungsmäßig manchmal noch viel zu streng gewesen“ (I 51:32-51:59).

Ihrem eigenen Anspruch folgend habe Michelle Ahrens sich immer bemüht, ihren Kindern Angebote zu unterbreiten (III 59:32-1:03:40). Hierbei hätten sich die Möglichkeiten im Lauf der Zeit erweitert. Sie habe mit ihrer Tochter bereits aus vielfältigen Mutter-Kind-Angeboten wählen können (I 50:37-51:04), die sie gerne genutzt habe, da sie ein „ziemlich neugieriger Mensch“ sei und „gern alles [...] ausprobier[e]“ (III 50:59-51:08). Soweit finanziell realisierbar, hätte sie mit ihren Kindern Ausflüge in verschiedene Städte unternommen, um den Kindern kulturell etwas zu bieten. Die Kinder 


\subsection{Geschlossene Tradierungslogiken}

hätten die Möglichkeit haben sollen, vielfältige Eindrücke zu sammeln, damit sie „ein kleines bisschen multikulturell“ aufwachsen und lernen, andere Meinungen zu akzeptieren (I 52:20-52:45 III 1:11:23-1:11:32). Michelle Ahrens erinnert z.B. eine von der Kirche organisierte Gruppenreise ins Ausland, die sie mit den Kindern unternommen habe (II 01:29-02:08 und III 44:04-44:13). Retrospektiv merkt sie selbstkritisch an, dass sie bei diesem Ausflug zu stringent auf den Aspekt der Sparsamkeit geachtet und ihrer Tochter manche realisierbaren Wünsche nicht erfüllt habe (II 01:29-02:08). Dies ist zum einen ein Beispiel dafür, wie stark Michelle Ahrens manche Orientierungen ihrer Kindheit trotz aller Abgrenzungsbemühungen verinnerlicht hat, und zum anderen belegt es, wie stringent sie bei der Erziehung der Tochter an ihren Vorstellungen festhält; ein erstes Indiz für ein geschlossenes Erziehungsarrangement, in dem sich die von Michelle Ahrens thematisierte Strenge als ein rigoroses Festhalten an den einmal entwickelten Richtlinien beschreiben lässt.

Ebenso wie bei den Eltern spielt die religiöse Gemeinschaft für Michelle Ahrens eine wichtige Rolle, allerdings in einer erweiterten Weise. Die Kirchengemeinde stellt neben den Kontaktmöglichkeiten für sie auch eine Ressource dar, auf die sie zurückgreift, um ihren Bezugsrahmen der Horizonterweiterung bei der Erziehung zu realisieren. Dieser bezieht sich nicht nur auf das Kennenlernen vielfältiger kultureller Elemente, sondern auch ganz praxisnah auf den kommunikativen Austausch. Sie habe z.B. das ehrenamtliche Engagement ihrer Kinder im kirchlichen Bereich begrüßt, weil die Kinder hierüber in Kontakt mit anderen Menschen gekommen seien und „andere Meinungen“ kennengelernt hätten (I 53:07-53:15). Das sei ihr wichtig gewesen, um die Kinder zur Toleranz $\mathrm{zu}$ erziehen, eine Einstellung, die ihre eigenen Eltern ihr gegenüber in ihrer Kindheit nicht praktiziert hätten. Auch hier merkt sie retrospektiv an, dass sie selbst wahrscheinlich ihren Kindern gegenüber nicht immer so tolerant gewesen sei: „Meine Tochter wird mit Sicherheit auch sagen: ,Du hast meine Meinung auch nicht akzeptiert.' Das ist mit Sicherheit auch ganz oft so gewesen“ (III 1:11:38-1:11:43). Ihr selbst ist demnach die eigene strikte Haltung zumindest partiell bewusst.

Der kommunikative Austausch und die aktive Herstellung einer Familiengemeinschaft spielen eine wichtige Rolle im Erziehungsarrangement von Michelle Ahrens. Sie habe Gelegenheiten für Gespräche geschaffen, indem sie auf eine gemeinsame Kaffeezeit einmal in der Woche bestanden (III 57:30-57:51) und zusammen mit ihren Kindern bestimmte Hausarbeiten erledigt habe:

„Und ich hab immer so als Regel gehabt, ich möchte, dass meine Kinder [...] mithelfen den Geschirrspüler einzuräumen [...] nicht weil sie mir praktisch helfen sollen, sondern weil ich die Erfahrung gemacht habe, und das weiß ich von 'ner Kundin, dass ähm manchmal dann mehr erzählt wird. $/ / \mathrm{hmh} / /$ Bei Tisch ist das gar nich unbedingt so. //hmh// Dann kommen sie nach Hause und haben Hunger [...]. Und wenn man dann noch 'ne halbe Stunde länger zusammen ist, dann kommen manchmal erst Sachen zum Tragen, die dann erzählt werden, die einen irgendwo drücken. 


\section{Familiale Tradierungsprozesse in einer Drei-Generationen-Perspektive}

$/ / \mathrm{hmh} / /$ Und deswegen war es mir immer wichtig, dass wir gemeinsame Sachen, dass wir was gemeinsam machen, wie gemeinsam Essen kochen“ (III 56:33-57:15).

Zwar zieht auch Michelle Ahrens, genau wie Frau Mahler ihrerzeit, ihre Kinder zu Hausarbeiten heran, was aber anderen Zwecken dient und nicht im Zeichen der mütterlichen Entlastung, sondern der kindlichen Förderung steht. Michelle Ahrens schafft beiläufig Gelegenheiten, um sich über den Alltag und ggf. Probleme ihrer Kinder zu informieren. Darüber hinaus habe sie ihre Kinder dazu angehalten, praktische Haushaltsfertigkeiten einzuüben, um ihre Selbstständigkeit zu fördern. Ansonsten habe es nicht „so extrem feste Regeln“ gegeben (III 53:54-54:57, 55:30-56:32). Verbindende Rituale, wie z.B. zu Weihnachten und Geburtstagen, seien aber wichtig gewesen (III 44:15-46:24, 46:35-46:50), und zielen letztlich auf die Herstellung von Gemeinschaft:

„Wenn die Kinder Geburtstage haben //hmh// oder einer bei uns aus der Familie, [...] dann nehmen wir uns vor diese ganzen alten Kochbücher, Backbücher $/ / \mathrm{hmh} / /$ und gucken zusammen, was wir denn machen wollen. Wie sich das nun letztendlich entscheidet, manchmal sagen sie auch: ,Oh Mama, du machst dann ja doch, was du willst', ne.@(.)@ Aber ähm wir gucken das zusammen an. Und das ist ein Ritual, was [...] so auch die Gemeinsamkeit pflegt, //hmh// ein Fest gemeinsam gestalten, aussuchen, planen, machen, kümmern, sich kümmern, $/ / \mathrm{hmh} / /$ wer macht das, wer macht jenes." ( III 49:50-50:21).

Obwohl es vermeintlich um gemeinsame Entscheidungen geht, behält Michelle Ahrens das Zepter fest in der Hand. Sie steuert Entscheidungen im Bedarfsfall in ihrem Sinne und setzt sie durch. Die Kritik der Kinder kann hieran anscheinend nichts ändern, was unterstreicht, dass geschlossene Erwartungshorizonte die Erziehung von Michelle Ahrens zumindest in den für sie zentralen Punkten prägen, auch wenn sie im Vergleich zu ihren Eltern auf ganz andere Weise umgesetzt werden. Die von Michelle Ahrens proklamierte Horizonterweiterung stellt eine absolute Maxime dar, an der sich die Kinder orientieren sollen, ohne sie zu verändern.

Wie schon an den Schilderungen der eigenen Kindheit ersichtlich, ist es Michelle Ahrens im Unterschied zur eigenen Mutter möglich, familiale Konflikte zu thematisieren. So habe es "mit Sicherheit Konflikte" gegeben:

„[E]s sind ja immer so Phasen, //hmh// also es immer so gewesen, wenn die dann so 'nen Schub gemacht haben, dann war immer sechs Wochen Kampf [...]. //hmh// Aber ich habe auch immer gesagt, diese sechs Wochen, die stehe ich $D U R C H$, weil ich genau weiß, dann habe ich wieder ein halbes Jahr Ruhe. //hmh// Dann sind die Fronten ja geklärt //hmh// und dann=und dann geht es. Da bin aber ich so. Mein Mann ist da eher ein bisschen anders, ne. Der sagt dann: ,Ach, na ja, dann lassen wir mal gut 


\subsection{Geschlossene Tradierungslogiken}

sein' und (mh,mh,mh). Und ich hab immer gesagt: ,Nee', weil da tue ich mir und vor allem meinen Kindern keinen Gefallen, weil erstmal //hmh// wachse ich da dran (.) und die wachsen da auch dran. Und wenn ich das dann alles nur so wischiwaschi mache, dann $=$ dann finde ich das nicht gut. Wie= wie, dann klärt sich das ja auch im Prinzip nicht" (III 52:20-53:04).

Michelle Ahrens vertritt offenbar kein so idealistisches Familienbild wie ihre Mutter und betrachtet Konflikte als Bestandteil des Familienlebens, die ein Lernpotenzial beinhalten. Sie kommuniziert zugleich, im Unterschied zu ihrem Mann besser in der Lage zu sein, konsequent an ihren Überzeugungen festzuhalten. Hier manifestiert sich abermals, dass Flexibilität nicht zentral in ihrem Erziehungsarrangement verankert ist.

Im Gegensatz zu ihren eigenen Eltern ist es für Michelle Ahrens aber elementar wichtig, ihre Vorstellungen nicht autoritär durchzusetzen; sie wählt andere Mittel, wie sich am Beispiel gemeinsamer Einkaufstouren illustrieren lässt. Sie habe z.B. eine gute Lösung gefunden, mit dem Kleidungsstil der Tochter umzugehen. Wenn sie mit ihrer Tochter einkaufen gehe, lasse sie sie zunächst für eine Zeit alleine, damit die Tochter sich in Ruhe umschauen könne. Dann schaue sie sich die Auswahl der Tochter an und sage „immer ganz ehrlich“ ihre Meinung. Anschließend biete sie an, für ihre Tochter einige Kleidungsstücke zur Auswahl herauszusuchen (III 1:05:59-1:08:16):

„Und komischerweise die nimmt sie dann. //hmh// Also dann hatte sie das Gefühl, sie hat eigentlich 'ne eigene Entscheidung getroffen $/ / \mathrm{hmh} / /$ und hat aber vorher auch ihre eigene Wahl gehabt. Und manchmal sage ich dann auch: ,Mensch, das sieht jetzt richtig gut aus"“ (III 1:07:10-1:07:20).

Michelle Ahrens setzt nicht auf offene Kritik und Verbote, sondern versucht, ihre Vorstellungen über positive Verstärkungen durchzusetzen, wobei ihre Steuerungsversuche zugleich verschleiert werden.

In anderen Bereichen habe sie sich mit den Entscheidungen der Kinder abgefunden. Sie habe sie z.B. nie gezwungen, zur Kirche zu gehen und ihre Entscheidung akzeptiert, als diese ihr Engagement in kirchennahen Organisationen aufgegeben hätten (III 57:52-58:04). Wenn es jedoch um den Bezugsrahmen der Horizonterweiterung geht, zeigt sich Michelle Ahrens weniger verständnisvoll. Es habe keine Gebote, wohl aber immer Angebote gegeben (III 59:32-59:37). Es sei ihr wichtig gewesen „den Tellerrand offen [zu] halten“ und mehrere Wege aufzuzeigen, damit es zu einer „persönliche[n] Weiterentwicklung" komme (III 1:03:13-1:03:34). Ihr Sohn habe sich diesbezüglich empfänglicher gezeigt als ihre Tochter (III 1:03:38):

„Und dann [...] sagte [ich, C.R.]: ,Mensch, dann mach doch [mit dem Theaterspiel, C.R.] weiter. Ist doch prima'. [...] ,Ach, ich hab keinen Bock mehr'. //hmh// Und dann [...]: ,Warum hab ich das nicht weitergemacht? Warum kann ich kein Klavier spielen?‘ [...], Weil du es nicht wolltest', ne. Sonja ist dann etwas anders, jedes Kind ist unterschiedlich. //hmh// Sie 


\section{Familiale Tradierungsprozesse in einer Drei-Generationen-Perspektive}

kriegt dann oft genug Angebote von mir, [...] dann hat sie aber keinen Bock dazu. //hmh// Und dann kommt ein Jahr, ein oder zwei Jahre später: ,Hätt' ich man““ (III 1:03:40-1:04:10).

Michelle Ahrens zwingt ihre Tochter nicht, die Angebote wahrzunehmen, weil dies nicht ihre „Art“ sei (III 1:09:16-1:10:55), es wird aber deutlich, dass sie die Entscheidungen der Tochter missbilligt und nicht verstehen kann, warum der eigene Bezugsrahmen der Horizonterweiterung für die Tochter nicht auf die von Michelle Ahrens angedachte Weise umgesetzt wird. Zum Unverständnis gesellt sich ein impliziter Vorwurf an die Tochter, die ihr gebotenen Möglichkeiten nicht genutzt zu haben. Michelle Ahrens' Vorstellungen haben einen hohen Verbindlichkeitscharakter, sodass der Bezugsrahmen der Horizonterweiterung keine Gestaltungsoffenheit für die Kinder impliziert, wie sich denken ließe. Michelle Ahrens richtet zwar ihre Erwartungen in Auseinandersetzung mit den eigenen Kindheitserfahrungen auf andere Inhalte als ihre Eltern, die für die Kinder entworfenen Horizonte weisen aber eine ähnliche Geschlossenheit auf wie ihrerzeit bei den Eltern.

Im Unterschied zu ihren Eltern wählt sie allerdings andere Wege der Umsetzung. Sie habe z.B. immer „Augen und Ohren offen“ gehalten, um den Kindern Perspektiven der Entwicklung aufzeigen zu können, was diese auch zu schätzen wüssten. Es sei ihr immer daran gelegen gewesen, ihren Kindern zu vermitteln, dass sie sie moralisch unterstütze und für sie da sei (III 1:01:56-1:02:09). Um ihre Kinder adäquat unterstützen zu können, habe sie „Rat von außen“ eingeholt und sich umfassend informiert (III 1:00:30-1:00:52, 1:05:04-1:05:16). Wie sich zeigt, ist Michelle Ahrens sehr aktiv, wenn es darum geht, den Bezugsrahmen der Horizonterweiterung umzusetzen und eine optimale Förderung ihrer Kinder zu gewährleisten. Dies gilt auch für Erziehungstipps generell:

„Ich habe mich dann immer mh an Bekannten und an Freunden orientiert, die ihre Kinder an der etwas längeren Leine gelassen haben, //hmh// und die äh ungefähr zehn Jahre älter sind wie ich. $/ / \mathrm{hmh} / /$ Und die Kinder dann auch. Und dann hab ich immer geguckt, wie ist das zehn Jahre später, kommen die wieder nach Hause zurück? Wie ist das Verhältnis? // $\mathrm{hmh} / /$ Und wenn ich das gesehen habe, das ist dann $O K A Y, / / \mathrm{hmh} / /$ dann habe ich gesagt, das kannst du dann so ein bisschen nachmachen, das war für mich dann immer so 'ne Leitlinie, //ja, hmh// weil ich es nicht so haben wollte wie zu Hause" (III 58:25-58:53).

Da sich für Michelle Ahrens eine Wiederholung der selbst erfahrenen Erziehung verbietet, ist sie auf eine stärkere Reflexion des Erziehungshandelns und Anregungen von außen angewiesen. Hierbei ist es ihr besonders wichtig, ein gutes Verhältnis zu den Kindern zu haben, weswegen sie auf eine offen autoritäre Praxis wie in ihrer Kindheit verzichtet. Die Kinder „an der etwas längeren Leine“ zu lassen, bedeutet in diesem Fall, ihnen verschiedene Optionen zur Auswahl sowie das Gefühl zu geben, selbst entscheiden zu können. Dies ist aber nicht mit einer freien Wahl gleichzusetzen, da Michelle Ahrens konsequent an der Vermittlung der ihr wichtigen Inhalte arbeitet. 


\subsection{Geschlossene Tradierungslogiken}

Michelle Ahrens ist um eine symmetrischere Gestaltung der intergenerationellen Beziehungen bemüht und gewährt ihren Kindern im Unterschied zu ihren eigenen Eltern größere Mitspracherechte. Das Schaffen von Wahloptionen kann aber nicht über die deutlich erkennbar steuernde Komponente in Michelle Ahrens Erziehungsarrangement hinwegtäuschen. Auch sind die an die Kinder herangetragenen Vorstellungen in zentralen Bereichen als geschlossen zu betrachten. Allerdings erfahren die emotionale Unterstützung und Förderung der Kinder im Generationenverlauf eine größere Aufmerksamkeit, und das emotionale Wohlbefinden der Kinder wird stärker beachtet. Dies zeigt sich insbesondere an der großen Bedeutung, die Michelle Ahrens dem kommunikativem Austausch zuschreibt. Die Erziehung wird in der zweiten Generation insgesamt kindzentrierter und Michelle Ahrens verschiebt den familialen Bezugsrahmen. Statt eines idyllischen, harmonischen Familienlebens ist für sie die Horizonterweiterung zentral. Auf einer höheren Abstraktionsebene lässt sich aber auch konstatieren, dass trotz dieser Transformationen eine gemeinsame Linie, nämlich die der Milieuorientierung zu erkennen ist. Sowohl Mutter als auch die Tochter orientieren sich an Vorstellungen, die sie als Standard des sozialen Nahfeldes betrachten. Michelle Ahrens verweist sogar explizit auf ihr Umfeld als Orientierungsmaßstab. Die vorgenommenen Veränderungen lassen sich als Reaktion auf die gesellschaftlichen Verhältnisse sowie das Bemühen betrachten, den familialen Bezugsrahmen für sich selbst und ihre Kinder anschlussfähig zu halten.

\section{Aneignungsebene Sonja Ahrens $\left({ }^{*} 1989\right)^{169}$ : Zwischen Zufriedenheit und Kritik} Sonja Ahrens' Blick auf ihre Erziehung fällt in der Gesamtschau ambivalent aus. Zunächst stellt sie gleich zu Beginn des Interviews wiederholt fest, „nicht sehr streng“ erzogen worden zu sein (00:40-01:15). Zu diesem Urteil tragen diverse Einschätzungen der elterlichen Erziehung bei. Entscheidend hierfür ist sicherlich die Wahrnehmung, außer festgelegten Ausgehzeiten in der Kindheit und reglementiertem Süßigkeitenkonsum keine „so sta:rke Regeln“ gesetzt bekommen zu haben (1:33:07-1:33:31; 1:03:46-1:03:50). Aufgrund der eigenen jeweils schwierigen Kindheiten hätten die Eltern bei der Erziehung darauf geachtet, "dass es einem einfach gut geht, dass man sich (einfach) wohl fühlt. //hmh// Und ähm wie gesagt, es war nich so streng und nich so viele Verbote oder so was. Dass man einfach (auch) ein gutes, gemeinschaftliches Leben hat" (1:37:06-1:37:25). Die Erziehung habe zur Entwicklung ihrer Eigenständigkeit beigetragen (1:41:16-1:41:23). Diese positive Bilanzierung dürfte ein Element sein, das die Zufriedenheit mit der elterlichen Erziehung befördert und den Eindruck einer eher liberalen Erziehung verstärkt. Auch mit bestimmten Problemen habe sie sich an die Eltern wenden können. Als Kind habe sie aus Versehen die Kleidung einer Freundin zerrissen, die daraufhin eine finanzielle Entschädigung gefordert habe. Nach anfänglichem Zögern habe sie sich der Mutter anvertraut:

„Und dann als ich das dann endlich meiner Mutter gesagt habe, war ich super erleichtert. Und sie meinte: ,Ach das, äh das is doch nich schlimm

\footnotetext{
${ }^{169}$ Das Interview mit Sonja Ahrens wurde am 08.04.2009 geführt.
} 


\section{Familiale Tradierungsprozesse in einer Drei-Generationen-Perspektive}

und so. Also da war ich dann sehr@erleichtert@ Und ja mit so was konnte ich eigentlich auch immer zu meiner Mu-, zu meinen Eltern kommen, weil da wurde nie groß rumgemeckert oder so“ (13:21-14:20).

Sie erlebt, dass sie für (unbeabsichtigte) Missgeschicke nicht sanktioniert wird und nimmt die Eltern diesbezüglich als unterstützend und tolerant wahr. Sie erinnert sich ebenfalls positiv an die regelmäßig unternommenen, gemeinsamen Einkaufsausflüge mit der Mutter (18:15-18:42), die sie trotz regelmäßiger Reibereien zu Beginn der Ausflüge als „richtig schöne Trips“ klassifiziert (1:09:07-1:10:25). Sie würden sich gegenseitig Kleidungsvorschläge unterbreiten, wobei sie den mütterlichen Rat schätze: „Also ich hör dann mehr eigentlich auf ihre Meinung. //hmh// Ich bin auch nicht so ein Typ, der einfach was kauft, weil es mir jetzt nur gefällt, $/ / \mathrm{hmh} / /$ sondern ich brauche auch immer eine zweite@Meinung@“ (1:10:47-1:11:21). Offensichtlich scheint die von Michelle Ahrens beschriebene Taktik der Steuerung aufzugehen, da Sonja die Ratschläge der Mutter nicht als Bevormundung, sondern als wichtige Unterstützung wahrnimmt und schätzt. Auch die mit dem Bezugsrahmen der Horizonterweiterung verbundenen Städtetouren sind Sonja in guter Erinnerung (05:19-05:27), ein Hinweis, dass die auf den Bezugsrahmen rückführbaren Aktivitäten für sie positiv konnotiert sind. Sonja kann sich der mütterlichen Hilfe sicher sein, wenn es um die Umsetzung der Horizonterweiterung geht. Die Mutter habe sie sofort unterstützt, als ihr die Idee gekommen sei, ein Jahr im Ausland zu verbringen, wohingegen der Vater an dieser Stelle etwas zurückhaltender gewesen sei (1:07:15-1:07:50). Wie sich zeigt, hat Sonja durchaus Interesse daran, an den familialen Bezugsrahmen anzuschließen.

Die bis zu dieser Stelle recht positive Beurteilung der elterlichen Erziehung erfährt jedoch auch Einschränkungen. Zunächst einmal konstatiert Sonja relativ früh, ihre Mutter sei in einigen Bereichen „etwas strenger“ als der Vater gewesen (01:34-01:36). Dies liege daran, dass die Mutter stärker für die Erziehung zuständig und die zentrale Betreuungsperson gewesen sei, die die „Regelungen und grundlegenden Regeln halt und so [habe, C.R.] festmachen müssen" (01:51-02:03, 09:00-09:13). Auch wenn Sonja Ahrens das Familienleben im Großen und Ganzen als recht harmonisch betrachtet, habe es vor allem mit der Mutter „Konfliktpunkte“ gegeben (14:49-15:19). Dies wirkt sich auf die Einschätzung der Generationenbeziehungen aus. Das Verhältnis zum Vater sei „schon immer so ein bisschen" besser gewesen als zur Mutter (08:54-08:58). Die Mutter wird als unnachgiebiger wahrgenommen als der Vater, was Sonja u.a. auf die Kindheitserfahrungen der Mutter zurückführt und darauf, „dass sie dann hier die ersten Jahre halt ähm auch so 'n bisschen alleine 'n bisschen wirtschaften musste" (17:22-17:56). Sonja versucht ihre Aussage zu relativieren, indem sie eine Erklärung für das unterschiedliche Verhalten der Eltern anbietet - ein Indiz für starke Loyalitätsbindungen zu den Eltern, die das Äußern von Kritik erschwert. Die von Sonja erlebte stärkere Rigorosität der Mutter lässt sich umstandslos mit der mütterlichen Erziehungspraxis der geschlossenen Arrangements in Zusammenhang bringen, die insbesondere den familialen Bezugsrahmen betreffen. Diese Praxis erweist sich dann als unproblematisch, wenn die Tochter 


\subsection{Geschlossene Tradierungslogiken}

den mütterlichen Vorstellungen entsprechend handelt und z.B. einer organisierten Freizeitbeschäftigung nachgeht (29:15-29:42, 20:33-20:41). Sie habe auch zuerst die Mutter konsultiert, als sie den Entschluss gefasst habe, ein Auslandsjahr zu absolvieren, „weil sie muss erst mal, erst mal alles @genehmigen@“ (16:13-16:28). Sonja weiß, dass die Beurteilung der Mutter ausschlaggebend für eine Erlaubnis ist. Da es sich in diesem Fall um einen Wunsch handelt, der dem familialen Bezugsrahmen der Horizonterweiterung entspricht, verwundert es nicht, dass die Mutter für diesen Vorschlag „total offen“ gewesen sei: „Wenn du das machen willst, dann machst du das“ (16:28-16:45).

Als gut betrachtete Leistungen, wie z.B. selbst gebastelte Geschenke während der Kindheit, seien von der Mutter auch entsprechend positiv gewürdigt und sichtbar präsentiert worden. Das habe Sonja mit Stolz erfüllt (07:31-07:47), was zugleich zeigt, wie stark sich die Tochter nach der mütterlichen Anerkennung sehnt.

Problematisch wird das mütterliche Vorgehen für Sonja, wenn sie nicht dem familialen Bezugsrahmen und den mütterlichen Vorstellungen folgt, die mit hohen Ansprüchen an die Tochter einherzugehen scheinen. So habe ihre Theatergruppe eine Darbietung eingeübt, zu deren Aufführung auch die Mutter gekommen sei:

„Und sie war dann da und ähm die [Theaterpädagogen, C.R.] waren nich so gut, wie halt die andere davor war. $/ / \mathrm{hmh} / /$ [...] und meine Mutter hat immer nur den Kopf geschüttelt. //hm// Oh, das fand ich so: gemei:n und unangenehm, //hmh// wei:l ich hab das da ein halbes Jahr gemacht und das, was ich dann bekomme, is ein Kopfschütteln und das fand ich dann immer so fies. //hmh// O:h. (.) Auch wenn es da gar nich mir galt, sondern eher den [verantwortlichen Erwachsenen, C.R.]“ (21:04-21:35).

Die gewünschte Anerkennung für das eigene Bemühen wird zu Sonjas Enttäuschung seitens der Mutter verweigert, was die Entwicklung von Selbstvertrauen erschwert. Die Mutter ist aus Sonjas Sicht nicht bereit, ihre Ansprüche situationsspezifisch anzupassen. Das Ausmaß der töchterlichen Mithilfe im Haushalt habe ebenfalls nicht den mütterlichen Vorstellungen entsprochen und sei ein Konfliktpunkt gewesen:

„[U]nd so äh von der Grundschule eigentlich bis hin zum Gymnasium @ähm hat äh meine Mutter mir immer vorgehalten, dass ich im Haushalt sehr faul bin@.//hm//Das fand ich immer sehr@ungerecht@. Weil, ich weiß nich, wie das bei meinem Bruder war, aber ich finde eigentlich schon, dass ich immer mit angepackt habe und so" (14:50-15:11).

Sie habe z.B. samstags die Küche oder das Badezimmer putzen müssen (1:04:38-1:04:47). Für die mütterliche Idee, der Tochter mit elf, zwölf Jahren das Kochen beizubringen, habe sich Sonja damals wie heute nicht erwärmen können und sie versuche, diese Aufgabe bis heute zu umgehen, was der Mutter missfalle (1:04:55-1:05:37). Auch wenn Sonja den Forderungen der Mutter nachgekommen sei, bestimmte Dinge im Haushalt $\mathrm{zu}$ erledigen, und sich bemüht habe, sei die Mutter mit dem Ergebnis nicht immer zufrieden gewesen (59:31-59:52). Sonjas Sichtweise sowie ihre individuellen Präferenzen 


\section{Familiale Tradierungsprozesse in einer Drei-Generationen-Perspektive}

finden in ihrer Wahrnehmung keine Beachtung durch die Mutter, vielmehr hält diese an ihrer Einschätzung fest, wodurch der Konflikt nicht gelöst wird. In seltenen Fällen mische sich die Mutter auch zu sehr in Sonjas Privatsphäre ein, was ein Streitfaktor sei (09:53-10:41).

Wenn Sonja Entscheidungen trifft, die mit den mütterlichen Vorstellungen der Horizonterweiterung konfligieren, wird dies in anderen Fällen zwar hingenommen, trifft aber auf elterliche Missbilligung und Unverständnis. Sonja habe ihr Engagement in einer kirchlichen Gruppe eingestellt, weil sie mit der neuen Leiterin nicht zurecht gekommen sei. Dies hätten die Eltern nicht verstehen können (22:47-23:52). Nachdem Sonja das Theaterspiel und später eine Sportart aufgegeben habe, sei sie aufgefordert worden, sich eine andere organisierte Freizeitbeschäftigung zu suchen (29:44-29:53, 1:12:21-1:12:23, 1:12:58-1:13:16). Die Eltern, vermutlich insbesondere die Mutter, halten an ihren Vorstellungen zur Umsetzung des Bezugsrahmens der Horizonterweiterung fest. Der Tochter verbleiben zwar insofern Gestaltungsräume, als sie sich die Form der Freizeitbeschäftigung aussuchen darf, die grundsätzliche Forderung, einer organisierten Form der Beschäftigung nachzugehen, ist hingegen nicht verhandelbar.

Für Sonja wiederum ist es schwierig, der Forderung in der gewünschten Form zu folgen, da die elterlichen Beweggründe, die für die Ausformung und Umsetzung des Bezugsrahmens ausschlaggebend sind, auf Erfahrungen basieren, die mit ihren eigenen wenig bis keine Überschneidungspunkte aufweisen. Dabei orientiert Sonja sich durchaus am familialen Bezugsrahmen, wie ihr Interesse für das Auslandsjahr sowie ihr mehrjähriges Engagement in einer Kirchengruppe belegen. Sie scheint darüber hinaus den Aspekt der Horizonterweiterung auf einer anderen als von der Mutter vorgesehenen Ebene umzusetzen. Sie sei früher jede Woche in die Bücherei gegangen, um sich Bücher auszuleihen (1:17:55-1:18:19), worin auch eine Form der Horizonterweiterung zu sehen ist; die aber anders als bei der Mutter nicht zwangsläufig mit der außerhäuslichen Sphäre verbunden ist.

Obwohl Sonja das Verhältnis zu ihren Eltern in einer Gesamtbilanz als „recht gut“ bezeichnet, zeigen die bisherigen Ausführungen auch verschiedene Facetten, die die intergenerationellen Beziehungen belasten. Zu dem Wunsch einer stärkeren Anerkennung ihrer Leistung durch die Mutter gesellt sich der nach Nähe. Neben den als schön erlebten Einkaufs- und Wochenendtouren habe Sonja noch das gemeinsame abendliche Stricken als „ganz toll“ empfunden (07:50-08:16). Sie habe allerdings institutionalisierte familiale Freizeitaktivitäten vermisst:

„Und ja (6) das, was mich früher immer so 'n bisschen äh gestört hatte, war, dass ähm ich von anderen äh Freunden immer mitbekommen habe, dass die ähm jeden Sonntag, gerade bei meiner besten Freundin war das so, die Familie immer eine Freizeitaktivität gemacht hat. $/ / \mathrm{hmh} / /$ Und das war bei uns nich so. Also wir haben auch ab und zu mal was zusammen gemacht, aber es war nie so richtig geregelt. $/ / \mathrm{hmh} / /$ Das wir jetzt wirklich einmal in der Woche was zusammen machen" (03:47-04:23). 


\subsection{Geschlossene Tradierungslogiken}

Zwar würde sonntags gemeinsam gegessen und bis vor kurzem habe es eine gemeinsame Kaffeezeit gegeben (30:52-31:06, 1:32:11-1:32:34), was ihr aber anscheinend nicht ausreicht. Zusammen mit den obigen Ausführungen deutet sich eine Ambivalenz zwischen dem Wunsch nach Gemeinschaft und Nähe bei gleichzeitigem Streben nach anerkannten Freiräumen an. Eine weitere Kritik bezieht sich auf die im Gegensatz zum Vater reserviertere Haltung der Mutter Sonja gegenüber (1:08:16-1:08:46). Hierin ist ein weiteres Indiz für den Wunsch nach einer größeren Nähe zu sehen. Die Mutter-TochterBeziehungen ihrer Freundinnen als Vergleichsmaßstab heranzuziehen, ist zugleich ein Verweis auf Sonjas Milieuorientierung. Im Unterschied zu den Vorgängergenerationen bezieht sich diese Milieuorientierung jedoch auf die Vorstellungen der Eltern-KindBeziehungen und nicht auf die Horizonterweiterung bzw. das Verbleiben im vertrauten Rahmen. Sonja formuliert in diesem Zusammenhang eine deutliche Kritik:

„Und früher dachte ich immer, oder is mir nur so vorgekommen, als ob $@()$.$@ meine Eltern, oder gerade auch meine Mutter, mich gar nich richtig$ ernst nimmt. //hmh// [...] Fand ich, kam bei anderen Müttern äh etwas besser rüber $/ / \mathrm{hmh} / /$ oder bei anderen Eltern. Ich weiß, ähm 'ne Freundin von mir, ähm die hat 'n sehr, sehr gutes Verhältnis zu ihrer Mutter und hab ich immer das Gefühl gehabt, dass ihre Mutter sie einfach ernster nimmt, als meine das immer gemacht hat" (24:09-24:56).

An anderer Stelle führt sie aus, sie habe den Eindruck gehabt, dass ihre Meinung „nich so richtig ernst genommen " und ihr nicht richtig zugehört worden sei, wenn sie Kritik geäußert habe (1:20:34-1:21:34). Sonjas Erinnerung, die Eltern hätten ihr eine bestimmte Freizeitsportart nicht gestattet, was die Mutter retrospektiv selbst nicht mehr verstehen könne (19:43-20:31), zielt in eine ähnliche Richtung. Letztlich sind die elterlichen Vorstellungen ausschlaggebend, die zumindest in der damaligen Situation nicht flexibel gewesen sind. Die intergenerationelle Machtbalance ist im Zweifelsfall eher asymmetrisch konzipiert, obwohl den Kindern zumindest vordergründig Entscheidungsräume gelassen werden.

Zusammenfassend stellt sich das Erziehungsarrangement aus Sonjas Sicht in bestimmten Bereichen als geschlossen dar. Wenn sie den elterlichen Erwartungen nicht entspricht, wird ihr deutlich die elterliche Missbilligung kommuniziert. So lange sie allerdings den Vorgaben folgt, kann sie sich der Unterstützung durch die Eltern sicher sein.

Zusammenfassende Betrachtung des familialen Bezugsrahmens In dieser Familie durchläuft der familiale Bezugsrahmen von der ältesten zur mittleren Generation eine Transformation von der Orientierung an einem idyllischen und harmonischen Familienleben hin zur Fokussierung auf die Horizonterweiterung. In der jüngsten Generation spielt die Horizonterweiterung weiterhin eine Rolle, jedoch in schwächerer Ausprägung. Dafür rückt verstärkt die Gestaltung der intergenerationellen Beziehungen ins Zentrum der Aufmerksamkeit. Auf einer höheren Abstraktionsebene lässt sich 


\section{Familiale Tradierungsprozesse in einer Drei-Generationen-Perspektive}

allerdings eine Milieuorientierung in allen drei Generationen erkennen, die in jeder Generation eine spezifische Ausprägung erfährt.

Für Frau Mahler nimmt die Orientierung an einem harmonischen, idyllischen Familienleben aufgrund der eigenen Biografie einen zentralen Stellenwert ein. Der Wunsch nach Zugehörigkeit zu einer (familialen) Gemeinschaft ist so dominant, dass Frau Mahler sich äußerst anpassungsbereit, genügsam und gehorsam verhält. Konflikte werden als mögliche Bedrohung des familialen Bezugsrahmens weitgehend ausgeblendet. Ein wesentlicher Bestandteil des familialen Bezugsrahmens bildet ein stark idealisiertes Mutterbild, wobei die Anwesenheit der Mutter zum Garanten für ein harmonisches Familienleben avanciert. Für ihre Tochter, Michelle Ahrens, ist ein Anschließen hieran schon allein aufgrund der als wenig idyllisch und harmonisch erfahrenen Kindheit kaum möglich. Stattdessen wird die Horizonterweiterung zum zentralen Thema. Die als einengend erlebte häusliche Atmosphäre lässt für Michelle Ahrens Freiräume außerhalb der Kernfamilie bedeutsam werden. Die Förderung der persönlichen Entwicklung wird für sie elementar, um den Bezugsrahmen der Horizonterweiterung umzusetzen. Diese Leitlinie, verstanden als Förderung der individuellen Weiterentwicklung, ist während der eigenen Familienphase bereits eine allgemein anerkannte Richtlinie, die auch in dem sie umgebenden Milieu eine wichtige Rolle spielt. Die Kindesorientierung sowie -förderung werden in das Erziehungssetting integriert und die Verregelung des kindlichen Alltags nimmt ab; insbesondere autoritäre Anweisungen und Verbote haben als Elemente der Erziehung ausgedient.

Für Sonja ist die Horizonterweiterung eine im Bedarfsfall zu aktivierende Einstellung, die nicht so prominent wie bei ihrer Mutter im Vordergrund steht. Zentraler sind für sie Fragen des intergenerationellen Umgangs. Das Streben nach familialer Nähe sowie die Anerkennung ihrer Leistungen und Ansichten bilden für sie zentrale Themen. Letzteres spiegelt zugleich ihren Wunsch nach größeren eigenen Gestaltungsräumen. Insgesamt wird das emotionale Wohlbefinden in der jüngsten Generation zu einem wichtigen Thema.

\subsubsection{Familie Nolte/Gümper: Repräsentative Höchstleistungen}

Biografische Eckdaten Frau Nolte wird 1932 in Pommern geboren und lebt seit 1946 in Niedersachsen. Sie hat eine jüngere Schwester. Sie besucht bis zur zehnten Klasse das Gymnasium und danach die Höhere Handelsschule. Herr Nolte wird 1929 als viertes Kind einer Akademikerfamilie geboren. Er hat eine akademische Ausbildung. Beide sind evangelisch und kirchlich engagiert. Das Paar heiratet 1955 und zieht in in die niedersächsische Teilregion des Untersuchungsgebiets. Sie haben sieben Kinder.

Iris Gümper wird 1959 als zweites Kind geboren. Sie verlässt nach der zehnten Klasse das Gymnasium und absolviert eine Ausbildung im sozialen Bereich und ist in der Jugendarbeit tätig. Nach der Ausbildung zieht sie in eine mittelgroße Stadt in der Nähe ihres Herkunftsortes, in der sie heute noch lebt. Ihr Ehemann ist neun Jahre älter und betreibt ein eigenes Ladengeschäft. Das Paar heiratet 1992. Im selben Jahr wird Lea 
Gümper geboren. Sie besucht das Gymnasium und verbringt ein Schuljahr im Ausland. Sie ist Leistungssportlerin.

Aneignungsebene Frau Nolte $\left({ }^{*} 1932\right)^{170}$ : Akzeptierte Ordnungsmuster Frau Nolte berichtet gleich zu Beginn ihres Interviews, dass sie aus einem „Beamtenhaushalt" stamme (00:47-00:50, 01:27-01:32); ihr Vater habe sich diese Position erarbeitet (0:51-01:26). Sie hebt an dieser Stelle die Aufstiegsorientierung des Vaters hervor, die im Verlauf des Interviews über die Attribute Anstrengung, Disziplin und Genauigkeit (01:23:-01:26, 37:06-37:25) weiter ausdifferenziert wird und insgesamt auf die Leistungsorientierung des Vaters abhebt. Somit wird der Bezugsrahmen der Leistung gleich zu Beginn des Interviews eingeführt, der anschließend an vielen Stellen relevant werden wird und von Frau Nolte eine besondere Ausprägung in Form repräsentativer Höchstleistungen erhält. Das väterliche Verhalten dient ihr als Vorbild, das sie ungebrochen übernimmt.

Die Erziehung durch die Eltern, insbesondere durch den Vater, ist für Frau Nolte durch deutlich formulierte Erwartungen geprägt, deren Nichtbeachtung konsequent bestraft worden sei:

„Es war bei uns ganz, ganz wichtig, dass wir ehrlich sind, dass wir also nich schwindelten, sonst hat er sogar auch mal, nicht 'ne Peitsche genommen, aber 'nen Klopper genommen, 'n Teppichklopper, is heute alles nich mehr möglich. Aber das war also ganz, ganz, ganz wichtig, dass man also ehrlich war. Das war auch wichtig, dass man pünktlich wa:r und auch nach Möglichkeit gehorsam war, eben preußische Tugenden“ (01:59-02:25).

Die zum Ausdruck kommende strikte Haltung des Vaters wird in keiner Weise kritisiert oder retrospektiv in Frage gestellt. Dennoch fühlt sich Frau Nolte veranlasst, die rigide Beschreibung des väterlichen Verhaltens zu relativieren:

„Wir hatten aber 'ne wunderschöne Kindheit. [...] Wir hatten eigentlich alles, was ein Kind haben wollte. Wir konnten zum Schwimmen gehen, wir hatten ganz in der Nähe 'n Schlittschuhteich [...] Und das war wunderschön. Wir hatten 'nen gro:ßen äh großen Freundeskreis, ja mit dem wir alles Mögliche unternommen haben“ (02:25-02:59).

Diese Ausführungen sind sicherlich teilweise der Interviewsituation geschuldet. Um die Gefahr einer potenziellen negativen Rezeption in der Gegenwart zu minimieren, fügt sie positiv konnotierte Beschreibungen ihrer Kindheit an, die auch aktuelle Zuhörer auf diese Weise betrachten können, und relativiert so das Bild des strengen Vaters. Zugleich verdeutlicht sie, dass ihr als Kind jenseits der väterlichen Erwartungen eigene Erfahrungsräume zugestanden und eröffnet werden, wofür sie ihre Dankbarkeit ausdrückt.

\footnotetext{
${ }^{170}$ Das Interview mit Frau Nolte wurde am 13.02.2008 geführt.
} 


\section{Familiale Tradierungsprozesse in einer Drei-Generationen-Perspektive}

Die Eröffnung von Möglichkeitsräumen ist auch der Aspekt, den Frau Nolte in Bezug auf die NS-Jugendorganisation der Jungmädel betont. Als Beispiele nennt sie Reisen und sportliche Wettkämpfe (02:59-06:23). Sie berichtet davon, wie in der Gruppe verschiedene Orientierungen eingeübt worden seien, wie Ehrlichkeit, Pflichtbewusstsein, Zuverlässigkeit und Fleiß (04:12-04:36, 05:58-06:21), die zu dem vom Elternhaus erwarteten Eigenschaften kompatibel sind, und die Frau Nolte offensichtlich internalisiert. Im Rahmen ihrer Schilderungen zu ihrer Mitgliedschaft in der NS-Jugendorganisation lässt sich ihre Orientierung am Bezugsrahmen der repräsentativen Höchstleistungen erkennen. Sie habe an Sportwettbewerben teilgenommen. Gerade als sie die Möglichkeit gehabt habe, die Wettbewerbe zu gewinnen, seien diese aufgrund der Kriegssituation eingestellt worden und sie habe ihre Talente nicht öffentlich beweisen können (05:25-05:52). Das rechtfertigt sie, indem sie zugleich die Botschaft vermittelt, es habe weder an ihren Fähigkeiten noch am Willen gelegen, sondern vielmehr an den äußeren Umständen, auf die sie keinen Einfluss gehabt habe. Ähnlich verhält es sich mit ihrer Darstellung zur schulischen Bildung. Sie habe trotz guter Leistungen das Abitur nicht ablegen können, was sie mit der prekären finanziellen Situation der Familie in der Nachkriegszeit begründet (32:40-33:10). Frau Nolte verschweigt die missglückte Umsetzung des familialen Bezugsrahmens nicht, was auf die starke Dominanz eines weiteren Bezugsrahmens, den der Ehrlichkeit, verweist. Dieser verhindert, ausschließlich die gelungene Umsetzung der repräsentativen Höchstleistungen zu thematisieren. Um aber trotzdem eine möglichst positive Außendarstellung zu gewährleisten, sind die eben beschriebenen Rechtfertigungs- und Relativierungsstrategien notwendig.

Das Kriegsende ist für Frau Nolte mit der Abwesenheit des Vaters, der Flucht der übrigen Familie sowie diversen Entbehrungen verbunden. In ihren Schilderungen zeigt sie auf, wie ihre Mutter während des Krieges und nach Kriegsende Probleme mit der Kleidungs- und Nahrungsmittelversorgung gelöst habe (22:30-22:40, 07:42-08:03, 30:2330:54). Auch berichtet sie von diversen Arbeiten, zu denen sie, ihre Schwester und ihre Mutter während und nach der Flucht herangezogen worden seien (09:51-10:12, 27:43-27:54). Die Mutter wird als Person eingeführt, die durch Fleiß, Engagement und Aktivität Schwierigkeiten bewältigt, nicht resigniert und somit zentrale Orientierungen der Familie vorlebt. Über die Arbeiten beklagt sich Frau Nolte auch retrospektiv nicht, sondern zeigt im Gegenteil die hiermit für sie verbundenen positiven Seiten auf. Damit unterstreicht sie ihre Arbeits- oder Leistungsorientierung sowie ihr Bestreben, Herausforderungen aktiv zu begegnen.

Nach Kriegsende kehren Frau Nolte, ihre Mutter, Großmutter und Schwester zunächst in den inzwischen besetzten Heimatort zurück, um den Vater wiederzufinden. Erst aus der Retrospektive wird dies von Frau Nolte als unüberlegt betrachtet (12:3913:03, 16:22-16:33). Hier ist eine latente Orientierung erkennbar, die zentral für ihre Familie ist: Angesichts der unbekannten Situation reagiert die Familie, indem sie auf das Bekannte zurückgreift, was in diesem Falle bedeutet, in den Heimatort zurückzukehren. Dort findet die Familie wieder zusammen, aber das Leben im nun besetzten Ort 


\subsection{Geschlossene Tradierungslogiken}

erweist sich als schwierig. Die Familie versucht in bestimmten Bereichen an die vertrauten Routinen anzuknüpfen, indem Frau Nolte z.B. zum Konfirmationsunterricht geht. Insbesondere das Verhalten des Pastors, der trotz der widrigen Umstände seinen Pflichten als Gemeindevorstand nachgekommen sei, beeindruckt Frau Nolte. Hierin sieht sie retrospektiv die Relevanz des Glaubens für ihr Leben begründet (18:40-19:34). Die Religion scheint darüber hinaus eine Halt gebende Funktion eingenommen zu haben, die auch nach dem Verlassen des Heimatortes und der Ankunft in Niedersachsen eine Rolle spielt. Frau Nolte schließt sich einer religiösen Jugendgruppe an und lernt dort ihren späteren Mann kennen.

Die Schilderungen des väterlichen Verhaltens in der Nachkriegszeit lässt starke Loyalitäten erkennen. Der Vater habe versucht, ihr trotz der prekären finanziellen Verhältnisse Konsumwünsche in einem gewissen Rahmen zu erfüllen (31:23-32:18). Sie charakterisiert ihren Vater folgendermaßen: „, [M] ein Vater war streng, aber er war un:heimlich gutmütig und gü:tig“" (32:21-32:25). Lediglich die erwünschte Schulausbildung habe er ihr verwehren müssen: „Ich äh musste mit der zehnten Klasse abgehen. Ich musste. Hab geheult. Aber mein Vater sagte: ,Wir haben kein Geld. Ich kann dich nicht studieren lassen. ' [...] $\mathrm{Na}$ ja. Und dann bin ich auf die Höhere Handelsschule gegangen. Und das hat mir GUTgetan“ (32:40-33:10). Das väterliche Verhalten wird mit der finanziellen Situation der Familie begründet und auf diese Weise von Frau Nolte entschuldigt und gerechtfertigt. Durch die nachfolgende positive Bilanzierung wird die väterliche Entscheidung zudem als richtig etikettiert. Gleichzeitig verdeutlicht Frau Nolte, dass sie der schulischen Bildung einen hohen Stellenwert einräumt und nicht ihre Leistungen, sondern die allgemeinen Umstände der Zeit sie daran gehindert hätten, das Abitur zu erlangen.

Insgesamt übernimmt Frau Nolte die von den Eltern an sie herangetragenen Erwartungen. Eine Orientierung am familialen Bezugsrahmen ist für sie eine nicht zu hinterfragende Selbstverständlichkeit, an der sie auch in ihrem späteren Leben festhält. Sie betont z.B., wie ihr in ihrem weiteren Leben im Zuge ihres ehrenamtlichen Engagements ein Aufstieg in mit hohem Sozialprestige verbundene, öffentliche Positionen gelungen sei (54:43-1:04:15). Dabei konkurriert sie mit Personen, die über symbolisches Kapital in Form von schulischen und akademischen Titeln verfügen. Sie bemüht sich um herausragende Leistungen, um sich adäquat beteiligen zu können (1:04:23-1:05:07). Auf diese Weise versucht sie u.a., zumindest teilweise das fehlende Abitur zu kompensieren. Die Ausführungen verdeutlichen, wie stark Frau Noltes Aufstiegsorientierung ist, die sie über Höchstleistungen und ihre öffentliche Präsentation umsetzt. Die in ihrer Herkunftsfamilie zentralen Orientierungen Fleiß, Strebsamkeit und Ehrgeiz unterstützen die Umsetzung dieses Zieles.

Vermittlungsebene Frau Nolte $\left({ }^{* 1932)}\right.$ : Strenge Ordnung und Kontrolle Frau Nolte orientiert sich bei der Erziehung der eigenen Kinder an den eigenen Kindheitserfahrungen und entwirft für ihre Kinder einen geschlossenen Erwartungshorizont, der 


\section{Familiale Tradierungsprozesse in einer Drei-Generationen-Perspektive}

kaum Möglichkeiten zur Veränderung enthält. Im Zentrum ihrer Erziehung steht das Einhalten der vorgegebenen Bahnen bzw. einer strengen Ordnung. Hierbei handelt es sich um Elemente, die sie aus ihrer Kindheit kennt:

„Ja, und da musste natürlich das bei uns, bei so vielen Kindern, musste das alles ge:nau gehen. Nu hatte ich ja Ordnung und so weiter, hatte ich ja du-, durch meinen Vater äh gelernt. Mein V-, äh mein Mann war auch ziemlich streng" (40:44-40:57).

Die elterliche Erziehung hat für Frau Nolte Vorbildcharakter, die sie bis heute verteidigt, und die sie mit einer Kritik an der vermuteten aktuellen Erziehung verbindet: „Die war super. Ich will da nichts anderes machen.//hmh//@(.)@ Dieses Laissez-faire von heute ist auch nicht immer gut" (1:34:03-1:34:13). Die von den Eltern übernommenen Erziehungsvorstellungen werden mit denen des Ehemannes verbunden. Das ist relativ unproblematisch, da es offenbar große Übereinstimmungen gibt.

Wichtig sei es ihr gewesen, „dass all Kinder gesund im Trott langliefen @(.)@“(1:49:09_ 1:49:13). Zu den Maximen dieses „Trotts“ zählen Ehrlichkeit, Fleiß, Zuverlässigkeit und Pünktlichkeit (46:49-47:01, 42:28-42:42) - Elemente, die wichtige Eckpfeiler des eigenen Aufwachsens gebildet haben. Die Kinder sollen sich in von außen gesetzte Ordnungsmuster einfügen, wodurch sie gleichzeitig lernen, die eigenen Bedürfnisse zurückzustellen. Das sind wichtige Voraussetzungen für die Internalisierung des Bezugsrahmen der repräsentativen Höchstleistungen, der einen zentralen Bestandteil der vorgegebenen Bahnen darstellt. Im Bereich der musikalischen Ausbildung wird den Kindern z.B. kein Verhandlungsspielraum gewährt: „Sie mussten alle [Klarinette, C.R.] spielen. Sie mussten. [...] Also das ist auch was mit Strenge und Ordnung " (43:50-44:17). Kreative und soziale Fähigkeiten sowie die Ausbildung einer kritischen Haltung finden demgegenüber kaum Beachtung und werden nicht explizit gefördert.

Regelmäßiges Üben für Schule und Musikunterricht gehören zu den obligatorischen Pflichten der Kinder (1:47:44-1:47:51). Den Kindern wird verdeutlicht, gute Leistungen seien nicht nebenbei zu erbringen, sondern sind mit Anstrengungen und Durchhaltevermögen verbunden. Hierbei verhält Frau Nolte selbst sich nicht immer vorbildhaft. Sie gibt z.B. das Erlernen eines Musikinstruments auf, sobald sie merkt, dass sie nicht an die Leistungen der Kinder heranreichen kann. Sie begründet das mit der Auslastung durch ihre vielfältigen Aufgaben (42:46-43:50). Hier manifestiert sich abermals die oben beschriebene Rechtfertigungs- und Relativierungsstrategie Frau Noltes. Zugleich wird ein weiteres Muster erkennbar. Anstrengungen erfolgen in den Bereichen, wo eine Umsetzung des familialen Bezugsrahmens der repräsentativen Höchstleistungen erreichbar scheint. Auch die Kinder dürfen offensichtlich in seltenen Ausnahmefällen auf diese Strategie zurückgreifen. Für Iris Gümper wird die Regel, regelmäßig Klarinettenunterricht zu nehmen, außer Kraft gesetzt. Allerdings ist dabei der Wunsch der Tochter in Verbindung mit dem Ratschlag der Musiklehrerin ausschlaggebend und nicht ausschließlich das Interesse des Kindes (44:20-44:47). Wichtiger jedoch dürfte es 


\subsection{Geschlossene Tradierungslogiken}

sein, dass die Tochter beim Erlernen des zweiten Instruments ungleich erfolgreicher ist und hierdurch an den familialen Bezugsrahmen der repräsentativen Höchstleistungen anknüpfen kann: „Und dann hat sie [Harfe, C.R.] gelernt. Das konnte hier niemand. Und da war sie so eine, die da brillieren konnte. [...] Das war ganz toll“ (44:48-45:03).

Abgesehen hiervon wird auf die individuellen Bedürfnisse der Kinder selten eingegangen. Von den Kindern wird erwartet, ein zweites Musikinstrument zu erlernen, das sie sich aber selbst aussuchen dürfen (44:03-44:11). In der Regel werden den Kindern aber keine Wahlmöglichkeiten gelassen. Bei abweichendem oder unerwünschtem Verhalten greift Frau Nolte rigoros ein und zeigt wenig Toleranz. So wird z.B. die Freizeitgestaltung reglementiert, wenn sie mit den elterlichen Vorgaben kollidiert. Die Kinder dürfen einer populären Sportart nicht nachgehen, weil das nach Ansicht des Vaters die Feinmotorik der Hände und damit die musikalische Ausbildung beeinträchtige (45:49-46:04). Als einige ihrer Kinder eine eigene Kindersprache entwickeln, unterbindet Frau Nolte das umgehend (51:03-51:52). Ihre Tochter, Iris Gümper, darf nicht an Leichtathletikwettkämpfen teilnehmen, weil die Termine sich mit dem als wichtiger eingestuften Gottesdienst überschneiden (46:05-46:31). Hier ergibt sich ein Interessenkonflikt zwischen den beiden Teilkomponenten des familialen Bezugsrahmens, den Höchstleistungen und der Repräsentativität, die auf die Außendarstellung abzielt. Letztere genießt in diesem Fall eine höhere Priorität als die Förderung der kindlichen Leistungen. Die beschriebenen Verbote zielen darauf, die Kinder in die von den Eltern festgelegte Ordnung einzupassen.

Im Fokus der Aufmerksamkeit stehen die schulischen und musikalischen Leistungen der Kinder. Bereiche wie der sportliche Sektor sind demgegenüber nachrangig. Diese enge Begrenzung revidiert Frau Nolte später, wofür es mehrere Gründe gibt. Zum einen stärkt der Aufstieg in prestigeträchtige ehrenamtliche Positionen zweifelsohne Frau Noltes Selbstbewusstsein und sie kann sich von der ihrem Ehemann zugeschriebenen Leistungshierarchie lösen. Mit der Einbeziehung des sportlichen Sektors sowie der Weiterbildung - welche die Möglichkeit eines Aufstiegs umschließt - sind Leistungsbereiche angesprochen, die in ihrer Herkunftsfamilie relevant gewesen sind. An dieser Stelle lässt sich gleichsam von einer Art Rückbesinnung sprechen. Zum anderen führt die Konzentration auf Schule und Musik in der Familie zu einigen Problemen. Eines der Kinder erlangt nicht den gewünschten Schulabschluss, erreicht aber durch persönliches Engagement eine relativ gut abgesicherte Leitungsposition (53:42-53:59, 1:11:33-1:11:47). In der Folge avanciert der Aspekt der Sicherheit bzw. Beständigkeit für Frau Nolte zu einem betonenswerten Gütekriterium (1:13:15-1:13:23). Die anderen Kinder erfüllen zwar die in sie gesetzten Erwartungen im schulischen Bereichen, schaffen es aber nicht alle, abgesicherte berufliche Positionen zu bekleiden (1:11:48-1:13:14). All das sind Gründe, die zu einer Umorientierung beitragen. Schließlich wird die ehemals eindeutige Leistungshierarchie auch in Auseinandersetzung mit den Ansichten der inzwischen erwachsenen Kinder und ihren Erziehungsmaximen abgemildert (1:48:30-1:48:59).

Die sich an dieser Stelle andeutende zunehmende Gelassenheit in der Gegenwart dürfte jedoch auch mit der Tatsache verbunden sein, in der Gegenwart keine Erziehungsver- 


\section{Familiale Tradierungsprozesse in einer Drei-Generationen-Perspektive}

antwortung mehr zu haben - weder für die Kinder noch für die Enkelkinder. Trotzdem bleibt der Bezugsrahmen im Zeitverlauf erhalten. Zwar ändern sich die Bereiche, für die er gilt, bzw. es vollzieht sich eine Reorientierung auf die in ihrer Kindheit wichtigen Sektoren, das Ziel der repräsentativen Höchstleistungen wird allerdings jeweils mit Konsequenz verfolgt und es gibt kaum Handlungsspielräume. Das zeigt sich insbesondere in der eigenen Elternphase.

Die Einhaltung des vorgegebenen Rahmens wird konsequent eingefordert und von den Kindern wird Gehorsam verlangt. Zum festgelegten Rahmen gehört die Regel, pünktlich zu den Mahlzeiten zu Hause zu sein, wie Frau Nolte es aus dem eigenen Elternhaus kennt und worauf sie akribisch achtet (1:32:01-1:32:07, 1:46:42-1:47:06, 1:47:35-1:47:40). Frau Nolte rückt in diesem Zusammenhang weniger die Kontakt- und Austauschmöglichkeiten ins Zentrum ihrer Ausführungen als vielmehr die Ordnungsstrukturen. Sie strukturiert durch die gemeinsamen Mahlzeiten stark den Tagesablauf ihrer Kinder, die auf alltäglicher Basis dazu angehalten werden, den von den Eltern vorgegebenen Bahnen zu folgen, die kaum Raum für Ausnahmen zulassen. Nebenbei wird deutlich, wie zentral für Frau Nolte die Versorgung der Kinder und die Vorgabe von Ordnungsstrukturen als elterliche Pflichten sind.

Eine weitere aus der Kindheit übernommene Praxis stellt der regelmäßige Gottesdienstbesuch dar (1:17:43-1:18:17), der auch für die Kinder obligatorisch (46:26- 46:29) und ein weiteres Beispiel für die zeitliche Strukturierung des Wochenablaufs ist. $\mathrm{Zu}$ den Regelmäßigkeiten des Jahresablaufs gehören ferner die alljährlichen Urlaube. Die gemeinsamen Familienfeste werden ebenfalls kurz thematisiert, aber eher beiläufig behandelt (1:31:02-1:31:28, 1:34:54-1:35:29). Sowohl die Reisen als auch die Feste haben anscheinend vor allem eine Strukturierungs- und Ordnungsfunktion, da deren Inhalte, hiermit verbundene Erlebnisse und Erinnerungen nicht weiter ausgeführt werden.

Die Orientierung an einer strengen Ordnung impliziert Regelmäßigkeiten, die dem Familienleben und dem Tagesablauf eine Struktur und somit den Familienmitgliedern auch Orientierungshilfen geben. Es verhindert jedoch auch Flexibilität und lässt kaum Spielraum für eine individuelle Entwicklung und Entfaltung von Interessen und Fähigkeiten. Das verweist auf ein geschlossenes Erziehungssetting, das auf eine unveränderte Weitergabe des familialen Bezugsrahmen zielt und mit strikten Erwartungen verbunden ist.

Kontrolle ist ein wesentliches Mittel zur Umsetzung der Regeln. Es wird kontrolliert, ob die Hausaufgaben ordentlich gemacht wurden, ob die Kinder geübt haben oder wer die Freunde sind (1:47:45-1:47:52, 1:32:10-1:32:22). Auch wenn Frau Nolte im Allgemeinen um die Lenkung der Kinder bemüht ist, kann sie nicht überall präsent sein. Sie lässt den Kindern kontrollierte Freiräume, wie z.B. das unbeaufsichtigte Spielen im elterlichen Garten (41:55-42:19). Die älteren Kinder habe sie nicht in den Kindergarten geschickt (41:23-41:44, 50:52-50:57). Zum einen dürfte das mit einem bestimmten Bild der Mutterrolle zusammenhängen, wonach es vor allem deren Aufgabe ist, sich um die Kinder zu kümmern. Zum anderen bedeutet es aber auch, die alleinige Kontrolle über die Erziehung der Kinder zu behalten und nicht teilweise Personen außerhalb der 


\subsection{Geschlossene Tradierungslogiken}

Familie zu überlassen. Das Bemühen, die Deutungshoheit zu behalten, lässt sich auch in Bezug auf das Verhältnis zwischen Schule und Elternhaus erkennen. Frau Nolte kritisiert, in der Schule habe es einige Lehrer gegeben, die sie als „68er“ etikettiert, die gegen die Eltern und eine strenge Ordnung eingestellt gewesen seien. Das hätte zu einigen Aufarbeitungsbemühungen zu Hause geführt (1:37:29-1:37:44). Frau Nolte sieht die elterliche Autorität untergraben und versucht dem entgegenzuwirken, wobei sie in ihren Ausführungen nicht konkreter werden möchte (1:39:11-1:39:32). Deutlich wird aber die parallele Existenz verschiedener Erziehungsvorstellungen, wobei Frau Nolte von den eigenen Vorstellungen abweichende Praktiken strikt ablehnt und wenig Bereitschaft zeigt, sich auf Neuerungen einzulassen. Dass die elterliche Kontrolle nicht umfassend gewesen ist, ist an den von Frau Nolte angedeuteten gelegentlichen Ausbrüchen der Kinder abzulesen (1:49:19-1:49:27), wobei es ihr aber wichtig ist, diese als Ausnahmen hinzustellen.

Das leitet über zur familialen Außendarstellung. Die Wahrnehmung durch das Umfeld ist generell eine wichtige Komponente bei der Erziehung der Kinder. Dazu zählt neben der eben beschriebenen Reglementierung der Kinder auch das öffentliche Präsentieren der kindlichen Leistungen als Bestandteil des Familienlebens. Frau Nolte vertritt die Ansicht, es sei für Kinder wichtig, ihre Leistungen öffentlich vorzuführen und sich darzustellen; die Außendarstellung sei also im Interesse der Kinder (45:04-45:41). Wie die Kinder das beurteilen, wird dabei nicht berücksichtigt.

Emotionale Zuneigung oder körperliche Nähe bilden randständige Themen innerhalb des Interviews. Ihnen wird anscheinend keine große Bedeutung zugeschrieben und Frau Nolte räumt der Beziehungsebene generell kaum Aufmerksamkeit ein. Dies zeigt sich z.B. daran, dass Frau Nolte zwar durchaus das Konkurrenzverhältnis zwischen ihrer Tochter, Iris Gümper, und einem ihrer Söhne bemerkt, diesem aber nicht entgegenwirkt (44:20-44:38). Da diese Rivalitäten geeignet sind, den familialen Bezugsrahmen der repräsentativen Höchstleistungen zu fördern, schreitet Frau Nolte vielleicht bewusst nicht ein.

Verständnisvolle Zuwendung, das Eingehen auf die kindlichen Bedürfnisse oder das Fördern der Kinder durch Ermutigungen scheinen nicht zum Erziehungsrepertoire Frau Noltes zu gehören. Aufschlussreich ist in diesem Zusammenhang ihre Metapher vom Garten als ,tollem Kindermädchen': „Ach so, wir hatten äh 'n tolles Kindermädchen: Das war unser Garten. Keine Person //ah// unser Garten. Wir haben die Kinder da rausgesteckt. [...] Und es war wu:nderschön. Ein Paradies für unsere Kinder“ (41:5542:19). Etwas später führt Frau Nolte aus:

„Nun hatte ich ja wenig Zeit. Ich hab sie dann runter in den Garten gepackt. Und [...] im Sommer [...] sind die nackend da rumgelaufen wie Adam und Eva. Und [...] ich hab immer drauf geachtet, dass alle drei äh Türen zu waren. [...] Dann hab ich immer gesehen, wie unsere beiden [jüngsten Kinder, C. R.], die ja nun allein da unten waren, die brauchten ja auch Unterhaltung, und dann haben die sich platt auf die Erde [...] gelegt, 


\section{Familiale Tradierungsprozesse in einer Drei-Generationen-Perspektive}

und haben äh durch die $=$ das Garagentor äh rausgeguckt auf die Straße. Da konnten sie nich viel sehen, $/ / \mathrm{hmh} / /$ aber die, die Füße konnten sie sehen. Das war doch toll, ne“ (51:53-52:38).

Sie zeichnet das Bild eines idyllischen, quasi natürlichen, mit Freiheiten verknüpften Aufwachsens jenseits gesellschaftlicher $Z$ wänge, wobei die Freiheiten jedoch relativ sind und der Aktionsradius von Erwachsenen abgesteckt wird. In erster Linie handelt es sich um eine teilweise romantisierte und verklärte Sicht eines Erwachsenen auf die Kindheit. Die Perspektive der Kinder wird kaum berücksichtigt. Zwar bemerkt Frau Nolte, dass die Kinder Unterhaltung gebraucht hätten, die sie sich auch gesucht hätten, allerdings ist diese ebenso wie die Möglichkeit zwischenmenschlicher Kontakte stark limitiert. Frau Nolte legt ihren Fokus eindeutig auf das Abstecken eines Rahmens. Zwischenmenschliche Kontakte und Zuwendung als Attribute, die das „Kindermädchen“ Garten nicht bieten kann, finden keine besondere Beachtung. Symbolisch manifestieren sich hier die von Frau Nolte festgelegte „strenge Ordnung“ sowie die von ihr festgesetzten Limitierungen für die Kinder, denen kaum Gestaltungsraum bleibt.

Die Erziehung der Kinder fasst sie in erster Linie als elterliche Aufgabe auf und greift nicht auf die Unterstützung der Großeltern zurück (1:45:15-1:45:20). Das entspricht den eigenen Kindheitserfahrungen und wird auf diese Weise in der eigenen Familienphase fortgeführt. Die Großeltern, die nicht in unmittelbarer Nähe leben, werden regelmäßig besucht, sind aber nicht im Erziehungsalltag präsent (1:22:54-1:24:25). Frau Nolte selbst ist ebenfalls nicht aktiv in die Erziehung ihrer Enkel eingebunden (1:53:38-1:53:45), womit sie die ihr aus Kindheit und eigener Familienzeit bekannte Linie fortsetzt. Damit wird gleichzeitig das Konfliktpotenzial zwischen Frau Nolte und ihren Kindern gemindert.

Interessant ist in diesem Zusammenhang, dass Frau Nolte ihre eigenen Eltern als liebevoll beschreibt (1:34:24-1:34:28). Diese Zuschreibung spiegelt sich aber nicht unmittelbar in den Erzählungen und Beschreibungen Frau Noltes über ihre Kindheit. Es handelt sich vielmehr um eine bilanzierende Charakterisierung, die vermutlich in Auseinandersetzung mit der gewachsenen Bedeutung dieser Kategorie retrospektiv entstanden ist und erst in der Gegenwart einen gewissen Stellenwert erlangt hat.

Insgesamt zeichnet sich trotz einiger Ausnahmen ein stark asymmetrisches Machtverhältnis ab. Die Kinder haben kaum Mitspracherechte, es wird Gehorsam gefordert, und die Entscheidungen der Eltern sind selten verhandelbar. Die für die Kinder entworfenen Erwartungshorizonte speisen sich zu einem großen Teil aus den eigenen Kindheitserfahrungen und zeichnen sich durch eine weitestgehende Geschlossenheit aus. Die Bedürfnisse, Fähigkeiten und Wünsche der Kinder stehen nicht im Zentrum der Aufmerksamkeit.

Aneignungsebene Iris Gümper $(* 1959)^{171}$ : Ablehnen und Anknüpfen an den familialen Bezugsrahmen Die Auseinandersetzung mit dem familialen Bezugsrahmen

\footnotetext{
${ }^{171}$ Das Interview mit Iris Gümper wurde am 28.02.2008 geführt.
} 


\subsection{Geschlossene Tradierungslogiken}

der repräsentativen Höchstleistungen zieht sich als roter Faden durch das Interview mit der Tochter, Iris Gümper. Im Verlauf des Interviews wird ihre ambivalente Positionierung zu diesem Bezugsrahmen deutlich, der einerseits aus einer strikten Ablehnung, andererseits in einer starken Orientierung an eben diesem Rahmen besteht. Auch aus Iris Gümpers Perspektive ist eine strenge Ordnung das zentrale Merkmal der elterlichen Erziehung. Sie beschreibt das Erziehungssetting ähnlich wie ihre Mutter, bewertet es aber nicht positiv, sondern empfindet es als Korsett und Zwang.

Als zentrale elterliche Maxime nennt Iris Gümper: „Gut dazustehen, gute Ausbildung, gut in der Schule sein. [...] Man musste immer gut sein“ (II 51:54-52:04). Ihre Mutter, Frau Nolte, habe stark auf die Entwicklung der Kinder geachtet und sei bei abweichendem Verhalten eingeschritten. Wenn ihre schulischen Leistungen, z.B. ihre Lesekompetenz, oder die musikalischen Fertigkeiten nicht den Erwartungen entsprochen hätten, dann hätte sie intensiv üben müssen, was sie als Drill empfunden habe. Ihre Lernschwierigkeiten und Überforderungen habe sie mit niemanden besprechen können (I 05:18-06:22). Aus dieser Perspektive bringen die Eltern wenig Verständnis für die Probleme und Bedürfnisse der Tochter auf und sie erfährt wenig Unterstützung. Stattdessen schreiben die Eltern die Schuld für die aus ihrer Sicht inadäquaten Leistungen der Tochter und ihrem vermeintlich fehlenden Willen zu:
„Und dann kann ich mich dran erinnern, als es auf die weiterführende Schule ging, dass meine Mutter jeden Abend mit mir Diktate übte. Üb- te, übte, übte. Ich heulte. Mir, das hab ich auch noch das Gefühl in Augen, ich war so müde, dass mir das schon alles wehtat, $/ / \mathrm{hmh} / /$ aber ich musste üben, üben, üben, bis endlich null Fehler unten waren“(I 07:17-07:32).

Ihr wird vermittelt, sie müsse sich lediglich mehr anstrengen. Das widerspricht auf den ersten Blick den beschriebenen Rechtfertigungsstrategien der Mutter, die für ein Verfehlen des familialen Bezugsrahmens i.d.R. äußere Umstände anführt. Das lässt sich damit erklären, dass Frau Nolte, dem familialen Bezugsrahmen folgend, gegenüber Dritten und in der Retrospektive anders argumentieren dürfte als während ihrer Familienphase gegenüber ihren Kindern. Iris Gümper jedenfalls nimmt die an sie gerichteten Erwartungen als relativ geschlossen wahr.

Die elterliche Erziehung zielt darauf, die Kinder in tradierte Ordnungsmuster einzufügen. Wichtige Elemente zur Umsetzung dieser Vorgaben sind Kontrolle und Korrekturen:

„Sie haben uns so erzogen nach den Benimm-Dich-Regeln. $/ / \mathrm{hmh} / /$ Was man so machte, wie man sich verhält in der Öffentlichkeit, zu fremden Personen, wie man grüßt. Wir haben auch noch gelernt, wie man Knicks macht und Diener macht, wie man die Hand gibt und nich mit links. Also da wurde sehr drauf geachtet, wie wir das machen. Und wenn wir das nich richtig funktionierten, dann haben wir immer von den Eltern von 


\section{Familiale Tradierungsprozesse in einer Drei-Generationen-Perspektive}

hinten so 'n Griff gekriegt, ne. So (.) dahin oder dahin oder dahin. Also wir wurden richtig dirigiert" (I 29:39-30:15).

Gerade die Wortwahl des ,Dirigierens' und ,Funktionierens' illustriert, dass den Kindern keine Wahloptionen gelassen werden und die Eltern entschlossen und steuernd intervenieren. Den Eltern ist es sehr wichtig, wie das kindliche Verhalten gegenüber Dritten wirkt. Sie hätten viel Wert auf das Urteil des Umfeldes gelegt und hätten die kindlichen Leistungen nach außen präsentiert (I 13:01-13:19). Das hat Iris Gümper als unangenehm in Erinnerung. Die Eltern hätten die Familie „öffentlich gemacht“ (II 31:00-31:04) und die „heile Familie“ (I 31:34-31:37) nach außen präsentieren wollen. Hierdurch entsteht für die Kinder ein starker Druck, sich den Vorgaben der Eltern entsprechend zu verhalten.

Die Rituale, auf die die Eltern viel Wert gelegt hätten, seien auf die Vermittlung der elterlichen Vorstellungen ausgerichtet gewesen. Iris Gümper erinnert sich z.B. an die regelmäßigen Gebete vor den Mahlzeiten, die interessanterweise von ihrer Mutter überhaupt nicht erwähnt werden. Obwohl sie und ihre Geschwister die Gebete gekannt hätten, hätten sie sie nicht aufsagen können, wenn sie von den Eltern dazu aufgefordert worden seien (I 24:03-24:47). Aus dieser Perspektive sind die Rituale nicht an den Wünschen und Bedürfnissen der Kinder ausgerichtet. Mit dem Abfragen entsteht praktisch eine Prüfungssituation, durch die ein Leistungsdruck aufgebaut wird. Die Gestaltung des Weihnachtsfestes ist ebenfalls durch die elterlichen Vorstellungen eines regelmäßigen Ablaufs gekennzeichnet. Sie und ihre Geschwister hätten das als "total stressig“ (I 24:50-24:54) empfunden. Iris Gümper erinnert folgendes Gestaltungsschema: Nach dem Essen

„mussten wir wieder in das andere Zimmer gehen, ins Esszimmer [...] Und mussten warten, bis nun das Glöckchen läutet, bimmel, bimmel, das Christkind war da, und wir durften dann ins Weihnachtszimmer rein. Und dann hatte jeder unter 'm Weihnachtsbaum so 'n Häufchen, wo dann ein weißes Laken drüber gedeckt war und da waren dann die Geschenke drunter. Aber vorher mussten wir bitteschön alle Lieder singen, die Weihnachtsgeschichte hören von vorne bis hinten. ,Es begab sich zu der Zeit, dass ein Gebot vom Kaiser Augustus ausging', kenn ich alles auswendig, //hm// @(.)@ weil wir die dann später aufsagen mussten, immer, kam jeder einmal dran. Und mussten wir alles können“ (I 26:53-27:32).

Der Ablauf des Weihnachtsfestes orientiert sich nicht primär an den kindlichen Bedürfnissen. Vielmehr zeigt sich an der wiederholten Verwendung des Verbes ,müssen die deutlich erkennbare intergenerationelle Machtasymmetrie und der elterliche Zwang, dem die Kinder unterliegen. Auch das Weihnachtsfest dient dazu, den Kindern beizubringen, sich in von außen gesetzte Ordnungsstrukturen einzupassen. Belohnung in Form von Geschenken gibt es erst nach einer erbrachten Leistung. Ein weiterer Verweis auf die Dominanz des Bezugsrahmens der repräsentativen Höchstleistungen, auch 


\subsection{Geschlossene Tradierungslogiken}

wenn die Höchstleistungen in diesem Falle vor dem kleinen Publikum der Familie im Privaten zu erbringen sind.

Das Verhältnis zu den Eltern empfindet Iris Gümper als distanziert. Die Mutter habe sich zwar gut um die Kinder gekümmert, aber es sei stärker eine Versorgung gewesen. Liebevolle Zuneigungsgesten hingegen habe es wenig gegeben (I 02:44-03:48). Der emotionalen Seite der Beziehungen wird aus Sicht von Iris Gümper eher ein marginaler Stellenwert eingeräumt, womit die Erinnerungen von Mutter und Tochter sich in diesem Punkt ähneln.

In klassischer Weise wird der Vater als strafende Instanz eingeführt: „Was die Erziehung angeht, @(.)@, ich sag das ruhig, die war sehr autoritär. Also mit Drohungen, wenn du das und das machst, dann kommt Vater nach Hause und dann gibt's (.), gibt's was, so ungefähr" (I 02:18-02:30). Verständnisvolle Zuwendung und Nachgiebigkeit sind für sie keine dominanten Merkmale der elterlichen Erziehung. Die väterliche Maxime: „Erst kommt der Beruf, dann die Familie“ (I 33:59-34:14) unterstreicht, dass die Bedürfnisse der Familie und damit auch die der Kinder keine oberste Priorität gehabt haben. Iris Gümper erlebt in ihrer Umgebung durchaus andere Arten, die intergenerationellen Beziehungen zu gestalten, was dazu beiträgt, die im Vergleich dazu distanziertere Eltern-Kind-Beziehung im eigenen Elternhaus als Manko zu empfinden. Iris Gümper habe sich zu Hause nicht wohlgefühlt und sei lieber bei Freundinnen gewesen, in deren Elternhäusern es eine liebevolle Zuwendung gegeben habe (II 03:47-04:10). In der Folge zeichnet sich das Verhältnis zu den Eltern nicht unbedingt durch vertrauensvolle Nähe aus. Probleme habe sie eher mit den Geschwistern besprochen und nicht mit den Eltern (II 24:56-25:16, 28:45-29:48).

Im schulischen Kontext erlebt Iris Gümper einen anderen Umgang mit Kindern. In ihrer Schulzeit habe sie von einigen Lehrern starke Unterstützung erhalten, die alles aus ihr „rausholten“ (I 09:46-10:41). Unterstützung, sowohl praktischer als auch emotionaler Art, werden als leistungsfördernde Hilfen eingeführt, die zugleich die Umsetzung des familialen Bezugsrahmens erleichtern. In Bezug auf die elterliche Erziehung hingegen wird diese Form der positiven Bestärkung nicht erwähnt. Sie wird vielmehr als maßregelnd erlebt, was Iris Gümper eher als leistungshemmend wahrnimmt. Ungeachtet dessen habe Iris Gümper jedoch immer darum gekämpft, das zu tun, was ihr Spaß mache (II 33:22-33:47). Freude wird somit von ihr als ein weiteres Kriterium eingeführt, das eine Leistungsorientierung begünstigt.

Entsprechend dieser - zumindest retrospektiv - recht kritischen Betrachtung des elterlichen Verhaltens fallen Iris Gümpers Reaktionen aus. Sie versucht auf verschiedene Weise der mütterlichen Kontrolle zu entgehen. Sie habe z.B. gern im Garten gespielt (I 01:58-02:14), wodurch sie etwas Freiraum erlangen konnte, wenn auch einen kontrollierten. Mit ihren Freundinnen habe sie sich lieber außerhalb des Elternhauses getroffen, weil ihr die vielen Auflagen und Verbote der Mutter unangenehm gewesen seien (I 03:59-04:21).

In der Pubertät habe sie sich vehement gegen die strengen elterlichen Regeln gewehrt (I 13:23-13:32). Selbst Strafen hätten sie nicht davon abgehalten, gegen die el- 


\section{Familiale Tradierungsprozesse in einer Drei-Generationen-Perspektive}

terlichen Vorschriften aufzubegehren. Verstöße, wie abendliche Unpünktlichkeit, seien von den Eltern rigoros geahndet worden, u.a. mit körperlichen Strafen (I 13:21-13:49, 15:15-19:16, 27:34-28:03). ${ }^{172}$ Das unterstreicht, wie sehr den Eltern an der Einhaltung einer strengen Ordnung gelegen ist.

Iris Gümper betrachtet ihre Reaktion auf das Familienleben als Verweigerungshaltung und Ablehnung der strengen Ordnung sowie der Maxime des Strebens nach repräsentativen Höchstleistungen. Ein Grund hierfür ist sicherlich, dass die Leistungen von Iris Gümper eher selten Gegenstand der Anerkennung waren. Darüber hinaus hätten die Eltern ihr einen Bruder als Vorbild präsentiert, der sehr gute Leistungen erbracht habe (I 9:24-09:35). Das verstärkt ihre Ablehnung und sie beschreibt ein ausgeprägtes Konkurrenzverhältnis zu diesem Geschwisterteil (I 05:07-05:12, 30:20-31:20).

Eine weitere Strategie, mit der nahezu unveränderlichen Geschlossenheit der elterlichen Erwartungen umzugehen, besteht darin, sich - zumindest retrospektiv - eine besondere und vor allem aktive Rolle zuzuschreiben. Das gilt sowohl für ihre Kindheit als auch den weiteren Lebensweg. Sie kennzeichnet ihre Reaktionen, sowohl in Bezug auf die elterlichen als auch auf die von ihr im weiteren Lebensverlauf angenommenen gesellschaftlichen Erwartungen, als oftmals abweichend vom vermeintlichen Durchschnitt, sowohl im positiven als auch vordergründig negativen Sinn. Sie sei früher das „schwarze Schaf" (I 04:46-04:52) der Familie gewesen, was sie allerdings mit einer positiven Deutung versieht. Sie habe die Anforderungen nicht eingesehen und nicht befolgen wollen (I 04:38-04:50). Sie sei eine Kämpfernatur und der Maßstab ihres Handelns sei ihr Wohlergehen gewesen. Das bezieht sie ausdrücklich auch auf ihre Kindheit (II 33:26-34:26). Hierdurch möchte sie unterstreichen, der Situation nicht völlig hilflos gegenübergestanden und sich gezielt von den Erwartungen und dem Normierungsdruck der Eltern distanziert zu haben. Es sei ihr z.B. gelungen, ihren Willen durchzusetzen und mit dem Lernen des ersten Musikinstruments aufzuhören. Sie habe auch vorgeschlagen, welches Instrument sie alternativ erlernen könne (I 11:49-12:02).

Diese Verweigerungshaltung gilt aber nicht für alle Bereiche. Dort, wo sie nicht im Schatten eines anderen Geschwisterteils steht, ist sie um herausragende Leistungen bemüht und bringt sich mit sehr viel Energie ein. Ihre Erfolge hierbei thematisiert sie auch sehr deutlich. Iris Gümper bedient sich dabei einer ähnlichen Strategie wie ihrer Mutter. Dort, wo sie den familialen Bezugsrahmen nicht umsetzen kann, greift sie auf Rechtfertigungen und Relativierungen zurück. Darüber hinaus weicht sie auf andere Felder aus, in denen sie nach außen repräsentierbare Bestleistungen erbringen kann. Das gilt z.B. für ihren Musikinstrumentenwechsel. Sie wählt ein Instrument, das keines ihrer Geschwisterteile und auch sonst niemand in ihrem Umfeld spielt, und ist mit diesem sehr erfolgreich (I 11:38-12:37). Ebenso verhält es sich im schulischen Bereich. Zwar ist sie in der Schule nicht sonderlich leistungsstark (09:33-09:41) und verlässt vorzeitig

\footnotetext{
${ }^{172}$ Die von Iris Gümper thematisierten körperlichen Züchtigungsmittel werden von ihrer Mutter, Frau Nolte, nicht angesprochen, was damit zusammenhängen könnte, dass Schläge heute nicht mehr als adäquates Erziehungsmittel betrachtet werden und Frau Nolte deswegen diesen Aspekt auslässt.
} 


\subsection{Geschlossene Tradierungslogiken}

das Gymnasium (I 19:20-19:25), beendet aber dafür ihre Ausbildung mit einem sehr guten Abschluss, womit sie die Eltern habe beeindrucken können (I 23:29-23:57). Sie kritisiert, im sportlichen Bereich von den Eltern an der Entfaltung und öffentlichen Darstellung ihrer Leistungen gehindert worden zu sein (II 52:10-52:24). Das unterstreicht nochmals ihre Orientierung am familialen Bezugsrahmen.

Insgesamt beschreibt Iris Gümper eine rebellische Kindheit. Sie berichtet aber auch, ihr Leben sei - vor allem in Bezug auf die Wahl ihres Ausbildungsplatzes - „irgendwie von zu Hause dirigiert worden." Ihr erster Ausbildungsplatz im sozialen Bereich sei ihr vom Vater vermittelt worden. Auch wenn sie den Ausbildungsplatz wechselt, bleibt sie in diesem Arbeitsbereich, obwohl sie ursprünglich ein anderes Berufsfeld anvisiert habe. Sie sei in ihren Beruf „automatisch gerutscht“ (II 34:32-34:46). Das verdeutlicht, dass die Steuerung durch die Eltern und die eigene Auseinandersetzung mit Regeln und Erziehungsinhalten miteinander verbunden sind und sich auf das Resultat der Erziehung auswirken. Weder gelingt es den Eltern, ihre Vorstellungen ungebrochen durchzusetzen, noch kann sich Iris Gümper den Regeln und Anforderungen des Elternhauses komplett entziehen. Wenn sie z.B. an den familialen Bezugsrahmen anknüpfen kann und Mitspracherechte erhält, bemüht sie sich erfolgreich um Bestleistungen. In diesen Fällen bereitet es Iris Gümper keine Probleme, sich an einer bestimmten Ordnung zu orientieren sowie fleißig und ehrgeizig zu sein, wie das Beispiel des zweiten Musikinstruments zeigt. Ein wesentliches Motiv für ihre Bemühungen ist der Wunsch nach elterlicher Anerkennung und Wertschätzung, was daran ersichtlich wird, dass Iris Gümper die positiven elterlichen Reaktionen auf ihre guten Leistungen stets hervorhebt (I 12:59-13:08, 23:44-23:57, 40:38-40:56, II 31:57-32:02). Ihre Ablehnung bezieht sich folglich in erster Linie auf den unflexiblen Grundsatz, eine strenge Ordnung einzuhalten, der die Umsetzung des familialen Bezugsrahmens flankiert, und weniger auf den Bezugsrahmen selbst.

Am Beispiel von Iris Gümper zeigt sich eindrücklich, dass Erziehung aus einem Wechselspiel von Vermittlung und Aneignung besteht. Wie ihre Schilderungen darüber hinaus belegen, garantiert ein asymmetrisches Machtverhältnis zwischen Eltern und Kindern nicht automatisch ein reibungsloses Einordnen der Kinder in die von den Eltern gesetzten Regeln. Vielmehr tragen die rigoros geschlossenen Erwartungen, mit denen sie konfrontiert wird, sowie die unausgeglichene Machtbalance zu einem massiven Abwehrverhalten Iris Gümpers bei und verhindern die Internalisierung einiger Regeln, wie die strikte Orientierung an einem vorgegebenen Ordnungsmuster. Die ungleiche Machtverteilung befördert Konflikte und das Aufbegehren gegen die elterliche Ordnung. Verstärkend kommt hinzu, dass sie in ihrem Umfeld durch Lehrer und andere Eltern (I 09:46-10:41, II 03:47-04:10) alternative Umgangsweisen mit Kindern erlebt.

Trotz dieser Rebellionen und Bemühungen, sich von den elterlichen Vorstellungen und Wünschen zu distanzieren, gelingt ihr das nicht gänzlich. Die Orientierung an repräsentativen Höchstleistungen bildet trotz der zwiespältigen Kindheitserfahrungen einen wesentlichen Referenzpunkt für Iris Gümpers Ausführungen, auch was ihren weiteren Lebensweg sowie ihre eigene Mutterschaft betrifft. Sie schildert, wie sie im Rahmen 


\section{Familiale Tradierungsprozesse in einer Drei-Generationen-Perspektive}

ihrer beruflichen Tätigkeit durch persönliches Engagement in eine Führungsposition gelangt sei (II 35:40-36:04). Hier zeigt sie ähnlich wie ihre Mutter eine starke Aufstiegsorientierung. Von den beruflichen Werdegängen ihrer Geschwister berichtet Iris Gümper entlang des Kriteriums der erreichten Positionen - ähnlich wie ihre Mutter. Sie präsentiert die geschwisterlichen Leistungen (I 34:54-36:47) und erstellt quasi eine Statusrangfolge, was auf ein immer noch bestehendes Konkurrenzverhältnis hinweist.

Im Gegensatz zu ihrer Mutter setzt sich Iris Gümper aber zugleich kritisch mit dem familialen Bezugsrahmen der repräsentativen Höchstleistungen auseinander und setzt andere Akzente. Für sie sind Bestleistungen dann leicht zu erbringen, wenn sie mit dem Aspekt der Freude verbunden werden. Emotionale Unterstützung und die die Schaffung einer Atmosphäre, in der sich das Individuum wohlfühlen kann, sind für sie weitere fördernd wirkende Faktoren.

Vermittlungsebene Iris Gümper (* 1959): Das optimale Umfeld schaffen Diese Neuausrichtungen schlagen sich in ihrem eigenen Erziehungssetting nieder. Die Auseinandersetzung mit den eigenen Erziehungserfahrungen und die hieraus resultierenden Veränderungen werden dabei zweifelsohne von den pädagogischen Theorien beeinflusst, die Iris Gümper während ihrer Ausbildung kennenlernt. Dennoch orientiert sie sich am familialen Bezugsrahmen der repräsentativen Höchstleistungen, indem sie auf eine möglichst früh einsetzende Talentförderung der Tochter achtet. Sie bemüht sich aber, die leitenden Grundsätze ihrer Erziehung anders als ihre Eltern zu gestalten. Sie lässt ihre Tochter, Lea, an verschiedenen Lern- und Freizeitangeboten teilnehmen, um festzustellen, wo die Begabungen der Tochter liegen. Iris Gümpers Maxime lautet:
„[U]nd man muss den Kindern die Vielfalt des Lebens öffnen und ganz viele Fenster //hmh// zum Sehen geben, was, wo denn die Interessen sind. Ich kann ja nich sagen als Mutter, und das haben ja meine Eltern getan, so das tut dem Kind gut, da hat's Talent und das is es, wenn, wenn das Kind sich nich ausprobieren kann" (I 1:03:57-1:04:12).

Es steht nicht vorab fest, auf welchem Gebiet ihre Tochter Bestleistungen erbringen soll, sondern ist das Resultat einer Suchphase nach individuellen Fähigkeiten. Dem Kind Wahlmöglichkeiten zu gewähren, bedeutet jedoch nicht automatisch, das Kind allein entscheiden zu lassen. Ausschlaggebend für die Sportartwahl ist in diesem Fall z.B. Talent gekoppelt mit dem Expertenrat eines Trainers (I 1:04:46-1:05:04). Der zentrale Unterschied zu dem Vorgehen der eigenen Eltern besteht darin, dass statt willkürlicher Vorgaben die individuellen Fähigkeiten und Sachargumente Grundlage der Entscheidung sind.

Neben der Beachtung der individuellen Fähigkeiten spielen die Wünsche der Tochter eine wichtige Rolle. Es sei z.B. der Wunsch ihrer Tochter gewesen, ein bestimmtes Musikinstrument zu erlernen (I 1:00:26-1:00:34). Als die Musiklehrerin das Instrument habe wechseln wollen, habe Lea das abgelehnt und Iris Gümper habe das akzeptiert 


\subsection{Geschlossene Tradierungslogiken}

(I 1:00:50-1:01:35). Das verweist auf ein weiteres zentrales Merkmal: Freiwilligkeit, die auch in anderen Bereichen, wie den gemeinsamen Festritualen zu Ostern und Weihnachten, maßgeblich ist. Da es in diesen Bereichen große Übereinstimmungen zwischen den Vorstellungen von Tochter und Mutter gibt, dürfte es Iris Gümper an diesen Stellen leicht fallen, der Tochter Wahlmöglichkeiten einzuräumen.

In einigen Bereichen, wie z.B. der Verantwortlichkeit für das eigene Handeln, gibt es wiederum keinen Gestaltungsspielraum, sondern grundsätzliche Regeln. Als die Tochter z.B. versehentlich einen (vermeintlich) kostenpflichtigen Vertrag abgeschlossen habe, sei sie von ihnen gezwungen worden, das Problem selbst zu lösen. Iris Gümper und ihr Ehemann hätten ihr zwar Ratschläge gegeben, aber die Tochter habe diese allein umsetzen müssen. Iris Gümper bilanziert in diesem Zusammenhang: „Da muss sie lernen, da muss sie durch. Also ich denk da auch immer durch, durch, durch mit allen Konsequenzen //hmh// (die es da gibt)“ (I 1:08:51-1:10:30). Hier zeigt sich Iris Gümper ähnlich konsequent wie ihre Mutter. Weitere Beispiele sind die festgesetzten abendlichen Schlafenszeiten während der Kindheit und die grundsätzliche Ablehnung eines eigenen Fernsehers im Zimmer der Tochter. Lea solle mit den Eltern absprechen, was gemeinsam im Fernsehen angeschaut werden könne (II 1:08:03-1:08:22). Hierdurch ist Iris Gümper informiert und behält sich eine gewisse Kontrolle vor. Zugleich fördert das den kommunikativen Austausch sowie die familialen Kontakte.

Iris Gümper übernimmt für ihre Erziehung nicht den eng begrenzten Leistungskanon ihrer Mutter, sondern erweitert das Spektrum. Sie misst der ganzheitlichen Bildung einen hohen Stellenwert zu. Schulische und musische Ausbildung sind zwar wichtig, ihnen sind aber andere Bereiche beigeordnet. Die sportlichen Talente werden früh gefördert (I 1:03:48-1:03:52, 1:04:14-1:04:45), was mit dem familialen Bezugsrahmen der repräsentativen Höchstleistungen harmoniert, da Lea in diesem Bereich in der Folgezeit Bestleistungen erbringt. Zusätzlich legt Iris Gümper großen Wert darauf, ihrer Tochter Kenntnisse im kulturellen Bereich zu vermitteln. Sie habe mit der Tochter Museen, Opern und Theateraufführungen besucht. Wichtig ist ihr in diesem Zusammenhang nicht nur die Rezeption, sondern eine vertiefte Auseinandersetzung mit den einzelnen Bereichen, z.B. durch elterliche Erklärungen und konkrete Anschauung (I 59:27-1:00:25, 1:06:14-1:06:41). Bildung umfasst für Iris Gümper viele Facetten und alle Sinne sollen geschult werden. Sie wird nicht allein der Schule überlassen, sondern auch von ihr als Mutter stark gefördert. Hierbei achtet sie nicht nur auf die kognitiven Fähigkeiten, sondern auch auf künstlerische und soziale Aspekte (I 51:49-52:35; 58:56-59:24). Für Iris Gümper ist z.B. das Selbstbewusstsein der Tochter eine betonenswerte Eigenschaft (I 1:11:28-1:12:05, 1:17:49-1:18:05), die sie durch ihre Unterstützung und Anerkennung fördert. Die Erziehung der Tochter ist für Iris Gümper mit einem starken elterlichen Einsatz und einer intensiven Beschäftigung mit dem Kind verbunden. Im Unterschied zu ihrer Mutter zeigt Iris Gümper eine stärkere Offenheit für neue Erziehungsimpulse und wendet diese auch an. Die von ihr als wichtig etikettierten Zie- 


\section{Familiale Tradierungsprozesse in einer Drei-Generationen-Perspektive}

le und Regeln werden allerdings mit einer ähnlichen Konsequenz verfolgt wie ihrerzeit von Frau Nolte.

Anders als ihre Mutter achtet Iris Gümper jedoch weniger auf eine strenge Ordnung und Kontrolle, sondern legt ihre Schwerpunkte auf Unterstützung, Kommunikation und ein anregendes Umfeld. Sie betrachtet es als ihre mütterliche Aufgabe, sich über die diversen kindbezogenen Angebote zu informieren und ihrem Kind verschiedene Optionen zu unterbreiten. Dies gilt nicht nur für den sportlichen Bereich. Kindergarten, Grundschule und die weiterführende Schule werden sorgfältig ausgewählt (I 54:28-54:54, 55:13-59:24, 1:20:24-1:24:40). Iris Gümper legt bei den jeweiligen Entscheidungen sehr großen Wert auf das pädagogische Konzept und die gesamte Atmosphäre. An der letztlich gewählten Grundschule habe ihr z.B. der liebevolle Umgang mit den Kindern, die Wertschätzung der Kinder sowie die gesamte Atmosphäre, die ein „Urvertrauen“ vermittelt habe, gefallen (II 36:41-37:43). Im Unterschied zur Erziehung durch die eigenen Eltern geht es weniger um das Einpassen in die gegebenen Verhältnisse als vielmehr um das Finden oder Schaffen eines optimalen Umfeldes, in dem sich die Begabungen bestmöglich entfalten können und das Erbringen von hohen Leistungen unterstützt wird. Das erfordert gleichzeitig ein starkes mütterliches Engagement, um einen ausreichenden Überblick über die verschiedenen Angebote zu erhalten.

Zentral ist in diesem Zusammenhang Iris Gümpers Betonung, die Tochter an dem Entscheidungsprozess beteiligt zu haben, indem sie auf die Argumente und Ansichten der Tochter eingegangen sei (I 1:00:50-1:01:35, 1:22:54-1:24:30). Kommunikation ist insgesamt ein wichtiges Merkmal, das die Regelsetzung einrahmt. Absprachen werden gemeinsam getroffen und Regeln erklärt (II 1:05:29-1:05:35). Das Kind soll die Regeln verstehen, was die Akzeptanz der Regeln erleichtern dürfte. Zugleich dient es da$\mathrm{zu}$, das kindliche Urteilsvermögen sowie die Eigenständigkeit zu trainieren, indem die Tochter angehalten wird, sich mit verschiedenen Argumenten auseinanderzusetzen und Optionen abzuwägen. Es entsteht der Eindruck, als seien die Regeln und Absprachen prinzipiell gestaltbar. Das gemeinsame Mittagessen eines Elternteils mit der Tochter (II 1:00:51-1:01:28) schafft ebenfalls regelmäßige Gelegenheiten des kommunikativen Austauschs. Im Unterschied zu den Intentionen der eigenen Eltern geht es Iris Gümper nicht um das Einüben von Pünktlichkeit und festgesetzten Regeln, sondern um das Wohlbefinden der Tochter, die mittags einen Ansprechpartner haben soll.

$\mathrm{Zu}$ den gemeinsamen Gesprächsthemen zählt u.a. die selbst erfahrene Erziehung von Iris Gümper (II 55:28-56:05). Das könnte dazu dienen der Tochter über einen Vergleich die für sie besseren Rahmenbedingungen vor Augen zu führen, die sie nutzen solle. Die sportlichen Leistungen der Tochter bilden ein weiteres Thema. Die Eltern würden ihre Tochter animieren, auf ihre sportlichen Leistungen zu achten und regelmäßig zu trainieren (I 1:13:34-1:13:50). Fleiß ist somit in dieser Familie ein Thema der intergenerationellen Kommunikation. Auch die Regel, erst nach Erledigung der Hausaufgaben fernsehen zu dürfen (II 1:02:17-1:02:34), verweist darauf. Allerdings erscheint Fleiß als Bezugsrahmen in dieser Generation beiläufiger vermittelt zu werden als bei Frau Nolte. 


\subsection{Geschlossene Tradierungslogiken}

Iris Gümper betont zudem, keinen Zwang auszuüben, sondern eher darauf zu achten, wie sie die Lernfreude bei Lea wecken könne, z.B. durch entsprechende Angebote wie die Auswahl eines optimalen Umfeldes. Das Erbringen von Bestleistungen wird in dieser Konstellation weniger mit Anstrengungen assoziiert als bei ihrer Mutter. Vielmehr manifestiert sich hier Iris Gümpers Überzeugung, ein anregendes Umfeld führe selbstverständlicher und müheloser zu Bestleistungen. D.h. auch Iris Gümper greift steuernd ein, aber auf eine gänzlich andere Art als ihre Mutter.

Es gibt auch intergenerationelle Auseinandersetzungen. Iris Gümper habe z.B. versucht, ihre Tochter von dem Entschluss abzubringen, den Musikunterricht aufzugeben (1:02:29-1:03:53). Statt aber direkten $Z$ wang anzuwenden, habe sie sich zunächst bemüht, ein anderes Unterrichtsumfeld für ihre Tochter zu finden (1:02:29- 1:03:08); sie versucht also zu intervenieren. Als dies nicht gelingt, arrangiert sie sich schließlich mit der Entscheidung ihrer Tochter, allerdings nicht ohne das zu rechtfertigen. Die Tochter würde so vielen Aktivitäten nachgehen, dass es schließlich erforderlich geworden sei, die Musik aufzugeben (I 1:03:08-1:03:53). Ähnlich wie für ihre Mutter ist die nicht erfolgreiche Umsetzung des familialen Bezugsrahmen für Iris Gümper rechtfertigungsbedürftig, wozu auf andere Leistungen verwiesen wird.

Generell sei es Iris Gümper wichtig gewesen, Konflikte auszutragen. Spannungen zwischen den Familienmitgliedern sollen thematisiert und kommunikativ aufgearbeitet werden. Die Etablierung dieser Art von Streitkultur soll verhindern, dass Konflikte zugunsten einer vermeintlichen Harmonie verdrängt werden (II 1:10:03- 1:10:45). Trotz aller Unterstützung und Förderung soll die Kindheit der Tochter nicht als realitätsferner, idyllischer Schonraum konzeptualisiert werden. Die Tochter soll sich auch mit den unerfreulichen und traurigen Aspekten des Lebens auseinandersetzen (I 52:26-53:41).

Obwohl Iris Gümpers Kindheitserinnerungen an die in der Familie praktizierten Rituale eher negativ gefärbt sind, empfindet sie einige Rituale bei der Erziehung der Tochter dennoch als wichtig. Nachdem ihre Tochter mit dem Musikunterricht begonnen habe, habe sie erstmals ihre Eltern zu Weihnachten eingeladen und es seien Musikstücke vorgespielt worden. Iris Gümper resümiert:
„Und dann sind Werte (.) wiedergekommen, so Rituale, die wir zu Hause gelernt haben. Da dachte ich auch, //hmh// na ja, aber ich fand es für mein Kind dann auf eINMAL doch wieder wichtig. Aber ich hab immer drauf geachtet, dass sie es nich muss. $/ / \mathrm{hmh} / /$ Und wenn sie nich will, dann kein Zwang" (I 1:02:13-1:02:28).

Iris Gümper betrachtet diese Entwicklung skeptisch und verbindet damit ambivalente Gefühle, u.a. weil sich hier ein Widerspruch zu ihrer zuvor beschriebenen Haltung gegenüber den Erziehungspraktiken der Eltern ergibt, insbesondere was das Vorführen der kindlichen Leistungen betrifft. Um diesen aufzulösen, hebt sie die besondere Akzente bei der konkreten Umsetzung hervor. Sie achtet auf eine besondere Gestaltung des Weihnachtsfestes, wodurch es mit der Aura des Außergewöhnlichen umgeben wird (I 


\section{Familiale Tradierungsprozesse in einer Drei-Generationen-Perspektive}

1:01:40-1:02:12, II 00:01-01:42). Die Leistungspräsentation erfolgt auf freiwilliger Basis und statt starrer Ordnungsstrukturen steht das Wohlbefinden der Familienmitglieder im Zentrum.

Weitere von der Mutter übernommene Rituale sind das Eierfärben, das Backen von Osterzöpfen bzw. Weihnachtskeksen und Pfefferkuchenhäusern zu den entsprechenden Jahreszeiten. Ebenso wie ihre Mutter gestaltet Iris Gümper diese Rituale gemeinsam mit der Tochter, die aktiv beteiligt wird: „Das sind so Rituale, ne, //hmh// von Anfang an. Sobald die ihre Hände bewegen konnte und irgendwas machen konnte, haben wir solche Rituale gemacht“ (I 1:10:31-1:11:07). Unübersehbar stehen diese Rituale für Iris Gümper im Zeichen der optimalen Kindesförderung, indem Lernanreize geschaffen werden, die über den intellektuellen Bereich hinaus reichen. Einem ähnlichen Zweck dienen die gemeinsam mit der Tochter gestalteten Jahreszeitentische (1:06:14-1:06:41). Es wird eine besondere Atmosphäre geschaffen und eine vielfältige Bildung angestrebt. Details erfahren eine besondere Wertschätzung. Ebenso wird die Wahrnehmung für jahreszeitliche Veränderungen geschärft und ein ganzheitliches Lernen unterstützt. Hier zeigt sich abermals, dass Iris Gümper bei der Erziehung ihrer Tochter auf einen vielfältigeren Kanon achtet als ihre Mutter. Durch die diversen Rituale erhält der Alltag eine besondere Ordnungsstruktur, die aber weniger als strenge Ordnung begriffen, sondern als kindbezogen und entwicklungsfördernd aufgefasst wird. Zugleich dient die Kombination der Rituale als Distinktionsmittel gegenüber den eigenen Eltern, indem einige Rituale wie zu Weihnachten anders gehandhabt und neue wie die Jahreszeitentische hinzugefügt werden. Ähnliches gilt für die Abgrenzung von anderen Familien, die über die besondere Betonung der ganzheitlichen Bildung und die Aufmerksamkeit für Details inszeniert wird. Iris Gümpers Erläuterungen, sie habe bewusst einen anderen Umgang mit ihrer Tochter gewählt als andere, vermeintlich elitäre Mütter, dienen ebenfalls als Distinktionsmittel - insbesondere, indem sie herausstellt, wie erfolgreich ihre Art der Erziehung gewesen sei (I 53:09-53:12, 1:11:28-1:11:32).

Schließlich sei noch das abendliche Vorlesen angesprochen. Hierdurch habe Iris Gümper bei ihrer Tochter die Lesefreude wecken wollen. Das habe aber nicht zum erwünschten Ziel geführt (I 1:06:45-1:07:43). Im Unterschied zur eigenen Kindheit versucht Iris Gümper die Lesekompetenz der Tochter durch gemeinsame, positiv zu erlebende Aktivitäten zu fördern. Die Anregung hierzu stamme aus einem Buch, den Iris Gümper aufgrund des geringen Erfolges des Ratschlags kritisiert (I 1:06:49-1:05:56). Gleichzeitig betont sie, dass ihre Tochter zwar nicht gern lese, eine Lehrerin sie aber beruhigt habe, dass manche Kinder sich auch hinter Büchern verstecken würden (I 1:07:22-1:07:27). Ihre Tochter hingegen "geht raus, offen in die Welt $/ / \mathrm{hmh} / /$ und lernt Freundinnen kennen und lernt dies kennen, macht das, probiert das. Warum muss man dann zu Hause sitzen und lesen, //hmh// ne? Also jeder hat da so seins" (I 1:07:33-1:07:43). Hier bedient sich Iris Gümper abermals derselben Strategie wie ihre Mutter. Sie kompensiert ihren vermeintlichen Fehlschlag, indem sie sogleich ein Beispiel für einen Erziehungserfolg präsentiert. 


\subsection{Geschlossene Tradierungslogiken}

Das emotionale Wohlbefinden und die emotionale Unterstützung nehmen einen zentralen Stellenwert im Erziehungssetting ein. Ihr Leitmotiv lautet:

„Und der Meinung war ich immer fr-, als Lea klein war, heute noch, man muss'n Kind auf' $m$ Schoß haben, Blick nach vorne, in die Welt, so is das Leben, $/ / \mathrm{hmh} / /$ ihr aber immer Geborgenheit und Sicherheit schenken, (.) dass sie weiß, man hält zu ihr, man unterstützt sie, man beschützt sie, aber sie muss die ganzen Risiken und Gefahren in ihrem Leben kennenlernen“ (I 53:18-53:38).

In dieser Äußerung manifestiert sich das Bemühen, Rückhalt und emotionale Unterstützung zu gewähren, ohne in eine überbehütende Haltung abzugleiten. Zuwendung und Unterstützung sollen nicht verhindern, dass das Kind seine eigenen Erfahrungen sammelt und Eigenständigkeit erlernt. Das Austarieren dieser beiden Pole schlägt sich in der Erziehungspraxis nieder. Der Tochter Wahlmöglichkeiten anzubieten, sie in Entscheidungsprozesse einzubinden und auf die Gestaltung des Erziehungsumfeldes zu achten, dienen u.a. dem Zweck, das emotionale Wohlbefinden der Tochter zu fördern. Ein weiteres Beispiel ist die konsequente Unterstützung der Tochter, als sie eines Fehlverhaltens beschuldigt wird. Das Vertrauen, das Iris Gümper ihrer Tochter hier entgegenbringt, bietet dieser emotionalen Rückhalt, um so mehr, als die Beschuldigungen sich später als falsch erwiesen hätten (I 1:14:20-1:17:45). Die Tatsache, dass Iris Gümper voller Stolz von den Leistungen ihrer Tochter im sportlichen Bereich berichtet sowie ihre sozialen Fähigkeiten und ihr Urteilsvermögen hervorhebt (I 1:11:28-1:14:05), lässt sich ebenfalls als Bestärkung und Unterstützung der Tochter begreifen. Hierbei scheint Iris Gümper selbst gar nicht zu realisieren, wie stark sie auf den familialen Bezugsrahmen der repräsentativen Höchstleistungen abhebt. Auch die Etablierung fester Mutter-Tochter-Zeiten in der Vergangenheit, die trotz der berufsbedingten nachmittäglichen Abwesenheit der Mutter den intergenerationellen Austausch hätten gewährleisten sollen, dienen dazu, der Tochter ein Gefühl der Wertschätzung zu vermitteln. Zugleich habe es aber auch festgelegte Zeiten gegeben, die für die mütterlichen Eigeninteressen reserviert gewesen seien (II 1:00:12-1:00:30). Iris Gümper ist auch darauf bedacht, die Tochter nicht zu sehr an sich zu binden:

„Ich habe von Anfang an gesagt: Wenn ich mein Kind von Anfang an nich weggebe, hab ich irgendwann so ein Kind am Rockzipfel sitzen. Das is nur, ich bin die Henne und das Kind kriege ich nich los, es klammert so. Und dann haben wir das schon, als wir umgezogen sind, also Lea war noch nicht 'n Vierteljahr, haben wir sie schon zu Freunden weggeben zum Schlafen, woanders schlafen“ (I 48:40-48:59).

Standen Lea und ihr emotionales Wohlbefinden in den vorherigen Ausführungen im Mittelpunkt, geht es nun stärker um die eigene Person. Iris Gümper möchte sich nicht 


\section{Familiale Tradierungsprozesse in einer Drei-Generationen-Perspektive}

nur über ihre Mutterschaft definieren und achtet auf ihre eigenen Bedürfnisse. Im Mittelpunkt stehen das Abwägen von Nähe und Distanz sowie der verschiedenen Bedürfnisse. Für die Tochter spielt das ebenfalls eine Rolle, wie das Anbringen einer Spielzeugalarmanlage an ihrer Kindertür zeigt (II 1:10:50-1:11:05). Die Tochter ist bemüht, ihre Bedürfnisse zu verteidigen und etwas Distanz einzufordern.

Iris Gümper erweitert in Auseinandersetzung mit ihren eigenen ambivalenten Kindheitserfahrungen den familialen Bezugsrahmen der repräsentativen Höchstleistungen, ohne ihn aufzugeben. Sie verändert vielmehr ihr Erziehungsarrangement, um die aus ihrer Sicht optimalen Voraussetzungen für die Weitergabe eben dieses Bezugsrahmens zu schaffen. Die Verwendung von Erziehungsbestandteilen, die gemeinhin als liberal gelten dürften, sollte nicht darüber hinwegtäuschen, dass der für die Tochter entworfene Erwartungshorizont eine hohe Geschlossenheit aufweist, indem er deutlich konturiert und artikuliert an sie herangetragen wird.

\section{Aneignungsebene Lea Gümper $\left({ }^{*} 1992\right)^{173}$ : Die Nutzung der bereitgestellten}

Ressourcen Eine der wesentlichsten Erziehungserfahrungen ist für Lea die elterliche Unterstützung, die sowohl praktischer, organisatorischer, finanzieller als auch emotionaler Art ist (04:49-06:21). Die Eltern ermöglichen ihr die Teilnahme an außerschulischen Bildungs- und Freizeitangeboten, finanzieren ein Auslandsjahr und fahren sie zu Wettkämpfen. Mit Problemen könne sie sich jederzeit an sie wenden. Lea schätzt den Rat der Mutter, die während der als schwierig empfundenen Anfangsphase ihres Auslandsaufenthalts eine wichtige Ansprechpartnerin für sie gewesen sei (07:30-07:50, 08:32-09:11). Letzteres unterstreicht, welch hohen Stellenwert der kommunikative Austausch als Unterstützungsleistung einnimmt. Die Eltern würden sich für ihre sportlichen Leistungen interessieren, seien wegen der eingeschränkte Trainingsmöglichkeiten während des Auslandsjahres besorgt gewesen und hätten sie ermuntert, sportliche Tiefphasen zu überwinden (10:01-10:21, 1:13:53-1:14:57). Das illustriert nachdrücklich, wie sehr die Eltern auf Leas Leistungsniveau achten und sie ermuntern, sich am Bezugsrahmen der repräsentativen Höchstleistungen zu orientieren. Hierbei erfolgt früh eine Lenkung durch die Eltern, indem sie Lea zur sportlichen Frühförderung in dem Sektor schicken, in dem sie heute erfolgreich ist. Lea davon zu erzählen (16:34-17:00), könnte als Motivationsanreiz sowie als Beleg sich selbst wie der Tochter gegenüber dienen, richtig gehandelt zu haben. Lea sieht hierin allerdings weniger eine Lenkung als vielmehr eine Förderung ihrer Talente. Die Eltern, insbesondere die Mutter, hätten ihr in diesem Zusammenhang auch von der eigenen Kindheit berichtet, in der das sportliche Leistungspotenzial von den Eltern nicht gefördert worden sei. Leas Schlussfolgerung lautet, die Eltern wüssten, wie es sich anfühle, wenn man nicht das machen könne, was man gerne möchte (26:25-27:01). Da ihr selbst die Möglichkeit geboten wird, ihr sportliches Talent zu entfalten, lässt sich ihr diesbezügliches Engagement quasi als Erfüllung eines elterlichen Auftrages interpretieren, wobei Lea es als ihren Entschluss ansieht,

${ }^{173}$ Das Interview mit Lea Gümper wurde am 24.09.2008 geführt. 


\subsection{Geschlossene Tradierungslogiken}

im Sportsektor nach repräsentativen Höchstleistungen zu streben. Sie betrachtet es als Chance, die sie nutzen möchte. Hiermit fügt sie der Leistungsorientierung eine neue Facette hinzu.

Auch wenn der Bezugsrahmen im Interview mit Lea keine so zentrale Rolle spielt wie bei ihrer Mutter und Großmutter, ist dennoch deutlich zu erkennen, dass Lea ihn übernimmt. Sie habe immer den Ansporn gehabt, die Beste sein zu wollen (26:05-26:20). In ihrer Sportmannschaft habe sie inzwischen eine Art Vorbildfunktion, weil sie so ehrgeizig sei. Im Rahmen dieser Funktion habe sie für eine gewisse Disziplin in der Mannschaft gesorgt und aktuell sei es ihr besonders wichtig, nach ihrem Auslandsjahr wieder ihr altes Leistungsniveau zu erreichen (29:28-31:38). Im schulischen Bereich hingegen gelingt es ihr nicht so gut, an den familialen Bezugsrahmen anzuknüpfen. Das führt sie u.a. auf die hohe zeitliche Beanspruchung durch den Leistungssport zurück. Zudem seien in einigen Kursen sehr viele herausragende Schüler, sodass sie es schwer habe, sich gut zu positionieren (32:06-33:00). Sie bedient sich hier einer ähnlichen Strategie wie Mutter und Großmutter, indem sie äußere Umstände anführt, die die Entfaltung ihres Leistungspotenzials behindern würden.

Lea betrachtet ihre Eltern als Vorbilder (1:27:12-1:27:16), die das, was sie ihr vermitteln wollten, auch selber vorlebten und ihr hierdurch eine Orientierung gäben, wie z.B. das Streben nach repräsentativen Höchstleistungen. Das dürfte ein wichtiger Grund dafür sein, dass Lea die elterliche Erziehung im Allgemeinen akzeptieren und den familialen Bezugsrahmen annehmen kann.

Die Machtbalance zwischen den Generationen wird als deutlich ausgeglichener als in der Vorgängergeneration beschrieben. Entscheidungen werden nicht einfach getroffen und Regeln gesetzt, sondern erklärt. Gespräche und Diskussionen sind Teil der Erziehung. Wenn die Mutter mit ihrem Verhalten nicht einverstanden gewesen sei, habe sie Lea die Gründe hierfür dargelegt. Zusätzlich habe die Mutter in der Vergangenheit manchmal mit Belohnungsanreizen gearbeitet, um ihr Ziel zu erreichen, was Lea akzeptabel findet (22:50-24:14).

Einzig in Bezug auf den Musikunterricht und die abendlichen Ausgehzeiten habe es Konflikte gegeben. Der Musikunterricht sei besonders vom Großvater unterstützt worden, das Üben ihres Musikinstruments habe sie aber manchmal eher als Zwang empfunden. Vor allem weil sie oft keine Lust gehabt habe, allein in ihrem Zimmer zu üben (1:15:27-1:16:40). Den Musikunterricht aufzugeben, sei nicht ganz so einfach durchzusetzen gewesen. Ihre Mutter habe nicht so schnell wie ihr Vater eingesehen, dass Lea aufgrund ihrer hohen Arbeitsbelastung den Musikunterricht aufgeben wolle (1:29:34-1:29:58). Aus Leas Perspektive fällt es der Mutter hier schwer, sich an die eigene Maxime zu halten und ihrer Tochter Wahlmöglichkeiten zu lassen. Schließlich habe Lea aber ihren Wunsch durchsetzen können. Die abendlichen Ausgehzeiten sind aktuell immer mal wieder Diskussionspunkte, wobei die Eltern ihr keine strengen Grenzen setzten und Lea sich auch hier durchsetzen könne (1:22:05-1:24:08). 


\section{Familiale Tradierungsprozesse in einer Drei-Generationen-Perspektive}

Die existierenden Regeln geben Lea wenig Anlass zur Kritik. Die meisten Regeln sind für sie nachvollziehbar und werden von ihr nicht als einengend, sondern eher als hilfreich erlebt:
„Na ja und dann muss ich noch so dazu sagen, dass ich ein sehr tempera- mentvoller Mensch bin, //hmh//@also dass@, zum Beispiel früher, ähm hat meine Mama mich auch oft [...] ins Zimmer geschickt, wenn ich halt sozusagen überreagiert habe. Und [...] was jetzt meine Methode war, ich hab mich auf den Fußboden gelegt und mit den ähm sozusagen Händen auf den Boden getrommelt, damit da dann halt meine ganze Wut rausging. Und das hat meine Mama mir auch gesagt, dass ich das dann machen sollte. Damit ich dann halt nich-, damit ich meine Wut dann an irgendwas aus- lasse. //hmh// Ähm na ja und das hat dann meistens auch geklappt. Und dann bin ich halt wieder sozusagen als normaler Mensch, sag ich jetzt mal, wieder rausgekommen. Und dann war auch mit mir alles in Ordnung. Also das war halt immer 'ne gute erzieherische Maßnahme" (02:17-03:04).

Regeln werden eher als am Kind ausgerichtete Ratschläge wahrgenommen, die helfen, konflikthafte Situationen zu bewältigen. Lea schreibt ihrer Mutter aufgrund ihres beruflichen Hintergrunds eine hohe Erziehungskompetenz zu (03:05-03:18), was die Annahme der elterlichen Regeln zusätzlich stark unterstützt. Es verhindert, die Maßnahmen der Mutter als willkürlich, ungerecht oder anmaßend zu empfinden. Sie werden im Gegenteil als vernünftig, richtig und annehmbar etikettiert.

Ein wesentliches Kennzeichen des Alltags ist seine zeitliche Verregelung durch eine Vielzahl an Terminen (06:32-06:39, 11:02-11:04). Es sind weniger die Eltern, die hier Ansprüche stellen, als vielmehr die Anforderungen durch den Sport. Da Lea der Sport sehr wichtig ist, sie also einen starken Eigenantrieb hat, wird die Terminfülle als unumgänglich akzeptiert und es gibt an dieser Stelle keine Reibungspunkte mit den Eltern. Generell betrachtet Lea die Umsetzung des familialen Bezugsrahmens der repräsentativen Höchstleistungen als stark eigenmotiviert, sodass kaum Regeln von außen gesetzt werden müssen.

Die Frage nach eigenen Wahlfreiheiten betrachtet Lea differenzierter als ihre Mutter. Früher habe sie wenig allein entscheiden dürfen. Die Entscheidungsspielräume seien erst im Laufe der Zeit gewachsen, und inzwischen dürfe sie selbst entscheiden (1:29:091:29:33). Lea beschreibt somit eine Praxis, in der die Spielräume allmählich zunehmen und die Eigenständigkeit schrittweise eingeübt wird, was die Gefahr minimiert, das Kind zu überfordern. Interessant ist, dass Lea Entscheidungen, wie z.B. den Wechsel des Sportvereins, als ihren Entschluss darstellt (04:59-05:04), was aber zugleich im Einklang mit den elterlichen Wünschen steht. Möglicherweise stellt das ein Muster dar, indem eine etwaige Lenkung durch die Eltern durch die Betonung der eigenen Wahl überblendet wird, was wiederum die Akzeptanz der getroffenen Entscheidung erklärt.

In Zusammenhang mit den Entscheidungsspielräumen hätten die Eltern ihr früh beigebracht, dass das „Lustige sozusagen [nicht immer, C.R.] das Beste“ sei (1:29:17 


\subsection{Geschlossene Tradierungslogiken}

-1:29:21), was als ein Appell an die Vernunft zu lesen ist. Die Eltern bemühen sich, der Tochter zu vermitteln, dass eine Zurückstellung aktueller Bedürfnisse längerfristig vorteilhaft sein kann, was Lea anscheinend akzeptiert; zumindest gibt es keinen Hinweis des Aufbegehrens. Zugleich wird hierdurch ein anderer Blickwinkel auf Iris Gümpers Maxime, Leistungsorientierung mit Freude zu koppeln, erkennbar. Aus Leas Perspektive wird $\operatorname{im} Z$ weifelsfall der Leistungsorientierung Vorrang eingeräumt.

In einigen Bereichen gibt es keine Verhandlungsspielräume. So seien z.B. Wutausbrüche Leas, wenn sie beim Spielen verloren habe, nicht hingenommen worden, bestimmte Spiele seien nicht gespielt worden und zu Silvester habe es kein Feuerwerk gegeben (22:50-24:14, 15:26-15:34, 1:09:11-1:09:22). Das Verhalten der Eltern zeichnet sich demzufolge bei grundsätzlichen Einstellungen als konsequent aus. Zwar erklären sie Lea ihre Beweggründe, ihre Entscheidungen sind in diesen Fällen aber unumstößlich. Da Lea dies nicht kritisiert, ist das für sie offenbar akzeptabel. Generell betrachtet Lea das elterliche Verhalten als konsequent. So müsse sie z.B. die Folgen tragen, wenn sie gegen Regeln verstoße (1:33:50-1:34:12). Das beurteilt Lea ebenso positiv wie den Umstand, dass die Eltern ihr sagen würden, „wo es langgeht“ (1:27:17-1:27:20). Zudem behalten sich die Eltern ein gewisses $\mathrm{Ma}$ an Kontrolle vor, indem sie z.B. über die Freundschaften Leas informiert sein möchten (1:25:50-1:26:15), was Lea aber nicht stört.

Diese Beispiele stehen auf den ersten Blick im Widerspruch zu den vor allem von der Mutter aber auch von Lea angesprochenen Wahlfreiheiten. Für Lea selbst ergeben sich jedoch keine Widersprüche, da auch diese feststehenden Regeln von ihr nicht als einengend empfunden werden, sondern ihr im Gegenteil eine Orientierung ermöglichen. Auf einer übergeordneten Ebene geht es um die Balance zwischen Wahloptionen bei gleichzeitiger Orientierungshilfe. Hieraus ergibt sich ein differenzierter Blick auf die Frage der Wahlfreiheiten, und es zeigt sich eine elterliche Steuerung im Bereich zentraler Grundsätze und Erwartungen, die nicht verhandelbar sind.

Im Allgemeinen werden aber Regeln weniger als von außen auferlegte Pflichten empfunden, sondern eher positiv aufgefasst, da sie zu Leas Gunsten zugeschnitten sind. Beispiele hierfür sind die Regel, eine Freundin mit in den Urlaub nehmen zu dürfen, die Anwesenheit des Vaters, wenn sie früher mittags nach Hause gekommen ist, die Hilfe bei den Hausaufgaben durch den Vater oder die Wochenenden als „großes Familiending“, an denen die Mutter gekocht habe (01:18-01:50, 11:11-11:36). Über die Regeln werden nicht einseitig Forderungen an Lea gestellt; sie sind im Gegenteil mit Anforderungen an die Eltern verbunden.

Auch Lea spricht verschiedene Rituale oder Traditionen an, die ihr wichtig sind. Hierzu zählen u.a. die österlichen und weihnachtlichen Rituale sowie die Gestaltung der Geburtstage (13:01-13:14, 15:38-16:14). Sie hebt die besondere Ausgestaltung des Tages hervor, das Schaffen einer außeralltäglichen Atmosphäre. Diese Tage stellen kleine Höhepunkte dar, die den Jahreslauf strukturieren. Aus diesem Grund werden die Rituale auch positiv erinnert. Zudem steht nicht das Einhalten einer strengen Ordnung im Zentrum, sondern die besondere Atmosphäre. Zu Weihnachten führt sie für die Groß- 


\section{Familiale Tradierungsprozesse in einer Drei-Generationen-Perspektive}

eltern Musikstücke auf (13:55-14:23, 17:26-17:36). Das sind für Lea Gelegenheiten, ihr Können zu demonstrieren, wodurch dieses Ritual für sie positiv besetzt ist. Generell betrachtet Lea die Präsentation ihrer Leistungen als eigenmotiviert, sodass hier kein Konfliktpotenzial entsteht. Für Lea ist der Kontakt zu ihren Großeltern mütterlicherseits wichtig, die sie früher regelmäßig besucht habe. Heute sei der Kontakt aufgrund der eigenen vielfältigen Verpflichtungen sporadischer (58:03-59:51). Lea übernimmt offenbar nicht die ambivalente Sicht auf die Großeltern von ihrer Mutter. Es sind keine kritischen Distanzierungen erkennbar.

Familienfeiern nehmen für Lea einen hohen Stellenwert ein (14:25-14:41), worin sich ihre Einstellung von der der Mutter unterscheidet. Ging es der Mutter in der Vergangenheit eher darum, sich abzugrenzen und aus der Familiengemeinschaft zu lösen, steht für Lea die Einbindung in und Teilhabe an eben dieser Gemeinschaft im Zentrum. Weihnachten ist für sie z.B. ausschließlich „Familienzeit“ (13:01-13:14). Das abendliche Vorlesen und die „Mutter-Tochter-Zeiten“ sind Zeiten, in denen die Mutter, die tagsüber nicht zu Hause ist, ihre Aufmerksamkeit ganz auf ihre Tochter konzentrieren kann, was von Lea sehr geschätzt wird. Beim Vorlesen sei Lea im Laufe der Zeit stärker gefordert worden, indem auch sie vorgelesen habe, was einen positiven Effekt auf ihre Lesefähigkeiten gehabt habe (03:40-04:30). Sie verbindet mit diesem Ritual also zusätzlich einen spielerisch umgesetzten Lerneffekt. Nach der „Mutter-Tochter-Zeit“ habe die Zeit begonnen, die die Mutter für sich nutzen konnte. Hierüber lernt Lea, die Bedürfnisse anderer zu respektieren. Die gemeinsamen Urlaube stehen im Zeichen der Entspannung und werden von Lea allgemein als positiv erlebt (55:07-55:16). Die Betonung der Entspannung ist sicherlich auch dem Umstand geschuldet, dass Leas Alltag durch viele Anforderungen und Termine geprägt ist.

Die Beziehungsebene ist für Lea ein wichtiges Thema, das sie gleich zu Beginn des Interviews anspricht. Sie habe ein gutes Verhältnis zu den Eltern und die Eltern hätten in ihrer Kindheit viel Zeit mit ihr verbracht bzw. Zeitfenster für sie reserviert (03:36-03:40, 00:46-01:57). Lea sieht sich im Zentrum der elterlichen Aufmerksamkeit und bilanziert das als positiv.

Insgesamt wird ein recht nahes Verhältnis zwischen Lea und ihren Eltern erkennbar. Das wird durch den kommunikativen Austausch sowie das Achten auf gemeinsame Familienzeiten gefördert. Lea beurteilt das Verhältnis von Nähe und Distanz zwischen ihr und ihren Eltern differenziert auf zwei Ebenen. Zum einen stellt sie ihren Status als Einzelkind ins Zentrum der Betrachtung. Da sie keine Geschwister habe, hätten ihre Eltern in ihrer Kindheit viel Zeit mit ihr verbracht (1:33:17-1:33:45). Aus diesem Blickwinkel gleichen die Eltern ein Defizit aus und Lea profitiert von diesem Arrangement der Nähe. Ihre Feststellung, sie habe früh gelernt, dass die Eltern nicht immer für sie da sein könnten (12:28-13:00), verweist auf das Bemühen, eine Balance zwischen Nähe und Distanz herzustellen, das von den Eltern ausgeht und für Lea einsichtig ist. Das ist mit dem Ziel verbunden, Lea zum eigenständigen Handeln zu befähigen. Die Mitgliedschaft im Sportverein sichert den Kontakt zu Gleichaltrigen, was ebenfalls dazu beiträgt, die Eltern-Kind-Beziehung nicht zu eng zu gestalten. Das wird auch von Lea 


\subsection{Geschlossene Tradierungslogiken}

so gesehen, und die Freundschaften mit anderen Vereinsmitgliedern haben für sie eine hohe Bedeutung. Zum anderen ist Lea zwar insgesamt mit der elterlichen Erziehung zufrieden, sie empfindet aber manchmal die Aufmerksamkeit der Mutter ihr gegenüber als zu intensiv. Lea sei immer unterstützt worden, auch wenn sie keine Hilfe gewollt habe und die Mutter sorge sich manchmal zu sehr um ihr Wohlergehen (1:18:01-1:18:10, 1:36:05-1:36:15). Hier ist es Lea, die um etwas Distanz in den Familienbeziehungen bemüht ist, um sich eigene Gestaltungsspielräume zu schaffen.

Zusammenfassende Betrachtung des familialen Bezugsrahmens Auch wenn es im Generationenverlauf unterschiedliche Akzentuierungen gibt, bleibt der familiale Bezugsrahmen der repräsentativen Höchstleistungen gut erkennbar. Veränderungen beziehen sich vor allem auf den Hintergrund, der den familialen Bezugsrahmen einfasst. Während für Frau Nolte der familiale Bezugsrahmen schon fast einen verpflichtenden Charakter aufweist, benennt Iris Gümper Bedingungen, die eine Umsetzung des familialen Bezugsrahmen erleichtern: Unterstützung durch die ältere Generation und die Freude der Jüngeren. Lea betrachtet ähnlich wie ihre Mutter Unterstützung als wichtigen Faktor, der die Umsetzung des familialen Bezugsrahmens begünstigt. Im Unterschied $\mathrm{zu}$ ihrer Mutter stellt für Lea die Leistungsorientierung weniger eine Belastung als vielmehr eine Chance dar. Die Umarbeitung der den familialen Bezugsrahmen begleitenden Vorstellungen fällt zwischen ältester und mittlerer Generation wesentlich stärker aus als zwischen mittlerer und jüngster Generation, wo sich eher sanfte Modifikationen vollziehen. Hiermit einhergehend ist das Konfliktpotenzial zwischen ältester und mittlerer Generation wesentlich höher als zwischen der mittleren und jüngsten Generation. Die Umarbeitungen beeinflussen dabei die konkreten Erziehungspraxen.

\subsubsection{Einordnung der Tradierungslogik unter Berücksichtigung der gesellschaftlichen Bezüge}

Die Erziehungssettings der Familien Nolte/Gümper und Mahler/Ahrens weisen in den einzelnen Generationen diverse Gemeinsamkeiten auf. Die Erziehungsarrangements, die Frau Nolte und Frau Mahler - als Vertreterinnen der ältesten Generation - für ihre Kindheit beschreiben, sind aus dem Blickwinkel der Erziehungsstilforschung (Baumrind 1989 und 1991) als autoritär zu klassifizieren. Die Erwartung von Gehorsam sowie die Orientierung an einer strengen Ordnung bilden in beiden Familien die Grundmuster der Erziehung, an die sie bei der Erziehung der eigenen Kinder bruchlos anschließen.

Ihre Töchter, Michelle Ahrens und Iris Gümper, setzen sich äußerst kritisch mit der elterlichen Erziehung auseinander und verändern entsprechend ihre eigenen Erziehungsarrangements. Der bedingungslose Gehorsam hat ausgedient und den Kindern werden Freiräume zugestanden. In beiden Familien existieren Regeln, die in der Familie Gümper zudem nicht einfach gesetzt, sondern erklärt werden. Einmal etabliert sind die Regeln allerdings verbindlich. Die Kindzentrierung nimmt zu, und dem kommunikativen Austausch zwischen den Generationen wird vermehrt Aufmerksamkeit gewidmet. 


\section{Familiale Tradierungsprozesse in einer Drei-Generationen-Perspektive}

Das Erziehungssetting von Iris Gümper weist in vielen Bereichen Züge auf, die sich sehr viel deutlicher als bei Michelle Ahrens als autoritativ (Baumrind 1991) bezeichnen lassen.

Mit den Veränderungen in den Erziehungssettings sind nahezu unvermeidlich Veränderungen der intergenerationellen Machtbalancen verbunden. Während die Kindheiten der ältesten und mittleren Generation eindeutig durch asymmetrisch konzipierte intergenerationelle Machtverhältnisse im Sinne eines Befehlshaushalts (Bois-Reymond 1994) geprägt sind, ändert sich dies bei der Erziehung der jüngsten Generation. Die Beziehungen zwischen Sonja bzw. Lea und ihren Eltern sind symmetrischer konzeptionalisiert und ihnen werden Mitspracherechte zugestanden. In der Familie Ahrens werden Sonjas Entschlüsse aber unterschwellig unterlaufen, sobald ihre Entschlüsse nicht mit den elterlichen Einstellungen im Einklang stehen. Unabhängig von einer etwaigen Lenkung durch die Mutter betrachtet Lea wiederum die Entscheidungsfindung i.d.R. als partnerschaftlich. Michelle Ahrens bemüht sich, Nähe zu ihren Kindern über einen kommunikativen Austausch herzustellen, allerdings gelingt ihr das aus Sicht ihrer Tochter nicht immer optimal. In der Familie Gümper wird das Familienklima von Mutter und Tochter als unterstützend und durch Nähe geprägt beschrieben. Die Erziehungssettings in beiden Familien weisen zentrale Elemente eines Verhandlungshaushalts (Bois-Reymond 1994) auf, wobei diese Merkmale in der Familie Gümper insgesamt stärker ausgesprägt sind.

Durch die Heranziehung der Erziehungstypologien Baumrinds (1991) und Bois-Reymonds (1994) lassen sich einige wichtige Elemente der jeweiligen Erziehungsarrangements herausarbeiten, und es entsteht der Eindruck eines grundlegenden Wandels. Allerdings bleiben weitere Merkmale unberücksichtigt, die ebenso zentral sind. Eine Verlagerung des Blickwinkels, der die Bezugsrahmen sowie die Art, wie diese an die nächste Generation herangetragen werden, einschließt, fördert demgegenüber erstaunliche Kontinuitäten zu Tage. Unter Rückgriff auf Kosellecks Termini von Erfahrungsraum und Erwartungshorizont (1989, 349-359) zeigt sich, dass die älteste Generation aus dem Erfahrungsraum der eigenen Kindheit einen ebenso gearteten Erwartungshorizont für die nächste Generation ableitet, während die mittlere Generation aufgrund des eigenen Erfahrungsraums in Abgrenzung zu den Eltern zwar die Erwartungshorizonte für die nachfolgende Generation modifiziert, diese dann aber mit einer ähnlich konsequenten Erwartungshaltung wie die eigenen Eltern an die nächste Generation heranträgt. Michelle Ahrens und Iris Gümper verändern viele Elemente des Erziehungssettings und arbeiten den familialen Bezugsrahmen um bzw. versehen ihn mit neuen Akzenten. Anschließend richten sie ihr Bestreben darauf, den einmal etablierten Bezugsrahmen in der von ihnen entwickelten Ausprägung an die nächste Generation weiterzureichen. In Anlehnung an Olsons u.a. Typologie (1989, 47-51) lässt sich Geschlossenheit als wesentliches Charakteristikum dieser Tradierungslogik herausarbeiten. Im Vergleich zur flexibel erscheinenden Tradierungslogik sind die einzelnen Erziehungselemente in der mittleren Generation sehr viel direkter und konsequenter darauf angelegt, den jeweiligen familialen Bezugsrahmen an die nächste Generation weiterzugeben. 


\subsection{Geschlossene Tradierungslogiken}

Als Erziehende versuchen sowohl die älteste als auch die mittlere Generation, ihre Vorstellungen bruchlos auf die die nächste Generation zu übertragen. Sie bedienen sich aber unterschiedlicher Mittel und haben jeweils andere Grenzen und Vorstellungen. Frau Mahler und Frau Nolte folgen bei der Erziehung ihrer Kinder den ihnen bekannten Bahnen, von denen sie nicht abweichen. An die Kinder werden Forderungen herangetragen, die Frau Mahler und Frau Nolte aus ihrer Kindheit vertraut sind, und sie betrachten den kindlichen Gehorsam quasi als natürliches Element der intergenerationellen Beziehungen. Die hierbei entstehenden Konflikte, die ihre Kinder ansprechen, werden von ihnen jeweils dethematisiert.

Auch im Erziehungssetting von Michelle Ahrens spielen Forderungen und Ansprüche an die Kinder eine wichtige Rolle. Diese werden in Auseinandersetzung mit den eigenen Kindheitserfahrungen entwickelt und sind genau konturiert. Allerdings verzichtet sie auf eine autoritäre Durchsetzung ihrer Vorstellungen und fordert keinen unbedingten Gehorsam. Gleichwohl versucht Michelle Ahrens ihre Ziele konsequent durchzusetzen. Statt auf körperliche Strafen setzt sie auf den Ausdruck ihrer Missbilligung und ggf. auf das Ignorieren der töchterlichen Wünsche. Im positiven Sinne setzt sie zudem Anreize in Form von Angeboten, um ihre Kinder zu einer Übernahme der von ihr gewünschten Orientierungen zu bewegen. Weder Frau Mahler noch Michelle Ahrens haben Diskussionen mit den Kindern zentral in ihren Erziehungssettings verankert. Das ist nachvollziehbar, da intergenerationelle Aushandlungsprozesse dazu beitragen könnten, ihre festgeschriebenen Ziele und Vorstellungen aufzuweichen.

Iris Gümper verfügt ebenfalls über eine genau konturierte Erwartungshaltung gegenüber ihrer Tochter. Hierzu setzt sie konsequenter als Michelle Ahrens darauf, ihrer Tochter Angebote zu unterbreiten. Um den familialen Bezugsrahmen der repräsentativen Höchstleistungen zu vermitteln, achtet sie insbesondere auf die Etablierung eines unterstützenden und anregenden Umfeldes, in dem sich ihre Tochter wohl und angenommen fühlen kann. Eine Atmosphäre der Nähe, das Schaffen optimaler Förderbedingungen, Erklärungen und Diskussionen bilden die Eckpfeiler ihrer Erziehung. Allerdings gibt es auch feste Regeln, deren Einhaltung verlangt wird. Die Wünsche der Tochter werden i.d.R. nicht ignoriert. Wenn sie allerdings den Zielen von Iris Gümper widersprechen, versucht sie die töchterlichen Wünsche durch Diskussionen zu kanalisieren. Die Orientierung am familialen Bezugsrahmen wird von Iris Gümper in der Gesamtschau ähnlich energisch und kompromisslos verfolgt wie von ihrer Mutter, allerdings mit gänzlich anderen Mitteln.

Als jeweils aneignende Generation nehmen Frau Mahler, Frau Nolte, Michelle Ahrens und Iris Gümper die an sie herangetragenen Erwartungen sowie die Vermittlungssettings als geschlossen wahr. Für die älteste Generation ist das unproblematisch; sie fügen sich in die geforderten Ordnungsstrukturen ein und übernehmen sie. In der nachfolgenden Generation ändert sich das.

Michelle Ahrens Kindheitserfahrungen stehen im Widerspruch zum mütterlichen Bezugsrahmen des idyllischen, harmonischen Familienlebens. Eine Orientierung an vor- 


\section{Familiale Tradierungsprozesse in einer Drei-Generationen-Perspektive}

gegebenen Bahnen ist für sie prekär und sie transformiert ihn zur Horizonterweiterung. Iris Gümper empfindet die Art und Weise, wie die Eltern die Orientierung am familialen Bezugsrahmen einfordern, als Zwang und Belastung und reagiert mit einer (partiellen) Verweigerungshaltung. Beide Frauen bemängeln die fehlende Förderung der individuellen Stärken der Kinder. Die Geschlossenheit des Erziehungssettings wird von beiden ausführlich kritisiert.

Für Sonja Ahrens, als Vertreterin der jüngsten Generation, ist die Geschlossenheit der an sie herangetragenen Erwartungen problematisch, da Abweichungen auf subtile Art sanktioniert werden. Das unbedingte Befolgen des an sie herangetragenen Bezugsrahmens der Horizonterweiterung ist im Grunde für sie nicht nachvollziehbar, da ihr die Möglichkeiten für eine individuelle Weiterentwicklung im Gegensatz zu ihrer Mutter nie versperrt gewesen sind. Durch die Geschlossenheit des Vermittlungssettings fühlt sich Sonja eingeengt, was zu Konflikten führt. Die aus ihrer Sicht fehlende Würdigung und Akzeptanz ihrer Entscheidungen erschwert ein umstandsloses Anknüpfen an den Bezugsrahmen. Zugleich erklärt es, warum für sie statt der Horizonterweiterung die intergenerationellen Beziehungen ins Zentrum der Aufmerksamkeit rücken. Hierbei ist anzumerken, dass alle drei Generationen der Familie Mahler/Ahrens als aneignende Generationen ein Defizit an emotionaler Zuwendung beklagen. Dieses Thema erfährt im Zeitverlauf eine zunehmende Bedeutung. Die Milieuorientierung bleibt in allen drei Generationen erhalten.

Für Lea ist eine Orientierung am familialen Bezugsrahmen größtenteils unproblematisch. Zum einen erfährt sie eine starke Unterstützung durch die Eltern, die es ihr erleichtern, an den familialen Bezugsrahmen anzuschließen. Zum anderen weist sie ihrer Mutter einen Expertenstatus in Erziehungsfragen zu, was es ihr ermöglicht, die mütterlichen Handlungen zu akzeptieren.

In der Gesamtschau weisen die Erwartungshaltungen der vermittelnden Generationen eine hohe Geschlossenheit auf, aber die Mittel zur Erreichung des jeweils anvisierten Ziels ändern sich. Es könnte sein, dass diese Tradierungslogik sich in den vorgestellten Familien im Stadium der Transformation befindet und sich in den nächsten Generationen der Umgang mit den Erwartungshorizonten verändern wird, was aber erst die Zukunft zeigen wird. Die mittlere Generation ist weniger bereit, die elterlichen Erwartungen anzunehmen und verändert in weitaus stärkerem Ausmaß die Tradierungsinhalte und/oder -formen, sodass mit einer geschlossenen Tradierungslogik im Vergleich zur flexibel erscheinenden ein größerer Wandel verbunden ist. Das hält die mittlere Generation jedoch nicht davon ab, ähnlich wie die eigenen Eltern geschlossene Erwartungshorizonte, die als kennzeichnende Konstante dieser Tradierungslogik erhalten bleibt, für die eigenen Kinder zu entwerfen.

Das Zusammenspiel von familialer und gesellschaftlicher Ebene Die in den Familien Mahler/Ahrens und Nolte/Gümper zu beobachtenden Neujustierungen der Bezugsrahmen sowie der praktizierten Erziehung lassen sich unter Heranziehung der 


\subsection{Geschlossene Tradierungslogiken}

gesellschaftlichen Entwicklung erklären und als Versuche der jeweils aneignenden Generationen verstehen, die entsprechenden familialen Bezugsrahmen im Zeitverlauf anschlussfähig zu halten.

Um die vertrauten Bahnen bzw. eine strenge Ordnung aufrechtzuerhalten, ist es nur folgerichtig, wenn Frau Nolte und Frau Ahrens die Kinder stark kontrollieren, Regelübertretungen ahnden, Freiräume und Mitspracherechte der Kinder so gering wie möglich halten und einen strikten Gehorsam verlangen. Das sind klassische Elemente eines Befehlshaushalts, wie er in den 1950er und 1960er Jahren in allen sozialen Schichten weit verbreitet war (Bois-Reymond 1994, 148). Frau Mahler und Frau Nolte können somit sicher sein, keine Außenseiterposition bei der Erziehung der Kinder einzunehmen. Das dürfte den Eindruck verstärken, das eigene Erziehungssetting als bewährtes Modell $\mathrm{zu}$ betrachten und nicht weiter zu hinterfragen. Allerdings ist auch festzustellen, dass es sich bei der Forderung nach kindlichem Gehorsam sowie der kindlichen Unterordnung - trotz der feststellbaren Verbreitung - um Erziehungswerte handelt, die seit Anfang der 1950er Jahre im Rückgang befindlich sind (Reuband 1997, 134). Diese Verschiebungen werden jedoch weder von Frau Mahler noch von Frau Nolte aufgegriffen. Der Vorteil dieses beharrenden Verhaltens besteht darin, dass das Festhalten an bekannten Grundsätzen Sicherheit und Orientierung bietet, gerade in Zeiten, in denen bestehende Grundsätze und Einstellungen an Verbindlichkeit verlieren und brüchig werden. Andererseits birgt das Festhalten an vertrauten Maximen die Gefahr, die Anschlussfähigkeit an die veränderte Verhältnisse zu verlieren, da die bekannten Muster an Tragfähigkeit einbüßen. Das Beibehalten der überlieferten Ordnung dient u.a. dem Zweck, mögliche Veränderungen zu vermeiden und die prinzipielle Offenheit der kindlichen Zukunft zu kanalisieren, um die hiermit verbundenen Unwägbarkeiten so gering wie möglich zu halten.

Der sich abzeichnende gesellschaftliche Wandel, der sich u.a. in der Verlagerung der Erziehungswerte sowie im Wertewandel allgemein manifestiert (Meulemann 1996b, Klages 1984, 2001b Inglehart 1979, 1989, 1998), erklärt zugleich zumindest teilweise, warum die mittlere Generation, verkörpert durch Michelle Ahrens und Iris Gümper, die elterliche Erziehung kritisiert und ablehnt. Das Befolgen strikter Ordnungsmuster wird von beiden Frauen nicht mehr als Orientierungshilfe empfunden, die Sicherheit bietet, sondern als Limitierung. Sie wachsen in einer Zeit auf, in der alte Ordnungsvorstellungen an Bindekraft verlieren und öffentlich in Frage gestellt werden, was wiederum auf den individuellen Alltag zurückwirkt. Durch die Protagonisten der antiautoritären Bewegung ${ }^{174}$ werden Erziehungsgrundsätze in die Öffentlichkeit getragen, die sich radikal von denen ihrer Eltern unterscheiden. Auch wenn bereits zuvor verschiedene Erziehungskonzepte parallel existierten (Wurzbacher 1958 und Baumert 1954), wird spätesens mit dieser Debatte einer breiten Öffentlichkeit bewusst, dass es ganz un-

\footnotetext{
${ }^{174}$ Einen Überblick über die Pädagogik der Studentenbewegung findet sich bei Jansa (Jansa 2000). Insbesondere die Erziehungsziele Selbstständigkeit und Mündigkeit werden durch die antiautoritäre Bewegung in die Öffentlichkeit getragen (Jansa 2000, 240).
} 


\section{Familiale Tradierungsprozesse in einer Drei-Generationen-Perspektive}

terschiedliche Erziehungsmaximen gibt, was auch Iris Gümper und Michelle Ahrens registriert haben. Beide Frauen erleben in ihrem unmittelbaren persönlichen Umfeld außerhalb der Familie Beispiele für einen anderen Umgang zwischen Erwachsenen und Kindern, der durch großzügigere Erziehungspraxen und emotionale wie praktische Unterstützung geprägt ist. Diese Verschiebungen werden aber in den eigenen Elternhäusern nicht aufgegriffen, was zu Konflikten führt und dazu beiträgt, die grundlegenden Elemente der elterlichen Erziehung nicht zu übernehmen, sondern andere Formen des intergenerationellen Umgangs zu wählen. Auch andere inhaltliche Schwerpunkte der elterlichen Erziehung sind für die nächste Generation nicht mehr ohne Weiteres anschlussfähig. Während Frau Nolte eine Konzentration auf die schulische und musikalische Ausbildung mit Bezug auf ihre eigenen Erfahrungen noch als Erfolgsgaranten für ihre Kinder betrachtet, bewertet Iris Gümper dies bereits anders. Zum einen erlebt sie die Limitierung auf einen engen Leistungskanon als hemmend. Zum anderen sieht sie an den Berufslaufbahnen ihrer Geschwister und sich selbst, dass diese Fokussierung nicht zwangsläufig mit Erfolg verbunden ist, was auf eine weitere gesellschaftliche Entwicklung verweist: Eine hohe schulische Ausbildung und kulturelle Bildung garantieren nicht mehr zwangsläufig das Erreichen bestimmter gesellschaftlicher Positionen.

Für Frau Mahler ist das Familienmodell der Hausfrauenehe alternativlos. Dies impliziert eine „soziale Festschreibung der Frauenrolle auf den Lebensbereich der Familie“ (Sommerkorn/Liebsch 2002, 103), womit Frau Mahler dem Trend der Zeit folgt. Die eigenen biografischen Erfahrungen, zu der die Orientierung an einem harmonischen und idyllischen Familienleben bei gleichzeitiger Ausblendung familialer Konflikte zählen, verstärken dieses Bestreben.

Michelle Ahrens hingegen betrachtet dieses Familienmodell nicht mehr ohne Weiteres als anschlussfähig. Im Zuge der Bildungsexpansion rückt der Stellenwert der Bildung in den Mittelpunkt des öffentliches Interesses, was zugleich die Frage der eigenen beruflichen Ausbildung aufwirft. Die Ehe als Lebensmodell büßt zunehmend an Bedeutung und Attraktivität ein. Andere Formen des Zusammenlebens nehmen ebenso zu wie die Scheidungsziffern (siehe Kap.1.1.2). In der Folge wird die Frage der eigenständigen finanziellen Absicherung des Lebensunterhaltes durch Erwerbsarbeit für Frauen zunehmend wichtiger. Angesichts dieser Veränderungen ist das mütterliche Lebensmodell für Michelle Ahrens nicht mehr selbstverständlich und die eigene Berufstätigkeit erhält für sie einen zentralen Stellenwert. Ihre Berufsorientierung steht dabei im Einklang mit der zunehmenden Doppelorientierung von Frauen (Sommerkorn/Liebsch 2002, 112). Zugleich ist hierin auch ein Ausdruck ihres Bestrebens nach einer Horizonterweiterung bzw. persönlichen Weiterentwicklung zu sehen, die ebenfalls mit einer gesamtgesellschaftlichen Entwicklung korrespondiert: der zunehmenden Bedeutung von Selbstentfaltungswerten (siehe Kap. 1.2.3), in die sich die persönliche Horizonterweiterung einreihen lässt.

Die eigenen, negativ konnotierten Erziehungserfahrungen sind zusammen mit den gesellschaftlichen Veränderungsprozessen für Michelle Ahrens und Iris Gümper wirkmächtige Gründe, die dazu führen, diverse Elemente ihrer Erziehungssettings zu verän- 


\subsection{Geschlossene Tradierungslogiken}

dern, um ihre jeweiligen Bezugsrahmen an die nächste Generation vermitteln zu können. Beide Mütter verzichten auf eine autoritäre Gestaltung der intergenerationellen Beziehungen. Unabhängig davon, dass die Eltern in einigen Fällen versuchen, die Entscheidungsfreiheiten der Kinder zu kanalisieren, werden den Kindern im Vergleich zur Vorgängergeneration mehr Freiheiten eingeräumt und kein unmittelbarer Zwang ausgeübt. Hierin spiegelt sich eine gesamtgesellschaftliche Tendenz, im Zuge derer die ElternKind-Beziehungen zunehmend partnerschaftlich gestaltet werden (Baader/Sager 2008, 305). Iris Gümpers Grundsätze, ihre Tochter an Entscheidungen zu beteiligen und über verschiedene Wahlmöglichkeiten zu diskutieren, verweisen zudem auf ein verändertes Eltern- bzw. Kinderbild. Während Frau Nolte noch die Definitions- und alleinige Entscheidungsmacht bei den Eltern sah, denen allgemein die Kompetenz für angemessene Entscheidungen zugesprochen wurde, räumt Iris Gümper ihrer Tochter größere Machtbefugnisse ein und betrachtet das Kind allgemein stärker als Experten seiner selbst. Das wird durch eine gesamtgesellschaftliche Entwicklung begünstigt, in deren Verlauf das Kind zunehmend als „Akteur in eigener Sache“ (Schütze 2002, 83) betrachtet wird. Hierbei ist es für sie als Mutter unabdingbar, das eigene Erziehungshandeln beständig zu reflektieren und zu entscheiden, welche Befugnisse dem Kind eingeräumt werden können, ohne es zu überfordern. Zugleich erweitert Iris Gümper den Bereich, auf den sie bei der Erziehung ihrer Tochter besonders achtet. Das korrespondiert mit der familialen Strategie, Energien auf die Bereiche zu konzentrieren, in denen das Erreichen von repräsentativen Höchstleistungen möglich erscheint. Gleichzeitig spiegelt sich darin eine allgemeine Entwicklung. Das Spektrum der als förderungswürdig betrachteten Bereiche wird vielfältiger und Aspekte wie Kreativität und soziale Kompetenzen erfahren eine neue Wertschätzung. ${ }^{175}$

Michelle Ahrens' Intention, die Kinder zur Eigenständigkeit zu erziehen, steht ebenfalls im Einklang mit der gesamtgesellschaftlichen Tendenzen (siehe Kap. 1.2.4). Zur Umsetzung dieses Zieles wäre ein autoritäres Erziehungsarrangement kontraproduktiv, das einseitig auf Gehorsamserwartungen setzt.

Die Qualität der Generationenbeziehungen rückt im Zeitverlauf stärker in den Mittelpunkt der Aufmerksamkeit. Während das emotionale Wohlbefinden für Frau Nolte keine besonders herausgehobene Stellung eingenommen hat, bildet es für Iris Gümper ein zentrales Thema. Das manifestiert sich u.a. in ihren Anstrengungen, ein Umfeld für ihre Tochter zu schaffen, in dem sie sich geborgen fühlen kann. Auch wenn Sonja Ahrens gelegentlich eine mangelnde emotionale Fürsorge ihrer Mutter beklagt, ist nicht $\mathrm{zu}$ übersehen, dass Michelle Ahrens ebenfalls versucht, sich über das emotionale Wohlergehen ihrer Kinder zu informieren. Die stärkere Beachtung der intergenerationellen Beziehungsqualität lässt sich mit einem weiteren gesellschaftlichen Trend verbinden: dem zunehmenden Fokus auf die emotionale Qualität der Beziehungen, die Schülein

\footnotetext{
${ }^{175}$ Die vorgenommenen Modifikationen Iris Gümpers wirken wiederum auf die Einschätzungen ihrer Mutter, Frau Nolte, zurück, die anscheinend einige Erweiterungen des familialen Bezugsrahmens übernimmt.
} 


\section{Familiale Tradierungsprozesse in einer Drei-Generationen-Perspektive}

mit dem Begriff der Beziehungsrevolution (Schülein 1990, 86) umschreibt. Im Zuge dessen erfahren die Anpassung der Eltern an kindliche Bedürfnisse sowie die empathische Zuwendung zum Kind eine enorme Bedeutungssteigerung (Schülein 1990, 134/135) Aspekte, die Sonja mit ihrer Kritik am mütterlichen Verhalten einfordert. Relativierend ist jedoch anzumerken, dass Frau Mahler und Frau Nolte ihre Aufmerksamkeit auf sechs bzw. sieben Kinder verteilen mussten; Michelle Ahrens und Iris Gümper hingegen können sich auf zwei bzw. ein Kind konzentrieren.

In der Familie Gümper werden dadurch neue Problemlagen geschaffen. Ein sowohl für Iris Gümper als auch für Lea wichtiges Anliegen ist die richtige Balance von Nähe und Distanz zu finden. Die Eltern wollen Lea bestmöglich unterstützen, ohne dabei die Entwicklung von Leas Eigenständigkeit zu beeinträchtigen. In diesem Zusammenhang wird es wichtiger, Lea zu eigenverantwortlichem Verhalten zu erziehen, um trotz der aufgegebenen Maxime der strengen Ordnung den familialen Bezugsrahmen der repräsentativen Höchstleistungen zu tradieren. Auch das entspricht einer gesamtgesellschaftlichen Entwicklung: Die Generationenbeziehungen werden gleichberechtigter, das eigene Verhalten und seine Auswirkungen auf das Gegenüber werden stärker reflektiert und der Erziehungswert der Selbstverantwortung löst den des Gehorsams ab (BoisReymond 1994, 144). Das sind Merkmale des Verhandlungshaushaltes, der mittlerweile den des Befehlshaushalts als dominierende Form der Erziehung in der Gesellschaft weitgehend abgelöst hat, was u.a. auf die veränderten gesellschaftlichen Erfordernisse zurückgeführt wird (Ecarius 2002, 54).

Darüber hinaus sind Michelle Ahrens und Iris Gümper in der Lage, intergenerationelle Konflikte zu thematisieren. Dies lässt sich ebenfalls mit einer allgemeinen Entwicklung rückkoppeln, in deren Folge sich die familiale Streitkultur verändert und Konflikte mit den Eltern thematisierbar werden (Zinnecker 1985, 165/166, 170, 186).

Da für Michelle Ahrens und Iris Gümper die elterlichen Vermittlungssettings in vielen Bereichen nicht praktikabel sind, sind sie darauf angewiesen, sich außerfamilial Erziehungsrat einzuholen. Michelle Ahrens realisiert dies über das soziale Nahfeld. Iris Gümper kommt über ihre Berufsausbildung mit neuen Ideen der Erziehung in Kontakt, greift aber offensichtlich gelegentlich auch auf Ratgeber zurück. Die steigende Zahl an Veröffentlichungen im Bereich der Erziehungsratgeber ${ }^{176}$ verweist hierbei auf eine gesamtgesellschaftlich gestiegene Nachfrage. ${ }^{177}$

Hiermit einhergehend wird die optimale Kindesförderung zunehmend bedeutsamer. Michelle Ahrens reagiert hierauf, indem sie ihren Kindern diverse Angebote unterbreitet und darum bemüht ist, sich über die verschiedenen Möglichkeiten zur Förderung der

\footnotetext{
${ }^{176}$ Laut Berg werden Anfang der 1990er Jahre jährlich zwischen 40 bis 60 Erziehungsratgeber veröffentlicht (Berg 1991, 713). Höffer-Mehlmer und Schmid weisen ebenfalls auf das wachsende Angebot von Erziehungsratgebern hin (Höffer-Mehlmer 2003, 7, Schmid 2010, 13).

${ }^{177}$ Für Hopfner ist die Nachfrage nach Erziehungsratgebern Ausdruck eines Verlustes von Selbstverständlichkeiten in Zusammenspiel mit steigenden gesellschaftlichen Ansprüchen an die Erziehung (Hopfner 2001, 76).
} 


\subsection{Geschlossene Tradierungslogiken}

Kinder zu informieren. Iris Gümper setzt sich intensiv und kritisch mit den verschiedenen pädagogischen Angeboten auseinander, die für ihre Tochter in Frage kommen könnten. An die Eltern stellen sich in diesem Zusammenhang neue Anforderungen: Im Zuge der gestiegenen schulischen und außerschulischen Angebote für Kinder erhalten die Eltern größere Auswahlmöglichkeiten. Dies bedeutet zugleich, dass Eltern im zunehmenden Maße gezwungen werden, sich zu informieren, wenn sie eine bestmögliche Förderung für ihre Kinder erreichen wollen (siehe Kap. 1.2.4). Hierin unterscheidet sich die Situation der mittleren Generation grundlegend von der ihrer Eltern. Während der Familienzeit von Frau Nolte und Frau Mahler waren die Freizeit- und Bildungsmöglichkeiten für Kinder weit weniger ausdifferenziert, sodass sich für diese Generation weniger Auswahlprobleme ergaben, zumal die Vorstellungen auf bestimmte Bereiche fixiert waren. Ein vielfältiges pädagogisches Angebot wäre eventuell sogar hinderlich, wenn es darum geht, die Kinder in die gegebenen Verhältnisse einzupassen.

Neben den Chancen, die sich ergeben, wächst zugleich der Druck auf die Eltern. Ihnen wird verstärkt die Verantwortlichkeit zugeschrieben, für eine optimale Entwicklungsatmosphäre zu sorgen (siehe Kap. 1.2.4). Das Erbringen von Leistungen ist weniger einseitig eine selbstverständliche Pflicht der Kinder, als vielmehr mit Anstrengungen auf beiden Seiten verbunden. Ferner setzt die Wahrnehmung der Angebote entsprechende finanzielle Mittel der Eltern voraus, sodass sich insgesamt betrachtet neue Ungleichheiten ergeben. Im Falle der Familie Gümper sind die entsprechenden finanziellen Mittel vorhanden, um sich verschiedene pädagogische Zusatzangebote leisten und von ihnen profitieren zu können.

Durch die zusätzlichen Freizeitangebote ist Leas Woche stärker zeitlich verplant als der Alltag Iris Gümpers während ihrer Kindheit, was einem weiteren gesellschaftlichen Trend entspricht, dem der Institutionalisierung von Kindheit. Die Anzahl der speziell an Kinder adressierten Freizeitangebote ist enorm gestiegen, die kindliche Freizeit wird stärker verplant, von Erwachsenen organisiert, kontrolliert und ist zweckgebunden. Gleichzeitig werden die Möglichkeiten eines selbst organisierten Kinderlebens geringer (Peuckert 2008, 150-153).

Das Eröffnen von Wahlmöglichkeiten ist allerdings nicht mit Beliebigkeit gleichzusetzen. Für Iris Gümper z.B. existieren feststehende Grundsätze, mit denen ein allgemeiner Erziehungsrahmen abgesteckt wird. Hierdurch werden Verbindlichkeiten geschaffen, die als Orientierungspunkte für Erziehende und Erzogene dienen. Diese Orientierungshilfen werden auch als solche von Lea wahrgenommen und positiv als Stützen verortet. Hierin manifestiert sich der Wunsch nach Strukturen angesichts der zunehmenden Vielfalt von Handlungsmöglichkeiten. Sonjas Wunsch nach mehr institutionalisierten, gemeinsamen Familienzeiten entspringt ebenfalls dem Bedürfnis nach Strukturen und Verbindlichkeiten. 


\subsection{Diffuse Tradierungslogiken}

\subsubsection{Familie Beyer/Schmidt: Familiale Bindungen und Verantwortlichkeiten}

Biografische Eckdaten Frau Beyer wird 1949 in einem kleinen Ort im heutigen Thüringen geboren. Der Ort liegt zu DDR-Zeiten innerhalb des Sperrgebietes. Ihre Eltern sind Inhaber eines kleinen Ladengeschäftes. Frau Beyer verfügt über eine kaufmännische Ausbildung und ist während ihrer Familienphase erwerbstätig. 1970 heiratet sie den vier Jahre älteren Herrn Beyer, der als Handwerker ebenfalls selbstständig einen kleinen Betrieb führt. Das Paar hat zwei Töchter und drei Söhne. Zwei Kinder werden Anfang der 1970er Jahre geboren und drei in den 1980er Jahre. Die Familie lebt im Elternhaus von Herrn Beyer, in dem auch der inzwischen verstorbene Vater von Herrn Beyer eine eigene Wohnung gehabt hat. Die Eltern Frau Beyers leben bis zu ihrem Tod in der Nachbarschaft.

Birgit Schmidt, Jahrgang 1971, ist das älteste Kind. Sie absolviert eine handwerkliche Ausbildung und arbeitet seit der Wende mit kurzen Unterbrechungen bei verschiedenen Arbeitgebern. Sie heiratet 1992 den vier Jahre älteren Thomas Schmidt. Das Paar bezieht im Haus von Birgit Schmidts Schwiegermutter eine eigene Wohnung, in der die Familie bis heute lebt. 1992 wird das erste Kind Magdalene geboren, 1998 folgt der Sohn Dirk. Magdalene verfügt inzwischen über einen Realschulabschluss und hat eine Lehre begonnen. Dirk geht mittlerweile zur Realschule. Beide Familien sind katholisch.

Aneignungsebene Frau Beyer (1949) ${ }^{178}$ : Der geschützte Rahmen der Familie Für Frau Beyer spielen die besonderen Lebensumstände ihrer Kindheit eine besondere Rolle. Sie wächst im sogenannten Sperrgebiet innerhalb der DDR, also an der innerdeutschen Grenze, in einer katholischen Familie auf. Das hat aus ihrer Sicht ihre Erziehung ebenso beeinflusst wie die gesellschaftlichen Rahmenbedingungen der DDR insgesamt:

„Ja und äh diese ganze politische Situation es war doch ein äh guter $\mathrm{Zu}$ sammenhalt in der Familie, man hat sich auch gegenseitig geschützt äh vor dem, was von außen hätte kommen können, was hätte passieren können durch unbedachte Äußerungen [...]. Und das hat schon äh unsere Erziehung auch geprägt und der Zusammenhalt war ganz einfach äh doch auch da“" (I 05:32-06:04).

Die äußeren Umstände lassen aus Sicht Frau Beyers die Familie näher zusammenrücken, und die familialen Bindungen und Sorgebeziehungen erhalten einen zentralen Stellenwert. Gleichzeitig lassen die Familienmitglieder eine große Vorsicht in den zwischenmenschlichen Beziehungen walten:

„[I]n der Familie, da gehört ja dann auch die Verwandtschaft dazu, da musste schon ein, ein ziemlich großes Vertrauensverhältnis auch äh herrschen

\footnotetext{
${ }^{178}$ Die Interviews mit Frau Beyer wurden am 08.02.2008 und am 15.02.2008 geführt.
} 


\subsection{Diffuse Tradierungslogiken}

damals hier bei uns. [...] Aber man musste schon wissen in der Familie, [...] wem kann man mal was anvertrauen. Mit wem kann ich äh über ganz gewisse Dinge reden, über ganz gewisse Probleme. Wer kann mir eventuell auch mal wo weiterhelfen, das war einfach damals so" (I 38:22-38:55).

$\mathrm{Zu}$ denjenigen, die das familiale Vertrauen genießen, besteht offenbar ein enges Verhältnis. Zugleich deutet sich eine gewisse Tendenz zur Abschottung gegenüber denjenigen an, die nicht zu diesem Kreis zählen, was wiederum ganz konkrete Folgen für den Alltag hat. Frau Beyer erinnert sich an die im Wohnort stationierte Grenzkompanie und eine gewisse Spaltung des Dorfes in Alteingesessene und „die Roten [...], [d]ie mit äh Kirche und Glauben so nichts am Hut hatten“ (III 18:04-19:39). Ihre Eltern hätten Freundschaften ihrer Tochter mit Kindern aus Offiziersfamilien nicht unbedingt gern gesehen (I 29:53-30:03, III 19:42-19:59). Auch sei Frau Beyer zur Verschwiegenheit ermahnt worden: „Und ähm es hieß auch immer, was hier zu Hause gesprochen wird, das sagst du nicht in der Schule. Und denke dran, wir haben Westverwandte und Westkontakt, ähm das soll man hier nich so laut sagen, und äh das könnte Probleme geben“ (III 20:56-21:13). Die Eltern fordern ihre Tochter zu einem reflektierten, eigenkontrollierten und verantwortlichen Handeln auf. In anderen Bereichen hätten die Eltern versucht, die Kinder zu schützen, indem sie ihnen z.B. verschwiegen hätten, wenn Personen in den Westen geflohen oder verhaftet worden seien (I 39:18-42:00). Einerseits hätten die Eltern sie nicht belasten wollen, andererseits erfordern die äußeren Umstände aber ganz offensichtlich Anpassungen, was dazu führt, dass die elterliche Erziehung zwischen der Aufforderung zur Eigenverantwortung und Selbstkontrolle sowie dem Bemühen, die Kinder zu beschützen, oszilliert.

Aber auch wenn die Eltern den Kindern bestimmte Dinge vorenthalten hätten, habe Frau Beyer mitbekommen, wenn es Schwierigkeiten gegeben habe, was sie als „recht bedrückend oder auch beklemmend" empfunden habe (I 42:14-42:23). Das elterliche Verhalten ist nicht nur eine Entlastung, sondern wirkt unbeabsichtigt auch verstörend. Frau Beyer erinnert staatliche Maßnahmen, die in Umsiedlungen einiger Familien des Dorfes in andere Gebiete bestanden hätten, die diese Ängste wachgehalten und eine einschüchternde Wirkung entfaltet hätten (I 26:24-28:39) ${ }^{179}$ : „[U]nd man war dann schon sehr eingeschüchtert und hatte auch die ganzen DDR-Jahre Angst vor solchen Maßnahmen" (I 28:34-28:39). Die staatlichen Aktivitäten erscheinen als willkürlich und unberechenbar, sodass die Familie bestrebt gewesen sei, möglichst wenig aufzufallen: „[S]o hat man sich zumindest immer nach außen hin angepasst gezeigt, //hmh// um ganz einfach Ruhe zu haben, das einem so was nicht passierte" (I 28:40-28:48). Diese Maxime hat unmittelbare Folgen für ihre Alltagsgestaltung. Sie sei bei den Pionieren und in der FDJ gewesen, „weil das ganz einfach äh (.) so war und weil der Staat das auch eigentlich so gefordert hat $/ / \mathrm{hmh} / /$ und dass die Eltern dann gesagt haben: ,Mensch, mach

\footnotetext{
${ }^{179}$ Frau Beyer bezieht sich hier auf die staatlichen Umsiedlungsaktionen der Jahre 1952 und 1961. Ein ausführlicher Überblick zu diesen Maßnahmen findet sich bei Bennewitz/Potratz (1994).
} 


\section{Familiale Tradierungsprozesse in einer Drei-Generationen-Perspektive}

diesen Quatsch mit, //hmh// mach das MIT. Dass wir hier äh keine Probleme kriegen““ (I 32:17-33:03). Das verdeutlicht ein erzieherisches Dilemma. Die Eltern fordern die Kinder zur Partizipation an staatlichen Organisationen auf, wobei sie ihre Ablehnung eben dieser nicht verheimlichen und damit den $Z$ wangscharakter betonen.

Ein Grund für die Zugeständnisse ist in den spezifischen Lebensumständen der Familie zu sehen, die aus Sicht Frau Beyers die Gefahr staatlicher Repressionen erhöhen. Zum einen sei das die katholische Konfession und zum anderen aufgrund des Betreibens eines eigenen Betriebes durch die Eltern die Zuordnung zur Klasse der „Sonstigen“ innerhalb des staatlichen Klassensystems (I 03:23-04:57), die für Frau Beyer prekär ist. In ihrer Selbstwahrnehmung bedeutet das, nicht eindeutig in der staatlichen Gesellschaft verortet zu sein, sondern als "Sonstige“ dazwischen zu stehen. Aufgrund der gesellschaftlichen Rahmenbedingungen verursachen eindeutige Zuordnungen, wie etwa die zum katholischen Glauben, ebenso Probleme wie aus ihrer Sicht nicht eindeutige $\mathrm{Zu}$ ordnungen wie die zur Gruppe der „Sonstigen“. Diese Lokalisierung ,dazwischen“ zeigt sich auch symbolisch in der Beschreibung ihres Wohnortes als zwischen zwei Grenzen (I 00:56-01:10). Es deutet sich ein grundsätzliches Dilemma der Familie an: Sie kann sich selbst nicht eindeutig verorten, weder geografisch noch sozial, sie nimmt stattdessen eine Zwischenposition ein, die u.a. eine Orientierung erschwert und Eindeutigkeiten verschwimmen lässt. Die Familie reagiert mit Kompromissen auf die gesellschaftlichen Veränderungen, die wiederum selbst gelegentlich zu Widersprüchen führen. Sie befindet sich in einem Zwiespalt zwischen alten und neuen Verhältnissen, den sie nicht befriedigend aufösen kann.

Auch wenn Frau Beyer viele für die Familie problematische Aspekte der gesellschaftlichen Rahmenbedingungen aufzählt, beurteilt sie die DDR-Vergangenheit nicht ausschließlich negativ. Sie bilanziert sie als eine „verrückte Zeit“, die trotz oder gerade wegen der Schwierigkeiten aber auch „zusammengeschweißt“ und hierdurch insbesondere die familialen Beziehungen gestärkt habe (I 43:11-43:16): „Der Familienverbund w-, das war doch viel, viel enger als das heute ist, $/ / \mathrm{hmh} / /$ ne. Heute braucht man ${ }^{\circ}$ das nicht mehr ${ }^{\circ}$. Und da hat das auch doch an Wert verloren“ (I 43:17-43:32). Retrospektiv kann sie den Lebensumständen in der DDR positive Seiten abgewinnen und verbindet das zugleich mit einer Gegenwartskritik, in deren Folge das familiale Zusammenleben aus ihrer Perspektive entscheidenden Veränderungen unterworfen ist. Sie bedauert den Bedeutungsrückgang des familialen Zusammenhaltes, der für sie ein zentraler Bezugsrahmen ist.

Ihren Vater beschreibt sie als sehr kindorientiert und fürsorglich. Seine generelle Intention habe darin bestanden, dass es seine Kinder einmal besser haben sollten als er selbst (III 53:03-53:42). Er betrachtet es offensichtlich als seine Aufgabe, die entsprechenden Bedingungen zu schaffen. Es handelt sich somit weniger um einen Auftrag an die nächste Generation als vielmehr um eine elterliche Verpflichtung:

„Aber mein Vater hat immer gesagt: ,Wir hatten nie die Möglichkeit, äh viel zu lernen oder etwas zu studieren [...]. Und ich möchte meinen Kin- 


\subsection{Diffuse Tradierungslogiken}

dern das bieten, äh was ich ihnen bieten kann an Bildung, an Ausbildung, äh was in unseren Kräften hier steht““ (I 02:38-02:58).

Der Vater sei bemüht gewesen, seine Kinder außerhalb des schulischen Rahmens zu fördern. Er habe z.B. den Kindern privat Musikunterricht erteilen lassen (I 04:57-05:13). Die väterliche Fürsorge beschränkt sich nicht nur auf die Kindheitsphase von Frau Beyer, sondern erstreckt sich weit in die Zukunft. Der Vater habe eine Obstplantage angelegt und verbindet das mit dem emotionalen Appell, einen Platz im Familiengedächtnis zu erhalten (I 55:33-55:53), wodurch er gleichzeitig das familiale Zusammengehörigkeitsgefühl unterstreicht.

Eine besondere Ausprägung des Bezugsrahmens der familialen Fürsorge findet sich in der Beziehung zum elf Jahre jüngeren Bruder. Der elterliche Wunsch, ein drittes Kind zu bekommen, habe geraume Zeit auf sich warten lassen:

„Und weil man ja doch auch hier verhältnismäßig religiös is, hat meine Mutter gesagt: ,Wenn's Gottes Wille ist, dann kriegen wir noch ein drittes Kind.' U:nd ähm meine Tante im Westen [...] wusste auch, dass ähm meine Eltern noch diesen Kinderwunsch hatten. Und da hat sie mal ein Bild geschickt, so ein großes Heiligenbild, das ist der heilige (Gerhard) Majella. Und das is so der Schutzpatron der Mütter. //hmh// [...] Und mein Vater hat dann für dieses Bild [...] einen tollen Rahmen gekauft. (.) Und das Bild hat er mir übers Bett gehängt und hat gesagt: ,Und du betest jetzt immer schön.' //hmh// Und tatsächlich, als meine Mutter [zweiundvierzig, C.R.] wurde, ist mein Bruder geboren“ (III 01:52-03:05).

Zum einen zeigt sich hier die feste Verankerung der Religiosität im Familienalltag. Zum anderen erhält Frau Beyer die Gelegenheit, im Rahmen ihrer Möglichkeiten in Form des Gebets ihren Beitrag zur familialen Fürsorge zu leisten und sie zugleich einzuüben. Nach der Geburt des Bruders sei sie zumindest partiell für ihn verantwortlich gewesen: „Das ging äh erstmal nachmittags, wenn die Schule aus war, den, den Jungen mit verwahren. $/ / \mathrm{hmh} / /$ Spazieren gehen. Oder ich hatte dann auch schon so: die Aufgabe äh mit Windeln zu waschen“ (III 07:10-07:23). In der Folge habe sie eine ganz besondere Beziehung zu ihrem jüngeren Bruder gehabt: „Das is wIE, nich wie mein Kind, aber das is wirklich wie nochmal so 'n Stück von mir, ne. $/ / \mathrm{hmh} / /$ Das is eigentlich äh mit das Größte, was ich so äh, an=äh was ich mich in=an meine Kindheit so erinnere" (III 06:19-06:33). Den mit der Kinderbetreuung verbundenen Aufgaben sei sie gern nachgekommen: „[I]ch hab das auch nie als, als Arbeit empfunden oder als, äh: als lästig. //hmh// Das hat mir ganz einfach Spaß gemacht, schon so ein kleines bisschen äh Mutter spielen zu können“ (III 08:55-09:07 ). Frau Beyer kann in ihrer Darstellung problemlos an den Bezugsrahmen der familialen Sorge und Verantwortung anknüpfen. Er ist für sie in doppelter Weise im Alltag verankert: Einmal ist sie Nutznießerin der familialen Fürsorge und einmal ist sie diejenige, die einem anderen Fürsorge zuteil werden lassen kann. 


\section{Familiale Tradierungsprozesse in einer Drei-Generationen-Perspektive}

Das Verhältnis der Eltern zu den Kindern beschreibt Frau Beyer als emotional zugewandt. Sie habe „sehr liebe Eltern“ gehabt (I 01:16-01:23). Hierbei nimmt sie eine Differenzierung zwischen dem mütterlichen und väterlichen Verhalten ihr gegenüber vor, das sie mit bestimmten Grundeinstellungen der Eltern in Zusammenhang bringt. Die Mutter sei „ein bisschen äh geizig oder auch mehr sparsam“ (I 01:24-01:30) gewesen, was sich auch im konkreten Erziehungsverhalten niedergeschlagen habe. Die Mutter habe sie in Bezug auf die Kleidung „so 'n bisschen knapp gehalten“ und ihr z.B. nicht die sehnlichst gewünschten und als modisch empfundenen Perlonstrümpfe gekauft (I 58:54-59:35). Die Mutter verfällt offensichtlich in keinen verwöhnenden Erziehungsmodus. Die Wünsche der Kinder führen nicht dazu, bestimmte Grundeinstellungen außer Kraft zu setzen.

Das mit der mütterlichen Sparsamkeit verbundene Erziehungsverhalten führt gelegentlich zu kleineren Konflikten: „[I]ch hab immer keine, wenn’s ma’ Rouladen gab, den Speck nich essen können in den Rouladen. Hab das immer raus gelesen. Und dann sagte se: ,Was auf den Tisch kommt, wird gegessen'. Hab ich gesagt: ,Wenn ich ma Kinder hab, die müssen keinen Speck essen“" (I 1:03:13-1:03:34). Die Mutter formuliert zwar eindeutige Anweisungen, die jedoch keinen unüberwindbaren Befehlscharakter haben, da sie die Tochter nicht davon abhalten, sich gegen die Anordnungen aufzulehnen. Auch wenn sich die Generationenbeziehungen an dieser Stelle durchaus asymmetrisch zugunsten der Mutter darstellen, verbleiben Frau Beyer Gestaltungsspielräume, indem sie ihren Unmut äußern und der Mutter mehr oder weniger direkt vorwerfen kann, die kindlichen Bedürfnisse zu vernachlässigen. Das mütterliche Verhalten führt zu dem Vorsatz, gegenüber den eigenen Kindern „großzügiger" und kindzentrierter zu handeln (I 1:00:51-1:01:16). Trotz der geäußerten Kritik kann Frau Beyer der mütterlichen Sparsamkeit aber auch positive Seiten abgewinnen: „[D]as kommt ja doch der Familie zugute, wenn man einteilt, wenn man aufteilt oder zuteilt“ (I 07:31-07:46). Zudem sei dies den damaligen Verhältnissen geschuldet gewesen, die die elterliche Generation geradezu dazu gezwungen habe, zu improvisieren und durch sparsames Wirtschaften das Auskommen der Familie sicherzustellen (I 1:01:17-1:01:55). Frau Beyer sieht somit das mütterliche Verhalten nicht nur im Einklang mit dem familialen Bezugsrahmen der Fürsorge, sondern zugleich als ein Element, das diesen Rahmen unterstützt und nicht lediglich der mütterlichen Willkür entspringt.

Im Gegensatz zur mütterlichen Sparsamkeit wird der Vater als „eher großzügig“ charakterisiert (I 01:31-01:40). Er habe Frau Beyers Wünsche nicht so leicht ausschlagen können (I 59:38-59:43). Gelegentlich verbündet er sich mit der Tochter und unterläuft auf diese Weise die Erziehungsmaximen seiner Frau:

„Und dann hab ich immer so manchmal so zu meinem Papa gesagt: ,Papa, 'wolln wir nich mal 'n Glas Pfirsiche', das war so 'ne herrliche Frucht, ne, 'n Glas Pfirsiche aufmachen?' ,Oh', hat er da gesagt, ,Mama schimpft, wenn wir das machen. 'Und dann haben wir uns immer hinten ins Lager gestellt, in die Ecke so hinter so dem Regal und da war noch so 'n Fenster nach 


\subsection{Diffuse Tradierungslogiken}

draußen, und dann haben wir so ein = Papa ein Glas Pfirsiche aufgemacht und dann haben wir jeder 'ne Gabel genommen und haben die Pfirsiche so rausgegessen und dann das, die, die, den $\mathrm{Saft}^{\circ}$ aus dem Glas so getrunken und das Glas entsorgt. ,Aber sag das nicht zu Mama', hat er immer gesagt, ne“ (I 52:31-53:11).

Der Vater unterwandert auch im mütterlichen Beisein deren Absichten: „Und wenn dann mal ein Glas Kirschen, so Kompott, aufgemacht wurde und ich wollte noch 'n Schälchen, dann sagte meine Mutter: ,Du hast schon eins gekriegt.' Dann sagte mein Papa: ,Gib das doch dem Kind. Das is 'n Zeichen, dass der Körper das braucht" “(I 53:30-53:47). Der Vater ist gegenüber der Tochter sehr nachgiebig und orientiert sich nicht wie seine Frau an einer absoluten Gerechtigkeitsvorstellung. ${ }^{180}$

In Anbetracht der Ausführungen ist es nicht verwunderlich, dass Frau Beyer eine besonders starke Verbundenheit zu ihrem Vater beschreibt, was sie damit erklärt, „dass die Jungens äh mehr zur Mutter gehen und die Mädchen eher zum Vater" (I 49:06-49:36). $\mathrm{Da}$ sie vermutlich den Anspruch internalisiert hat, zu beiden Elternteilen ein gleichermaßen gutes Verhältnis haben zu müssen, bedient sie sich eines Allgemeinplatzes, um die besondere Bindung zum Vater zu rechtfertigen. Aufgrund der elterlichen Schwerpunktsetzungen ist Frau Beyer gleichzeitig mit zwei widersprüchlichen Orientierungen konfrontiert: Großzügigkeit und Sparsamkeit.

Die praktische Verankerung des Bezugsrahmens im Alltag dürfte dazu beigetragen haben, dass er sich für Frau Beyer als anschlussfähig darstellt und sie mit der elterlichen Erziehung zufrieden ist.

„Es gab da nichts äh: pf:, eigentlich nichts, was ich als Gebot oder Verbot empfunden hätte. Es war eigentlich das ähm ganz normale Familienleben, es = was ganz einfach bei uns dazugehört, ohne dass da groß das angeordnet wurde oder dass da groß drüber gesprochen wurde“ (III 47:45-48:06).

Hierzu zählt Frau Beyer diverse religiöse Praxen wie das Morgen- und Abendgebet, die Gebete vor den Mahlzeiten, den sonntäglichen und wochentäglichen Kirchgang sowie das Einhalten des Freitagsgebots (III 48:10-51:09). Des Weiteren habe es „die ganz normalen Regeln“ gegeben (III 51:28-51:31). Das seien neben Pünktlichkeit und Streben nach guten schulischen Leistungen das Achten auf Höflichkeitsregeln gewesen. $\mathrm{Zu}$ Letzterem habe der Appell „Benimm Dich“ gezählt. Gegenüber Lehrern hätten sie nie „frech sein“ oder „ausfallend“ werden dürfen (III 51:28-52:31). In dieser Familie wird ein grober Verhaltensrahmen für die Kinder entworfen, ohne den kindlichen Alltag detailliert zu verregeln. Das trägt dazu bei, dass die Regeln „nicht als Zwang empfunden“ werden (III 51:00-51:09) und Frau Beyer sich in die grob abgesteckte Rahmenstruktur der Familie einfügen kann. Diese als Basisstandards klassifizierbaren Regeln dürften

\footnotetext{
${ }^{180}$ Die absolute Gerechtigkeitsvorstellung der Mutter wird von Frau Beyer besonders betont. Ihre Mutter habe z.B. akribisch darauf geachtet, die Inhalte der Westpakete genau zwischen ihren Kindern aufzuteilen (I 1:04:14-1:05:25).
} 


\section{Familiale Tradierungsprozesse in einer Drei-Generationen-Perspektive}

auch in anderen Familien gegolten haben, sodass sie als normal und selbstverständlich erscheinen.

Die Großmutter ist für Frau Beyer eine wichtige Bezugsperson. Sie habe Frau Beyer mit in die Kirche genommen und sie in die diversen kirchlichen Riten des Lebenslaufs und des Kirchenjahres eingeführt (I 44:34-47:01). Ähnlich wie in den Familien mit flexibel erscheinenden Erziehungsarrangements stellt auch hier das Vorleben einen gewichtigen Transmissionsriemen (Schönpflug/Bilz 2009) für die Weitergabe religiöser Praktiken dar. Die Großmutter habe viel mit ihren Enkeln gespielt und gesungen, für sie Kinderspielzeug aus Alltagsmaterialien hergestellt sowie für eine behagliche Atmosphäre gesorgt (I 44:34-47:01). Die Enkelkinder stehen im Zentrum der großmütterlichen Aufmerksamkeit, die ihr Verhalten stark auf die kindlichen Bedürfnisse ausrichtet, was einen weiteren zentralen Faktor darstellt, um das generelle Erziehungssetting und die hierüber transportierten Orientierungen annehmen zu können.

Manchmal habe Frau Beyer den gesetzten Regeln, wie z.B. dem Besuch der Frühmesse nicht Folge leisten wollen: „Und wenn man dann auch mal nich aufstehen wollte, dann hat meine Mutter gesagt: , ${ }^{\circ}$ (Geh man in die) Kirche. Es is doch keiner da, der den Engel des Herrn beten kann o'“ (III 49:35-49:44). Die Mutter kann ihre Vorstellungen ohne Befehle durchsetzen, indem sie an das Gewissen der Tochter appelliert. Sie wählt somit im Vergleich zu direkten Anweisungen die eher indirekte Form des Überredens.

Insgesamt wird die Tradierung des Bezugsrahmens einerseits durch das gewählte Erziehungssetting sowie die gesellschaftlichen Rahmenbedingungen unterstützt. Andererseits führen die elterlichen Reaktionen auf die gesellschaftlichen Kontextbedingungen gelegentlich zu einer Verwässerung mancher Erziehungsinhalte. Auch die teilweise entgegengesetzten Prioritäten - Sparsamkeit vs. Großzügigkeit - tragen zu einer partiellen Diffusität der anzustrebenden Orientierungen bei. Dennoch lässt sich für Frau Beyer familiale Sorge und Verantwortlichkeit als zentraler Bezugsrahmen festhalten, den sie mit Nachgiebigkeit und starker Empathie verbindet. Hinzu gesellen sich religiöse Praxen sowie tradierte Formen des Familienlebens als relevante Bestandteile, die auch bei der Erziehung der eigenen Kinder bedeutsam sind.

Vermittlungsebene Frau Beyer (*1949): Das Umsorgen der Kinder Retrospektiv sieht Frau Beyer Parallelen zur selbst erfahrenen Erziehung:

„Und alles, was ich vom Elternhaus mitbekommen hab, ähm was mir besonders gefallen hat, das hab ich auch immer versucht [...] an meine Kinder weiterzugeben. Und was mir nicht so gefallen hat oder was ich als Manko dann später empfunden habe, äh das habe ich versucht, äh meinen Kindern auch zu bieten“( (I 07:09-07:30).

Insbesondere habe ihr gefallen, wie der Vater „mit uns umgegangen ist und ähm ganz besonders auch mit mir" (I 49:06-49:22). Frau Beyer rekurriert auf die väterliche Fürsorge und Nachgiebigkeit, die sich aus ihren Erinnerungen als charakteristisch für den 


\subsection{Diffuse Tradierungslogiken}

Vater herausarbeiten lassen und für die Erziehung ihrer Kinder Leitmotive darstellen. Eine besondere Betonung erfährt dabei die empathische Hinwendung zu den Kindern: "Ja sicherlich, also man, man freut sich mit den Kindern [...]. Man leidet aber auch mit den Kindern, wenn was nich so, äh nich so gut läuft, //hmh// wenn was nich so gut is“ (III 1:15:09-15:22). Die jüngeren Kinder hätten gesundheitliche Probleme gehabt, was teils auch zu Schwierigkeiten im sozialen Umfeld geführt und Frau Beyer belastet habe (III 1:15:30-1:18:55):

„[U]nd sicherlich is es, äh wenn, wenn bei einem Kind alles in Ordnung is und alles gut läuft und so, wie bei der Ältesten, da gab es eigentlich so gar keine Probleme. Mit dem Johannes war das schon anders, der hat dann doch auch schon mehr Zuwendung von mir gekriegt, $/ / \mathrm{hmh} / /$ ne, weil der viel mehr Trost brauchte, wegen seiner Mentalität oder weil der auch so sensibel ist“ (III 1:19:53-1:20:18).

Beim jüngeren Sohn sei sie aufgrund seiner gesundheitlichen Situation ebenfalls „manchmal zu mehr Zugeständnisse[n]" bereit gewesen (III 1:20:20-1:20:54). Im Unterschied zur eigenen Mutter orientiert sich Frau Beyer an einer relativen Gerechtigkeitsvorstellung. Die Kinder werden nicht strikt gleich behandelt, sondern erhalten bedarfsorientiert in unterschiedlichem Maße Fürsorge und Zuwendung. Das führt bei den Kindern zu Geschwisterkonkurrenzen:
„Aber (.) sicherlich äh (.) äh gibt es unter den, wenn man mehr Kinder hat, auch immer wieder so kleinere Eifersüchteleien. //hmh// Dass der eine sagt: ,Ja: immer kriegt die alles' oder ,immer kriegt der alles [...•]'. Und das, $/ / \mathrm{hmh} / /$ äh ich denke, das gibt es überall, oder das kann man auch äh bestimmt nicht vermeiden, //hmh//ne“ (III 1:18:56-1:19:25).

Frau Beyer versucht, die Reaktionen der Kinder unabhängig von ihrem Verhalten quasi als selbstverständlich und unvermeidlich hinzustellen. Trotzdem ahnt sie, dass sie potenziell geschwisterliche Rivalitäten fördert, worauf ihre entschuldigende Aussage verweist, es sei den anderen Kindern gegenüber „nicht böse gemeint“ (1:20:55-1:20:58), wenn ein Kind im Bedarfsfall mehr Aufmerksamkeit erhalte. Die familiale Fürsorge, die letztlich dem Zweck dient, die familiale Gemeinschaft zu stärken, untergräbt in der Art der Umsetzung genau dieses Ziel.

Frau Beyer habe sich darum bemüht, ein „schönes Familienleben mit den Kindern“ zu realisieren (III 1:09:54-1:10:02). Sie habe viel Zeit mit den Kindern verbracht und sie und ihr Ehemann seien bemüht gewesen, den Mangel an Konsumgütern durch kreative Improvisationen auszugleichen (III 1:10:03-1:11:59). Zu den gemeinsamen Aktivitäten habe gelegentlich das gemeinsame Frühstück im Bett gezählt und Frau Beyer habe ihren Kindern „ganz viel vorgelesen. $/ / \mathrm{hmh} / /$ Abends ähm $\mathrm{Be}=$ äh $=$ Bettgeschichten oder hab dann auch dicke Märchenbücher, jeden Abend ein Märchen oder eine Geschichte“ (III 2:05:34-2:06:10). Neben Familienausflügen ins Umland hätten sie mit ihren Kindern 


\section{Familiale Tradierungsprozesse in einer Drei-Generationen-Perspektive}

sonntags gelegentlich Wallfahrtsorte und die dortigen Gottesdienste besucht, an die sich jeweils ein Picknick angeschlossen habe (I 11:33-13:15, III 1:12:49-1:14:52). Ähnlich wie ihr Vater lässt sich Frau Beyer von dem Motiv leiten, den Kindern etwas zu bieten. Aus ihrer Sicht kann sie dieses Vorhaben aufgrund des gestiegenen ökonomischen Wohlstands im Unterschied zu ihren Eltern auf einem höheren Niveau realisieren (I 11:13-11:30). Die Bildungsorientierung hingegen scheint im Vergleich zur Vorgängergeneration an Bedeutung zu verlieren. Das könnte daran liegen, dass Frau Beyer aufgrund der Zuordnung zur Klasse der „Sonstigen“ im staatlichen Bildungssystem ohnehin wenig Chancen für ihre Kinder sieht. Es könnte aber auch ihrer Einstellung geschuldet sein, im Gegensatz zu ihrer eigenen Mutter etwas großzügiger gegenüber ihren Kindern zu sein und sich stärker in sie hineinzuversetzen (I 1:00:51-1:01:16). Das ist mit einer starken Kindzentrierung und Nachgiebigkeit verbunden, in deren Folge möglichst wenig Forderungen an die Kinder gestellt werden. Frau Beyer erwähnt weder Erwartungen, die sich auf den schulischen Bereich beziehen, noch den Kindern zugeteilte Aufgaben im häuslichen Bereich.

Ansonsten habe sie das Familienleben in weiten Teilen ähnlich wie ihre Eltern gestaltet, zumindest was das Aufwachsen der beiden größeren Kinder betreffe. Während deren Kindheit seien „die Uhren auch noch anders“ (III 1:38:31-1:38:33) gegangen:

„Da war das genau wie zu meiner Kindheit oder äh bei mir zu Hause. Mit dem Beten, mit der Kirche sonntags, //hmh//mit dem Mittag, Sonntagmittag, halb zwölf wird gegessen //hmh// oder Samstagmittag: Wir trinken Kaffee zusammen oder auch in der Woche je nach dem. Dann waren die Kinder einfach da“" (III 1:38:34-1:38:52).

Der Familienalltag verläuft für sie in tradierten Bahnen und die Familienmitglieder halten sich an die vorgegebenen Ordnungsmuster. Der gewohnte Ablauf wird erst während der Jugendzeit der jüngeren Kinder, die in die Nachwendezeit fällt, brüchig:

„Dann kam das mit den Discos. //hmh// Dann äh haben sie abends, samstags abends hier gelegen und waren todmüde. Um zehn ruft 'n Freund an: ,Ey' und dann waren sie putzmunter auf einmal und dann ging das los. (.) $\mathrm{Ja}$, und dann sind die natürlich am nächsten Morgen, Sonntagmorgen nich aufgestanden zur Kirche. //hmh// Dann sind die mitunter auch nich zum Mittagessen aufgestanden. $/ / \mathrm{hmh} / /$ Und DAS war für meinen Mann und für mich wirklich erstmal schlimm, [...] Aber dann, hab gesagt zu meinem Mann: ,Was soll's'. [...] Wenn das jetzt so ist, und wenn man andere äh Familien gehört hat: Es war da genauso. //hmh// Das, was ich eigentlich immer für unnormal gehalten habe, das war auf einmal normal. // hmh// (.) Und das äh, DAS tut mir eigentlich ein bisschen leid. //hmh// Und ich finde, da geht auch viel äh Familienleben kaputt. [...] Dann frage ich mich jetzt schon: Wie ist das nachher? Wie geben die das an ihre Kinder weiter? //hmh// (.) Ist das nachher gar nich mehr so wichtig, $/ / \mathrm{hmh} / /$ dass 


\subsection{Diffuse Tradierungslogiken}

man irgendwann mal zusammen sitzt oder was zusammen macht, ne?“ (III 1:41:35-1:43:37).

Äußere Einflüsse setzen die tradierte Alltagsstruktur außer Kraft, worauf Frau Beyer letztlich in der ihr vertrauten Weise mit Nachgiebigkeit reagiert und die von ihr abgelehnte Entwicklung hinnimmt. Zudem wirkt sie auf ihren Mann ein, sich ebenfalls mit den Veränderungen abzufinden und nimmt damit eine Vermittlerposition zwischen Vater und Kindern ein. ${ }^{181}$ Letztlich ist ihr die Wahrung eines friedlichen Zusammenlebens wichtiger als das Beharren auf tradierten Ordnungsmustern - zumal das eine wichtige Voraussetzung für die Aufrechterhaltung des familialen Zusammenhalts ist. Auch in den konkreten Erziehungsansichten manifestiert sich ihre Nachgiebigkeit, die sie zugleich rechtfertigt:
„Aber ich bin auch der Meinung, dass man äh mit übertriebener oder über- zogener Härte und Strenge auch nichts erreicht. //hmh// Da macht man vielleicht eher was kaputt, als dass man irgendwas zum Positiven wendet. $/ / \mathrm{hmh} / /$ Und äh so wie es is (.) jetzt, is es nicht unbedingt äh befriedigend. $/ /{ }^{\circ} \mathrm{hm} \% / /(1)$ Aber ich, äh es gibt auch Dinge, die ich nicht ändern kann“ (III 1:47:56-1:48:25).

Wie die Beispiele zeigen, insistiert Frau Beyer nicht darauf, die von ihren Vorstellungen abweichenden Orientierungen ihrer Kinder zu korrigieren. Sie werden aber auch nicht unbedingt akzeptiert, wie sich an ihren Missfallensäußerungen erkennen lässt. Hiermit vermeidet sie zwar konfrontative Auseinandersetzungen, es verbleiben aber latente Spannungen, die wiederum klare Positionierungen untergraben und auf diese Weise zur Diffusität beitragen.

Frau Beyer benennt einige Entwicklungen, mit denen sie unzufrieden ist. Während die älteren Kinder „ein bisschen äh die DDR-Mentalität mitbekommen“ hätten und ein bisschen bescheidener seien, seien die jüngeren Kinder stärker von der „westliche[n] Freiheit", beeinflusst, hätten sich etwas vom Glauben entfernt und würden sonntags auch mal ausschlafen statt zur Kirche zu gehen (I 14:45-15:34). Auch die Familienfeiern seien zu DDR-Zeiten anders gewesen:

„Es war eigentlich auch immer ungezwungen und [...] die Leute hatten alle gleich viel oder alle gleich wenig. $/ / \mathrm{hmh} / /$ Und es war auch eine andere Zufriedenheit. Es war eine Ehrlichkeit da (.) und auch eine andere Herzlichkeit, als man das heute oftmals äh auch äh in den Familien so findet. Es war eigentlich keiner auf den anderen neidisch" (III 30:21-30:49).

Frau Beyer assoziiert mit der Wende nicht nur positive Veränderungen. Für sie haben die gestiegenen Konsummöglichkeiten und Freiheiten auch eine korrumpierende Wirkung, die die Gemeinschaft, vor allem die der Familie, bedroht und eine Orientierung

\footnotetext{
${ }^{181}$ Diese Vermittlungsfunktion übernimmt sich auch bei anderen Gelegenheiten ein (II 21:04-22:04 ).
} 


\section{Familiale Tradierungsprozesse in einer Drei-Generationen-Perspektive}

an individuellen Bedürfnissen befördert. Sie konstatiert ein Aufbrechen der vormaligen Gleichheit in und zwischen den Familiengruppen, die sie ebenfalls als gemeinschaftsbedrohend einstuft, ohne dem aktiv entgegenwirken zu können. Diese allgemeine Gegenwartskritik erfüllt eine wichtige Funktion. Frau Beyer kann die an den Kindern kritisierten Eigenschaften den allgemeinen gesellschaftlichen Entwicklungen zuschreiben. Auf diese Weise lassen sich potenziell Konflikte mit den Kindern vermeiden, was die intergenerationellen Beziehungen entlastet. Vor diesem Hintergrund wird auch verständlich, warum Frau Beyer die positiven Aspekte ihrer Kindheit betont, nicht nur Kritik an den systembedingten Umständen ihres Aufwachsens übt und insgesamt zu einer ambivalenten Einschätzung ihres Familienlebens und ihrer eigenen Kindheit während der DDR-Zeit kommt.

Der Großelterngeneration, insbesondere mütterlicherseits, wird ein fester Platz im Familienleben eingeräumt, und Frau Beyer ist an einem regelmäßigen Kontakt zwischen ihren Kindern und ihren Eltern gelegen, den sie durch einen wöchentlichen „OmaOpa-Tag“ (III 45:56-46:31) sichergestellt habe. Insgesamt misst sie der Großelternschaft eine hohe Bedeutung zu. Die eigene Großmutter beschreibt sie als diejenige, die die Enkel umsorgt und die familialen Gepflogenheiten an die nächste Generation vermittelt, womit sie ein klassisches Großelternbild skizziert (Büchner/Brake 2010, 213). In diese Traditionslinie reiht sie sich selbst ein. Gegenüber den Enkelkindern ist sie nachgiebig. Es werde ihnen nichts verboten (III 2:08:49-2:08:55) und Frau Beyer ist ihnen emotional sehr zugewandt; sie registriert z.B., dass ihre Enkelin Magdalene gerade „den allerersten Liebeskummer hinter sich" habe (III 2:11:48-2:12:00). Neben Geborgenheit möchte sie ihnen ein „Gefühl für den Nächsten“, also Verantwortungsbewusstsein, sowie ein regionales Geschichtsbewusstsein vermitteln (III 2:15:24-2:16:44). Zudem sind ihr jahreszeitliche Rituale wichtig:

„[D]as mach ich dann aber auch schon wieder mit den Enkeln, //hmh// ne. (.) Muss sagen, äh, ich sage auch manchmal zu meinen Kindern: ,Sagt mal, haben wir das hier zu Hause nich auch so gemacht? (.) Hat euch das nich auch Spaß gemacht, wenn wir so was gemacht haben?‘ [...] Aber ich finde gerade, das is das, was den Kindern Spaß macht und was //ja, hmh, $\mathrm{hmh} / /$ die sich auch merken, ne“ (III 1:35:07-1:35:55).

Frau Beyer missbilligt das Desinteresse ihrer Kinder und sieht sich verpflichtet, bestimmte Aufgaben im Zweifelsfall selbst zu übernehmen, um Tradtionen für die Enkel zu erhalten. Frau Beyer ist bereit, selbst Abstriche zu machen, um das Wohlbefinden der Enkel sicherzustellen bzw. um intergenerationelle Familienkonflikte zu vermeiden (I 48:20-48:22, II 31:05-31:26). Sie bemüht sich, das familiale Zusammenleben zu managen. Sei es, indem sie als Vermittlerin bei familialen Konflikten auftritt (II 23:20-24:44), oder sich für die Lösung der Probleme ihrer Kinder verantwortlich fühlt und sie nicht „im Stich lassen“ möchte (II 32:50-32:55). Ihre großmütterlichen Hilfeleistungen betrachtet sie als elementar. Sie fürchtet, die jüngere Generation sei nicht so belastbar (II 32:56-33:04), weswegen die Anwesenheit einer „Pufferoma“ (II 33:12-34:34:12) wichtig 


\subsection{Diffuse Tradierungslogiken}

sei, um das Familienleben nicht zu gefährden. Den Großeltern schreibt sie eine zentrale Unterstützungsfunktion zu: „Also 'ne Oma is denke ich schon 'ne ganz besondere Person. (.) Der Opa is mehr für's Verwöhnen der Kinder da. Die Oma sicherlich auch, aber die Oma is dann auch mehr für das Praktische da @(.)@“ (II 03:30-03:46).

In einer Gesamtbilanz lässt sich festhalten, dass Frau Beyer den familialen Bezugsrahmen der familialen Sorge und Gemeinschaft weitergeben möchte, in der Art der Umsetzung jedoch recht nachgiebig ist. Zudem ist die Art und Weise, wie sie selbst den familialen Bezugsrahmen ausfüllt, tendenziell kontraproduktiv für die Etablierung eben dieses Rahmens in der nächsten Generation.

Aneignungsebene Birgit Schmidt $(* 1971)^{182}$ : Überschaubare Pflichten Die familialen Beziehungen spielen bereits in Birgit Schmidts Eingangserzählung eine dominierende Rolle. $\mathrm{Zu}$ ihrem jüngeren Bruder habe sie eine besonders enge Bindung gehabt:

„Aber dann hier mein anderer Bruder, der dann kam, da war ich ja dann neun, wo Mirko geboren is. Das war so: (.), Mutti hat immer gesagt, das is wie dein Kind, //hmh// den hab ich großgezogen. Den hab ich vom=vom ersten Tag an groß gezogen. Mit dem bin ich abends ins Bett gegangen, egal ob ich noch Fernseh gucken wollte oder nich. Der is ohne mich nich ins Bett gegangen. [...] Für den hätt ich alles gemacht“ (I 02:00-2:27).

Unverkennbar ist hier eine Tradierungslinie der familialen Fürsorge, die von Birgit Schmidt offenbar gerne wahrgenommen wird. Allerdings hat diese Tradierung von besonders intensiven Bindungen zu einem Familienmitglied bei Birgit Schmidt anders als bei der Mutter auch eine deutlich artikulierte Kehrseite. Sie habe zu ihrem Bruder Johannes „nie so das Verhältnis“ gehabt. Ähnliches gelte für das Verhältnis zur jüngeren Schwester. Sie und ihr zwei Jahre jüngerer Bruder sowie ihre Schwester seien wie „Feuer und Wasser“ gewesen und hätten sich „gezofft“ (I 01:44-01:59, 02:49-03:38). Auch wenn Birgit Schmidt einvernehmliche Momente mit diesem Bruder beschreibt, etwa das Zusammenhalten, wenn es Konflikte mit den Eltern gegeben habe (I 34:58-35:17), sind die Beziehungen zum Bruder durch deutliche Ambivalenzen geprägt: „[M]an kann, wenn man will, kann man ja viel zusammen machen. Man kann aber auch ganz schön gemein zueinander sein“ (I 04:10-4:18). Die Beziehung zur jüngeren Schwester ist ebenfalls deutlich reservierter: „Ich hab sie nich vernachlässigt, aber ich hab lange nicht so alles mitgemacht wie mit Mirko" (I 51:44-51:50). Ein Grund dafür ist sicherlich in der Ungleichbehandlung durch die Eltern zu sehen, die von Birgit Schmidt registriert wird. Ihre Schwester habe u.a. durch die gute Beziehung zum Vater größere Freiräume gehabt (I 38:18-38:46). ${ }^{183}$ Diese Wahrnehmung fördert geschwisterliche Rivalitäten und belastet die intragenerationellen Beziehungen. Zudem halten die Eltern offensichtlich nicht

\footnotetext{
${ }^{182}$ Das Interview mit Birgit Schmidt wurde am 23.02.2008 geführt.

${ }^{183}$ Die Gründe für den unterschiedlichen Umgang mit den Kindern sind sicherlich vielfältig und dürften das Resultat von individuellen, familialen und gesellschaftlichen Entwicklungsprozessen sein.
} 


\section{Familiale Tradierungsprozesse in einer Drei-Generationen-Perspektive}

an bestimmten Grundlinien der Erziehung fest, sondern ändern ihr Verhalten situationsbedingt, was wiederum das Potenzial der geschwisterlichen Eifersucht steigert.

Für Birgit Schmidt sind die familialen Beziehungen ambivalent belegt. Einerseits haben sie für sie eine große Bedeutung, sind teils positiv besetzt und ein wesentlicher Bestandteil, um das Charakteristische des Familienlebens zu beschreiben, andererseits gibt es negative Facetten, wie die ausgeprägten Geschwisterkonkurrenzen, die belastend wirken. Diese Ambivalenz existiert in abgemilderter Form bis in die Gegenwart:

„Und dann muss ich natürlich sagen, heute ziehe ich Ulmendorf vor vor Bergdorf. $/ / \mathrm{hmh} / /$ Es is mir einfach hier zu stressig im Haus. Wenn ich hierher komme und wir sind hier mal so ein paar Stunden, das is mir zu stressig. Ich hab es zu Hause so schön IDYLLISCH, RUHIG. [...] Aber na ja, @(.)@ das hab ich vorher ja auch nich gewusst. Da hat man das nich so empfunden früher, da war $/ / \mathrm{hmh} / /$ man mit drinne. //hmh// Aber wenn man denn zwischen raus is und was anderes sieht, is es doch anders, $/ / \mathrm{hmh} / /$ ne. Und dann vermisst man das dann auch nich mehr, dieses $=$ dieses $=$ diesen Trubel. //hmh// Ich komme gerne nach Hause, aber ich fahre auch gerne wieder weg" (I 05:20-06:11).

Auch wenn sie den Kontakt zur Familie teilweise als anstrengend erlebt und die ihr zur Verfügung stehenden Rückzugsmöglichkeiten schätzt, sind ihr zwischenmenschliche Kontakte elementar wichtig. Sie habe z.B. eine Anstellung relativ schnell wieder aufgegeben, weil sie alleine ohne weitere Kollegen gearbeitet habe: „[A]ber dieses Einsame. //hmh// Ich brauche auch Leute um mich“ (I 14:26-14:30).

Trotz dieser erkennbaren Ambivalenzen fällt Birgit Schmidts Blick auf ihre Kindheit relativ positiv aus:

„Eigentlich hatten wir immer ein schönes, harmonisches Familienleben. Also (.) wir hatten viele Freiheiten. Es war nich so, wie das, wie = wie man das so manchmal von anderen hört, dass man immer hunderte von Arbeiten machen muss. Nö, also da war es eigentlich relativ locker bei uns. Gut, man musste was machen, is ja logisch. Äh, aber wenn nich, haben wir uns auch mal schön im Bad versteckt. Wenn's an's Abwaschen ging, dann musst ich immer auf Toilette. @Wenn dann@meine Mutter fertig war, hat Papa immer gerufen: ,Du kannst kommen. Wir sind fertig.' Weil, also äh aber so im Großen und Ganzen war es relativ locker. //hmh// Gab keine großen Verpflichtungen, gab keinen großen Ärger“ (I 00:57-01:41).

Die Eltern werden als nachgiebig beschrieben: Sie zwingen die Kinder nicht, an sie gestellte Aufgaben zu erfüllen. Im Gegenteil: Die Kinder können sich alltäglichen Anforderungen entziehen, ohne Sanktionen fürchten zu müssen. Diese den Kindern sehr zugewandte und nachgiebige elterliche Erziehungshaltung trägt sicherlich dazu bei, mit 


\subsection{Diffuse Tradierungslogiken}

dem Familienleben zufrieden zu sein. Die mit dem Wort „eigentlich“ angedeutete Relativierung bezieht sich vermutlich auf die geschwisterlichen Konflikte und Konkurrenzen, die sich aus der Gestaltung des Familienlebens ergeben.

Wie die Passage zeigt, erweist sich vor allem der Vater als Verbündeter seiner Tochter. Er wird als derjenige wahrgenommen, der zwar die Vermeidungsstrategien der Tochter durchschaut, das aber ganz offensichtlich toleriert und somit die elterlichen Intentionen letztlich untergräbt und vereitelt. Dieses Bündnis zeigt sich auch an anderer Stelle, wenn es um Forderungen geht, die der im Haus wohnende Großvater, der Vater von Herrn Beyer, an Birgit Schmidt heranträgt:

„Der hat uns auch, der hat es auch fertiggebracht und hat uns in den Ferien frühs aus'm Bette geschmissen. //hmh// Wir sollen aufstehen, wir sollen was tun. Straße fegen, Hof fegen, egal was. [...] Also da hat dann mein Vater auf'n Tisch gehauen. //hmh// Weil da habe ich gesagt: ,Ich glaube, ich spinne. Was kommt der in mein Zimmer und will mich hier zum Bette rausschmeißen. //hmh// Wenn ich Ferien habe, habe ich Ferien“" (I 44:07-44:57).

Diese Episode erlaubt Rückschlüsse auf das innerfamiliale Machtgefüge. Der Vater ergreift Partei für seine Tochter und unterbindet großväterliche Eingriffe in die Erziehung. Birgit Schmidt kann ihre Wünsche selbstbewusst vorbringen und darf Forderungen widersprechen. Ihr wird keine passive Rolle als Befehlsempfängerin zugeschrieben, die sich den Anordnungen der älteren Generationen unterzuordnen hat. Auch verschieben sich im Vergleich zur Vorgängergeneration die Prioritäten. Während sich in den Kindheitserinnerungen Frau Beyers keinerlei Anzeichen für eine Ablehnung der an sie herangetragenen Aufgaben oder für den Verweis auf die eigenen Bedürfnisse finden, werden letztere von Birgit Schmidt deutlich artikuliert. Im Unterschied zur eigenen Mutter ist es ihr möglich, ihr Eigeninteresse als Argument einzubringen. Das verweist auf veränderte Vorstellungen in Bezug auf das Verhältnis von Arbeit und Freizeit, indem der Freizeit im Vergleich zur Vorgängergeneration ein größerer Stellenwert zugeschrieben wird.

Neben den alltäglichen Kontakten zum Großvater väterlicherseits habe es regelmäßige Kontakte zu den Großeltern mütterlicherseits gegeben, die allerdings mangels Zeit und Gelegenheiten nicht viel mit ihren Enkeln hätten unternehmen können (I 1:26:441:26:57). Hier deuten sich steigende Ansprüche in den intergenerationellen Beziehungen an. Während für Frau Beyer die Partizipation am großmütterlichen Alltag noch selbstverständlich und ausreichend ist, scheinen sich bei ihrer Tochter die Erwartungen an die Großeltern stärker auf eine kindorientierte Freizeitgestaltung zu richten.

Auch wenn Birgit Schmidt in ihrer Kindheit und Jugend in einigen Fällen gut in der Lage ist, Arbeitsaufgaben abzuwehren, ist sie nicht pflichtenlos. Sie habe für die Familie Botengänge erledigt und aus ihrer Sicht verantwortungsvolle Aufgaben wie das Kassieren von Rechnungen für den Großvater übernommen. Sie sei gern „der Laufbursche“ gewesen, da sie so weniger zu häuslichen Arbeiten herangezogen worden sei 


\section{Familiale Tradierungsprozesse in einer Drei-Generationen-Perspektive}

(1:18:35-1:20:46). Die beschriebenen Aufgaben dürften zudem deswegen für Birgit Schmidt reizvoll gewesen sein, da sie zwischenmenschliche Kontakte und kommunikativen Austausch beinhalten. Das kommt ihrer bereits aufgezeigten Gemeinschaftsorientierung entgegen und fördert sie zugleich. Durch die für den Großvater erledigten Aufgaben erfährt sie zusätzlich eine ihr Selbstbewusstsein stärkende Wertschätzung, die in Zusammenhang mit der Geschwisterkonkurrenz gebracht wird. Ihr Großvater habe die jüngere Schwester nicht mit diesen Aufgaben betrauen können (I 1:19:37-1:19:54). Das zielt darauf, sich selbst als verlässlicher und kompetenter im Vergleich zur Schwester darzustellen. Es zeigt auch, dass die Beziehungen zum Großvater nicht nur konfliktbehaftet gewesen sind, sondern auch positive Aspekte aufweisen, was beispielhaft die Ambivalenz der Generationenbeziehungen (Lüscher/Liegle 2003, 286, 288) illustriert. Die Pflichten, die mit Anreizen verbunden sind, werde von Birgit Schmidt gern ausgeführt. Das betrifft z.B. Aufgaben, für die sie Geld erhalten habe (I 1:20:48-1:21:48). In der Gesamtschau unterstreicht das die gestiegene Bedeutung und Beachtung der eigenen Interessen und Bedürfnisse.

In potenziellen Konfliktsituationen bemüht sich Birgit Schmidt, ihre Mutter als Vermittlerin zu gewinnen und hierdurch unliebsame Aussprachen und letztlich ein Stück Verantwortung zu delegieren. Als Birgit Schmidt ledig schwanger geworden sei, habe sie das zunächst der Mutter erzählt, die geschockt gewesen sei. Da Birgit Schmidt Bedenken hat, diese Neuigkeit dem Vater mitzuteilen, versucht sie, die Mutter dazu zu bringen, diese Aufgabe für sie zu übernehmen (06:40-07:31). Auch wenn der Vater in Bezug auf Arbeitsverpflichtungen gegenüber seiner Tochter eher großzügig ist, gilt das nicht für das Einhalten moralischer Standards. Die hier gezogenen Grenzen sind nicht so einfach zu verschieben. Birgit Schmidt nimmt ihren Vater diesbezüglich als ,sehr altmodisch“ wahr (I 53:45-53:48). Sie fürchtet die Auseinandersetzung mit ihm (I 53:48-53:57), weswegen sie diese Aufgabe an die Mutter übertragen möchte, die offensichtlich in dieser Hinsicht weniger dogmatisch ist. Der Entschluss zu heiraten (I 08:12-09:05) stimmt den Vater schließlich versöhnlich (I 55:42-55:46). Die Einstellung ihres Vaters erklärt Birgit Schmidt mit Verweis auf die damaligen Rahmenbedingungen:

„[J]a es waren andere, //hmh// es waren andere Zeiten. //hmh// Es war noch so 'n bisschen die Zeit, wo: erst geheiratet wurde und dann Kinder kamen, //ach so, hmh//, ne. Es war so DDR, kindmäßig war: Erst wurde geheiratet und dann wurde man schwanger, dann kriegte man Kinder. $/ / \mathrm{hmh} / /$ Das war, das ging da schon los, dass äh immer mehr schon vorher Kinder auch hatten $/ / \mathrm{hmh} / /$ oder, aber mei-, da war mein Vater sehr altmodisch" (I 53:14-53:45).

Auch wenn ihr Verweis auf die DDR-Verhältnisse an dieser Stelle nicht unbedingt zutrifft, ist in ihrer Argumentation ein Mittel zu sehen, um die persönlichen Beziehungen zum Vater zu entlasten, indem seine Einstellung an die jeweiligen Rahmenbedingungen rückgebunden und somit praktisch entschuldigt und für sie akzeptabel wird. 


\subsection{Diffuse Tradierungslogiken}

Die gesellschaftlichen Rahmenbedingungen sind auch in anderer Hinsicht für die Betrachtung der intergenerationellen Beziehungen relevant. Es werden weniger die Eltern als vielmehr der Staat als reglementierend wahrgenommen. Als Kinder hätten sie zu Hause immer ihre Meinung äußern dürfen (I 1:38:51-1:39:45), was allerdings keine großen Auswirkungen gehabt habe, da die Entscheidungsmöglichkeiten durch äußere Faktoren begrenzt gewesen seien:

„Wir waren dann ja, es gab ja nich so viel zu entscheiden zu DDR-Zeiten. $/ / \mathrm{hmh} / /$ Das waren ganz andere Zeiten, das kann man mit heute gar nich vergleichen. //hmh// Das waren zwei Welten, //hmh// ne. Das gab's ja so gar nich. Es gab ja normalerweise offiziell nich mal ARD und ZDF, //hmh// jetzt im Fernsehen, ne. //ja, hmh// Man kann das heute nicht mehr mit, mit damals vergleichen. Es gab ja auch nichts im Laden zu kaufen. Außer Butter und Milch und Brot. //hmh// Also obstmäßig gab’s nur Äpfel. //hmh// Deshalb, man konnte das (nee), man kann das heute gar nich mehr so sagen, wie alleine entscheiden. Das gab's ja noch nich. Wir, wir sind, der Staat hat ja für uns entschieden $/ / \mathrm{hmh} / /$ im großen Sinne. [...] Deshalb man, man wurde gar nich, man kam gar nicht so in die Verlegenheit //hmh// als Kind, was entscheiden zu müssen“ (I 1:39:46-1:41:00).

Für Birgit Schmidt sind es weniger die Eltern, die Grenzen setzen und die Spielräume einschränken, als vielmehr ein als autoritär wahrgenommener Staat. Durch diese Sichtweise werden abermals die intergenerationellen Beziehungen zu den Eltern von Konfliktpotenzialen entlastet.

Trotz der generellen Nachgiebigkeit der Eltern habe es gelegentlich körperliche Bestrafungen (I 34:51-34:55) und Fernsehverbote (I 1:34:08-1:34:19) gegeben, was auf die nicht eindeutige elterliche Erziehungspraxis verweist, die eben nicht nur nachgiebig und kindorientiert gewesen ist, sondern bisweilen auch autoritäre Züge aufweist. Mit dem Verweis auf das anstrengende kindliche Verhalten (I 42:17-43:11) entschuldigt sie retrospektiv diese Praxen, was dazu dient, die intergenerationellen Beziehungen zu entlasten.

Als konkrete Regel erinnert Birgit Schmidt die Verpflichtung zum sonntäglichen Kirchgang und zur Schülermesse (I 1:31:29-1:31:58). Die Eltern hätten Wert auf die Einhaltung der allgemeinen Höflichkeitsregeln gelegt (I 1:36:39-1:37:45). Letzteres ist als Maßnahme zu betrachten, die letztlich die Fokussierung auf die zwischenmenschlichen Bindungen auch außerhalb der Familie unterstützen kann, indem ein Sozialkontakte förderndes Verhalten eingeübt wird. Insgesamt scheint der Alltag von Birgit Schmidt keinen großen Einschränkungen durch die Eltern zu unterliegen, was zu einer positiven Bewertung der Eltern-Kind-Beziehung beiträgt:„Ich hatte ein gutes Verhältnis zu meinen Eltern, ich hatte ein supergutes Verhältnis. Ich durfte ja alles" (I 1:44:09-1:44:14). Bis in die Gegenwart könne sie auf die elterliche Unterstützung vertrauen (I 1:25:10-1:25:59, 1:28:59-1:30:16) und es finden sich keinerlei Hinweise, dass die Eltern einen Leistungsdruck im schulischen oder außerschulischen Bereich auf die 


\section{Familiale Tradierungsprozesse in einer Drei-Generationen-Perspektive}

Kinder ausüben. Die Mutter habe sich Zeit genommen und den Kindern bei den Hausaufgaben geholfen (I 1:26:00-1:26:09), aber anscheinend keine weitergehenden Forderungen an sie gestellt. Auch habe die Mutter jahreszeitliche Rituale wie Adventsstunden für die Kinder gestaltet (I 1:35:45-1:36:09). All dies sind Aspekte, die die positive Beurteilung der eigenen Kindheit verstärken und dazu beitragen, das elterliche Verhalten als freigiebig, an den aktuellen Bedürfnissen ausgerichtet sowie kindorientiert zu erleben. Einzig der Umstand, kein Taschengeld erhalten zu haben, lässt sich in gewisser Weise als kontrollierend betrachten. Sie habe als Kind dann Geld von ihrer Mutter bekommen, wenn sie es gebraucht habe (I 1:21:49-1:22:06). Hierdurch lässt sich von der Mutter die Geldverwendung überwachen, was aber zugleich bedeutet, in diesem Punkt die Entwicklung eines eigenverantwortlichen Umgangs im finanziellen Bereich eher zu behindern.

Insgesamt ergibt sich für die Kindheit von Birgit Schmidt eine mehrfache Diffusität, die sich auf die Tradierung auswirkt. Die elterliche Ausgestaltung des familialen Bezugsrahmens führt zu Konflikten zwischen den Geschwistern und ist damit für das intendierte Ziel, die innerfamilialen Bindungen zu stärken, teilweise kontraproduktiv. In der Folge sind die inter- und intragenerationellen Beziehungen mit Ambivalenzen verbunden und teilweise nicht eindeutig für Birgit Schmidt zu verorten. Schließlich werden zwar Regeln gesetzt, wenn auch wenige, die Einhaltung aber nicht immer konsequent durchgesetzt. Ebenso ist die elterliche Nachgiebigkeit zwar kennzeichnend für das Erziehungssetting, wird aber nicht durchgängig angewendet.

Vermittlungsebene Birgit Schmidt (*1971): Partielle Regellosigkeit Bei der Erziehung der eigenen Kinder orientiert sich Birgit Schmidt in vielen Aspekten an der selbsterfahrenen Erziehung. Die familialen Bindungen bilden auch im Zusammenhang mit der eigenen Familienphase ein wichtiges Thema. Die Schwiegermutter habe Magdalene „so ziemlich großgezogen“ (I 09:55-10:00). Retrospektiv stellt Birgit Schmidt fest, sie habe „nich viel gehabt von dem Kind als Baby“ (I 10:59-11:03). In der Folge sei ihr die Tochter als Kind auch „richtig fremd geworden“ (I 10:36-10:39):

„Die [Tochter, C.R.] hat sonntags zwar mit uns Mittag gegessen, wenn wir alle zu Hause waren, die hat auch mit uns 'nen Ausflug gemacht, //hmh// aber wenn es dann ans Schlafen ging, (ging), dann ging sie wieder runter. $/ / \mathrm{hmh} / / \mathrm{Zu}$ Oma. Immer. Die ging schon in die Schule, da hat sie noch bei Oma geschlafen. Die hat ja fast bis zehn Jahre bei Oma geschlafen“ (I 1:05:25-1:05:42).

Diese Alltagsgestaltung - die Tochter verbringt den größten Teil des Tages bei der Großmutter - wirkt sich auf die Generationenbeziehungen aus:

„Oma war sowieso, wenn Oma was gesagt hat, hat sie [die Tochter, C.R.] das sofort gemacht. Wenn ich was gesagt habe, ich konnte sagen oder nich, $/ / \mathrm{hmh} / /$ das hat sie dann gar nich interessiert. $/ / \mathrm{hmh} / /$ Sie hat mich dann 


\subsection{Diffuse Tradierungslogiken}

auch irgendwo nicht mehr als Mutter wahrgenommen, $/ / \mathrm{hmh} / /$ so akzeptiert, ne. Oma war für sie das Nonplusultra“ (I 1:03:49-1:04:07).

Birgit Schmidts Rollenerwartungen, in denen ihr als Mutter gegenüber der Tochter eine von dieser anerkannte Autorität zukommt, wird durch die familiale Beziehungskonstellation unterwandert. Für das Zusammenleben von Eltern und Tochter hat das Betreuungsarrangement insgesamt weitreichende Folgen:

„Aber wenn wir in den Urlaub gefahren sind, (sind) wir ja ohne Oma in den Urlaub gefahren. [...] [D] esto länger wir geblieben sind, desto mehr kriegte Magdalene Heimweh. //hmh, hmh// Wir mussten regelrecht nach Hause fahren, also zwei Wochen Urlaub war nich drinne. [...] Die war irgendwo nur auf Oma fixiert. //hmh// Die war regelrecht auf Oma fixiert. (.) Wie gesagt, bis dass sie (2), bis dass Dirk geboren is“ (I 1:04:17-1:05:19).

Die Großmutter ist aus dieser Perspektive die zentrale Bezugsperson für Magdalene, was wiederum die Beziehungsqualität zwischen Tochter und Mutter sowie zwischen Mutter und Schwiegermutter beeinflusst. Anflüge eines konkurrenzhaften Verhältnisses zur Schwiegermutter sind unverkennbar und wirken sich auf die Gestaltung des familialen Zusammenlebens aus. Die Haushalte seien „von Anfang an“ getrennt gewesen (I 11:28-11:42): „Kann jeder für sich machen, was er will. Kann jeder essen, wann er will. [...] Jung und alt, das geht mal zusammen, aber nich nur" (I 49:35-49:52). Trotz dieser proklamierten Trennung ist die Schwiegermutter in der Praxis fest in die familiale Aufgabenteilung eingebunden. Birgit Schmidt beschreibt die Zusammenarbeit mit der Schwiegermutter als ein gegenseitiges Abhängigkeitsverhältnis: „Sie ist auf mich genauso angewiesen wie ich auf sie angewiesen bin" (II 15:30-15:33). Zugleich manifestiert sich hierin die Reziprozität der Generationenbeziehungen, auf die Birgit Schmidt Wert legt: „Eine Hand wäscht irgendwo die andere“ (II 05:51-05:53). Im Unterschied zur eigenen Mutter ist sie stärker auf die Wahrung der eigenen Interessen bedacht, die sie auch durchsetzt. Sie habe z.B. einen lang ersehnten Familienurlaub trotz einer Erkrankung der Schwiegermutter nicht abgesagt (II 01:53-05:47). Zusammenfassend sind die Beziehungen zwischen Birgit Schmidt und ihrer Schwiegermutter durch deutliche Ambivalenzen gekennzeichnet. Die Unterstützung durch die Schwiegermutter wird sowohl als Hilfe in Anspruch genommen, aber auch manchmal als Belastung erlebt, wobei Birgit Schmidt durchaus anerkennt, dass es umgekehrt genauso sein dürfte (II 06:21-06:25). Einerseits ist ihr die Zusammenarbeit wichtig. Sie ist nicht nur eine Hilfe, sondern leistet darüber hinaus einen wichtigen Beitrag zur Aufrechterhaltung der familialen Gemeinschaft und des familialen Zusammenhalts. Andererseits finden sich Abgrenzungsbemühungen und der Wunsch nach Eigenständigkeit und Unabhängigkeit. Der Versuch, beides zu realisieren, forciert das ambivalente Erleben der Generationenbeziehungen.

Die als belastend wahrgenommenen Elemente führen zu mehr oder weniger versteckten Rivalitäten, die sich konkret in der Beziehungsgestaltung zu den Kindern niederschlagen und über diese ausagiert werden mit unmittelbaren Folgen für das Erziehungsarrangement. Die Betreuung des zweiten Kindes betrachtet sie als ihre Aufgabe: „Na 


\section{Familiale Tradierungsprozesse in einer Drei-Generationen-Perspektive}

ja, und dann hab ich immer zu meiner Schwiegermutter gesagt: ,Und jetzt kriege ich 'n Kind für mich. //hmh// Jetzt kriege ich mein Kind"“ (I 12:01-12:08). In der Folge habe sie ihre Vorstellungen der Kompetenzzuständigkeiten auch durchgesetzt: „Und dann hab ich dann Dirk gekriegt. Und [...] das is wirklich MEIN Kind. //hmh// Da is (.), der wäre auch nie so zu Oma gegangen. Das war nich (.), der wol-, ging nur zu mir. Das war mein Kind“ (I 12:20-12:37). Birgit Schmidt überträgt ihre Konflikte aus der Herkunftsfamilie auf ihre eigene Familie, wobei die Rollen anders verteilt werden. Aus primär intragenerationellen Auseinandersetzungen zwischen den Geschwistern in der Herkunftsfamilie werden intergenerationelle zwischen ihr und der Schwiegermutter. Im Unterschied zu ihrer eigenen Mutter reagiert sie aber nicht nur auf äußere Einflüsse, sondern wehrt sich durch aktive Steuerungsversuche.

Die Ungleichbehandlung der Kinder ist ein zentraler Bestandteil des Erziehungssettings. Das stellt in gewisser Hinsicht eine Parallele zur Erziehung durch die Vorgängergeneration dar, ist aber in den beiden Generationen unterschiedlich motiviert. Während die Mutter, Frau Beyer, die unterschiedlich ausfallende mütterliche Aufmerksamkeitsverteilung mit den Bedürfnissen der Kinder begründet, ist das in der nächsten Generation anders. Birgit Schmidt ist diejenige, die mit der Gestaltung der Eltern-KindBeziehung unzufrieden ist und das beim zweiten Kind verändert. Insgesamt seien sowohl die Art der Beziehung als auch der konkrete Umgang mit dem Sohn anders konturiert als mit der Tochter:
„Aber es war halt doch nich so dieses Verhältnis so wie nachher mit Dirk, ne. //hmh// Da war sie dann auch manchmal eifersüchtig. [...] Wenn Dirk frühs runterkommt, der muss mich erst mal drücken, wenn der frühs auf- steht. //hmh// Und wenn Magdalene runterkommt: ,Mo::rgen. ' Das is auch so ein bisschen so ein Morgenmuffel. Und Dirk überhaupt nich. Sie sagt nur: ,Guten Morgen', und schnappt ihr Frühstück und geht in die Schule. [...] Sie kommt jetzt manchmal und sagt: ,Oh Mama, oh Mama, oh Mama.' Jetzt ja. Aber früher. Jetzt geht sie auch nicht mehr so viel nach ${ }^{\circ} \mathrm{Oma}$ runter. ${ }^{\circ} / / \mathrm{hmh} / / \mathrm{Das}$ is dann irgendwo vielleicht auch zuviel ge- wesen. $/ / \mathrm{hmh} / /$ Oma mit ihrer Omaliebe hat sie vielleicht auch zu sehr erdrückt, //hmh// ne“(I 1:07:26-1:08:25).

Diente der besondere Fokus auf die familialen Bindungen in der Kindheit Frau Beyers u.a. noch als Schutz gegen potenzielle Bedrohungen von außen, verlagert sich der Schwerpunkt aktuell auf die Arbeitsteilung zwischen Birgit Schmidt und ihrer Schwiegermutter sowie auf das familiale Beziehungsnetz. Die konkrete Gestaltung der familialen Beziehungen führt dazu, dass Magdalene sich verstärkt um die mütterliche Aufmerksamkeit bemüht, wodurch gleichzeitig die Bedeutung der Großmutter als Bezugsperson aus Birgit Schmidts Perspektive relativiert wird. Hierdurch mag die Aufmerksamkeit der Kinder für die intergenerationellen Beziehungen erhöht werden, es belastet aber zugleich die inter- und intragenerationellen Bindungen. Trotz der erkennbaren Ambivalenzen und Schwierigkeiten erfahren die familialen Bindungen im Familienalltag eine 


\subsection{Diffuse Tradierungslogiken}

besondere Aufmerksamkeit und werden aktiv bearbeitet. Die lebensweltliche Verankerung ist somit auch in dieser Familie ein Tradierungskanal, wenn auch die konkrete Umsetzung nicht intendierte und den familialen Bezugsrahmen konterkarierende Konflikte nach sich zieht.

Fester Bestandteil des Familienlebens ist das nachmittägliche Kaffeetrinken:

„Und nachmittags, wenn ich da komme, dann trinkt man erst mal Kaffee. $[\ldots][\mathrm{U}]$ nd dann müssten = müssen die Kinder erst mal alles loswerden, was sie so am Tage $/ / \mathrm{hmh} / /$ so hatten, dann müssen wir noch mal die Hausaufgaben angucken vom Dirk. Magdalene macht ja alles alleine, da brauch ich mich ja gar nich kümmern“ (I 27:44-28:07).

Birgit Schmidt legt somit ebenfalls Wert auf gemeinsame Familienzeiten. Die Mahlzeiten dienen als Gelegenheiten zu einem zwanglosen kommunikativen Austausch über den Alltag. Hierin ist eine beiläufige Strategie zu sehen, um die familialen Bindungen zu stärken. Die Kinder können das thematisieren, was sie aktuell beschäftigt, was es ihnen erleichtern dürfte, dieses Angebot mitsamt dem ihm inhärenten Bezugsrahmen aufzunehmen. Ein ähnlicher Effekt lässt sich für die gemeinsamen Freizeitunternehmungen annehmen:

„Wir machen so was auch mit Kindern. Wir fahren auch sonntags dann viel ins Bad. [...] Weil der Kleine schw-, geht gerne Schwimmen. //hmh// [...] Fällt dann das Mittag zu Hause aus. Dann essen wir dann im Bad ein bisschen was" (I 32:04-32:21).

An dieser Passage ist ebenfalls gut erkennbar, dass sich die mütterliche Aufmerksamkeit stark auf den Sohn richtet. Das lässt sich sicherlich teilweise mit dem Alter der Kinder erklären, ist aber auch ein Ausdruck für die besonders engen Bindungen zum Sohn. Die Freizeitunternehmungen, die mal mit und mal ohne Kinder stattfinden, sowie Familienurlaube sind für Birgit Schmidt ein wichtiger Ausgleich zum Arbeitsleben:

„[D]ann gönnen wir uns auch dann immer mal was, //hmh// ne. [...] Wir gönnen uns nun nich gerade große Luxusurlaube, $/ / \mathrm{hmh} / /$ so, aber dann gönnen wir uns mal, und wenn wir nur 'n Tag irgendwo hinfahren, ne. Und das muss auch irgendwo sein" (I 33:01-33:20).

Stand für ihre eigene Mutter, Frau Beyer, noch die Maxime, den Kindern bei der Freizeitgestaltung etwas zu bieten, im Vordergrund, geht dies bei Birgit Schmidt über zu der Einstellung, sich etwas zu gönnen, was die Erwachsenen ausdrücklich mit einschließt. Das verweist auf eine veränderte Wahrnehmung der Elternrolle. Lässt sich in der Vorgängergeneration noch eine starke Kindzentrierung mit Tendenzen zur Überbehütung feststellen, die sich in einer großen Fürsorglichkeit sowie in der eigenen Bedürfnislosigkeit manifestiert, ändert sich das in der Folgegeneration. Birgit Schmidt ist es möglich, die eigenen Interessen zu berücksichtigen und die vormals starke Fokussierung auf die 


\section{Familiale Tradierungsprozesse in einer Drei-Generationen-Perspektive}

Kinder scheint nachzulassen. Die Mutter wird somit im Generationenverlauf zunehmend als Person mit eigenen Bedürfnissen wahrgenommen, die auch artikulierbar und umsetzbar sind.

Den Kindern würden kaum Verbote oder Regeln gesetzt:

„Nee, ${ }^{\circ}$ ich glaube meine Kinder haben überhaupt gar keine Verbote oder Regeln.`@Die wachsen, glaube ich wild auf, wenn ich so drüber nachdenke.@ Wenn du manchmal so mitkriegst, was, was andere so machen müssen. [...] (Weil) eigentlich haben meine Kinder eigentlich 'n lockeres Leben. //hmh// Di:e haben eigentlich keinerlei Verpflichtungen, keinerlei Aufgaben, die sie jetzt regelmäßig jede Woche oder was $/ / \mathrm{hmh} / /$ machen müssen. //hmh// Eigentlich nicht. //hmh// Wenn sie auch immer der Meinung sind, sie müssen so viel tun. Aber das machen sie eigentlich erst dann, wenn ich zu Hause bin. //hmh// Dass sie dann, dass ich sage: ,Hier los, ab, hoch, wischen.' [...] Und dann macht sie [die Tochter, C.R.] das hin und her und dann macht das von einer Ecke zur anderen und dann ärger ich mich hinterher, dass ich überhaupt was gesagt habe. (Dann sagt zum Beispiel____ ): Du müsstest viel härter mit deinen Kindern umgehen. Viel strenger müsstest du sein.' Das krieg ich dann auch immer vorgehalten. $/ / \mathrm{hmh} / / \mathrm{A}$ :ber was ich als, wo sie kleiner waren, versäumt habe, kann ich jetzt auch nich mehr reindreschen. [...] Also eigentlich leben @meine Kinder regelfrei@“(II 15:44-18:20).

Indem wenig Regeln gesetzt werden, wird zugleich das intergenerationelle Konfliktpotenzial minimiert. Es könnte sich folglich um eine implizite Strategie der Konfliktvermeidung handeln. An die Kinder werden wenig Anforderungen gestellt, und sie unterliegen kaum Zwängen. All das sind Elemente, die dazu beitragen, die intergenerationellen Beziehungen insgesamt als positiv zu bewerten und dadurch Tradierungsprozesse zu fördern. Allerdings bleibt in diesem relativ regelfreien Setting u.U. vage, was überhaupt vermittelt werden soll, was wiederum eine Tradierung erschwert. Im Erziehungsarrangement von Birgit Schmidt ist ähnlich wie bei ihrer Mutter eine große Nachgiebigkeit zu erkennen. Die von ihr aufgestellten Anforderungen an die Kinder werden nicht mit Nachdruck verfolgt, sondern nur gelegentlich artikuliert und noch seltener wirklich eingefordert.

Auch wenn den Kindern relativ große Freiheiten eingeräumt werden, bleiben trotzdem Asymmetrien im intergenerationellen Machtverhältnis erhalten. Das zeigt sich an den gelegentlich ausgesprochenen mütterlichen Anweisungen, deren Befolgung erwartet wird. D.h. es gibt durchaus Forderungen, die aber nicht konkret geregelt und dadurch transparent werden. Stattdessen ist ihnen ein willkürliches und situationsspezifisches Element inhärent, das u.U. die Entwicklung von Eigenständigkeit und Selbstorganisation untergräbt. Auch andere Elemente des Erziehungsarrangements verweisen auf die asymmetrische Machtbalance. Gelegentlich greife sie auf Sanktionen aus dem autoritären Erziehungsrepertoire zurück, wenn sie sich nicht mehr zu helfen wisse (I 


\subsection{Diffuse Tradierungslogiken}

35:36-35:48 ). D.h. es gibt Grenzen, die die Kinder nicht überschreiten dürfen, wobei autoritäre Verhaltensweisen, auch wenn sie vorkommen, als Ausnahme eingeordnet werden.

In der obigen Passage fällt auf, dass es eher mit der Tochter als mit dem Sohn zu Konflikten kommt; die Beziehung zur Tochter folglich als konflikthafter erlebt wird. Das mag dazu beitragen, die Beziehung zum Sohn im Gegenzug als besonders eng $\mathrm{zu}$ betrachten. Allerdings richtet sich das mütterliche Augenmerk im Bedarfsfall auch auf die Tochter und ihr emotionales Wohlbefinden: Magdalene habe gerade „ihre erste große Liebe verloren“, was Birgit Schmidt als Mutter „unheimlich weh“ getan habe (I 20:16-20:36).

Auch wenn Birgit Schmidt konstatiert, ihre Kinder wüchsen „regelfrei“ auf, existieren Regeln. Ihre Kinder seien ebenso wie sie selbst zu Freundlichkeit, Höflichkeit und Hilfsbereitschaft erzogen worden (II 18:52-19:10). Hiermit zeichnet Birgit Schmidt eine Tradierungslinie von der selbst erfahrenen zu der von ihr praktizierten Erziehung und pro-soziale Orientierungen bleiben in dieser Familie relevant. In anderen Bereichen gibt es Unterschiede, z.B. in Bezug auf den sonntäglichen Gottesdienstbesuch:

„Heute is man da auch lockerer mit den Kindern. Heute stehen sie nich mal auf, wenn sie nich gefeiert haben.@(.)@ Da is dann mein Mann ein bisschen stren(ger). Aber der is ja nie da. Der meckert dann immer erst, wenn er zu Mittag erst nach Hause kommt. //hmh//, Wart ihr in der Kirche?‘ (1) Gucken sie sich nich mal an. Einer da, einer da. //hmh// ,Nö.‘ (.) Da is dann mein Mann ein bisschen strenger. //hmh// (Ach) bei uns is aber auch schon um halb neune frühs Kirche" (I 1:34:41-1:35:11).

Der Vater ist gegenüber seinen Kindern beharrlicher. Bei Birgit Schmidt hingegen zeigt sich abermals ihre Nachgiebigkeit. Zwar widerspricht sie ihrem Ehemann nicht, sie unterstützt seine Forderungen aber auch nicht, sondern bringt Verständnis für die Kinder auf.

Im Bereich der schulischen Bildung werden keine überdurchschnittlichen Erwartungen an die Kinder herangetragen. Der Sohn würde trotz guter schulischer Leistungen nicht zum Gymnasium gehen, da er dies aus Angst vor im Vergleich zur Grundschule schlechteren Noten nicht wolle (I 22:52-23:09): „Und zwingen bringt nichts. //hmh// Dann wird's gar nichts. Ne, das, der geht jetzt auf die Regelschule nach Großdorf. [...] Magdalene is auch in Großdorf gewesen“ (I 1:09:14-1:09:39). Vordergründig wird dem Sohn die Entscheidungsfreiheit gelassen. Da es aber von elterlicher Seite anscheinend keine Initiative zum Abwägen der verschiedenen Argumente gibt, sondern vielmehr der Hinweis erfolgt, dass auch die Tochter zu derselben Schule gegangen sei, liegt die Vermutung nahe, als wäre die mütterliche Zustimmung zum Entschluss des Sohnes auch ein wenig der Angst vor Veränderungen geschuldet. Diese Interpretation wird durch eine weitere Episode gestützt. Birgit Schmidt habe eine Mutter-Kind-Kur beantragt, die auch genehmigt worden sei: „Ich war überrascht [...]. Oh ne, und dann haben wir uns mit@abgefunden@, nun fahren wir auch.Oh:, mir graut’s.@Oh, na ja@. Wir fahren ja 


\section{Familiale Tradierungsprozesse in einer Drei-Generationen-Perspektive}

auch ganz schön weit“(I 21:03-21:17). Hier zeigt sich ebenfalls eine Verunsicherung angesichts unbekannter Situationen. Das lässt sich in eine familiale Entwicklungslinie einordnen. Während ihr Großvater noch stark darauf bedacht gewesen ist, die sich bietenden Chancen für die Kinder zu ergreifen, zumindest was den Bildungsbereich betrifft, verändert sich diese Haltung bereits bei seiner Tochter, Frau Beyer. In deren Wahrnehmung dominiert das eher notgedrungene Reagieren auf äußere, gesellschaftliche Impulse und die eigenaktive Gestaltung tritt verschiedentlich in den Hintergrund. Thre Tochter, Birgit Schmidt, schließlich zögert ebenfalls, die sich den Familienmitgliedern bietenden Möglichkeiten positiv als Chancen zu begreifen und zu nutzen, zumindest wenn es darum geht, für die Familie unbekannte Bereiche zu erschließen.

\section{Aneignungsebene Magdalene Schmidt $\left({ }^{*} 1992\right)^{184}$ : Zwischen Konflikt und Nähe} Die elterliche Erziehung wird von Magdalene ambivalent beurteilt. Einerseits sei die Erziehung eine Mischung aus „nicht zu streng [...], aber auch nicht zu locker“ gewesen (27:09-27:16), andererseits seien sie teilweise „ziemlich streng“ (30:21-30:24) gewesen. Diese etwas widersprüchliche Einordnung steht in direktem Zusammenhang damit, wie Magdalene die familialen Beziehungen erlebt, die ähnlich wie bei ihrer Mutter bereits in der Eingangserzählung einen breiten Raum einnehmen. Sie thematisiert ihr Familienleben anhand der Beziehungsqualität zu einzelnen Familienmitgliedern. Eine herausgehobene Position nimmt die Großmutter väterlicherseits ein, die im selben Haus wohnt. Sie wird von ihr als zentrale Bezugs- und Betreuungsperson des Alltags beschrieben. Sie habe bei ihrer Großmutter gegessen und geschlafen (00:38-01:05), die Großmutter habe ihr bei den Hausaufgaben geholfen (37:23-37:40) und sie habe in der Vergangenheit den Alltag weitgehend bei ihr verbracht:

„(Also) früher hab ich dann auch immer sehr viel bei meiner Oma gelebt. $/ / \mathrm{hmh} / /$ Ich hab ja, eigentlich war ich schon [...] ihr Kind praktisch so. $/ / \mathrm{hmh} / /$ Und das, ja das, hat sie mich bestimmt auch öfters mal bevorzugt (.) gegenüber den anderen, weil ich mit ihr auch sehr viel Kontakt hatte“ (6:01-06:27).

Magdalene bringt eine besonders enge Bindung zwischen Großmutter und Enkelin zum Ausdruck, die auf Wechselseitigkeit beruht und durch den Umstand verstärkt wird, dass die Großmutter immer für sie da gewesen sei: „Und deshalb hab ich auch schon guten Draht zu meiner Oma. //hmh// Und das merk ich halt jetzt auch noch, dass, ich bin halt ihre liebste Enkelin von allen $/ / \mathrm{hmh} / /$ und so. U:nd das is schon ganz schön“ (01:06-01:21). Ähnlich wie ihre Mutter richtet Magdalene ein besonderes Augenmerk auf die Beurteilung der Beziehungsqualität zu den einzelnen Familienmitgliedern sowie hiermit verbunden auf die Frage von Bevorzugung und Benachteiligung. Was das Verhältnis zur Großmutter betrifft, profitiert Magdalene von der Beziehungskonstellation,

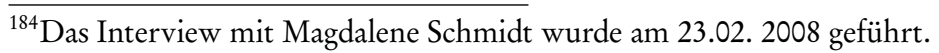




\title{
4.3 Diffuse Tradierungslogiken
}

was die emotionale Bindung zur Großmutter steigert. Die Großmutter sei bis heute eine wichtige Ansprechpartnerin für Magdalene:

\begin{abstract}
„Und auch wenn meine Eltern keine Zeit haben zum Reden oder so, kann ich auch immer zu ihr kommen. //hmh// (.) Ja, und das is immer schon ziemlich hilfreich. Sie versteht mich da auch immer noch und das is dann immer ganz gut. //hmh// Weil, manchmal will halt man doch nich immer über alles mit der Freundin reden, auch was Familie betrifft. Und wenn man halt dann mit der Mutter redet, dann kann man auch noch 'n Rat von der Oma holen. //hmh// Und das is auch immer sehr hilfreich teilweise. $/ / \mathrm{hmh} / /$ Oder auch wenn in der Familie n-, so Kleinigkeiten sind oder so, wenn ich mi-, mit meinem Bruder gestritten habe oder irgendwie so was, $/ / \mathrm{hmh} / /$ dann is sie halt auch immer dann da und dann sagt sie: ,Ja, das hast du gut gemacht' oder ,das war halt völlig falsch' oder so. Meine Oma spielt da schon große Rolle. //hmh// Zwar is das jetzt nich mehr, nich mehr immer so, weil ich hab mich ja auch im Laufe der Zeit verändert und nehme das jetzt auch nich manchmal so ernst, setze da manchmal eher meinen eigenen Kopf durch. $/ / \mathrm{hmh} / /$ Aber im Nachhinein hat sie dann meistens doch schon noch recht" (01:32-02:44).
\end{abstract}

Magdalene sucht den intergenerationellen Austausch und die Großmutter wird in der Grundtendenz als kompetente Ratgeberin betrachtet. Die einzelnen Generationen sind durch Kommunikationsprozesse miteinander verbunden, wodurch eine gemeinsame familiale Lebenswelt hergestellt wird. Magdalene beschreibt ein Vertrauensverhältnis zu Mutter und Großmutter, was sich darin manifestiert, dass sie bestimmte Angelegenheiten, u.a. familiale, lieber mit ihnen bespreche als mit einer Freundin. Sie orientiert sich offenbar an der Maxime, familiale Themen auch in der familialen Sphäre zu belassen. Somit scheint sich eine für die Kindheit ihrer Großmutter mütterlicherseits, Frau Beyer, gültige Orientierung bis heute zu erhalten; allerdings mit einem wichtigen Unterschied. Während für Frau Beyer die familiale Abschottung in ihrer Kindheit eine schlichte Notwendigkeit zum Schutz der Familie gegenüber befürchteten staatlichen Sanktionen darstellte, fällt dieses Argument als Begründung in der jüngsten Generation weg. An dieser Stelle wird entweder eine familiale Tradition fortgeführt, obwohl sich die Basis dafür fundamental geändert hat, oder die Art der Außendarstellung, der Magdalene folgt, verbietet es, bestimmte Themen außerhalb der familialen Sphäre zu präsentieren. Zugleich zeigt Magdalene einen Entwicklungsprozess. Dem Rat der Großmutter nicht immer zu folgen, zeugt von dem Bestreben, eigene Entscheidungen zu treffen und darüber Eigenständigkeit zu erlangen. Sie reflektiert allerdings das eigene Handeln und betrachtet die großmütterlichen Ratschläge retrospektiv als sinnvoll. Die Erfahrungen der älteren Generation werden letztlich als wertvoll und anschlussfähig betrachtet. Insgesamt wird ersichtlich, wie die familialen Beziehungen sich lebensphasenabhängig verändern. Schließlich deutet sich in der zitierten Passage an, dass Magdalene durch ihr Verhalten Mutter und Großmutter potenziell in ein Konkurrenzverhältnis zueinander bringt. 


\section{Familiale Tradierungsprozesse in einer Drei-Generationen-Perspektive}

Intensive Konkurrenz prägt auch die Beziehungen zum Bruder, zumindest in der Vergangenheit. Sie sei anfangs sehr eifersüchtig auf ihn gewesen, da die Mutter bei ihrem Sohn die Betreuung selbst übernommen habe und im Unterschied zu ihr viel Zeit für ihn gehabt habe (06:27-06:39). Magdalene und ihr Bruder hätten sich früher gegenseitig „geärgert und immerzu gezankt“ (03:36-04:21). Magdalene vermutet, dass das konflikthafte Verhältnis mit der mütterlichen Bevorzugung des Sohnes zusammenhänge, da diese einen „sehr großen Bezug“ zu ihm gehabt habe (32:11-32:37). Die Verantwortung für Streitigkeiten sei ausschließlich ihr zugeschrieben worden und die Eltern hätten nur sie bestraft (31:32-31:56).

Das als ungerecht empfundene elterliche Verhalten belastet zeitweise besonders die Mutter-Tochter-Beziehung. Magdalene habe zu ihrer Mutter früher „gar nicht so 'nen guten Kontakt" gehabt, wobei die Beurteilung nicht durchgängig negativ ausfällt; in letzter Zeit würden sie sich gut verstehen und „über alles“ reden (33:04-33:29). Ähnliches gelte für das Verhältnis zum Bruder (03:36-03:52). Magdalene beschreibt, ähnlich wie beim Verhältnis zur Großmutter, einen Entwicklungsprozess, dem die Beziehungen zu Bruder und Mutter unterliegen und die sich im Zeitverlauf verbessern. Auch mit ihrem Vater verstehe sie sich gut, Mutter oder Großmutter seien aber bedeutsamere Ansprechpartnerinnen, wenn es z.B. um Beziehungsprobleme gehe (02:48-03:21).

Die von Magdalene vorgenommene Charakterisierung der familialen Beziehungen belegt, wie wichtig ihr der kommunikative Austausch, das gegenseitige Verständnis sowie die Unterstützung innerhalb der Familie sind. Die Beschreibung eines für sie positiven Entwicklungsverlaufs der familialen Beziehungen verweist darauf, dass ihr besonders an familialer Harmonie gelegen ist. Hierbei ergeben sich für sie in der Umsetzung deutliche Ambivalenzen. Zum einen erlebt sie unmittelbar familiale Solidarität, zum anderen aber auch innerfamiliale Konkurrenzen und Zurücksetzungen. Letzteres ist ein Faktor, der dazu beiträgt, die Erziehung widersprüchlich zu bewerten und zumindest teilweise als zu streng zu klassifizieren. Das Knüpfen besonderer Bindungen zu einzelnen Familienmitgliedern ist im Alltag Magdalenes unmittelbar präsent und wird von der älteren Generation vorgelebt, was den Tradierungsprozess dieses Bezugsrahmens unterstützt. Die ebenfalls empfundenen Konkurrenzen und Ungerechtigkeiten bewirken keine Abkehr von diesem Bezugsrahmen, sondern scheinen die Aufmerksamkeit für sowie das Streben nach engen familialen Bindungen noch zu stärken. Magdalene ist sehr daran gelegen, „einen guten Draht zu allen“ zu haben und zu erhalten (37:55-38:08). Hierbei bezieht sie sich nicht nur auf den engen Familienkreis, sondern auch auf den weiteren Verwandtenkreis wie z.B. ihre Cousins, zu denen sie einen „guten Kontakt“ habe (04:34-05:06). Allerdings führt die Art, wie in der Familie besonders enge Beziehungen zu einzelnen Familienmitgliedern realisiert werden, zu einem Dilemma. Die Etablierung enger Bindungen zwischen bestimmten Familienmitgliedern geht auf Kosten der Beziehungen zu anderen Familienmitgliedern, was dem Ziel entgegensteht, über besonders enge Bindungen zwischen einzelnen Familienmitgliedern den familialen Zusammenhalt zu stärken. 


\subsection{Diffuse Tradierungslogiken}

Gekoppelt mit der besonderen Aufmerksamkeit für die zwischenmenschliche Beziehungsebene findet sich eine besondere Sensibilisierung Magdalenes für die emotionale Ebene. Es ist ihr wichtig, Ansprechpartner zu haben, wenn es ihr z.B. schlecht geht (04:59-05:44). Im Gegenzug fühle sie wiederum mit anderen mit. So freue sie sich inzwischen für ihre Großmutter, wenn sie in den Urlaub fahre und „mal rauskommt“; es nehme sie aber auch sehr mit, wenn die Großmutter irgendwelche Probleme habe (10:24-11:32). Generell sei sie sehr empathisch: „Das is eh immer so, wenn irgendwer was hat und so, dann fühl ich auch immer schon gleich mit und so. //hmh// Ich äh, bin ich halt so; kann ich nich anders" (12:37-12:47).

Magdalene berichtet von gemeinsamen Zeiten der Familie, die als eine Voraussetzung betrachtet werden können, um familiale Bindungen und Gemeinschaft herzustellen. Es gebe regelmäßig gemeinsame Mahlzeiten. Einzig der Vater sei berufsbedingt nicht immer anwesend. Sonntags würden alle Mahlzeiten gemeinsam eingenommen. (25:56-26:20). Auch gehe die Familie sonntags regelmäßig in die Kirche und im Anschluss würden sie häufig etwas gemeinsam unternehmen (25:56-26:37). Interessant ist die zwischen Mutter und Tochter differierende Sichtweise auf den Gottesdienstbesuch. Während die Mutter erzählt, die Kinder gingen nicht jeden Sonntag zur Kirche, ist die Tochter der Meinung, die Familie nehme regelmäßig am sonntäglichen Gottesdienst teil. Das verweist darauf, dass sich die religiösen Praxen und deren Bedeutung im Generationenverlauf verändert haben. Für die Mutter, Birgit Schmidt, ist der wöchentliche Gottesdienstbesuch Bestandteil des religiösen Lebens, auch wenn sie selbst diese Regel nicht immer einhält. Für die Tochter wiederum ist zwar der regelmäßige, nicht aber unbedingt der wöchentliche Kirchgang Element des religiösen Lebens. Die eigene Religiosität wird im Zeitverlauf im abnehmenden Maße mit der Häufigkeit des Gottesdienstbesuches in Verbindung gebracht. Hierdurch verliert die Quantität der Gottesdienstbesuche an Relevanz für den Ausdruck der eigenen Religiosität, ohne dass der Kirchgang als solcher obsolet werden würde. Die von der Mutter beschriebenen Konflikte um den sonntäglichen Gottesdienstbesuch werden von Magdalene nicht thematisiert. Sie könnten von ihr als nicht bedeutsam eingestuft werden, da sie aus Magdalenes Sicht keine weiteren Konsequenzen nach sich ziehen. Das ist wiederum ein Indiz für die elterliche Nachgiebigkeit. Zudem trägt es dazu bei, das Bekenntnis zur Religion als ein freiwilliges Element des Alltags zu betrachten, was die Internalisierung erleichtern dürfte.

Im familialen Alltag gebe es kaum Regeln für sie und ihren Bruder:

„Wir können halt machen, was wir wollen so. //hmh// Ab:er es wird dann halt schon gesagt, dass wir das nich machen sollen. Aber so richtig Regeln, dass wir dann so bestraft werden oder so, gibt es eigentlich nich. //hmh// Halt, dass wir dann, wenn wir, wenn ich jetzt so zum Beispiel weggehe $/ /{ }^{\circ} \mathrm{hmh} / / /$ oder mein Bruder auch zum Freund oder so; dass wir dann, wann wir wieder zu Hause sein sollen. //hmh// Aber so eigentlich größer eigentlich nich“ (25:23-25:49). 


\section{Familiale Tradierungsprozesse in einer Drei-Generationen-Perspektive}

Das Erziehungssetting besteht aus Magdalenes Sicht aus einem Minimum an Regeln. Es gibt kaum Forderungen und Verpflichtungen. Allerdings wird das Verhalten der Kinder im Bedarfsfall durchaus korrigiert. Es scheint, als würden Regeln oder Verbote eher ad hoc ausgesprochen und erst im Bedarfsfall kommuniziert. Insgesamt wird Magdalenes Alltag aus ihrer Sicht kaum von den Eltern reglementiert und sie hat viele Freiheiten. Die mütterlichen Schilderungen unterstützen diese Schlussfolgerung.

Magdalene nimmt das Erziehungsverhalten von Mutter und Vater unterschiedlich wahr. Da Magdalene den Vater als dominanter betrachtet, habe sie sich eher getraut, mit dem Bruder zu streiten, wenn lediglich die Mutter anwesend gewesen sei. Sie habe vor ihrem Vater „mehr Respekt“ gehabt als vor der Mutter, vor allem während der Pubertät (15:45-17:51). Der Vater wird offensichtlich als durchsetzungsfähigere Autorität betrachtet und ein nachgiebiges Verhalten in erster Linie mit der Mutter assoziiert. Der Vater zeigt den Kindern anscheinend eher Grenzen auf, weswegen Magdalene Machtkämpfe eher mit der Mutter führt.

Im schulischen Bereich ist es eher der Vater, der Forderungen an seine Tochter heranträgt. Er achte stärker auf die schulischen Noten als die Mutter, was dazu führe, dass sie von schlechten Noten eher der Mutter als dem Vater berichte (33:46-34:10). Wenn sie schlechte Noten bekommen habe, würde sie folgende Taktik anwenden: „Aber dann sammel ich meistens, sammel die guten Noten, wenn ich 'ne schlechte dazwischen habe. Und dann sieht er, ach, Geschichte 'ne zwei, Englisch 'ne zwei, und dann Chemie 'ne vier. Ach ja, okay, das sieht er dann nicht so verbissen" (34:45-35:04). Dem Vater geht es offenbar nicht um Höchstleistungen, sondern darum, einen gewissen Standard sicherzustellen. Die Mutter wiederum freue sich zwar, wenn Magdalene gute Noten schreibe, kritisiere sie aber nicht für schlechte (36:16-36:24). Der Vater zeigt sich in Bezug auf den schulischen Bereich insgesamt ambitionierter als die Mutter. ${ }^{185}$ Hierbei könnte es sich um eine väterliche Tradierungslinie handeln. Während der Grundschule habe die Großmutter väterlicherseits Magdalene bei den Hausaufgaben betreut und darauf bestanden, dass die Hausaufgaben sofort erledigt worden seien. Auch wenn Magdalene das teilweise ,ziemlich nervig“ gefunden habe, habe sie die Forderungen eingesehen und erfüllt (37:23-37:39). Die Großmutter trägt somit zumindest in einem gewissen Maße ein Pflicht- und Leistungsbewusstsein an ihre Enkelin heran. Dieses Anliegen scheint auch aufzugehen. Mittlerweile würden ihre Hausaufgaben nicht mehr kontrolliert und Magdalene sehe ein, dass sie selbst davon profitiere, wenn sie die schulischen Aufgaben erledige (36:39-37:20). Insgesamt beschreibt Magdalene ähnlich wie ihre Mutter und Großmutter mütterlicherseits teilweise Unterschiede im elterlichen Erziehungsverhalten. Es gibt aber auch Gemeinsamkeiten. Den Eltern sei es wichtig gewesen, ihr zu vermitteln, Verantwortung für sich und andere zu übernehmen und hilfsbereit zu sein; Verhaltensweisen, die sie auch bei den Eltern unmittelbar erlebt habe (27:38-28:07) und die tradiert werden.

${ }^{185}$ Wenn es allerdings Problem in der Schule gäbe, würden sich beide Eltern für ihre Tochter einsetzen (35:32-36:05). 
Zusammenfassende Betrachtung des familialen Bezugsrahmens In der Familie Beyer/Schmidt bildet die Aufmerksamkeit für die Qualität der familialen Beziehungen in allen drei Generationen einen zentralen Bezugsrahmen. Frau Beyer erlebt die Eltern, insbesondere den Vater, sowie die Großmutter als liebevoll, fürsorglich und ihr emotional zugewandt. Diese Attribute überträgt sie auf die Gestaltung der Beziehung zu ihrem jüngeren Bruder. Hierdurch werden besondere Bindungen und Verantwortlichkeiten zwischen einzelnen Familienmitgliedern geschaffen, die letztlich insgesamt der Stärkung der familialen Gemeinschaft dienen, unabhängig davon, ob dies explizit intendiert wurde oder nicht. Während der eigenen Familienphase setzt sich dieses Prinzip fort. Die Aufmerksamkeit richtet sich dabei in unterschiedlichem Umfang auf die Kinder, was zu Geschwisterkonkurrenzen führt.

Die Fokussierung auf die Beziehungsqualitäten ist für Birgit Schmidt als Vertreterin der mittleren Generation zwiespältig. Einerseits knüpft sie an den familialen Bezugsrahmen an, indem sie ähnlich wie ihrerzeit die Mutter eine enge emotionale Bindung zum jüngeren Bruder entwickelt und sich ihm gegenüber sehr fürsorglich zeigt. Die besonders engen Bindungen an ein Geschwisterteil bedingen im Gegenzug konflikthaftere Beziehungen zu den anderen Geschwistern, wodurch die familiale Gemeinschaft nur partiell gestärkt wird. Dennoch hält Birgit Schmidt an diesem Gestaltungsprinzip während der eigenen Familienphase fest.

Auch für die Tochter, Magdalene, sind die Qualitäten der familialen Beziehungen von besonderer Bedeutung. Sie beschreibt ein nahes Verhältnis zur Großmutter sowie für die Vergangenheit intragenerationelle Konflikte mit ihrem Bruder, die sie als Folge der mütterlichen Ungleichbehandlung betrachtet. Die Beziehung zur Mutter ist dadurch in der Vergangenheit ebenfalls angespannt. Magdalene beschreibt eine positive Entwicklung der Beziehungen zu Mutter und Bruder im Zeitverlauf und kann somit die negativen Effekte der jeweiligen Beziehungsgestaltung überwinden. Die in der Vorgängergeneration potenziell gefährdete familiale Gemeinschaft kann durch Lernprozesse der jüngsten Generation in der aktuellen Familienphase offensichtlich wieder gestärkt werden. Magdalene geht empathisch auf die Familienmitglieder ein, worin eine Parallele zum Verhalten ihrer Großmutter mütterlicherseits zu sehen ist.

\subsubsection{Einordnung der Tradierungslogik unter Berücksichtigung der gesellschaftlichen Bezüge}

Das elterliche Erziehungsverhalten weist im Generationenverlauf zunehmend permissive Züge auf. ${ }^{186}$ Die jeweils an die Kinder herangetragenen Forderungen und Regelsetzungen nehmen von der ältesten zur jüngsten Generation deutlich ab. Die intergenerationellen Machtbalancen in dieser Familie weisen von Anfang an keine sonderlich starken Asymmetrien auf. Vorstellungen werden in allen drei Generationen weniger über Befehle als vielmehr über Appelle an die jüngere Generation herangetragen. Ein-

${ }^{186} \mathrm{Zu}$ den Spezifika des permissiven Erziehungsstils siehe Kap. 1.2.2. 


\section{Familiale Tradierungsprozesse in einer Drei-Generationen-Perspektive}

zig Birgit Schmidt scheint als Erziehende in einigen Ausnahmefällen ihren Kindern unmissverständlich Arbeitsanweisungen zu erteilen. Auch wenn die Familie weniger einem Befehlshaushalt zuzurechnen ist, entzieht sich die beschriebene Strategie einer einfachen Zuordnung zum Gegenentwurf, dem Verhandlungshaushalt. ${ }^{187}$ Der Appellcharakter des elterlichen Verhaltens ist nicht gleichzusetzen mit einem mehr oder weniger gleichberechtigten Aushandeln von Regeln, da er auf subtilere Art als bei direkten Befehlen die intergenerationellen Asymmetrien überdeckt.

Entscheidend für die Einordnung dieser Familie ist, dass die Erziehungsarrangements diffus erscheinen, was sich auch unmittelbar auf die jeweilige Gestaltung des familialen Bezugsrahmens auswirkt. In dieser Familie wird ähnlich wie bei den Familien, die einer flexibel erscheinenden Tradierungslogik folgen, ein eher grober Rahmen für die Kinder abgesteckt. Im Falle Frau Beyers erscheint dieses Arrangement während ihrer Kindheit auch unproblematisch, da den Eltern offenbar wenig Anlass gegeben wird, ihr die Grenzen dieses Rahmens zu verdeutlichen. Das verändert sich allerdings in der Folgegeneration. Die elterlichen Vorstellungen werden im Falle von Birgit Schmidt nicht immer umgesetzt, was aber - und hierin liegt der entscheidende Punkt - keine sonderlich energischen Versuche der Eltern zur Folge hat, auf eine Einhaltung der gesetzten Regeln zu insistieren. Vielmehr bemühen sich die Eltern, Konflikte zu vermeiden, was u.U. bedeutet, nicht auf den eigenen Vorstellungen zu beharren, wodurch diese leicht an Konturiertheit verlieren können. Auch Birgit Schmidt als Erziehende erlegt ihren Kindern kaum Pflichten und Regeln auf. Ähnlich wie in ihrer eigenen Kindheit finden sich Beispiele, die zeigen, dass sie nicht mit Nachdruck auf die Einhaltung der elterlichen Forderungen besteht. Die erziehenden Generationen in dieser Familie zeichnen sich insgesamt durch eine große Nachgiebigkeit aus. Ferner finden sich auf Erziehungsseite in allen Generationen Beispiele, wie jeweils ein Elternteil an die Kinder adressierte Forderungen des anderen Elternteils unterläuft und auf diese Weise zuvor an die Kinder herangetragene Erwartungen in ihrer Deutlichkeit zumindest relativiert.

Der Bezugsrahmen der familialen Sorge und ausgeprägten Bindungsnähe wird in allen drei aneignenden Generationen nicht unwesentlich durch äußere Faktoren verstärkt. Die Orientierung am Bezugsrahmen ist folglich zumindest partiell erzwungen. In der ältesten Generation resultiert dies aus der Angst vor staatlichen Repressionen, in den beiden Folgegenerationen führt die Gestaltung des familialen Beziehungsgefüges zu einem Gefühl der Ungleichbehandlung, das wiederum das Bemühen verstärkt, familiale Allianzen zu bilden. Als gesellschaftlich forciert betrachtete Notwendigkeiten werden in dieser Familie im Generationenverlauf zu familialen Besonderheiten umgearbeitet.

Der Bezugsrahmen der familialen Fürsorge und Bindungsnähe ist in der gewählten Umsetzung eng mit einer nicht unerheblichen Verantwortungsdelegation verbunden. Der Bezugsrahmen enthält somit unter der Hand eine weitere, nicht intendierte Zielsetzung, die für die aneignenden Generationen, die sich ausschließlich auf die emotionale Qualität der Beziehungsebenen konzentrieren, offenbar nicht explizit zu Tage tritt.

${ }^{187} \mathrm{Zu}$ den Modellen von Befehls- und Verhandlungshaushalt siehe Kap. 1.2.2. 


\subsection{Diffuse Tradierungslogiken}

Diese unbemerkte Funktion des familialen Bezugsrahmens verstärkt den Eindruck der Diffusität. Spätestens mit Beginn der Familienphase Frau Beyers hat die Umsetzung des Bezugsrahmens zwiespältige Folgen. Neben einer partiellen Stärkung von familialen Bindungen tritt als Kehrseite auch eine ausgeprägte Konkurrenz zwischen bestimmten Familienmitgliedern hervor, sodass der Bezugsrahmen in seiner Auswirkung diffus erscheint. Im Vergleich zu der flexibel erscheinenden Tradierungslogik unterliegen die Inhalte der Tradierung in diesem Setting stärkeren generationellen Umarbeitungen und Veränderungen.

Das Zusammenspiel von gesellschaftlicher und familialer Ebene Die jeweiligen Ausprägungen des familialen Bezugsrahmens sowie die Formen der Vermittlung und Aneignung in dieser Familie lassen sich an verschiedenen gesellschaftliche Einflüsse rückbinden. Die Furcht vor staatlichen Repressionen forciert während der Kindheit von Frau Beyer die familiale Anpassungsbereitschaft. Die von ihr erwähnten Zwangsumsiedlungen und die Art ihrer Durchführung erfüllen damit den staatlicherseits intendierten Zweck. Die staatlichen Interventionen dienen dazu, eine culture of secrecy zu etablieren (Berdahl 1999, 69). Weder erfahren die Bürger, nach welchen Kriterien der Staat bei seinen Maßnahmen, wie z.B. den Z wangsumsiedlungen, vorgeht, noch ist für die Bürger ersichtlich, in welchen Fällen der Staat eingreift und in welchen er sie gewähren lässt. Das staatliche Handeln unterliegt einer nicht einsichtigen Willkür, die bei ihnen eine Art vorauseilenden Gehorsam auslöst (Berdahl 1999, 63-70). Genau dieser Mechanismus lässt sich bei der Familie Beyer beobachten, was zugleich bedeutet, dass in die Lebenswelt der Familie zumindest partiell ein Handeln gegen die eigenen Orientierungen einzieht, wodurch vormals klare Einstellungen aufgeweicht werden. Für die aneignende Generation, die nun im Alltag Widersprüchlichkeiten ausgesetzt ist, bedeutet das zugleich potenziell einen Verlust von Orientierungssicherheit. Die Familie hält z.B. an der tradierten Religiosität mit ihren Praxen fest und öffnet sich zugleich für die Mitgliedschaft in den staatlichen Jugendorganisationen. Letzteres ist dem Bestreben geschuldet, potenzielle Angriffsflächen zu minimieren.

Das besonders nachgiebige und kindorientierte Verhalten des Vaters gegenüber seinen Kindern lässt sich als Versuch betrachten, die staatlicherseits bedingten Einschränkungen und Zwänge für die Kinder zu kompensieren. Frau Beyer scheint dieses Verhalten gegenüber den Kindern während ihrer eigenen Familienphase noch zu verstärken, wodurch der Eindruck einer Überkompensation entsteht. Das führt gelegentlich dazu, dass sie ihren Kindern die Problemlösung und damit die Verantwortlichkeit ab- und gleichzeitig eine überbehütende Haltung einnimmt. Reubands These des begrenzten Einflusses des Gesellschaftssystems auf die familiale Sphäre (Reuband 1995, 236) ist ähnlich wie bei der Familie Cronert insofern zutreffend, als der familiale Bezugsrahmen nicht aufgegeben wird. Die gesellschaftlichen Rahmenbedingungen sind aber trotzdem relevant, da sie den Stellenwert des familialen Bezugsrahmens noch erhöhen. 


\section{Familiale Tradierungsprozesse in einer Drei-Generationen-Perspektive}

Der für die Kindheit Frau Beyers beschriebene Anpassungsdruck wird in Bezug auf die eigene Familienphase nicht mehr so explizit thematisiert. Die Familie hält an ihrer Religiosität und den hiermit verbundenen Praxen fest, ohne staatlichen Sanktionen ausgesetzt zu sein, was die Notwendigkeit zur Anpassung relativiert. Auf diese Weise kann der regelmäßige Kirchgang an die Kinder als selbstverständliches Alltagsritual herangetragen werden. Ebenso selbstverständlich ist die Tradierung der gemeinsamen Familienmahlzeiten. Diese Praxen werden erst im Zuge der mit der Wende verbundenen gesellschaftlichen Umbrüche von den jüngeren Kindern in Frage gestellt. Für die älteren hingegen sind sie während ihrer Jugendphase, die in die DDR-Zeit fällt, noch selbstverständlich. Hier zeigt sich eindrücklich, dass die Jugendphasen und die hiermit verbundenen Orientierungen und Wahlmöglichkeiten innerhalb der Kindergruppe der mittleren Generation einem gravierenden Wandel unterliegen. ${ }^{188}$

Birgit Schmidt ist in der Lage, klar und deutlich ihre Bedürfnisse zu artikulieren und auf eine Beachtung dieser zu insistieren. Damit unterscheidet sich ihr Verhalten deutlich von dem ihrer Mutter, der ein solches Verhalten eher fremd zu sein scheint. Dies ist ein deutliches Indiz, dass Birgit Schmidt die auch in der DDR sich vollziehenden Verschiebungen in den Wertpräferenzen (Gensicke 1992b, 688 und 1995, 73) in ihren Alltag integriert. Das ausgewogene Verhältnis von Arbeit und Freizeit wird für Birgit Schmidt zu einem wichtigen Thema. Neben der von ihr artikulierten Arbeits- und Pflichtorientierung nimmt für sie eine Freizeit- und Erholungsorientierung als Ausgleich einen hohen Stellenwert ein. Für sie rückt die Balance von Pflicht- und Selbstentfaltungswerten in den Mittelpunkt der Betrachtung.

Die stärkere Fokussierung auf die eigenen Bedürfnisse wirkt sich auch auf ihr Verhalten als Mutter aus. Sie widmet sich zwar ihren Kindern und zeigt eine große Kindorientierung, die sich in einem Minimum an Regeln und Anforderungen manifestiert, ohne die starke Aufopferungsbereitschaft ihrer Mutter zu übernehmen. Stattdessen erfahren die eigenen Anliegen eine stärkere Beachtung. Die Aufmerksamkeit für die eigene Selbstentfaltung trägt zu einer Relativierung des Mutterideals bei, indem die Mutterrolle von überhöhten Erwartungen entlastet wird.

Magdalene wiederum achtet auf das eigene emotionale Wohlbefinden und auf das der anderen. Bei ihr findet sich somit eine Synthese aus Fürsorge, wie sie bei ihrer Großmutter zu finden ist, sowie Beachtung der eigenen Bedürfnisse, wie sie von ihrer Mutter praktiziert wird. Sie kombiniert auf diese Weise zwei gesellschaftliche Trends, indem sie die für die östlichen Bundesländer postulierte Familienorientierung (Uhlendorff 2004, 80 und Meulemann 1996b, 356 und 358), die offenbar bis in die Gegenwart zu finden ist, mit der im Zeitverlauf gestiegenen Relevanz von Selbstentfaltungswerten (Klages 2001b, 730/731) kombiniert.

\footnotetext{
${ }^{188}$ Unterschiede lassen sich auch auf anderen Ebenen ausmachen. Für Bürgel manifestieren sie sich darin, dass die um 1970 Geborenen die Wende positiver einschätzen als die um 1980 Geborenen, da die Wende jeweils andere Folgen für die einzelnen Gruppen gehabt habe (Bürgel 2006, 171, 172).
} 


\subsection{Familiale Tradierungslogiken im Überblick}

\subsection{Familiale Tradierungslogiken im Überblick}

Wie die Fallbeispiele eindrücklich zeigen, finden sich in den einzelnen Familien Tradierungsprozesse. Das ist sicherlich teilweise auch der Sampleauswahl geschuldet. Die Studie ist in einem christlich geprägten Milieu durchgeführt worden, von dem anzunehmen ist, dass der Familie ein besonderer Stellenwert zugeschrieben wird. Folglich ist es wahrscheinlich, auf Familien zu treffen, in denen ein regelmäßiger intergenerationeller Austausch stattfindet - eine zentrale Voraussetzung für Tradierungsprozesse. Die Einbeziehung von drei Generationen in die Untersuchung hat dazu beigetragen, dass keine Familie vertreten ist, in denen die älteste und mittlere Generation kaum oder keinen Kontakt mehr zueinander hat. Auch fehlen Familien mit niedrigen Bildungsabschlüssen, die zu einer größeren Heterogenität des Samples hätten beitragen können.

Die in die Studie einbezogene Gruppe erweist sich somit in mehrerer Hinsicht als relativ homogen. Um so interessanter ist es, dass sich trotzdem verschiedene Tradierungslogiken herausarbeiten lassen: eine geschlossene, eine flexibel erscheinende sowie eine diffuse. In Bezug auf Tradierungsprozesse ist die Gruppe folglich gar nicht so homogen, wie angesichts der Samplezusammensetzung zu vermuten wäre. Vier Familien lassen sich innerhalb dieses Samples einer flexibel erscheinenden Tradierungslogik zuordnen. ${ }^{189}$ Drei Familien folgen einer überwiegend geschlossenen Logik und zwei Familien einer diffusen. Eine Familie kann aufgrund des vorliegenden Interviewmaterials nicht eindeutig verortet werden. Geschlossene Tradierungslogiken dominieren im westlichen und flexibel erscheinende im östlichen Teilgebiet. Diffuse Tradierungslogiken fanden sich innerhalb dieses Samples nur in der östlichen Region. In Bezug auf den Bildungsstand sind die einzelnen Teilgruppen heterogen zusammengesetzt. Innerhalb der Gruppe, die einer geschlossenen Tradierungslogik folgt, gibt es sowohl mittlere Ausbildungsniveaus als auch Akademiker, wobei letztere dominieren. Ähnlich heterogen ist die Gruppe der flexibel erscheinenden Tradierungslogiken. Bei den Familien, die einer diffusen Tradierungslogik folgen, sind mittlere Bildungsabschlüsse vorhanden. Bei der nicht eindeutig zurechenbaren Familie ist der Vater in der ältesten Generation ein Akademiker und die nachfolgende Generation verfügt über einen mittleren Bildungsabschluss.

Geschlossene Tradierungslogiken mit dezidiert autoritären Elementen finden sich in diesem Sample verstärkt in kinderreichen Familien. Die Familie Beyer, die einer diffusen Tradierungslogik folgt, bildet hier eine Ausnahme. ${ }^{190}$ In den übrigen kinderreichen Familien könnten die autoritären Elemente dazu dienen, die Organisation des Familienalltags zu erleichtern, worauf z.B. Frau Nolte auch hinweist. Insgesamt lässt sich die

\footnotetext{
${ }^{189}$ Für eine Familie gilt dies nur eingeschränkt, da es sich bei ihr um keine genealogische Linie handelt und deswegen eine direkte Tradierungslinie über drei Generationen nicht nachgezeichnet werden kann.

${ }^{190}$ In dieser Familie finden sich keine ausgesprochen autoritären Erziehungselemente.
} 


\section{Familiale Tradierungsprozesse in einer Drei-Generationen-Perspektive}

Familiengröße als Faktor betrachten, der dazu beitragen kann, autoritäre Elemente in der Erziehung anzuwenden, wenn dem auch nicht zwangsläufig so sein muss.

Es fällt auf, dass in keiner Familie ein Wechsel von einer geschlossenen zu einer flexibel erscheinenden Tradierungslogik oder umgekehrt erfolgt. Insgesamt lässt sich keine eindeutige Änderung der Tradierungslogik feststellen. Hierbei ist allerdings die Samplezusammensetzung zu bedenken. Würde die Untersuchung auf weitere Regionen, Bildungsmilieus sowie Familien mit einer starken geographischen Mobilität ausgeweitet, ließen sich vermutlich weitere Tradierungslogiken sowie Wechsel zwischen diesen erfassen.

Berteaux/Berteaux-Wiames Ansatz (1991), dass Tradierungsprozesse sich nicht allein in einer unveränderten Weitergabe erschöpfen, sondern Inhalte im Zuge von Vermittlung und Aneignung transformiert werden, hat sich als elementar erwiesen, um Tradierungsprozesse in den einzelnen Familien zu identifizieren. Inhalte und Formen der Tradierung werden von jeder Generation auf je spezifische Weise bearbeitet, was Berteaux/Berteaux-Wiames Annahme unterstreicht, Transmissionsprozesse zum Äquivalenten seien häufiger zu finden als Transmissionsprozesse zum Identischen (Berteaux/ Berteaux-Wiame 1991, 38). Dies wirkt sich jedoch je nach Tradierungslogik unterschiedlich aus. Während mit der flexibel erscheinenden Logik eher kontinuierliche, konsensuale Weiterentwicklungen verbunden sind, führen die geschlossene sowie diffuse Tradierungslogik eher zu konflikthaften Auseinandersetzungen, die mit stärkeren Umarbeitungen und Veränderungen der Erziehungsarrangements sowie der familialen Bezugsrahmen verbunden sind, ohne dass sich allerdings die dahinter stehende Logik der diffusen oder geschlossenen Erwartungshaltungen gegenüber den Kindern im Generationenverlauf verändern würde.

Die Ergebnisse dieser Studie widersprechen dem immer wieder aufkommenden Allgemeinplatz, wonach vor allem Frauen, quasi als ,Hüterinnen der Tradition' für Tradierungsprozesse zuständig seien. Wie das Fallbeispiel Cronert/Jäger demonstriert, sind auch Männer an Tradierungsprozessen beteiligt. Knafo/Schwartz hingegen betrachten einige Werte wie self-enhancement und self-transcendence in ihrer quantitativ durchgeführten Untersuchung als gender-typed (Schwartz/Knafo 2009, 259). Diese generalisierende Feststellung lässt sich in der Form in der vorliegenden qualitativ angelegten Studie nicht wiederfinden. Die Beispiele Cronert/Jäger sowie Nolte/Gümper mit ihren familialen Bezugsrahmen des familialen Zusammenhalts bzw. dem Streben nach repräsentativen Höchstleistungen belegen, dass Männer durchaus Orientierungen weitergeben, die sich dem Bereich self-transcendence zuordnen lassen, und Frauen Orientierungen tradieren, die in die Kategorie des self-enhancement fallen. Wie die Fallbeispiele zeigen, werden die jeweiligen familialen Bezugsrahmen nicht zuletzt von der Familiengeschichte und von historischen Kontexten beeinflusst und eine singuläre Konzentration auf eine Kategorie, wie z.B. das Geschlecht, reicht nicht aus, um familiale Tradierungsprozesse $\mathrm{zu}$ erfassen. 


\subsection{Familiale Tradierungslogiken im Überblick}

Interessant ist der Untersuchungsansatz von Schönpflug/Bilz, die in ihren Studien auf transmission belts, wie etwa einen empathischen Erziehungsstil, fokussieren (Schönpflug/Bilz 2001, 2009). Übertragen auf die vorliegende Studie ließe sich annehmen, dass vor allem flexibel erscheinende Tradierungslogiken erfolgreich sein dürften. Wie sich aber herausgestellt hat, sind in diesem Sample Tradierungsprozesse nicht auf ein bestimmtes Erziehungssetting oder eine bestimmte Tradierungslogik beschränkt, sondern finden sich in den unterschiedlichsten Erziehungsarrangements. Der elterliche Motivationsgrad, bestimmte Inhalte weiterzugeben, sowie die Bereitschaft auf Aneignungsseite, den elterlichen Einfluss zu akzeptieren (Schönpflug/Bilz 2009, 229/230), sind zweifellos für Tradierungsprozesse wichtige Einflussfaktoren. Das konkrete Verhalten der vermittelnden wie der aneignenden Generation wird jedoch erst verständlich, wenn der jeweilige sozio-historische Kontext in die Überlegungen einbezogen wird. Letzterer beeinflusst entscheidend, inwiefern bestimmte Tradierungsinhalte und -modi für die nächste Generation praktikabel sind. Die Anschlussfähigkeit ist folglich zumindest für das hier untersuchte Sample ein zentrales Kriterium für die Annahme oder Modifikation von Inhalten und Formen der Tradierung.

Im Vergleich zu Büchner/Brake, die die Bildungsbedeutsamkeit der Familie untersuchen, die Transmission des Familienhabitus ins Zentrum stellen und verschiedene Transmissionsvarianten herausarbeiten können (Büchner/Brake 2006, 256), werden in der vorliegenden Studie die verschiedenen Tradierungsprozesse zu Logiken gruppiert, die nicht auf den Familienhabitus, sondern auf familiale Bezugsrahmen und ihre Bearbeitung fokussieren. Es wurde eine Systematik zur Erfassung von Tradierungsprozessen erarbeitet, die sich durch die Einbeziehung weiterer Familien und Milieus sicherlich noch erweitern lässt.

Innerhalb der einzelnen Logiken sind allerdings auch Unterschiede feststellbar, die sich vor allem an den jeweiligen Bezugsrahmen sowie den Veränderungen in den konkreten Vermittlungs- und Aneignungsprozessen im Generationenverlauf festmachen lassen. Um diese Unterschiede zu erklären, ist es hilfreich, sich Halbwachs' Annahme zu vergegenwärtigen, dass jedes Individuum zugleich in verschiedenen Gruppen verankert ist (Halbwachs 1991, 64/65). Bezogen auf die Frage nach Tradierungsprozessen bedeutet dies, Individuen sind nicht nur familiale Akteure, sondern zugleich auch Mitglieder in anderen Gruppen. Hierauf richtet sich das Augenmerk bei der Analyse im folgenden Abschnitt. Wie die Fallbeispiele nahelegen, sind im Zusammenhang dieser Studie Bildungsstand, Generation und Region weitere wichtige Gruppenzuordnungen, deren systematische Betrachtung zu einem differenzierten Bild von Tradierungsprozessen beiträgt. 



\section{Bildungsstatus, Generation und Region: Familiale Tradierungslogiken und ihre gesellschaftliche Einbettung}

Nachdem für einzelne Familien in vertikaler Perspektive ihre Bezugsrahmen sowie die unterschiedlichen Tradierungslogiken über drei Generationen en détail herausgearbeitet worden sind, ändert sich in diesem Kapitel die Analyseebene. Nun stellt sich in horizontaler Perspektive die Frage, inwiefern sich der Bildungsstand, die jeweilige generationelle Verortung sowie die Zugehörigkeit zu einer der beiden Regionen auf die Entwicklung der familialen Bezugsrahmen sowie der jeweiligen Erziehungsarrangements auswirken. Die Genderperspektive, als weitere wichtige Kategorie, steht nicht im Mittelpunkt der Fragestellung, weswegen dieser Punkt nicht in einem eigenen Abschnitt behandelt wird. Sofern sich genderspezifische Aspekte aufzeigen lassen, werden sie innerhalb der genannten Kategorien berücksichtigt. Als Grundlage der im folgenden Kapitel vorgenommenen Analysen dienen vielfach Aussagen aus den Fallbeispielen des vorherigen Kapitels, die aus diesem Grunde nicht noch einmal gesondert belegt werden. Es werden aber auch Aspekte und Mitglieder aus Familien eingebunden, die bisher nicht eigens vorgestellt worden sind, und für die exemplarische Belege angeführt werden.

\subsection{Bildungsmilieu}

Innerhalb dieses Samples folgen sowohl Familien mit einem hohen als auch Familien mit einem mittleren Bildungsstand einer geschlossenen Tradierungslogik. Ähnliches gilt für die flexibel erscheinende Tradierungslogik. Die jeweilige Tradierungsform lässt sich folglich nicht einfach mit einem bestimmten Bildungsstand verknüpfen. Dieses Ergebnis $\mathrm{zu}$ generalisieren ist aufgrund der Samplegröße sowie -zusammensetzung schwierig. Es ist durchaus denkbar, dass sich ein anderes Bild ergibt, wenn die Fallzahl erhöht wird, eine größere Heterogenität der Bildungsabschlüsse sowie verschiedene Sozialmilieus in die Untersuchung einbezogen werden. Innerhalb des vorliegenden Samples scheinen jedoch die jeweilige Familiengeschichte und die spezifischen Erfahrungen in den einzelnen Herkunftsfamilien für die gewählte Tradierungslogik zentraler zu sein als der Bildungsstand. Die beiden thüringischen Familien, die einer diffusen Tradierungslogik folgen, verfügen über ein mittleres Bildungsniveau. Darüber hinaus sind die Eltern der ältesten Generation jeweils als selbstständige Unternehmer tätig. Dieser Status ist mit der Etablierung eines sozialistischen Systems in der DDR gefährdet bzw. muss aufgegeben werden. Hier ist anzunehmen, dass diese Bedrohung verunsichernd wirkt und zur Diffusität der Tradierung beiträgt. 


\section{Bildungsstatus, Generation und Region}

Es lassen sich allerdings Zusammenhänge erkennen, die die Inhalte betreffen. In allen Familien sind Religiosität, Bildungsorientierung und familialer Zusammenhalt relevant, sie werden aber in ganz unterschiedlicher Weise betont. Was in einigen Familien den zentralen Bezugsrahmen bildet, ist auch für andere Familien wichtig, steht für diese aber nicht so prominent im Vordergrund.

\subsubsection{Religiosität}

Was bereits an den Fallbeispielen deutlich geworden ist, gilt für die meisten Familien des Samples: Sie betrachten sich selbst als religiös. Wie bereits die Kirchenmitgliedszahlen nahelegen, handelt es sich hierbei um kein repräsentatives Ergebnis. Ende der 1990er Jahre gehörten ca. 35\% der Westdeutschen sowie 22\% der Ostdeutschen „einer Gliedkirche der EKD an" und $42 \%$ der Westdeutschen sowie 4 bis $5 \%$ der Ostdeutschen waren Mitglieder der katholischen Kirche (Wolf 2003, 53). ${ }^{191}$ Insbesondere für Ostdeutschland stellen die Ergebnisse dieser Studie folglich eher eine Ausnahme dar. Insofern repräsentiert der Befund eine Besonderheit der untersuchten Region.

Interessanter als die hohe Bedeutung, die allgemein der Religion in den Familien dieser Region beigemessen wird, sind deshalb die unterschiedlichen Funktionen, die sie in den einzelnen Familien erfüllt. Die Religiosität prägt jeweils das familiale Selbstverständnis, wobei, je nach Bildungsstand, unterschiedliche Schwerpunkte erkennbar sind. In Familien, in denen mittlere Bildungsabschlüsse dominieren, steht die Teilhabe an sozialen Praxen im Vordergrund, worüber zugleich eine Gemeinschaftseinbindung hergestellt wird. In Akademikerfamilien wird die Gestaltung der familialen Religiosität zugleich als Distinktionsmittel genutzt. Frau Dienel setzt sich beispielsweise über ihre intellektuelle Beschäftigung mit Glaubensaspekten von dem von ihr angenommenen Durchschnitt ab, und die Familien Dienel und Friese ${ }^{192}$ betrachten den Gottesdienstbesuch als Beitrag, um die eigene Vorbildfunktion zu unterstreichen (1:02:01-1:02:06, 1:04:37-1:05:26). Die beschriebenen Arten der Distinktion sind vor allem in der ältesten Generation zu finden und schwächen sich im Generationenverlauf ab.

Diese Unterschiede wirken sich auch auf die jeweiligen Erziehungssettings aus. In den Familien mit mittlerem Ausbildungsniveau stehen in erster Linie die religiösen Praxen, die die Kinder übernehmen sollen, im Vordergrund. Eine Rückkopplung mit aus der Religion ableitbaren Orientierungen erfolgt eher unterschwellig, wie anhand der Familie Cronert/Jäger herausgearbeitet werden konnte. In Akademikerfamilien hingegen treten die mit der Religion verbundenen Orientierungen, die den Kindern vermittelt werden sollen, stärker in den Vordergrund. Gelegentlich werden diese Zusammenhänge

\footnotetext{
${ }^{191}$ Die Mitgliedszahlen lassen sich als Indikator für die Bedeutung der Religion auffassen, wobei zu beachten ist, dass die individuelle Religiosität sich nicht ausschließlich an der Kirchenmitgliedschaft festmachen lässt, wie auch an den Fallbeispielen deutlich geworden ist.

${ }^{192}$ Das Interview mit Herrn Friese fand am 21.01.2009 statt. Gegen Ende des Interviews war zusätzlich seine Frau anwesend.
} 
auch explizit formuliert und sind Gegenstand der Reflexion, wie das Fallbeispiel Dienel/ Ebel nachdrücklich zeigt.

\subsubsection{Bildung}

In allen untersuchten Familien findet sich eine Bildungsorientierung. Dies ist nicht weiter verwunderlich, da davon auszugehen ist, dass alle Eltern das Beste für ihre Kinder wünschen - auch in schulischer Hinsicht. Ähnlich wie in Bezug auf die Religiosität besteht der mit dem jeweiligen Bildungsstand koppelbare Unterschied darin, wie dies umgesetzt wird. In Akademikerfamilien werden eine erfolgreiche schulische Karriere sowie außerschulische Leistungen im kulturellen oder ggf. im sportlichen Bereich schlichtweg vorausgesetzt. In Familien mit mittlerem Ausbildungsniveau hingegen werden sie zwar gewünscht, aber nicht selbstverständlich erwartet.

Bestimmte Bereiche, wie etwa die musische Bildung, sind zudem in der familialen Lebenswelt der Akademikerfamilien dieses Samples fest verankert, beispielsweise in Form des gemeinsamen Musizierens zu Weihnachten. Darüber hinaus werden die Leistungen der Kinder durchaus aufmerksam verfolgt, und es wird bei Leistungseinbrüchen interveniert. Miriam Friese berichtet z.B. davon, wie ihre Eltern sie überredet hätten, in die zuvor von ihr aufgegebene Sportart zurückzukehren und wieder zu trainieren, da sie ein „Potenzial“ gesehen hätten, das sich ausbauen lasse (Miriam Friese, 22:54-23:27). ${ }^{193}$ Bei Leistungsrückgängen würden die Eltern unterstützend eingreifen: „Und wenn ich mal 'nen kleinen Durchhänger habe, dann versuchen meine Eltern auch so 'n bisschen mich wieder neu zu motivieren und zu sagen, komm, das schaffst du" (Miriam Friese, 03:14-03:24). Wie das Fallbeispiel von Frau Dienel exemplarisch gezeigt hat, betonen Vertreterinnen der ältesten Generation, die zwar nicht aus Akademikerfamilien stammen, denen aber eine schulische Bildung ermöglicht worden ist, die über das zu ihrer Zeit übliche Maß hinausgeht, darüber hinaus den hohen Stellenwert des Lesens in ihrer Kindheit sowie die über ihre Schulzeit hinaus andauernde Bildungsorientierung.

In den Familien, die über ein mittleres Ausbildungsniveau verfügen, scheinen Leistungsrückgänge hingegen eher hingenommen zu werden. Die Eltern wünschen zwar, dass ihre Kinder strebsam und bildungsorientiert sind, scheinen hierbei aber stärker als Akademikerfamilien auf eine intrinsische Motivation ihrer Kinder zu setzen. Bei Friederike Jäger, die offensichtlich eine gute Sportlerin war, finden sich keine Hinweise, dass ihre Eltern ein besonderes Augenmerk auf die Entwicklung ihrer sportlichen Leistungen gelegt hätten. Stattdessen seien für sie vielmehr die Siegesprämien ein Ansporn gewesen, gut bei den Wettkämpfen abzuschneiden (I 29:29-30:49).

Auch in der Außendarstellung gibt es Unterschiede. Während in Akademikerfamilien die schulischen und außerschulischen, sportlichen wie musischen Leistungen der Kinder im Interview scheinbar en passant erwähnt oder ausdrücklich hervorgehoben werden, verweisen die übrigen Familien kaum auf die Fähigkeiten ihrer Kinder, auch wenn sie

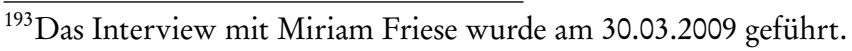




\section{Bildungsstatus, Generation und Region}

vorhanden sind. Friederike Jäger erwähnt z.B. in ihrem Interview, eine gute Schülerin gewesen sowie von der Schule für externe Sportwettkämpfe eingesetzt worden zu sein (II 24:31-24:50, 31:28-32:12). Im Interview mit ihrem Vater, Herrn Cronert, wiederum findet sich kein Verweis auf diese Leistungen seiner Tochter, obwohl sie offenbar über dem Durchschnitt gelegen haben. Die töchterlichen Leistungen werden nicht in die familiale Präsentation eingebaut.

Die elterlichen Erwartungshaltungen schlagen sich in der Kindergeneration nieder und werden von dieser internalisiert. Dies zeigt sich insbesondere bei der jüngsten Generation. Während für diejenigen, die nicht aus einer Akademikerfamilie stammen, der Gymnasialbesuch keine Selbstverständlichkeit darstellt, wie das Fallbeispiel von Sonja Ahrens zeigt, betrachten Akademikerkinder den Gymnasialbesuch als vorgezeichneten Weg. Das lässt sich exemplarisch an einer Äußerung von Miriam Friese belegen: „Meine Geschwister sind alle auf's Gymnasium gegangen und von daher hab ich es auch nich anders äh, und meine Eltern sind auch auf's Gymnasium gegangen, hab ich es auch nich anders ähm, ja mitgekriegt. //hmh// Und für mich war das so'n selbstverständlicher Weg“ (55:04-55:19).

\subsubsection{Familiale Gemeinschaft}

Der familiale Zusammenhalt spielt in allen Familien eine wichtige Rolle und es lassen sich keine nennenswerten statusbezogenen Besonderheiten ausmachen. Die gemeinsamen Familienmahlzeiten lassen sich als klassisches Ritual bezeichnen, auf das alle Familien, gleichgültig, welcher Tradierungslogik sie folgen, zu allen Zeiten achten. Hierbei mögen sich die Routinen sowie aufgrund der zunehmenden außerfamilialen Termine der einzelnen Familienmitglieder die Häufigkeiten der gemeinsamen Mahlzeiten ändern, nicht aber die Bedeutung, die ihnen die Familien beimessen. Sie dienen dazu, die familiale Gemeinschaft zu stützen, oder um Audehm aufzugreifen, den „kollektiven Zusammenhalt [zu, C.R.] festigen“ (Audehm 2007, 205). ${ }^{194}$ Für die aktuelle Eltern- und Kindergeneration mag dies auch als eine Reaktion auf die Diskussion um Vereinzelung und Individualisierung gewertet werden, denen die Familien mit der besonderen Betonung der familialen Mahlzeiten bewusst etwas entgegensetzen möchten. Die Familienurlaube, die verstärkt von der mittleren und jüngsten Generation thematisiert werden, dürften u.a. demselben $Z$ weck dienen.

\subsection{Intragenerationelle Gemeinsamkeiten und intergenerationelle Unterschiede}

Abgesehen von den Unterschieden, die sich aus den Tradierungslogiken ergeben, finden sich in horizontaler Perspektive innerhalb der einzelnen familialen Generationen

\footnotetext{
${ }^{194}$ Für Audehm dienen Tischrituale, worunter gemeinsame Familienmahlzeiten zu verstehen sind, dazu „die Familie als Einheit in Differenzen“ darzustellen (Audehm 2007, 205)
} 


\subsection{Intragenerationelle Gemeinsamkeiten und intergenerationelle Unterschiede}

einige intragenerationelle Gemeinsamkeiten, die sich als generationelle Bezugsrahmen bezeichnen lassen. Über diese sowie die hiermit verbundenen Konsequenzen für die jeweiligen Erziehungsarrangements markieren die Akteure zugleich direkt oder indirekt Generationendifferenzen. ${ }^{195}$

In dieser Studie wurden die einzelnen Generationen gemäß ihrer familialen Position verortet. Im Falle der drei Frauen, die Ende der 1940er bzw. Anfang der 1950er Jahre geboren wurden, bedeutet das, dass eine Interviewpartnerin der ältesten und zwei der mittleren Generation zugewiesen wurden. Bei ihnen sind die im Folgenden als generationstypisch markierten Positionen nicht so stark ausgeprägt wie bei den anderen Mitgliedern ihrer jeweiligen Generation. Sie lassen sich somit quasi als generationelle Grenzgängerinnen betrachten. Das deutet auf eher langsame und allmähliche Übergangsprozesse zwischen den Generationen hin, was die Orientierungsmuster betrifft, selbst wenn diese in horizontaler Perspektive als gesellschaftliche konzipiert werden. Innerhalb der jüngsten Generation lassen sich zwei Untergruppen ausmachen. Während die Geburtsjahre der meisten zwischen Ende der 1980er und Anfang der 1990er Jahre liegen, gibt es zwei Vertreter der mittleren Generation im östlichen Teilgebiet, die Mitte bis Ende der 1970er Jahre geboren wurden. Letztere sind somit teilweise in der DDR-Gesellschaft aufgewachsen und haben die Wende als Jugendliche erlebt, wohingegen die übrigen Angehörigen der jüngsten Generation die DDR nicht mehr selbst erlebt haben. Folglich verfügen die beiden Teilgruppen über teils unterschiedliche Erfahrungen, weswegen sich die Aussagen zur jüngsten Generation in erster Linie auf die Ende der 1980er bis Mitte der 1990er Jahre Geborenen beziehen.

\subsubsection{Generationendifferenz und generationelle Topoi}

Die älteste Generation thematisiert in ihren Erinnerungen explizit oder implizit verschiedene Einstellungen, die sie zumindest unterschwellig als Kennzeichen ihrer Generation betrachten. Die meisten Vertreter der Großelterngeneration berichten von den prekären wirtschaftlichen Verhältnissen der Nachkriegszeit, die die Kindheit bzw. Jugend geprägt haben. Nahezu alle sprechen einen Mangel an - entweder bezogen auf Nahrungsmittel oder auf Güter des täglichen Bedarfs (z.B. Frau Dienel IV 23:18-23:33, 24:18-25:09, Cronert II 01:26-01:53, 22:31-22:42, Frau Grothe ${ }^{196}$ I 38:50-40:18, Frau Nolte 30:23-30:54). Sofern die Familien über eigenes Land verfügen, müssen die meisten bei der Bewirtschaftung dieser Fläche mithelfen. Insbesondere die Vertreterinnen der ältesten Generation, die in einem dörflichen Umfeld aufwachsen, erinnern sich, zur Mithilfe herangezogen worden zu sein, sei es im Haushalt, im Bereich der Landwirtschaft oder bei der Betreuung jüngerer Geschwister (siehe z.B. das Fallbeispiel von

\footnotetext{
${ }^{195}$ Einige dieser Aspekte führen die Akteure selbst im Sinne einer „Selbstthematisierungsformel“ (Jureit 2006, 9) ein, um sich einer generationellen Gruppe zuzuordnen. In anderen Fällen wird die generationelle Zuordnung aus den Interviews rekonstruiert.

${ }^{196}$ Die Interviews mit Frau Grothe wurden am 27.08.2009 und am 07.09.2009 geführt.
} 


\section{Bildungsstatus, Generation und Region}

Frau Mahler und Frau Beyer, Frau Grothe I 00:47-01:37, 32:40-32:44, Frau Siebert ${ }^{197}$ I 45:23-46:18). Hierbei thematisieren die Frauen in stärkerem Maße die Mithilfe in Familie und Haushalt als die Männer. Herr Cronert und Herr Friese berichten z.B. eher am Rande von Hilfsleistungen. Stattdessen stellen sie stärker ihre familienbezogenen Tätigkeiten in ihrer Jugend als vollwertige Unterstützungsleistungen dar (Herr Cronert I 08:07-08:15, Herr Friese 2:01:49-2:01:57). In nahezu jedem Interview mit Angehörigen der ältesten Generation finden sich Aussagen, die im Grundtenor darauf abzielen, dass die Verhältnisse während der Kindheit bescheidener gewesen seien. Die äußeren Rahmenbedingungen tragen aus Sicht der Akteure dazu bei, sich mit dem Mangel zu arrangieren, eine genügsame Einstellung auszubilden und Orientierungen wie Fleiß, Sparsamkeit und Bescheidenheit zu internalisieren. Fleiß und Sparsamkeit werden von den Akteuren praktisch mit körperlichen Anstrengungen und Entbehrungen gleichgesetzt, über die sich die Orientierungen zugleich manifestieren.

Eng verbunden mit der Fleißorientierung ist ein bestimmtes Bildungsverständnis in dieser Generation. Viele berichten von im weitesten Sinne haushaltbezogenen und landwirtschaftlichen Fertigkeiten und Kenntnissen, die sie über ihre Mithilfe bei verschiedenen Aufgaben erlernt hätten, wobei in manchen Fällen ein Vergleich zur Gegenwart gezogen wird, der zumindest unterschwellig ein Bedauern über das Fehlen dieser Kenntnisse bei den Jüngeren enthält (Frau Grothe I 1:20:22-1:20:39). Neben den landwirtschaftlichen Kenntnissen wird in einigen Fällen auch naturkundliches Wissen angesprochen, das auf gemeinsam mit Erwachsenen unternommenen Wanderungen oder Waldspaziergängen vermittelt wird (Herr Friese 1:41:44-1:43:10, Frau Dienel IV 05:11-05:17). Hier manifestiert sich ein Lernen über sinnliche, zum Alltag gehörende, in einigen Fällen zwar angeleitete, aber dennoch eigene Erfahrungen. Auch finden sich Anspielungen auf einen kulturellen Kanon in Form von Gedichten, musischer oder ritueller Natur (Frau Grothe I 1:17:47-1:19:15, Frau Dienel IV 21:12-22:04, IV 41:56-43:11, Fallbeispiel Frau Beyer). Wenn implizit Vergleiche zur Gegenwart erfolgen, in der diese Fertigkeiten nicht mehr vermittelt würden, wird hier ein Kanon geteilten Wissens innerhalb der Großelterngeneration suggeriert, über den zugleich eine generationelle Gemeinschaft konstruiert wird. Für die älteste Generation gehört ein bestimmtes Faktenwissen kultureller, naturkundlicher und praktischer Art untrennbar zu ihrem Verständnis von Bildung. Auch wenn die einzelnen Interviewpartner bezogen auf die jeweiligen Kanonkategorien ganz unterschiedliche Inhalte benennen, erwecken sie die Illusion, in der Vergangenheit habe es einen Kanon geteilten Wissens gegeben, der heute in dieser Form nicht mehr vorhanden sei. ${ }^{198}$ Hierdurch wird praktisch ein (vermeintlich) gemeinsamer Bildungskanon als Kennzeichen der eigenen Generation eingeführt.

\footnotetext{
${ }^{197}$ Das Interview mit Frau Siebert stammt vom 28.04.2010.

${ }^{198}$ Implizit kritisiert die Großelterngeneration, in der heutigen Generation gebe es weniger Wissen oder Bildung. Diese Haltung ist allerdings nicht ausschließlich für diese Generation charakteristisch, sondern ein generell wiederkehrendes Phänomen beim Blick auf die jüngeren Generationen, wie Wineburgs Ausführungen nahelegen (2001, 179/180).
} 


\subsection{Intragenerationelle Gemeinsamkeiten und intergenerationelle Unterschiede}

Der beschriebene Bildungsanspruch korrespondiert mit dem in öffentlichen Institutionen wie der Schule vertretenen Bildungsverständnis dieser Zeit - der Orientierung an absoluten Bildungswerten -, das sich erst im Zuge der anschließenden Reformen verändert hat (Gass-Bolm 2005, 213). ${ }^{199}$

Das Aufwachsen in wirtschaftlich prekären Zeiten führt jedoch nicht dazu, einen negativen Blick auf die eigene Kindheit zu entwickeln. Vielmehr bewerten nahezu alle Vertreter der ältesten Generation die Kindheit trotz der gegebenen Schwierigkeiten als eine schöne Zeit. Als typisch für die älteste Generation kann folgendes Zitat gelten: „Es war alles nicht so wie heute. Aber es war doch eine schöne Kindheit" (Frau Grothe, I 02:42-02:46). Hierin ist sicherlich ein Beispiel für den von Pohl thematisierten Positivitätsbias (Pohl 2010, 82) zu sehen, der dazu führt, die Vergangenheit positiver $\mathrm{zu}$ betrachten als sie evtl. ihrerzeit erlebt worden ist. Es ist aber auch ein Beispiel für Reubands Festellung, dass bestimmte Merkmale des Alltags deswegen nicht als problematisch angesehen werden, weil sie als charakteristisch für das gesamte Milieu und aus diesem Grunde vielleicht als einschränkend, nicht aber als grundsätzliches Manko betrachtet werden (Reuband 1992, 107/108).

In den einzelnen Interviews werden über Vergleiche mit der Gegenwart direkt oder indirekt Verlustszenarien skizziert, in denen die Vertreter der ältesten Generation die Bedeutungsabnahme der einen oder anderen soeben beschriebenen Orientierung und Verhaltensstandards in der Gegenwart bedauern. ${ }^{200}$ Das betrifft aus Sicht der Großeltern vor allem die Enkel und ihre Erziehung. Frau Siebert kritisiert z.B. die Anspruchshaltung der heutigen Kinder, bezieht dies aber nicht konkret auf ihre Enkel:

„Na ja, an und für sich, es war ja gut, dass unsere, a:Iso die Eltern streng mit einem waren $/ / \mathrm{hmh} / /$, ne. Denn (es is sonst) heute ist es doch gar nicht mehr so. Heute ist es doch meistens, da erziehen die Kinder doch ihre Eltern, wenn ich das so sehe, $/ / \mathrm{hmh}, \mathrm{hmh} / /$ ne. Meine jüngste Tochter $[\ldots]$ hat $[\ldots]$ gesagt: ,Wir waren heute morgen mit dem Kindergarten zum 70. Geburtstag. Wir haben uns geschämt wie sonst was.' Die rufen schon von weitem: ,Ich will nur grüne Brause, ich will nur rote.' //hmh// Also die stellen schon immer gleich Ansprüche, ne. $/ / \mathrm{hmh} / /$. Das haben wir doch nicht gemacht“ (II 19:47-20:34).

\footnotetext{
${ }^{199}$ Gass-Bolm zeichnet die in den 1950er und 1960er Jahren geführte Debatte um das Bildungskonzept der Gymnasien nach (2005, 176-214) und zeigt einen Wandel auf, für die er folgende Schlagworte benennt: „Relativierung absoluter Bildungswerte“, „Pluralisierung“, „,verstärktes Denken von den Schülern aus“, „Pragmatisierung der Bildungsmetaphysik“, „Intellektualisierung und Theoretisierung anstelle von Charakterbildung und Wertevermittlung“ sowie „Wandel von einem sozialkonservativen zu einem emanzipatorischen Bildungsbegriff“" $(2005,213 / 214)$.

${ }^{200}$ Es werden nicht alle oben angesprochenen Bereiche von allen Interviewteilnehmern thematisiert. Es fällt allerdings als Gesamttendenz im Vergleich zu den beiden jüngeren Generationen auf, dass diese Bereiche verstärkt in die Interviews eingebracht werden und zumindest ein Bedeutungsrückgang direkt oder indirekt bedauernd festgestellt wird.
} 


\section{Bildungsstatus, Generation und Region}

Gelegentlich wird im Vergleich zur eigenen Kindheit der Überfluss kritisiert, in dem die Enkel heute aufwachsen würden. Frau Dienel koppelt das z.B. mit der Befürchtung, der Überfluss könnte die Ausbildung der kindlichen Fantasie behindern (IV 26:29-26:27:01). Im Umkehrschluss bedeutet das, die eher bescheidenen Verhältnisse der Nachkriegszeit hätten Eigenschaften wie Kreativität, Improvisationstalent und Eigeninitiative gefördert. Der wirtschaftlich angespannten Nachkriegssituation werden auf diese Weise retrospektiv positive Seiten abgewonnen.

Ein Blick auf die Darstellungen der nachfolgenden Generationen zeigt, dass die Klage über sie etwas zu pessimistisch ausfällt. Die von der ältesten Generation betonte Sparsamkeit wird zwar in der mittleren Generation seltener erwähnt und nicht so prominent in den Vordergrund gerückt, was sicherlich mit dem im Vergleich zur unmittelbaren Nachkriegszeit gestiegene Wohlstand zusammenhängt. Eine strikte Sparsamkeit ist nicht mehr zwangsläufig erforderlich und steht deswegen weniger im Zentrum der Aufmerksamkeit. Das ist jedoch nicht unbedingt mit einem Verlust dieses Wertes gleichzusetzen.

Auch die von der ältesten Generation für sich reklamierte Orientierung des Fleißes ist in der nachfolgenden Generation nicht verschwunden. Sie kommt allerdings weniger im Gewand einer besonders betonten Tugend daher als vielmehr als eine selbstverständliche Leistungsorientierung und Pflichterfüllung. Die Mithilfe in Haushalt und Familie hat z.B. in der mittleren Generation während ihrer Kindheit bei Weitem noch nicht ausgedient. Es ergibt sich aber im Vergleich zur vorherigen Generation ein deutlich heterogeneres Bild. Während etwa Frank Grothe ${ }^{201}$ aufgrund der familialen sowie gesellschaftlichen Rahmenbedingungen der DDR viele Aufgaben in der Nebenerwerbslandwirtschaft wahrnehmen muss (I 06:45-07:43), scheint Georg Friese, ${ }^{202}$ der in einer westlichen Akademikerfamilie aufwächst, kaum Verpflichtungen zur familialen Mithilfe zu haben. Für die Frauen der mittleren Generation ergibt sich ein ähnlich heterogenes Bild. Diejenigen, die mehrere Geschwister haben, wie Michelle Ahrens und Iris Gümper, sind noch stark in die Betreuung der Kinder und die Mithilfe im Haushalt eingebunden. Für Heike Ebel und Friederike Jäger hingegen scheinen sich die haushaltlichen Verpflichtungen in Grenzen zu halten.

Wie aus den Fallbeispielen bereits ersichtlich, gehen in der jüngsten Generation viele einer organisierten Freizeitbeschäftigung im musikalischen oder sportlichen Bereich nach oder engagieren sich in religiösen Jugendgruppen. Drei Vertreterinnen der jüngsten Generation haben zudem die Möglichkeit eines Auslandsjahres genutzt, um ihren sprachlichen und kulturellen Horizont zu erweitern. Insgesamt zeigt die jüngste Generation eine hohe Motivation, sich im Rahmen ihrer Möglichkeiten weiterzubilden und Kompetenzen zu erlangen, die sie als nützlich für ihre Zukunft betrachten. Die Wertorientierung Fleiß hat somit keineswegs ausgedient, wie sich im Anschluss an die Ausführungen der ältesten Generation vermuten ließe. Vielmehr manifestiert sich Fleiß bei

${ }^{201}$ Die Interviews mit Frank Grothe wurden wurden am 09.10.2009 und 25.02.2010 geführt.

${ }^{202}$ Georg Friese wurde am 11.02.2009 interviewt. 


\subsection{Intragenerationelle Gemeinsamkeiten und intergenerationelle Unterschiede}

der jüngsten Generation in anderer Form. Er ist weniger an unmittelbare körperliche Anstrengung oder familiale Mithilfe gebunden als vielmehr an eine generelle Leistungsorientierung, deren Ergebnisse nicht unmittelbar ersichtlich sind, sondern auf die $\mathrm{Zu}-$ kunft zielen und sich in der Erlangung von Fähigkeiten niederschlagen. Auch der Wert der Sparsamkeit ist nicht obsolet geworden. Zwar wird er von der jüngsten Generation seltener thematisiert und nicht ins Zentrum gestellt, was sich mit den veränderten gesellschaftlichen Rahmenbedingungen erklären lässt. Während Sparsamkeit für die älteste Generation in Kindheit und Jugend aufgrund der prekären Nachkriegsverhältnisse noch eine schlichte Notwendigkeit war, hat sich die finanzielle Situation der heutigen Familien deutlich verbessert. Dennoch finden sich Interviewpassagen, die das Thema Sparsamkeit im weiteren Sinne anschneiden: Janina Ebel muss z.B. Kleidung, die nicht unbedingt notwendig ist, selber bezahlen (44:25-45:58). Die Erziehung konzentriert sich weniger auf eine Sparsamkeit als wirtschaftliche Notwendigkeit, als vielmehr auf die Ausbildung von Kompetenzen im Umgang mit den vorhandenen finanziellen Ressourcen angesichts der vielfältigen Konsummöglichkeiten. Fleiß und Sparsamkeit sind nicht verschwunden, erhalten aber im Generationenverlauf einen anderen Stellenwert und Ausdruck.

Die Klage über den Verlust von Fleiß und Sparsamkeit spiegelt in erster Linie die Sichtweise der älteren Generation auf ihre Nachfolger. Fleiß und Sparsamkeit bilden für die älteste Generation wichtige Topoi, über die sie sich - ausgehend von der Gegenwart - von den Jüngeren, insbesondere der jüngsten Generation, abgrenzen und sich selbst innerhalb einer generationellen Gemeinschaft verorten. Eine generationelle Vergemeinschaftung erfolgt hier nicht in Abgrenzung von einer älteren, wie etwa bei Mannheim (1964/1928) beschrieben, sondern im Gegenteil in Abgrenzung zu einer jüngeren Generation. Gerade der Umstand, dass die Gegenwart und die Erziehung der Enkel den Vergleichsmaßstab bilden, zeigt, dass generationsstiftende Zuordnungen nicht nur in die Jugendzeit fallen, sondern auch zu späteren Zeitpunkten des Lebens erfolgen können, worauf bereits Rosenthal verweist (1997, 62 und 2000, 165). Die Jugendzeit ist hierbei keinesfalls unwichtig, die ihr zugeschriebene Bedeutung für eine generationelle Vergemeinschaftung erfolgt allerdings offensichtlich erst retrospektiv.

In der mittleren Generation gerät die Freizeit stärker in den Fokus der Aufmerksamkeit. Acht Protagonisten der mittleren Generation thematisieren ausdrücklich ihre Spiele im Freien mit Gleichaltrigen. Das dient - ähnlich wie in der ältesten Generation Fleiß und Sparsamkeit - als Topos, um eine Generationendifferenz zur jüngeren Generationen zu markieren und über diese Abgrenzung eine eigene generationelle Zugehörigkeit zu konstruieren. Während es für die mittlere Generation während ihrer Kindheit noch selbstverständlich und wichtig gewesen sei, mit anderen Kindern gemeinsam im Freien zu spielen, sei das bei ihren Kindern nicht mehr so. Gisela Hildner ${ }^{203}$ erinnert sich z.B., viel mit anderen Kindern draußen gespielt zu haben und „nich wie jetzt im Zimmer gehockt“ zu haben (IV 05:20-05:41). Ähnlich äußert sich Friederike Jäger, der

\footnotetext{
${ }^{203}$ Die Interviews mit Gisela Hildner wurden am 07., 14. und 21.04.2010 geführt.
} 


\section{Bildungsstatus, Generation und Region}

es wichtig gewesen sei, „[s]o oft es ging raus“ zu gehen, und die diesen Wunsch bei ihren Kindern vermisst (I 10:10-11:00). Frank Grothe thematisiert ebenfalls die gemeinsamen Spiele mit anderen Kindern: „[M]an hat noch mehr miteinander gespielt wie heute" (I 08:19-08:47). Die mittlere Generation beschreibt hier das Phänomen der Verhäuslichung (Zinnecker 1990) der Kindheit. Diese Veränderung löst bei der mittleren Generation Verwunderung bzw. Bedauern aus. Sie zeichnet somit ebenfalls eine Verlustgeschichte, allerdings anderer Art als die älteste Generation. Die gemeinsamen Spiele im Freien lassen sich als Metapher betrachten, die für verschiedene der mittleren Generation wichtige Aspekte steht. Das Spielen im Freien ist mit dem Aneignen von Freiräumen verbunden, die (zumindest ein Stück weit) der Kontrolle Erwachsener entzogen sind. Es eröffnen sich Möglichkeiten der Selbstständigkeit in Form eigener Entscheidungen und Gestaltungen. Das dürfte vor allem für diejenigen bedeutsam sein, die aus Familien mit einer geschlossenen Tradierungslogik stammen. Wenn die eigenen Kinder nun aus Sicht der Erwachsenen wenig Interesse zeigen, draußen zu spielen und der elterlichen Aufsicht zu entkommen, deutet das u.a. die Befürchtung an, die Kinder könnten weniger als ihrerzeit sie selbst danach streben, Eigenständigkeit zu erlangen. Zugleich wird indirekt kommuniziert, dass die Beziehungen zwischen Eltern und Kindern relativ gut sind, da diese sich kaum um Abgrenzung bemühen.

Zudem enthält das Thema eine soziale Komponente. Die mittlere Generation bringt zum Ausdruck, aktiv eine Gemeinschaftseinbindung gesucht zu haben. Über die Spiele im Freien mit anderen Kindern vollziehen sich Vergemeinschaftungsprozesse, in deren Zuge soziale Fähigkeiten eingeübt werden. Bei den eigenen Kindern besteht offensichtlich die Sorge, sie könnten weniger an Gleichaltrigenkontakten interessiert sein, worin die Erwachsenen die Gefahr zu erblicken scheinen, dass die sozialen Fähigkeiten ihrer Kinder leiden und sie möglicherweise sozial vereinsamen könnten. Diese Interpretation erfährt eine Verstärkung durch die elterliche Skepsis gegenüber Computer und Internet, die z.B. Friederike Jäger (11:01-11:27), Heike Ebel (III 43:17-43:34) und Frank Grothe (I 08:45-08:56) äußern.

Darüber hinaus bedeuten die Kinderspiele im Freien nicht zuletzt freie Zeit. Über den Vergleich mit der Gegenwart, in der einige Kinder sehr viele Termine haben, wird der Verlust von Freizeit sowie ein hiermit verbundener erhöhter Stresspegel beklagt. Dabei erfolgt eine Verklärung der eigenen Kindheit. Denn auch wenn die mittlere Generation bis auf wenige Ausnahmen in ihrer Kindheit nicht so viele außerhäusliche Termine wahrnehmen musste, waren doch viele stark in familiale Aufgaben eingebunden, und ihre freie Zeit war ebenfalls begrenzt. Im Vergleich zur Vorgängergeneration wird über die Metapher des Spielens im Freien das Verhältnis von Arbeit und Freizeit thematisiert sowie der Stellenwert der Freizeit hervorgehoben.

Gemeinschaftsverlust sowie Beschleunigung als zentrale Topoi zu betrachten, die Rehberg zufolge gerade den DDR-Bürgern zur Verarbeitung der Wende dienen (Rehberg 2006, 219), erscheint angesichts dieser Ausführungen nicht plausibel. Im östlichen Teilgebiet wird das Thema des Spiels im Freien vielleicht etwas prominenter in den Vor- 


\subsection{Intragenerationelle Gemeinsamkeiten und intergenerationelle Unterschiede}

dergrund gerückt, es findet sich aber ebenso in der westlichen Region. Innerhalb dieses Samples lassen sich die Klage über Gemeinschaftsverlust sowie Beschleunigung vielmehr als Reaktionen der mittleren Generation auf gesellschaftliche Veränderungen im Zeitverlauf lesen, die dazu dienen, sich über (vermeintlich) andere Erfahrungen einer generationellen Gemeinschaft zuzurechnen und sich dabei insbesondere von der jüngeren Generation abzugrenzen.

Für letztere ist die Gefahr eines Gemeinschaftsverlustes weniger ein Thema, da sie sich in familiale und außerfamiliale Netzwerke eingebunden sieht. In einigen Fällen werden die mit den diversen Verpflichtungen verbundenen Belastungen zwar beklagt, es werden aber auch die positiven und bereichernden Aspekte der schulischen und außerschulischen Aktivitäten hervorgehoben, wie die Beispiele von Lea Gümper und Janina Ebel zeigen. Das unterstreicht nochmals, dass die mit dem Topos des Spielens im Freien transportierten Orientierungen von Gemeinschaft, Freizeit und dem Streben nach Eigenständigkeit in erster Linie Themen der mittleren Generation sind, die dazu dienen sich einer Gemeinschaft zuzuordnen. Zwar werden Kinderspiele als Bestandteil des Alltags auch von der Vorgängergeneration erwähnt, sie werden aber von der ältesten Generation in einen anderen Zusammenhang gesetzt: „Und da waren alle so in dem Alter so wie wir waren, ne. $/ / \mathrm{hmh} / /$ Dann haben wir viel Mal Völkerball oder abends so was gespielt, $/ / \mathrm{hmh} / /$ ne. Wir haben ja auch gespielt, also wir haben nicht nur was getan“ (Frau Siebert I 46:31-46:42). Ähnlich äußert sich Frau Grothe: „Und trotzdem, ich muss sagen, wir hatten aber auch noch Zeit zum Spielen. Nicht dass wir nur Arbeitstiere waren“ (I 1:02:11-1:02:17). Das Spielen dient der ältesten Generation weniger als Abgrenzungskriterium von den jüngeren Generationen, sondern vielmehr als Beleg für die trotz mancher Entbehrungen schöne Zeit der eigenen Kindheit - wobei auch diese Bilanzierung eine verklärende Komponente enthalten mag, wie sich mit Verweis auf den von Pohl beschriebenen Positivitätsbias (Pohl 2010, 82) vermuten lässt.

Es finden sich auch Aussagen, über die sich die mittlere Generation von ihren Eltern abgrenzt, vor allem was bestimmte Ordnungsstrukturen und Elemente des Erziehungsarrangements betrifft. Allerdings ist die Streuung der angeschnittenen Themen breiter als bei der Abgrenzung zur jüngeren Generation.

Innerhalb der jüngsten Generation schließlich lassen sich keine nennenswerten Distanzierungsbemühungen von den älteren Generationen feststellen, wohl aber von bestimmten allgemeinen Diskursen. Die Angehörigen der jüngsten Generation erinnern sich überwiegend, dass die Eltern auf das Einhalten der allgemeinen Höflichkeitsformen geachtet hätten, wie die Fallbeispiele von Janina Ebel und Magdalene Schmidt exemplarisch gezeigt haben. Dieser Aspekt ist sicherlich auch in den vorherigen Generationen relevant gewesen, wurde aber im Vergleich zu anderen Themen nicht so stark hervorgehoben. ${ }^{204}$ Die jüngste Generation könnte an dieser Stelle auf einen allgemeinen Diskurs reagieren, in dem der Verfall der Höflichkeits- und Anstandsregeln beklagt wird. Diese

\footnotetext{
${ }^{204}$ Das Einhalten der allgemeinen Höflichkeitsformen könnte für die älteren Generationen noch als Selbstverständlichkeit gelten, die nicht gesondert betont werden muss.
} 


\section{Bildungsstatus, Generation und Region}

Klage findet sich gelegentlich auch in Äußerungen der mittleren und ältesten Generation. Indem die Höflichkeitsformen als Bestandteil der Erziehung betrachtet werden, distanziert sich die jüngste Generation von diesem vermeintlichen Verlust. Zugleich folgt sie einem aktuellen Trend, wie das öffentlich diskutierte gegenwärtig wieder steigende Interesse an der Kenntnis dieser Umgangsformen als Gegenreaktion auf den beklagten Verfall zeigt. Möglicherweise wird dieser Aspekt aber auch deswegen thematisiert, weil er als eine Art Minimalkonsens und eine der wenigen verbleibenden Forderungen mit verpflichtendem Charakter wahrgenommen wird.

Ein weiteres Thema ist die elterliche Erziehung. Obwohl die Kindergeneration einerseits die Erziehung als sehr freiheitlich und ungezwungen beschreibt, möchte sie andererseits nicht den Eindruck erwecken, die Eltern hätten eine laissez-faire-Erziehung praktiziert. Wie die Fallbeispiele gezeigt haben, charakterisieren die meisten Angehörigen der Kindergeneration die Erziehung zunächst als ,nicht sehr streng'. Das schränken sie aber anschließend ein, indem sie sich an eine gelegentlich strengere Erziehung der Eltern und einige feste Regeln erinnern. Diese Relativierungen lassen sich als Reaktion auf einen öffentlichen Diskurs verstehen, der durch die Klage geprägt ist, Kindern würden keine Grenzen und Regeln mehr gesetzt. Der implizit mitschwingende Vorwurf lautet, Kinder würden in der Folge zu Egoisten erzogen und der gesellschaftliche Zusammenhalt sei gefährdet. Diesen plakativen Verfallsszenarien tritt die Kindergeneration entgegen, indem sie darauf verweist, dass ihr Regeln gesetzt worden seien und sie in der Folge nicht als unerzogen gelten könne.

In der Gesamtschau betonen die einzelnen Generationen jeweils spezifische Aspekte und Orientierungen. Diese lassen sich als Elemente betrachten, die dazu dienen, sich in die Gemeinschaft einer Generation einzuschreiben. Interessanterweise grenzen sich hierzu die beiden älteren Generationen in erster Linie von der jüngeren ab, wohingegen die jüngste Generation sich nicht dezidiert von der älteren absetzt. Das ist eine wichtige Erkenntnis, thematisieren klassische Texte wie der Mannheims (1964/1928) doch eher die Abgrenzung der jüngeren von einer älteren Generation. Demgegenüber weisen die Ergebnisse dieser Studie darauf hin, dass generationelle Selbstverortungen anscheinend besonders in späteren Lebensphasen relevant werden und das Einschreiben in eine generationelle Gemeinschaft oftmals ex post erfolgt. Aus dem vorhandenen Portfolio an Orientierungen werden in den einzelnen Generationen jeweils bestimmte besonders hervorgehoben, die sich folglich als generationell geprägte Bezugsrahmen betrachten lassen: In der ältesten Generation sind das Fleiß und Sparsamkeit, in der mittleren die Gemeinschaft der Gleichaltrigen, das Verhältnis von Arbeit und Freizeit sowie das Streben nach Eigenständigkeit und in der jüngsten Generation Leistungsorientierung sowie die Aufmerksamkeit für das Sozialverhalten. Insbesondere die Veränderungen zwischen der ältesten und mittleren Generation lassen sich in Zusammenhang mit dem Wertewandel der 1960er und 1970er Jahre bringen. Dies heißt jedoch nicht, dass bestimmte Werte verloren gingen. Vielmehr werden im Generationenverlauf aus dem Spektrum der vorhandenen Werte jeweils bestimmte besonders betont, ohne dass die anderen gänzlich obsolet würden. Die aufgezeigten Veränderungen sind zudem unweigerlich mit struk- 


\subsection{Intragenerationelle Gemeinsamkeiten und intergenerationelle Unterschiede}

turellen Entwicklungen wie der allgemeinen Wohlstandsentwicklung verbunden, die es erst ermöglichen, Orientierungen wie Fleiß und Sparsamkeit zugunsten anderer Bezugsrahmen in den Hintergrund treten zu lassen. Die zunehmende Individualisierung, Pluralisierung und Entkopplung, in deren Zuge die Möglichkeiten zur Gestaltung der eigenen Orientierungen zunehmen (Vester u.a., 1993, 38/39), stellen weitere Faktoren dar, die die beschriebenen Neuakzentuierungen begünstigen. Die beschriebenen Ergebnisse spiegeln Vesters u.a. Befunde, wonach Kinder sich selten vollständig von den elterlichen Traditionen lösen und stattdessen die tradierten „Deutungs-, Handlungs- und Ausdrucksmuster" weiterentwickeln, die milieuspezifischen Orientierungen in ihren Grundzügen erhalten bleiben, „in ihrer Ausgestaltung jedoch modernisierungsfähig“ sind (Vester u.a. 1993, 183 und 188).

\subsubsection{Erziehungsarrangements: Von der selbstverständlichen Ordnungs- zur Angebotsstruktur}

Ein weiteres Kennzeichen, das die älteste Generation auszeichnet, ist das Sich-Einfügen in gegebene Ordnungsstrukturen. Die von Herrn und Frau Friese geäußerte Devise, sich so zu benehmen, dass die Eltern nicht blamiert werden (1:02:23- 1:03:46, 47:22-1:47:41), kann als allgemeine Leitlinie für die älteste Generation gelten, jedoch in unterschiedlich stark ausgeprägter Form. Generell werden Anweisungen der Eltern selten offen und direkt kritisiert oder missachtet. Unabhängig von der jeweils konkreten Ausgestaltung der Eltern-Kind-Beziehungen werden die Eltern als Autoritäten wahrgenommen, denen die letztliche Entscheidungsbefugnis zugebilligt wird, wie bereits die Fallbeispiele illustrieren. Exemplarisch für diese Grundhaltung lässt sich die Äußerung Frau Webers ${ }^{205}$ anführen, die zugleich zu erkennen gibt, dass der ältesten Generation das Autoritätshandeln der Eltern bewusst ist: „Da kamen wir gar nicht auf die IDEE aufzubegehren. //hmh// Da muss ich sagen, da wurden wir als Kinder auch schön klein gehalten. //ja// (2) Und fanden das auch in Ordnung" (II 23:17-23:34). Zwar finden sich in den Interviews diverse Beispiele dafür, wie sich die Angehörigen der ältesten Generation über elterliche Anweisungen hinwegsetzen, das steht für diese Generation allerdings nicht im Widerspruch dazu, die elterliche Autorität grundsätzlich anerkannt zu haben. Die Regelübertretungen erfolgen eher verdeckt, eine offene Konfrontation mit den Eltern hingegen wird nicht aktiv angestrebt. Wie bereits an den Fallbeispielen ersichtlich geworden ist, ist die regelmäßige Gottesdienstteilnahme eine Selbstverständlichkeit und ein elementarer Bestandteil der eigenen Religiosität. Darüber hinaus beschreibt die älteste Generation für ihr Aufwachsen das Vorherrschen einer konservativen Sexualmoral, die von ihr meist nicht hinterfragt wird und mehr oder weniger unverändert in die Erziehung der eigenen Kinder einfließt (z.B. Frau Dienel IV 47:10-47:40, 1:27:51-1:28:09; Herr Friese 2:22:51-2:23:04). Das Einfügen in die vorgefundenen Ordnungsstrukturen ließe sich tendenziell als eine Form der Unterdrückung deuten, wird aber von den Angehöri-

${ }^{205}$ Das Interview mit Frau Weber stammt vom 18.02.2009. 


\section{Bildungsstatus, Generation und Region}

gen der Großelterngeneration keinesfalls in dieser Weise wahrgenommen. Vielmehr stehen für sie die Gemeinschaftseinbindung und Milieuorientierung im Vordergrund, die ihnen neben Reglementierungen auch Unterstützung und Zusammenhalt bietet: „Also das wär, diese, (diese) Gemeinschaft, dieses gemeinschaftliche Leben, das GEBEN, aber auch das Nehmen, //hmh// das hängt dann aber eng zusammen" (1:05:27-1:05:37). Laut Herrn Cronert sei der Zusammenhalt im Verwandten- und Bekanntenkreis während der Nachkriegszeit und auch noch zu DDR-Zeiten stark und die gegenseitige Hilfe selbstverständlich gewesen (Herr Cronert II 1:25:44-1:27:15) ${ }^{206}$ Der Zusammenhalt wird als Vorteil beschrieben, der aus Sicht der Interviewpartner im Vergleich zur Gegenwart früher größer gewesen sei. Pointiert kommt diese Ansicht in der Äußerung von Frau Siebert zum Ausdruck: „Ja, früher waren=waren wir alle, ich will mal sagen, praktisch wie verwandt, ne. //ja// Jeder hat sich um den anderen auch gekümmert. //hmh// Das ist doch heute nicht mehr" (I 1:25:37-1:25:47).

Insgesamt entsteht der Eindruck, dass die älteste Generation das sie umgebende Milieu während ihrer Kindheit als geschlossener wahrnimmt als die heutige Kindergeneration. Das wird aber nicht unbedingt negativ gedeutet, denn das Einfügen in die bestehende Ordnung bietet vor allem Orientierungs- und Verhaltenssicherheit. Das wird auch von den Befragten in beiden Teilregionen so wahrgenommen: „Aber dadurch war es ja auch, muss ich ehrlich sagen, es war ja auch leicht dadurch. $/ / \mathrm{hmh} / / \mathrm{Man}$ war es gewohnt. $/ / \mathrm{hmh} / /$ Man fühlte sich verpflichtet, ja al:so, es war also. Es erforderte ja gar keine Anstrengung mehr. Man lebte in der Gemeinschaft, mit der Gemeinschaft" (Herr Friese 1:04:07-1:04:22). Da die Standards vorgegeben werden, ist zwar die Wahl eingeschränkt, es enthebt den Einzelnen aber auch des $Z$ wanges, verschiedene Optionen gegeneinander abwägen und selbst Entscheidungen treffen sowie verantworten zu müssen.

Der somit von der ältesten Generation umrissene Wertekanon lässt sich an Ingleharts $(1979,1989,1998)$ und Klages' $(1984,2001 b, 2002)$ Studien zum Wertewandel rückbinden. Die Angehörigen der Großelterngeneration verinnerlichen im Zuge ihres Aufwachsens die Orientierungen, die sich in die Kategorie der Pflicht- und Akzeptanzwerte einordnen lassen. Hiermit einhergehend stehen die eigenen Gefühlslagen selten im Mittelpunkt der Betrachtung, wenn die Angehörigen der Großelterngeneration von ihrer Kindheit erzählen. Auch finden sich kaum Reflexionen, die die Frage nach den eigenen Entwicklungschancen und ihre (familienbedingten) Begrenzungen betreffen. In den wenigen Fällen, in denen das geschieht, werden verhinderte Optionen zwar bedauert, das führt aber selten zu eindeutig gegen die Eltern gerichtete Anklagen. Insgesamt zeichnet die Großelterngeneration in ihren Schilderungen das Bild einer akzeptierenden Generation, deren Konturen sich insbesondere über einen Vergleich mit der Gegenwart ergeben.

Diese akzeptierende Grundhaltung schlägt sich auch in der retrospektiven, tendenzi-

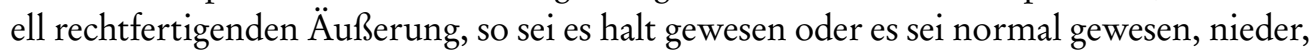
die in dieser oder ähnlicher Form in verschiedenen Interviews zu finden ist und von den

\footnotetext{
${ }^{206}$ Das habe erst mit den Veränderungen der Lebensumstände nach der Wende abgenommen.
} 


\subsection{Intragenerationelle Gemeinsamkeiten und intergenerationelle Unterschiede}

Befragten auf unterschiedliche Bereiche ihres kindlichen Alltags wie die wirtschaftliche Situation, die Religiosität sowie die elterlichen Vorgaben bezogen werden und sich als Topos dieser Generation bezeichnen lässt. Frau Grothe formuliert z.B. in Bezug auf die Versorgung mit Kleidung: „Und von wegen immer was Neues haben, das gab's nich. Einer musste vom anderen das auftragen, nich. //hmh// Das war ja so"(I 39:19-39:26). Die religiösen Praxen werden in ähnlicher Weise bilanziert: „[A]ber hier waren sie alle katholisch. Und wir haben das gar nicht als, als was Strenges empfunden, $/ / \mathrm{hmh} / /$ das war eben so. $/ / \mathrm{hmh} / /$ Wir gingen sonntags zur, (um, zur) Kirche und dann war das gut" (Frau Grothe II 11:09-11:20). Frau Mahler kommentiert ihre kindliche Verpflichtung, im Haushalt zu helfen oder den sonntäglichen Kirchgang mit den Sätzen: „Das war einfach so" bzw. „[d]as war so" (Frau Mahler 10:10-10:27 und 47:09-47:15). Frau Dienel wiederum verweist auf die Normalität der knappen finanziellen Mittel während ihrer Kindheit: „Und dass eben die anderen Kinder, unsere Freunde, eigentlich auch nicht viel hatten und das deswegen normal war" (IV 25:09-25:14). Ein eindrückliches und mit der Gegenwart gekoppeltes Beispiel für die betonte Bereitschaft, sich in die vorgefundenen Ordnungsstrukturen einzufügen, findet sich auch bei Frau Weber: „Also wir waren sehr, ich muss sagen, (__ anpassungsfähig, //hmh// ohne Kopf durchzusetzen, //hmh// weil wir eben Verständnis hatten für alles. $/ / \mathrm{hmh}, \mathrm{hmh} / /$ Das geht nicht (.) und dann ging das nicht. Heute ist man da selbstbewusster" (II 20:32-20:48).

Insgesamt sind bei der Erziehung der eigenen Kinder, also der mittleren Generation, kaum dezidierte Absetzbewegungen vom selbst erfahrenen Erziehungsstil zu bemerken. Es werden zwar einige Elemente der Erziehung verändert, nicht aber das Erziehungssetting der eigenen Eltern radikal in Frage gestellt. Vielmehr scheint es den Vertretern der ältesten Generation bei der Erziehung der nächsten Generation viele Anknüpfungspunkte zu bieten. Die Übernahme der elterlichen Erziehungspraxen bildet eine Gemeinsamkeit innerhalb dieser Generation. Allerdings gibt es aufgrund der jeweiligen Tradierungslogiken erhebliche Unterschiede in Bezug auf die konkrete Erziehungsgestaltung. Hierbei fällt auf, dass sich die bereits in der Kindheit der ältesten Generation erkennbaren Unterschiede der familialen Erziehungsarrangements während der eigenen Familienphasen noch verstärken, wie den Fallbeispielen des vorherigen Kapitels zu entnehmen ist. Vertreter der ältesten Generation, die in Familien aufwachsen, in denen liberale Elemente der Erziehung bereits zu finden sind, übernehmen besonders diese bei der Erziehung der eigenen Kinder und bauen sie aus, wohingegen diejenigen, die in autoritär geprägten Verhältnissen aufwachsen, eben diese beibehalten. In der Folge driften die familialen Erziehungssettings der Familien des Samples bei der Erziehung der eigenen Kinder weiter auseinander.

Diese Ergebnisse deuten darauf hin, dass sich nicht erst in der Folge der Ereignisse um 1968 die Erziehungsverhältnisse radikal zugunsten einer egalitäreren Erziehung ändern, da bereits einige Vertreter der ältesten Generation unter Bedingungen aufwachsen, die nicht mehr als ausgesprochen autoritär eingestuft werden können. Reubands (1995, 1997) Untersuchungen, die als langfristigen Trend den Abbau autoritärer Praxen seit Be- 


\section{Bildungsstatus, Generation und Region}

ginn des 20.Jahrhunderts aufzeigen, unterstützen diese Annahme. Und Baumert findet schon in den 1950er Jahren neben autoritären Familienstrukturen mit einer deutlichen Vormachtstellung des Mannes Familien, in denen sich Tendenzen der Gleichrangigkeit in den Familienbeziehungen sowie ein verändertes Verhältnis zum Kind zeigen (Baumert 1954, 167/168). ${ }^{207}$ Das legt den Schluss nahe, dass die Ereignisse um 1968 als Katalysatoren für die Verbreitung liberalerer Erziehungseinstellungen gewirkt haben können, aber nicht als Auslöser. ${ }^{208}$ Die älteste Generation, deren Familienphase Ende der 1950er bis in die 1970er Jahre fällt, kann potenziell auf verschiedene, gesellschaftlich und familial vorfindbare Leitbilder zurückgreifen, und eine dezidiert autoritäre Erziehung ist nicht zwangsläufig anzunehmen. Vor diesem Hintergrund wird die vorgefundene Heterogenität der Erziehungsarrangements innerhalb dieses Samples verständlich.

Die mittlere Generation erinnert, ähnlich wie ihre Eltern, bestimmte Rahmenbedingungen der Erziehung, die darin bestehen, sie als Kinder in das sie umgebende Milieu mit seinen Wertsetzungen einzubinden. Die zentralen Orientierungen, die an alle Vertreter der mittleren Generation herangetragen werden, sind die Partizipation an religiösen Praxen sowie eine konservative Sexualmoral. In der Art und Weise, wie die mittlere Generation die Umsetzung wahrnimmt, ergeben sich allerdings gewichtige Unterschiede. Die Spannweite reicht von „Richtlinien“ (Georg Friese 10:52-10:57), die einzuhalten gewesen seien, über einen grob abgesteckten Rahmen (Fallbeispiel Friederike Jäger), innerhalb dessen sich die mittlere Generation entfalten kann, bis zu (geduldeten) Überschreitungen dieser Rahmen (Fallbeispiel Birgit Schmidt). Die Devise, sich so zu benehmen, dass das Ansehen der Eltern nicht beschädigt wird, gilt in dieser expliziten Form nur noch in einigen Familien, die einer geschlossenen Tradierungslogik folgen. Die bereits aus der Großelternperspektive rekonstruierte zunehmende Pluralität der Erziehungsarrangements wird durch die Erinnerung der mittleren als aneignenden Generation bestätigt, wobei in der Gesamtschau die Kindorientierung zunimmt. Diese Entwicklung korrespondiert mit der für die 1960er/1970er Jahre beschriebenen Heterogenität der Erziehungssettings (Schütze/Geulen 1983, 45/46) sowie der gesamtgesellschaftlich zu beobachtenden wachsenden Kindzentrierung.

Die mittlere Generation stellt sich selbst nicht mehr als so passiv hinnehmend und akzeptierend dar wie die älteste Generation. Sie erweckt im Gegenteil eher den Eindruck, aktiv die eigenen Interessen zu vertreten und durchzusetzen. Hiermit einher geht eine höhere Bereitschaft, Konflikte während der Kindheit und Jugend zu thematisieren. Das Konfliktniveau innerhalb der verschiedenen Familien fällt dabei sehr unterschiedlich aus. Beispiele für stark ausgeprägte intergenerationelle Konflikte finden sich vor allem in Familien, die einer geschlossenen Tradierungslogik folgen, wenn auch nicht in allen.

${ }^{207}$ Diese Ergebnisse widersprechen der Studie Schaffners, der für die Nachkriegszeit ausgesprochen autoritäre Familienkonstellationen mit einem väterlichen Machtmonopol beschreibt (Schaffner 1949). Eine kritische Auseinandersetzung mit dieser Untersuchung liefert König (1974, 220-220).

${ }^{208}$ In Bezug auf Veränderungen im Diskurs um die Frühsozialisation schätzt Gebhardt den Anteil der '68er-Bewegung ebenso skeptisch ein (Gebhardt 2009a, 175/176). 


\subsection{Intragenerationelle Gemeinsamkeiten und intergenerationelle Unterschiede}

So ist das Konfliktniveau in den beiden vorgestellten Fallbeispielen zwischen Michelle Ahrens bzw. Iris Gümper und ihren Eltern sehr hoch. Georg Friese wiederum, der ebenfalls in einer Familie heranwächst, die einer geschlossenen Tradierungslogik folgt, berichtet kaum über Konflikte. Während Michelle Ahrens und Iris Gümper sich während ihrer Kindheit oft als fremdbestimmt wahrnehmen sowie unter dem Konformitätsbzw. Leistungsdruck der Eltern leiden und dies auch thematisieren können, scheint Georg Friese eine Fremdbestimmung oder gar einen von den Eltern ausgehenden Leistungsdruck auszublenden. Er ist eher darum bemüht, sich als selbstbestimmt agierend darzustellen (00:57-03:28). Diese Divergenzen könnten das Resultat einer genderdifferenten Sozialisation sein, die es den Frauen ermöglicht, ihre Gefühle und insbesondere ihr Leiden zu thematisieren, wohingegen dies dem männlichen Geschlechtsrollenklischee deutlich widerspricht.

Insgesamt wird die Wahrnehmung der elterlichen Erziehung im Vergleich zur Vorgängergeneration heterogener. Zwar finden sich bereits in der Vorgängergeneration Unterschiede, die sich darauf beziehen, wie die elterliche Erziehung beschrieben wird, die Beurteilung fällt jedoch ähnlich aus: Die elterliche Erziehung wird weitgehend akzeptiert. Für die mittlere Generation hingegen ist es nicht mehr unbedingt selbstverständlich, sich in die gegebenen Ordnungsstrukturen widerspruchslos einzufügen. Wie sich an dieser Stelle zeigt, hängt die Wahrnehmung und Beurteilung der erfahrenen Erziehung wesentlich von den jeweils dominanten „Erwartungen und Erfahrungen von ,Normalität"“ sowie davon ab, was in der jeweiligen Bezugsgruppe als typisch und allgemein gültig für die jeweilige Zeit betrachtet wird (Reuband 1992, 108). Während die älteste Generation trotz der in der Analyse herausgearbeiteten Unterschiede kaum alternative Erziehungsformen und -inhalte in ihrem Umfeld während der eigenen Kindheit thematisiert, ändert sich das in der folgenden Generation. Die Begrenzungen des zuvor fest umrissenen sozialen Milieus beginnen aufzuweichen. Einzig der regelmäßige Kirchgang wird bis auf wenige Ausnahmen weiterhin als elementarer Bestandteil des Alltags akzeptiert und verbleibt als kleinster gemeinsamer Nenner und einendes Band zwischen den Angehörigen des sozialen Milieus. Die Erziehungssettings selbst hingegen weisen eine zunehmende Heterogenität auf. Während einige Eltern an den tradierten Bahnen der Erziehung festhalten und Veränderungen strikt zu vermeiden suchen, integrieren andere Eltern liberale Elemente in ihr Erziehungsarrangement, die in Ansätzen bereits in ihrer Kindheit zu finden sind, und bauen diese weiter aus. Diese Liberalisierungstendenzen müssen auch Kinder bemerken, die aus Familien stammen, welche einer geschlossenen Tradierungslogik folgen. Sie können direkt in ihrem Umfeld erleben, dass das Einfügen in tradierte Ordnungsvorstellungen nicht (mehr) unbedingt selbstverständlicher Bestandteil der Erziehung sein muss. Diese Pluralisierungstendenzen dürften die Neigung erhöhen, gegen allzu strikte elterliche Vorgaben aufzubegehren und Regelungen zu hinterfragen. Die größere Bandbreite der Reaktionen in der mittleren als aneignenden Generation ergibt sich damit aus der zunehmenden Heterogenität der elterlichen Erziehungssettings im unmittelbaren Nahfeld. 


\section{Bildungsstatus, Generation und Region}

Bei der Erziehung der jüngsten Generation werden die Erziehungssettings der einzelnen Familien insofern wieder homogener, als autoritäre Elemente in der Erziehung deutlich an Bedeutung verlieren. In einigen Familien haben sie ohnehin im untersuchten Zeitraum eine eher marginale Rolle gespielt. In den Familien, in denen autoritäre Traditionen zu finden sind, werden diese von der mittleren Generation nicht für die Erziehung der eigenen Kinder übernommen. Das wird auch deutlich kommuniziert, wie die Fallbeispiele im vorherigen Kapitel bereits gezeigt haben. Georg Frieses Ansicht, die Erziehung sei heute deutlich „lockerer" geworden (06:29-06:40), kann als typisch für die mittlere Generation gelten. Das betrifft z.B. die vormals festen Ordnungsstrukturen. So wird der regelmäßige Gottesdienstbesuch, wie bereits für einzelne Familien beschrieben, von einer Pflicht zu einer Option für die Kinder transformiert. Auch hat die konservative Sexualmoral offensichtlich ausgedient. Folglich vollzieht sich auf breiter Ebene eine Loslösung von bestimmten Elementen des engen Werte- und Verhaltenskanons, der zumindest in Ansätzen bei der eigenen Erziehung noch eine Rolle gespielt hat. Andere Elemente hingegen behalten ihren Stellenwert oder erfahren eine gesteigerte Aufmerksamkeit. In allen Familien wird auf die schulischen Leistungen der Kinder geachtet. Damit verlagert sich der Normierungsdruck bzw. wird auf ein Element gebündelt. Das Primat der optimalen Kindesförderung sowie der weitgehende Verzicht auf autoritäre Erziehungsstrukturen sind weitere gesellschaftliche Trends (Beck-Gernsheim 1990a, 169 und Büchner 2010, 533), die von der mittleren Generation des Untersuchungssamples aufgegriffen werden.

Wie die Fallbeispiele eindrücklich gezeigt haben, werden den Kindern mehr Freiräume und eigene Wahlmöglichkeiten eingeräumt. Ihnen wird der elterliche Wille nicht direkt aufgezwungen, sondern es werden ihnen zumindest vordergründig Entscheidungsoptionen eingeräumt. Als Tendenz zeichnet sich ab, dass Grenzen weniger absolut gesetzt werden, sondern als relative Größen in Abhängigkeit von den jeweiligen Umständen im intergenerationellen Dialog deutbar werden. Die beschriebenen Erziehungskomponenten sind geeignet das Einüben von Eigenständigkeit zu unterstützen. Die mittlere Generation des Samples orientiert sich damit an einer gesamtgesellschaftlichen Entwicklung; der Favorisierung des Erziehungsziels „Selbständigkeit und freier Wille“ (Reuband 1997, 134).

Die allgemeine Lockerung der Erziehungsarrangements ist mit einer abnehmenden Reglementierung des kindlichen Verhaltens verbunden. Lediglich die Wahrung eines bestimmten Sozialverhaltens, die ein gewisses Maß an Hö̈lichkeit und Respekt gegenüber anderen beinhaltet, verbleibt als allgemeine Verhaltensregel. Insgesamt nehmen verbindliche Regelungen ab, Grenzen werden durchlässiger und die Wahloptionen steigen. Die aktuelle Elterngeneration des Samples präsentiert sich diesbezüglich sehr tolerant, indem sie ihren Kindern in vielen Bereichen Entscheidungsmöglichkeiten einräumt. Die Kinder als „Akteur[e] in eigener Sache“ (Schütze 2002, 83) wahrzunehmen, findet hier ihren lebensweltlichen Niederschlag. Das bedeutet zugleich, dass die Kinder selbst als stärker verantwortlich für ihr eigenes Handeln betrachtet werden, was wiederum die Eltern ein Stück weit entlastet und ein Grund sein dürfte, warum sich die aktuelle El- 


\subsection{Intragenerationelle Gemeinsamkeiten und intergenerationelle Unterschiede}

terngeneration im Vergleich zu ihren Vorgängern als großzügiger beschreiben kann, zumindest in Bezug auf bestimmte Bereiche. Gleichzeitig avanciert die Förderung des Kindes (Beck-Gernsheim 1990a, 169) zum neuen Beurteilungsmaßstab der Erziehung, sodass sich in der Gesamtschau die elterlichen Verantwortlichkeiten lediglich verschieben.

Des Weiteren rückt der kommunikative Austausch mit den Kindern in der mittleren Generation stärker ins Zentrum der jeweiligen Erziehungssettings. Zwar ist die intergenerationelle Kommunikation zweifelsohne auch in der Vorgängergeneration wichtig, die mittlere Generation reflektiert dieses Thema allerdings stärker und entwickelt verschiedene Strategien, um ein hohes Maß an kommunikativem Austausch mit den Kindern sicherzustellen, der über Gespräche während der gemeinsamen Familienmahlzeiten hinausgeht. Hierin manifestiert sich eine zunehmende Kindorientierung und Berücksichtigung ihres (emotionalen) Wohlbefindens, was mit gesamtgesellschaftlichen Entwicklungen korrespondiert (siehe Kap. 1.2.4).

Im Unterschied zu den beiden älteren Generationen und in der Folge ihrer Erziehung betont die Kindergeneration weniger die Ordnungsstrukturen als vielmehr die vielfältigen Freiheiten, die ihnen eingeräumt werden. Sie erinnern kaum Vorschriften, und die familialen Aufgaben und Pflichten halten sich in Grenzen. Ähnlich verhält es sich mit Verboten. An ihre Stelle treten in vielen Fällen oftmals außerfamiliale Anforderungen, sei dies in Form schulischer Aufgaben oder diverser Freizeitaktivitäten. Das existierende Angebot führt gleichzeitig zu neuen Zwängen, die (relativ) unabhängig von den Eltern durch die gesellschaftlichen Rahmenbedingungen entstehen. Die jüngste Generation kann aus einer Vielzahl von Bildungs- und Freizeitmöglichkeiten wählen, zugleich steigt der allgemeine Druck, eines dieser Angebote wahrzunehmen und hierdurch über das schulische Angebot hinaus Zusatzqualifikationen zu erwerben. Bei der Realisierung außerschulischer Bildungs- und Freizeitangebote fühlt sich die jüngste Generation i.d.R. von den Eltern unterstützt, die offensichtlich das Primat der optimalen Kindesförderung (Beck-Gernsheim 1990a, 169) internalisiert haben. Die Eltern sind fast immer bereit, sportliche oder musikalische Hobbys finanziell zu unterstützen, wie die Fallbeispiele exemplarisch gezeigt haben. Darüber hinaus berichten fast alle Vertreter der jüngsten Generation von gemeinsamen Familienurlauben, womit sie zugleich zum Ausdruck bringen, dass die Eltern ihren Kindern „tolle Erlebnisse geboten“ (Miriam Friese 01:15-01:17) haben sowie viele Möglichkeiten eröffnen, neue Eindrücke zu gewinnen und den eigenen Horizont zu erweitern. Diese elterlichen Leistungen werden von der jüngsten Generation gewürdigt und geschätzt. Die gemeinsamen Reisen werden von einigen Vertretern der jüngsten Generation explizit als Beitrag zur eigenen Persönlichkeitsentwicklung gedeutet. Anika Jäger betrachtet die Reisen z.B. als Katalysator, der ihr „multikulturelles Denken“ gefördert habe (06:30-06:35). Insgesamt zeigt sich die jüngste Generation hoch motiviert, sich im Rahmen ihrer jeweiligen Möglichkeiten weiterzubilden und Kompetenzen zu erlangen, die sie als nützlich für ihre Zukunft betrachten. Hieraus spricht ein ziel- und zweckorientiertes Denken, das aktuelle Erfah- 


\section{Bildungsstatus, Generation und Region}

rungsmöglichkeiten aus dem Blickwinkel eines zukünftigen Ertrages beurteilt, das für die jüngste Generation in beiden Teilgebieten gilt.

Die Kindergeneration schätzt die Beziehung zu den Eltern im Großen und Ganzen positiv ein. Hierzu trägt sicherlich der Eindruck bei, in vielen Bereichen große Entscheidungsspielräume und Mitspracherechte eingeräumt zu bekommen. Diese Möglichkeiten nutzt die Kindergeneration, womit sie die Rolle des Kindes als „Akteur in eigener Sache" (Schütze 2002, 83) an- und sich in der Folge als sehr eigenständig wahrnimmt. Konflikte spielen insgesamt eher eine marginale Rolle. Damit reiht sich die jüngste Generation in die positive Beurteilung der Eltern-Kind-Beziehungen ein, wie sie sich in den neueren Shell Jugendstudien finden (Langness/Leven/Hurrelmann 2006, 57-60). Einen Sonderstatus nehmen hierbei diejenigen ein, die ihre Jugend während der Wende erlebt haben, die sogenannten Mauerfallkinder. Sie erinnern im Unterschied zu den übrigen Vertretern der jüngsten Generation größere Auseinandersetzungen mit den Eltern. Diese lassen sich als eine Folge der mit der Wende einhergehenden Veränderungen interpretieren. Sie beschreiben eine Jugend, in der die bis dato gültigen Strukturen, insbesondere der regelmäßige Kirchgang, deutlich an normierender Kraft einbüßen und vormals wenig präsente Freizeitangebote in Konkurrenz zu den bisherigen Ordnungsstrukturen treten.

Wie die Fallbeispiele gezeigt haben, betrachten die Kinder ihre Eltern in vielen Bereichen als undogmatisch. ${ }^{209}$ Die Kinder setzen sich selbstbewusst und kreativ mit den gesetzten Regeln auseinander und können ihre Eltern oftmals durch Diskussionen davon überzeugen, ihren Standpunkt zu ändern. Janinas erfolgreiches Durchsetzen der abendlichen Internetnutzung zu Kommunikationszwecken ist ebenso ein Beispiel hierfür wie Leas letztlich wirkungsvolle Argumente dafür, den Musikunterricht aufzugeben. Die Beziehungen zu den Eltern werden nicht, wie noch gelegentlich in der Vorgängergeneration, als angstgeprägt beschrieben. Die Elterngeneration überträgt nicht umstandslos die eigenen Erfahrungen auf die Erziehung der Kinder, sondern ist in bestimmten Bereichen bereit, sich den veränderten Gegebenheiten anzupassen sowie den Wünschen und Interessen der Kinder entgegenzukommen. Wie die Beispiele demonstrieren, kann die weitgehende Durchsetzung des Verhandlungshaushalts in den Erziehungssettings (Ecarius 2002, 221) bis in die Gegenwart Gültigkeit beanspruchen und ist prägend für die aktuelle Erziehung.

In der Gesamtschau ändern sich die Erziehungssettings im Generationenverlauf. Je nach Tradierungslogik starten die Familien von verschiedenen Ausgangsniveaus, was die Kindzentrierung, die Beachtung des emotionalen Wohlbefindens sowie die Mitspracherechte der Kinder betrifft. In Familien, die einer geschlossenen Tradierungslogik folgen, sind diese drei Elemente in der ältesten Generation am schwächsten ausgeprägt. In der folgenden Generation nehmen sie in allen Familien unabhängig von der jeweiligen Tradierungslogik zu. Dies impliziert zugleich die aktive Teilhabe von vermittelnder wie aneignender Generation am Tradierungsprozess. Für die Entwicklung der Erzie-

${ }^{209}$ Die in den Fallbeispielen beschriebenen Ausnahmen ändern nichts an diesem Grundtenor. 


\subsection{Intragenerationelle Gemeinsamkeiten und intergenerationelle Unterschiede}

hungsarrangements lassen sich folglich deutlich generationelle Einflüsse konstatieren, die mit den in Kapitel 1.2.3 bis 1.2.5 beschriebenen gesamtgesellschaftlichen Entwicklungen korrespondieren.

Um die Tradierungsvorgänge unter Einbeziehung der Anpassungen angemessen zu verstehen, ist eine Berücksichtigung des Zusammenspiels von Erfahrung und Erwartung, Vermittlung und Aneignung nützlich. Überträgt man das Konstrukt von Erfahrungsraum und Erwartungshorizont (Koselleck 1989, 349-359) auf die Erziehung, so ist zweierlei feststellbar. Bezogen auf das konkrete Erziehungsarrangement zeigt sich, dass sich die mittlere Generation mit den eigenen Erziehungserfahrungen auseinandersetzt und in der Folge teilweise stark veränderte Erziehungssettings für die eigenen Kinder entwickelt. Das gilt insbesondere für diejenigen, die aus Familien stammen, die einer geschlossenen Tradierungslogik folgen. Allerdings finden sich auch starke Kontinuitäten, die sich darauf beziehen, wie die Erwartungen an die nachfolgende Generation gestaltet werden. Vertreter der mittleren Generation, die in Familien aufgewachsen sind, die einer flexibel erscheinenden Tradierungslogik folgen, entwerfen für ihre Kinder Erwartungshorizonte, die sie selbst wie auch ihre Kinder als flexibel wahrnehmen. Diejenigen, die in Familien erzogen wurden, die einer geschlossenen Tradierungslogik folgen, entwerfen trotz aller Liberalisierungstendenzen für ihre Kinder relativ klar umrissene Erwartungshorizonte. Familien, in denen eine diffuse Tradierungslogik dominiert, verfolgen die Umsetzung ihrer Erwartungshorizonte nicht mit Nachdruck. Die eigenen Erziehungserfahrungen beeinflussen zwar die jeweiligen Erziehungsarrangements der nachfolgenden Generationen, determinieren sie jedoch nicht. Ähnliches gilt für die Inhalte, wobei die Bezugsrahmen und die Qualität der Erwartungshaltungen ein hohes $\mathrm{Maß}$ an Kontinuität aufweisen. Schneewinds Feststellung, es gebe eine gewisse Kontinuität in der Erziehung (Schneewind 1996, 126 und Schneewind/Ruppert 1995, 153), scheint innerhalb dieses Samples vor allem für das Ausmaß der Direktheit und Konsequenz der für die Kinder entwickelten Erwartungshorizonte zu gelten. Die Familien integrieren gesellschaftliche Entwicklungen in ihre Erziehungssettings und gewährleisten auf diese Weise eine Anschlussfähigkeit an die Bezugsrahmen im Generationenverlauf.

\subsubsection{Familienleitbilder: Von der Dethematisierung zur Thematisierung von Konflikten}

Auch wenn die älteste Generation gegenwärtig mehr oder weniger direkt beklagt, bestimmte wünschenswerte Orientierungen seien im Zeitverlauf verlorengegangen, geht das in den seltensten Fällen einher mit einer offen an die Kinder oder Enkelkinder adressierten Anklage. Vielmehr reagieren die Vertreter der ältesten Generation entweder mit einer verständnisvollen oder mit einer mehr oder weniger (erzwungenen) resignativen Akzeptanz. Ein ausdrucksvolles Beispiel hierfür stellt der Umgang des Ehepaares Friese mit der Frage des Zusammenlebens von Kindern und Enkeln mit ihren Lebenspartnern dar. Ihren eigenen Kindern hätten sie nicht erlaubt, mit ihren Lebenspartnern unverheiratet zusammenzuwohnen. Die Enkel hingegen würden heute teil- 


\section{Bildungsstatus, Generation und Region}

weise mit ihren ledigen Lebenspartnern in einer gemeinsamen Wohnung leben (Frau und Herr Friese 2:22:11-2:22:36), womit sich das Ehepaar abfinden muss. Frau Friese bilanziert: „Ja, ja, also das, da haben sich die Zeiten geändert. Da kann man gar nichts jetzt dagegen sagen. //ja, hmh// Also so liberal sind wir auch@(.)@“ und Herr Friese ergänzt: „Es bleibt uns nichts, wenn wir keinen Krieg wollen@(.)@“ (Frau und Herr Friese 2:23:50-2:24:07). Wenn die Großelterngeneration doch einmal Kritik an Kindern oder Enkeln übt, so wird diese anschließend meist wieder relativiert (Frau Dienel IV 2:01:15-2:01:48, Beyer, Frau Grothe II 1:05:12-1:05:47). Die bedauerten (vermeintlichen) Verluste bestimmter Orientierungen werden weniger den Kindern oder Kindeskindern angelastet, als vielmehr in Zusammenhang mit einer allgemeinen gesellschaftlichen Entwicklung gebracht. Hierin ist eine Strategie zu sehen, Kritik nach außen zu verlagern, um Harmonie nach innen gewährleisten zu können. Das Streben nach Harmonie dürfte auch der Grund sein, warum die Großeltern nicht versuchen, die Machtbalancen zu verschieben, die in ihrer Darstellung zugunsten der Kinder und Enkel ausfällt. Die Großeltern folgen einer Konfliktvermeidungsstrategie und mischen sich im Zweifelsfall nicht in die Erziehung der Enkelkinder ein, da die Orientierung an einem harmonischen Familienleitbild für sie wichtiger ist als das Durchsetzen bestimmter Orientierungen. Wie zentral dieses Familienbild in der ältesten Generation verankert ist, zeigt sich auch an anderen Stellen. Es erklärt, warum die Großelterngeneration kaum Kritik an den eigenen Eltern äußert und die eigene Kindheit als relativ konfliktfrei darstellt. Vor dem Hintergrund der hohen Bedeutung eines harmonischen Familienleitbildes wird verständlich, dass die Vertreter der ältesten Generation auch in Bezug auf die eigene Elternphase Konflikte eher dethematisieren. Zwar wird nicht gesagt, es habe keine Streitigkeiten mit den Kindern gegeben, es fällt aber auf, dass derartige Bereiche im Interview eher ausgespart oder umgangen werden. Die Orientierung an einem harmonischen Familienleitbild wird in Anbetracht des Bezugsrahmens der Akzeptanz verständlich. Darüber hinaus erwähnen viele Vertreter der ältesten Generation, i.d.R. nicht geschlagen worden zu sein und erwähnen solche Praxen auch nicht in Bezug auf die Erziehung der eigenen Kinder, was ebenfalls auf das internalisierte Leitbild zurückzuführen ist. Zugleich dürfte der ältesten Generation bewusst sein, dass Schläge inzwischen allgemein nicht mehr als legitimes Erziehungsmittel betrachtet werden. Dies ist ein weiterer Grund, warum die älteste Generation bei der Präsentation ihrer Lebensgeschichte diesen Aspekt marginalisiert oder dethematisiert.

Während die Großelterngeneration familiale Konflikte noch als potenzielle Bedrohung auffasst, die ihrer Orientierung an einem harmonischen Familienleitbild widerspricht, sind für ihre Kinder familiale Auseinandersetzungen ein selbstverständlicher Bestandteil ihres Familienlebens und werden in ihr Familienleitbild integriert. Die mittlere Generation thematisiert familiale Konflikte in der Kindheit explizit. Auch berichten mehrere Vertreter der mittleren Generation davon, geschlagen worden zu sein. Das beschriebene Konfliktniveau sowie Art und Ausmaß der elterlichen Strafpraxen variieren hierbei in hohem Maße in Abhängigkeit von der jeweiligen Tradierungslogik. Bei der Erziehung der eigenen Kinder räumt die mittlere Generation ihren Kindern nach eige- 


\subsection{Intragenerationelle Gemeinsamkeiten und intergenerationelle Unterschiede}

nem Bekunden generell ein Widerspruchs- und Mitspracherecht ein. Intergenerationelle Auseinandersetzungen avancieren auf diese Weise - von der mittleren Generation gefördert - zu einem wesentlichen Bestandteil der Familienkultur und werden nicht mehr als Ausdruck einer zu vermeidenden Fehlentwicklung betrachtet. Sie werden ganz im Gegenteil zu einem elementaren Bestandteil, der als notwendig für die kindliche (und auch familiale) Entwicklung betrachtet wird. Hier deutet sich zugleich eine weitere Veränderung an, die sich auf die elterliche Aufgabe bezieht, die Kinder zu fördern. Einige Frauen der mittleren Generation beklagen, während ihrer Kindheit nicht ausreichend von ihren Eltern bei der Entwicklung ihrer Fähigkeiten unterstützt worden zu sein. Diese Erfahrung führt im Zusammenspiel mit der gesellschaftlichen Entwicklung, in denen den jeweiligen elterlichen Förderleistungen eine höhere Bedeutung zukommt, die einen emotionalen Rückhalt einschließen (Beck-Gernsheim 1990a, 168/169 und Schülein 1990, 134/135), dazu, dass die mittlere Generation die emotionale wie praktische Unterstützung ihrer Kinder besonders stark beachtet. Emotionaler Rückhalt sowie die Schaffung einer Umgebung, in der die Kinder sich optimal entwickeln können, werden auf diese Weise zu einem integralen Bestandteil des elterlichen Rollenverständnisses.

In der mittleren Generation scheinen die Väter ihr Rollenbild im Vergleich zur Vorgängergeneration zu modifizieren. Die ältere Vätergeneration sieht sich zwar auch an der Kindererziehung beteiligt, aber sowohl Herr Cronert (II 59:26-59:29) als auch Herr Friese (1:29:57-1:30:15) erwähnen, nicht so stark in die praktische Umsetzung der Kindererziehung im Alltag involviert gewesen zu sein. Diese Relativierung findet sich bei den Vätern der mittleren Generation nicht mehr. Zwar mögen auch sie de facto nicht so stark im Erziehungsalltag präsent sein, sie fühlen sich aber uneingeschränkt zuständig für die Erziehung ihrer Kinder. Sie scheinen somit bestimmte gesellschaftliche Entwicklungen, nach der sich Väter zunehmend als Erzieher ihrer Kinder betrachten (Friebertshäuser/Matzner/Rothmüller 2007, 185), in ihr Selbstverständnis als Vater integriert zu haben. In der ältesten Generation finden sich noch Hinweise, dass sowohl Herr Cronert als auch Herr Friese für sich im Zweifelsfall bei intergenerationellen Konflikten ein Entscheidungsmonopol beanspruchen und sich damit während ihrer Familienphase noch auf ein weit verbreitetes Leitbild beziehen (Plötz 2006, 62 und 65). In der folgenden Vätergeneration verblasst dieses Leitbild.

Die jüngste Generation schließlich verbindet in ihren Beschreibungen des familialen Zusammenlebens zwei Aspekte. Zum einen werden die Beziehungen zu den Eltern insgesamt als recht gut und einvernehmlich dargestellt, wobei i.d.R. insbesondere die elterlichen Unterstützungsleistungen materieller wie emotionaler Art gewürdigt werden. ${ }^{210}$ Zum anderen werden aber auch intergenerationelle Konflikte thematisiert, die allerdings je nach Familie unterschiedlich intensiv ausfallen. Diese Konflikte werden als selbstverständlicher Bestandteil des Familienlebens betrachtet: „Dass man mal 'ne Meinungsverschiedenheit hat oder was, das is denke ich, in jeder Familie so. //hmh//

\footnotetext{
${ }^{210}$ Die Ausnahmen wurden in den Fallbeispielen ausführlich vorgestellt, schwächen allerdings die allgemeine Tendenz nicht.
} 


\section{Bildungsstatus, Generation und Region}

Oder dass es auch mal Streit gibt, das ist bei uns auch so. Das gehört aber auch dazu, ohne wäre es@langweilig@“" (Hannah Grothe I 06:18-06:29). ${ }^{211}$ Im Vergleich zu einigen Vertretern der Vorgängergeneration wird das Konfliktniveau auf einem deutlich niedrigeren Niveau angesiedelt. Das Zubilligen einer größeren Eigenständigkeit dürfte diese Entwicklung begünstigt haben. Im Generationenverlauf verliert ein Familienleitbild an Bedeutung, das die Harmonie in den Mittelpunkt stellt. Stattdessen werden Auseinandersetzungen als integraler Bestandteil des Familienlebens in das Familienleitbild integriert. Konflikte werden nicht als Bedrohung, sondern als Element des Alltags aufgefasst.

\subsection{Regionale Besonderheiten und Bezüge}

Innerhalb des Samples lassen sich regionale Unterschiede in den beiden Teilgebieten feststellen, die besonders bei den beiden älteren Generationen recht markant sind. Aber auch in der jüngsten Generation hat noch keine vollständige Angleichung stattgefunden. In den Familien im östlichen Teilgebiet spielt der familiale Zusammenhalt in je familienspezifisch unterschiedlichen Varianten als zentraler Bezugsrahmen eine wichtige Rolle und erhält sich im weiteren Generationenverlauf. Innerhalb der westlichen Teilgruppe herrscht eine größere Heterogenität zwischen den Familien. Das Spektrum der zentralen Bezugsrahmen reicht vom Streben nach repräsentativen Höchstleistungen (Nolte/Gümper) über das Knüpfen sozialer Kontakte (Familie Friese) bis zur religiösen Alltagsprägung (Familie Dienel/Ebel). Die Großeltern im östlichen Teilgebiet sind im Gegensatz zu denen in der westlichen Region bis heute stärker in die Betreuung ihrer Enkel eingebunden.

Im westlichen Teilgebiet sind die Mütter der ältesten Generation überwiegend nicht berufstätig bzw. unterbrechen ihre Erwerbsarbeit für einen längeren Zeitraum, um sich ganz der Betreuung ihrer Kinder zu widmen. Die Mütter im östlichen Teilgebiet sind mehrheitlich ins Berufsleben eingebunden. In der nachfolgenden Müttergeneration nimmt diese deutliche Differenz zwar tendenziell ab, ist aber weiterhin noch zu erkennen. Im westlichen Teil finden sich zwei Familien, in denen sich die Mütter ganz auf die Bereiche Familie und Haushalt konzentrieren. Im östlichen Teil gibt es lediglich eine Mutter, die sich aufgrund des Verlustes ihres Arbeitsplatzes zeitweilig für dieses Modell entscheidet.

Geschlossene Tradierungslogiken, die im Falle der ältesten Generation mit autoriären Erziehungselementen gekoppelt werden, finden sich in erster Linie in den westlichen Familien. Hiermit einhergehend kritisiert vor allem die mittlere Generation im westlichen Teilgebiet die elterliche Erziehung. Bei der Erziehung der jüngsten Generation gibt es ebenfalls einige regionale Differenzen. Eine bezieht sich auf die optimale Kindesförderung. Zunächst einmal ist allgemein davon auszugehen, dass alle Eltern ihre

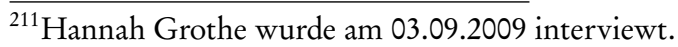




\subsection{Regionale Besonderheiten und Bezüge}

Kinder optimal fördern möchten. Hierzu werden allerdings in Ost und West tendenziell verschiedene Wege eingeschlagen. Während im westlichen Teilgebiet die umfassende Kindesförderung eine stark verinnerlichte elterliche Maxime bildet, wird die Förderung der kindlichen Entwicklung im östlichen Teilgebiet weniger als Projekt verstanden, das von den Eltern gründlich durchdacht und geplant werden muss. Hiermit korrespondierend wird die Lektüre von Erziehungsratgebern bzw. die Suche nach Erziehungstipps lediglich von einigen Müttern im Westen thematisiert.

Auch wenn Freundschaften in der jüngsten Generation generell wichtig sind, was sicherlich zum Teil der aktuellen Lebensphase geschuldet ist, nimmt im Westen der Freundeskreis einen größeren Raum in den Interviews ein, wohingegen im Osten die Verwandtschaft eine stärkere Rolle spielt. Im westlichen Teilgebiet sind darüber hinaus gelegentlich Klagen über die vielen Termine und die hiermit verbundene zeitliche Auslastung zu finden. Zudem schätzt es die jüngste Generation zwar generell im Mittelpunkt der elterlichen Aufmerksamkeit zu stehen, allerdings wird die Kindzentrierung im Westen manchmal als zu stark erlebt. Im östlichen Teilgebiet wird das Thema der Hilfsbereitschaft tendenziell etwas stärker betont.

Neben der jeweiligen Familiengeschichte lassen sich die jeweils unterschiedlichen gesellschaftlichen Rahmenbedingungen vor der Wende in den beiden Teilgebieten als wichtige Elemente betrachten, um diese Unterschiede zu erklären.

\subsubsection{Familienleitbilder}

Einen Erklärungsansatz dafür, dass sich geschlossene Tradierungslogiken mit ausgesprochen autoritären Zügen vor allem im westlichen Teil des Untersuchungsgebietes finden, liefern die in den beiden Teilgebieten jeweils präferierten und staatlich geförderten Familienmodelle, die sich auf die Gestaltung des Familienlebens auswirken. In der Bundesrepublik wird in den 1950er und 1960er Jahren das Modell der Hausfrauenehe propagiert und staatlich unterstützt (Sommerkorn/Liebsch 2002, 103). Dieses enthält eine klare Aufgabenteilung, wonach der Ehemann den Familienunterhalt verdient und die Frau sich auf die Führung des Haushaltes und die Kindererziehung konzentriert. Die Festschreibung auf die familiale Sphäre und die Erziehung der Kinder prägt folglich das mütterliche Selbstverständnis und findet sich auch in diesem Sample. Von diesem Modell geht offensichtlich zugleich ein starker Normierungsdruck aus, der dazu führt, dass die Mutter zugleich in besonderer Weise verantwortlich für Erfolg oder Misserfolg der Erziehung wird. ${ }^{212}$ (Vermeintliche) Fehlentwicklungen der Kinder erweisen sich in der Folge insbesondere für die Mütter als problematisch. Deswegen muss eine Orientierung der Kinder an den gewünschten Normen erreicht und abweichendes Verhalten der Kinder unterbunden werden. Hierzu greifen einige Familien des Samples auf geschlossene Tradierungslogiken zurück, die stark autoritäre Erziehungselemente enthalten. In der DDR wiederum wird eine Doppelorientierung der Mütter an Familie und Beruf

\footnotetext{
${ }^{212}$ Bowlbys Bindungstheorie verstärkt diese Sichtweise (Schütze 2000, 95/96).
} 


\section{Bildungsstatus, Generation und Region}

propagiert (Schlegel 1999, 382), der die Mütter des Samples mehrheitlich folgen. Um die Betreuung der Kinder während der Arbeitszeit zu gewährleisten, müssen neben der Mutter weitere Personen in die Kindeserziehung eingebunden werden. Diese Konstellation begünstigt die Entwicklung eines im Vergleich zur westlichen Vergleichsgruppe anderen Selbstverständnisses. Die Mütter definieren sich nicht ausschließlich über die familiale Sphäre und können sich zugleich zumindest in einem gewissen Umfang von dem Druck entlastet sehen, allein für die Entwicklung der Kinder verantwortlich gemacht zu werden. Beide Aspekte mögen dazu beitragen, den Kindern innerfamilial mehr Freiräume zuzugestehen.

In der folgenden Generation verlagert sich der Druck, dem sich die Mütter im Westen ausgesetzt sehen. Nun wird erklärungsbedürftig, warum sie keiner bzw. einer Erwerbstätigkeit nachgehen. Während für die Mütter im Osten die eigene Erwerbsarbeit eine Selbstverständlichkeit darzustellen scheint, die nicht weiter begründungsbedürftig für sie ist, fühlt sich die aktuelle Müttergeneration im westlichen Teil des Untersuchungsgebiets dazu veranlasst, die Entscheidung für oder gegen eine Erwerbsarbeit eigenständig im Interview anzuschneiden. Hierbei argumentiert sie u.a. jeweils mit dem Kindeswohl, das für die einen durch die Nichtberufstätigkeit und für die anderen durch die Berufstätigkeit gefördert wird (Heike Ebel I 22:22-22:43, IV 07:49-08:06, Michelle Ahrens III 02:50-03:35, 05:11-05:30). Die Auseinandersetzung mit der Frage der eigenen Erwerbsarbeit, wie sie die westlichen Mütter vornehmen, lässt sich als Reaktion auf die von Peuckert konstatierte Tatsache betrachten, dass für heutige Mütter keine einheitlichen Rollenvorstellungen mehr existieren (Peuckert 2007, 49).

Interessant und erklärungsbedürftig ist in diesem Zusammenhang, dass sich die Situation für die westlichen Mütter diffuser bzw. widersprüchlicher darstellt, was in ihrem Rechtfertigungsbedürfnis zum Ausdruck kommt. Offensichtlich wirken an dieser Stelle noch systembedingte Unterschiede aus der eigenen Kindheit der Interviewpartnerinnen in Ost und West nach. Für die Interviewpartnerinnen im östlichen Teilgebiet zählt zumindest eine partielle mütterliche Erwerbsarbeit zu den eigenen Kindheitserfahrungen. Sie scheinen dies in der Folge als ein erprobtes Modell zu werten, das sie für die eigene Familienphase übernehmen. Die Interviewpartnerinnen aus dem westlichen Teil des Samples hingegen können aufgrund der eigenen Familiengeschichten nicht auf solche Erfahrungen zurückgreifen, weshalb eine weibliche Erwerbsarbeit in ihrer eigenen Familiengeschichte eine Art ,Neuland' darstellt, das sie sich erst erschließen müssen. Zusätzlich sehen sie sich aufgrund der eigenen Kindheitserfahrungen offensichtlich stärker mit der Forderung konfrontiert, sich ganz auf die Kinder zu konzentrieren. Sie scheinen sich im Vergleich zu den Müttern im östlichen Teil einem stärkerem Druck ausgesetzt zu sehen, für die Entwicklung ihrer Kinder verantwortlich zu sein. ${ }^{213}$

Die Diskussion um eine weibliche Erwerbsarbeit ist darüber hinaus aufgrund der unterschiedlichen systembedingten historischen Entwicklungen im westlichen Teil offen-

${ }^{213} \mathrm{Im}$ Vergleich zu den eigenen Müttern nimmt der äußere Verantwortlichkeitsdruck zwar ab, verbleibt aber auf einem höheren Niveau als im Osten. 


\subsection{Regionale Besonderheiten und Bezüge}

sichtlich anders gerahmt. Die mütterliche Erwerbsarbeit stellt für die befragten Mütter in der westlichen Teilregion einen Bereich dar, in dem kindliche und eigene Bedürfnisse und in der Konsequenz der Fokus der Kindzentrierung mit dem der eigenen Selbstentfaltung potenziell kollidieren. In dieser Diskussion wirken vermutlich gesellschaftliche Diskurse der bundesrepublikanischen Geschichte nach (Sommerkorn/Liebsch 2002), in der u.a. die Frage der ökonomischen Eigenständigkeit der Frau, die eine Selbstentfaltung erst ermöglicht, eine zentrale Rolle einnimmt. In diesem Zusammenhang stellen die eigenen Interessen für die mittlere Frauengeneration bis heute offenbar kein legitimes Argument dar, weswegen die Interviewpartnerinnen auf das Kindeswohl zur Begründung der eigenen Entscheidung zurückgreifen. Für die Mütter im östlichen Teilgebiet wiederum ist die eigene Erwerbsarbeit eher eine Selbstverständlichkeit bzw. gesellschaftliche Notwendigkeit, worin sich ein ,Erbe' des DDR-Systems manifestiert.

\subsubsection{Familie und Gesellschaftssystem}

Die vergleichsweise großzügigere Gestaltung der Erziehung im Osten durch die älteste Generation könnte ferner als eine (implizite) Reaktion auf einen als autoritär empfundenen Staat verstanden werden. Um diesem möglichst wenig Einflussmöglichkeiten auf das Familienleben zu gewähren, streben die Familien nach einem engen Zusammenhalt.

Der Verzicht auf autoritäre Erziehungssettings kann hierbei als Versuch betrachtet werden, die Wahrscheinlichkeit für intergenerationelle Spannungen zu minimieren, die den familialen Zusammenhalt potenziell gefährden könnten. Die Eltern des Samples sind - teils aus Angst vor staatlichen Repressionen, wie sie von Frau Beyer thematisiert werden - darauf bedacht, dass sich ihre Kinder außerhalb der Familie in einer bestimmten Art und Weise verhalten und bestimmte Themen, die zu Hause diskutiert werden, sowie bestimmte familiale Praxen nicht nach außen kommunizieren. Um das zu erreichen, erscheinen für sie Erklärungen hilfreicher als schlichte Verbote oder Anweisungen. Weiterhin ist der weitgehende Verzicht auf autoritäre Erziehungselemente als ein Versuch zu lesen, die als systembedingt betrachteten Einschränkungen der individuellen Möglichkeiten im wirtschaftlichen wie sozialen Bereich für die Kinder auszugleichen.

Durch die Errichtung des Sperrgebiets wird die Bevölkerung zudem in gewisser Weise isoliert. Der Zuzug von Personen unterliegt der staatlichen Kontrolle, und die Kontaktmöglichkeiten mit Personen aus anderen Regionen sind erschwert, sodass insgesamt von einer eingeschränkten Mobilität der Bevölkerung gesprochen werden kann. Hierdurch wird das Veränderungspotenzial minimiert, das sich durch den Austausch mit Bevölkerungskreisen aus anderen Regionen ergeben könnte. Die Beschränkungen der Mobilität stellen folglich eine Barriere für gesellschaftliche Wandlungsprozesse in der Region dar. Anders als im westlichen Teilgebiet ist damit auch die Wahrscheinlichkeit geringer, dass die Kinder mit Vorstellungen in Kontakt kommen, die den elterlichen Einstellungen widersprechen. Entsprechend seltener sind die Eltern gezwungen, den Kindern Grenzen zu setzen und Konflikte mit ihnen auszutragen. In der Folge können sich die vermittelnden Generationen in Bezug auf Erziehung und Tradierungslogik entsprechend großzü- 


\section{Bildungsstatus, Generation und Region}

gig präsentieren, da die Gefahr von ungewollten Veränderungen durch eine Freiräume gewährende Erziehung begrenzt ist. ${ }^{214}$

Die mittlere Generation trägt nicht unwesentlich zu den vergleichsweise großzügigeren Vermittlungssettings im östlichen Teilgebiet bei. In der Selbstpräsentation zeigt sie sich tendenziell weniger widerspruchsbereit, was die elterlichen Erziehungsvorgaben betrifft. Ihre Kritik bezieht sich vielmehr auf die gesellschaftlichen Rahmenbedingungen des DDR-Systems. Die Mitgliedschaft in politischen Jugendorganisationen, politische Indoktrination, Konsummangel, staatliche Reglementierungen sowie die Begrenzungen des Sperrgebiets (Frank Grothe I 10:31-11:07; 56:41-59:43; Fallbeispiele Friederike Jäger und Birgit Schmidt) werden von der mittleren Generation, die in der DDR aufgewachsen ist, tendenziell in stärkerem Maße als Probleme thematisiert als z.B. Konflikte mit den Eltern. Da die Vertreter der mittleren Generation weniger als ihre Altersgenossen im Westen gegen ihre Eltern aufbegehren, müssen ihre Eltern wiederum viel weniger auf autoritäre Elemente in der Erziehung zurückgreifen, um ihre Vorstellungen durchzusetzen. Die Schilderungen führen zu dem Eindruck, dass die als problematisch wahrgenommenen Aspekte des DDR-Systems zu einem stärkeren Zusammenschluss innerhalb der Familie geführt haben. Die Externalisierung der Kritik auf die äußeren Rahmenbedingungen erfüllt hierbei eine wichtige Funktion, indem die familialen Beziehungen entlastet werden. Weiterhin kann auf diese Weise das staatliche System mit seinen Auswirkungen auf die Gestaltung des Alltags anstelle der Eltern als reglementierende Autorität wahrgenommen werden. Aus dieser Perspektive kanalisiert und beschränkt das System der DDR für die mittlere Generation die Handlungsmöglichkeiten in weitaus stärkerem Umfang als die Eltern. Vor diesem Hintergrund ist es verständlich, wenn die Kinder die Leistungen ihrer Eltern in besonderer Weise anerkennen. Die von Friederike Jäger im Fallbeispiel beschriebene Dankbarkeit für das Engagement und die Leistungen der Eltern ist kein Einzelfall. Auch andere Interviewpartner der mittleren Generation, die in der DDR aufgewachsen sind, heben die elterlichen Unterstützungsleistungen hervor. Frank Grothe findet z.B., er könne nicht dankbar genug sein für das, was seine Mutter ihren Kindern unter den gegebenen Umständen ermöglicht habe (I 1:27:30-1:27:38).

Im westlichen Teilgebiet zeigen sich die Vertreter der mittleren Generation in stärkerem Maße bereit, sich den elterlichen Vorgaben zu widersetzen. Im Zuge des Wertewandels (Meulemann 1996b und Klages 1984, 2001b, 2002) steht das überlieferte Ordnungsgefüge im Westen zur Disposition. Diese Infragestellung wird dabei vor allem von den Protagonisten der mittleren Generation aufgegriffen, die in Familien mit einer geschlossenen Tradierungslogik aufwachsen. Im Gegensatz zum östlichen Teilgebiet sind es im westlichen Teil unmittelbar die Eltern, die als autoritär empfunden werden. Diese versuchen entgegenzusteuern, indem sie auf die ihnen bekannten Elemente einer autoritären Erziehung zurückgreifen. Darüber hinaus lassen sich die stärkeren Konflikte der mittle-

${ }^{214}$ Der Kontakt zu denjenigen, die in die Region ziehen, in erster Linie Grenzposten, wird von der Dorfgemeinschaft auf ein Minimum beschränkt, wie das Fallbeispiel von Friederike Jäger zeigt. 


\subsection{Regionale Besonderheiten und Bezüge}

ren Generation mit ihren Eltern während ihres Aufwachsens als Ausdruck einer veränderten Streitkultur (Zinnecker 1985, 165/166, 186) deuten, die offensichtlich vor allem im westlichen Teilgebiet zu finden ist. Im Zuge dieser Veränderung werden Konflikte thematisierbar. Das korrespondiert mit einer gesamtgesellschaftlichen Entwicklung, in deren Zuge sich spätestens in den 1980er Jahren eine neue Form der Protestkultur, wie die Friedens- und Umweltbewegung, etabliert, die ihre Kritik öffentlich formuliert.

Im Osten bringt das Leben im Sperrgebiet zudem einige Besonderheiten mit sich. Als Folge der räumlichen Begrenzungen wohnen entweder mehrere Generationen gemeinsam in einem Haus oder in unmittelbarer Nachbarschaft zueinander. Das verstärkt die wechselseitige Bezogenheit der Familienmitglieder aufeinander. Der erhöhte familiale Zusammenhalt lässt sich somit als eine Reaktion auf die Rahmenbedingungen innerhalb der DDR betrachten, der interessanterweise bis in die Gegenwart nachwirkt. Dies ist ein starkes Indiz dafür, dass sich dieser Bezugsrahmen in der Vergangenheit als erfolgreich erwiesen hat und bis heute für die Familien anschlussfähig ist. Hierauf verweist insbesondere auch die starke Bedeutung der Verwandtschaft für die jüngste Generation im Osten. Die im Vergleich zum westlichen Teilgebiet höhere Generationensolidarität ist auch in anderen Teilen der ostdeutschen Bundesländer zu finden, wie Szydliks (2000, 192) und Meulemanns (1996b, 356 und 358) Ausführungen belegen. Die bereits in der Vergangenheit höhere geographische Mobilität der Familienmitglieder im westlichen Teilgebiet trägt $\mathrm{zu}$ einer Reduzierung der alltäglichen verwandtschaftlichen Kontakte bei, wodurch zugleich der Stellenwert des familialen Beziehungsnetzes relativiert wird. Im Westen steigen zudem im Zuge des allgemeinen gesellschaftlichen Wandels die Orientierungsmöglichkeiten. Die stärkere Ausdifferenzierung der familialen Bezugsrahmen im Westen lässt sich als Ausdruck dieser Entwicklung betrachten.

\subsubsection{Optimale Kindesförderung und elterliche Ratsuche}

Die Tatsache, dass die mittlere Generation im Westen in höherem Maße außerfamilialen Erziehungsrat einholt und einen besonderen Fokus auf die optimale Förderung ihrer Kinder legt, verweist auf einen paradoxen Befund. Die aktuelle Elterngeneration im Osten kann offenbar besser an die eigenen Erziehungserfahrungen und die Erziehungsarrangements der eigenen Eltern anschließen, obwohl sie einen Systemumbruch erlebt hat. Die Eltern und insbesondere die Mütter im Westen hingegen, für die kein Systemwechsel zwischen ihrer Kindheit und der eigenen Familienphase liegt, können trotz der nachweisbaren Tradierungslinien nicht so problemlos an die eigenen Erziehungserfahrungen anknüpfen. Offensichtlich wirken die spezifischen Sozialisationserfahrungen in Ost und West bis heute nach und beeinflussen die jeweilige Erziehung. Es entsteht der Eindruck, als hätten die Eltern im westlichen Teilgebiet in stärkerem Ausmaß die Idee des Wettbewerbs internalisiert, mit der sie selbst bereits aufgewachsen sind. Zugleich scheinen sie auch einen erhöhten Wettbewerbsdruck zu verspüren und sich stärker an dem Leitbild der optimalen Kindesförderung (Beck-Gernsheim 1990a, 168/169) zu orientieren. 


\section{Bildungsstatus, Generation und Region}

Ein Blick auf die jeweils unterschiedlichen Rahmenbedingungen in den beiden Teilgebieten vor der Wende ist hilfreich, um diesen Widerspruch zu verstehen. Ein Grund für die Unterschiede sind sicherlich die Erziehungsdiskurse der vergangenen Jahrzehnte in Westdeutschland, in deren Verlauf eine Vielzahl von (teils widersprüchlichen) Aspekten in die Diskussion eingeführt worden ist, die eine durchaus polarisierende Wirkung entfaltet haben. Von diesen seien im Folgenden einige schlaglichtartig angeführt, ohne einen Anspruch auf Vollständigkeit zu erheben. Spätestens in den 1960er Jahren rückt das Bildungssystem ins Zentrum der öffentlichen Aufmerksamkeit, und es werden Reformen gefordert und öffentlich diskutiert. ${ }^{215}$ Gegen Ende der 1960er Jahre begehrt die antiautoritäre Bewegung medienwirksam gegen eine als konservativ und repressiv empfundene Erziehung auf. ${ }^{216}$ In den 1970ern hat die Diskussion um die kompensatorische Erziehung Konjunktur. ${ }^{217}$ Schließlich folgt Ende der 1970er Jahre als Replik auf die antiautoritäre Erziehungsbewegung der Aufruf „Mut zur Erziehung“ (1978), der ebenfalls nicht unwidersprochen bleibt (Herrmann 1978 und Tübinger Erklärung 1978). ${ }^{218}$ Über Bowlbys Bindungstheorie (1975) rückt die Qualität der intergenerationellen Beziehungen ins Zentrum der Betrachtung. Publikationen wie „Die Familienkonferenz“ (Gordon 1972) schließlich lenken das Augenmerk auf die Bedeutung der kommunikativen Ebene. Ende der 1970er/Anfang der 1980er Jahre gibt eine vermeintliche Leistungsverweigerung der Jugend Anlass zur Besorgnis. ${ }^{219}$ Spätestens seit den 1990er Jahren erhalten Forderungen nach Grenzsetzungen und Disziplin wieder eine erhöhte Aufmerksamkeit. ${ }^{220}$ Diese Auflistung dürfte genügen, um zu zeigen, dass öffentlich geführte Diskurse um die Erziehung das Aufwachsen und den weiteren Lebensweg der mittleren Generation in Westdeutschland begleiten. Positiv ausgedrückt, beziehen sich die Debatten auf die Frage, wie Erziehung verbessert werden kann; negativ formuliert, werden diverse Defizite konstatiert. Die öffentliche Präsenz teilweise ganz unterschiedlicher Ansichten sowie

${ }^{215}$ Einen Überblick über diese Entwicklung mit ihren Folgen findet sich bei Tenorth (2010, 287-310).

${ }^{216}$ Es lassen sich zwei Phasen der antiautoritären Bewegung unterscheiden: 1. Die antiautoritäre Phase von Juni 1967 - Sommer 1968 und 2. die proletarische Phase von Herbst 1968 - Winter 1969 (Jansa 1999, 69-78). Während in der frühen Periode noch die Bedürfnisse des Kindes im Mittelpunkt stehen (Seifert 1969), verschiebt sich die Blickrichtung während der zweiten Phase zum Kollektiv und die Kinder sollen dazu erzogen werden, den Klassenkampf zu führen (Werder 1972, 14 und 1977, 23).

${ }^{217}$ Eine kurze, kritische Auseinandersetzung mit der kompensatorischen Erziehung liefert BoisReymond (1975). Auch wenn die Familien dieses Samples zweifelsohne nicht zur Zielgruppe dieses Ansatzes gehörten, wird durch die Debatte die Aufmerksamkeit auf die Rahmenbedingungen des Lernens gelenkt.

${ }^{218}$ Diese Entwicklungen werden von Göppel als „Wellen der Erziehungskritik“ bezeichnet, zu denen er weiter z.B. die antipädagogische Bewegung der 1980er Jahre und die Kritik an der antiautoritären Erziehung in den 1990er Jahren zählt (Göppel 2010, 29-31).

${ }^{219}$ Eindrückliche Beispiele für diese Sorge finden sich in den von Fend zitierten elterlichen Abiturreden von 1982 (Fend 1988, 17-23).

${ }^{220}$ Hierbei handelt es sich um die aktuelle Phase der Erziehungskritik, die sich in Erziehungsratgebern niederschlagen, wie z.B. bei Bueb (2006) (Göppel 2010, 31) oder Gaschke (2001). 


\subsection{Regionale Besonderheiten und Bezüge}

die Betonung verschiedenster Elemente der Erziehung forcieren eine Reflexion der eigenen Erziehungserfahrungen sowie des eigenen Erziehungshandelns. Zugleich dürfte die Vielfalt der angesprochenen Themen sowie der teilweise konträren Auffassungen verunsichernd wirken ${ }^{221}$ und ein unreflektiertes Anschließen an die Erziehungspraxen der eigenen Kindheit erschweren. Erst in Auseinandersetzung mit den öffentlich verhandelten Erziehungsfragen und über die Integration ausgewählter Aspekte dieser Debatten in das eigene Erziehungssetting kann die mittlere Generation eine Anschlussfähigkeit an die familialen Tradierungslinien herstellen. Für die mittlere Generation im östlichen Teil des Untersuchungsgebietes stellt sich die Situation wiederum anders dar. Ihr Aufwachsen wird nicht von einer ähnlich ausgeprägten, öffentlich geführten Diskussion um Erziehung begleitet. Zwar werden sie mit einer ,Erziehung zur sozialistischen Persönlichkeit ${ }^{\circ 22}$ sowie ideologischen Maximen konfrontiert, die allerdings in den Familien des östlichen Untersuchungsgebietes sogleich wieder relativiert werden:

„[D]adurch, dass deine Eltern zu Hause anders gesprochen haben, dadurch dass du ARD und ZDF kriegtest, dadurch dass du äh Verwandte hattest im, im Westen, mit denen du dich ein-, zwei- oder auch dreimal im Jahr getroffen hast, hast du doch ganz anders diskutiert. [...] Du hast überhaupt nicht so dieses FeIndbild gehabt, //hmh// wie man versucht hat, das aufzubauen hier. //hmh// Das, das gab's ja gar nicht. War natürlich auch ein - wie soll ich sagen - nicht ein Konflikt, aber du, du bist damit aufgewachsen, dass deine Eltern dir was anderes gesagt haben, wie das, was einige Lehrer versuchten, dir einzubläuen, ne. //hmh// Du wusstest, dass das eine (.) ideologisch versucht wurde, dir einzutrichtern $/ / \mathrm{hmh} / /$ und dass die, die Realität ganz anders aussah. $/ / \mathrm{hmh} / /$ Mein Cousin war nicht mein Feind //hmh, hmh// und meine Onkel und Tante schon gar nicht" (Frank Grothe I 57:47-58:52).

Auch findet sich in der östlichen Gruppe im Vergleich zur westlichen niemand, der die eigene Erziehung als repressiv erlebt hätte. Vielmehr werden die Eltern durchgängig als beschützend und die eigene Entwicklung unterstützend wahrgenommen. Die elterlichen Erziehungsarrangements werden als ausreichend betrachtet, um einen eigenen Platz in der Gesellschaft zu finden. All diese Einschätzungen erleichtern es, an die elterlichen Erziehungssettings anzuschließen und einzelne Elemente zu übernehmen, wie Gisela Hildners Bilanzierung prägnant zeigt: „Aber einmal war der Glaube die Grundlage und dann, ich sag mal Bauchgefühl. //hmh// So wie wir erzogen wurden, haben wir das auch wieder an unsere Kinder weitergegeben“ (II 10:18-10:25). ${ }^{223}$ Hieraus spricht

\footnotetext{
${ }^{221}$ Eine Orientierungsunsicherheit in Bezug auf die Erziehung als Folge der gesellschaftlichen Veränderungen in den letzten 50 Jahren wird z.B. auch von Göppel konstatiert $(2010,38)$.

${ }^{222}$ Die Erziehung in der Familie wurde als gesellschaftlicher Auftrag proklamiert und die Eltern sollten ihre Kinder ,sozialistisch“ erziehen (Busch 1972, 92/93).

${ }^{223}$ Gisela Hildner stellt insofern eine Ausnahme in diesem Sample dar, als ihre Kinder bereits Ende der 1970er Jahre geboren wurden. Da zu dieser Zeit von einer staatlichen Kontrolle des Büchermarktes
} 


\section{Bildungsstatus, Generation und Region}

ein im Vergleich zu den westlichen Müttern stärker pragmatisch ausgerichteter Umgang mit den eigenen Erziehungserfahrungen sowie der Gestaltung des eigenen Erziehungssettings. Die elterliche Erziehung bietet der nachfolgenden Generation offenbar ein anschlussfähiges Repertoire an Erziehungsmitteln, auf das sie sehr viel selbstverständlicher zurückgreifen als die Vergleichsgruppe im westlichen Teilgebiet. Die öffentlich geführten Debatten um Erziehung, die spätestens mit der Wende auch die mittlere Generation im Osten erreichten, können dieses Vertrauen in die selbst erfahrene Erziehung offensichtlich nicht erschüttern. Im Vergleich zur westlichen Gruppe scheint sich diese Gruppe durch eine größere Beharrungstendenz im Bereich Erziehung auszuzeichnen. Alheits Befund, in Ostdeutschland gebe es „veränderungsresistente Grundstimmungen“ (Alheit 2005, 35), scheint somit auch auf den Bereich der familialen Erziehung zuzutreffen. In diesem Fall ist das nicht unbedingt negativ konnotiert, sondern kann auch als Ressource verstanden werden, um Unsicherheiten zu begegnen.

In Anbetracht der größeren Aufmerksamkeit, mit der die mittlere Generation - vor allem die Mütter - im westlichen Teilgebiet sich der Förderung der kindlichen Fähigkeiten und der Schaffung eines optimalen Umfeldes für ihre Kinder widmet, wozu im Westen auch eher als im Osten auf Erziehungsratgeber zurückgegriffen wird, ist die Angst vor einer sozialen Deklassierung in der westlichen Vergleichsgruppe vermutlich stärker verankert. Obgleich die Gefahr eines sozialen Abstiegs für die östliche Teilgruppe angesichts der seit der Wende zu verzeichnenden Arbeitslosenzahlen in Ostdeutschland ${ }^{224}$ stärker empfunden werden müsste, ist in diesem Sample offensichtlich das Gegenteil der Fall. Dieser auf den ersten Blick ebenfalls erstaunliche Befund lenkt das Augenmerk auf die Lebensgeschichten der beiden Teilgruppen und lässt vermuten, dass unterschiedliche Erfahrungen vorhanden sein müssen, die mit den jeweils unterschiedlichen politischen Systemen in Verbindung stehen und bis in die Gegenwart bedeutsam sind. Von der Wirtschaftskrise der 1970er Jahre sind beide Staaten betroffen, allerdings unterscheidet sich der Umgang mit der Krise und ihren Folgeerscheinungen in den beiden Gebieten. In der Bundesrepublik ist die Krise Gegenstand öffentlicher Verhandlungen und Maßnahmen, wie z.B. der Forderung zum Konsumverzicht, etwa durch einen autofreien Sonntag (Steiner 2005, 188). Die DDR-Führung, die „in ihrer eigenen Propaganda die ostdeutsche Wirtschaft als unbeeinflusst von westlichen Krisen und erfolgreich bei der Hebung des Lebensstandards darstellte“, konnte nicht auf eine offene Strategie zur Bekämpfung setzen und versuchte, einen Konsumverzicht verschleiert durchzusetzen (Steiner 2005, 188). Spätestens seit den 1980er Jahren ist in Westdeutschland neben ei-

auszugehen ist, könnte ein Verzicht auf Erziehungsratgeber in diesem Fall auch hierauf zurückzuführen sein. Allerdings erwähnen auch die Eltern der östlichen Teilregion, deren Kinder nach der Wende geboren wurden, keine Heranziehung von Erziehungsratgebern oder eine verstärkte Verunsicherung in Erziehungsfragen.

${ }^{224}$ Wie Rehberg mit Referenz auf Reißig feststellt, sind allein zwischen 1989 und 1992 ca. 4 Millionen Arbeitsplätze in Ostdeutschland weggefallen (Reißig zit. n. Rehberg 2006, 211). 2006 beträgt die Arbeitslosenquote in Ostdeutschland 18,3 Prozent gegenüber acht Prozent im Westen (Rehberg 2006, 213). 


\subsection{Regionale Besonderheiten und Bezüge}

nem Anstieg der Arbeitslosenzahlen eine Erosion des Normalarbeitsverhältnisses zu beobachten (Scheller 2005, 40 und Steiner 2005, 189). Das DDR-System wiederum setzte auf eine möglichst umfassende Integration der erwerbsfähigen Bevölkerung in den Arbeitsmarkt in Vollzeitbeschäftigung. Dieses Ziel war nach offizieller Lesart auch bis 1989 nahezu komplett realisiert (Scheller 2005, 69, 71). Während der westlichen Gruppe in ihrer Kindheit und Jugend durch die öffentliche Diskussion vor Augen geführt wird, dass eine finanzielle Absicherung über Erwerbsarbeit nicht mehr zwangsläufig für jedes Gesellschaftsmitglied gewährleistet ist, muss sich die östliche Teilgruppe mit dieser Problematik während ihres Heranwachsens nicht auseinandersetzen. Die östliche Teilgruppe ist in Folge der Kindheits- und Jugenderfahrungen weniger stark getrieben von der Angst eines sozialen Abstiegs. Die mittlere Generation im Westen hingegen verspürt offenbar einen erhöhten Wettbewerbsdruck und sie internalisiert die Maxime, die individuellen Fähigkeiten zu optimieren. Beides beeinflusst die Erziehung, indem nach optimalen Wegen der Kindesförderung gesucht wird.

In der jüngsten Generation wird in erster Linie im Westen über einen zu stark ausgefüllten Terminkalender und eine manchmal zu starke Kindzentrierung geklagt. Lea Gümper, Janina Ebel und Miriam Friese bleibt z.B. aufgrund ihrer vielfältigen Zusatztermine kaum Freizeit. Janina Ebel kritisiert die teils zu starke Ängstlichkeit in der elterlichen Erziehung und Lea Gümper stört sich gelegentlich an der zu großen Fürsorglichkeit der Mutter (Janina Ebel, 32:55-33:52, Lea Gümper 1:36:05-1:36:25). Im östlichen Teilgebiet finden sich diese Klagen nicht. Das hängt mit den beschriebenen Unterschieden im Erziehungsverhalten in den beiden Teilregionen zusammen. Es sind insbesondere die westlichen Eltern, die einen starken Fokus auf die kindliche Entwicklung und optimale Förderung haben. Somit wird verständlich, warum eher die westlichen Kinder über verplante Freizeit und eine manchmal zu starke Kindzentrierung klagen. Die stärkere Betonung der Hilfsbereitschaft im östlichen Teilgebiet (siehe Fallbeispiele Anika Jäger und Magdalene Schmidt) lässt sich als ein Erbe der DDR-Gesellschaft deuten. Die Eltern und Großeltern der jüngsten Generation im östlichen Teilgebiet sind im Alltag stärker auf eine innerfamiliale Solidarität wie auch auf die gegenseitige Hilfe im Bekannten- und Freundeskreis angewiesen gewesen. Die hieraus resultierende Wertschätzung der Hilfsbereitschaft wird an die jüngste Generation weitergegeben und von dieser aufgenommen. Die stärkere Bedeutung, die die jüngste Generation im östlichen Teilgebiet den familialen Beziehungen beimisst, lässt sich ebenfalls mit dem Fortwirken des Bezugsrahmens des familialen Zusammenhalts erklären.

\subsubsection{Großelternschaft}

Im thüringischen Teilgebiet sind die Großeltern im Unterschied zu ihrer Vergleichsgruppe in Niedersachsen viel stärker in den Erziehungsalltag ihrer Enkel eingebunden. Der durch die gesellschaftlichen Rahmenbedingungen in der DDR forcierte familiale Zusammenhalt findet auch hier seinen Niederschlag und beeinflusst bis in die Gegenwart die Gestaltung der Großelternschaft. Hiermit verbunden, sehen sich die Familien 


\section{Bildungsstatus, Generation und Region}

in Ost und West in unterschiedliche Traditionen eingebettet, was die Beteiligung der Großeltern an der Kindesbetreuung und -erziehung betrifft. Während die älteste Generation im östlichen Teilgebiet während ihrer Kindheit mehrheitlich eine starke Alltagspräsenz ihrer Großeltern erinnert, ist dies in der westlichen Teilgruppe seltener der Fall. Die älteste Generation schreibt sich hierüber jeweils in bestimmte Familientraditionen ein, die zu der Ausgestaltung der eigenen Großelternschaft passt. In der nächsten Generation setzt sich das fort. Während die Großeltern für die Protagonisten der mittleren Generation im Osten verstärkt zentrale Bezugspersonen darstellen (siehe Fallbeispiel Friederike Jäger) oder zumindest im Alltag sehr präsent sind (siehe Fallbeispiel Birgit Schmidt), betrachten die Vertreter der mittleren Generation im Westen die Großeltern mehrheitlich als liebe und warmherzige Personen, die allerdings nicht so stark in den Alltag eingebunden sind. Heike Ebel charakterisiert z.B. die Großeltern als „liebe (.) Knuddeltiere im Hintergrund“ (IV 11:18-11:22). Diese jeweils unterschiedlichen Kindheitserfahrungen prägen die Erwartungen an die eigenen Eltern als Großeltern. Während für diejenigen, die einen alltäglichen Kontakt zu ihren Großeltern in der Kindheit gehabt haben, die Einbeziehung der eigenen Eltern eine problemlose Selbstverständlichkeit darstellt, erwartet die mittlere Generation im westlichen Teilgebiet ein solches Engagement nicht. Die jeweiligen Ansichten der mittleren Generation beeinflussen auf diese Weise, wie die Großelternschaft in ihrer aktuellen Familienphase ausgefüllt wird. Für die jüngste Generation ist das jeweils praktizierte Arrangement selbstverständlich und akzeptabel. Sie betrachten die Beziehungen zu ihren Großeltern mehrheitlich als gut und sind ihnen emotional sehr zugewandt. Ein distanziertes Verhältnis zu den Großeltern findet sich nur selten. Das hier gezeichnete Bild korrespondiert in hohem Maße mit den Ergebnissen von Höpflinger/Hummel/Hugentobler (2006, 55-57).

In der ältesten Generation finden sich regional unterschiedliche Vorstellungen, wie die Rolle der Großelternschaft auszufüllen ist, die mit spezifischen Bildern vom Alter gekoppelt sind. Für die Großeltern im thüringischen Untersuchungsgebiet ist es mehrheitlich eine Selbstverständlichkeit, sich in die Alltagsorganisation ihrer Enkel einzubringen. Dieses Engagement wird selten als ein Faktor betrachtet, der mit den eigenen Interessen kollidieren könnte. Im niedersächsischen Teilgebiet sind die Großeltern in Notfällen ebenfalls bereit, ihre Hilfe bei der Betreuung der Enkel anzubieten. Sie gehört aber nicht zu den alltäglichen Aufgaben, und die Großeltern artikulieren stärker ihre eigenen Interessen. Der Unterschied zwischen dem östlichen und westlichen Teilregionen lässt sich exemplarisch an den beiden folgenden Aussagen ablesen, die jeweils eine Maximalposition widerspiegeln. Für Frau Nolte (West) stehen in ihrer jetzigen Lebensphase die eigenen Angelegenheiten im Vordergrund, was sie folgendermaßen rechtfertigt: „Da hab ich gesagt: Ich bin, glaube ich, eine gro-, eine gute Mutter gewesen. Aber ich bin keine, keine gute Großmutter" (1:50:51-1:50:58). Sie verdeutlicht, dass sie ihren Beitrag zur Familienarbeit während ihrer Elternphase geleistet hat und sich nun erlauben kann, stärker ihre eigenen Interessen zu verfolgen. Frau Grothe (Ost) wiederum ist stark im Alltag ihrer Enkel präsent: „[A]ber die Oma muss immer da sein. //hmh// Wenn der Kleine aus der Schule kommt, und ich bin, ich koche noch die ganze Woche [..] und 


\subsection{Regionale Besonderheiten und Bezüge}

wenn der Kleine aus der Schule kommt, dann ruft er: ,Oma““ (I 19:31-19:47). Wie stark sie sich bis heute in den Erziehungsalltag ihrer Enkelkinder eingebunden sieht, verdeutlicht folgende Passage: „Ja, diesen Kleinen, den ziehe ich ja nun auch noch groß. Die Hannah auch schon. $/ / \mathrm{hmh} / /$ Und is gut, ich muss auch immer sagen, gut, wer noch 'ne Oma im Hause hat" (I 1:42:36-1:42:46). Im Unterschied zur Position von Frau Nolte wird nicht nur die Betreuung, sondern auch die Erziehung als selbstverständliche Aufgabe angenommen. Diese beiden Pole des Spektrums großelterlichen Selbstverständnisses verdeutlichen, dass das Verhältnis von familialen und eigenen Interessen von den aktuellen Großeltern unterschiedlich aufgefasst wird. Während im Osten die Familie verstärkt als lebenslange Wirtschafts- und Sozialgemeinschaft verstanden wird, zu der die Großeltern - sofern es ihre Kräfte erlauben - ihren Teil in Form der Unterstützung beitragen, wird das familiale Engagement im Westen stärker lebensphasenspezifisch aufgefasst. Nach einer Periode des starken familialen Engagements während der eigenen Familienphase folgt ein Abschnitt, der stärker den persönlichen Neigungen vorbehalten bleibt. Dies lässt sich als Ausdruck eines im Westen stärker vorangeschrittenen Individualisierungsprozesses (Beck 1986 und Beck/Beck-Gernsheim 1990b) verstehen, in dem familiale Bindungen eher zeitweise als durchgängig das gesamte Leben bestimmen.

Ertragreich ist in diesem Zusammenhang die Einbeziehung der Resilienzforschung. Strukturen langer Dauer sowie Traditionen helfen den Individuen, abrupte Veränderungen im Zuge eines Systemumbruchs besser zu bewältigen (Bagus 2012, 68). Sofern sich die Verarbeitungsstrategien bewährt haben, stellen sie eine Ressource für den Umgang mit erneuten Umbrüchen dar (Bagus 2012, 82). Das im östlichen Teilgebiet praktizierte Modell, die Großeltern in den Alltag der Enkel einzubinden, könnte ebenfalls eine Strategie im Rahmen eines Resilienzpotenzials sein. Die hier zu erkennenden familialen Bindungen sind keine neuen, durch die Wende verursachten Phänomene, sondern stellen bereits in der Vergangenheit bewährte Traditionen in dieser Teilregion dar, die durch die gesellschaftlichen Rahmenbedingungen der Vergangenheit verstärkt worden sind und sich für die Familien bewährt haben. Die Einbeziehung der Großeltern in den Familienalltag bildet für die Familien eine Ressource, auf die sie in Reaktion auf den Systemumbruch zurückgreifen. Gefördert wird das nicht zuletzt durch die im Vergleich zur westlichen Teilregion eher geringe Mobilität der Familien, die dazu führt, dass die Großeltern eher als im westlichen Untersuchungsraum in unmittelbarer Nähe der Enkel leben.

Im westlichen Teilgebiet wird eine Facette betont, die sich in dieser Form nur hier findet. Frau Dienel thematisiert z.B. ausführlich das Nachlassen der körperlichen Fähigkeiten im Alter, die den großelterlichen Handlungsspielraum begrenzen und verknüpft das mehr oder weniger direkt mit der an die Kinder gerichtete Forderung, hierauf Rücksicht zu nehmen (II 26:05-31:14). In der Gesamtschau dominiert im Osten eine Sicht auf das Alter und die Großelternschaft, in der Großeltern als Teil der familialen Gemeinschaft betrachtet werden. Hieraus resultieren selbstverständliche Pflichten, die nicht als belastend empfunden werden. Zudem werden familiale und eigene Interessen weniger in einem Spannungsverhältnis zueinander wahrgenommen, sondern als einander ergän- 


\section{Bildungsstatus, Generation und Region}

zend. Im Westen hingegen wird Großelternschaft als eine Phase konzipiert, in der die persönlichen Wünsche nach einer Periode des Verzichts in den Vordergrund rücken und von den jüngeren Generationen Rücksichtnahme gefordert wird. Zwischen familialen und eigenen Interessen werden potenziell eher Diskrepanzen wahrgenommen.

Die aufgezeigten regionalen Unterschiede in Bezug auf Großelternschaft korrespondieren mit den Ergebnissen von Herlyn/Kistner, die verstärkt in Ostdeutschland familienorientierte Großmütter finden (Herlyn/Kistner 1997, 10). In der vorliegenden Studie bezieht sich das allerdings ausschließlich auf die praktische Involviertheit in die alltäglichen Betreuungs- und Erziehungsleistungen ${ }^{225}$, die im Osten deutlich ausgeprägter ist, und gilt auch - sofern vorhanden - für die Großväter. Herlyn/Kistners Ergebnis, wonach die Großmütter mehrheitlich angeben, sich nicht in die Erziehung der Enkel einzumischen (Herlyn/Kistner 1997, 9 und 12), ist de facto für viele Großeltern im Osten durch die alltägliche Betreuung der Enkel außer Kraft gesetzt. Sie sind in die Erziehung der Enkel praktisch involviert.

Was die Großeltern in Ost und West hingegen eint, ist die Internalisierung bestimmter positiver Stereotype zur Großelternschaft, wie sie von Höpflinger/Hummel/Hugentobler (2006, 21) oder Chvojka (2003) beschrieben werden. Nahezu alle Großeltern geben an, in Bezug auf ihre Enkel weniger streng und eher verwöhnend zu sein. In Lüschers Terminologie $(2008,51 / 52)$ würden die meisten Großeltern für sich die Rolle der Gefährten reklamieren.

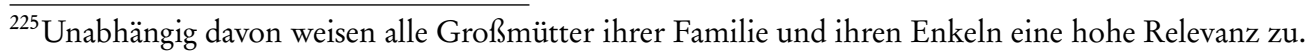




\section{Familiale Tradierungsprozesse im gesellschaftlichen Wandel: Fazit und Ausblick}

Die Frage, ob angesichts des gesellschaftlichen Wandels in den Familien noch Tradierungsprozesse stattfinden, lässt sich aufgrund der Fallbeispiele eindeutig bejahen. Die populäre Annahme des Werteverfalls im Generationenverlauf sowie die Vermutung, familiale Tradierungsprozesse seien angesichts des gesellschaftlichen Wandels der letzten Jahrzehnte obsolet geworden, lässt sich mit den Ergebnissen dieser Studie nicht bestätigen. Vielmehr sollte deutlich geworden sein, dass Tradierungsprozesse nach wie vor in den Familien eine wichtige Rolle spielen. Sie bestehen aus einem komplexen Geflecht aus Vermittlung und Aneignung, Erfahrung und Erwartung, dem Anknüpfen an sowie der Weiterentwicklung des Bestehenden und werden von der familialen wie gesellschaftlichen Sphäre beeinflusst. Hierbei wirkt die jeweilige Verankerung in weiteren Gruppen, wie z.B. die soziale, regionale und generationelle Zugehörigkeit, auf die Inhalte und Modi der Weitergabe ein.

Auch wenn die Genderperspektive nicht im Zentrum der Fragestellung stand, ließen sich dennoch einige genderbezogene Aspekte herausarbeiten. So können die Frauen der mittleren Generation, die sich als fremdbestimmt wahrnehmen und einem Konformitäts- und Leistungsdruck ausgesetzt sehen, dies eher thematisieren als diejenigen Männer der mittleren Generation, die sich in einer ähnlichen Lage befunden haben. Eine systematische Einbeziehung der Genderperspektive in das im Zuge dieser Arbeit entwickelte Analyseinstrumentarium erscheint vielversprechend, um weitere Facetten der Ver- und Bearbeitung von Tradierungsprozessen zu erfassen.

Angesichts der Samplezusammensetzung, in das nur mittlere und höhere Bildungsabschlüsse sowie Familien, die in einem regelmäßigen Austausch miteinander stehen, aufgenommen worden sind, ist zu vermuten, dass die vorgestellten Tradierungslogiken noch kein umfassendes Bild darstellen. Insbesondere ließen sich weder klare Wechsel der Tradierungslogiken noch deutliche Brüche herausarbeiten. Die Einbeziehung weiterer Milieus kann dazu beitragen, die vorgestellte Systematik zu erweitern.

Wie gezeigt werden konnte, spielt die generationelle Zugehörigkeit bei der Aneignung und Gestaltung von Tradierungsprozessen eine wichtige Rolle. Eine weiterführende Anschlussmöglichkeit in diesem Zusammenhang besteht darin, die Generationenperspektive weiter zu differenzieren, indem nach der Bedeutung der Geschwister sowie der PeerGroup für Tradierungsprozesse gefragt wird. Schließlich wurde deutlich, dass innerhalb dieses Samples drei Generationen im Austausch miteinander stehen, wobei die jeweilige Großelterngeneration in unterschiedlicher Weise in den Alltag ihrer Enkelkinder eingebunden ist. Inwiefern die Großeltern ihren Enkelkindern andere Orientierungen 


\section{Familiale Tradierungsprozesse im gesellschaftlichen Wandel: Fazit/Ausblick}

vermitteln oder eine Vermittlungsposition zwischen Eltern und Kindern einnehmen, stellt eine spannende Anschlussfrage dar.

Für die Untersuchung von Tradierungsprozessen hat sich Halbwachs' Gedächtnistheorie $(1985,1991)$, die die Gruppenbezogenheit der Erinnerungen herausstellt, in mehrfacher Hinsicht als ein hilfreiches theoretisches Gerüst erwiesen. Über die Bezugsrahmen, die wichtige Bausteine der Theorie darstellen, lassen sich in den einzelnen Interviews die jeweils relevanten Orientierungen der Individuen erfassen. Zugleich bietet die Theorie die Möglichkeit, den Einzelnen in verschiedenen Gruppen zu verorten. Hierin liegt eine Stärke der Halbwachs'schen Theorie, da jedes Individuum gleichzeitig in verschiedene Gruppen eingebunden ist und somit zugleich verschiedene Bezugsrahmen in sich vereint. Durch eine systematische Einbeziehung verschiedener Gruppenzugehörigkeiten konnten diese wechselseitigen Verflechtungen berücksichtigt werden.

Von Interesse für diese Studie ist zunächst einmal die Gruppe der Familie, die Halbwachs (1985) selbst durch seine Ausführungen zum Familiengedächtnis in den Blick nimmt. Die Einbeziehung einer familial-pädagogischen Generationenperspektive in Halbwachs' Überlegungen ermöglicht es, sowohl Vermittlungs- als auch Aneignungsprozesse zu berücksichtigen, wodurch die Wechselseitigkeit des Tradierungsprozesses deutlich hervortritt. Die Tradierungsinhalte und -formen werden von jeder Generation bearbeitet und mit jeweils eigenen Schwerpunktsetzungen versehen. Das unterstreicht die Erkenntnis von Berteaux/Berteaux-Wiame (1991), die Transmissionsprozesse zum Äquivalenten, worunter situationsspezifische Anpassungen von Tradierungsinhalten zu verstehen sind, als Regelfälle annehmen, die weitaus häufiger anzutreffen sind als Transmissionsprozesse zum Identischen, die eine unveränderte Weitergabe darstellen.

Wie die Fallbeispiele eindrücklich zeigen, finden sich in den einzelnen Familien familiale Bezugsrahmen, die in den Generationen je spezifische Ausprägungen erfahren. Der Bezugsrahmen der Vorgängergeneration geht i.d.R. nicht verloren, wird aber mit jeweils anderen Schwerpunktsetzungen und teils mit weiteren Rahmungen versehen. I.d.R. finden sich in den Familien des Samples weder klare Brüche mit den Bezugsrahmen der Vorgängergeneration, noch übernimmt die aneignende Generation den Bezugsrahmen der Vorgänger, ohne ihn zu bearbeiten. Bei Tradierung handelt es sich um einen dynamischen, intergenerationellen Prozess, in dem die Familienmitglieder Kontinuität und Wandel bearbeiten und miteinander verbinden. Zugespitzt lässt sich dieser Vorgang auf folgende Formel bringen: Tradierung stellt einen Prozess der kontinuierlichen Veränderung bzw. der veränderlichen Kontinuität dar.

Innerhalb dieses Samples lassen sich drei Tradierungslogiken identifizieren: eine geschlossene, eine flexibel erscheinende sowie eine diffuse. Indem Vermittlung und Aneignung als Grundlage für die Analyse herangezogen sowie Kosellecks (1989) Termini von Erfahrungsraum und Erwartungshorizont auf Tradierungsprozesse übertragen worden sind, sind die Unterschiede zwischen den einzelnen Logiken deutlich hervorgetreten. In allen Familien hat die jeweils vermittelnde Generation in Auseinandersetzung mit den eigenen Erziehungserfahrungen Erwartungshorizonte für die eigenen Kinder entwor- 
fen. Die Kinder haben sich mit diesen Erwartungen auseinandergesetzt, ohne sie zwingend zu übernehmen. Im Zuge von Vermittlung und Aneignung sind offene Horizonte verblieben, die von den Akteuren auch genutzt worden sind. Inwiefern und in welchem Ausmaß es zu Modifikationen der Tradierungsinhalte und -modi in den Folgegenerationen gekommen ist, hing stark von der jeweiligen Anschlussfähigkeit der Bezugsrahmen für die Lebensgestaltung der nachfolgenden Generationen ab. Die Unterschiede zwischen den Tradierungslogiken haben sich in erster Linie aus dem Umgang mit den für die jeweiligen familialen Bezugsrahmen entwickelten Erwartungshorizonten ergeben.

In Familien, die einer flexibel erscheinenden Tradierungslogik folgen, vollziehen sich die Prozesse der Vermittlung und Aneignung im Vergleich zu den anderen Familien relativ reibungslos und konfliktfrei. Die jeweiligen für die Kinder entworfenen Erwartungshorizonte werden in ein Gesamtsetting integriert, das den Eindruck erweckt, den Kindern verblieben Spielräume. Dies wird von der vermittelnden Generation deutlich kommuniziert und von der aneignenden Generation auch in dieser Weise wahrgenommen. Die eigenen Erziehungserfahrungen dienen der ältesten Generation als Grundlage für die Entwicklung von Erwartungshorizonten für die nachfolgende Generation, ohne diese zwangsläufig exakt zu kopieren. Die Bezugsrahmen der Eltern werden zwar übernommen, aber mit neuen Schwerpunkten versehen. Die verwendeten Erziehungsarrangements sind auf die Vermittlung des Bezugsrahmens ausgelegt, werden aber mit einem beiläufig erscheinenden Charakter versehen. Für die eigenen Kinder wird ein grober Rahmen abgesteckt, innerhalb dessen für die einzelnen Akteure Spielräume verbleiben. Die aneignende mittlere Generation kann auf diese Weise den Eindruck gewinnen, selbstständig Entscheidungen zu treffen, auch was den familialen Bezugsrahmen betrifft. Dieser Umstand erleichtert es, die Bezugsrahmen anzunehmen. Wesentliche Elemente der Vermittlung bilden eine Milieueinbindung, die als beiläufig und quasi selbstverständlich deklariert wird, sowie das Vorleben der zu vermittelnden Bezugsrahmen. Die Maßstäbe, die die Kinder verinnerlichen sollen, werden auch an das eigene Verhalten angelegt. Dieses Muster setzt sich zwischen der mittleren und der jüngsten Generation fort, wobei eventuell die Gestaltungsräume der aneignenden Generation noch zunehmen. Autoritäre Elemente werden bereits für die Kindheit der ältesten Generation selten beschrieben. In den folgenden Generationen hält dieser Trend an und autoritäre Elemente verschwinden fast gänzlich aus den Erziehungsarrangements. Dies trägt dazu bei, dass die jeweils aneignende Generation die Erziehungssettings als flexibel und sich selbst als relativ eigenbestimmt wahrnehmen kann. Hiermit zusammenhängend werden die intergenerationellen Beziehungen von allen Beteiligten als positiv beschrieben. Indem die Bezugsrahmen als in gewisser Hinsicht flexibel aufgefasst werden, wird es für die aneignenden Generationen zudem leichter, eine Anschlussfähigkeit an diese herzustellen. Gesellschaftliche Veränderungen können auf diese Weise leichter aufgenommen und in die jeweiligen Bezugsrahmen integriert werden. Die Gestaltungsräume erlauben es, die Bezugsrahmen mit neuen Akzentuierungen zu versehen, ohne Konflikte mit der Vorgängergeneration zu verursachen. Die Familien in diesem Sample, die einer flexi- 


\section{Familiale Tradierungsprozesse im gesellschaftlichen Wandel: Fazit/Ausblick}

bel erscheinenden Tradierungslogik folgen, sind relativ erfolgreich bei der Vermittlung ihrer Bezugsrahmen.

Die geschlossene Tradierungslogik zeichnet sich durch sehr genau konturierte Erwartungshaltungen gegenüber den Kindern aus und die Erziehungsarrangements sind direkt auf die Vermittlung des familialen Bezugsrahmens fokussiert. Dieses Vorgehen erweist sich im Vergleich zur vorherigen Logik als störungs- und konfliktanfälliger, da die jeweils aneignende Generation das Fehlen von eigenen Gestaltungsmöglichkeiten nicht unbedingt akzeptiert. Die älteste Generation überträgt die eigenen Erziehungserfahrungen unverändert auf die für die Kinder entworfenen Erwartungshorizonte. Ähnliches gilt für die Modi der Vermittlung, die als ausgesprochen autoritär einzustufen sind. Das Fehlen von Gestaltungsspielräumen führt zu Konflikten mit der mittleren Generation, die die elterlichen Erwartungen teilweise ablehnt, da sie unter den gegebenen Umständen keine Anschlussfähigkeit an diese erblicken kann. In der Folge werden die Bezugsrahmen in stärkerem Maße als bei der vorherigen Logik modifiziert und mit gänzlich anderen Zusatzrahmungen als in der Vorgängergeneration versehen. Die auf diese Weise entstandenen Bezugsrahmen wiederum werden ähnlich stringent wie einstmals von den Eltern praktiziert an die jüngste Generation herangetragen, allerdings werden die deutlich formulierten Ziele mit anderen Mitteln verfolgt. Befehle, Forderung eines unbedingten Gehorsams sowie strenge Ordnungsmuster haben ausgedient und werden durch ein engmaschiges Arrangement von Angeboten und Unterstützungsleistungen ersetzt, die darauf zielen, die kindliche Entwicklung in die gewünschte Richtung zu lenken. Das Umfeld, in dem die Kinder aufwachsen, wird nicht beiläufig, sondern sehr bewusst gestaltet. Inwiefern die Kinder sich in dieses Setting einfügen, hängt stark von der Anschlussfähigkeit für diese ab. Wird das Arrangement als hilfreich und unterstützend erlebt, wird es von der jüngsten Generation akzeptiert; wird es jedoch als einengend erlebt, kommt es zu Konflikten.

Kennzeichnend für die diffuse Tradierungslogik ist, dass auch hier Erwartungshaltungen gegenüber den Kindern existieren. Bei der Umsetzung dominiert aber eine große Nachgiebigkeit, die gekoppelt mit diversen Relativierungen den Tradierungsprozess erschwert. Auch bei dieser Logik spielt die Auseinandersetzung mit den eigenen Erziehungserfahrungen für die Entwicklung der Erwartungshorizonte für die nächste Generation eine Rolle. Die älteste Generation orientiert sich stark an der selbst erfahrenen Erziehung. Der Bezugsrahmen der eigenen Erziehung wird als Erwartung an die nächste Generation herangetragen, wobei er etwas weniger klar konturiert als bei den beiden vorherigen Logiken erscheint. Die Tradierung dieses Rahmens wird jedoch weder rigoros verfolgt, noch durch ein der aneignenden Generation Spielräume gewährendes Regelwerk unterstützt. Vielmehr überlagern im Zweifelsfall Nachgiebigkeit und empathisches Einfühlen in die Kindergeneration die Versuche, den Bezugsrahmen an die nächste Generation weiterzugeben. Dieses Muster setzt sich in der nächsten Generation fort. Die mittlere Generation versucht, im Zuge der Aneignung den Bezugsrahmen den eigenen Bedürfnissen anzupassen. Zwischen den Familienmitgliedern kommt es zu 
Konflikten, die deutlich wahrgenommen, aber letztendlich nicht für alle Beteiligten zufriedenstellend gelöst werden. Die älteste Generation ist hierbei durchaus bereit, Konflikte aufzulösen, indem sie die nachfolgenden Generationen von Verantwortung zu entlasten versucht. Zum Erziehungsarrangement dieser Tradierungslogik zählen zwar in allen Generationen vereinzelt autoritäre Elemente, die darin bestehen, dass Forderungen an die Kinder gelegentlich deutlich formuliert werden und im Anspruch kaum Verhandlungsspielräume aufweisen, sie werden aber im Konfliktfall nicht durchgesetzt. Die älteste Generation trägt die Maßstäbe, die sie für das eigene Verhalten etabliert, nicht konsequent an die nächste Generation heran. Die jeweils aneignenden Generationen sind gleichwohl bemüht, an diese Bezugsrahmen anzuschließen, wobei in jeder Generation Modifikationen zu finden sind. Allerdings führt die jeweils konkrete Umsetzung des Bezugsrahmens in den beiden jüngeren Generationen zu Konflikten mit bestimmten Familienmitgliedern, sodass der Bezugsrahmen in diesem Falle eine ambivalente Qualität aufweist.

Wie die Fallbeispiele zeigen, finden in den Familien unabhängig von der jeweils geltenden Tradierungslogik Prozesse der Vermittlung und Aneignung statt. Aus diesem Grunde lässt sich nicht sagen, dass eine der Logiken Tradierungsprozesse verhindere und somit ein ineffektiver transmission belt (Schönpflug/Bilz 2009) sei. Allerdings sind die Chancen für die Weitergabe von Tradierungsinhalten, wenn auch in leicht veränderter Form, im Rahmen der flexibel erscheinenden Tradierungslogiken etwas günstiger als bei den anderen Logiken. Die Beurteilung der intergenerationellen Beziehungen fällt in den Familien, die einer flexibel erscheinenden Logik folgen, insgesamt positiver aus. Dies kann als wichtige Voraussetzung für die Annahme familialer Tradierungsinhalte betrachtet werden.

An der Vermittlung sind sowohl Mütter als auch Väter aktiv beteiligt. Die populäre Annahme, Tradierung sei eine weibliche Sphäre, lässt sich mit diesem Sample nicht bestätigen. Auch die einfache Zuordnung, wonach in weiblichen Dyaden stärker mit weiblichen Geschlechtsrollenstereotypen assoziierte Werte und in männlichen Dyaden eher Werte, die mit männlichen Geschlechtsrollenstereotypen korrespondieren, tradiert werden, ist in dieser Form zu pauschalisierend. Vielmehr hängt es vom jeweiligen familialen und gesellschaftlichen Kontext sowie dem jeweiligen Milieu ab, was in diesem Falle ein katholisches ist, welche Orientierungen tradiert werden. In diesem Sample lassen sich in weiblichen sowie in männlich-weiblichen Tradierungslinien durchaus Orientierungen bzw. Bezugsrahmen herausarbeiten, die nicht den gängigen Geschlechtsrollenstereotypen entsprechen (siehe Fallbeispiele Cronert/Jäger und Nolte/Gümper).

Die Individuen sind jedoch nicht nur familiale Akteure, sondern gehören zugleich diversen anderen Gruppen an. Im Rahmen dieser Studie lassen sich die Familienmitglieder zusätzlich anhand des Bildungsstands, der Region sowie der Generation einordnen. Die jeweilige räumliche, soziale und generationelle Verortung der Interviewpartner ist hilfreich, um die unterschiedliche Gewichtung der Tradierungsinhalte in den einzelnen Familien sowie die Veränderungen von Inhalten und Formen der Tradierung im Zeitverlauf zu verstehen. 


\section{Familiale Tradierungsprozesse im gesellschaftlichen Wandel: Fazit/Ausblick}

Zwar lassen sich in diesem Sample keine Zusammenhänge zwischen Bildungsstand und Tradierung in dem Sinne herstellen, dass ein bestimmter sozialer Status Tradierungsprozesse begünstigen und ein anderer Tradierungsprozesse bremsen oder eine Tradierungslogik in einigen sozialen Kreisen höher im Kurs stehen würde als eine andere, wohl aber hat der soziale Status einen Einfluss auf den jeweiligen familialen Wertemix bzw. den Stellenwert, den bestimmte Orientierungen einnehmen.

Interessant sind die regionalen Unterschiede, die sich in den Familien bis in die Gegenwart erhalten haben. Die höhere Bedeutung des familialen Zusammenhalts und der stärkeren Einbeziehung der Großeltern in den Erziehungsalltag im ostdeutschen Teilsample lässt sich nur damit erklären, dass die unter den Bedingungen des DDR-Systems geformten familialen Strategien sich bis heute als anschlussfähig für die Familien dieser Region erwiesen haben. Wie sich zeigt, ist der Einfluss des politischen Systems auf die familiale Erziehung begrenzt (Reuband 1995, 236), aber nicht gänzlich bedeutungslos. Über das politische System der DDR werden keine neuen Bezugsrahmen in den Familien etabliert, die hiermit einhergehenden gesellschaftlichen Rahmenbedingungen wirken sich aber auf die Tradierungsprozesse aus. In den untersuchten Familien werden die existierenden familialen Bezugsrahmen weder durch eine politische Beeinflussung, wie etwa die Propagierung sozialistischer Erziehungskonzepte, noch durch die gesellschaftlichen Lebensumstände gebrochen. Stattdessen werden die mit den allgemeinen Rahmenbedingungen verbundenen Auswirkungen in den familialen Bezugsrahmen integriert. In der Folge werden die existierenden familialen Bezugsrahmen nicht durch das politische System und die hiermit einhergehende politische Propaganda überlagert, vielmehr führt es in den Familien zu einer Verstärkung der bereits vorhandenen familialen Bezugsrahmen. Erst durch die Verknüpfung von familialer und politischer Sphäre ergibt sich ein für familiale Tradierungsprozesse relevantes Gesamtbild. Die Familien folgen durchaus ihrer eigenen Agenda, die jedoch von den gesamtgesellschaftlichen Verhältnissen beeinflusst wird und bestimmte Optionen eröffnet und andere verschließt. Durch die politischen Rahmenbedingungen werden nicht unbedingt neue Orientierungen in den Familien erschaffen, sie beeinflussen aber, wie die Familien mit den existierenden Inhalten umgehen.

Die insgesamt geringer erscheinende Verunsicherung der mittleren Generation im ostdeutschen Teilsample, was Erziehungsfragen betrifft, sowie die schwächer ausgeprägte Fokussierung auf die Leistungsoptimierung, was mit dem stärkeren Anknüpfen an die selbst erfahrene Erziehung durch die Eltern zusammenhängt, lässt sich ebenfalls mit den jeweils spezifischen Rahmenbedingungen verbinden, die durch das politische System der DDR hervorgebracht worden sind und die den familialen Zusammenhalt forciert haben. Die allgemeinen Lebensbedingungen im Sperrgebiet förderten die enge familiale Vernetzung und den intergenerationellen Austausch, der als eine zentrale Voraussetzung für Tradierungsprozesse zu betrachten ist. Die im Vergleich zum westlichen Teilgebiet geringeren intergenerationellen Spannungen begünstigen hierbei die Übernahme von Tradierungsinhalten durch die aneignende Generation. Die in der Ge- 
samtschau vorherrschende Zufriedenheit mit der elterlichen Erziehung in der mittleren Generation erleichtert ihre Fortführung sowie die Beibehaltung der zentralen Orientierungen. Familiale und gesellschaftliche Ebene sind eng aufeinander bezogen, wobei die gesellschaftlichen Verhältnisse von den Familien in einer der jeweiligen familialen Eigenlogik folgenden Weise verarbeitet werden. Die gesellschaftlichen Gegebenheiten werden von den Familien in ihre Rahmungen integriert, ohne dass sie die Gestaltung der familialen Sphäre determinieren würden. Diese Feststellung gilt ebenso für das westliche Teilgebiet, in dem die Familien im Generationenverlauf die gesellschaftlichen Entwicklungen in ihre Bezugsrahmen integrieren, womit die generationelle Verortung thematisiert ist.

Besonders markant sind die im Generationenverlauf erkennbaren Verschiebungen, die die Erziehungssettings betreffen. Je nach Tradierungslogik von relativ unterschiedlichen Niveaus aus startend, gleichen sich in der mittleren Generation diverse Elemente der Erziehung an - und zwar unabhängig von der jeweils verfolgten Tradierungslogik. Im Zeitverlauf nimmt die Kindorientierung deutlich zu. Gleiches gilt für die Beachtung des emotionalen Wohlbefindens der Kinder. Zudem werden den Kindern in verstärktem Ausmaße Mitspracherechte eingeräumt. Diejenigen Vertreter, die aus Familien mit einer geschlossenen Tradierungslogik stammen, grenzen sich hierüber bewusst von den Erziehungssettings der Eltern ab und nehmen im Vergleich zu den anderen Protagonisten ihrer Generation die stärksten Veränderungen in ihren Erziehungsarrangements vor. Die übrigen führen die selbst erfahrene Erziehung fort bzw. modifizieren sie. In Anbetracht dieses relativ einheitlichen Verlaufs sind diese Angleichungen als Ausdruck einer generationell bedingten Entwicklung zu deuten, die sich nur unter Einbeziehung des allgemeinen gesellschaftlichen Wandels erklären lässt. Doch bleiben Unterschiede bestehen, die sich auf die Art und Weise beziehen, wie die Erwartungshaltungen für die nächste Generation gestaltet werden. Die Art des Umgangs mit diesen Erwartungen ist weniger einer generationellen Zugehörigkeit geschuldet, sondern bleibt vielmehr der familial etablierten Tradierungslogik verhaftet.

Die beiden älteren Generationen markieren direkt oder indirekt Generationendifferenzen, wobei eine deutliche Abgrenzung zu der jeweils jüngeren Generation erfolgt. In der jüngsten Generation hingegen finden sich keine Distanzierungen von der älteren Generation. Dies lässt sich als eine Reaktion auf gesellschaftliche Veränderungsprozesse deuten, wobei die eigene Kindheit und Jugend erst retrospektiv mit einer besonderen Bedeutung aufgeladen wird und als Folie für eine horizontale Vergemeinschaftung dient. Diese wird zumindest in diesem Sample erst in späteren Jahren bedeutsam und dient dazu, gesellschaftliche Veränderungsprozesse zu verarbeiten. Eine weitere Funktion besteht darin, wahrgenommene Unterschiede zwischen den Generationen zu erklären und über das latent vorhandene Argument der Generationendifferenz potenzielle Konflikte $\mathrm{zu}$ minimieren.

Es finden sich familiale Bezugsrahmen, die sich über die drei untersuchten Generationen erstrecken. Dabei bearbeitet jede Generation die familialen Bezugsrahmen auf spezifische Weise und versieht sie mit jeweils besonderen Akzentuierungen, die sich mit 


\section{Familiale Tradierungsprozesse im gesellschaftlichen Wandel: Fazit/Ausblick}

den jeweils existierenden gesellschaftlichen Rahmenbedingungen verknüpfen lassen. Jede Generation rückt in Verbindung mit dem familialen Bezugsrahmen und dem eigenen Erziehungssetting jeweils bestimmte Orientierungen in den Mittelpunkt, die für andere Generationen keine bzw. eine untergeordnete Rolle spielen.

Hiermit ist die gesellschaftliche Entwicklung mit ihren Auswirkungen auf familiale Tradierungsprozesse ins Zentrum der Betrachtung gerückt. Zunächst einmal ist der strukturelle Wandel zu nennen, der sich auf das Familienleben auswirkt. Eine wichtige Veränderung ist in der sinkenden Kinderzahl pro Familie zu sehen (Schäfers 2004). ${ }^{226}$ Hierüber wird es möglich, die einzelnen Kinder stärker in das Zentrum der Aufmerksamkeit zu rücken und sich ihnen intensiver zu widmen. Der steigende ökonomische Wohlstand im Untersuchungszeitraum ist ein weiterer Faktor, der es den Eltern (erst) erlaubt, eine als optimal verstandene Kindesförderung (Beck-Gernsheim 1990a, 168/ 169) zu realisieren. Hinzu gesellt sich ein zunehmendes Kontingent an freier Zeit, das es den Eltern ermöglicht, sich verstärkt ihren Kindern zu widmen sowie sich umfassend über Fördermöglichkeiten (Beck-Gernsheim 1990a, 170 und Peuckert 2007, 52) zu informieren. Letzteres ist eng verbunden mit der Ausbreitung der Informations- und Wissensgesellschaft. Im Zuge der medialen Entwicklung steigt die Verfügbarkeit und Rezeptionsmöglichkeit pädagogischen Wissens (Berg 1991, Schmid 2010 und Höffer-Mehlmer 2003) und schafft damit erst die Voraussetzung, sich wissenschaftlich sowie populärwissenschaftlich aufbereitete Erkenntnisse über Erziehung in Form öffentlicher Diskurse sowie Erziehungsratgeber anzueignen. Zusätzlich fördert die mediale Entwicklung die Verbreitung verschiedener Orientierungsoptionen in Fragen der Erziehung, wodurch tendenziell verschiedene Modelle und Ziele der Erziehung gleichberechtigt nebeneinander stehen können. Die noch in der ältesten Generation zu findenden festgefügten Ordnungsstrukturen weichen auf und pluralisieren sich, was zugleich die prinzipielle Offenheit der elterlichen Erziehung fördert und erklärt, warum Vertreter der mittleren Generation die aktuelle Erziehung als „lockerer“ bezeichnen.

Eine große Rolle für die Veränderungen innerhalb der Bezugsrahmen sowie ihrer Modi der Tradierung spielt der Mitte der 1960er Jahre in Westdeutschland einsetzende Wertewandel (Meulemann 1996b, Klages 1984, 2001b, 2002), in dessen Zuge sich auch zentrale Erziehungswerte (Reuband 1997, 134) verändern. Das erklärt zum einen, warum die älteste Generation, deren Kindheit und Jugend in der Zeit vor dem Wertewandel liegt, sich stark bei den von ihnen gewählten Formen und Modi der Tradierung an der selbst erfahrenen Erziehung orientiert und kaum grundlegende Änderungen vornimmt. Dies vollzieht sich erst in der mittleren Generation, deren Kindheit und Jugend mit dem Wertewandel zusammenfällt, in dessen Zuge Selbstentfaltungswerte (Klages 2001b, 730) an Bedeutung gewinnen. Zugleich erklärt es, warum einige Protagonisten der mittleren Generation im westlichen Teil des Untersuchungsgebiets ein weitaus hö-

${ }^{226}$ Dieser bereits zu Beginn des 20. Jahrhunderts einsetzende Trend ist in den Familien des Samples deutlich zu erkennen. Während in der ältesten Generation noch Familien mit vier oder mehr Kindern zu finden sind, ist in der mittleren Generation die Ein- oder Zwei-Kind-Familie die Norm. 
heres intergenerationelles Konfliktniveau erinnern als die Vertreter im östlichen Teil. Während im westlichen Teil in einigen Familien elterliche und kindliche Vorstellungen des intergenerationellen Umgangs sowie anzustrebender Orientierungen aufgrund des Wertewandels kollidieren, fehlt diese Komponente im östlichen Teilgebiet, da dort wesentliche Veränderungen der Wertorientierungen erst rund zehn Jahre später einsetzen (Gensicke 1992b, 692 und Klages/Gensicke 1993, 222). Insgesamt werden aber die Wertvorstellungen nicht obsolet, sondern erhalten je generationell spezifische Ausformungen. Das Milieu erweist sich insofern als anpassungsfähig, als es die Integration und Veränderung der existierenden Orientierungen erlaubt (Vester 1993).

Die recht hohe Zufriedenheit der jüngsten Generation mit den elterlichen Erziehungsarrangements lässt sich als eine Folge der mit dem Wertewandel verbundenen Veränderungen begreifen, in deren Zuge Autonomiewerte (Meulemann 1996b, 224 und 227) in der Erziehung einen zunehmenden Stellenwert erhalten. Die jüngste Generation kann sich selbst als relativ eigenständig und mit großen Freiräumen ausgestattet wahrnehmen, was sich positiv auf die intergenerationellen Beziehungen in Form abnehmender Konfliktintensitäten auswirkt.

Insgesamt birgt der Wertewandel ein hohes Potenzial, um die Akzentverlagerungen innerhalb der familialen Bezugsrahmen und insbesondere die Umgestaltungen innerhalb der familialen Erziehungsarrangements zu erklären. Hieraus allerdings einseitig eine Dominanz des Wandels abzuleiten, wäre ein Fehlschluss. Die einzelnen Generationen integrieren die Veränderungen in die vorhandenen Bezugsrahmen, um sie im familialen Generationenverlauf anschlussfähig zu halten. Demselben Zweck dienen die Weiterentwicklungen und Neuausrichtungen der Erziehungssettings. Hierdurch wird eine intergenerationelle Kontinuität der familialen Bezugsrahmen hergestellt.

Angesichts der aufgezeigten Tradierungslinien kann nicht von massiven Generationenkonflikten, in deren Zuge sich radikale Neuorientierungen ergeben würden, die Rede sein. Zwar werden insbesondere in der mittleren Generation teilweise recht ausgeprägte Konflikte mit den Eltern beschrieben, diese führen aber i.d.R. nicht dazu, mit den familialen Bezugsrahmen gänzlich zu brechen.

Eine populäre Lesart der Individualisierungsthese (Beck 1986, Beck-Gernsheim 1990b), wonach die Menschen zunehmend aus den Bindungen, wie etwa den familialen, herausgelöst werden, ist in dieser Radikalität für den Untersuchungsraum ebenfalls nicht haltbar, sondern das Gegenteil ist der Fall. Für die jüngste Generation scheinen die familialen Bindungen und Unterstützungsleistungen wichtiger denn je zu sein. Während sich in der mittleren Generation in einigen Fällen Distanzierungsbemühungen von der Herkunftsfamilie feststellen lassen, die jedoch in keiner Familie generell gelten, werden von der jüngsten Generation die intergenerationelle Verbundenheit und die Unterstützungsleistungen durch die Eltern durchweg herausgestellt. Ein Grund hierfür könnte die von Schülein als „Beziehungsrevolution“ $(1990,79-88)$ bezeichnete Entwicklung darstellen, in deren Zuge die emotionale Qualität der zwischenmenschlichen Bindungen an Bedeutung zugenommen hat. Was hingegen an der von Beck und Beck/ Beck-Gernsheim $(1986,1994)$ vertretenen Individualisierungsthese durchaus zutrifft, ist 


\section{Familiale Tradierungsprozesse im gesellschaftlichen Wandel: Fazit/Ausblick}

die Tatsache, dass sich - bei gleichzeitiger Etablierung neuer Zwänge - alte Zwänge auflösen, wie etwa die Forderung, sich in die überlieferten Bahnen einzufügen. Die aktuelle Elterngeneration kann aus einer Vielzahl von Erziehungsrichtungen und pädagogischen Angeboten auswählen, steht aber gleichzeitig unter Druck, die bestmögliche Förderung für das eigene Kind (Beck-Gernsheim 1990a, 170/171, Peuckert 2007, 52 und Engelbert u.a. 2000, 7) zu finden. Der jüngsten Generation wiederum stehen (theoretisch) vielfältige Bildungsangebote und Möglichkeiten zur Entfaltung der eigenen Potenziale zur Verfügung, gleichzeitig steht sie unter dem Zwang, die eigenen Fähigkeiten bestmöglich zu entwickeln, um die eigenen Zukunftschancen zu verbessern.

Im Rahmen dieser Studie konnte ein detailliertes Bild familialer Tradierungsprozesse gezeichnet werden. Durch die konsequente Verknüpfung von Halbwachs' Überlegungen zum kollektiven Gedächtnis mit der Generationenperspektive in vertikaler und horizontaler Ebene, die den Zusammenhang von Vermittlung und Aneignung, Erfahrungsraum und Erwartungshorizont sowie die jeweils historischen Kontexte berücksichtigt, konnte ein Analyseinstrumentarium entworfen werden, das Tradierungsprozesse in ihrer Vielschichtigkeit erfasst und als Basis für weitere Forschungsfragen genutzt werden kann. Es wäre z.B. interessant, mit dem entwickelten Analysetool in einigen Jahren die Studie fortzusetzen und zu untersuchen, wie sich die Tradierungslogiken in der Zukunft entwickeln, ob die sich abzeichnenden Angleichungen der jüngsten Generation im östlichen und westlichen Teilgebiet sich weiter fortsetzen oder ob Unterschiede erkennbar bleiben. Auch bietet das hier entworfene Analysegerüst eine gute Ausgangsbasis, um weitere Facetten des Tradierungsprozesses zu erfassen, wie beispielsweise die Genderperspektive oder weitere soziale Milieus. 


\section{Danksagung}

Zahlreiche Personen haben mich bei der Realisierung dieses Forschungsvorhabens unterstützt. Mein besonderer Dank gilt meinen InterviewpartnerInnen, die mir bereitwillig Einblicke in ihre Lebens- und Familiengeschichte gewährt haben, sowie all denjenigen, die mir behilflich waren, Kontakte zu Familien zu vermitteln. Ohne ihre Hilfe hätte diese Studie nicht in der vorliegenden Form geschrieben werden können.

Prof. Dr. Margret Kraul hat die verschiedenen Entwicklungsphasen der Studie kontinuierlich begleitet, sich stets Zeit genommen, um neue Ideen zu besprechen und diese mit konstruktiven Anmerkungen und Anregungen versehen. Hierfür möchte ich mich ebenso herzlich bedanken wie auch bei Prof. Dr. Dr. Peter Alheit und Prof. Dr. Regina Bendix für ihre Betreuung.

Für die Gewährung eines Stipendiums im Rahmen des DFG-Graduiertenkollegs „Generationengeschichte“ bin ich sehr dankbar. Das ermöglichte es mir, mich auf meine Forschungsfrage zu konzentrieren. Zugleich eröffneten sich mir durch den institutionellen Rahmen des Kollegs zahlreiche Gelegenheiten des interdisziplinären Austauschs, die meine Arbeit bereichert haben. In den Kolloquien von Prof. Dr. Margret Kraul und Prof. Dr. Klaus-Peter Horn traf ich auf engagierte GesprächspartnerInnen, von denen ich wichtige Impulse für meine Forschungsarbeit erhielt. Ihnen gilt mein herzlicher Dank. Das Kurzzeitstipendium der Sozialwissenschaftlichen Fakultät der GeorgAugust-Universität Göttingen ermöglichte es mir, die Schriftfassung der Studie mit der notwendigen Sorgfalt überarbeiten zu können.

Dr. Franka Maubach hat mich mit ihrer Aufgeschlossenheit und ihrem Engagement motiviert, kontinuierlich an der Studie weiterzuarbeiten. Die inhaltlichen Diskussionen mit ihr regten mich dazu an, meine Ideen zu ordnen und zu vertiefen. Durch ihre gründliche und kritische Lektüre gewann der Text an Prägnanz, indem sie zielsicher undeutliche Passagen identifizierte. Der regelmäßige Austausch mit Catharina Keßler und Michaela Nietert über unsere jeweiligen Projekte waren eine weitere wichtige Hilfe. Ihrer aufmerksamen Lektüre der verschiedenen Teilkapitel verdanke ich diverse Anregungen und die gemeinsamen Diskussionsrunden sorgten für eine kontinuierliche inhaltliche Auseinandersetzung. Sehra Duran, Anne Hild, Vânia Morais, Julia Setter, Anna Stisser und Sabrina Wagner unterstützten mich, indem sie sich bereitwillig meine Ideen anhörten, Textauszüge kritisch kommentierten und/oder Kürzungspotenzial aufzeigten. Prof. Dr. Axel Dessecker, Dr. Anne-Katrin Hillebrandt, Anne Kirchberg, Kerstin Lenz und Lisa Peppler brachten ihre Zeit und ihr Wissen ein, um das Manuskript einer kritischen Gesamtlektüre zu unterziehen. Ihnen allen gilt mein besonderer Dank.

Ohne die motivierende Unterstützung durch Lars und meine Mutter hätte ich die vorliegende Studie nicht realisiert, wofür ich ihnen zutiefst danke. Lars interessierte 


\section{Danksagung}

sich stets für meine Ideen und ermutigte mich beständig, an der Studie weiterzuarbeiten. Von unschätzbarem Wert waren sein technisches Wissen und seine Hilfe in Bezug auf sämtliche Aspekte, die die Software und das Textlayout betrafen. 


\section{Literatur}

Ahnert, L./Lamb, M. E. (2001): The East German Child Care System. Associations With Caretaking and Caretaking Beliefs, and Children's Early Attachment and Adjustment. In: American Behavioral Scientist 44, 1843-1863. http: //abs . sagepub . com/content/44/11/1843 (Zugriff: 08/2011).

Alheit, P. (2005): Modernisierungsblockaden in Ostdeuschland? In: Aus Politik und Zeitgeschichte 40, 32-40.

Alheit, P./Bast-Haider, K./Drauschke, P. (2004): Die zögernde Ankunft im Westen. Biographien und Mentalitäten in Ostdeutschland. Frankfurt, New York.

Alheit, P./Dausien, B. (2000): Die biographische Konstruktion der Wirklichkeit. Überlegungen zur Biographizität des Sozialen. In: Hoerning, E. M. (Hg.): Biographische Sozialisation. Stuttgart. 257-283.

Alheit, P./Hoerning, E. M. (1989): Biographie und Erfahrung. Eine Einleitung. In: Alheit, P./Hoerning, E. M. (Hg.): Biographisches Wissen. Beiträge zu einer Theorie lebensgeschichtlicher Erfahrung. Frankfurt, New York. 8-23.

Andresen, S./Brumlik, M./Koch, C. (2010): Vorwort. In: Andresen, S./Brumlik, M./ Koch, C. (Hg.): Das ElternBuch: Wie unsere Kinder geborgen aufwachsen und stark werden. 0-18 Jahre. Weinheim. 10-13.

Assmann, J. (1988): Kollektives Gedächtnis und kulturelle Identität. In: Assmann, J./ Hölscher, T. (Hg.): Kultur und Gedächtnis. Frankfurt am Main. 9-19.

- (2002): Das kulturelle Gedächtnis. München.

Audehm, K. (2007): Erziehung bei Tisch. Zur sozialen Magie eines Familienrituals. Bielefeld.

Baader, M. S. (2006): Vaterschaft im Spannungsverhältnis zwischen alter Ernährerrolle, neuen Erwartungen und Männlichkeitsstereotype. Die Thematisierung von Vaterschaft in aktuellen Print-Medien. In: Bereswill, M./Scheiwe, K./Wolde, A. (Hg.): Vaterschaft im Wandel. Multidisziplinäre Analysen und Perspektiven aus geschlechtertheoretischer Sicht. Weinheim, München. 117-136.

Baader, M. S./Sager, C. (2008): Sozialgeschichte der Generationen. In: Faulstich-Wieland, H./Faulstich, P. (Hg.): Erziehungswissenschaft. Ein Grundkurs. Reinbek bei Hamburg. 291-306.

Baddeley, A. (2009): What is memory. In: Baddeley, A./Eyseneck, M. W./Anderson, M. C. (Hg.): Memory. New York. 1-18.

Bagus, A. (2012): Regionale Entwicklungspfade im ostdeutschen Transformationsprozess: Beispiele aus dem Thüringer Eichsfeld und der Prignitz in Brandenburg. In: Bagus, A. (Hg.): Erfahrung kultureller Räume im Wandel. Transformationsprozesse in ostdeutschen und osteuropäischen Regionen. (SFB 580 Mitteilungen 42). Jena. 67-87. 


\section{Literatur}

Baumert, G. (1952): Jugend der Nachkriegszeit. Lebensverhältnisse und Reaktionsweisen. Darmstadt.

- (1954): Deutsche Familien nach dem Kriege. Darmstadt.

Baumrind, D. (1989): Rearing Competent Children. In: Damon, W. (Hg.): Child Development Today and Tomorrow. San Francisco, London. 349-378.

- (1991): Parenting Styles and Adolescent Development. In: Lern, R. M./Petersen, A. C./Brooks-Gunn, J. (Hg.): Encyclopedia of adolescence. Volume II. London. 746-758.

Beck, U. (1986): Risikogesellschaft. Auf dem Weg in eine andere Moderne. Frankfurt am Main.

- (1990): Freiheit oder Liebe. Vom Ohne-, Mit- und Gegeneinander der Geschlechter innerhalb und außerhalb der Familie. In: Beck, U./Beck-Gernsheim, E. (Hg.): Das ganz normale Chaos der Liebe. Frankfurt am Main. 20-64.

Beck, U./Beck-Gernsheim, E. (1990): Riskante Chancen - Gesellschaftliche Individualisierung und soziale Lebens- und Liebesformen. In: Beck, U./Beck-Gernsheim, E. (Hg.): Das ganz normale Chaos der Liebe. Frankfurt am Main. 7-19.

- (1994): Individualisierung in modernen Gesellschaften - Perspektiven und Kontroversen einer subjektorientierten Soziologie. In: Beck, U./Beck-Gernsheim, E. (Hg.): Riskante Freibeiten. Frankfurt am Main. 10-39.

Beck-Gernsheim, E. (1990a): Alles aus Liebe zum Kind. In: Beck, U./Beck-Gernsheim, E. (Hg.): Das ganz normale Chaos der Liebe. Frankfurt am Main. 135-183.

- (1990b): Von der Liebe zur Beziehung? Veränderungen im Verhältnis von Mann und Frau in der individualisierten Gesellschaft. In: Beck, U./Beck-Gernsheim, E. (Hg.): Das ganz normale Chaos der Liebe. Frankfurt am Main. 65-104.

Behrens, P. (2009): Regionale Identität und katholisches Milieu. Die Eichsfelder Katholiken zwischen Kriegsende und Mauerbau. In: Rürup, M. (Hg.): Praktiken der Differenz. Diasporakulturen in der Zeitgeschichte. Göttingen. 175-196.

Bengston, V. L. (1975): Generation and Family Effects in Value Socialization. In: American Sociological Review 40/3, 358-371.

Benner, D. (2004): Erziehung und Tradierung. Grundprobleme einer innovatorischen Theorie und Praxis der Überlieferung. In: Vierteljahrsschrift für wissenschaftliche Pädagogik 80/2-3, 163-181.

Bennewitz, I./Potratz, R. (1994): Zwangsaussiedlungen an der innerdeutschen Grenze. Analysen und Dokumente. Berlin.

Berdahl, D. (1999): Where the World Ended. Re-Unification and Identity in the German Borderland. Berkeley, Los Angeles, London.

Berg, C. (1991): „Rat geben“. Ein Dilemma pädagogischer Praxis und Wirkungsgeschichte. In: Zeitschrift für Pädagogik 37/5, 709-731.

Bertaux, D./Bertaux-Wiame (1991): „Was du ererbt von deinen Vätern ...“. Transmissionen und soziale Mobilität über fünf Generationen. In: Bios 4, 13-40. 
Bertram, H. (1994): Wertwandel und Werttradierung. In: Bien, W. (Hg.): Eigeninteresse oder Solidarität. Beziehungen in modernen Mehrgenerationenfamilien. Opladen. 113-135.

- (1996): Familienwandel und Generationenbeziehungen. In: Buba, H. P./Schneider, N. F. (Hg.): Familie. Zwischen gesellschaftlicher Prägung und individuellem Design. Opladen. 61-79.

- (2000): Die verborgenen familiären Beziehungen in Deutschland: Die multilokale Mehrgenerationenfamilie. In: Kohli, M./Szydlik, M. (Hg.): Generationen in Familie und Gesellschaft. Opladen. 97-121.

Bien, W. (1994): Leben in Mehrgenerationenkonstellationen: Regel- oder Sonderfall? In: Bien, W. (Hg.): Eigeninteresse oder Solidarität. Beziebungen in modernen Mehrgenerationenfamilien. Opladen. 3-27.

Böhm, W. (2005): Wörterbuch der Pädagogik. Stuttgart.

Böhnisch, L (2005): Familie und Bildung. In: Tippelt, R. (Hg.): Handbuch Bildungsforschung. Wiesbaden. 283-292.

Boehnke, K./Welzel, C. (2006): Wertetransmission und Wertewandel: Eine explorative Drei-Generationen-Studie. In: Zeitschrift für Soziologie der Erziehung und Sozialisation 26/4, 341-360.

Bohnenkamp, B./Manning, T./E.-M., Silies (2009): Argument, Auftrag, Mythos und Konstrukt. Generationelle Erzählungen in interdisziplinärer Perspektive. In: Bohnenkamp, B./Manning, T./Silies, E.-M. (Hg.): Generation als Erzäblung. Neue Perspektiven auf ein kulturelles Deutungsmuster. Göttingen. 9-29.

Bohnsack, R. (1997): „Orientierungsmuster“: Ein Grundbegriff qualitativer Sozialforschung. In: Schmidt, F. (Hg.): Methodische Probleme der empirischen Erziehungswissenschaft. Hohengehren. 49-61.

- (2001): Typenbildung, Generalisierung und komparative Analyse. Grundprinzipien der dokumentarischen Methode. In: Bohnsack, R./Nentwig-Gesemann, I./Nohl, A.-M. (Hg.): Die dokumentarische Methode und ibre Forschungspraxis. Grundlagen qualitativer Sozialforschung. Opladen. 225-252.

- (2007): Rekonstruktive Sozialforschung. Einführung in qualitative Methoden. Opladen, Farmington Hills.

Bohnsack, R./Nentwig-Gesemann, I. (2006): Typenbildung. In: Bohnsack, R./Marotzki, W./Meuser, M. (Hg.): Hauptbegriffe qualitativer Sozialforschung. Opladen, Farmington Hills. 162-166.

Bohnsack, R./Nentwig-Gesemann, I./Nohl, A.-M. und (2001): Einleitung: Die dokumentarische Methode und ihre Forschungspraxis. In: Bohnsack, R./Nentwig- Gesemann, I./Nohl, A.-M. (Hg.): Die dokumentarische Methode und ihre Forschungspraxis. Grundlagen qualitativer Sozialforschung. Opladen. 9-24.

Bois-Reymond, M. du (1975): Kompensatorische Erziehung. In: Speichert, H. (Hg.): Kritisches Lexikon der Erziehungswissenschaft und Bildungspolitik. Reinbek bei Hamburg. 201-203. 


\section{Literatur}

Bois-Reymond, M. du/unter Mitarbeit von Torrance, K. (1994): Die moderne Familie als Verhandlungshaushalt. Eltern-Kind-Beziehungen in West- und Ostdeuschland und in den Niederlanden. In: Bois-Reymond, M. du u.a. (Hg.): Kinderleben. Modernisierung von Kindheit im interkulturellen Vergleich. Opladen. 137-219.

Bourdieu, P. (1987): Die feinen Unterschiede. Kritik der gesellschaftlichen Urteilskraft. Frankfurt am Main.

Bowlby, J. (1972): Mutterliebe und kindliche Entwicklung. München, Basel.

- (1975): Bindung. Eine Analyse der Mutter-Kind-Beziehung. München.

Brake, A. (2003): Familie - Arbeit - Freizeit: Was zählt? Optionen der Lebensqualität in den Vorstellungen junger Erwachsene. Opladen.

- (2006): Das Sichtbare und das Unsichtbare. Bildungsstrategien als Strategien des Habitus. In: Büchner, P./Brake, A. (Hg.): Bildungsort Familie. Transmission von Bildung und Kultur im Alltag von Mehrgenerationenfamilien. Wiesbaden. 81-108.

Brake, A./Büchner, P. (2007): Großeltern in Familien. In: Ecarius, J. (Hg.): Handbuch Familie. Wiesbaden. 199-219.

Brezinka, W. (1990): Grundbegriffe der Erziehungswissenschaft. München.

Brüggen, F. (1996): Tradition. In: Lenzen, D. (Hg.): Band 2. Reinbek bei Hamburg. $1528-1532$.

Brumlik, M. (Hg.) (2007): Vom Missbrauch der Disziplin. Antworten der Wissenschaft auf Bernhard Bueb. Weinheim, Basel.

Bude, H. (2006): Lebenskonstruktion. In: Bohnsack, R./Marotzki, W./Meuser, M. (Hg.): Hauptbegriffe qualitativer Sozialforschung. Opladen, Farmington Hills. 109.

Bueb, B. (2006): Lob der Disziplin. Eine Streitschrift. Berlin.

Büchner, P. (2006): Zur Einführung: Die Familie als Bildungsort. In: Büchner, P./Brake, A. (Hg.): Bildungsort Familie. Transmission von Bildung und Kultur im Alltag von Mehrgenerationenfamilien. Wiesbaden. 11-20.

- (2010): Kindheit und Familie. In: Krüger, H.-H./Grunert, C. (Hg.): Handbuch Kindheits- und Jugendforschung. Wiesbaden. 519-541.

Büchner, P./Brake, A. (2006): Transmission von Bildung und Kultur in Mehrgenerationenfamilien im komplexen Netz gesellschaftlicher Anerkennungsbeziehungen. Resümee und Ausblick. In: Büchner, P./Brake, A. (Hg.): Bildungsort Familie. Transmission von Bildung und Kultur im Alltag von Mehrgenerationenfamilien. Wiesbaden. 255-277.

Büchner, P./Brake, A. (Hg.) (2006): Bildungsort Familie. Transmission von Bildung und Kultur im Alltag von Mehrgenerationenfamilien. Wiesbaden.

Büchner, P./Fuhs, B. (1996): Der Lebensort Familie. Alltagsprobleme und Beziehungsmuster. In: Büchner, P./Fuhs, B./Krüger, H.-H. (Hg.): Vom Teddybär zum ersten Kuß. Wege aus der Kindheit in Ost- und Westdeutschland. Opladen. 159-200.

Bürgel, T. (2006): Ausprägungen einer „prekären Jugendgeneration“ im Osten Deutschlands. Zum Generationsselbstverständnis der 20-25jährigen Deutschen im Ost-WestVergleich. In: Bürgel, T. (Hg.): Generationen in den Umbrüchen postkommunistischer Gesellschaften. Erfahrungstransfers und Differenzen vor dem Generationenwechsel in Russland und Ostdeutschland. (SFB 580 Mitteilungen 20). Jena. 168-181. 
Busch, F. W. (1972): Familienerziehung in der sozialistischen Pädagogik der DDR. Düsseldorf.

Chvojka, E. (2003): Geschichte der Großelternrollen vom 16. bis zum 20. Jahrhundert. Wien, Köln, Weimar.

Claessens, D. (1972): Famile und Wertsystem. Eine Studie zur „Zweiten, sozio-kulturellen Geburt" des Menschen und der Belastbarkeit der „Kernfamilie“. Berlin.

Claessens, D./Menne, F. W. (1970): Zur Dynamik der bürgerlichen Familie und ihrer möglichen Alternativen. In: Lüschen, G./Lupri, E. (Hg.): Soziologie der Familie. Kölner Zeitschrift für Soziologie und Sozialpsychologie. Sonderheft 14. 169-198.

Duncker, C. (1998): Dimensionen des Wertewandels in Deutschland. Eine Analyse anhand ausgewählter Zeitreihen. Frankfurt am Main.

Ecarius, J. (1998): Generationsbeziehungen und Generationenverhältnisse. Analyse zur Entwicklung des Generationenbegriffs. In: Ecarius, J. (Hg.): Was will die jüngere mit der älteren Generation? Generationenbeziehungen in der Erziehungswissenschaft. Opladen. 41-66.

- (2001): Familie zwischen Tradierung und Wandel. Generationsbeziehungen und familiale Aufträge in drei Generationen. In: Allmendinger, J. (Hg.): Gute Gesellschaft? Verhandlungen des 30. Kongresses der Deutschen Gesellschaft für Soziologie. Teil A. Opladen. 558-572.

- (2002): Familienerziehung im historischen Wandel. Eine qualitative Studie über Erziehung und Erziehungserfahrungen von drei Generationen. Opladen.

- (2003): Biografie, Lernen und Familienthemen in Generationsbeziehungen. In: Zeitschrift für Pädagogik 49/4, 534-549.

- (2007): Familienerziehung. In: Ecarius, J. (Hg.): Handbuch Familie. Wiesbaden. 137-156.

- (2008): Generation, Erziehung und Bildung. Eine Einführung. Stuttgart.

Ehmer, J. (2003): Generationen in der historischen Forschung: Konzepte und Praktiken. In: Künemund, H./Szydlik, M. (Hg.): Generationen. Multidisziplinäre Perspektiven. Wiesbaden. 59-80.

Engelbert, A. u. a. (2000): Postmoderne Familienkindheit? Anforderungen, Risiken, Chancen. In: Herlth, A. u.a. (Hg.): Spannungsfeld Familienkindheit. Neue Anforderungen, Risiken und Chancen. Opladen. 7-22.

Engstler, H./Menning, S. (2003): Die Familie im Spiegel der amtlichen Statistik. Lebensformen, Familienstrukturen, wirtschaftliche Situation der Familien und familiendemographische Entwicklung in Deutschland. Bonn.

Erll, A. (2005): Kollektives Gedächtnis und Erinnerungskulturen. Stuttgart, Weimar.

Fend, H. (1969): Sozialisierung und Erziehung. Weinheim, Berlin, Basel.

- (1988): Sozialgeschichte des Aufwachsens. Bedingungen des Aufwachsens und Jugendgestalten im zwanzigsten Jahrhundert. Frankfurt am Main.

- (2009): Was die Eltern ihren Kindern mitgeben - Generationen aus Sicht der Erziehungswissenschaft. In: Künemund, H./Szydlik, M. (Hg.): Generationen. Multidisziplinäre Perspektiven. Wiesbaden. 81-103. 


\section{Literatur}

Flick, U. (2002): Qualitative Sozialforschung. Eine Einführung. Reinbek bei Hamburg. Friebertshäuser, B./Matzner, M./Rothmüller, N. (2007): Famile: Mütter und Väter. In: Ecarius, J. (Hg.): Handbuch Familie. Wiesbaden. 179-198.

Friederichs, J. (1968): Vater- und Mutterbild. In: Pfeil, E. (Hg.): Die 23jährigen. Eine Generationenuntersuchung am Geburtenjabrgang 1941. Tübingen. 129-137.

Fritzsche, Y. (2000): Moderne Orientierungsmuster: Inflation am „Wertehimmel“. In: Deutsche Shell (Hg.): Jugend 2000. Band 1. Opladen. 93-156.

Fuchs, W. (1984): Biographische Forschung. Opladen.

Fuhs, B. (2007): Zur Geschichte der Familie. In: Ecarius, J. (Hg.): Handbuch Familie. Wiesbaden. 17-35.

Gaschke, S. (2001): Die Erziehungskatastrophe. Kinder brauchen starke Eltern. Stuttgart, München.

Gass-Bolm, T. (2005): Das Gymnasium 1945-1980. Göttingen.

Gebhardt, M. (2009a): Die Angst vor dem kindlichen Tyrannen. Eine Geschichte der Erziehung im 20. Jahrhundert. München.

- (2009b): Die Last der „Lebensbemeisterung“: Ein Sozialisationsmuster des 20. Jahrhunderts und seine lange Wirkung. In: Seegers, L./Reulecke, J. (Hg.): Die „Generation der Kriegskinder". Historische Hintergründe und Deutungen. Gießen. 33-57.

Gensicke, T. (1992a): Mentalitätsentwicklungen im Osten Deutschlands seit den 70er Jahren. Vorstellung und Erläuterung von Ergebnissen einiger empirischer Untersuchungen in der DDR und in den neuen Bundesländern von 1977 bis 1991. Speyer.

- (1992b): Werte und Wertewandel im Osten Deutschlands. In: Klages, H./Hippler, H.-J./Herbert, W. (Hg.): Werte und Wandel. Ergebnisse und Methoden einer Forschungstradition. Frankfurt am Main. 672-694.

- (1995): Deutschland im Wandel. Sozialer Wandel und Wertewandel in Deutschland vor und nach der Wiedervereinigung. Speyer.

- (2000): Deutschland im Übergang. Lebensgefühl, Wertorientierungen, Bürgerengagement. Speyer.

- (2002a): Deutschland am Ausgang der neunziger Jahre: Lebensgefühl und Werte. In: Klages, H./Gensicke, T. (Hg.): Wertewandel und bürgerschaftliches Engagement an der Schwelle zum 21. Jabrbundert. Speyer. 21-51.

- (2002b): Individualität und Sicherheit in neuer Synthese? Wertorientierungen und gesellschafltiche Aktivität. In: Deutsche Shell (Hg.): Jugend 2002. Zrwischen pragmatischem Idealismus und robustem Materialismus. Frankfurt am Main. 139-212.

- (2006): Jugend und Religiosität. In: Shell Deutschland Holding (Hg.): Jugend 2006. Eine pragmatische Generation unter Druck. Frankfurt am Main. 203-239.

Gerhards, J./Hölscher, M. (2003): Kulturelle Unterschiede zwischen Mitglieds- und Beitrittsländern der EU. Das Beispiel Familien- und Gleichberechtigungsvorstellungen. In: Zeitschrift für Soziologie 32/3, 206-225.

Giddens, A. (1993): Traditionen in der post-traditionalen Gesellschaft. In: Soziale Welt 44/4, 445-485. 
Göppel, R. (2010): Pädagogik und Zeitgeist. Erziehungsmentalitäten und Erziehungsdiskurse im Wandel. Stuttgart.

Gohlke, H./Büchner, P. (2006): Das familiale Bildungserbe als Produkt gelebter Generationenbeziehungen. Über das Spannungsverhältnis zwischen Weitergabe und Übernahme von Bildung und Kultur. In: Büchner, P./Brake, A. (Hg.): Bildungsort Familie. Transmission von Bildung und Kultur im Alltag von Mehrgenerationenfamilien. Wiesbaden. 143-178.

Gordon, T. (1972): Familienkonferenz. Die Lösung von Konflikten zwischen Eltern und Kind. Hamburg.

Grob, A./Weisheit, W./Gomez, V. (2009): Similarity of Life Goals in the Family: A Three-Generation Study. In: Schönpflug, U. (Hg.): Cultural Transmission. Psychological, Developmental, Social, and Methodological Aspects. Cambridge u.a. 338-369.

Groß, J./Wößner, G. (2008): Kultur, Erziehung, Tradierung - Die Vermittlung kulturell geprägter Erziehungsinhalte. In: Recht der Jugend und des Bildungswesens 56/2, 135-152.

Gukenbiehl, H. L. (1995): Familie. In: Schäfers, B. (Hg.): Grundbegriffe der Soziologie. Opladen. 69-72.

Gysi, J. (1989): Familienleben in der DDR. Zum Alltag von Familien mit Kindern. Berlin.

- (1990): Die Zukunft von Familie und Ehe. Familienpolitik und Familienforschung in der DDR. In: Burkart, G. (Hg.): Sozialisation im Sozialismus. Lebensbedingungen in der DDR im Umbruch. 1. Beibeft der Zeitschrift für Sozialisationsforschung und Erziebungssoziologie. Weinheim. 33-41.

Hämmerle, C. (2010): Vaterlosigkeit - ein fragwürdiges Konzept der neueren Geschichtswissenschaft? Grundsätzliche Überlegungen mit Blick auf das 19. und frühe 20. Jahrhundert. In: Thomä, D. (Hg.): Vaterlosigkeit. Geschichte und Gegenwart einer fixen Idee. Frankfurt am Main. 124-141.

Halbwachs, M. (1985): Das Gedächtnis und seine sozialen Bedingungen. Frankfurt am Main.

- (1991): Das kollektive Gedächtnis. Frankfurt am Main.

Haumann, W. (2006): Generationen-Barometer 2006. Eine Studie des Instituts für Demoskopie Allensbach/ hg. v. Forum Familie stark machen. Freiburg, München.

Helsper, W. u. a. (2009): Jugend zwischen Familie und Schule. Eine Studie zu pädagogischen Generationsbeziehungen. Wiesbaden.

Hentig, H. v. (2009): Das Ethos der Erziehung. Was ist in ihr elementar? In: Zeitschrift für Pädagogik 55/4, 509-527.

Herlyn, I./Kistner, A. (1997): Großmuttersein heute. In: Zeitschrift für Franenforschung $15 / 3,5-21$.

Herrmann, U. (1978): „Mut zur Erziehung“. Anmerkungen zu einer proklamierten Tendenzwende in der Erziehungs- und Bildungspolitik. In: Zeitschrift für Pädagogik 24/2, 221-234. 


\section{Literatur}

Hille, B. (1985): Familie und Sozialisation in der DDR. Opladen.

Hillmann, K.-H. (2001): Zur Wertewandelsforschung: Einführung, Übersicht und Ausblick. In: Oesterdiekhoff, G. W./Jegelka, N. (Hg.): Werte und Wertewandel in westlichen Gesellschaften. Resultate und Perspektiven der Sozialwissenschaften. Opladen. 15-39.

Hirst, W./Manier, D. (2002): The Diverse Forms of Collective Memory. In: Echterhoff, G./Saar, M. (Hg.): Kontexte und Kulturen des Erinnerns. Maurice Halbwachs und das Paradigma des kollektiven Gedächtnisses. Konstanz. 37-58.

Höffer-Mehlmer, M. (2003): Elternratgeber. Zur Geschichte eines Genres. Baltmannsweiler.

- (2007): Sozialisation und Erziehungsratschlag. Elternratgeber nach 1945. In: Gebhardt, M./Wischermann, C. (Hg.): Familiensozialisation seit 1933 - Verhandlungen über Kontinuität. Stuttgart. 71-85.

Höpflinger, F./Hummel, C./Hugentobler, V. (2006): Enkelkinder und ihre Grosseltern. Intergenerationelle Beziehungen im Wandel. Zürich.

Hofer, M. (2002): Familienbeziehungen in der Entwicklung. In: Hofer, M./Wild, E./ Noack, P. (Hg.): Lehrbuch Familienbeziehungen: Eltern und Kinder in der Entwicklung. Göttingen u. a. 4-27.

Hofer, M./Pikowsky, B. (2002): Familien mit Jugendlichen. In: Hofer, M./Wild, E./ Noack, P. (Hg.): Lebrbuch Familienbeziehungen. Eltern und Kinder in der Entwicklung. Göttingen u.a. 241-264.

Hollstein, B. (2005): Reziprozität in familialen Generationenbeziehungen. In: Adloff, F./Mau, S. (Hg.): Vom Geben und Nehmen. Zur Soziologie der Reziprozität. Frankfurt am Main. 187-209.

Hopfner, J. (2001): Wie populär ist pädagogisches Wissen? Zum Verhältnis von Ratgebern und Wissenschaft. In: Neue Sammlung, Vierteljabres-Zeitschrift für Erziebung und Gesellschaft 44/1, 73-88.

Hurrelmann, K. u.a. (2006): Eine pragmatische Generation unter Druck - Einführung in die Shell Jugendstudie 2006. In: Shell Deutschland Holding (Hg.): 15. Shell Jugendstudie. Jugend 2006. Eine pragmatische Generation unter Druck. Frankfurt am Main. 31-48.

Inglehart, R. (1977): The Silent Revolution. Changing Values and Political Styles Among Western Publics. Princeton, New Jersey.

- (1979): Wertwandel in den westlichen Gesellschaften: Politische Konsequenzen von materialistischen und postmaterialistischen Prioritäten. In: Klages, H./Kmieciak, P. (Hg.): Wertwandel und gesellschaftlicher Wandel. Frankfurt am Main, New York. 279-316.

- (1989): Kultureller Umbruch. Wertwandel in der westlichen Welt. Frankfurt am Main, New York.

- (1998): Modernisierung und Postmodernisierung. Kultureller, wirtschaflicher und politischer Wandel in 43 Gesellschaften. Frankfurt am Main, New York. 
Jansa, A. (1999): Pädagogik, Politik, Ästhetik. Paradigmenwechsel um '68. Frankfurt am Main.

- (2000): Die Pädagogik der Studentenbewegung in ihrer Auswirkung auf das Generationenverhältnis und den gesellschaftlichen Umgang mit Kindern. In: Lingelbach, K. C. and Zimmer, H. (Red.): Jahrbuch für Pädagogik: Das Jahrhundert des Kindes? Frankfurt am Main. 223-246.

Jureit, U. (2006): Generationenforschung. Göttingen.

- (2009): Generationen-Gedächtnis. Überlegungen zu einem Konzept kommunikativer Vergemeinschaftungen. In: Seegers, L./Reulecke, J. (Hg.): Die „Generation der Kriegskinder". Historische Hintergründe und Deutungen. Gießen. 125-137.

Karstein, U. (2009): Familiale Einheit und generationelle Differenz. Zur kommunikativen Konstruktion historischer Generationen am Beispiel ostdeutscher Familien. In: Bohnenkamp, B./Manning, T./Silies, E.-M. (Hg.): Generation als Erzäblung. Neue Perspektiven auf ein kulturelles Deutungsmuster. Göttingen. 53-71.

Kaufmann, F.-X. (1988): Familie und Modernität. In: Lüscher, K./Schultheis, F./Wehrspaun, M. (Hg.): Die „postmoderne Familie“. Familiale Strategien und Familienpolitik in einer Übergangszeit. Konstanz. 391-415.

Kellerhals, J./Ferreira, C./Perrenoud, D. (2002): Kinship Cultures and Identity Transmissions. In: Current Sociology 50/2, 213-228.

Keppler, A. (1992): Tischgespräche. Frankfurt am Main.

Klages, H. (1984): Wertorientierungen im Wandel. Rückblick, Gegenwartsanalyse, Prognosen. Frankfurt am Main, New York.

- (1992): Die gegenwärtige Situation der Wert- und Wertwandelsforschung - Probleme und Perspektiven. In: Klages, H./Hippler/H.-J. Herbert, W. (Hg.): Werte und Wandel. Ergebnisse und Methoden einer Forschungstradition. Frankfurt am Main. 5-39.

- (2001a): Brauchen wir eine Rückkehr zu traditionellen Werten? In: Aus Politik und Zeitgeschichte B29, 7-14.

- (2001b): Werte und Wertewandel. In: Schäfers, B./Zapf, W. (Hg.): Handwörterbuch zur Gesellschaft Deutschlands. Bonn. 726-738.

- (2002): Zerfällt das Volk? Von den Schwierigkeiten der modernen Gesellschaft mit Gemeinschaft und Demokratie. In: Klages, H./Gensicke, T. (Hg.): Wertewandel und bürgerschaftliches Engagement an der Schwelle zum 21. Jabrbundert. Speyer. 1-20.

Klages, H./mit Gensicke, T. (1993): Wertewandel in den neuen Bundesländern. Fakten und Deutungsmodelle. In: Klages, H.: Traditionsbruch als Herausforderung. Perspektiven der Wertewandelsgesellschaft. Frankfurt am Main, New York. 215-238.

Klenke, D. (2003): Der Eichsfelder Katholizismus zwischen SED-Diktatur und westlicher Demokratie. Grossbodungen.

Kluckhohn, C. (1952): Values and Value-Orientations in the Theory of Action. In: Parsons, T./Shils, E. A. (Hg.): Toward a General Theory of Action. Cambridge, Massachusetts. 388-433. 


\section{Literatur}

Kmieciak, P. (1976): Wertstrukturen und Wertwandel in der Bundesrepublik Deutschland. Göttingen.

Knafo, A./Schwartz, S. H. (2009): Accounting for Parent-Child Value Congruence: Theoretical Considerations and Empirical Evidence. In: Schönpflug, U. (Hg.): Cultural Transmission. Psychological, Developmental, Social, and Methodological Aspects. Cambridge u.a. 240-268.

König, R. (1974/1955): Familie und Autorität: Der deutsche Vater im Jahre 1955. In: König, R. (Hg.): Materialien zur Soziologie der Familie. Köln. 214-230.

Kohli, M. (2007): Von der Gesellschaftsgeschichte zur Familie. Was leistet das Konzept der Generationen? In: Lettke, F./Lange, A. (Hg.): Generationen und Familie. Analysen - Konzepte - gesellschaftliche Spannungsfelder. Frankfurt am Main. 47-68.

Kohli, M./Szydlik, M. (2000): Einleitung. In: Kohli, M./Szydlik, M. (Hg.): Generationen in Familie und Gesellschaft. Opladen. 7-18.

Koller, H.-C. (2008): Grundbegriffe, Theorien und Methoden der Erziehungswissenschaft. Eine Einführung. Stuttgart.

Korte, M. (2001): Engramm. In: Pethes, N./Ruchatz, J. (Hg.): Gedächtnis und Erinnerung. Ein interdisziplinäres Lexikon. Reinbek bei Hamburg. 142.

Kortendiek, B. (2004): Familie: Mutterschaft und Vaterschaft zwischen Traditionalisierung und Modernisierung. In: Becker, R./Kortendiek, B. (Hg.): Handbuch Frauenund Geschlechterforschung. Theorie, Methoden, Empirie. Wiesbaden. 384-401.

Koselleck, R. (1989): Vergangene Zukunft. Zur Semantik geschichtlicher Zeiten. Frankfurt am Main.

Krah, K./Büchner, P. (2006): Habitusgenese und Religiosität in Mehrgenerationenfamilien. Zum Stellenwert religiöser Praxisformen im Rahmen der intergenerationalen Weitergabe und Aneignung von Bildung und Kultur. In: Büchner, P./Brake, A. (Hg.): Bildungsort Familie. Transmission von Bildung und Kultur im Alltag von Mehrgenerationenfamilien. Wiesbaden. 109-141.

Kraul, M. (2003a): Sisyphos oder „Was will denn eigentlich die ältere Generation mit der jüngeren?". Generationen und Tradierung in der Erziehung. In: Beillerot, J./Wulff, C. (Hg.): Erziehungswissenschaftliche Zeitdiagnosen: Deutschland und Frankreich. Münster u.a. 31-43.

- (2003b): „Was will denn eigentlich die ältere Generation mit der jüngeren?“. Erziehung als Tradierung und Innovation. In: Neue Sammlung, Vierteljabres-Zeitschrift für Erziehung und Gesellschaft 44/3, 283-297.

Kraul, M./Radicke, C. (2012): Familiale Erziehung zwischen Tradierung, intergenerationeller Dynamik und Aneignung. Dimensionen religiöser Praxen. In: Owetschkin, D. (Hg.): Tradierungsprozesse im Wandel der Moderne. Religion und Familie im Spannungsfeld von Konfessionalität und Pluralisierung. Essen. 137-161.

Kreher, S. (2002): Continuity and change over the generations Trials and tribulations of an East German family. In: History of the family 7, 183-205.

Kron, F. W. (2001): Grundwissen Pädagogik. München, Basel. 
Krüger, T. (2001): Elaboration. In: Pethes, N./Ruchatz, J. (Hg.): Gedächtnis und Erinnerung. Ein interdisziplinäres Lexikon. Reinbek bei Hamburg. 137/138.

Lamnek, S. (2010): Qualitative Sozialforschung. Weinheim, Basel.

Lange, A./Lettke, F. (2007): Schrumpfung, Erweiterung, Diversität. Konzepte zur Analyse von Familien und Generationen. In: Lettke, F./Lange, A. (Hg.): Generationen und Familie. Frankfurt am Main. 14-43.

Langness, A./Leven, I./Hurrelmann, K. (2006): Jugendliche Lebenswelten. Familie, Schule, Freizeit. In: Shell Deutschland Holding (Hg.): 15. Shell Jugendstudie. Jugend 2006. Eine pragmatische Generation unter Druck. Frankfurt am Main. 49-102.

Lebegern, R. (2004): Zur Geschichte der Sperranlagen an der innerdeutschen Grenze 1945 - 1990. Erfurt.

Lehmann, A. (1991): Der Schicksalsvergleich - Eine Gattung des Erzählens und eine Methode des Erinnerns. In: Bönisch-Brednich, B./Brednich, R. W./Gerndt, H. (Hg.): Erinnern und Vergessen. Vorträge des 27. Deutschen Volkskundekongresses Göttingen 1989. Göttingen. 197-207.

Lehmann, R. (2009): Transformation, Generation, Erinnerung: Ostdeutsche Perspektiven auf Vergangenheit und Gegenwart vor dem Hintergrund gesellschaftlicher Transformation. In: Balla, B./Sterbling, A. (Hg.): Europäische Entwicklungsdynamik. Hamburg. 127-143.

Lehner, F. (1979): Die „Stille Revolution“: Zur Theorie und Realität des Wertwandels in hochindustrialisierten Gesellschaften. In: Klages, H./Kmieciak, P. (Hg.): Wertwandel und gesellschaftlicher Wandel. Frankfurt, New York. 317-327.

Lenz, K./Böhnisch, L. (1997): Zugänge zu Familien. Ein Grundlagentext. In: Böhnisch, L./Lenz, K. (Hg.): Familien. Eine interdisziplinäre Einführung. Weinheim, München. 9-63.

Lettke, F./Lange, A. (2007): Vorwort. In: Lettke, F./Lange, A. (Hg.): Generationen und Familie. Analysen - Konzepte - gesellschaftliche Spannungsfelder. Frankfurt am Main. 9-13.

Leven, I./Quenzel, G./Hurrelmann, K. (2010): Familie, Schule, Freizeit: Kontinuitäten im Wandel. In: Shell Deutschland Holding (Hg.): Jugend 2010. Eine pragmatische Generation behauptet sich. Bonn. 53-128.

Lewin, K./Lippitt, R./White, R. K. (1939): Patterns of aggressive behavior in experimentally created 'social climates'. In: The Journal of Social Psychology; Political, Racial and differential Psychology 10/2, 271-299.

Liebau, E. (1997): Generation - ein aktuelles Problem? In: Liebau, E. (Hg.): Das Generationenverhältnis. Über das Zusammenleben in Familie und Gesellschaft. Weinheim, München. 15-37.

Liebenwein, S. (2008): Erziehung und soziale Milieus. Elterliche Erziehungsstile in milieuspezifischer Differenzierung. Wiesbaden.

Liegle, L./Lüscher, K. (2004): Das Konzept des „Generationenlernens“. In: Zeitschrift für Pädagogik 50/1, 38-55. 


\section{Literatur}

Liegle, L./Lüscher, K. (2008): Generative Sozialisation. In: http: //www . kurtluescher . de/downloads//KL_LL_Generative_Sozialisation.pdf (Zugriff: 12/2011).

Lüscher, K. (1993): Generationenbeziehungen. Neue Zugänge zu einem alten Thema. In: Lüscher, K./Schultheis, F. (Hg.): Generationenbeziehungen in „postmodernen" Gesellschaften. Konstanz. 17-47.

- (2005): Ambivalenz - Eine Annäherung an das Problem der Generationen. Die Aktualität der Generationenfrage. In: Jureit, U./Wildt, M. (Hg.): Generationen. Zur Relevanz eines wissenschaftlichen Grundbegriffs. Hamburg. 53-78.

- (2008): Großelternschaft - eine soziologische Annäherung. In: Klosinski, G. (Hg.): Großeltern heute - Hilfe oder Hemmnis? Analysen und Perspektiven für die pädagogischpsychologische Praxis. Tübingen. 33-58.

Lüscher, K./Liegle, L. (2003): Generationenbeziehungen in Familie und Gesellschaft. Konstanz.

Lukesch, H. (1996): Leitbilder in der Familienerziehung. In: Vaskovics, L. A./Lipinski, H. (Hg.): Familiale Lebenswelten und Bildungsarbeit (1). Opladen. 153-184.

Mannheim, K. (1964/1928): Das Problem der Generationen. In: Wolff, K. H. (Hg.): Karl Mannheim. Wissenssoziologie. Auswabl aus dem Werk. Neuwied, Berlin. 509-565.

Markowitsch, H. J. (2009): Das Gedächtnis. Entwicklung, Funktionen, Störungen. München.

Marotzki, W. (2000): Qualitative Biographieforschung. In: Flick, U./Kardorff, E. v./ Steinke, I. (Hg.): Qualitative Forschung. Ein Handbuch. Reinbek bei Hamburg. 175-186.

Matthes, J. (1985): Karl Mannheims „Das Problem der Generationen“ neu gelesen. Generationen- „Gruppen“ oder „gesellschafltiche Regelung von Zeitlichkeit“? In: Zeitschrift für Soziologie 14/5, 363-372.

Matzner, M. (2004): Vaterschaft aus der Sicht von Vätern. Wiesbaden.

Meulemann, H. (1996a): Wandel der Erziehungswerte für Familie und Schule in Westund Ostdeuschland. In: Schacht, K. (Hg.): Bildung und Erzieung nach 1945. Wiesbaden. 59-79.

- (1996b): Werte und Wertewandel. Zur Identität einer geteilten und wieder vereinten Nation. Weinheim, München.

- (2002): Werte und Wertwandel im vereinten Deutschland. In: Aus Politik und Zeitgeschichte $\mathrm{B} 37 / 38,13-22$.

Meuser, M. (2006): Interpretatives Paradigma. In: Bohnsack, R./Marotzki, W./Meuser, M. (Hg.): Hauptbegriffe qualitativer Sozialforschung. Opladen, Farmington Hills. 92-94.

Mitterauer, M. (1997): „Das moderne Kind hat zwei Kinderzimmer und acht Großeltern“. Die Entwicklung in Europa. In: Mitterauer, M./Ortmayr, N. (Hg.): Familie im 20. Jahrbundert. Traditionen, Probleme, Perspektiven. Frankfurt am Main. 13-51.

Mollenhauer, K. (1976): Theorien zum Erziehungsprozeß. München.

- (1996): Familie - Familienerziehung. In: Lenzen, D. (Hg.): Pädagogische Grundbegriffe. Band 1. Reinbek bei Hamburg. 603-613. 
Moller, S. (2010): Das kollektive Gedächtnis. In: Gudehus, C./Eichenberg, A./Welzer, H. (Hg.): Gedächtnis und Erinnerung. Ein interdisziplinäres Handbuch. Stuttgart. 85-92.

Müller, H.-R./Borg, K./Falkenreck, D. (2010): Das Familienfoto. Annäherungen an den verborgenen Bildungssinn familialer Selbstpräsentationen. In: Müller, H.-R./Ecarius, J./Herzberg, H. (Hg.): Familie, Generation, Bildung. Beiträge zur Erkundung eines informellen Lernfeldes. Opladen, Farmington Hills. 53-67.

Müller-Schneider, T. (2001): Wertewandel, Erlebnisorientierung und Lebensstile. Eine gesellschaftsgeschichtliche und modernisierungstheoretische Interpretation. In: Oesterdiekhoff, G. W./Jegelka, N. (Hg.): Werte und Wertewandel in westlichen Gesellschaften. Resultate und Perspektiven der Sozialwissenschaften. Opladen. 91-106.

Nave-Herz, R. (2000): Wandel der Familie: eine familiensoziologische Perspektive. In: Schneewind, K. A. (Hg.): Familienspsychologie im Aufwind. Brückenschläge zwischen Forschung und Praxis. Göttingen u.a. 19-31.

- (2007): Familie heute. Wandel der Familienstrukturen und Folgen für die Erziehung. Darmstadt.

Nelson, K. (2006): Über Erinnerungen reden: Ein soziokultureller Zugang zur Entwicklung des autobiographischen Gedächtnisses. In: Welzer, H./Markowitsch, H. J. (Hg.): Warum Menschen sich erinnern können. Fortschritte der interdisziplinären Gedächtnisforschung. Stuttgart. 87-94.

Nentwig-Gesemann, I. (2001): Die Typenbildung der dokumentarischen Methode. In: Bohnsack, R./Nentwig-Gesemann, I./Nohl, A.-M. (Hg.): Die dokumentarische Methode und ibre Forschungspraxis. Grundlagen qualitativer Sozialforschung. Opladen. 275-300.

Neugarten, B. L./Weinstein, K. K. (1964): The Changing American Grandparent. In: Journal of Marriage and Family 26/2, 199-204.

Noelle-Neumann, E./Petersen, T. (2001): Zeitenwende. Der Wertewandel 30 Jahre später. In: Aus Politik und Zeitgeschichte B29, 15-22.

Nohl, A.-M. (2006): Interview und dokumentarische Methode. Anleitungen für die Forschungspraxis. Wiesbaden.

Nothelle-Wildfeuer, U. (2008): Die Sozialprinzipien der Katholischen Soziallehre. In: Rauscher, A. (Hg.): Handbuch der katholischen Soziallehre. Berlin. 143-163.

Oesterdiekhoff, G. W. (2001): Soziale Strukturen, sozialer Wandel und Wertewandel. Das Theoriemodell von Ronald Inglehart in der Diskussion seiner Grundlagen. In: Oesterdiekhoff, G. W./Jegelka, N. (Hg.): Werte und Wertewandel in westlichen Gesellschaften. Resultate und Perspektiven der Sozialwissenschaften. Opladen. 41-54.

Oesterdiekhoff, G. W./Jegelka, N. (2001): Einführung. In: Oesterdiekhoff, G. W./Jegel$\mathrm{ka}, \mathrm{N}$. (Hg.): Werte und Wertewandel in westlichen Gesellschaften. Resultate und Perspektiven der Sozialwissenschaften. Opladen. 7-14.

Ollick, J. K. (1999): Collective Memory: The Two Cultures. In: Sociological Theory $17 / 3,333-348$. 
Olson, D. H. u.a. (1989): Families. What makes them work. Newbury Park, London, New Delhi.

Ortlepp, W. (2002): Gedächtnis und Generation. Überlegungen zu Halbwachs' Gedächtnisbegriff und Mannheims Generationenbegriff. In: Kraul, M./Marotzki, W. (Hg.): Biographische Arbeit. Perspektiven erziehungswissenschaftlicher Biographieforschung. Opladen. 308-325.

Oswald, H. (1989): Intergenerative Beziehungen (Konflikte) in der Familie. In: Markefka, M./Nave-Herz, R. (Hg.): Handbuch der Familien-und Jugendforschung. Band 2: Jugendforschung. Neuwied, Frankfurt am Main. 367-381.

[o.V.] (1978a): Mut zur Erziehung. Beiträge zu einem Forum am 9./10. Januar 1978 im Wissenschaftszentrum Bonn-Bad Godesberg. Stuttgart.

- (1978b): Tübinger Erklärung zu den Thesen des Bonner Forums „Mut zur Erziehung“. In: Zeitschrift für Pädagogik 24/2, 235-240.

- (2006): Zusammenfassung. In: Shell Deutschland Holding (Hg.): 15. Shell Jugendstudie. Jugend 2006. Eine pragmatische Generation unter Druck. Frankfurt am Main. 15-30.

Palmowski, J. (2009): Inventing a Socialist Nation. Heimat and the Politics of Everyday Life in the GDR, 1945-1990. Cambridge u.a.

Papastefanou, C./Hofer, M. (2002): Familienbildung und elterliche Kompetenzen. In: Hofer, M./Wild, E./Noack, P. (Hg.): Lehrbuch Familienbeziehungen. Eltern und Kinder in der Entwicklung. Göttingen, u.a. 168-191.

Peuckert, R. (2007): Zur aktuellen Lage der Familie. In: Ecarius, J. (Hg.): Handbuch Familie. Wiesbaden. 36-56.

- (2008): Familienformen im sozialen Wandel. Wiesbaden.

Pfeifer, W. (1993): Etymologisches Wörterbuch des Deutschen. M-Z. Berlin.

Pfeil, E. (1968): Familiengründung und Vorstellungen vom Familienleben. In: Pfeil, E. (Hg.): Die 23jäbrigen. Eine Generationenuntersuchung am Geburtenjabrgang 1941. Tübingen. 61-99.

Piefke, M./Markowitsch, H. J. (2010): Neuroanatomische und neurofunktionelle Grundlagen von Gedächtnis. In: Gudehus, C./Eichenberg, A./Welzer, H. (Hg.): Gedächtnis und Erinnerung. Ein interdisziplinäres Handbuch. Stuttgart. 11-21.

Plötz, K. (2006): „Heimkehrer“, die „natürliche Ordnung“ und „vollständige Familien“. Väter in der bundesdeutschen Nachkriegszeit. In: Bereswill, M./Scheiwe, K./Wolde, A. (Hg.): Vaterschaft im Wandel. Multidisziplinäre Analysen und Perspektiven aus geschlechtertheoretischer Sicht. Weinheim, München. 57-74.

Pohl, R. (2007): Das autobiographische Gedächtnis. Die Psychologie unserer Lebensgeschichte. Stuttgart.

- (2010): Was ist Gedächtnis/Erinnerung? In: Gudehus, C./Eichenberg, A./Welzer, H. (Hg.): Gedächtnis und Erinnerung. Ein interdisziplinäres Handbuch. Stuttgart. 75-84.

Pollmer, K./Hurrelmann, K. (1992): Framilientraditionen und Erziehungsstile in Ostund Westdeutschland im Vergleich. In: Kind, Jugend, Gesellschaft. Zeitschrift für Kinderund Jugendschutz 37/1, 2-7. 
Pottmeyer, H. J. (1996): Modernisierung in der katholischen Kirche am Beispiel der Kirchenkonzeption des I. und II. Vatikanischen Konzils. In: Kaufmann, F.-X./Zingerle, A. (Hg.): Vatikanum II und Modernisierung. Historische, theologische und soziologische Perspektiven. Paderborn u.a. 131-146.

Qvortrup, J. (1993): Die soziale Definition von Kindheit. In: Markefka, M./Nauck, B. (Hg.): Handbuch der Kindheitsforschung. Neuwied, Kriftel, Berlin. 109-124.

Radebold, H./Bohleber, W./Zinnecker, J. (Hg.) (2009): Transgenerationale Weitergabe kriegsbelasteter Kindheiten. Interdisziplinäre Studien zur Nachhaltigkeit historischer Erfahrungen über vier Generationen. Weinheim, München.

Radicke, C. (2013): Familiale Tradierungsprozesse. Die Gegenwart von Vergangenheit und Zukunft. In: Gerland, K./Möckel, B./Ristau, D. (Hg.): Generation und Erwartung. Konstruktionen zwischen Vergangenheit und Zukunft. Göttingen. 222-243.

Rehberg, K.-S. (2006): Ost - West. In: Lessenich, S./Nullmeier, F. (Hg.): Deutschland. Eine gespaltene Gesellschaft. Bonn. 209-233.

Reuband, K.-H. (1988): Von äußerer Verhaltenskonformität zu selbständigen Handeln. Über die Bedeutung kultureller und struktureller Einflüsse für den Wandel in den Erziehungszielen und Sozialisationsinhalten. In: Luthe, H. O./Meulemann, H. (Hg.): Wertewandel - Faktum oder Fiktion? Bestandsaufnahmen und Diagnosen aus kultursoziologischer Sicht. Frankfurt am Main, New York. 73-97.

- (1992): Veränderungen in den familialen Lebensbedingungen Jugendlicher seit der Jahrhundertwende. Eine Analyse auf der Basis retrospektiver Daten. In: Zeitschrift für Sozialisationsforschung und Erziehungssoziologie 12/2, 99-113.

- (1995): Autoritarismus und Familie - Zum Wandel familialer Sozialisationsbedingungen Jugendlicher in Ost- und Westdeutschland. In: Reuband, K.-H./Pappi, F. U./Best, H. (Hg.): Die deutsche Gesellschaft in vergleichender Perspektive. Opladen. 221-242.

- (1997): Aushandeln statt Gehorsam. Erziehungsziele und Erziehungspraktiken in den alten und neuen Bundesländern im Wandel. In: Böhnisch, L./Lenz, K. (Hg.): Familien. Eine interdisziplinäre Einführung. Weinheim, München. 129-153.

Reulecke, J. (2003): Einführung: Lebensgeschichten des 20. Jahrhunderts - im „Generationencontainer"? In: Reulecke, J. (Hg.): Generationalität und Lebensgeschichte im 20. Jabrbundert. München. VII-XV.

Roseann, G./Silverstein, M. (1996): Familiy Complexity and the Grandparent Role. In: Generations 20/1, o.S.. http://search. ebscohost. com/login. aspx?direct= true\&db=pbh\&AN=9605072138\&site=ehost-live (Zugriff: 08/2011).

Rosenbaum, H. (1978): Einleitung. In: Rosenbaum, H. (Hg.): Seminar: Familie und Gesellschaftsstruktur. Materialien zu den sozioökonomischen Bedingungen von Familienformen. Frankfurt am Main. 9-54.

- (1982a): Die Bedeutung historischer Forschung für die Erkenntnis der Gegenwart dargestellt am Beispiel der Familiensoziologie. In: Mitterauer, M./Sieder, R. (Hg.): Historische Familienforschung. Frankfurt am Main. 40-63. 


\section{Literatur}

Rosenbaum, H. (1982b): Formen der Familie. Untersuchungen zum Zusammenhang von Familienverhältnissen, Sozialstruktur und sozialem Wandel in der deutschen Gesellschaft des 19. Jahrhunderts. Frankfurt am Main.

- (2001): Kindheitsbiographien und -autobiographien in der Sozialgeschichte von Familie und Kindheit. In: Behnken, I./Zinnecker, J. (Hg.): Kinder - Kindheit - Lebensgeschichte. Seelze-Velber. 744-757.

Rosenthal, G. (1995): Erlebte und erzählte Lebensgeschichte. Gestalt und Struktur biographischer Selbstbeschreibungen. Frankfurt a. M.

- (1997): Zur interaktionellen Konstitution von Generationen. Generationenabfolgen in Familien von 1890 bis 1970 in Deutschland. In: Mansel, J./Rosenthal, G./Tölke, A. (Hg.): Generationen-Beziehungen, Austausch und Tradierung. Opladen. 57-73.

- (2000): Historische und familiale Generationenabfolge. In: Kohli, M./Szydlik, M. (Hg.): Generationen in Familie und Gesellschaft. Opladen. 162-178.

- (2002): Introduction. Family history: Life stories. In: History of the Family 7/2, $175-182$.

- (2005): Interpretative Sozialforschung. Eine Einführung. Weinheim, München.

- (2009): Die Biographie im Kontext der Familien- und Gesellschaftsgeschichte. In: Völter, B. u.a. (Hg.): Biographieforschung im Diskurs. Wiesbaden. 46-64.

Schacter, D. L. (1999): Wir sind Erinnerung. Gedächtnis und Persönlichkeit. Reinbek bei Hamburg.

Schäfer, J. (2013): Ökumenisches Heiligenlexikon. 2. Vatikanisches Konzil. In: http: // www . heiligenlexikon.de/Glossar/2__Vatikanisches_Konzil.htm (Zugriff: 01/2013).

Schäfers, B. (2004): Sozialstruktur und sozialer Wandel in Deutschland. Stuttgart.

Schaffner, B. (1949): Father Land. New York.

Schaub, H./G., Zenke K. (1995): Wörterbuch zur Pädagogik. München.

Scheller, G. (2005): Die Wende als Individualisierungsschub? Umfang, Richtung und Verlauf des Individualisierungsprozesses in Ostdeutschland. Wiesbaden.

Schlegel, U. (1999): Geschlechter- und Frauenforschung. In: Friedrich, W./Förster, P./ Starke, K. (Hg.): Das Zentralinstitut für Jugendforschung Leipzig 1966-1990. Geschichte, Methoden, Erkenntnisse. Berlin. 373-395.

Schleiermacher, F. D. E. (2000/1826): Texte zur Pädagogik. Kommentierte Studienausgabe. Band 2. Herausgegeben von Winkler, M. und Brachmann, J. Frankfurt am Main.

Schmid, M. (2010): Erziehungsratgeber und Erziehungswissenschaft. Zur Theorie-Praxis-Problematik populärpädagogischer Schriften. Bad Heilbrunn.

Schmidt, G. R. (1970): Tradition. In: Horney, W./Ruppert, J.P./Schultze, W. (Hg.): Pädagogisches Lexikon in zwei Bänden. Zweiter Band K - Z. Gütersloh. 1202-1203.

Schmidtchen, G. (1997): Wie weit ist der Weg nach Deutschland? Sozialpsychologie der Jugend in der postsozialistischen Welt. Opladen. 
Schmidt-Lauber, B. (2001): Das qualitative Interview oder: Die Kunst des Reden-Lassens. In: Göttsch, S./Lehmann, A. (Hg.): Methoden der Volkskunde. Positionen, Quellen, Arbeitsweisen der Europäischen Ethnologie. Berlin. 165-186.

Schneewind, K. A. (1980): Elterliche Erziehungsstile: einige Anmerkungen zum Forschungsgegenstand. In: Schneewind, K. A./Herrmann, T. (Hg.): Erziebungsstilforschung. Theorien, Methoden und Anwendung in der Psychologie elterlichen Erziebungsverhaltens. Bern, Stuttgart, Wien. 19-30.

- (1992): Familien zwischen Rhetorik und Realität: eine familienpsychologische Perspektive. In: Schneewind, K. A./Rosenstiel, L. v. (Hg.): Wandel der Familie. Göttingen, Toronto, Zürich. 9-35.

- (1996): Gesellschaftliche Veränderungswahrnehmung und Wandel des elterlichen Erziehungsstils im Generationenvergleich. In: Buba, H. P./Schneider, N. F. (Hg.): Familie. Zwischen gesellschaftlicher Prägung und individuellem Design. Opladen. 117-128.

- (2000): Kinder und elterliche Erziehung. In: Lange, A./Lauterbach, W. (Hg.): Kinder in Familie und Gesellschaft zu Beginn des 21. Jabrbunderts. Stuttgart. 187-208.

- (2010): Familienpsychologie. Stuttgart.

Schneewind, K.A./Ruppert, S. (1995): Familien gestern und heute: ein Generationenvergleich über 16 Jahre. München.

Schönpflug, U. (2001): Introduction: Cultural Transmission - A Multidisciplinary Research Field. In: Journal of Cross-Cultural Psychology 32/2, 131-134. http : / / jcc . sagepub . com/content/32/2/131 (Zugriff: 08/2011).

- (2009): Theory and Research in Cultural Transmission: A Short History. In: Schönpflug, U. (Hg.): Cultural Transmission. Psychological, Developmental, Social, and Methodological Aspects. Cambridge u.a. 9-30.

Schönpflug, U./Bilz, L. (2001): Intergenerational Transmission of Values: The Role of Transmission Belts. In: Journal of Cross-Cultural Psychology 32/2, 174-185. http: // jcc. sagepub.com/content/32/2/174 (Zugriff: 08/2011).

- (2009): The Transmission Process: Mechanisms and Contexts. In: Schönpflug, U. (Hg.): Cultural Transmission. Psychological, Developmental, Social, and Methodological Aspects. Cambridge u.a. 212-239.

Schröder, H. J. (2005): Topoi des autobiographischen Erzählens. In: Hengartner, T./ Schmidt-Lauber, B. (Hg.): Leben - Erzäblen. Beiträge zur Erzäbl- und Biographieforschung. Berlin, Hamburg. 17-42.

Schülein, J. A. (1990): Die Geburt der Eltern. Über die Entstehung der modernen Elternposition und den Prozeß ihrer Aneignung und Vermittlung. Opladen.

Schütz, A. (2004/1953): Common-Sense und wissenschaftliche Interpretation menschlichen Handelns. In: Strübing, J./Schnettler, B. (Hg.): Methodologie interpretativer Sozialforschung. Klassische Grundlagentexte. Konstanz. 157-197.

Schütze, F. (1987): Das narrative Interview in Interaktionsfeldstudien I. Kurseinheit 1. Kurs. Hagen. 


\section{Literatur}

Schütze, Y. (2000): Wandel der Mutterrolle - Wandel der Familienkindheit? In: Herlth, A. u.a. (Hg.): Spannungsfeld Familienkindheit. Neue Anforderungen, Risiken und Chancen. Opladen. 92-105.

- (2002): Zur Veränderung im Eltern-Kind-Verhältnis seit der Nachkriegszeit. In: NaveHerz, R. (Hg.): Kontinuität und Wandel der Familie in Deutschland. Eine zeitgeschichtliche Analyse. Stuttgart. 71-97.

Schütze, Y./Geulen, D. (1983): Die „Nachkriegskinder“ und die „Konsumkinder“: Kindheitsverläufe zweier Generationen. In: Preuss-Lausitz, U. u.a. (Mitverf.): Kriegskinder, Konsumkinder, Krisenkinder. Zur Sozialisationsgeschichte seit dem Zweiten Weltkrieg. Weinheim, Basel. 29-52.

Schuman, H./Scott, J. (1989): Generations and Collective Memories. In: American Sociological Review 54/3, 359-381.

Schwab, U. (2007): Religion. In: Ecarius, J. (Hg.): Handbuch Familie. Wiesbaden. 500-517.

Schwartz, S. (1992): Universals in the Content and Structur of Values: Theoretical Advances and Empirical Tests in 20 Countries. In: Zanna, M. P. (Hg.): Advances in Experimental Social Psychology. Volume 25. San Diego u.a. 1-65.

- (1996): Value Priorities and Behavior: Applying a Theory of Integrated Value Systems. In: Seligman, C./Olson, J. M./Zanna, M. P. (Hg.): The Psychology of Values: The Ontario Symposium, Volume 8. Mahwah, N.J. 1-24.

Schwenk, B. (1996): Erziehung. In: Lenzen, D. (Hg.): Pädagogische Grundbegriffe. Band 1. Reinbek bei Hamburg. 429-439.

Seifert, M. (1969): Antiautoritäre Erziehung. In: Fraiberg, S. H. (Hg.): Das verstandene Kind. Hamburg. 305-317.

Shils, E. (1981): Tradition. Chicago.

Sieder, R. (1987): Sozialgeschichte der Familie. Frankfurt am Main.

Siegel, D. J. (2006): Entwicklungspsychologische, interpersonelle und neurobiologische Dimensionen des Gedächtnisses. Ein Überblick. In: Welzer, H./Markowitsch, H. J. (Hg.): Warum Menschen sich erinnern können. Fortschritte der interdisziplinären Gedächtnisforschung. Stuttgart. 19-49.

Sommerkorn, I. N./Liebsch, K. (2002): Erwerbstätige Mütter zwischen Beruf und Familie: Mehr Kontinuität als Wandel. In: Nave-Herz, R. (Hg.): Kontinuität und Wandel der Familie in Deutschland. Eine zeitgeschichtliche Analyse. Stuttgart. 99-130.

Statistisches Bundesamt Deutschland (2010): Alleinerziehende in Deutschland. Ergebnisse des Mikrozensus 2009. Wiesbaden.

- (2011a): Bevölkerung. In: https : / / www . destatis . de / DE / ZahlenFakten / Indikatoren/LangeReihen/Bevoelkerung/lrbev06 .html (Zugriff: 03/2011).

- (2011b): Geburtenentwicklung. In: http://www. destatis.de/jetspeed/portal/ cms / Sites / destatis / Internet/DE/Content/Statistiken/Bevoelkerung/ AktuellGeburtenentwicklung, templateId=renderPrint . psml\#Geburtenziffer (Zugriff: 03/2011). 
Stecher, L./Zinnecker, J. (2007): Kulturelle Transferbeziehungen. In: Ecarius, J. (Hg.): Handbuch Familie. Wiesbaden. 389-405.

Steiner, A. (2005): Zwischen Wirtschaftswundern, Rezession und Stagnation. Deutschdeutsche Wirtschaftsgeschichte 1945 bis 1989. In: Kleßmann, C./Lautzas, P. (Hg.): Teilung und Intergration. Die doppelte deutsche Nachkriegsgeschichte. Bonn. 177-191.

Sünkel, W. (1996): Der pädagogische Generationenbegriff. Schleiermacher und die Folgen. In: Liebau, E./Wulf, C. (Hg.): Generation. Versuche über eine pädagogisch-anthropologische Grundbedingung. Weinheim. 280-285.

- (1997): Generation als pädagogischer Begriff. In: Liebau, E. (Hg.): Das Generationenverhältnis. Über das Zusammenleben in Familie und Gesellschaft. Weinheim, München. 195-204.

Szydlik, M. (2000): Lebenslange Solidarität? Generationenbeziehungen zwischen erwachsenen Kindern und Eltern. Opladen.

Tenorth, H.-E. (2010): Geschichte der Erziehung. Einführung in die Grundzüge ihrer neuzeitlichen Entwicklung. Weinheim, München.

Tenorth, H.-E./Tippelt, R. (Hg.) (2007): Beltz Lexikon Pädagogik. Weinheim, Basel.

Thome, H. (2005): Wertewandel in Europa aus Sicht der empirischen Sozialforschung. In: Joas, H./Wiegandt, K. (Hg.): Die kulturellen Werte Europas. Frankfurt am Main. 386-443.

Thon, C. (2010): Das Erbe der Emanzipation? Bildungsaufstiege von Frauen über drei Generationen. In: Müller, H.-R./Ecarius, J./Herzberg, H. (Hg.): Familie, Generation, Bildung. Beiträge zur Erkundung eines informellen Lernfeldes. Opladen, Farmington Hills. 149-165.

Troll, L./Bengston, V. (1979): Generations in the Family. In: Burr, W. R. (Hg.): Contemporary theories about the family. Volume I. New York. 127-161.

Tulving, E. (2006): Das episodische Gedächtnis: Vom Geist zum Gehirn. In: Welzer, H./Markowitsch, H. J. (Hg.): Warum Menschen sich erinnern können. Fortschritte der interdisziplinären Gedächtnisforschung. Stuttgart. 50-77.

Tyrell, H. (1988): Ehe und Familie - Institutionalisierung und Deinstitutionalisierung. In: Lüscher, K./Schultheis, F./Wehrspaun, M. (Hg.): Die „postmoderne Familie“. Familiale Strategien und Familienpolitik in einer Übergangszeit. Konstanz. 145-156.

Uhlendorff, H. (2003): Großeltern und Enkelkinder: Sozialwissenschaftliche Perspektiven und Forschungsergebnisse hinsichtlich einer selten untersuchten Beziehung. In: Psychologie in Erziebung und Unterricht 50, 111-128.

- (2004): After the Wall: Parental attitudes to child rearing in East and West Germany. In: International Journal of Behavioral Development 28/1, 71-82.

- (2009): Grosseltern und Enkelkinder. In: http : / / www . bagso . de / fileadmin / Aktuell/Aktivitaeten/Grosseltern_BAGSO_Prof_Uhlendorff .pdf (Zugriff: 17. 12. 2009).

Vester, M. u.a. (1993): Soziale Milieus im gesellschaftlichen Strukturwandel. Zwischen Integration und Ausgrenzung. Köln. 


\section{Literatur}

Vierzigmann, G./Kreher, S. (1998): „Zwischen den Generationen“ - Familiendynamik und Familiendiskurse in biographischen Erzählungen. In: Berliner Journal für Soziologie 8/1, 23-37.

Wachsmuth, I. (2008): NS-Vergangenheit in Ost und West. Berlin.

Wahl, K. (1997): Familienbilder und Familienrealität. In: Böhnisch, L./Lenz, K. (Hg.): Familien. Eine interdisziplinäre Einfübrung. Weinheim, München. 99-112.

- (2006): Soziale Gebrauchsweisen von Informationsquellen am Bildungsort Familie. Die familialen Voraussetzungen von information literacy in ihrer Wechselwirkung mit anderen Bildungsorten. In: Büchner, P./Brake, A. (Hg.): Bildungsort Familie. Transmission von Bildung und Kultur im Alltag von Mehrgenerationenfamilien. Wiesbaden. 225-254.

Weigel, S. (2002): Generation, Genealogie, Geschlecht. Zur Geschichte des Generationskonzepts und seiner wissenschaftlichen Konzeptualisierung seit Ende des 18. Jahrhunderts. In: Musner, L./Wunberg, G. (Hg.): Kulturwissenschaften. Forschung - Praxis - Positionen. Wien. 161-190.

Weisbrod, B. (2005): Generation und Generationalität in der Neueren Geschichte. In: Aus Politik und Zeitgeschichte 8, 3-9.

Welzer, H. (2001): Das gemeinsame Verfertigen von Vergangenheit im Gespräch. In: Welzer, H. (Hg.): Das soziale Gedächtnis. Geschichte, Erinnerung, Tradierung. Hamburg. 160-178.

- (2002): Das kommunikative Gedächtnis. Eine Theorie der Erinnerung. München.

- (2010): Erinnerung und Gedächtnis. Desiderata und Perspektiven. In: Gudehus, C./ Eichenberg, A./Welzer, H. (Hg.): Gedächtnis und Erinnerung. Ein interdisziplinäres Handbuch. Stuttgart. 1-10.

Welzer, H./Markowitsch, H. J. (2006): Reichweiten und Grenzen interdisziplinärer Gedächtnisforschung. In: Welzer, H./Markowitsch, H. J. (Hg.): Warum Menschen sich erinnern können. Fortschritte der interdisziplinären Gedächtnisforschung. Stuttgart. 9-15.

Welzer, H./Moller, S./Tschugnall, K. (2002): „Opa war kein Nazi“. Nationalsozialismus und Holocaust im Familiengedächtnis. Frankfurt am Main.

Werder, L. v. (1972): Von der antiautoritären zur proletarischen Erziehung. Frankfurt am Main.

- (1977): Bedeutung und Entwicklung der Kinderladenbewegung in der Bundesrepublik. In: Werder, L. v. (Hg.): Was kommt nach den Kinderläden? Erlebnis-Protokolle. Berlin. 7-58.

Wilk, L. (2000): Veränderte Familienformen - postmoderne kindliche Lebenswelten? In: Herlth, A. u.a. (Hg.): Spannungsfeld Familienkindheit. Neue Anforderungen, Risiken und Chancen. Opladen. 23-46.

Wimmer, M. (1998): Fremdheit zwischen den Generationen. Generative Differenz, Generationsdifferenz, Kulturdifferenz. In: Ecarius, J. (Hg.): Was will die jüngere mit der älteren Generation? Generationenbeziehungen in der Erziehungswissenschaft. Opladen. 81-113. 
Wineburg, S. (2001): Sinn machen: Wie Erinnerung zwischen Generationen gebildet wird. In: Welzer, H. (Hg.): Das soziale Gedächtnis. Geschichte, Erinnerung, Tradierung. Hamburg. 179-204.

Winkler, M. (1998): Friedrich Schleiermacher revisited: Gelegentliche Gedanken über Generationenverhältnisse in pädagogischer Sicht. In: Ecarius, J. (Hg.): Was will die jüngere mit der älteren Generation? Generationenbeziehungen in der Erziehungswissenschaft. Opladen. 115-138.

- (2000): Erziehung. In: Krüger, H.-H./Helsper, W. (Hg.): Einfübrung in Grundbegriffe und Grundfragen der Erziehungswissenschaft. Opladen. 53-69.

Winterhager-Schmid, L. (2000): „Groß“ und „klein“ - Zur Bedeutung der Erfahrung mit Generationendifferenz im Prozeß des Heranwachsens. In: Winterhager-Schmid, L. (Hg.): Erfahrung mit Generationendifferenz. Weinheim. 15-37.

Wohlrab-Sahr, M./Karstein, U./Schmidt-Lux, T. (2009): Forcierte Säkularität. Religiöser Wandel und Generationendynamik im Osten Deutschlands. Frankfurt, New York.

Wolf, C. (1995): Religiöse Sozialisation, konfessionelle Milieus und Generation. In: Zeitschrift für Soziologie 24/5, 345-357.

- (2003): Religion und Familie in Deutschland. In: Zeitschrift für Evangelische Ethik 47, 53-71.

Wurzbacher, G. (1958): Leitbilder gegenwärtigen deutschen Familienlebens. Stuttgart.

Ziegler, M. (2000): Das Soziale Erbe. Eine soziologische Fallstudie über drei Generationen einer Familie. Wien, Köln, Weimar.

Zimbardo, P. G. (1992): Psychologie. Berlin u.a.

Zinnecker, J. (1985): Kinheit. Erziehung. Familie. In: Fischer, A./Fuchs, W./Zinnecker, J. (Hg.): Jugendliche und Erwachsene '85: Generationen im Vergleich. Band 3. Jugend der fünfziger Jahre - heute. Hg. vom Jugendwerk der Dt. Shell. Opladen. 97-292.

- (1990): Vom Straßenkind zum verhäuslichten Kind. Kindheitsgeschichte im Prozeß der Zivilisation. In: Behnken, I. (Hg.): Stadtgesellschaft und Kindheit im Prozeß der Zivilisation. Opladen. 142-162.

- (1998): Die Tradierung kultureller System zwischen den Generationen. Die Rolle der Familie bei der Vermittlung von Religion in der Moderne. In: Zeitschrift für Soziologie der Erziehung und Sozialisation 18/4, 343-356.

- (2003): „Das Problem der Generationen“. Überlegungen zu Karl Mannheims kanonischen Text. In: Reulecke, J. (Hg.): Generationalität und Lebensgeschichte im 20. Jahrbundert. München. 33-58.

- (2009): Die „transgenerationale Weitergabe“ der Erfahrung des Weltkrieges in der Familie. Der Blickwinkel der Familien-, Sozialisations- und Generationenforschung. In: Radebold, H./Bohleber, W./J., Zinnecker (Hg.): Transgenerationale Weitergabe kriegsbelasteter Kindheiten. Interdisziplinäre Studien zur Nachbaltigkeit historischer Erfabrungen über vier Generationen. Weinheim, München. 141-154.

Zinnecker, J./Georg, W. (1996): Die Weitergabe kirchlich-religiöser Familienerziehung und Orientierung zwischen Eltern- und Kindergeneration. In: Zinnecker, J./Silberei- 


\section{Literatur}

sen, R. K. (Hg.): Kindheit in Deutschland. Aktueller Survey über Kinder und ibre Eltern. Weinheim, München. 347-356.

Zinnecker, J./Hasenberg, R. (1999): Religiöse Eltern und religiöse Kinder: Die Übertragung von Religion auf die nachfolgende Generation in der Familie. In: Silbereisen, R. K./Zinnecker, J. (Hg.): Entwicklung im sozialen Wandel. Weinheim. 445-457.

Zinnecker, J./Strozda, C./Georg (1996): Kirchlich-religiöse Praxis und religiöse Erziehung. In: Zinnecker, J./Silbereisen, R. K. (Hg.): Kindheit in Deutschland. Aktueller Survey über Kinder und ibre Eltern. Weinheim, München. 331-346. 


\section{Anhang}

\subsection{Sampleübersicht}

Die personenbezogenen Angaben wurden gemäß der auf S. 78 beschriebenen Vorgehensweise verändert.

\begin{tabular}{|c|c|c|c|}
\hline Familie / Generation & Großeltern & Eltern & Kinder \\
\hline \multicolumn{4}{|l|}{ Niedersachsen } \\
\hline Dienel/Ebel & $\begin{array}{l}\text { Frau Dienel } \\
* 1939\end{array}$ & $\begin{array}{l}\text { Heike Ebel } \\
* 1965\end{array}$ & $\begin{array}{l}\text { Janina } \\
* 1991\end{array}$ \\
\hline Friese & $\begin{array}{l}\text { Herr Friese } \\
* 1928\end{array}$ & $\begin{array}{l}\text { Georg Friese } \\
* 1956\end{array}$ & $\begin{array}{l}\text { Miriam } \\
* 1992\end{array}$ \\
\hline Mahler/Ahrens & $\begin{array}{l}\text { Frau Mahler } \\
* 1938\end{array}$ & $\begin{array}{l}\text { Michelle Ahrens } \\
* 1961\end{array}$ & $\begin{array}{l}\text { Sonja } \\
* 1989\end{array}$ \\
\hline Nolte/Gümper & $\begin{array}{l}\text { Frau Nolte } \\
* 1932\end{array}$ & $\begin{array}{l}\text { Iris Gümper } \\
* 1959\end{array}$ & $\begin{array}{l}\text { Lea } \\
* 1992\end{array}$ \\
\hline Sonntag/Klein & $\begin{array}{l}\text { Frau Sonntag } \\
* 1937\end{array}$ & $\begin{array}{l}\text { Sabine Klein } \\
* 1965\end{array}$ & $\begin{array}{l}\text { Laura } \\
* 1994\end{array}$ \\
\hline \multicolumn{4}{|l|}{ Thüringen } \\
\hline Beyer/Schmidt & $\begin{array}{l}\text { Frau Beyer } \\
* 1949\end{array}$ & $\begin{array}{l}\text { Birgit Schmidt } \\
* 1971\end{array}$ & $\begin{array}{l}\text { Magdalene } \\
* 1992\end{array}$ \\
\hline Cronert/Jäger & $\begin{array}{l}\text { Herr Cronert } \\
* 1936\end{array}$ & $\begin{array}{l}\text { Friederike Jäger } \\
* 1969\end{array}$ & $\begin{array}{l}\text { Anika } \\
* 1989\end{array}$ \\
\hline Grothe & $\begin{array}{l}\text { Frau Grothe } \\
* 1929\end{array}$ & $\begin{array}{l}\text { Frank Grothe } \\
* 1960\end{array}$ & $\begin{array}{l}\text { Hannah } \\
* 1991\end{array}$ \\
\hline Siebert/Hildner & $\begin{array}{l}\text { Frau Siebert } \\
* 1923\end{array}$ & $\begin{array}{l}\text { Gisela Hildner } \\
* 1953\end{array}$ & $\begin{array}{l}\text { Björn } \\
* 1979\end{array}$ \\
\hline Weber/Groß & $\begin{array}{l}\text { Frau Weber } \\
* 1924\end{array}$ & $\begin{array}{l}\text { Petra Groß } \\
* 1951\end{array}$ & $\begin{array}{l}\text { Silke } \\
* 1977\end{array}$ \\
\hline
\end{tabular}




\subsection{Transkripitionsregeln}

Die Transkription folgt in weiten Teilen den von Bohnsack (2007, 235) aufgestellten Regeln. Um eine gute Lesbarkeit zu gewährleisten, wurden sprachliche Überlappungen nicht kenntlich gemacht und die Interpunktion orientiert sich an der Schriftsprache.

\begin{tabular}{|c|c|}
\hline Zeichen & Bedeutung \\
\hline (.) & Pause bis zu einer Sekunde \\
\hline (3) & Anzahl der Sekunden \\
\hline ABER & betont gesprochenes Wort \\
\hline${ }^{\circ}$ aber ${ }^{\circ}$ & leise gesprochenes Wort \\
\hline jedo- & Abbruch eines Wortes \\
\hline $\mathrm{ja}=\mathrm{bitte}$ & $\begin{array}{l}\text { Wortverschleifung, schneller } \\
\text { Wortanschluss }\end{array}$ \\
\hline a::ber & $\begin{array}{l}\text { Dehnung eines Wortes; Anzahl der : } \\
\text { verweist } \\
\text { auf die Länge des gedehnten Wortes }\end{array}$ \\
\hline (ich) & unsichere Transkription \\
\hline$(\ldots)$ & $\begin{array}{l}\text { unverständliche Passage, Anzahl der } \\
\text { verweist auf deren Dauer }\end{array}$ \\
\hline @aber@ & lachend gesprochenes Wort \\
\hline$@() @$. & kurzes Lachen \\
\hline$@(4) @$ & Lachen, das vier Sekunden dauert \\
\hline$(($ und dann $($ stockend $)))$ & Kommentar zur Sprechweise \\
\hline
\end{tabular}


D ie Frage, welche Inhalte in welcher Form im Generationenverlauf innerfamilial tradiert werden, stellt ein periodisch wiederkehrendes und öffentlich verhandeltes Diskussionsthema dar. In dieser qualitativ angelegten Studie werden familiale Vermittlungs- und Aneignungsprozesse in einer Drei-GenerationenPerspektive unter Berücksichtigung der innerfamilialen Bearbeitung gesellschaftlicher Entwicklungsprozesse in zwei benachbarten Teilregionen untersucht. Hierbei gilt die Aufmerksamkeit nicht nur den Kontinuitäten, sondern auch den Anpassungen und Veränderungen von Formen und Inhalten familialer Tradierung. Die Verknüpfung einer generationellen Perspektive mit Halbwachs' Überlegungen zum kollektiven Gedächtnis eröffnet dabei mehrere Vergleichsoptionen. Neben der Erfassung familialer, generationeller und regionaler Gemeinsamkeiten und Unterschiede können auf diese Weise die Dynamiken familialer Tradierung herausgearbeitet werden.

\section{Universitätsverlag Göttingen}

